\title{
Regio- and Enantioselective Bromocyclization of Difluoroalkenes as a Strategy to Access Tetrasubstituted Difluoromethylene Containing Stereocenters
}

Authors: Edward Miller, Suhong Kim, Katarina Gibson, Jeffrey S. Derrick, F. Dean Toste*

Affiliations: Department of Chemistry, University of California, Berkeley, California 94720

*Correspondence to: fdtoste@ berkeley.edu

Table of Contents

$\begin{array}{ll}\text { General Information } & \text { S2 }\end{array}$

$\begin{array}{ll}\text { Synthesis of Brominating Agents } & \text { S3 }\end{array}$

$\begin{array}{lr}\text { Synthesis of Difluoroalkene Substrates } & \text { S3 }\end{array}$

$\begin{array}{lr}\text { Synthesis of } \mathrm{CF}_{2} \mathrm{Br} \text { Containing Heterocycles } & \text { S24 }\end{array}$

$\begin{array}{lr}\text { Derivatization of } \mathrm{CF}_{2} \mathrm{Br} \text { Heterocycles } & \mathrm{S} 39\end{array}$

$\begin{array}{lr}\text { Crystallographic Data } & \text { S47 }\end{array}$

$\begin{array}{ll}\text { References } & \text { S51 }\end{array}$

$\begin{array}{lr}\text { Chiral HPLC Traces } & \text { S53 }\end{array}$

$\begin{array}{lr}\text { NMR Spectra } & \text { S88 }\end{array}$ 


\section{General Information}

Unless otherwise noted, reagents were obtained from commercial sources and used without further purification. All reactions were carried out under $\mathrm{N}_{2}$ using Schlenk line techniques, unless otherwise stated. Dry and degassed tetrahydrofuran (THF), dichloromethane (DCM), diethyl ether, toluene, triethylamine, and dimethylformamide (DMF) were obtained by passage through activated alumina columns under argon. All other dried solvents were obtained by storage over $3 \AA$ or $4 \AA$ molecular sieves overnight. TLC analysis of reaction mixtures was performed on Merck silica gel 60 F254 TLC plates and visualized by UV and/or $\mathrm{KMnO}_{4}$. Preparative TLC was carried out on the same plates. Flash chromatography was carried out with Fischer Silica Gel $60 \AA$ А, 230 X 400 mesh. Nuclear magnetic resonance (NMR) spectra were recorded with Bruker AVQ-400, NEO-500, and AV-600 spectrometers. ${ }^{1} \mathrm{H}$ and ${ }^{13} \mathrm{C}$ chemical shifts are reported in ppm downfield of tetramethylsilane and referenced to residual solvent peak $\left(\mathrm{CDCl}_{3}: \delta \mathrm{H}=7.26 \mathrm{ppm}\right.$ and $\delta \mathrm{C}=$ $77.16 \mathrm{ppm} ; \mathrm{CD}_{2} \mathrm{Cl}_{2}: \delta \mathrm{H}=5.32 \mathrm{ppm}$ and $\delta \mathrm{C}=54.00 \mathrm{ppm} ; \mathrm{CD}_{3} \mathrm{NO}_{2}: \delta \mathrm{H}=4.33$ ppm, DMSO- $d_{6}$ : $\delta \mathrm{H}=2.50 \mathrm{ppm}$ and $\delta \mathrm{C}=39.5 \mathrm{ppm}$ ). Multiplicities are reported using the following abbreviations: $\mathrm{s}=$ singlet, $\mathrm{d}=$ doublet, $\mathrm{t}=$ triplet, $\mathrm{q}=$ quartet, $\mathrm{p}=$ pentet, $\mathrm{m}=$ multiplet, $\mathrm{bs}=$ broad singlet, $\mathrm{dd}=$ doublet of doublet, $\mathrm{dt}=$ doublet of triplet, $\mathrm{dq}=$ doublet of quartet, $\mathrm{td}=$ triplet of doublet, $\mathrm{tt}=$ triplet of triplet, $\mathrm{qt}=$ quartet of triplet, $\mathrm{qq}=$ quartet of quartet, $\mathrm{ddd}=$ doublet of doublet of doublet, $\mathrm{ddt}=$ doublet of doublet of triplet, dddt $=$ doublet of doublet of doublet of triplet resonance. Enantioselectivity was determined by chiral HPLC using Daicel OD-H, OJ-H, IA, IB, and IC columns (0.46 x $25 \mathrm{~cm})$. High Resolution Mass Spectra (HRMS) were obtained with a Perkin Elmer UHPLC-TOF operated by the Catalysis Center in the College of Chemistry, University of California, Berkeley (ESI) and from QB3/Chemistry Mass Spectrometry Facility (EI). X-Ray crystallographic analysis was carried at the College of Chemistry X-Ray Crystallographic Facility (CHEXRAY, University of California, Berkeley). Melting points measured on a Vernier Melt Station. 


\section{Synthesis of Bromination Reagents}

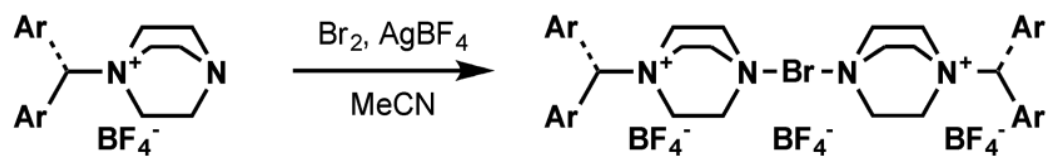

Novel brominating agents were prepared according to procedure for synthesizing $\left[\left(\mathbf{D A B}^{3}\right)_{2} \mathbf{B r}\right]\left(\mathbf{B F}_{4}\right)_{3}$ and $\left[\left(\mathbf{D A B}^{\mathbf{4}}\right)_{\mathbf{2}} \mathbf{B r}\right]\left(\mathbf{B F}_{4}\right)_{3} .{ }^{1}$ To a solution of parent DABCOnium $(2.0 \mathrm{mmol}, 1$ equiv.) in $70 \mathrm{~mL}$ of $\mathrm{MeCN}$, was added $210 \mathrm{mg}$ of $\mathrm{AgBF}_{4}$ (1.1 mmol, 0.55 equiv.). After covering the flask with aluminum foil to prevent exposure to light, $60 \mu \mathrm{L}$ of $\mathrm{Br}_{2}$ (1.2 mmol, 0.62 equiv.) was added, where an immediate precipitation of a yellow solid was observed. The solution was allowed to stir for $6 \mathrm{~min}$, after which the solution was filtered through Celite ${ }^{\circledR}$ into a mixture of $300 \mathrm{~mL}$ of 1:1 DCM/hexanes. The resulting suspension was placed into a $-20{ }^{\circ} \mathrm{C}$ freezer for 1 hour, and then vacuum filtered to yield brominating agents as white solids.

$\left[\left(\mathbf{D A B}^{1}\right)_{2} \mathbf{B r}\right]\left(\mathbf{B F}_{4}\right)_{3}: \mathrm{Ar}=\mathrm{Ph}$

${ }^{1} \mathbf{H}$ NMR $\left(600 \mathrm{MHz}, \mathrm{CD}_{3} \mathrm{NO}_{2}\right) \delta 7.59(\mathrm{t}, J=7.2 \mathrm{~Hz}, 2 \mathrm{H}), 7.56-7.49(\mathrm{~m}, 8 \mathrm{H}), 4.61(\mathrm{~s}, 4 \mathrm{H}), 3.78$ (ddt, $J=15.0,10.3,6.4 \mathrm{~Hz}, 24 \mathrm{H})$.

$\left[\left(\mathbf{D A B}^{2}\right)_{2} \mathbf{B r}\right]\left(\mathbf{B F}_{4}\right)_{3}: \mathrm{Ar}=3,5-t \mathrm{Bu}_{2} \mathrm{Ph}$

${ }^{1} \mathbf{H}$ NMR $\left(600 \mathrm{MHz}, \mathrm{CD}_{3} \mathrm{NO}_{2}\right) \delta 7.74(\mathrm{t}, J=1.8 \mathrm{~Hz}, 2 \mathrm{H}), 7.39(\mathrm{~d}, J=1.8 \mathrm{~Hz}, 4 \mathrm{H}), 4.69(\mathrm{~s}, 4 \mathrm{H})$, $3.92-3.80(\mathrm{~m}, 24 \mathrm{H}), 1.33(\mathrm{~s}, 36 \mathrm{H})$.

$\left[\left(\mathbf{D A B}^{\mathbf{5}}\right)_{2} \mathbf{B r}\right]\left(\mathbf{B F}_{4}\right)_{3}: \mathrm{Ar}=\left(3,4,5-\mathrm{F}_{3} \mathrm{Ph}\right)_{2}$

${ }^{1} \mathbf{H}$ NMR $\left(600 \mathrm{MHz}, \mathrm{CD}_{3} \mathrm{NO}_{2}\right) \delta 7.66-7.47(\mathrm{~m}, 8 \mathrm{H}), 5.71(\mathrm{~s}, 2 \mathrm{H}), 3.91-3.69(\mathrm{~m}, 24 \mathrm{H})$.

\section{Synthesis of Difluoroalkene Substrates}<smiles>Brc1cnc(N2CCOCC2)nc1</smiles><smiles>O=C(O)c1cnc(N2CCOCC2)nc1</smiles><smiles>C[C@H](OC(=O)[O-])C(C)(C)C</smiles><smiles>ClC(Cl)(Cl)c1cnc(N2CCOCC2)nc1</smiles>

2-morpholinopyrimidine-5-carboxylic acid (S1): To a solution of $1.7 \mathrm{~g}$ of 4-(5bromopyrimidin-2-yl)morpholine ${ }^{2}\left(6.9 \mathrm{mmol}, 1.0\right.$ equiv.) in $35 \mathrm{~mL}$ of anhydrous $\mathrm{THF}$ at $-78{ }^{\circ} \mathrm{C}$, was added $3.2 \mathrm{~mL}$ of an $n \mathrm{BuLi}$ solution $(2.5 \mathrm{M}$ in hexanes, $7.6 \mathrm{mmol}, 1.1$ equiv.) dropwise. After allowing the solution to stir for 30 min at this temperature, gaseous $\mathrm{CO}_{2}$ was bubbled through the solution for $20 \mathrm{~min}$. After stirring an additional hour, the solution was opened to air and allowed 
to warm to room temperature. The reaction was then acidified with $20 \mathrm{~mL}$ of aqueous $1 \mathrm{M} \mathrm{HCl}$ and diluted with $40 \mathrm{~mL}$ of DCM. The layers were separated, and the aqueous layer was extracted thrice with $50 \mathrm{~mL}$ of DCM each time. The combined organic layers were combined, dried with $\mathrm{Na}_{2} \mathrm{SO}_{4}$, and the solvent was removed under reduced pressure, yielding $570 \mathrm{mg}$ (39\%) of the title compound as a white solid, which was used without further purification.

${ }^{1}$ H NMR (600 MHz, DMSO-d $) \delta 12.84$ (bs, 1H), 8.78 (s, 2H), 3.85 - 3.80 (m, 4H), 3.69 - 3.64 $(\mathrm{m}, 4 \mathrm{H})$.

${ }^{13}$ C NMR (151 MHz, DMSO- $\left.d_{6}\right) \delta 165.4,161.8,159.6,158.0,65.9,44.0$.

HRMS (ESI+): calcd. for $\left[\mathrm{C}_{9} \mathrm{H}_{11} \mathrm{~N}_{3} \mathrm{O}_{3}+\mathrm{MeCN}+\mathrm{H}\right]^{+}:$251.1138, found: 251.1125.

MP: $>240{ }^{\circ} \mathrm{C}$

2-morpholinopyrimidine-5-carbonyl chloride•HCI (S2): To a solution of $500 \mathrm{mg}$ of $\mathbf{S 1}$ (2.4 mmol, 1.0 equiv.) in $12 \mathrm{~mL}$ of dry $\mathrm{DCM}$ at $0{ }^{\circ} \mathrm{C}$, was added 5 drops of DMF and $0.21 \mathrm{~mL}$ of oxalyl chloride (2.4 mmol, 1 equiv.) dropwise. After stirring for 1 hour, the solution was allowed to warm to room temperature and stirred for an additional 2 hours. The solvent was then removed under reduced pressure and the resulting salt was used in the next step without further purification.
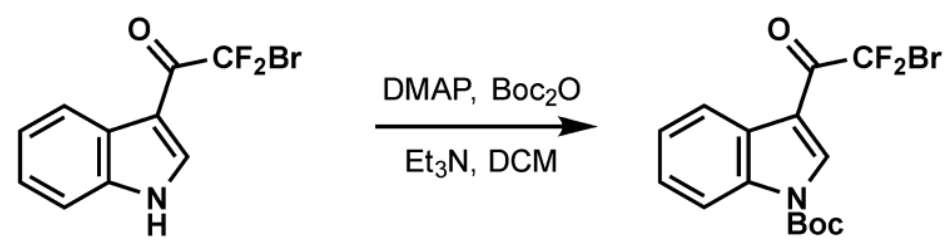

N-Boc-3-(2-bromo-2,2-difluoroacetyl)-indole (S3): To a solution of $1.1 \mathrm{~g}$ of 3(bromodifluoroacetyl)-indole ${ }^{3}(5.0 \mathrm{mmol}, 1.0$ equiv.) and $61 \mathrm{mg}$ of DMAP $(0.50 \mathrm{mmol}, 10 \mathrm{~mol} \%)$ in $120 \mathrm{~mL}$ of anhydrous DCM, $1.3 \mathrm{~mL}$ of $\mathrm{Boc}_{2} \mathrm{O}$ (5.5 mmol, 1.1 equiv.) was added, followed by $2.1 \mathrm{~mL}$ of $\mathrm{Et}_{3} \mathrm{~N}$ (15 mmol, 3.0 equiv.). The solution was stirred overnight, after which $40 \mathrm{~mL}$ of $\mathrm{H}_{2} \mathrm{O}$ was slowly added. The aqueous and organic layers were separated, and the aqueous layer was extracted with $50 \mathrm{~mL}$ of additional DCM. The organic layers were combined, dried with $\mathrm{Na}_{2} \mathrm{SO}_{4}$, and the solvent was removed under reduced pressure. The crude product was purified by flash chromatography (1:9 EtOAc/Hexanes) to yield $1.7 \mathrm{~g}$ (90\%) of N-Boc-3-(2-bromo-2,2difluoroacetyl)-indole as a colorless white solid.

${ }^{1} \mathbf{H}$ NMR $\left(500 \mathrm{MHz}, \mathrm{CDCl}_{3}\right) \delta 8.50(\mathrm{t}, J=2.0 \mathrm{~Hz}, 1 \mathrm{H}), 8.41-8.33(\mathrm{~m}, 1 \mathrm{H}), 8.20-8.13(\mathrm{~m}, 1 \mathrm{H})$, $7.49-7.38(\mathrm{~m}, 2 \mathrm{H}), 1.73(\mathrm{~s}, 9 \mathrm{H})$.

${ }^{13} \mathrm{C}$ NMR $\left(126 \mathrm{MHz}, \mathrm{CDCl}_{3}\right) \delta 178.1(\mathrm{t}, J=27.1 \mathrm{~Hz}), 148.7,136.4-134.2(\mathrm{~m}), 127.9,126.5$, $125.3,122.7,115.4,113.9(\mathrm{t}, J=318.3 \mathrm{~Hz}), 111.4,86.6,28.2$. (One missing resonance)

${ }^{19}$ F NMR $\left(470 \mathrm{MHz}, \mathrm{CDCl}_{3}\right) \delta-58.1$ (s).

HRMS (EI+): calcd. For $\left[\mathrm{C}_{14} \mathrm{H}_{14} \mathrm{BrF}_{2} \mathrm{NO}_{3}\right]^{+}: 373.0125$, found: 373.0128 .

MP: $124.2-124.9^{\circ} \mathrm{C}$ 

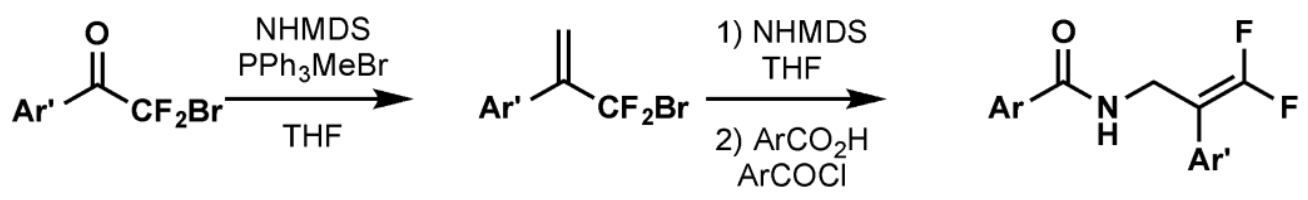

1a-j

1a-j: To a solution of $2.5 \mathrm{~g}$ of $\mathrm{PPh}_{3} \mathrm{MeBr}(7.0 \mathrm{mmol}, 1.4$ equiv.) in $55 \mathrm{~mL}$ of anhydrous THF at $-78^{\circ} \mathrm{C}, 3.5 \mathrm{~mL}$ of an NHMDS solution (2M in THF, $7 \mathrm{mmol}, 1.4$ equiv.) was added dropwise. After complete addition, the solution was warmed to $0{ }^{\circ} \mathrm{C}$ and stirred for an hour. A solution of the corresponding bromodifluoro acetophenone ${ }^{4}(5 \mathrm{mmol}, 1$ equiv.) in $4 \mathrm{~mL}$ of anhydrous THF was then added dropwise. After complete addition, the reaction was allowed to warm to room temperature, and stirred for an additional hour. After this, the reaction was quenched with $10 \mathrm{~mL}$ of aqueous $1 \mathrm{M} \mathrm{HCl}$, and diluted with $50 \mathrm{~mL}$ of $\mathrm{Et}_{2} \mathrm{O}$. The aqueous and organic layers were separated, and the aqueous layer was extracted with $20 \mathrm{~mL}$ of additional ether. The organic layers were combined, dried with $\mathrm{Na}_{2} \mathrm{SO}_{4}$, and the solvent was removed under reduced pressure. To the residue was added a minimal amount of DCM to dissolve the oil, then the solution was passed through a silica plug with 1:9 EtOAc/hexane as eluent. The solvent was removed under reduced pressure, and the resulting residue was purified once again by silica plug with 1/9 EtOAc/hexane as eluent. The solvent was then removed under reduced pressure, and the resulting oil was used in the next step without further purification.

To a stirred solution of allylic $\mathrm{CF}_{2} \mathrm{Br}$ ( $1 \mathrm{mmol}, 1$ equiv.) in dry $\mathrm{THF}$ ( $5 \mathrm{~mL}$ ), was added $0.60 \mathrm{~mL}$ of an NHMDS solution (2M in THF, $1.2 \mathrm{mmol}, 1.2$ equiv.) over $1 \mathrm{~min}$. After addition, the solution was heated to $40{ }^{\circ} \mathrm{C}$ and stirred overnight. After cooled to room temperature, the reaction was quenched with $\mathrm{Ar}_{1} \mathrm{CO}_{2} \mathrm{H}$ (1.1 mmol, 1.1 equiv.), where a solid precipitate is immediately observed. To this slurry, $\mathrm{Ar}_{1} \mathrm{COCl}$ (2.4 mmol, 2.4 equiv.) was added dropwise over $1 \mathrm{~min}$. After stirring for an additional 2 hours at ambient temperature, the reaction is quenched with saturated $\mathrm{NaHCO}_{3}(10 \mathrm{~mL})$ and diluted with $20 \mathrm{~mL}$ of diethyl ether. The aqueous and organic layers were separated, and the aqueous layer was extracted with $20 \mathrm{~mL}$ of diethyl ether. The organic layers were combined, dried with $\mathrm{Na}_{2} \mathrm{SO}_{4}$, and the solvent was removed under reduced pressure. The crude product was purified by flash chromatography ( $\mathrm{Et}_{2} \mathrm{O} /$ pentanes) to yield $\mathbf{1}$ as a colorless white solid. Trace benzoyl chlorides were removed from purified product by dissolving the mixture in $30 \mathrm{~mL} \mathrm{Et}_{2} \mathrm{O}$, and washing with $25 \%$ aqueous ammonia ( $3 \times 20 \mathrm{~mL}$ ). After drying of the organic layer with $\mathrm{Na}_{2} \mathrm{SO}_{4}$, the solvent was removed under reduced pressure to yield pure 1aj. 
<smiles>Cc1ccc(C(CNC(=O)c2ccccc2)=C(F)F)cc1</smiles>

1a: Title compound was synthesized by following the general procedure, yielding $145 \mathrm{mg}$ of $\mathbf{1 a}$ as a white solid (50\% yield over two steps).

${ }^{1} \mathbf{H}$ NMR $\left(500 \mathrm{MHz}, \mathrm{CDCl}_{3}\right) \delta 7.64(\mathrm{dt}, J=7.0,1.4 \mathrm{~Hz}, 2 \mathrm{H}), 7.47(\mathrm{tt}, J=7.4,1.4 \mathrm{~Hz}, 1 \mathrm{H}), 7.41-$ $7.36(\mathrm{~m}, 2 \mathrm{H}), 7.30$ (d, $J=7.8 \mathrm{~Hz}, 2 \mathrm{H}), 7.19$ (d, $J=7.9 \mathrm{~Hz}, 2 \mathrm{H}), 6.04$ (bs, 1H), 4.49 (dt, $J=5.3,2.4$ $\mathrm{Hz}, 2 \mathrm{H}), 2.34$ (s, 3H).

${ }^{13}$ C NMR (126 MHz, $\left.\mathrm{CDCl}_{3}\right) \delta 167.5,154.7$ (dd, $\left.J=293.1,291.5 \mathrm{~Hz}\right), 138.0,134.3,131.7,129.7$, 128.7, 128.3, $128.1(\mathrm{t}, J=3.5 \mathrm{~Hz}), 127.0,90.4(\mathrm{dd}, J=18.4,15.4 \mathrm{~Hz}), 37.5(\mathrm{t}, J=3.3 \mathrm{~Hz}), 21.3$.

${ }^{19}$ F NMR $\left(470 \mathrm{MHz}, \mathrm{CDCl}_{3}\right) \delta-87.6(\mathrm{~d}, J=35.2 \mathrm{~Hz}, 1 \mathrm{~F}),-87.7(\mathrm{~d}, J=35.5 \mathrm{~Hz}, 1 \mathrm{~F})$.

HRMS (ESI+): calcd. for [2( $\left.\left.\mathrm{C}_{17} \mathrm{H}_{15} \mathrm{~F}_{2} \mathrm{NO}\right)+\mathrm{Na}\right]^{+}:$597.2136, found: 597.2143.

MP: $67.8-69.3{ }^{\circ} \mathrm{C}$<smiles>COc1ccc(C(=O)NCC(Br)=C(F)F)cc1</smiles>

1b: Title compound was synthesized by following the general procedure, yielding $128 \mathrm{mg}$ of $\mathbf{1 b}$ as a white solid (40\% yield over two steps).

${ }^{1} \mathbf{H}$ NMR $\left(500 \mathrm{MHz}, \mathrm{CDCl}_{3}\right) \delta 7.61(\mathrm{dd}, J=8.9,2.1 \mathrm{~Hz}, 2 \mathrm{H}), 7.29(\mathrm{~d}, J=7.6 \mathrm{~Hz}, 2 \mathrm{H}), 7.18(\mathrm{~d}, J=$ $7.9 \mathrm{~Hz}, 2 \mathrm{H}), 6.87(\mathrm{dd}, J=8.9,2.0 \mathrm{~Hz}, 2 \mathrm{H}), 5.96(\mathrm{bs}, 1 \mathrm{H}), 4.47$ (dt, $J=5.5,2.4 \mathrm{~Hz}, 2 \mathrm{H}), 3.82$ (s, $3 \mathrm{H}), 2.34$ (s, 3H).

${ }^{13} \mathrm{C}$ NMR $\left(126 \mathrm{MHz}, \mathrm{CDCl}_{3}\right) \delta 167.0,162.4,154.7$ (dd, $\left.J=293.4,291.5 \mathrm{~Hz}\right), 137.9,129.6,128.8$, $128.4(\mathrm{t}, J=2.3 \mathrm{~Hz}), 128.1(\mathrm{t}, J=3.5 \mathrm{~Hz}), 126.6,113.9,90.5(\mathrm{dd}, J=18.4,15.2 \mathrm{~Hz}), 55.5,37.4(\mathrm{t}$, $J=3.1 \mathrm{~Hz}), 21.3$.

${ }^{19}$ F NMR $\left(470 \mathrm{MHz}, \mathrm{CDCl}_{3}\right) \delta-87.6(\mathrm{~d}, J=35.4 \mathrm{~Hz}, 1 \mathrm{~F}),-87.8(\mathrm{~d}, J=35.6 \mathrm{~Hz}, 1 \mathrm{~F})$.

HRMS (ESI+): calcd. for [2( $\left.\left.\mathrm{C}_{18} \mathrm{H}_{17} \mathrm{~F}_{2} \mathrm{NO}_{2}\right)+\mathrm{Na}\right]^{+}$: 657.2346, found: 657.2338.

MP: $109.8-112.5^{\circ} \mathrm{C}$ 
<smiles>O=C(NCC([Te]Cl)=C(F)F)c1ccc(F)cc1</smiles>

1c: Title compound was synthesized by following the general procedure, yielding $150 \mathrm{mg}$ of $\mathbf{1 c}$ as a white solid (49\% yield over two steps).

${ }^{1} \mathbf{H}$ NMR $\left(500 \mathrm{MHz} \mathrm{CDCl}_{3}\right) \delta 7.70-7.61(\mathrm{~m}, 2 \mathrm{H}), 7.29(\mathrm{~d}, J=7.7 \mathrm{~Hz}, 2 \mathrm{H}), 7.19(\mathrm{~d}, J=8.0 \mathrm{~Hz}$, $2 \mathrm{H}), 7.10-7.02(\mathrm{~m}, 2 \mathrm{H}), 5.98(\mathrm{bs}, 1 \mathrm{H}), 4.48(\mathrm{dt}, J=5.5,2.4 \mathrm{~Hz}, 2 \mathrm{H}), 2.34$ (s, 3H).

${ }^{13}$ C NMR $\left(151 \mathrm{MHz}, \mathrm{CDCl}_{3}\right) \delta 166.5,164.9(\mathrm{~d}, J=252.1 \mathrm{~Hz}), 154.7$ (dd, $\left.J=293.7,291.1 \mathrm{~Hz}\right)$, 138.0, $130.5(\mathrm{~d}, J=3.3 \mathrm{~Hz}), 129.7,129.3(\mathrm{~d}, J=8.9 \mathrm{~Hz}), 128.3(\mathrm{t}, J=3.0 \mathrm{~Hz}), 128.1(\mathrm{t}, J=3.3$ $\mathrm{Hz}), 115.8,115.7,90.4(\mathrm{dd}, J=18.9,15.2 \mathrm{~Hz}), 37.6(\mathrm{t}, J=3.1 \mathrm{~Hz}), 21.3$.

${ }^{19}$ F NMR $\left(470 \mathrm{MHz}, \mathrm{CDCl}_{3}\right) \delta-87.5(\mathrm{~d}, J=34.9 \mathrm{~Hz}, 1 \mathrm{~F}),-87.6(\mathrm{~d}, J=35.1 \mathrm{~Hz}, 1 \mathrm{~F}),-108.0(\mathrm{tt}$, $J=8.4,5.3 \mathrm{~Hz}, 1 \mathrm{~F})$.

HRMS (ESI+): calcd. for [2( $\left.\left.\mathrm{C}_{17} \mathrm{H}_{14} \mathrm{~F}_{3} \mathrm{NO}\right)+\mathrm{Na}\right]^{+}:$633.1946, found: 633.1946.

MP: $63.9-73.9^{\circ} \mathrm{C}$<smiles></smiles>

1d: Title compound was synthesized by following the general procedure, yielding $120 \mathrm{mg}$ of $\mathbf{1 d}$ as a white solid (40\% yield over two steps).

${ }^{1} \mathbf{H}$ NMR $\left(600 \mathrm{MHz}, \mathrm{CDCl}_{3}\right) \delta 7.54(\mathrm{~d}, J=8.2 \mathrm{~Hz}, 2 \mathrm{H}), 7.29(\mathrm{~d}, J=7.8 \mathrm{~Hz}, 2 \mathrm{H}), 7.18(\mathrm{~d}, J=7.8$ $\mathrm{Hz}, 4 \mathrm{H}), 6.00(\mathrm{bs}, 1 \mathrm{H}), 4.48$ (dt, $J=5.1,2.4 \mathrm{~Hz}, 2 \mathrm{H}), 2.36$ (s, 3H), 2.34 (s, 3H).

${ }^{13} \mathrm{C}$ NMR $\left(151 \mathrm{MHz}, \mathrm{CDCl}_{3}\right) \delta 167.4,154.7$ (dd, $\left.J=293.3,291.0 \mathrm{~Hz}\right), 142.2,137.9,131.5,129.6$, 129.4, $128.4(\mathrm{t}, J=2.8 \mathrm{~Hz}), 128.2(\mathrm{t}, J=3.4 \mathrm{~Hz}), 127.0,90.5(\mathrm{dd}, J=18.7,14.9 \mathrm{~Hz}), 37.4(\mathrm{t}, J=$ $2.9 \mathrm{~Hz}), 21.6,21.3$.

${ }^{19}$ F NMR $\left(470 \mathrm{MHz}, \mathrm{CDCl}_{3}\right) \delta-87.6(\mathrm{~d}, J=35.3 \mathrm{~Hz}, 1 \mathrm{~F}),-87.7(\mathrm{~d}, J=35.5 \mathrm{~Hz}, 1 \mathrm{~F})$.

HRMS (ESI+): calcd. for [2( $\left.\left.\mathrm{C}_{18} \mathrm{H}_{17} \mathrm{~F}_{2} \mathrm{NO}\right)+\mathrm{Na}\right]^{+}:$625.2448, found: 625.2408 .

MP: $93.9-95.6^{\circ} \mathrm{C}$ 
<smiles></smiles>

1e: Title compound was synthesized by following the general procedure, yielding $105 \mathrm{mg}$ of $\mathbf{1 e}$ as a sticky colorless oil (35\% yield over two steps).

${ }^{1} \mathbf{H}$ NMR $\left(600 \mathrm{MHz}, \mathrm{CDCl}_{3}\right) \delta 7.30(\mathrm{~d}, J=7.0 \mathrm{~Hz}, 2 \mathrm{H}), 7.29-7.23(\mathrm{~m}, 1 \mathrm{H}), 7.19(\mathrm{~d}, J=8.0 \mathrm{~Hz}$, 2H), $7.16-7.10(\mathrm{~m}, 3 \mathrm{H}), 5.68(\mathrm{bs}, 1 \mathrm{H}), 4.49(\mathrm{dt}, J=5.7,2.3 \mathrm{~Hz}, 2 \mathrm{H}), 2.35(\mathrm{~s}, 3 \mathrm{H}), 2.28(\mathrm{~s}, 3 \mathrm{H})$.

${ }^{13} \mathrm{C}$ NMR (151 MHz, $\left.\mathrm{CDCl}_{3}\right)$ 170.1, 154.6 (dd, $\left.J=293.6,290.8 \mathrm{~Hz}\right), 138.0,136.2,136.1,131.1$, 130.1, 129.6, 128.2 (t, $J=3.5 \mathrm{~Hz}), 126.7,125.8,90.5$ (dd, $J=18.9,14.8 \mathrm{~Hz}), 38.6-33.3$ (m), 21.3, 19.7. (One missing resonance)

${ }^{19}$ F NMR $\left(565 \mathrm{MHz}, \mathrm{CDCl}_{3}\right) \delta-87.9(\mathrm{~d}, J=35.6 \mathrm{~Hz}),-88.1(\mathrm{~d}, J=35.6 \mathrm{~Hz})$.

HRMS (ESI+): calcd. for [2( $\left.\left.\mathrm{C}_{18} \mathrm{H}_{17} \mathrm{~F}_{2} \mathrm{NO}\right)+\mathrm{Na}\right]^{+}:$625.2448, found: 625.2435 .<smiles>Cc1ccc(C(CNC(=O)c2cccs2)=C(F)F)cc1</smiles>

1f: Title compound was synthesized by following the general procedure, yielding $101 \mathrm{mg}$ of $\mathbf{1 f}$ as a white solid (34\% yield over two steps).

${ }^{1} \mathbf{H}$ NMR $\left(500 \mathrm{MHz}, \mathrm{CDCl}_{3}\right) \delta 7.43(\mathrm{dd}, J=5.0,1.2 \mathrm{~Hz}, 1 \mathrm{H}), 7.37(\mathrm{dd}, J=3.8,1.2 \mathrm{~Hz}, 1 \mathrm{H}), 7.29$ $(\mathrm{d}, J=7.2,2 \mathrm{H}), 7.18(\mathrm{~d}, J=7.9 \mathrm{~Hz}, 2 \mathrm{H}), 7.02(\mathrm{dd}, J=5.0,3.7 \mathrm{~Hz}, 1 \mathrm{H}), 5.92(\mathrm{bs}, 1 \mathrm{H}), 4.46(\mathrm{dt}, J=$ 5.6, $2.3 \mathrm{~Hz}, 2 \mathrm{H}), 2.34$ (s, 3H).

${ }^{13} \mathrm{C}$ NMR $\left(126 \mathrm{MHz}, \mathrm{CDCl}_{3}\right) \delta 161.9,154.7$ (dd, $\left.J=293.5,292.2 \mathrm{~Hz}\right), 138.5,138.0,134.8,130.3$, 129.7, 128.3, 128.1 (t, $J=3.4 \mathrm{~Hz}), 127.7,90.3$ (dd, $J=18.6,15.1 \mathrm{~Hz}), 37.4,21.3$.

${ }^{19}$ F NMR $\left(470, \mathrm{CDCl}_{3}\right) \delta-87.5(\mathrm{~d}, J=34.8 \mathrm{~Hz}, 1 \mathrm{~F}),-87.6(\mathrm{~d}, J=35.0 \mathrm{~Hz}, 1 \mathrm{~F})$.

HRMS (ESI+): calcd. for [2( $\left.\left.\mathrm{C}_{15} \mathrm{H}_{13} \mathrm{~F}_{2} \mathrm{NOS}\right)+\mathrm{Na}\right]^{+}:$609.1264, found: 609.1259 .

MP: $73.7-75.3{ }^{\circ} \mathrm{C}$ 
<smiles>COc1ccc(C(CNC(=O)c2ccccc2)=C(F)F)cc1</smiles>

1h: Title compound was synthesized by following the general procedure, yielding $143 \mathrm{mg}$ of $\mathbf{1 h}$ as a white solid (47\% yield over two steps).

${ }^{1} \mathbf{H}$ NMR $\left(500 \mathrm{MHz}, \mathrm{CDCl}_{3}\right) \delta 7.67-7.60(\mathrm{~m}, 2 \mathrm{H}), 7.49(\mathrm{tt}, J=7.4,1.3 \mathrm{~Hz}, 1 \mathrm{H}), 7.42-7.36(\mathrm{~m}$, $2 \mathrm{H}), 7.34(\mathrm{~d}, J=7.9,2 \mathrm{H}), 6.92(\mathrm{dt}, J=8.9,2.2 \mathrm{~Hz}, 2 \mathrm{H}), 6.04(\mathrm{bs}, 1 \mathrm{H}), 4.48(\mathrm{dt}, J=5.6,2.4 \mathrm{~Hz}$, $2 \mathrm{H}), 3.80(\mathrm{~s}, 3 \mathrm{H})$.

${ }^{13}$ C NMR $\left(126 \mathrm{MHz}, \mathrm{CDCl}_{3}\right) \delta 167.5,159.3,154.6(\mathrm{dd}, J=292.9,290.7 \mathrm{~Hz}), 134.3,131.7,129.5$ $(\mathrm{t}, J=3.5 \mathrm{~Hz}), 128.7,127.0,123.4(\mathrm{t}, J=3.0 \mathrm{~Hz}), 114.4,90.1(\mathrm{dd}, J=18.9,15.0 \mathrm{~Hz}), 55.4,37.5-$ $37.4(\mathrm{~m})$.

${ }^{19}$ F NMR $\left(470 \mathrm{MHz}, \mathrm{CDCl}_{3}\right) \delta-88.2(\mathrm{~d}, J=36.8 \mathrm{~Hz}, 1 \mathrm{~F}),-88.4(\mathrm{~d}, J=37.0 \mathrm{~Hz}, 1 \mathrm{~F})$.

HRMS (ESI+): calcd. for $\left[\mathrm{C}_{17} \mathrm{H}_{15} \mathrm{~F}_{2} \mathrm{NO}_{2}+\mathrm{MeCN}+\mathrm{Na}\right]^{+}: 367.1229$, found: 367.1219.

MP: $81.8-85.8^{\circ} \mathrm{C}$<smiles>Cc1ccc(C(CNC(=O)c2cnc(N3CCOCC3)nc2)=C(F)F)cc1</smiles>

1i: Synthesized on a $2.4 \mathrm{mmol}$ scale with crude $\mathbf{S 2}$ (2.4 mmol, 1.0 equiv.) used in place of $\mathrm{ArCO}_{2} \mathrm{H}$ and $\mathrm{ArCOCl}$, yielding $230 \mathrm{mg}$ of $\mathbf{1 i}$ as a white solid (26\% yield over two steps).

${ }^{1} \mathbf{H}$ NMR $\left(500 \mathrm{MHz}, \mathrm{CDCl}_{3}\right) \delta 8.56(\mathrm{~s}, 2 \mathrm{H}), 7.27(\mathrm{~d}, J=7.6,2 \mathrm{H}), 7.18(\mathrm{~d}, J=7.9,2 \mathrm{H}), 5.77$ (bs, $1 \mathrm{H}), 4.46(\mathrm{dt}, J=5.2,2.3 \mathrm{~Hz}, 2 \mathrm{H}), 3.88-3.84(\mathrm{~m}, 4 \mathrm{H}), 3.76-3.72(\mathrm{~m}, 4 \mathrm{H}), 2.34(\mathrm{~s}, 3 \mathrm{H})$.

${ }^{13} \mathrm{C}$ NMR $\left(151 \mathrm{MHz}, \mathrm{CDCl}_{3}\right) \delta 164.5,162.2,157.3,154.6(\mathrm{t}, J=292.2 \mathrm{~Hz}), 138.1,129.7,128.2$ $(\mathrm{t}, J=3.3 \mathrm{~Hz}), 116.1,91.0-89.4(\mathrm{~m}), 66.9,44.4,37.2(\mathrm{t}, J=3.3 \mathrm{~Hz}), 21.3$. (One missing resonance) ${ }^{19}$ F NMR $\left(470 \mathrm{MHz}, \mathrm{CDCl}_{3}\right) \delta-87.7(\mathrm{~s}, 2 \mathrm{~F})$.

HRMS (ESI+): calcd. for $\left[\mathrm{C}_{19} \mathrm{H}_{20} \mathrm{~F}_{2} \mathrm{~N}_{4} \mathrm{O}_{2}+\mathrm{MeCN}+\mathrm{H}\right]^{+}:$416.1892, found: 416.1902.

MP: $146.0-153.9^{\circ} \mathrm{C}$ 
<smiles></smiles>

$\mathbf{1 j}$ : Title compound was synthesized by following the general procedure, yielding $148 \mathrm{mg}$ of $\mathbf{1 j}$ as a white solid (36\% yield over two steps).

${ }^{1} \mathbf{H}$ NMR $\left(600 \mathrm{MHz}, \mathrm{CD}_{2} \mathrm{Cl}_{2}\right) \delta 8.16(\mathrm{~d}, J=8.4 \mathrm{~Hz}, 1 \mathrm{H}), 7.68(\mathrm{~s}, 1 \mathrm{H}), 7.62(\mathrm{~d}, J=7.3 \mathrm{~Hz}, 2 \mathrm{H})$, $7.54(\mathrm{~d}, J=8.0,1 \mathrm{H}), 7.47(\mathrm{~d}, J=7.4 \mathrm{~Hz}, 1 \mathrm{H}), 7.40-7.32(\mathrm{~m}, 3 \mathrm{H}), 7.26(\mathrm{t}, J=7.6 \mathrm{~Hz}, 1 \mathrm{H}), 6.21$ (bs, $1 \mathrm{H}), 4.48-4.45(\mathrm{~m}, 2 \mathrm{H}), 1.64(\mathrm{~s}, 9 \mathrm{H})$.

${ }^{13} \mathrm{C}$ NMR $\left(151 \mathrm{MHz}, \mathrm{CDCl}_{3}\right) \delta 167.6,154.7(\mathrm{t}, J=292.6 \mathrm{~Hz}), 149.4,135.5,134.3,131.7,129.0$, 128.7, 127.0, 125.3 (dd, $J=4.6,2.3 \mathrm{~Hz}), 125.0,123.2,119.9$ (d, $J=3.6 \mathrm{~Hz}), 115.6,111.8-111.1$ (m), 84.4, $83.3(\mathrm{dd}, J=23.1,17.2 \mathrm{~Hz}), 38.1$ (d, $J=4.4 \mathrm{~Hz}), 28.3$.

${ }^{19}$ F NMR $\left(470 \mathrm{MHz}, \mathrm{CDCl}_{3}\right) \delta-83.7(\mathrm{~d}, J=31.6 \mathrm{~Hz}, 1 \mathrm{~F}),-87.1(\mathrm{~d}, J=31.5 \mathrm{~Hz}, 1 \mathrm{~F})$.

HRMS (ESI+): calcd. for $\left[\mathrm{C}_{23} \mathrm{H}_{22} \mathrm{~F}_{2} \mathrm{~N}_{2} \mathrm{O}_{3}+\mathrm{MeCN}+\mathrm{H}\right]^{+}$: 476.1756, found: 476.1768.

MP: $108.1-110.2^{\circ} \mathrm{C}$<smiles>C=C(c1ccc2ccccc2c1)C(F)(F)F</smiles>

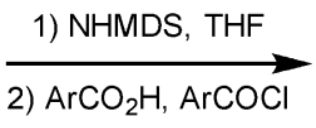<smiles>O=C(NCC(=C(F)F)c1ccc2ccccc2c1)c1ccccc1</smiles>

1g: To a stirred solution of $440 \mathrm{mg}$ of 2-(3,3,3-trifluoroprop-1-en-2-yl)naphthalene ${ }^{5}(2$ mmol, 1 equiv.) in dry THF (10 mL), was added $1.2 \mathrm{~mL}$ of an NHMDS solution (2M in THF, 2.4 mmol, 1.2 equiv.) over $1 \mathrm{~min}$. After addition, the solution was heated to $60^{\circ} \mathrm{C}$ and stirred overnight. After cooling to room temperature, the reaction was quenched with $270 \mathrm{mg}$ of benzoic acid (2.2 mmol, 1.1 equiv.), followed by addition of $0.56 \mathrm{~mL}$ of benzoyl chloride (4.8 mmol, 2.4 equiv.) over $1 \mathrm{~min}$. After stirring for an additional hour at ambient temperature, the reaction was quenched with saturated aqueous $\mathrm{NaHCO}_{3}(10 \mathrm{~mL})$ and diluted with $20 \mathrm{~mL}$ of diethyl ether. The aqueous and organic layers were separated, and the aqueous layer was extracted with $20 \mathrm{~mL}$ of diethyl ether. The organic layers were combined, dried with $\mathrm{Na}_{2} \mathrm{SO}_{4}$, and the solvent was removed under reduced pressure. The crude product was purified by flash chromatography (1:9 $\mathrm{Et}_{2} \mathrm{O} /$ pentanes) to yield $\mathbf{1 g}$ as a colorless white solid. Trace benzoyl chloride was removed from by dissolving the mixture in $30 \mathrm{~mL}$ of $\mathrm{Et}_{2} \mathrm{O}$, and washing with $25 \%$ aqueous ammonia $(3 \times 20 \mathrm{~mL})$. After drying of the organic layer with $\mathrm{Na}_{2} \mathrm{SO}_{4}$, the solvent was removed under reduced pressure to yield $210 \mathrm{mg}$ of pure $\mathbf{1 g}$ as a colorless solid (33\% yield). 
${ }^{1} \mathbf{H}$ NMR $\left(500 \mathrm{MHz}, \mathrm{CDCl}_{3}\right) \delta 7.89(\mathrm{~s}, 1 \mathrm{H}), 7.85(\mathrm{~d}, J=8.6 \mathrm{~Hz}, 1 \mathrm{H}), 7.84-7.80(\mathrm{~m}, 2 \mathrm{H}), 7.64-$ $7.60(\mathrm{~m}, 2 \mathrm{H}), 7.53(\mathrm{dt}, J=8.6,1.7 \mathrm{~Hz}, 1 \mathrm{H}), 7.51-7.47(\mathrm{~m}, 2 \mathrm{H}), 7.44(\mathrm{tt}, J=7.4,1.3 \mathrm{~Hz}, 1 \mathrm{H}), 7.35$ (t, $J=7.7 \mathrm{~Hz}, 2 \mathrm{H}), 6.10(\mathrm{bs}, 1 \mathrm{H}), 4.62(\mathrm{dt}, J=5.2,2.4 \mathrm{~Hz}, 2 \mathrm{H})$.

${ }^{13}$ C NMR $\left(126 \mathrm{MHz}, \mathrm{CDCl}_{3}\right) \delta 167.6,155.0(\mathrm{dd}, J=294.8,291.7 \mathrm{~Hz}), 134.2,133.4,132.8,131.7$, 128.8, 128.7, 128.7, 128.6, 128.2, 127.7, 127.7 (t, $J=3.6 \mathrm{~Hz}), 126.9,126.6,125.7$, (dd, $J=4.1,2.9$ $\mathrm{Hz}), 90.8$ (dd, $J=19.4,14.3 \mathrm{~Hz}), 37.6-37.4(\mathrm{~m})$.

${ }^{19} \mathbf{F}$ NMR $\left(470 \mathrm{MHz}, \mathrm{CDCl}_{3}\right) \delta-86.5(\mathrm{~d}, J=33.0 \mathrm{~Hz}, 1 \mathrm{~F}),-86.9(\mathrm{~d}, J=33.0 \mathrm{~Hz}, 1 \mathrm{~F})$.

HRMS (ESI+): calcd. for [2( $\left.\left.\mathrm{C}_{20} \mathrm{H}_{15} \mathrm{~F}_{2} \mathrm{NO}\right)+\mathrm{Na}\right]^{+}: 669.2136$, found: 669.2148 .

MP: $100.5-102.1^{\circ} \mathrm{C}$

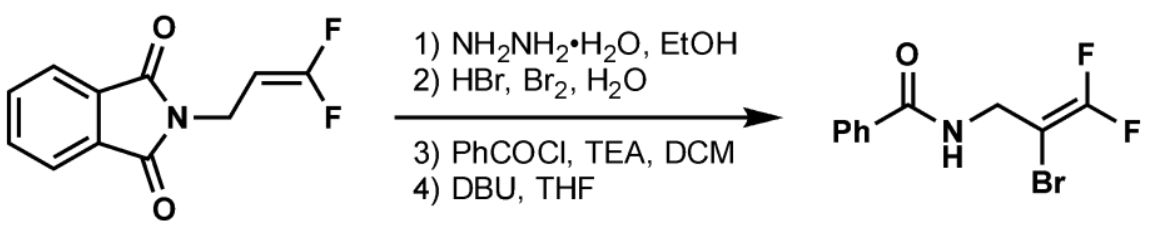

$\mathbf{N}$-(2-bromo-3,3-difluoroallyl)benzamide (S4): To a solution of $1.3 \mathrm{~g}$ of $\mathbf{N}$-(3,3difluoroallyl) phthalimide ${ }^{6}$ (5.6 mmol, 1.0 equiv.) in $16 \mathrm{~mL}$ of EtOH was added $0.27 \mathrm{~mL}$ of hydrazine monohydrate $\left(5.6 \mathrm{mmol}, 1.0\right.$ equiv.). After stirring the solution at $80^{\circ} \mathrm{C}$ overnight, the solids were filtered off and washed with an additional $20 \mathrm{~mL}$ of EtOH. To the filtrate was added $0.63 \mathrm{~mL}$ of $8.9 \mathrm{M} \mathrm{HBr}(5.6 \mathrm{mmol}, 1.0$ equiv.), and the solvent was removed under reduced pressure. After the resulting oil was dissolved in $22 \mathrm{~mL}$ of $\mathrm{H}_{2} \mathrm{O}, 0.43 \mathrm{~mL}$ of $\mathrm{Br}_{2}$ ( $8.4 \mathrm{mmol}, 1.5$ equiv.) was added and the resulting solution was stirred overnight. The solvent was then removed under reduced pressure and replaced with $28 \mathrm{~mL}$ of DCM. After the addition of $1.3 \mathrm{~mL}$ of BzCl (11

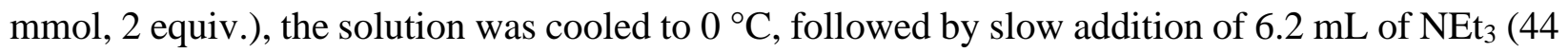
mmol, 8 equiv.). The solution was allowed to warm room temperature, allowed to stir for an additional 2 hours, and was then quenched by addition of $10 \mathrm{~mL}$ of $\mathrm{H}_{2} \mathrm{O}$. The layers were separated and the aqueous phase was extracted with DCM $(3 \times 5 \mathrm{~mL})$. The combined organic layers were dried over $\mathrm{Na}_{2} \mathrm{SO}_{4}$ and the solvent was removed under reduced pressure. The resulting oil was then dissolved in $55 \mathrm{~mL}$ of anhydrous THF and cooled to $0{ }^{\circ} \mathrm{C}$. To this solution was added $1.7 \mathrm{~mL}$ of DBU (11 mmol, 2.0 equiv.) dropwise. The solution to warm to room temperature and stirred for 2 hours, after which the solvent was removed under reduced pressure. The crude material was purified by flash chromatography (1:4 $\mathrm{Et}_{2} \mathrm{O} /$ pentane) to yield $420 \mathrm{mg}(27 \%)$ of the title compound as a white solid.

${ }^{1} \mathbf{H}$ NMR $\left(500 \mathrm{MHz}, \mathrm{CD}_{2} \mathrm{Cl}_{2}\right) \delta 7.82-7.75(\mathrm{~m}, 2 \mathrm{H}), 7.56-7.50(\mathrm{~m}, 1 \mathrm{H}), 7.46(\mathrm{t}, J=7.5 \mathrm{~Hz}, 2 \mathrm{H})$, 6.34 (bs, $1 \mathrm{H}), 4.39$ (dt, $J=5.9,2.4 \mathrm{~Hz}, 2 \mathrm{H})$.

${ }^{13}$ C NMR (126 MHz, $\left.\mathrm{CDCl}_{3}\right) \delta 167.5,154.2(\mathrm{dd}, J=287.6,280.5 \mathrm{~Hz}), 133.9,132.1,128.9,127.1$, $78.2(\mathrm{dd}, J=37.4,21.6 \mathrm{~Hz}), 39.7$. 
${ }^{19}$ F NMR $\left(470 \mathrm{MHz}, \mathrm{CD}_{2} \mathrm{Cl}_{2}\right) \delta-80.9(\mathrm{~d}, J=33.3 \mathrm{~Hz}, 1 \mathrm{~F}),-84.8(\mathrm{~d}, J=33.5 \mathrm{~Hz}, 1 \mathrm{~F})$.

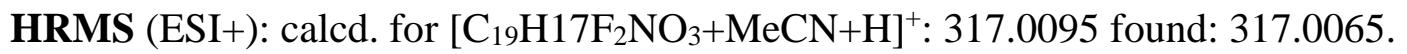

MP: $105.1-106.1^{\circ} \mathrm{C}$<smiles>O=C(NCC(Br)=C(F)F)c1ccccc1</smiles>
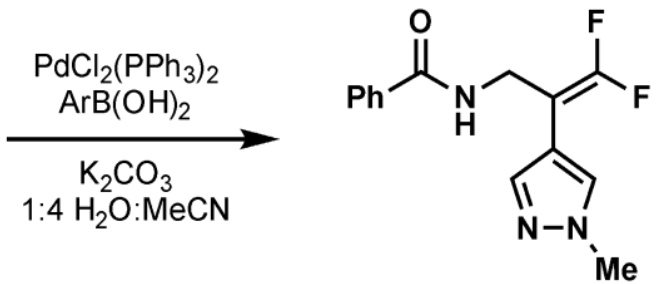

1k: A flame dried 2-dram vial was charged with $\mathbf{S 4}$ (96 mg, $0.35 \mathrm{mmol}, 1.0$ equiv.), $\mathrm{ArB}(\mathrm{OH})_{2}$ (96 mg, $0.53 \mathrm{mmol}, 1.5$ equiv.), $\mathrm{PdCl}_{2}\left(\mathrm{PPh}_{3}\right)_{2}(25 \mathrm{mg}, 0.035 \mathrm{mmol}, 10 \mathrm{~mol} \%$ ), and $\mathrm{K}_{2} \mathrm{CO}_{3}$ (97 mg, $0.7 \mathrm{mmol}, 2.0$ equiv.). The vial was exposed to vacuum and backfilled with $\mathrm{N}_{2}$ three times. Dry acetonitrile $(1.4 \mathrm{~mL})$ was added, followed by degassed $\mathrm{H}_{2} \mathrm{O}(0.35 \mathrm{~mL})$. The vial was heated to $70{ }^{\circ} \mathrm{C}$ for $3 \mathrm{~h}$. After complete conversion as determined by ${ }^{19} \mathrm{~F}$ NMR analysis, the reaction was cooled to room temperature, and $3 \mathrm{~mL}$ of $\mathrm{H}_{2} \mathrm{O}$ and $5 \mathrm{~mL}$ of DCM were added. The layers were separated and the aqueous phase was extracted with DCM ( $3 \times 5 \mathrm{~mL}$ ). The combined organic layers were dried over $\mathrm{Na}_{2} \mathrm{SO}_{4}$ and the solvent was removed under reduced pressure. The crude material was purified by flash chromatography $\left(1: 9 \mathrm{Et}_{2} \mathrm{O} /\right.$ pentanes to $\mathrm{Et}_{2} \mathrm{O}$ gradient $)$ to yield $45 \mathrm{mg}(47 \%)$ of $1 \mathbf{k}$ as a white solid.

${ }^{1} \mathbf{H}$ NMR $\left(500 \mathrm{MHz}, \mathrm{CD}_{2} \mathrm{Cl}_{2}\right) \delta 7.73-7.69(\mathrm{~m}, 2 \mathrm{H}), 7.54(\mathrm{~s}, 1 \mathrm{H}), 7.54(\mathrm{~s}, 1 \mathrm{H}), 7.51$ (tt, J= 7.4, $1.3 \mathrm{~Hz}, 1 \mathrm{H}), 7.43$ (tt, $J=6.7,1.4 \mathrm{~Hz}, 2 \mathrm{H}), 6.20$ (bs, $1 \mathrm{H}), 4.40$ (ddd, $J=5.7,2.6,1.7 \mathrm{~Hz}, 2 \mathrm{H}), 3.84$ $(\mathrm{s}, 3 \mathrm{H})$.

${ }^{13} \mathrm{C}$ NMR $\left(151 \mathrm{MHz}, \mathrm{CDCl}_{3}\right) \delta 167.6,154.4(\mathrm{dd}, J=294.0,288.5 \mathrm{~Hz}), 137.7(\mathrm{dd}, J=7.1,2.8 \mathrm{~Hz})$, 134.2, 131.9, 128.8, 128.6, 127.0, $113.0-112.8(\mathrm{~m}), 83.8(\mathrm{dd}, J=22.5,15.9 \mathrm{~Hz}), 39.3,36.4(\mathrm{dd}$, $J=5.6,1.9 \mathrm{~Hz})$.

${ }^{19}$ F NMR $\left(565 \mathrm{MHz}, \mathrm{CDCl}_{3}\right) \delta-84.0(\mathrm{~d}, J=37.9 \mathrm{~Hz}, 1 \mathrm{~F}),-92.0(\mathrm{~d}, J=37.7 \mathrm{~Hz}, 1 \mathrm{~F})$.

HRMS (ESI+): calcd. for $\left[\mathrm{C}_{14} \mathrm{H}_{13} \mathrm{~F}_{2} \mathrm{~N}_{3} \mathrm{O}+\mathrm{H}\right]^{+}:$: 278.1100, found: 278.1104.

MP: $157.4-161.0^{\circ} \mathrm{C}$ 
<smiles>O=C(NCC(=C(F)F)c1cc2ccccc2o1)c1ccccc1</smiles>

11: Prepared in analogous fashion to $\mathbf{1 k}$, yielding $66 \mathrm{mg}$ (60\%) of $\mathbf{1 1}$ as a white solid

${ }^{1} \mathbf{H}$ NMR $\left(600 \mathrm{MHz}, \mathrm{CD}_{2} \mathrm{Cl}_{2}\right) \delta 7.74-7.71(\mathrm{~m}, 2 \mathrm{H}), 7.57(\mathrm{~d}, J=7.6 \mathrm{~Hz}, 1 \mathrm{H}), 7.52-7.46(\mathrm{~m}, 2 \mathrm{H})$, $7.41(\mathrm{t}, J=7.6 \mathrm{~Hz}, 2 \mathrm{H}), 7.29$ (ddd, $J=8.4,7.3,1.4 \mathrm{~Hz}, 1 \mathrm{H}), 7.23(\mathrm{td}, J=7.5,1.0 \mathrm{~Hz}, 1 \mathrm{H}), 6.88$ (s, $1 \mathrm{H}), 6.52(\mathrm{bs}, 1 \mathrm{H}), 4.56(\mathrm{dt}, J=5.9,2.1 \mathrm{~Hz}, 2 \mathrm{H})$.

${ }^{13}$ C NMR (151 MHz MHz, $\left.\mathrm{CD}_{2} \mathrm{Cl}_{2}\right) \delta$ 167.6, 156.1 (dd, $\left.J=301.4,292.8 \mathrm{~Hz}\right), 155.1,149.4-149.0$ (m), 134.8, 132.1, 129.1, 129.1, 127.4, 125.1, 123.8, 121.5, 111.5, 105.8 (dd, $J=8.3,6.0 \mathrm{~Hz}), 85.6$ (dd, $J=26.4,12.2 \mathrm{~Hz}), 35.5$ (d, $J=4.3 \mathrm{~Hz})$.

${ }^{19}$ F NMR $\left(565 \mathrm{MHz}, \mathrm{CD}_{2} \mathrm{Cl}_{2}\right) \delta-78.7(\mathrm{~d}, J=16.8 \mathrm{~Hz}),-84.3(\mathrm{~d}, J=17.2 \mathrm{~Hz})$.

HRMS (ESI+): calcd. for $\left[\mathrm{C}_{18} \mathrm{H}_{13} \mathrm{~F}_{2} \mathrm{NO}_{2}+\mathrm{MeCN}+\mathrm{Na}\right]^{+}: 377.1072$, found: 377.1070 .

MP: $144.3-148.9^{\circ} \mathrm{C}$

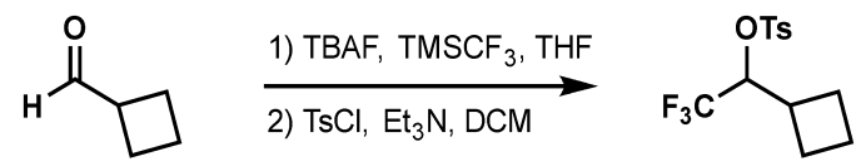

1-cyclobutyl-2,2,2-trifluoroethyl tosylate (S5): To a stirred solution of cyclobutylaldehyde ( $9.3 \mathrm{~mL}, 12 \mathrm{mmol}, 1.0$ equiv.) in anhydrous THF $(18 \mathrm{~mL})$, was added $2.1 \mathrm{~mL}$ of $\mathrm{TMSCF}_{3}$ (14.4 mmol, 1.2 equiv.). After cooling the solution to $0{ }^{\circ} \mathrm{C}$, a $1 \mathrm{M}$ solution of TBAF in THF ( $1.8 \mathrm{~mL}, 1.8 \mathrm{mmol}, 0.15$ equiv.) was added dropwise. The reaction was allowed to warm to room temperature and stirred for 4 hours. The reaction was then cooled to $0{ }^{\circ} \mathrm{C}$, diluted with $\mathrm{Et}_{2} \mathrm{O}(30 \mathrm{~mL})$ and quenched with $1 \mathrm{M} \mathrm{HCl}(15 \mathrm{~mL})$. After warming to room temperature, the aqueous and organic layers were separated, and the aqueous layer was extracted with $20 \mathrm{~mL}$ of $\mathrm{Et}_{2} \mathrm{O}$ twice. The organic layers were combined, dried with $\mathrm{Na}_{2} \mathrm{SO}_{4}$, and the solvent was removed under reduced pressure. The crude product was then dissolved in anhydrous DCM $(10 \mathrm{~mL})$, and added to a solution of TsCl (2.7 g, $14.4 \mathrm{mmol}, 1.2$ equiv.) in anhydrous DCM (14 mL). After complete addition, $2.0 \mathrm{~mL}$ of $\mathrm{Et}_{3} \mathrm{~N}$ (14.4 mmol, 1.2 equiv.) was added to the solution. After stirring the solution overnight, $\mathrm{Et}_{2} \mathrm{O}(40 \mathrm{~mL})$ was added and the resulting solid was removed by filtration. The solvent was removed under reduced pressure, and the residue was purified by column chromatography on $\mathrm{SiO}_{2}$ (hexanes to 1:9 EtOAc/hexanes gradient) to yield $2.6 \mathrm{~g}$ (70 \% over two steps) of title compound as a colorless oil.

${ }^{1} \mathbf{H}$ NMR $\left(500 \mathrm{MHz}, \mathrm{CD}_{2} \mathrm{Cl}_{2}\right) \delta 7.80(\mathrm{~d}, J=8.4 \mathrm{~Hz}, 2 \mathrm{H}), 7.38(\mathrm{~d}, J=8.1 \mathrm{~Hz}, 2 \mathrm{H}), 4.80(\mathrm{p}, J=6.4$ $\mathrm{Hz}, 1 \mathrm{H}), 2.83-2.71(\mathrm{~m}, 1 \mathrm{H}), 2.45(\mathrm{~s}, 3 \mathrm{H}), 2.07-1.69(\mathrm{~m}, 6 \mathrm{H})$. 
${ }^{13}$ C NMR (126 MHz, $\left.\mathrm{CD}_{2} \mathrm{Cl}_{2}\right) \delta 146.3,134.0,130.5,128.3,123.64$ (q, $\left.J=282.3 \mathrm{~Hz}\right), 79.5$ (q, $J=$ $31.9 \mathrm{~Hz}), 34.3,24.7,24.5,22.0,18.9$.

${ }^{19} \mathbf{F}$ NMR $\left(470 \mathrm{MHz}, \mathrm{CD}_{2} \mathrm{Cl}_{2}\right) \delta-75.5(\mathrm{~d}, J=6.3 \mathrm{~Hz})$.

HRMS (ESI+): calcd. for $\left[\mathrm{C}_{13} \mathrm{H}_{15} \mathrm{~F}_{3} \mathrm{O}_{3} \mathrm{~S}+\mathrm{MeCN}+\mathrm{Na}\right]^{+}$: 372.0852 , found: 372.0836 .

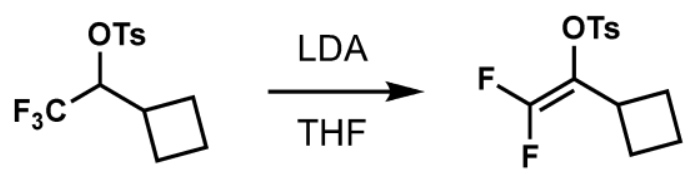

1-cyclobutyl-2,2-difluorovinyl tosylate (S6): To a stirred solution of $\mathbf{S 5}(2.6 \mathrm{~g}, 8.4 \mathrm{mmol}$, 1.0 equiv.) in anhydrous THF $(84 \mathrm{~mL})$ at $-78{ }^{\circ} \mathrm{C}$, was added $4.6 \mathrm{~mL}$ of a $2 \mathrm{M}$ solution of LDA in THF $\left(9.2 \mathrm{mmol}, 1.1\right.$ equiv.) dropwise. After stirring for 1 hour at $-78^{\circ} \mathrm{C}$, the solution was allowed to warm to room temperature and stirred overnight. The reaction was quenched with saturated aqueous $\mathrm{NH}_{4} \mathrm{Cl}(20 \mathrm{~mL})$ and then diluted with $\mathrm{Et}_{2} \mathrm{O}$. The aqueous and organic phase were separated, and the aqueous layer was extracted with $40 \mathrm{~mL}$ of $\mathrm{Et}_{2} \mathrm{O}$ twice. The organic layers were combined, dried with $\mathrm{Na}_{2} \mathrm{SO}_{4}$, and the solvent was removed under reduced pressure. The residue was purified by column chromatography (hexanes to 1:9 EtOAc/hexane gradient) to yield $1.4 \mathrm{~g}$ (58\%) of title compound as a colorless oil.

${ }^{1} \mathbf{H}$ NMR $\left(600 \mathrm{MHz}, \mathrm{CDCl}_{3}\right) \delta 7.83(\mathrm{~d}, J=8.4 \mathrm{~Hz}, 2 \mathrm{H}), 7.36(\mathrm{~d}, J=7.7 \mathrm{~Hz}, 2 \mathrm{H}), 3.29-3.18(\mathrm{~m}$, $1 \mathrm{H}), 2.46(\mathrm{~s}, 3 \mathrm{H}), 2.27-2.12(\mathrm{~m}, 2 \mathrm{H}), 2.10-2.02(\mathrm{~m}, 2 \mathrm{H}), 1.87$ (qt, $J=10.2,8.2 \mathrm{~Hz}, 1 \mathrm{H}), 1.81-$ $1.72(\mathrm{~m}, 1 \mathrm{H})$.

${ }^{13}$ C NMR $\left(151 \mathrm{MHz}, \mathrm{CDCl}_{3}\right) \delta 155.0(\mathrm{dd}, J=294.8,285.4 \mathrm{~Hz}), 145.6,133.3,129.9,128.3,115.4$ (dd, $J=45.0,12.2 \mathrm{~Hz}$ ), 33.0 - $32.9(\mathrm{~m}), 26.5-25.9(\mathrm{~m}), 21.9,18.6$.

${ }^{19} \mathbf{F}$ NMR $\left(565 \mathrm{MHz}, \mathrm{CDCl}_{3}\right) \delta-92.8(\mathrm{~d}, J=53.1 \mathrm{~Hz}, 1 \mathrm{~F}),-106.2(\mathrm{dd}, J=53.7,3.6 \mathrm{~Hz}, 1 \mathrm{~F})$.

HRMS (EI+): calcd. for $\left[\mathrm{C}_{13} \mathrm{H}_{14} \mathrm{~F}_{2} \mathrm{O}_{3} \mathrm{~S}\right]^{+}:$288.0632, found: 288.0633 .

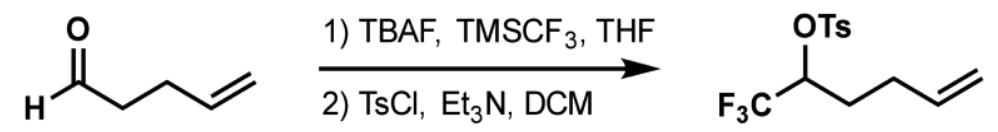

1,1,1-trifluorohex-5-en-2-yl tosylate (S7): Prepared in analogous fashion to S5, yielding $800 \mathrm{mg}$ of title compound as a colorless oil (21\% yield over two steps).

${ }^{1} \mathbf{H}$ NMR $\left(600 \mathrm{MHz}, \mathrm{CDCl}_{3}\right) \delta 7.82-7.79(\mathrm{~m}, 2 \mathrm{H}), 7.37-7.34(\mathrm{~m}, 2 \mathrm{H}), 5.76$ (ddt, $J=17.0,10.3$, $6.5 \mathrm{~Hz}, 1 \mathrm{H}), 5.12-5.05(\mathrm{~m}, 2 \mathrm{H}), 4.88(\operatorname{sextet} J=6.2 \mathrm{~Hz}, 1 \mathrm{H}), 2.46(\mathrm{~s}, 3 \mathrm{H}), 2.28-2.21(\mathrm{~m}, 1 \mathrm{H})$, $2.20-2.12(\mathrm{~m}, 1 \mathrm{H}), 1.92-1.86(\mathrm{~m}, 2 \mathrm{H})$. 
${ }^{13} \mathbf{C}$ NMR $\left(151 \mathrm{MHz}, \mathrm{CDCl}_{3}\right) \delta 145.6,135.9,133.5,130.0,128.1,123.1$ (q, $\left.J=281.4 \mathrm{~Hz}\right), 116.8$, $76.2(\mathrm{q}, J=33.1 \mathrm{~Hz}), 28.3,28.0,21.9$.

${ }^{19}$ F NMR $\left(565 \mathrm{MHz}, \mathrm{CDCl}_{3}\right) \delta-76.5(\mathrm{~d}, J=6.1 \mathrm{~Hz})$.

HRMS (EI+): calcd. for $\left[\mathrm{C}_{13} \mathrm{H}_{16} \mathrm{~F}_{3} \mathrm{O}_{3} \mathrm{~S}\right]^{+}:$309.0772, found: 309.0770.

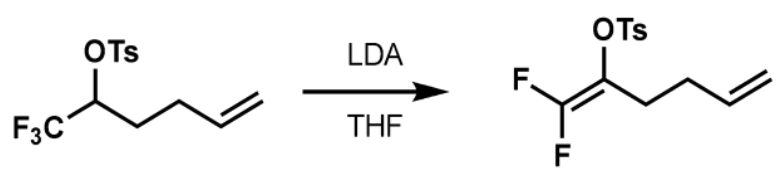

1,1,1-trifluorohex-5-en-2-yl tosylate (S8): Prepared in analogous fashion to S6 with 50\% yield. Product was inseparable from starting material. Mixture of starting material and difluoroalkene was taken onto the next step after column chromatography on $\mathrm{SiO}_{2}$.<smiles>FC(F)=C([Se-])I</smiles>

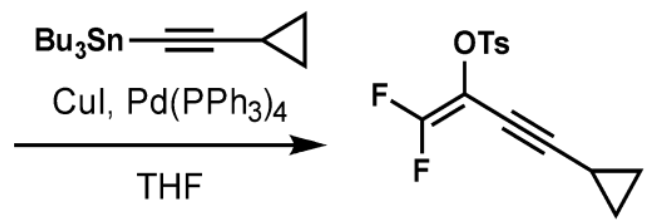

4-cyclopropyl-1,1-difluorobut-1-en-3-yn-2-yl tosylate (S9): To a $500 \mathrm{~mL}$ flame dried round bottom flask, was added $3.2 \mathrm{~g}$ of $\mathrm{Pd}\left(\mathrm{PPh}_{3}\right)_{4}(2.8 \mathrm{mmol}, 10 \mathrm{~mol} \%)$ and $3.2 \mathrm{~g}$ of $\mathrm{CuI}(2.8 \mathrm{mmol}$, $10 \mathrm{~mol} \%$ ). After the flask was evacuated and backfilled with $\mathrm{N}_{2}(3 \mathrm{x}), 200 \mathrm{~mL}$ of anhydrous THF was added, followed by $12 \mathrm{~g}$ of [2-(tri- $\boldsymbol{n}$-butylstannyl)-1-ethynyl]cyclopropane ${ }^{7}$ (33 mmol, 1.2 equiv.) and $9.9 \mathrm{~g}$ of 2,2-difluoro-1-iodovinyl tosylate ${ }^{8}(28 \mathrm{mmol}, 1.0$ equiv.). After stirring the solution at $80{ }^{\circ} \mathrm{C}$ for $90 \mathrm{~min}$, the reaction was allowed to cool to room temperature, and quenched with $\mathrm{H}_{2} \mathrm{O}(50 \mathrm{~mL})$. The reaction mixture was extracted with $\mathrm{Et}_{2} \mathrm{O}(2 \mathrm{x} 100 \mathrm{~mL})$, the combined organic layers dried with $\mathrm{Na}_{2} \mathrm{SO}_{4}$, and the solvent was removed under reduced pressure. The crude product was purified by column chromatography on $\mathrm{SiO}_{2}$ (hexanes to 1:9 EtOAc/hexanes gradient) to yield $5.2 \mathrm{~g}(63 \%)$ of title compound as a brown oil.

${ }^{1} \mathbf{H}$ NMR $\left(500 \mathrm{MHz}, \mathrm{CDCl}_{3}\right) \delta 7.84(\mathrm{~d}, J=8.4 \mathrm{~Hz}, 2 \mathrm{H}), 7.36(\mathrm{~d}, J=8.2 \mathrm{~Hz}, 2 \mathrm{H}), 2.47$ (s, 3H), 1.25 $-1.17(\mathrm{~m}, 1 \mathrm{H}), 0.83-0.73(\mathrm{~m}, 2 \mathrm{H}), 0.58-0.51(\mathrm{~m}, 2 \mathrm{H})$.

${ }^{13} \mathrm{C}$ NMR $\left(126 \mathrm{MHz}, \mathrm{CDCl}_{3}\right) \delta 160.9(\mathrm{dd}, J=301.5,292.8 \mathrm{~Hz}), 145.9,132.9,129.8,128.9,105.8$ $(\mathrm{dd}, J=8.8,5.0 \mathrm{~Hz}), 100.4(\mathrm{dd}, J=52.9,23.8 \mathrm{~Hz}), 61.6(\mathrm{dd}, J=8.8,2.4 \mathrm{~Hz}), 21.9,8.9,0.1$.

${ }^{19}$ F NMR $\left(470 \mathrm{MHz}, \mathrm{CDCl}_{3}\right) \delta-82.1(\mathrm{~d}, J=16.8 \mathrm{~Hz}, 1 \mathrm{~F}),-96.3(\mathrm{~d}, J=17.0 \mathrm{~Hz}, 1 \mathrm{~F})$.

HRMS (ESI+): calcd. for $\left[\mathrm{C}_{14} \mathrm{H}_{12} \mathrm{~F}_{2} \mathrm{O}_{3} \mathrm{~S}+\mathrm{MeCN}+\mathrm{Na}\right]^{+}:$362.0633, found: 362.0633. 
<smiles>[R]/C(F)=C(/F)[AsH2]</smiles><smiles>Nc1ccc(Br)cc1Br</smiles>

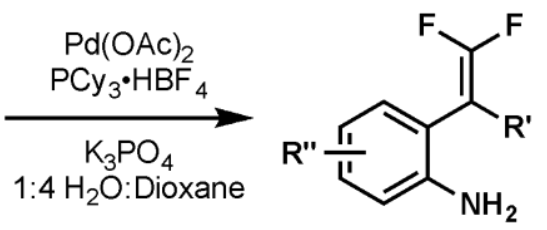

$3 a-j, 3 m$

3a-j, 3m: A $25 \mathrm{~mL}$ flame dried round bottom flask was charged with $\mathrm{Pd}(\mathrm{OAc})_{2}(22 \mathrm{mg}$, $0.10 \mathrm{mmol}, 10 \mathrm{~mol} \%), \mathrm{PCy}_{3} \cdot \mathrm{HBF}_{4}(74 \mathrm{mg}, 0.20 \mathrm{mmol}, 20 \mathrm{~mol} \%)$, the appropriate aryl boronic ester (1.0 mmol, 1.0 equiv.), and $\mathrm{K}_{3} \mathrm{PO}_{4}(640 \mathrm{mg}, 3.0 \mathrm{mmol}, 3.0$ equiv.). The flask was then evacuated and backfilled with $\mathrm{N}_{2}$ three times. Following this, a solution of the vinyl tosylate (1.0 mmol, 1.0 equiv.) in degassed 1:4 $\mathrm{H}_{2} \mathrm{O} / 1$,4-dioxane $(5.6 \mathrm{~mL})$ was added to the reaction flask dropwise. The reaction mixture was then heated to $80{ }^{\circ} \mathrm{C}$ and allowed to stir overnight. After cooling to room temperature, $10 \mathrm{~mL}$ of $\mathrm{H}_{2} \mathrm{O}$ and $10 \mathrm{~mL}$ of DCM were added to the reaction flask. The aqueous and organic layers were separated, and the aqueous layer was extracted with $20 \mathrm{~mL}$ of DCM twice. The organic layers were combined, dried with $\mathrm{Na}_{2} \mathrm{SO}_{4}$, and the solvent was removed under reduced pressure. The crude product was purified by flash chromatography (EtOAc/Hexanes) to yield the intermediate aniline as a dark brown oil and used immediately in the next step without further purification.

The intermediate aniline $(0.8 \mathrm{mmol}, 1.0$ equiv. $)$ was dissolved in $8 \mathrm{~mL}$ of dry DCM under $\mathrm{N}_{2}$. After cooling the solution to $0{ }^{\circ} \mathrm{C}$, the corresponding benzoyl chloride (1.0 mmol, 1.2 equiv.) was added, followed by $0.15 \mathrm{~mL}$ of $\mathrm{Et}_{3} \mathrm{~N}$ (1.2 mmol, 1.5 equiv.). After complete addition, the solution was stirred at room temperature for approximately 1 hour. The solvent was removed under reduced pressure, and the crude residue was purified by column chromatography on $\mathrm{SiO}_{2}$ $\left(\mathrm{Et}_{2} \mathrm{O} /\right.$ pentanes), to yield $\mathbf{3 a} \mathbf{a} \mathbf{j}, \mathbf{3 m}$.<smiles>CC(=C(F)F)c1ccccc1NC(=O)c1ccccc1</smiles>

3a: Title compound was synthesized by following the general procedure, yielding $249 \mathrm{mg}$ of 3a as a white solid (91\% yield over two steps).

${ }^{1} \mathbf{H}$ NMR $\left(500 \mathrm{MHz} \mathrm{CDCl}_{3}\right) \delta 8.27(\mathrm{~d}, J=8.3 \mathrm{~Hz}, 1 \mathrm{H}), 7.88-7.82(\mathrm{~m}, 2 \mathrm{H}), 7.79$ (bs, 1H), 7.58 (tt, $J=7.3,1.4 \mathrm{~Hz}, 1 \mathrm{H}), 7.52(\mathrm{ddt}, J=8.2,6.5,1.4 \mathrm{~Hz}, 2 \mathrm{H}), 7.40(\mathrm{ddd}, J=8.6,7.3,1.8 \mathrm{~Hz}, 1 \mathrm{H})$, $7.24(\mathrm{dd}, J=7.7,1.8 \mathrm{~Hz}, 1 \mathrm{H}), 7.19(\mathrm{td}, J=7.5,1.2 \mathrm{~Hz}, 1 \mathrm{H}), 1.95(\mathrm{t}, J=3.4 \mathrm{~Hz}, 3 \mathrm{H})$.

${ }^{13} \mathrm{C}$ NMR $\left(126 \mathrm{MHz}, \mathrm{CDCl}_{3}\right) \delta 165.6,152.8(\mathrm{dd}, J=290.8,286.6 \mathrm{~Hz}), 135.4,135.0,132.2,129.7$ (dd, $J=3.0,1.2 \mathrm{~Hz}), 129.2,129.1,127.0,125.1,122.6,84.4$ (dd, $J=23.3,19.0 \mathrm{~Hz}), 14.6$. (One missing resonance) 
${ }^{19}$ F NMR $\left(470 \mathrm{MHz}, \mathrm{CDCl}_{3}\right) \delta-87.3(\mathrm{~d}, J=40.6 \mathrm{~Hz}, 1 \mathrm{~F}),-90.9(\mathrm{~d}, J=41.1 \mathrm{~Hz}, 1 \mathrm{~F})$.

HRMS (ESI+): calcd. for $\left[\mathrm{C}_{16} \mathrm{H}_{13} \mathrm{~F}_{2} \mathrm{NO}+\mathrm{MeCN}+\mathrm{Na}\right]^{+}: 337.1123$, found: 337.1090 .

MP: $114.2-116.0^{\circ} \mathrm{C}$

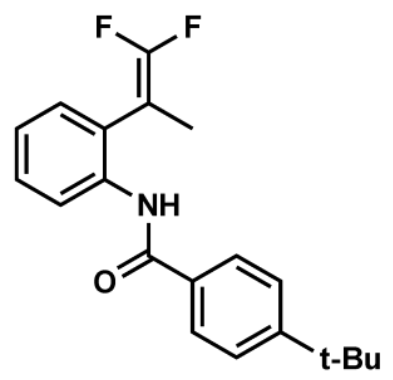

3b: Title compound was synthesized by following the general procedure, yielding $212 \mathrm{mg}$ of $\mathbf{3 b}$ as a white solid (64\% yield over two steps).

${ }^{1} \mathbf{H}$ NMR $\left(500 \mathrm{MHz}, \mathrm{CD}_{2} \mathrm{Cl}_{2}\right) \delta 8.22(\mathrm{~d}, J=8.3 \mathrm{~Hz}, 1 \mathrm{H}) 7.80(\mathrm{bs}, 1 \mathrm{H}), 7.78-7.75(\mathrm{~m}, 2 \mathrm{H}), 7.56$ $-7.53(\mathrm{~m}, 2 \mathrm{H}), 7.39$ (ddd, $J=8.5,7.3,1.7 \mathrm{~Hz}, 1 \mathrm{H}), 7.26(\mathrm{dd}, J=7.7,1.7 \mathrm{~Hz}, 1 \mathrm{H}), 7.20$ (td, $J=7.5$, $1.3 \mathrm{~Hz}, 1 \mathrm{H}), 1.94(\mathrm{t}, J=3.4 \mathrm{~Hz}, 3 \mathrm{H}), 1.36(\mathrm{~s}, 9 \mathrm{H})$.

${ }^{13} \mathrm{C}$ NMR $\left(126 \mathrm{MHz}, \mathrm{CD}_{2} \mathrm{Cl}_{2}\right) \delta 165.8,156.2,153.3(\mathrm{dd}, J=290.0,285.8 \mathrm{~Hz}), 136.2,132.6,130.1$ $(\mathrm{dd}, \mathrm{J}=3.0,1.4 \mathrm{~Hz}), 129.4,127.3,126.7(\mathrm{dd}, J=4.6,1.8 \mathrm{~Hz}), 126.4,125.3,123.0,85.0(\mathrm{dd}, J=$ 23.3, $18.9 \mathrm{~Hz}), 35.5,31.4,14.7$.

${ }^{19}$ F NMR $\left(470 \mathrm{MHz}, \mathrm{CD}_{2} \mathrm{Cl}_{2}\right) \delta-88.4(\mathrm{~d}, J=41.8 \mathrm{~Hz}, 1 \mathrm{~F}),-91.9(\mathrm{~d}, J=41.4 \mathrm{~Hz}, 1 \mathrm{~F})$.

HRMS (ESI+): calcd. for $\left[\mathrm{C}_{20} \mathrm{H}_{21} \mathrm{~F}_{2} \mathrm{NO}+\mathrm{MeCN}+\mathrm{Na}\right]^{+}: 393.1749$, found: 393.1726.

MP: $136.0-138.2{ }^{\circ} \mathrm{C}$<smiles>CC(=C(F)F)c1ccccc1NC(=O)c1cccc(C(F)(F)F)c1</smiles>

3c: Title compound was synthesized by following the general procedure, yielding $273 \mathrm{mg}$ of $\mathbf{3 c}$ as a white solid (80\% yield over two steps). 
${ }^{1} \mathbf{H}$ NMR $\left(500 \mathrm{MHz}, \mathrm{CDCl}_{3}\right) \delta 8.19(\mathrm{~d}, J=8.2 \mathrm{~Hz}, 1 \mathrm{H}), 8.13(\mathrm{~s}, 1 \mathrm{H}), 7.98(\mathrm{~d}, J=7.8 \mathrm{~Hz}, 1 \mathrm{H}), 7.83$ $(\mathrm{d}, J=6.6 \mathrm{~Hz}, 1 \mathrm{H}), 7.77(\mathrm{bs}, 1 \mathrm{H}), 7.66(\mathrm{t}, J=7.8 \mathrm{~Hz}, 1 \mathrm{H}), 7.41(\mathrm{ddd}, J=8.5,7.2,1.9 \mathrm{~Hz}, 1 \mathrm{H}), 7.28$ $-7.20(\mathrm{~m}, 2 \mathrm{H}), 1.96(\mathrm{t}, J=3.4 \mathrm{~Hz}, 3 \mathrm{H})$.

${ }^{13}$ C NMR $\left(126 \mathrm{MHz}, \mathrm{CDCl}_{3}\right) \delta 164.3,152.8(\mathrm{dd}, J=291.0,286.6 \mathrm{~Hz}), 135.9,134.9,131.9$ (q, $J=$ $33.1 \mathrm{~Hz}), 130.0,129.8,129.8,129.3,128.8(\mathrm{q}, J=3.6 \mathrm{~Hz}), 126.7-126.5(\mathrm{~m}), 125.7,124.5(\mathrm{q}, J=$ $3.7 \mathrm{~Hz}), 123.8(\mathrm{q}, J=272.6 \mathrm{~Hz}), 123.0,84.4(\mathrm{dd}, J=23.3,18.8 \mathrm{~Hz}), 14.6$.

${ }^{19} \mathbf{F}$ NMR $\left(470 \mathrm{MHz}, \mathrm{CDCl}_{3}\right) \delta-62.9(\mathrm{~s}, 3 \mathrm{~F}),-87.1(\mathrm{~d}, J=40.0 \mathrm{~Hz}, 1 \mathrm{~F}),-90.8(\mathrm{~d}, J=40.2 \mathrm{~Hz}, 1 \mathrm{~F})$. HRMS (ESI+): calcd. for [ $\left.\mathrm{C}_{17} \mathrm{H}_{12} \mathrm{~F}_{5} \mathrm{NO}+\mathrm{MeCN}+\mathrm{Na}\right]^{+}:$405.0997, found: 405.0989 .

MP: $91.9-94.7^{\circ} \mathrm{C}$<smiles>CC(=C(F)F)c1ccccc1NC(=O)c1ccc2c(c1)OCO2</smiles>

3d: Title compound was synthesized by following the general procedure, yielding $266 \mathrm{mg}$ of $\mathbf{3 d}$ as a white solid (78\% yield over two steps).

${ }^{1} \mathbf{H}$ NMR $\left(600 \mathrm{MHz}, \mathrm{CD}_{2} \mathrm{Cl}_{2}\right) \delta 8.15(\mathrm{~d}, J=8.2 \mathrm{~Hz}, 1 \mathrm{H}), 7.66(\mathrm{bs}, 1 \mathrm{H}), 7.38(\mathrm{t}, J=7.8 \mathrm{~Hz}, 1 \mathrm{H})$, $7.36-7.31(\mathrm{~m}, 2 \mathrm{H}), 7.26(\mathrm{~d}, J=7.7 \mathrm{~Hz}, 1 \mathrm{H}), 7.20(\mathrm{t}, J=7.5 \mathrm{~Hz}, 1 \mathrm{H}), 6.91(\mathrm{~d}, J=8.0 \mathrm{~Hz}, 1 \mathrm{H})$, $6.07(\mathrm{~s}, 2 \mathrm{H}), 1.93(\mathrm{t}, J=3.4 \mathrm{~Hz}, 3 \mathrm{H})$.

${ }^{13}$ C NMR $\left(126 \mathrm{MHz}, \mathrm{CDCl}_{3}\right) \delta 164.9,152.8(\mathrm{dd}, J=290.7,286.6 \mathrm{~Hz}), 151.0,148.5,135.5,129.7$ - $129.6(\mathrm{~m}), 129.2,129.2,126.1-126.0$ (m), 125.0, 122.6, 121.6, 108.5, 107.8, 102.0, 84.4 (dd, $J=23.3,19.0 \mathrm{~Hz}), 14.6$.

${ }^{19}$ F NMR $\left(470 \mathrm{MHz}, \mathrm{CDCl}_{3}\right) \delta-87.3(\mathrm{~d}, J=40.6 \mathrm{~Hz}, 1 \mathrm{~F}),-90.9(\mathrm{~d}, J=40.5 \mathrm{~Hz}, 1 \mathrm{~F})$.

HRMS (ESI+): calcd. for $\left[\mathrm{C}_{17} \mathrm{H}_{13} \mathrm{~F}_{2} \mathrm{NO}_{3}+\mathrm{MeCN}+\mathrm{Na}\right]^{+}: 381.1021$, found: 381.0992 .

MP: $99.9-101.5^{\circ} \mathrm{C}$ 


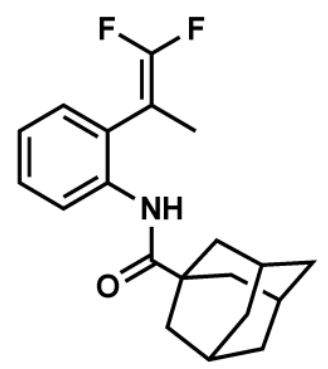

3e: Title compound was synthesized by following the general procedure, yielding $265 \mathrm{mg}$ of $\mathbf{3 e}$ as a white solid (80\% yield over two steps).

${ }^{1} \mathbf{H}$ NMR $\left(500 \mathrm{MHz}, \mathrm{CDCl}_{3}\right) \delta 8.15(\mathrm{dd}, J=8.2,0.9 \mathrm{~Hz}, 1 \mathrm{H}), 7.36(\mathrm{bs}, 1 \mathrm{H}), 7.32(\mathrm{ddd}, J=8.5,7.3$, $1.7 \mathrm{~Hz}, 1 \mathrm{H}), 7.17$ (dd, $J=7.7,1.8 \mathrm{~Hz}, 1 \mathrm{H}), 7.12(\mathrm{td}, J=7.5,1.2 \mathrm{~Hz}, 1 \mathrm{H}), 2.13-2.08(\mathrm{~m}, 3 \mathrm{H}), 1.94$ $(\mathrm{d}, J=3.0 \mathrm{~Hz}, 6 \mathrm{H}), 1.92$ (t, $J=3.4 \mathrm{~Hz}, 3 \mathrm{H}), 1.85-1.70(\mathrm{~m}, 6 \mathrm{H})$.

${ }^{13} \mathrm{C}$ NMR $\left(126 \mathrm{MHz}, \mathrm{CDCl}_{3}\right) \delta 176.0,152.7$ (dd, $\left.J=290.4,286.7 \mathrm{~Hz}\right), 135.5,129.5$ - $129.4(\mathrm{~m})$, 129.1, 125.7 (dd, $J=4.6,1.9 \mathrm{~Hz}), 124.5,122.4,84.3$ (dd, $J=23.1,19.1 \mathrm{~Hz}), 41.9,39.4,36.6,28.3$, 14.5 .

${ }^{19}$ F NMR $\left(376 \mathrm{MHz}, \mathrm{CDCl}_{3}\right) \delta-86.7(\mathrm{~d}, J=41.3 \mathrm{~Hz}, 1 \mathrm{~F}),-90.9(\mathrm{~d}, J=42.4 \mathrm{~Hz}, 1 \mathrm{~F})$.

HRMS (ESI+): calcd. for $\left[\mathrm{C}_{17} \mathrm{H}_{12} \mathrm{~F}_{5} \mathrm{NO}+\mathrm{MeCN}+\mathrm{Na}\right]^{+}: 685.3460$ found: 685.3488.

MP: $123.8-125.5^{\circ} \mathrm{C}$<smiles>O=C(Nc1ccccc1-c1ccccc1)c1ccccc1</smiles>

3f: Title compound was synthesized by following the general procedure, yielding $181 \mathrm{mg}$ of $\mathbf{3 f}$ as a white solid (54\% yield over two steps).

${ }^{1} \mathbf{H}$ NMR $\left(500 \mathrm{MHz}, \mathrm{CD}_{2} \mathrm{Cl}_{2}\right) \delta 8.37$ (d, $\left.J=8.3 \mathrm{~Hz}, 1 \mathrm{H}\right), 7.60$ (bs, 1H), $7.52-7.45$ (m, 2H), 7.43 $-7.30(\mathrm{~m}, 10 \mathrm{H}), 7.26(\mathrm{td}, J=7.6,1.3 \mathrm{~Hz}, 1 \mathrm{H})$.

${ }^{13} \mathrm{C}$ NMR (126 MHz, $\left.\mathrm{CD}_{2} \mathrm{Cl}_{2}\right) \delta 165.5,154.7$ (dd, $\left.J=299.4,290.8 \mathrm{~Hz}\right), 137.4-137.3(\mathrm{~m}), 135.3$, 132.7 (dd, $J=5.0,2.9 \mathrm{~Hz}), 132.3,132.2$ (t, $J=2.4 \mathrm{~Hz}), 130.1,129.7,129.1,128.8$ (t, $J=2.5 \mathrm{~Hz}$ ), 128.8, 127.2, 125.1, 124.2 (dd, $J=4.3,1.8 \mathrm{~Hz}), 122.6,93.1$ (dd, $J=20.8,17.0 \mathrm{~Hz}$ ).

${ }^{19}$ F NMR $\left(470 \mathrm{MHz}, \mathrm{CD}_{2} \mathrm{Cl}_{2}\right) \delta-82.3(\mathrm{~d}, J=25.1 \mathrm{~Hz}, 1 \mathrm{~F}),-86.5(\mathrm{~d}, J=25.1 \mathrm{~Hz}, 1 \mathrm{~F})$.

HRMS (ESI+): calcd. for $\left[\mathrm{C}_{21} \mathrm{H}_{15} \mathrm{~F}_{2} \mathrm{NO}+\mathrm{MeCN}+\mathrm{Na}\right]^{+}: 693.2136$ found: 693.2108 .

MP: $102.0-105.0^{\circ} \mathrm{C}$ 
<smiles>O=C(Nc1ccccc1C(=C(F)F)C1CCC1)c1ccccc1</smiles>

3g: Title compound was synthesized by following the general procedure, yielding $262 \mathrm{mg}$ of $\mathbf{3 g}$ as a white solid ( $84 \%$ yield over two steps).

${ }^{1} \mathbf{H}$ NMR $\left(600 \mathrm{MHz}, \mathrm{CD}_{2} \mathrm{Cl}_{2}\right) \delta 8.37(\mathrm{~d}, J=8.3 \mathrm{~Hz}, 1 \mathrm{H}), 7.80(\mathrm{~d}, J=8.0 \mathrm{~Hz}, 2 \mathrm{H}), 7.76(\mathrm{bs}, 1 \mathrm{H})$, $7.58(\mathrm{t}, J=7.4 \mathrm{~Hz}, 1 \mathrm{H}), 7.52(\mathrm{t}, J=7.6 \mathrm{~Hz}, 2 \mathrm{H}), 7.44(\mathrm{dd}, J=8.5,7.0 \mathrm{~Hz}, 1 \mathrm{H}), 7.20$ (dt, $J=15.7$, $7.5 \mathrm{~Hz}, 2 \mathrm{H}), 3.55-3.40(\mathrm{~m}, 1 \mathrm{H}), 2.10-1.89(\mathrm{~m}, 3 \mathrm{H}), 1.90-1.72(\mathrm{~m}, 2 \mathrm{H}), 1.59$ (q, J= 8.9, 8.4 $\mathrm{Hz}, 1 \mathrm{H})$.

${ }^{13} \mathrm{C}$ NMR $\left(151 \mathrm{MHz}, \mathrm{CD}_{2} \mathrm{Cl}_{2}\right) \delta 165.6,153.2(\mathrm{dd}, J=293.2,288.8 \mathrm{~Hz}), 137.2,135.5,132.5,131.6$ (dd, $J=2.7,1.5 \mathrm{~Hz}), 129.7,129.5,127.4,124.8,123.3-123.1(\mathrm{~m}), 122.1,91.9$ (dd, $J=20.6,14.3$ $\mathrm{Hz}), 34.3,28.0-27.4(\mathrm{~m}), 19.34$.

${ }^{19}$ F NMR $\left(470 \mathrm{MHz}, \mathrm{CD}_{2} \mathrm{Cl}_{2}\right) \delta-86.84(\mathrm{~d}, J=39.1 \mathrm{~Hz}, 1 \mathrm{~F}),-89.8(\mathrm{~d}, J=39.0 \mathrm{~Hz}, 1 \mathrm{~F})$.

HRMS (ESI+): calcd. for $\left[\mathrm{C}_{19} \mathrm{H}_{17} \mathrm{~F}_{2} \mathrm{NO}+\mathrm{MeCN}+\mathrm{Na}\right]^{+}: 377.1436$ found: 377.1425 .

MP: $114.8-116.5^{\circ} \mathrm{C}$

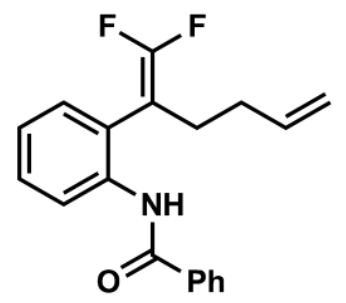

3h: Title compound was synthesized by following the general procedure, yielding $81 \mathrm{mg}$ of $\mathbf{3 h}$ as a white solid (23\% yield over two steps).

${ }^{1} \mathbf{H}$ NMR $\left(500 \mathrm{MHz}, \mathrm{CDCl}_{3}\right) \delta 8.36(\mathrm{~d}, J=8.2 \mathrm{~Hz}, 1 \mathrm{H}), 7.86(\mathrm{bs}, 1 \mathrm{H}), 7.85-7.78(\mathrm{~m}, 2 \mathrm{H}), 7.59-$ $7.55(\mathrm{~m}, 1 \mathrm{H}), 7.52-7.47(\mathrm{~m}, 2 \mathrm{H}), 7.41$ (ddd, $J=8.6,7.0,2.0 \mathrm{~Hz}, 1 \mathrm{H}), 7.23-7.15(\mathrm{~m}, 2 \mathrm{H}), 5.73$ (ddt, $J=16.9,10.2,6.5 \mathrm{~Hz}, 1 \mathrm{H}), 4.97$ (dq, $J=17.2,1.7 \mathrm{~Hz}, 1 \mathrm{H}), 4.91$ (dq, $J=10.2,1.4 \mathrm{~Hz}, 1 \mathrm{H})$, $2.50(\mathrm{tt}, J=7.2,2.3 \mathrm{~Hz}, 2 \mathrm{H}), 2.10$ (q, $J=7.0 \mathrm{~Hz}, 2 \mathrm{H})$.

${ }^{13} \mathrm{C}$ NMR $\left(126 \mathrm{MHz} \mathrm{CDCl}_{3}\right) \delta 165.5,153.1(\mathrm{dd}, J=291.3,289.2 \mathrm{~Hz}), 137.3,136.1,135.1,132.1$, $123.0-129.9(\mathrm{~m}), 129.2,129.0,127.1,124.7,123.9$ (dd, J= 4.5, $1.9 \mathrm{~Hz}), 122.3,116.5,87.9$ (dd, $J=22.2,16.3 \mathrm{~Hz}), 31.4(\mathrm{t}, J=2.7 \mathrm{~Hz}), 28.1$.

${ }^{19}$ F NMR $\left(565 \mathrm{MHz}, \mathrm{CDCl}_{3}\right) \delta-86.2(\mathrm{~d}, J=38.7 \mathrm{~Hz}, 1 \mathrm{~F}),-89.9(\mathrm{~d}, J=39.5 \mathrm{~Hz}, 1 \mathrm{~F})$.

HRMS (ESI+): calcd. for $\left[\mathrm{C}_{19} \mathrm{H}_{17} \mathrm{~F}_{2} \mathrm{NO}+\mathrm{MeCN}+\mathrm{Na}\right]^{+}: 649.2448$ found: 649.2419.

MP: $105.7-109.7^{\circ} \mathrm{C}$ 
<smiles>CCCCC(=C(F)F)c1ccccc1NC(=O)c1ccccc1</smiles>

3i: Parent aniline was synthesized by alternate literature procedure ${ }^{9}$. The aniline was reacted with benzoyl chloride according to the general procedure, yielding $176 \mathrm{mg}$ of $\mathbf{3 i}$ as a white solid (56\% yield over two steps).

${ }^{1} \mathbf{H}$ NMR $\left(500 \mathrm{MHz}, \mathrm{CDCl}_{3}\right) \delta 8.37(\mathrm{~d}, J=8.3 \mathrm{~Hz}, 1 \mathrm{H}), 7.84-7.79(\mathrm{~m}, 3 \mathrm{H}), 7.58(\mathrm{tt}, J=7.4,1.4$ $\mathrm{Hz}, 1 \mathrm{H}), 7.54-7.49$ (m, 2H), 7.41 (ddd, $J=8.6,6.6,2.4 \mathrm{~Hz}, 1 \mathrm{H}), 7.21-7.15$ (m, 2H), 2.33 (ddt, $J=7.7,4.6,2.3 \mathrm{~Hz}, 2 \mathrm{H}), 1.36-1.24(\mathrm{~m}, 4 \mathrm{H}), 0.83(\mathrm{t}, J=7.0 \mathrm{~Hz}, 3 \mathrm{H})$.

${ }^{13} \mathrm{C}$ NMR $\left(151 \mathrm{MHz}, \mathrm{CDCl}_{3}\right) \delta 165.4,153.1(\mathrm{dd}, J=291.4,288.5 \mathrm{~Hz}), 136.0,135.1,132.1,130.1$ $(\mathrm{d}, J=3.1 \mathrm{~Hz}), 129.2,129.1,127.0,124.7,124.3(\mathrm{~d}, J=4.4 \mathrm{~Hz}), 122.0,88.8(\mathrm{dd}, J=22.4,15.8$ $\mathrm{Hz}), 29.9$ (t, $J=2.5 \mathrm{~Hz}), 28.5,22.4,13.8$.

${ }^{19}$ F NMR $\left(470 \mathrm{MHz}, \mathrm{CDCl}_{3}\right) \delta-87.0(\mathrm{~d}, J=39.6 \mathrm{~Hz}, 1 \mathrm{~F}),-90.8(\mathrm{~d}, J=39.6 \mathrm{~Hz}, 1 \mathrm{~F})$.

HRMS (ESI+): calcd. for $\left[\mathrm{C}_{19} \mathrm{H}_{19} \mathrm{~F}_{2} \mathrm{NO}+\mathrm{MeCN}+\mathrm{Na}\right]^{+}: 379.1593$ found: 379.1556.

MP: $110.7-111.1^{\circ} \mathrm{C}$<smiles>CC(=C(F)F)c1cc2c(cc1NC(=O)c1ccccc1)OCCO2</smiles>

3j: Title compound was synthesized by following the general procedure, yielding $40 \mathrm{mg}$ of $\mathbf{3 j}$ as a white solid (12\% yield over two steps).

${ }^{1} \mathbf{H}$ NMR $\left(600 \mathrm{MHz}, \mathrm{CDCl}_{3}\right) \delta 7.81(\mathrm{~d}, J=7.0 \mathrm{~Hz}, 2 \mathrm{H}), 7.72(\mathrm{~s}, 1 \mathrm{H}), 7.61-7.54(\mathrm{~m}, 2 \mathrm{H}), 7.50(\mathrm{t}$, $J=7.5 \mathrm{~Hz}, 2 \mathrm{H}), 6.73(\mathrm{~s}, 1 \mathrm{H}), 4.27(\mathrm{ddt}, J=8.7,5.3,3.3 \mathrm{~Hz}, 4 \mathrm{H}), 1.89$ (t, $J=3.4 \mathrm{~Hz}, 3 \mathrm{H})$.

${ }^{13} \mathrm{C}$ NMR $\left(151 \mathrm{MHz}, \mathrm{CDCl}_{3}\right) \delta 165.5,152.9$ (dd, $\left.J=290.6,285.7 \mathrm{~Hz}\right), 143.6,141.0,135.1,132.0$, 129.1, 127.0, 117.7, 112.6, 84.1 (dd, $J=23.5,19.0 \mathrm{~Hz}$ ), 64.6, 64.5, 14.7. (Two missing resonances)

${ }^{19}$ F NMR $\left(565 \mathrm{MHz}, \mathrm{CDCl}_{3}\right) \delta-87.8(\mathrm{~d}, J=41.1 \mathrm{~Hz}, 1 \mathrm{~F}),-91.4(\mathrm{~d}, J=41.4 \mathrm{~Hz}, 1 \mathrm{~F})$.

HRMS (ESI+): calcd. for $\left[\mathrm{C}_{18} \mathrm{H}_{15} \mathrm{~F}_{2} \mathrm{NO}_{3}+\mathrm{MeCN}+\mathrm{Na}\right]^{+}: 395.1178$ found: 395.1157.

MP: $74.9-80.6^{\circ} \mathrm{C}$ 
<smiles>COc1ccc(C(=O)Nc2ccc(Cl)cc2C(C)=C(F)F)cc1</smiles>

31: Title compound was synthesized by following the general procedure on a $5.0 \mathrm{mmol}$ scale, yielding $846 \mathrm{mg}$ of $\mathbf{3 h}$ as a white solid (41\% yield over two steps).

${ }^{1}$ H NMR $\left(500 \mathrm{MHz}, \mathrm{CDCl}_{3}\right) \delta 8.24(\mathrm{~d}, J=8.9 \mathrm{~Hz}, 1 \mathrm{H}), 7.81-7.76(\mathrm{~m}, 2 \mathrm{H}), 7.67$ (bs, 1H), 7.34 $(\mathrm{dd}, J=8.8,2.5 \mathrm{~Hz}, 1 \mathrm{H}), 7.21(\mathrm{~d}, J=2.5 \mathrm{~Hz}, 1 \mathrm{H}), 7.03-6.96(\mathrm{~m}, 2 \mathrm{H}), 3.88(\mathrm{~s}, 3 \mathrm{H}), 1.93(\mathrm{t}, J=$ $3.4 \mathrm{~Hz}, 3 \mathrm{H})$.

${ }^{13} \mathrm{C}$ NMR $\left(151 \mathrm{MHz}, \mathrm{CDCl}_{3}\right) \delta 165.1,162.9,153.0(\mathrm{dd}, J=291.5,287.8 \mathrm{~Hz}), 134.3,129.7,129.5$ (m), 129.2, 128.9, 127.3, 126.8, 123.7, 114.4, 83.8 (dd, $J=23.8,18.7$ Hz), 55.7, 14.4.

${ }^{19}$ F NMR $\left(470 \mathrm{MHz}, \mathrm{CDCl}_{3}\right) \delta-86.2(\mathrm{~d}, J=37.9 \mathrm{~Hz}, 1 \mathrm{~F}),-90.0(\mathrm{~d}, J=37.4 \mathrm{~Hz}, 1 \mathrm{~F})$.

HRMS (ESI+): calcd. for $\left[\mathrm{C}_{17} \mathrm{H}_{14} \mathrm{ClF}_{2} \mathrm{NO}_{2}+\mathrm{MeCN}+\mathrm{Na}\right]^{+}: 401.0839$ found: 401.0822.

MP: $152.8-155.3^{\circ} \mathrm{C}$<smiles>COc1ccc(C(=O)Nc2ccc(Cl)cc2C(C#CC2CC2)=C(F)F)cc1</smiles>

3m: Title compound was synthesized by following the general procedure on a $4.5 \mathrm{mmol}$ scale, yielding $350 \mathrm{mg}$ of $\mathbf{3 m}$ as a white solid (20\% yield over two steps).

${ }^{1} \mathbf{H}$ NMR $\left(500 \mathrm{MHz} \mathrm{CDCl}_{3}\right) \delta 8.46(\mathrm{bs}, 1 \mathrm{H}), 8.38(\mathrm{~d}, J=8.8 \mathrm{~Hz}, 1 \mathrm{H}), 7.91(\mathrm{~d}, J=8.8 \mathrm{~Hz}, 2 \mathrm{H})$, 7.36 (dd, $J=8.9,2.5 \mathrm{~Hz}, 1 \mathrm{H}), 7.23(\mathrm{t}, J=2.2 \mathrm{~Hz}, 1 \mathrm{H}), 7.00$ (d, $J=8.8 \mathrm{~Hz}, 2 \mathrm{H}), 3.89$ (s, $3 \mathrm{H}), 1.43$ $-1.33(\mathrm{~m}, 1 \mathrm{H}), 0.87-0.81(\mathrm{~m}, 2 \mathrm{H}), 0.74-0.65(\mathrm{~m}, 2 \mathrm{H})$.

${ }^{13}$ C NMR $\left(126 \mathrm{MHz}, \mathrm{CDCl}_{3}\right) \delta 164.8,162.9,160.4(\mathrm{t}, J=300.8 \mathrm{~Hz}), 135.4(\mathrm{~d}, J=3.3 \mathrm{~Hz}), 129.9$ - 129.8 (m), 129.7, 129.3, 129.2, 129.1, 126.9, 123.4, 122.0 (dd, J= 4.0, 2.9 Hz), 114.2, 101.1 (t, $J=5.8 \mathrm{~Hz}$ ), 55.67, 8.91, 0.30. (One missing resonance)

${ }^{19}$ F NMR $\left(470 \mathrm{MHz}, \mathrm{CDCl}_{3}\right) \delta-76.2(\mathrm{~s}, 1 \mathrm{~F}),-77.9(\mathrm{~s}, 1 \mathrm{~F})$.

HRMS (ESI+): calcd. for [ $\left.\mathrm{C}_{21} \mathrm{H}_{16} \mathrm{ClF}_{2} \mathrm{NO}_{2}+\mathrm{MeCN}+\mathrm{Na}\right]^{+}: 451.0996$ found: 451.1004.

MP: $154.7-155.8^{\circ} \mathrm{C}$ 
<smiles>CC(=C(F)F)c1cccnc1N(C(=O)c1ccccc1)c1ccccc1</smiles>

3k-Bz: $234 \mathrm{mg}$ (85\% over two steps) of title compound isolated as white solid from reaction of $124 \mathrm{mg}$ of parent amino pyridine ( $0.73 \mathrm{mmol}, 1.0$ equiv.), $0.22 \mathrm{~mL}$ of BzCl (1.9 mmol, 2.6 equiv.), $0.31 \mathrm{~mL}$ of $\mathrm{Et}_{3} \mathrm{~N}$ (2.2 mmol, 3.0 equiv.) in $7.3 \mathrm{~mL}$ of DCM.

${ }^{1} \mathbf{H}$ NMR $\left(600 \mathrm{MHz}, \mathrm{CD}_{2} \mathrm{Cl}_{2}\right) \delta 8.48(\mathrm{dd}, J=4.7,1.9 \mathrm{~Hz}, 1 \mathrm{H}), 7.73(\mathrm{~d}, J=7.1 \mathrm{~Hz}, 4 \mathrm{H}), 7.57(\mathrm{~d}$, $J=7.8 \mathrm{~Hz}, 1 \mathrm{H}), 7.51-7.44(\mathrm{~m}, 2 \mathrm{H}), 7.35(\mathrm{t}, J=7.8 \mathrm{~Hz}, 4 \mathrm{H}), 7.28(\mathrm{dd}, J=7.7,4.7 \mathrm{~Hz}, 1 \mathrm{H}), 1.79$ $(\mathrm{t}, J=3.3 \mathrm{~Hz}, 3 \mathrm{H})$.

${ }^{13}$ C NMR $\left(151 \mathrm{MHz}, \mathrm{CD}_{2} \mathrm{Cl}_{2}\right) \delta 173.40,153.8(\mathrm{t}, J=289.0 \mathrm{~Hz}), 153.0,149.7,140.5(\mathrm{~d}, J=2.7$ $\mathrm{Hz}), 135.1,133.1,129.7,129.6$ (dd, $J=5.5,2.6 \mathrm{~Hz}), 129.0,123.8,85.2$ (dd, $J=26.0,16.9 \mathrm{~Hz})$, 13.4 .

${ }^{19}$ F NMR $\left(565 \mathrm{MHz}, \mathrm{CD}_{2} \mathrm{Cl}_{2}\right) \delta-87.5(\mathrm{~d}, J=35.4 \mathrm{~Hz}),-90.5(\mathrm{~d}, J=35.6 \mathrm{~Hz})$.

HRMS (ESI+): calcd. for [ $\left.\mathrm{C}_{22} \mathrm{H}_{16} \mathrm{~F}_{2} \mathrm{~N}_{2} \mathrm{O}_{2}+\mathrm{H}\right]^{+}: 379.1253$ found: 379.1227.<smiles>CC(=C(F)F)c1cccnc1NC(=O)c1ccccc1</smiles>

3k: To a solution of $230 \mathrm{mg}$ of $\mathbf{3 k - B z}(0.62 \mathrm{mmol}, 1.0$ equiv.) in $6.2 \mathrm{~mL}$ of anhydrous DMF, was added $270 \mathrm{mg}$ of piperazine ( $3.1 \mathrm{mmol}, 5.0$ equiv.). After the solution was allowed to stir overnight, the reaction mixture was diluted with $25 \mathrm{~mL}_{\text {of }} \mathrm{Et}_{2} \mathrm{O}$, and washed thrice with 30 $\mathrm{mL}$ of brine. The organic layer was then dried over $\mathrm{Na}_{2} \mathrm{SO}_{4}$, filtered and the solvent removed in vасио. The residue was purified by column chromatography $\left(1: 5 \mathrm{Et}_{2} \mathrm{O} /\right.$ pentane to $1: 1 \mathrm{Et}_{2} \mathrm{O} /$ pentane gradient) to yield $68 \mathrm{mg}(40 \%)$ of the title compound as a white solid.

${ }^{1} \mathbf{H}$ NMR $\left(600 \mathrm{MHz}, \mathrm{CDCl}_{3}\right) \delta 8.51(\mathrm{bs}, 1 \mathrm{H}), 8.37(\mathrm{~d}, J=3.0 \mathrm{~Hz}, 1 \mathrm{H}), 7.88(\mathrm{~d}, J=7.3 \mathrm{~Hz}, 2 \mathrm{H})$, $7.66(\mathrm{dd}, J=7.7,1.8 \mathrm{~Hz}, 1 \mathrm{H}), 7.56(\mathrm{t}, J=7.4 \mathrm{~Hz}, 1 \mathrm{H}), 7.47(\mathrm{t}, J=7.7 \mathrm{~Hz}, 2 \mathrm{H}), 7.21(\mathrm{dd}, J=7.7$, $4.8 \mathrm{~Hz}, 1 \mathrm{H}), 1.98(\mathrm{t}, J=3.3 \mathrm{~Hz}, 3 \mathrm{H})$.

${ }^{13} \mathrm{C}$ NMR $\left(151 \mathrm{MHz}, \mathrm{CDCl}_{3}\right) \delta 166.1,152.5(\mathrm{t}, J=287.5 \mathrm{~Hz}), 148.7,147.9,139.3(\mathrm{~d}, J=2.4 \mathrm{~Hz})$, $134.3,132.3,128.9,127.6,126.0,121.5,85.3(\mathrm{dd}, J=26.0,17.7 \mathrm{~Hz}), 13.3$.

${ }^{19}$ F NMR $\left(565 \mathrm{MHz}, \mathrm{CDCl}_{3}\right) \delta-89.1(\mathrm{~d}, J=40.1 \mathrm{~Hz}),-92.5(\mathrm{~d}, J=40.3 \mathrm{~Hz})$.

HRMS (ESI+): calcd. for $\left[\mathrm{C}_{15} \mathrm{H}_{12} \mathrm{~F}_{2} \mathrm{~N}_{2} \mathrm{O}+\mathrm{MeCN}+\mathrm{H}\right]^{+}: 275.0991$ found: 275.0963 .

MP: $60.1-62.6^{\circ} \mathrm{C}$ 


\section{Synthesis of $\mathrm{CF}_{2} \mathrm{Br}$ Containing Heterocycles}

\section{General Procedure for Racemic Bromocyclization}<smiles>O=C([Ga])NCC(Br)=C(F)F</smiles>

1a-I<smiles>[R]C=[R]</smiles>

3a-I
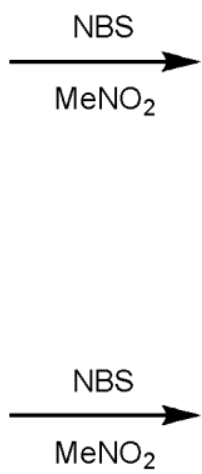<smiles>BrC(Br)(Br)C1(Br)CN=C(c2ccccc2)O1</smiles>

2a-I<smiles>[R]C1=Nc2ccc([R])cc2C([R])(C(F)(F)Br)O1</smiles>

$4 a-1$

To a solution of difluoroalkene ( $0.05 \mathrm{mmol}, 1.0$ equiv.) in $0.5 \mathrm{~mL}$ of $\mathrm{MeNO}_{2}, \mathrm{NBS}(13 \mathrm{mg}$, $0.075 \mathrm{mmol}, 1.5$ equiv.) was added. After stirring at room temperature overnight, the crude reaction mixture was loaded directly onto a preparatory TLC plate, and eluted with EtOAc/Hexanes. The product was isolated directly from the plate in analytical purity.<smiles>FC1(Br)CN=C(c2ccccc2)OC1(F)Br</smiles>

2a': $8 \mathrm{mg}$ of $\mathbf{2} \mathbf{a}^{\prime}(45 \%)$ isolated from reaction of $\mathbf{1 a}$ with NBS in nitromethane. In pure form, title compound decomposes over time.

${ }^{1} \mathbf{H}$ NMR $\left(500 \mathrm{MHz}, \mathrm{CDCl}_{3}\right) \delta 7.99(\mathrm{dd}, J=8.4,1.3 \mathrm{~Hz}, 2 \mathrm{H}), 7.58(\mathrm{~d}, J=8.1 \mathrm{~Hz}, 2 \mathrm{H}), 7.54-7.48$ $(\mathrm{m}, 1 \mathrm{H}), 7.43(\mathrm{t}, J=7.7 \mathrm{~Hz}, 2 \mathrm{H}), 7.20(\mathrm{~d}, J=8.0 \mathrm{~Hz}, 2 \mathrm{H}), 4.61(\mathrm{dt}, J=18.0,3.2 \mathrm{~Hz}, 1 \mathrm{H}), 4.52(\mathrm{dt}$, $J=18.0,3.7 \mathrm{~Hz}, 1 \mathrm{H}), 2.36(\mathrm{~s}, 3 \mathrm{H})$.

${ }^{13}$ C NMR $\left(126 \mathrm{MHz}, \mathrm{CDCl}_{3}\right) \delta 151.0,139.8,132.4,132.0,130.3,129.5,128.5,128.0,127.8,56.2$, 21.2. (Two missing resonances)

${ }^{19}$ F NMR (151 MHz, $\left.\mathrm{CDCl}_{3}\right) \delta-75.0(\mathrm{bs})$.

HRMS (ESI+): calcd. for $\left[\mathrm{C}_{17} \mathrm{H}_{14} \mathrm{BrF}_{2} \mathrm{NO}+\mathrm{MeCN}+\mathrm{H}\right]^{+}: 407.0565$ found: 407.0543. 


\section{General Procedure for the Enantioselective Bromocyclization to 2a-l:}<smiles>O=C([Al])NCC(Br)=C(F)F</smiles>

1a-I

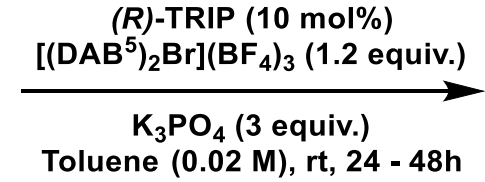

Toluene (0.02 M), rt, 24 - 48h

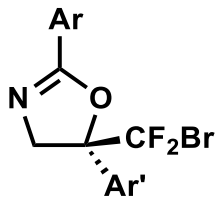

2a-I

To a flame-dried 2-dram vial was added amide substrate $(0.10 \mathrm{mmol}, 1.0$ equiv. $),(R)$ $\operatorname{TRIP}^{10}$ (7.6 mg, $\left.0.010 \mathrm{mmol}, 10 \mathrm{~mol} \%\right), \mathrm{K}_{3} \mathrm{PO}_{4}$ (67 mg, $0.3 \mathrm{mmol}, 3.0$ equiv.), and brominating agent $\left[\left(\mathbf{D A B}^{\mathbf{5}}\right)_{\mathbf{2}} \mathbf{B r}\right]\left(\mathbf{B F}_{4}\right)_{3} \quad(117 \mathrm{mg}, 0.12 \mathrm{mmol}, 1.2$ equiv. $)$. After the vial was evacuated and backfilled three times with $\mathrm{N}_{2}, 5.0 \mathrm{~mL}$ of dry, degassed toluene was added. The reaction was stirred at >800 rpm for until conversion stopped (24-48 h), as judged by TLC. Once complete, the reaction was filtered through Celite ${ }^{\circledR}$ with EtOAc as the eluent. The solvent was the removed under reduced pressure, and the crude product was purified by column chromatography ( $\mathrm{Et}_{2} \mathrm{O} /$ pentane) to yield the pure product.

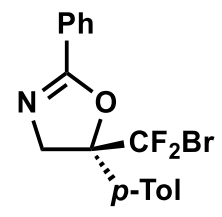

2a: Title compound was synthesized by following the general procedure for oxazolines, yielding $28 \mathrm{mg}$ of $\mathbf{2 a}$ as a white solid (75\% yield).

${ }^{1} \mathbf{H}$ NMR $\left(600 \mathrm{MHz}, \mathrm{CDCl}_{3}\right) \delta 8.06(\mathrm{~d}, J=7.4 \mathrm{~Hz}, 2 \mathrm{H}), 7.54(\mathrm{t}, J=7.4 \mathrm{~Hz}, 1 \mathrm{H}), 7.49-7.44(\mathrm{~m}$, $4 \mathrm{H}), 7.23(\mathrm{~d}, J=8.0 \mathrm{~Hz}, 2 \mathrm{H}), 4.78(\mathrm{~d}, J=15.5 \mathrm{~Hz}, 1 \mathrm{H}), 4.39$ (d, $J=15.5 \mathrm{~Hz}, 1 \mathrm{H}), 2.38(\mathrm{~s}, 3 \mathrm{H})$.

${ }^{13}$ C NMR (151 MHz, $\left.\mathrm{CDCl}_{3}\right) \delta 162.6,139.4,133.7,132.1,129.3,128.7,128.5,126.9,126.9$, $124.3(\mathrm{t}, J=312.7 \mathrm{~Hz}), 90.6(\mathrm{t}, J=24.7 \mathrm{~Hz}), 64.9,21.3$.

${ }^{19}$ F NMR $\left(565 \mathrm{MHz} \mathrm{CDCl}_{3}\right) \delta-59.4(\mathrm{~d}, J=168.8 \mathrm{~Hz}, 1 \mathrm{~F}),-60.4(\mathrm{~d}, J=168.2 \mathrm{~Hz}, 1 \mathrm{~F})$.

HRMS (ESI+): calcd. for $\left[\mathrm{C}_{17} \mathrm{H}_{14} \mathrm{BrF}_{2} \mathrm{NO}+\mathrm{MeCN}+\mathrm{Na}\right]^{+}: 407.0565$ found: 407.0540 .

MP: $74.3-78.0^{\circ} \mathrm{C}$ 


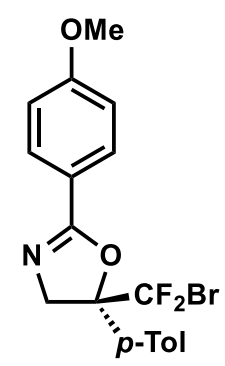

2b: Title compound was synthesized by following the general procedure for oxazolines, yielding $25 \mathrm{mg}$ of $\mathbf{2 b}$ as a white solid (63\% yield).

${ }^{1} \mathbf{H}$ NMR $\left(600 \mathrm{MHz}, \mathrm{CDCl}_{3}\right) \delta 8.03-7.95(\mathrm{~m}, 2 \mathrm{H}), 7.44(\mathrm{~d}, J=7.9 \mathrm{~Hz}, 2 \mathrm{H}), 7.22(\mathrm{~d}, J=8.0 \mathrm{~Hz}$, 2H), $7.00-6.93(\mathrm{~m}, 2 \mathrm{H}), 4.75(\mathrm{~d}, J=15.3 \mathrm{~Hz}, 1 \mathrm{H}), 4.35(\mathrm{~d}, J=15.3 \mathrm{~Hz}, 1 \mathrm{H}), 3.87(\mathrm{~s}, 3 \mathrm{H}), 2.37$ (s, 3H).

${ }^{13} \mathrm{C}$ NMR $\left(151 \mathrm{MHz}, \mathrm{CDCl}_{3}\right) \delta 162.7,162.3,139.4,133.8,130.3,129.3,126.9,124.4(\mathrm{t}, J=312.7$ $\mathrm{Hz}), 119.4,114.1,90.4(\mathrm{t}, J=24.6 \mathrm{~Hz}), 64.8,55.6,21.3$.

${ }^{19}$ F NMR $\left(565 \mathrm{MHz}, \mathrm{CDCl}_{3}\right) \delta-59.2(\mathrm{~d}, J=168.2 \mathrm{~Hz}, 1 \mathrm{~F}),-60.3(\mathrm{~d}, J=168.0 \mathrm{~Hz}, 1 \mathrm{~F})$.

HRMS (ESI+): calcd. for $\left[\mathrm{C}_{18} \mathrm{H}_{16} \mathrm{~F}_{2} \mathrm{NO}_{2}+\mathrm{H}\right]^{+}: 396.0405$ found: 396.0384 .

MP: $76.4-79.6^{\circ} \mathrm{C}$

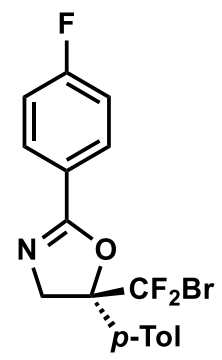

2c: Title compound was synthesized by following the general procedure for oxazolines, yielding $24 \mathrm{mg}$ of $2 \mathrm{c}$ as a colorless oil (68\% yield).

${ }^{1} \mathbf{H}$ NMR $\left(600 \mathrm{MHz}, \mathrm{CDCl}_{3}\right) \delta 8.08-8.03(\mathrm{~m}, 2 \mathrm{H}), 7.44(\mathrm{~d}, J=8.0 \mathrm{~Hz}, 2 \mathrm{H}), 7.23(\mathrm{~d}, J=8.5 \mathrm{~Hz}$, 2H), $7.18-7.13(\mathrm{~m}, 2 \mathrm{H}), 4.76(\mathrm{~d}, J=15.5 \mathrm{~Hz}, 1 \mathrm{H}), 4.38$ (d, $J=15.5 \mathrm{~Hz}, 1 \mathrm{H}), 2.38$ (s, 3H).

${ }^{13}$ C NMR $\left(151 \mathrm{MHz}, \mathrm{CDCl}_{3}\right) \delta 165.2(\mathrm{~d}, J=253.1 \mathrm{~Hz}), 161.6,139.5,133.5,130.8(\mathrm{~d}, J=9.1 \mathrm{~Hz})$, 129.3, 126.9, 124.2 (t, $J=312.7 \mathrm{~Hz}), 123.2$ (d, $J=3.0 \mathrm{~Hz}), 115.9$ (d, $J=22.1 \mathrm{~Hz}), 90.7$ (t, $J=24.7$ $\mathrm{Hz}), 64.9,21.3$.

${ }^{19}$ F NMR $\left(565 \mathrm{MHz}, \mathrm{CDCl}_{3}\right) \delta-59.5(\mathrm{~d}, J=168.3 \mathrm{~Hz}, 1 \mathrm{~F}),-60.5(\mathrm{~d}, J=168.3 \mathrm{~Hz}, 1 \mathrm{~F}),-107.0(\mathrm{p}$, $J=6.7 \mathrm{~Hz}, 1 \mathrm{~F})$.

HRMS (ESI+): calcd. for $\left[\mathrm{C}_{17} \mathrm{H}_{13} \mathrm{BrF}_{3} \mathrm{NO}+\mathrm{H}\right]^{+}: 384.0206$ found: 384.0210 . 


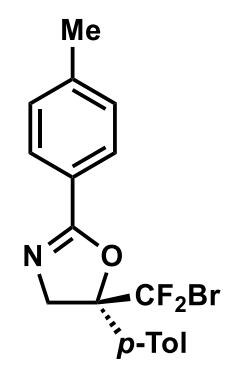

2d: Title compound was synthesized by following the general procedure for oxazolines, yielding $22 \mathrm{mg}$ of $\mathbf{2 d}$ as a colorless oil (58\% yield).

${ }^{1} \mathbf{H}$ NMR $\left(600 \mathrm{MHz}, \mathrm{CDCl}_{3}\right) \delta 7.94(\mathrm{~d}, J=8.1 \mathrm{~Hz}, 2 \mathrm{H}), 7.45(\mathrm{~d}, J=8.0 \mathrm{~Hz}, 2 \mathrm{H}), 7.27(\mathrm{~d}, J=8.1$ $\mathrm{Hz}, 2 \mathrm{H}), 7.22(\mathrm{~d}, J=8.0 \mathrm{~Hz}, 2 \mathrm{H}), 4.76(\mathrm{~d}, J=15.4 \mathrm{~Hz}, 1 \mathrm{H}), 4.37$ (d, $J=15.4 \mathrm{~Hz}, 1 \mathrm{H}), 2.42(\mathrm{~s}, 3 \mathrm{H})$, $2.37(\mathrm{~s}, 3 \mathrm{H})$.

${ }^{13}$ C NMR $\left(151 \mathrm{MHz}, \mathrm{CDCl}_{3}\right) \delta 162.7,142.6,139.4,133.8,129.4,129.3,128.5,126.9,124.3(\mathrm{t}$, $J=312.6 \mathrm{~Hz}), 124.1,90.4(\mathrm{t}, J=24.5 \mathrm{~Hz}), 64.9,21.8,21.3$.

${ }^{19}$ F NMR $\left(565 \mathrm{MHz}, \mathrm{CDCl}_{3}\right) \delta-59.2(\mathrm{~d}, J=168.6 \mathrm{~Hz}, 1 \mathrm{~F}),-60.3(\mathrm{~d}, J=168.0 \mathrm{~Hz}, 1 \mathrm{~F})$.

HRMS (ESI+): calcd. for $\left[\mathrm{C}_{18} \mathrm{H}_{16} \mathrm{BrF}_{2} \mathrm{NO}+\mathrm{H}\right]^{+}: 380.0456$ found: 380.0438 .

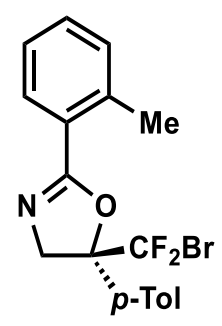

2e: Title compound was synthesized by following the general procedure for oxazolines, yielding $20 \mathrm{mg}$ of $2 \mathbf{e}$ as a white solid (53\% yield).

${ }^{1} \mathbf{H}$ NMR $\left(600 \mathrm{MHz}, \mathrm{CDCl}_{3}\right) \delta 7.94(\mathrm{dd}, J=8.2,1.4 \mathrm{~Hz}, 1 \mathrm{H}), 7.44(\mathrm{~d}, J=8.0 \mathrm{~Hz}, 2 \mathrm{H}), 7.40(\mathrm{td}, J=$ $7.5,1.5 \mathrm{~Hz}, 1 \mathrm{H}), 7.29$ (t, $J=7.2 \mathrm{~Hz}, 2 \mathrm{H}), 7.23$ (d, $J=7.6 \mathrm{~Hz}, 2 \mathrm{H}), 4.81(\mathrm{~d}, J=15.5 \mathrm{~Hz}, 1 \mathrm{H}), 4.42$ $(\mathrm{d}, J=15.5 \mathrm{~Hz}, 1 \mathrm{H}), 2.64(\mathrm{~s}, 3 \mathrm{H}), 2.38(\mathrm{~s}, 3 \mathrm{H})$.

${ }^{13} \mathrm{C}$ NMR $\left(151 \mathrm{MHz}, \mathrm{CDCl}_{3}\right) \delta 162.9,139.4,139.3,133.8,131.6,131.3,130.2,129.3,127.0$, 126.2, 125.9, $124.4(\mathrm{t}, J=313.0 \mathrm{~Hz}), 89.9$ (t, $J=24.5 \mathrm{~Hz}), 65.0,22.0,21.3$.

${ }^{19}$ F NMR $\left(565 \mathrm{MHz}, \mathrm{CDCl}_{3}\right) \delta-59.6(\mathrm{~d}, J=168.2 \mathrm{~Hz}, 1 \mathrm{~F}),-60.5(\mathrm{~d}, J=167.7 \mathrm{~Hz}, 1 \mathrm{~F})$.

HRMS (ESI+): calcd. for $\left[\mathrm{C}_{18} \mathrm{H}_{16} \mathrm{BrF}_{2} \mathrm{NO}+\mathrm{H}\right]^{+}: 380.04558$ found: 380.0457 .

MP: $62.5-64.9^{\circ} \mathrm{C}$ 


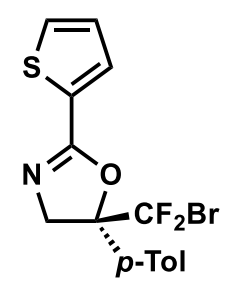

2f: Title compound was synthesized by following the general procedure for oxazolines, yielding $24 \mathrm{mg}$ of $\mathbf{2 f}$ as a white solid (69\% yield).

${ }^{1} \mathbf{H}$ NMR $\left(600 \mathrm{MHz}, \mathrm{CDCl}_{3}\right) \delta 7.77(\mathrm{~d}, J=3.7 \mathrm{~Hz}, 1 \mathrm{H}), 7.52(\mathrm{~d}, J=5.0 \mathrm{~Hz}, 1 \mathrm{H}), 7.43(\mathrm{~d}, J=7.9$ $\mathrm{Hz}, 2 \mathrm{H}), 7.23(\mathrm{~d}, J=8.0 \mathrm{~Hz}, 2 \mathrm{H}), 7.22(\mathrm{dd}, J=5.0,3.7 \mathrm{~Hz}, 1 \mathrm{H}), 4.75(\mathrm{~d}, J=15.4 \mathrm{~Hz}, 1 \mathrm{H}), 4.37$ (d, $J=15.4 \mathrm{~Hz}, 1 \mathrm{H}), 2.37$ (s, 3H).

${ }^{13} \mathrm{C}$ NMR $\left(151 \mathrm{MHz}, \mathrm{CDCl}_{3}\right) \delta 158.3,139.5,133.4,131.2,130.8,129.3,129.2,127.9,126.9$, $124.1(\mathrm{t}, J=312.6 \mathrm{~Hz}), 90.9(\mathrm{t}, J=24.7 \mathrm{~Hz}), 64.9,21.3$.

HRMS $\left(565 \mathrm{MHz}, \mathrm{CDCl}_{3}\right) \delta-59.3(\mathrm{~d}, J=168.9 \mathrm{~Hz}, 1 \mathrm{~F}),-60.4(\mathrm{~d}, J=168.6 \mathrm{~Hz}, 1 \mathrm{~F})$.

HRMS (ESI+): calcd. for $\left[\mathrm{C}_{15} \mathrm{H}_{12} \mathrm{BrF}_{2} \mathrm{NOS}+\mathrm{MeCN}+\mathrm{H}\right]^{+}$: 413.0129 found: 413.0142.

MP: $63.5-65.8^{\circ} \mathrm{C}$

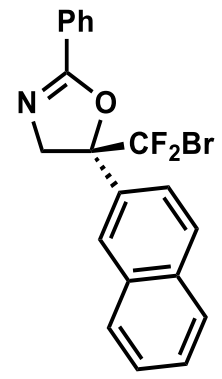

2g: Title compound was synthesized by following the general procedure for oxazolines, yielding $16 \mathrm{mg}$ of $\mathbf{2 g}$ as a white solid (37\% yield).

${ }^{1} \mathbf{H}$ NMR $\left(600 \mathrm{MHz}, \mathrm{CDCl}_{3}\right) \delta 8.11(\mathrm{~d}, J=8.0 \mathrm{~Hz}, 2 \mathrm{H}), 8.07(\mathrm{~s}, 1 \mathrm{H}), 7.90(\mathrm{~d}, J=8.6 \mathrm{~Hz}, 2 \mathrm{H}), 7.88$ $-7.85(\mathrm{~m}, 1 \mathrm{H}), 7.63(\mathrm{~d}, J=8.7 \mathrm{~Hz}, 1 \mathrm{H}), 7.59-7.48(\mathrm{~m}, 5 \mathrm{H}), 4.89(\mathrm{~d}, J=15.5 \mathrm{~Hz}, 1 \mathrm{H}), 4.52(\mathrm{~d}$, $J=15.5 \mathrm{~Hz}, 1 \mathrm{H})$.

${ }^{13} \mathrm{C}$ NMR $\left(151 \mathrm{MHz}, \mathrm{CDCl}_{3}\right) \delta 162.6,133.9,133.5,132.8,132.2,128.8,128.6,128.6,127.8$, 127.2, 126.9, 126.7, $124.2(\mathrm{t}, J=312.8 \mathrm{~Hz}), 124.0,90.8(\mathrm{t}, J=24.8 \mathrm{~Hz}), 65.0$. (Two missing resonances)

${ }^{19}$ F NMR $\left(565 \mathrm{MHz}, \mathrm{CDCl}_{3}\right) \delta-59.0(\mathrm{~d}, J=169.3 \mathrm{~Hz}, 1 \mathrm{~F}),-60.2(\mathrm{~d}, J=169.1 \mathrm{~Hz}, 1 \mathrm{~F})$.

HRMS (ESI+): calcd. for $\left[\mathrm{C}_{20} \mathrm{H}_{14} \mathrm{BrF}_{2} \mathrm{NO}+\mathrm{H}\right]^{+}: 402.0300$ found: 402.0274.

MP: $77.3-83.2{ }^{\circ} \mathrm{C}$ 


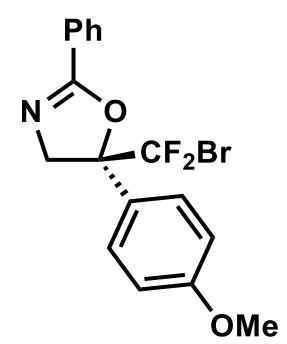

2h: Title compound was synthesized by following the general procedure for oxazolines, yielding $25 \mathrm{mg}$ of $\mathbf{2 h}$ as colorless oil (79\% yield).

${ }^{1} \mathbf{H}$ NMR $\left(600 \mathrm{MHz}, \mathrm{CDCl}_{3}\right) \delta 8.05(\mathrm{~d}, J=7.6 \mathrm{~Hz}, 2 \mathrm{H}), 7.54(\mathrm{t}, J=7.4 \mathrm{~Hz}, 1 \mathrm{H}), 7.50-7.43(\mathrm{~m}$, 4H), $6.94(\mathrm{~d}, J=8.5 \mathrm{~Hz}, 2 \mathrm{H}), 4.77(\mathrm{~d}, J=15.4 \mathrm{~Hz}, 1 \mathrm{H}), 4.39$ (d, $J=15.4 \mathrm{~Hz}, 1 \mathrm{H}), 3.83$ (s, 3H).

${ }^{13} \mathrm{C}$ NMR $\left(151 \mathrm{MHz}, \mathrm{CDCl}_{3}\right) \delta 162.5,160.4,132.1,128.7,128.5,128.5,128.4,126.9,124.4(\mathrm{t}$, $J=312.6 \mathrm{~Hz}), 114.0,90.4(\mathrm{t}, J=24.7 \mathrm{~Hz}), 64.8,55.5$.

${ }^{19}$ F NMR $\left(565 \mathrm{MHz}, \mathrm{CDCl}_{3}\right) \delta-59.5(\mathrm{~d}, J=168.2 \mathrm{~Hz}, 1 \mathrm{~F}),-60.5(\mathrm{~d}, J=168.0 \mathrm{~Hz}, 1 \mathrm{~F})$.

HRMS (ESI+): calcd. for $\left[\mathrm{C}_{17} \mathrm{H}_{14} \mathrm{BrF}_{2} \mathrm{NO}_{2}+\mathrm{H}\right]^{+}: 382.0249$ found: 382.0237.

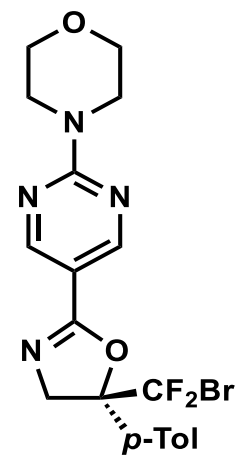

2i: Title compound was synthesized by following the general procedure for oxazolines, yielding $29 \mathrm{mg}$ of $\mathbf{2} \mathbf{i}$ as a white solid (62\% yield).

${ }^{1} \mathbf{H}$ NMR $\left(600 \mathrm{MHz}, \mathrm{CDCl}_{3}\right) \delta 8.88(\mathrm{~s}, 2 \mathrm{H}), 7.41(\mathrm{~d}, J=7.9 \mathrm{~Hz}, 2 \mathrm{H}), 7.22(\mathrm{~d}, J=7.9 \mathrm{~Hz}, 2 \mathrm{H}), 4.72$ $(\mathrm{d}, J=15.3 \mathrm{~Hz}, 1 \mathrm{H}), 4.34(\mathrm{~d}, J=15.3 \mathrm{~Hz}, 1 \mathrm{H}), 3.93(\mathrm{t}, J=4.9 \mathrm{~Hz}, 4 \mathrm{H}), 3.77(\mathrm{t}, J=4.8 \mathrm{~Hz}, 4 \mathrm{H})$, $2.37(\mathrm{~s}, 3 \mathrm{H})$.

${ }^{13} \mathbf{C ~ N M R}\left(151 \mathrm{MHz}, \mathrm{CDCl}_{3}\right) \delta 162.1,159.7,158.1,139.5,133.4,129.3,126.9,124.1(\mathrm{t}, J=312.7$ $\mathrm{Hz}), 109.9,90.3$ (t, $J=24.8 \mathrm{~Hz}), 66.9,64.6,44.5,21.3$.

${ }^{19}$ F NMR $\left(565 \mathrm{MHz} \mathrm{CDCl}_{3}\right) \delta-59.4(\mathrm{~d}, J=168.6 \mathrm{~Hz}, 1 \mathrm{~F}),-60.5(\mathrm{~d}, J=168.2 \mathrm{~Hz}, 1 \mathrm{~F})$.

HRMS (ESI+): calcd. for $\left[\mathrm{C}_{19} \mathrm{H}_{19} \mathrm{BrF}_{2} \mathrm{~N}_{4} \mathrm{O}_{2}+\mathrm{H}\right]^{+}$: 453.0732 found: 453.0703.

MP: $116.6-122.6{ }^{\circ} \mathrm{C}$ 


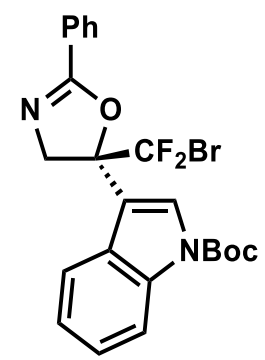

2j: Title compound was synthesized by following the general procedure for oxazolines, yielding $31 \mathrm{mg}$ of $\mathbf{2} \mathbf{j}$ as a white solid (62\% yield).

${ }^{1} \mathbf{H}$ NMR $\left(600 \mathrm{MHz}, \mathrm{CDCl}_{3}\right) \delta 8.17(\mathrm{~d}, J=8.4 \mathrm{~Hz}, 1 \mathrm{H}), 8.07-8.02(\mathrm{~m}, 2 \mathrm{H}), 7.83(\mathrm{~s}, 1 \mathrm{H}), 7.69(\mathrm{~d}$, $J=8.0 \mathrm{~Hz}, 1 \mathrm{H}), 7.56-7.53(\mathrm{~m}, 1 \mathrm{H}), 7.50-7.46(\mathrm{~m}, 2 \mathrm{H}), 7.36(\mathrm{ddd}, J=8.4,7.2,1.3 \mathrm{~Hz}, 1 \mathrm{H}), 7.29$ (ddd, $J=8.2,7.2,1.0 \mathrm{~Hz}, 1 \mathrm{H}), 4.82(\mathrm{~d}, J=15.5 \mathrm{~Hz}, 1 \mathrm{H}), 4.54(\mathrm{~d}, J=15.5 \mathrm{~Hz}, 1 \mathrm{H}), 1.69$ (s, 9H).

${ }^{13} \mathrm{C}$ NMR $\left(151 \mathrm{MHz}, \mathrm{CDCl}_{3}\right) \delta 162.7,149.5,135.7,132.1,128.7,128.6,127.6,126.8,125.7$, $125.1,124.5$ (t, $J=313.2 \mathrm{~Hz}), 123.4,121.0,116.3,115.7,88.7$ (t, $J=26.0 \mathrm{~Hz}), 84.9$, 64.2, 28.3.

${ }^{19}$ F NMR $\left(565 \mathrm{MHz}, \mathrm{CDCl}_{3}\right) \delta-59.0(\mathrm{~d}, J=167.7 \mathrm{~Hz}, 1 \mathrm{~F}),-60.1(\mathrm{~d}, J=168.1 \mathrm{~Hz}, 1 \mathrm{~F})$.

HRMS (ESI+): calcd. for $\left[\mathrm{C}_{23} \mathrm{H}_{21} \mathrm{BrF}_{2} \mathrm{~N}_{2} \mathrm{O}_{3}+\mathrm{H}\right]^{+}$: 491.0777 found: 491.0757.

MP: $100.6-106.9^{\circ} \mathrm{C}$

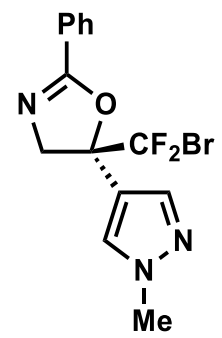

2k: Title compound was synthesized by following the general procedure for oxazolines, yielding $24 \mathrm{mg}$ of $\mathbf{2} \mathbf{k}$ as a colorless oil (68\% yield).

${ }^{1} \mathbf{H}$ NMR $\left(600 \mathrm{MHz}, \mathrm{CDCl}_{3}\right) \delta 8.01-7.97(\mathrm{~m}, 2 \mathrm{H}), 7.60(\mathrm{~s}, 1 \mathrm{H}), 7.54-7.51(\mathrm{~m}, 2 \mathrm{H}), 7.47-7.42$ $(\mathrm{m}, 2 \mathrm{H}), 4.66(\mathrm{~d}, J=15.5 \mathrm{~Hz}, 1 \mathrm{H}), 4.29$ (d, $J=15.5 \mathrm{~Hz}, 1 \mathrm{H}), 3.92(\mathrm{~s}, 3 \mathrm{H})$.

${ }^{13} \mathrm{C}$ NMR $\left(151 \mathrm{MHz}, \mathrm{CDCl}_{3}\right) \delta 162.6,137.8,132.1,129.3,128.7,128.5,126.8,123.9(\mathrm{t}, J=312.0$ $\mathrm{Hz}), 118.4,87.1$ (t, $J=25.2 \mathrm{~Hz}), 65.0,39.4$.

${ }^{19}$ F NMR $\left(565 \mathrm{MHz}, \mathrm{CDCl}_{3}\right) \delta-61.2(\mathrm{~d}, J=180.3 \mathrm{~Hz}),-61.7(\mathrm{~d}, J=164.3 \mathrm{~Hz})$.

HRMS (ESI+): calcd. for $\left[\mathrm{C}_{14} \mathrm{H}_{12} \mathrm{BrF}_{2} \mathrm{~N}_{3} \mathrm{O}+\mathrm{H}\right]^{+}: 356.0205$ found: 356.0177. 
<smiles>O=C(NC[C@@](O)(c1cc2ccccc2o1)C(F)(F)F)c1ccccc1</smiles>

2l• $\mathbf{H}_{2} \mathrm{O}$ : Title compound was synthesized by following the general procedure for oxazolines, yielding $14 \mathrm{mg}$ of $\mathbf{2 1 \cdot} \mathbf{H}_{2} \mathbf{O}$ as a white solid (36\% yield).

${ }^{1} \mathbf{H}$ NMR $\left(600 \mathrm{MHz}, \mathrm{CDCl}_{3}\right) \delta 7.64(\mathrm{dd}, J=8.2,1.2 \mathrm{~Hz}, 2 \mathrm{H}), 7.58(\mathrm{~d}, J=7.5 \mathrm{~Hz}, 1 \mathrm{H}), 7.52-7.48$ $(\mathrm{m}, 2 \mathrm{H}), 7.40-7.36(\mathrm{~m}, 2 \mathrm{H}), 7.32(\mathrm{ddd}, J=8.4,7.3,1.4 \mathrm{~Hz}, 1 \mathrm{H}), 7.27-7.22(\mathrm{~m}, 1 \mathrm{H}), 7.09(\mathrm{~d}, J=$ $1.0 \mathrm{~Hz}, 1 \mathrm{H}), 6.47$ (t, $J=6.3 \mathrm{~Hz}, 1 \mathrm{H}), 6.35(\mathrm{~s}, 1 \mathrm{H}), 4.45(\mathrm{dd}, J=14.6,6.0 \mathrm{~Hz}, 1 \mathrm{H}), 4.19$ (dd, $J=$ 14.6, $6.5 \mathrm{~Hz}, 1 \mathrm{H})$.

${ }^{13} \mathbf{C}$ NMR $\left(151 \mathrm{MHz}, \mathrm{CDCl}_{3}\right) \delta 171.3,155.4,152.8,132.7,132.6,128.9,127.9,127.3,125.0$, $124.6(\mathrm{t}, J=315.8 \mathrm{~Hz}), 123.4,121.9,111.6,108.7,81.0(\mathrm{t}, J=23.3 \mathrm{~Hz}), 45.0(\mathrm{~d}, J=2.4 \mathrm{~Hz})$.

${ }^{19}$ F NMR $\left(565 \mathrm{MHz}, \mathrm{CDCl}_{3}\right) \delta-56.9(\mathrm{~d}, J=164.5 \mathrm{~Hz}),-58.0(\mathrm{~d}, J=164.6 \mathrm{~Hz})$.

HRMS (ESI+): calcd. for $\left[\mathrm{C}_{18} \mathrm{H}_{14} \mathrm{~F}_{2} \mathrm{NO}_{3}+\mathrm{MeCN}+\mathrm{Na}\right]^{+}: 473.0283$ found: 473.0263.

MP: $145.9-148.2^{\circ} \mathrm{C}$

\section{General Procedure for the Enantioselective Bromocyclization to 4a-l}
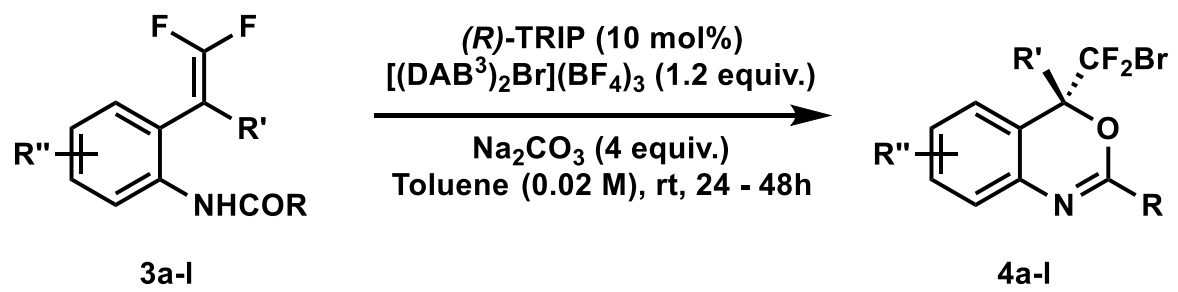

To a flame-dried 2-dram vial was added amide substrate ( $0.10 \mathrm{mmol}, 1.0$ equiv.), $(R)$-TRIP (7.6 mg, $0.010 \mathrm{mmol}, 10 \mathrm{~mol} \%$ ), $\mathrm{Na}_{2} \mathrm{CO}_{3}$ (44 mg, $0.4 \mathrm{mmol}, 4.0$ equiv.), and brominating agent $\left[\left(\mathbf{D A B}^{\mathbf{3}}\right)_{2} \mathbf{B r}\right]\left(\mathbf{B F}_{4}\right)_{3}$ (106 mg, $0.12 \mathrm{mmol}, 1.2$ equiv.). After the vial was evacuated and backfilled three times with $\mathrm{N}_{2}, 5.0 \mathrm{~mL}$ of dry, degassed toluene was added. The reaction was stirred at $>800$ rpm until conversion stopped (24-48 h), as judged by TLC. In cases where substantial starting material remained, an additional portion (70 $\mathrm{mg}, 0.08 \mathrm{mmol}, 0.8$ equiv.) of $\left[\left(\mathbf{D A B}^{\mathbf{3}}\right)_{2} \mathbf{B r}\right]\left(\mathbf{B F}_{4}\right)_{3}$ was added, and the reaction was allowed to stir for an additional $24 \mathrm{~h}$. Once complete, the reaction was filtered through Celite ${ }^{\circledR}$ with EtOAc as the eluent. The solvent was the removed under reduced pressure, and the crude product was purified by column chromatography ( $\mathrm{Et}_{2} \mathrm{O} /$ pentane) to yield pure $\mathbf{4 a}-\mathbf{l}$. 


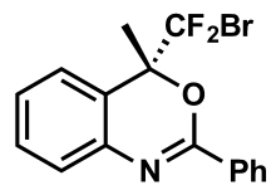

4a: Title compound was synthesized by following the general procedure for oxazines, yielding 29 $\mathrm{mg}$ of $\mathbf{4} \mathbf{a}$ as a colorless oil ( $80 \%$ yield).

${ }^{1} \mathbf{H}$ NMR $\left(600 \mathrm{MHz} \mathrm{CDCl}_{3}\right) \delta 8.22-8.17(\mathrm{~m}, 2 \mathrm{H}), 7.53(\mathrm{tt}, J=7.3,2.2 \mathrm{~Hz}, 1 \mathrm{H}), 7.47(\mathrm{t}, J=7.5$ $\mathrm{Hz}, 2 \mathrm{H}), 7.43(\mathrm{td}, J=7.6,1.5 \mathrm{~Hz}, 1 \mathrm{H}), 7.37(\mathrm{dd}, J=7.9,1.3 \mathrm{~Hz}, 1 \mathrm{H}), 7.32(\mathrm{dd}, J=7.8,1.6 \mathrm{~Hz}, 1 \mathrm{H})$, $7.28-7.24(\mathrm{~m}, 1 \mathrm{H}), 2.02(\mathrm{~d}, J=1.1 \mathrm{~Hz}, 3 \mathrm{H})$.

${ }^{13} \mathrm{C}$ NMR $\left(151 \mathrm{MHz}, \mathrm{CDCl}_{3}\right) \delta 155.0,139.2,131.9,131.8,130.8,128.5,128.3,127.2,126.2$, $125.8,125.3(\mathrm{t}, J=316.6 \mathrm{~Hz}), 121.5,82.6(\mathrm{t}, J=24.4 \mathrm{~Hz}), 23.9$.

${ }^{19}$ F NMR $\left(565 \mathrm{MHz}, \mathrm{CDCl}_{3}\right) \delta-59.5(\mathrm{~d}, J=166.5 \mathrm{~Hz}, 1 \mathrm{~F}),-60.9(\mathrm{~d}, J=166.7 \mathrm{~Hz}, 1 \mathrm{~F})$.

HRMS (ESI+): calcd. for $\left[\mathrm{C}_{16} \mathrm{H}_{12} \mathrm{BrF}_{2} \mathrm{NO}+\mathrm{H}\right]^{+}: 352.0143$ found: 352.0123 .

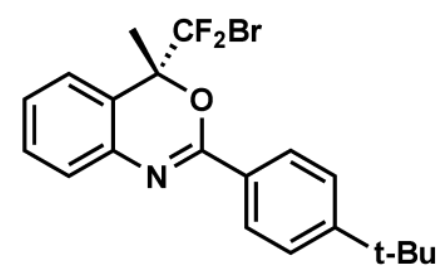

4b: Title compound was synthesized by following the general procedure for oxazines, yielding 34 mg of $\mathbf{4 b}$ as a colorless oil (89\% yield).

${ }^{1} \mathbf{H}$ NMR $\left(600 \mathrm{MHz}, \mathrm{CDCl}_{3}\right) \delta 8.12(\mathrm{~d}, J=8.4 \mathrm{~Hz}, 2 \mathrm{H}), 7.50(\mathrm{~s}, 2 \mathrm{H}), 7.42(\mathrm{t}, J=7.6 \mathrm{~Hz}, 1 \mathrm{H}), 7.36$ $(\mathrm{d}, J=7.8 \mathrm{~Hz}, 1 \mathrm{H}), 7.31(\mathrm{~d}, J=7.7 \mathrm{~Hz}, 1 \mathrm{H}), 7.28-7.22(\mathrm{~m}, 1 \mathrm{H}), 2.01(\mathrm{~s}, 3 \mathrm{H}), 1.36(\mathrm{~s}, 9 \mathrm{H})$.

${ }^{13} \mathrm{C}$ NMR $\left(151 \mathrm{MHz}, \mathrm{CDCl}_{3}\right) \delta 155.5,155.1,139.4,130.7,129.0,128.1,126.9,126.1,125.8$, 125.5, $125.4(\mathrm{t}, J=316.6 \mathrm{~Hz}), 121.5,82.5(\mathrm{t}, J=24.4 \mathrm{~Hz}), 35.2,31.3,23.9$.

${ }^{19}$ F NMR $\left(565 \mathrm{MHz}, \mathrm{CDCl}_{3}\right) \delta-59.4(\mathrm{~d}, J=166.6 \mathrm{~Hz}, 1 \mathrm{~F}),-60.8(\mathrm{~d}, J=166.5 \mathrm{~Hz}, 1 \mathrm{~F})$.

HRMS (ESI+): calcd. for $\left[\mathrm{C}_{20} \mathrm{H}_{20} \mathrm{BrF}_{2} \mathrm{NO}+\mathrm{H}\right]^{+}: 408.0769$ found: 408.0739. 
<smiles>CC1(C(F)(F)F)OC(c2cccc(C(F)(F)F)c2)=Nc2ccccc21</smiles>

4c: Title compound was synthesized by following the general procedure for oxazines, yielding 30 $\mathrm{mg}$ of $\mathbf{4 c}$ as a white solid (75\% yield).

${ }^{1} \mathbf{H}$ NMR $\left(600 \mathrm{MHz}, \mathrm{CDCl}_{3}\right) \delta 8.47(\mathrm{~s}, 1 \mathrm{H}), 8.37(\mathrm{~d}, J=7.9 \mathrm{~Hz}, 1 \mathrm{H}), 7.78(\mathrm{~d}, J=7.8 \mathrm{~Hz}, 1 \mathrm{H}), 7.61$ (t, $J=7.8 \mathrm{~Hz}, 1 \mathrm{H}), 7.49-7.42(\mathrm{~m}, 1 \mathrm{H}), 7.39$ (d, $J=7.2 \mathrm{~Hz}, 1 \mathrm{H}), 7.37-7.28(\mathrm{~m}, 2 \mathrm{H}), 2.04(\mathrm{~s}, 3 \mathrm{H})$.

${ }^{13} \mathrm{C}$ NMR (151 MHz, $\left.\mathrm{CDCl}_{3}\right) \delta 153.5,138.8,132.7,131.3,131.2(\mathrm{q}, J=32.8 \mathrm{~Hz}), 130.9,129.1$, $128.3(\mathrm{q}, J=3.7 \mathrm{~Hz}), 127.7,126.4,125.9,125.2(\mathrm{q}, J=3.6 \mathrm{~Hz}), 125.2(\mathrm{q}, J=316.6 \mathrm{~Hz}), 124.0(\mathrm{q}$, $J=272.4 \mathrm{~Hz}), 121.5,82.9(\mathrm{t}, J=24.6 \mathrm{~Hz}), 23.9$.

${ }^{19}$ F NMR $\left(565 \mathrm{MHz}, \mathrm{CDCl}_{3}\right) \delta-59.6(\mathrm{~d}, J=167.0 \mathrm{~Hz}, 1 \mathrm{~F}),-61.1(\mathrm{~d}, J=167.1 \mathrm{~Hz}, 1 \mathrm{~F}),-62.7(\mathrm{~s}$, $3 \mathrm{~F})$.

HRMS (ESI+): calcd. for $\left[\mathrm{C}_{17} \mathrm{H}_{11} \mathrm{BrF}_{5} \mathrm{NO}+\mathrm{H}\right]^{+}: 420.0017$ found: 420.0009.

MP: $79.2-82.9^{\circ} \mathrm{C}$

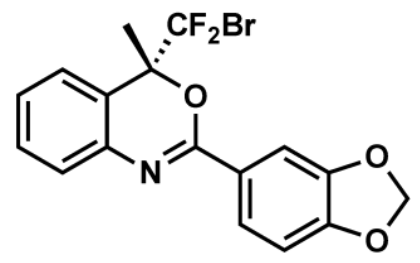

4d: Title compound was synthesized by following the general procedure for oxazines, yielding 31 $\mathrm{mg}$ of $\mathbf{4 d}$ as a white solid (85\% yield).

${ }^{1} \mathbf{H}$ NMR $\left(600 \mathrm{MHz}, \mathrm{CDCl}_{3}\right) \delta 7.79(\mathrm{dd}, J=8.3,1.7 \mathrm{~Hz}, 1 \mathrm{H}), 7.66(\mathrm{~d}, J=1.7 \mathrm{~Hz}, 1 \mathrm{H}), 7.42(\mathrm{td}, J=$ 7.6, $1.5 \mathrm{~Hz}, 1 \mathrm{H}), 7.34(\mathrm{~d}, J=7.8 \mathrm{~Hz}, 1 \mathrm{H}), 7.30(\mathrm{dd}, J=7.8,1.6 \mathrm{~Hz}, 1 \mathrm{H}), 7.24(\mathrm{td}, J=7.5,1.3 \mathrm{~Hz}$, $1 \mathrm{H}), 6.89(\mathrm{~d}, J=8.2 \mathrm{~Hz}, 1 \mathrm{H}), 6.04(\mathrm{~s}, 2 \mathrm{H}), 2.00$ (s, 3H).

${ }^{13}$ C NMR (151 MHz, $\left.\mathrm{CDCl}_{3}\right) \delta 154.6,151.0,148.0,139.3,130.7,126.9,126.0,125.8,125.3(\mathrm{t}$, $J=317.1 \mathrm{~Hz}), 123.7,121.3,108.4,108.3,101.8,82.7$ (t, $J=24.3 \mathrm{~Hz}$ ), 23.8. (One missing resonance)

${ }^{19}$ F NMR $\left(565 \mathrm{MHz}, \mathrm{CDCl}_{3}\right) \delta-59.4(\mathrm{~d}, J=166.9 \mathrm{~Hz}, 1 \mathrm{~F}),-60.8(\mathrm{~d}, J=166.6 \mathrm{~Hz}, 1 \mathrm{~F})$.

HRMS (ESI+): calcd. for $\left[\mathrm{C}_{17} \mathrm{H}_{12} \mathrm{BrF}_{2} \mathrm{NO}_{3}+\mathrm{H}\right]^{+}: 396.0042$ found: 398.0017.

MP: $130.4-132.4{ }^{\circ} \mathrm{C}$ 
<smiles>CC1(C(Br)(Br)Br)OC(Cl)=Nc2ccccc21</smiles>

4e: Title compound was synthesized by following the general procedure for oxazines, yielding 29 $\mathrm{mg}$ of $\mathbf{4 e}$ as a white solid (68\% yield).

${ }^{1} \mathbf{H}$ NMR $\left(600 \mathrm{MHz}, \mathrm{CDCl}_{3}\right) \delta 7.35(\mathrm{td}, J=7.6,1.6 \mathrm{~Hz}, 1 \mathrm{H}), 7.24-7.17(\mathrm{~m}, 3 \mathrm{H}), 2.10-2.03(\mathrm{~m}$, $3 \mathrm{H}), 2.02-1.98(\mathrm{~m}, 6 \mathrm{H}), 1.88(\mathrm{~s}, 3 \mathrm{H}), 1.79-1.72(\mathrm{~m}, 6 \mathrm{H})$.

${ }^{13}$ C NMR $\left(151 \mathrm{MHz}, \mathrm{CDCl}_{3}\right) \delta 165.3,139.1,130.5,126.7,125.8,125.7$ (d, J= 2.0 Hz), $125.2(\mathrm{t}$, $J=317.0 \mathrm{~Hz}), 121.3,81.9(\mathrm{t}, J=24.0 \mathrm{~Hz}), 39.3,39.2,36.8,28.3,23.7$.

${ }^{19}$ F NMR $\left(565 \mathrm{MHz} \mathrm{CDCl}_{3}\right) \delta-59.7(\mathrm{~d}, J=165.7 \mathrm{~Hz}, 1 \mathrm{~F}),-61.0(\mathrm{~d}, J=165.5 \mathrm{~Hz}, 1 \mathrm{~F})$.

HRMS (ESI+): calcd. for $\left[\mathrm{C}_{20} \mathrm{H}_{22} \mathrm{BrF}_{2} \mathrm{NO}+\mathrm{H}\right]^{+}: 410.0926$ found: 410.0895.

MP: $88.9-93.2{ }^{\circ} \mathrm{C}$<smiles>FC(Br)(Br)[C@]1(c2ccccc2)OC(c2ccccc2)=Nc2ccccc21</smiles>

4f: Title compound was synthesized by modification of the general procedure for oxazines. After stirring the reaction for 48 hours, an additional $70 \mathrm{mg}$ of brominating agent ( $0.08 \mathrm{mmol}, 0.8$ equiv.) was added, and the reaction was allowed to stir for an additional 24 hours. Following purification procedures, $30 \mathrm{mg}$ of $\mathbf{4 f}$ was isolated as a white solid (73\% yield).

${ }^{1} \mathbf{H}$ NMR $\left(600 \mathrm{MHz}, \mathrm{CDCl}_{3}\right) \delta 8.33-8.28(\mathrm{~m}, 2 \mathrm{H}), 7.61-7.54(\mathrm{~m}, 3 \mathrm{H}), 7.54-7.48(\mathrm{~m}, 2 \mathrm{H}), 7.43$ (t, $J=7.5 \mathrm{~Hz}, 2 \mathrm{H}), 7.39(\mathrm{dd}, J=8.1,1.5 \mathrm{~Hz}, 1 \mathrm{H}), 7.37-7.33(\mathrm{~m}, 3 \mathrm{H}), 7.30-7.25(\mathrm{~m}, 1 \mathrm{H})$.

${ }^{13} \mathrm{C}$ NMR $\left(151 \mathrm{MHz}, \mathrm{CDCl}_{3}\right) \delta 154.2,139.1,136.9,132.1,131.6,130.5,129.3,128.7,128.5$, 128.3, $127.6(\mathrm{t}, J=1.7 \mathrm{~Hz}), 126.9,126.5(\mathrm{t}, J=2.9 \mathrm{~Hz}), 126.4,124.2(\mathrm{dd}, J=316.4,313.5 \mathrm{~Hz})$, $121.8,88.8-84.1(\mathrm{~m})$.

${ }^{19}$ F NMR $\left(565 \mathrm{MHz}, \mathrm{CDCl}_{3}\right) \delta-53.5(\mathrm{~d}, J=174.4 \mathrm{~Hz}, 1 \mathrm{~F}),-55.2(\mathrm{~d}, J=174.3 \mathrm{~Hz}, 1 \mathrm{~F})$.

HRMS (ESI+): calcd. for $\left[\mathrm{C}_{21} \mathrm{H}_{14} \mathrm{BrF}_{2} \mathrm{NO}+\mathrm{H}\right]^{+}: 414.0300$ found: 414.0278.

MP: $93.5-98.6^{\circ} \mathrm{C}$ 


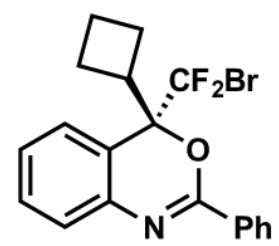

4g: Title compound was synthesized by following the general procedure for oxazines, yielding 29 $\mathrm{mg}$ of $\mathbf{4 g}$ as a white solid (72\% yield).

${ }^{1} \mathbf{H}$ NMR $\left(600 \mathrm{MHz}, \mathrm{CDCl}_{3}\right) \delta 8.25(\mathrm{~d}, J=7.3 \mathrm{~Hz}, 2 \mathrm{H}), 7.56(\mathrm{t}, J=7.3 \mathrm{~Hz}, 1 \mathrm{H}), 7.51(\mathrm{t}, J=7.5 \mathrm{~Hz}$, $2 \mathrm{H}), 7.40(\mathrm{td}, J=7.3,6.6,1.9 \mathrm{~Hz}, 1 \mathrm{H}), 7.36(\mathrm{~d}, J=7.3 \mathrm{~Hz}, 1 \mathrm{H}), 7.26-7.20(\mathrm{~m}, 2 \mathrm{H}), 3.47(\mathrm{p}, J=$ $8.9 \mathrm{~Hz}, 1 \mathrm{H}), 2.65(\mathrm{p}, J=10.0 \mathrm{~Hz}, 1 \mathrm{H}), 2.16-2.08(\mathrm{~m}, 1 \mathrm{H}), 1.95-1.84(\mathrm{~m}, 2 \mathrm{H}), 1.77-1.65$ (m, $2 \mathrm{H})$.

${ }^{13} \mathrm{C}$ NMR $\left(151 \mathrm{MHz}, \mathrm{CDCl}_{3}\right) \delta 154.8,139.6,132.1,131.9,130.4,128.6,128.2,127.0,126.4$, $125.7(\mathrm{~d}, J=3.1 \mathrm{~Hz}), 124.3(\mathrm{dd}, J=319.3,316.1 \mathrm{~Hz}), 119.7(\mathrm{~d}, J=1.9 \mathrm{~Hz}), 84.9(\mathrm{dd}, J=24.2,22.6$ $\mathrm{Hz}), 40.3,24.1,22.8$ (d, $J=2.8 \mathrm{~Hz}), 17.9$.

${ }^{19}$ F NMR $\left(565 \mathrm{MHz}, \mathrm{CDCl}_{3}\right) \delta-58.0(\mathrm{~d}, J=165.5 \mathrm{~Hz}, 1 \mathrm{~F}),-59.00(\mathrm{~d}, J=165.6 \mathrm{~Hz}, 1 \mathrm{~F})$.

HRMS (ESI+): calcd. for $\left[\mathrm{C}_{19} \mathrm{H}_{16} \mathrm{BrF}_{2} \mathrm{NO}+\mathrm{H}\right]^{+}: 392.0456$ found: 392.0436.

MP: $95.8-97.2^{\circ} \mathrm{C}$

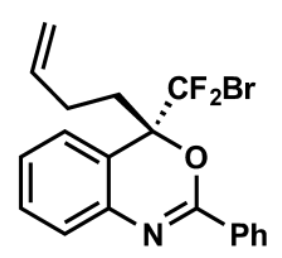

4h: Title compound was synthesized by modification of the general procedure for oxazines. After stirring the reaction for 48 hours, an additional $70 \mathrm{mg}$ of brominating agent ( $0.08 \mathrm{mmol}, 0.8$ equiv.) was added, and the reaction was allowed to stir for an additional 24 hours. Following purification procedures, $16 \mathrm{mg}$ of $\mathbf{4 h}$ was isolated as a colorless oil (43\% yield).

${ }^{1} \mathbf{H}$ NMR $\left(600 \mathrm{MHz}, \mathrm{CDCl}_{3}\right) \delta 8.21-8.16(\mathrm{~m}, 2 \mathrm{H}), 7.57-7.50(\mathrm{~m}, 1 \mathrm{H}), 7.51-7.45(\mathrm{~m}, 2 \mathrm{H}), 7.45$ $-7.41(\mathrm{~m}, 1 \mathrm{H}), 7.37(\mathrm{~d}, J=7.7 \mathrm{~Hz}, 1 \mathrm{H}), 7.27(\mathrm{dd}, J=5.8,1.2 \mathrm{~Hz}, 2 \mathrm{H}), 5.76$ (ddt, $J=16.8,10.2$, $6.5 \mathrm{~Hz}, 1 \mathrm{H}), 5.00-4.90(\mathrm{~m}, 2 \mathrm{H}), 2.52-2.35(\mathrm{~m}, 2 \mathrm{H}), 2.23-2.12(\mathrm{~m}, 1 \mathrm{H}), 2.00-1.87(\mathrm{~m}, 1 \mathrm{H})$.

${ }^{13} \mathrm{C}$ NMR $\left(151 \mathrm{MHz}, \mathrm{CDCl}_{3}\right) \delta 154.8,140.0,136.9,131.9,131.8,130.7,128.5,128.2,127.2$, 126.6, 125.7, $125.3(\mathrm{dd}, J=317.8,316.0 \mathrm{~Hz}), 119.2,115.6,85.5(\mathrm{t}, J=23.7 \mathrm{~Hz}), 34.1,28.0$.

${ }^{19}$ F NMR $\left(565 \mathrm{MHz}, \mathrm{CDCl}_{3}\right) \delta-59.2(\mathrm{~d}, J=166.6 \mathrm{~Hz}, 1 \mathrm{~F}),-61.0(\mathrm{~d}, J=166.6 \mathrm{~Hz}, 1 \mathrm{~F})$.

HRMS (ESI+): calcd. for $\left[\mathrm{C}_{19} \mathrm{H}_{16} \mathrm{BrF}_{2} \mathrm{NO}+\mathrm{H}\right]^{+}: 392.04558$ found: 392.0460 . 


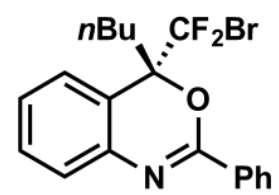

4i: Title compound was synthesized by following the general procedure for oxazines, yielding 32 $\mathrm{mg}$ of $\mathbf{4 i}$ as a white solid (79\% yield).

${ }^{1} \mathbf{H}$ NMR $\left(600 \mathrm{MHz}, \mathrm{CDCl}_{3}\right) \delta 8.19(\mathrm{~d}, J=7.4 \mathrm{~Hz}, 2 \mathrm{H}), 7.53(\mathrm{t}, J=7.4 \mathrm{~Hz}, 1 \mathrm{H}), 7.47(\mathrm{t}, J=7.6 \mathrm{~Hz}$, 2H), 7.42 (ddd, $J=8.4,5.1,3.5 \mathrm{~Hz}, 1 \mathrm{H}), 7.37$ (d, $J=7.8 \mathrm{~Hz}, 1 \mathrm{H}), 7.29-7.23(\mathrm{~m}, 2 \mathrm{H}), 2.37-2.24$ (m, 2H), $1.46-1.06(\mathrm{~m}, 4 \mathrm{H}), 0.84(\mathrm{t}, J=7.3 \mathrm{~Hz}, 3 \mathrm{H})$.

${ }^{13}$ C NMR $\left(151 \mathrm{MHz}, \mathrm{CDCl}_{3}\right) \delta 154.9,140.0,131.9,131.8,130.6,128.5,128.2,127.1,126.5$, $125.7,125.5(\mathrm{dd}, J=317.9,316.0 \mathrm{~Hz}), 119.6,85.8(\mathrm{t}, J=23.5 \mathrm{~Hz}), 34.6,25.8,22.8,14.0$.

${ }^{19} \mathbf{F}$ NMR $\left(565 \mathrm{MHz}, \mathrm{CDCl}_{3}\right) \delta-59.0(\mathrm{~d}, J=166.3 \mathrm{~Hz}, 1 \mathrm{~F}),-60.8(\mathrm{~d}, J=166.0 \mathrm{~Hz}, 1 \mathrm{~F})$.

HRMS (ESI+): calcd. for $\left[\mathrm{C}_{19} \mathrm{H}_{18} \mathrm{BrF}_{2} \mathrm{NO}+\mathrm{H}\right]^{+}: 394.0613$ found: 394.0610 .

MP: $61.5-64.5^{\circ} \mathrm{C}$<smiles>CC1(C(Br)(Br)Br)OC(c2ccccc2)=Nc2cc3c(cc21)OCCO3</smiles>

4j: Title compound was synthesized by following the general procedure for oxazines, yielding 25 $\mathrm{mg}$ of $\mathbf{4} \mathbf{j}$ as a white solid (62\% yield).

${ }^{1} \mathbf{H}$ NMR $\left(600 \mathrm{MHz}, \mathrm{CDCl}_{3}\right) \delta 8.15(\mathrm{~d}, J=7.0 \mathrm{~Hz}, 2 \mathrm{H}), 7.50(\mathrm{t}, J=7.3 \mathrm{~Hz}, 1 \mathrm{H}), 7.45(\mathrm{t}, J=7.4 \mathrm{~Hz}$, 2H), 6.90 (s, 1H), $6.82(\mathrm{~d}, J=1.5 \mathrm{~Hz}, 1 \mathrm{H}), 4.34-4.24$ (m, 4H), 1.95 (s, 3H).

${ }^{13}$ C NMR $\left(151 \mathrm{MHz}, \mathrm{CDCl}_{3}\right) \delta 153.8,145.1,142.7,133.6,132.0,131.6,128.4,128.1,125.4(\mathrm{~d}$, $J=316.9 \mathrm{~Hz}), 114.7,114.6,114.6,82.3(\mathrm{t}, J=24.5 \mathrm{~Hz}), 64.6,23.8$. (One resonance missing)

${ }^{19}$ F NMR $\left(565 \mathrm{MHz}, \mathrm{CDCl}_{3}\right) \delta-59.3(\mathrm{~d}, J=165.9 \mathrm{~Hz}, 1 \mathrm{~F}),-60.5(\mathrm{~d}, J=166.1 \mathrm{~Hz}, 1 \mathrm{~F})$.

HRMS (ESI+): calcd. for $\left[\mathrm{C}_{18} \mathrm{H}_{14} \mathrm{BrF}_{2} \mathrm{NO}_{3}+\mathrm{H}\right]^{+}: 410.0198$ found: 410.0183 .

MP: $84.6-89.2{ }^{\circ} \mathrm{C}$ 
<smiles>CC1(C(F)(F)F)OC(c2ccccc2)=Nc2ncccc21</smiles>

4k: Title compound was synthesized by modification of the general procedure for oxazines. After stirring the reaction for 48 hours, an additional $70 \mathrm{mg}$ of brominating agent ( $0.08 \mathrm{mmol}, 0.8$ equiv.) was added, and the reaction was allowed to stir for an additional 24 hours. Following purification procedures, $15 \mathrm{mg}$ of $\mathbf{4 k}$ was isolated as a white solid (41\% yield).

${ }^{1} \mathbf{H}$ NMR $\left(600 \mathrm{MHz}, \mathrm{CDCl}_{3}\right) \delta 8.60(\mathrm{~d}, J=4.8 \mathrm{~Hz}, 1 \mathrm{H}), 8.29(\mathrm{~d}, J=7.7 \mathrm{~Hz}, 2 \mathrm{H}), 7.66(\mathrm{~d}, J=7.6$ $\mathrm{Hz}, 1 \mathrm{H}), 7.57$ (t, $J=7.4 \mathrm{~Hz}, 1 \mathrm{H}), 7.48$ (t, $J=7.5 \mathrm{~Hz}, 2 \mathrm{H}), 7.21$ (dd, $J=7.8,4.9 \mathrm{~Hz}, 1 \mathrm{H}), 2.03$ (s, $3 \mathrm{H})$.

${ }^{13} \mathrm{C}$ NMR $\left(151 \mathrm{MHz}, \mathrm{CDCl}_{3}\right) \delta 159.0,151.8,151.4,134.6,132.8,131.0,129.0,128.6,124.5(\mathrm{t}$, $J=315.9 \mathrm{~Hz}), 122.2,116.9,83.5(\mathrm{t}, J=24.9 \mathrm{~Hz}), 23.8$.

${ }^{19}$ F NMR $\left(565 \mathrm{MHz}, \mathrm{CDCl}_{3}\right) \delta-60.6(\mathrm{~d}, J=169.0 \mathrm{~Hz}, 1 \mathrm{~F}),-62.1(\mathrm{~d}, J=169.0 \mathrm{~Hz}, 1 \mathrm{~F})$.

HRMS (ESI+): calcd. for $\left[\mathrm{C}_{15} \mathrm{H}_{11} \mathrm{BrF}_{2} \mathrm{~N}_{2} \mathrm{O}+\mathrm{H}\right]^{+}: 353.0096$ found: 353.0083.

MP: $55.2-60.2{ }^{\circ} \mathrm{C}$

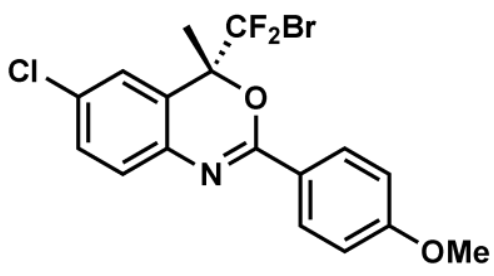

41: Title compound was synthesized by modification of the general procedure for oxazines. After stirring the reaction for 48 hours, an additional $70 \mathrm{mg}$ of brominating agent ( $0.08 \mathrm{mmol}, 0.8$ equiv.) was added, and the reaction was allowed to stir for an additional 24 hours. Following purification procedures, $44 \mathrm{mg}$ of $\mathbf{4 1}$ was isolated as a white solid (85\% yield).

${ }^{1} \mathbf{H}$ NMR $\left(600 \mathrm{MHz}, \mathrm{CDCl}_{3}\right) \delta 8.03(\mathrm{~d}, J=8.9 \mathrm{~Hz}, 2 \mathrm{H}), 7.27(\mathrm{dd}, J=8.4,2.3 \mathrm{~Hz}, 1 \mathrm{H}), 7.18(\mathrm{~d}, J=$ $7.7 \mathrm{~Hz}, 1 \mathrm{H}), 7.16$ (s, 1H), $6.89-6.84(\mathrm{~m}, 2 \mathrm{H}), 3.78$ (s, 3H), 1.89 (s, 3H).

${ }^{13} \mathrm{C}$ NMR $\left(151 \mathrm{MHz}, \mathrm{CDCl}_{3}\right) \delta 163.0,155.3,138.2,131.8,130.9,130.3,128.5,127.1,125.9$, $124.9(\mathrm{t}, J=316.5 \mathrm{~Hz}), 122.8,114.0,82.4(\mathrm{t}, J=24.6 \mathrm{~Hz}), 55.6,23.8$.

${ }^{19}$ F NMR $\left(565 \mathrm{MHz}, \mathrm{CDCl}_{3}\right) \delta-59.7(\mathrm{~d}, J=168.0 \mathrm{~Hz}, 1 \mathrm{~F}),-61.1(\mathrm{~d}, J=167.9 \mathrm{~Hz}, 1 \mathrm{~F})$.

HRMS (ESI+): calcd. for $\left[\mathrm{C}_{17} \mathrm{H}_{13} \mathrm{BrClF}_{2} \mathrm{NO}_{2}+\mathrm{H}\right]^{+}$: 415.9859 found: 415.9891 .

MP: $84.8-86.6{ }^{\circ} \mathrm{C}$ 


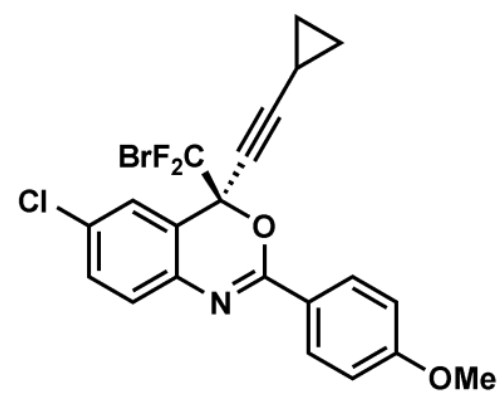

4m: To a flame-dried $100 \mathrm{~mL}$ round bottom flask, was added $390 \mathrm{mg}$ of $\mathbf{3 m}(1.0 \mathrm{mmol}$, 1.0 equiv.), (S)-TRIP (75 mg, $0.10 \mathrm{mmol}, 10 \mathrm{~mol} \%$ ), $\mathrm{NaHCO}_{3}$ (340 mg, $4.0 \mathrm{mmol}, 4.0$ equiv.), and $1.1 \mathrm{~g}$ of $\left[\left(\mathbf{D A B}^{\mathbf{3}}\right)_{2} \mathbf{B r}\right]\left(\mathbf{B F}_{4}\right)_{3}(1.2 \mathrm{mmol}, 1.2$ equiv.). After the flask was evacuated and backfilled three times with $\mathrm{N}_{2}, 50 \mathrm{~mL}$ of dry, degassed benzene was added. The reaction was stirred at $>800 \mathrm{rpm}$ for 48 hours, at which point an additional portion of $\left[\left(\mathbf{D A B}^{\mathbf{3}}\right)_{\mathbf{2}} \mathbf{B r}\right](\mathbf{B F} 4)_{3}(730$ $\mathrm{mg}, 0.80 \mathrm{mmol}, 0.8$ equiv.) was added, and the reaction was allowed to stir for an additional 24 hours. Once complete, the reaction was filtered through Celite ${ }^{\circledR}$ with EtOAc as the eluent. The solvent was then removed under reduced pressure, and the crude product was purified by column chromatography (1:9 $\mathrm{Et}_{2} \mathrm{O} /$ pentane to $1: 3 \mathrm{Et}_{2} \mathrm{O}$ /pentane gradient) to yield $270 \mathrm{mg}$ (60\% yield) of $\mathbf{4 m}$ as a yellow solid.

${ }^{1} \mathbf{H}$ NMR $\left(600 \mathrm{MHz}, \mathrm{CDCl}_{3}\right) \delta 8.18-8.14(\mathrm{~m}, 2 \mathrm{H}), 7.53(\mathrm{~s}, 1 \mathrm{H}), 7.41$ (dd, J= 8.4, $\left.2.4 \mathrm{~Hz}, 1 \mathrm{H}\right)$, $7.27(\mathrm{~d}, J=8.4 \mathrm{~Hz}, 1 \mathrm{H}), 7.00-6.95(\mathrm{~m}, 2 \mathrm{H}), 3.89(\mathrm{~s}, 3 \mathrm{H}), 1.43(\mathrm{tt}, J=8.3,5.1 \mathrm{~Hz}, 1 \mathrm{H}), 0.99-$ $0.80(\mathrm{~m}, 4 \mathrm{H})$.

${ }^{13} \mathrm{C}$ NMR $\left(151 \mathrm{MHz}, \mathrm{CDCl}_{3}\right) \delta 163.1,154.5,137.9,131.7,131.4,130.5,127.8,127.0,123.6$, $123.3(\mathrm{t}, J=316.6 \mathrm{~Hz}), 120.1,114.0,95.0,80.0(\mathrm{t}, J=28.1 \mathrm{~Hz}), 69.4(\mathrm{~d}, J=4.1 \mathrm{~Hz}), 55.6,8.9,8.9$, -0.3 .

${ }^{19}$ F NMR $\left(565 \mathrm{MHz}, \mathrm{CDCl}_{3}\right) \delta-60.0(\mathrm{~d}, J=165.0 \mathrm{~Hz}, 1 \mathrm{~F}),-61.5(\mathrm{~d}, J=165.2 \mathrm{~Hz}, 1 \mathrm{~F})$.

HRMS (ESI+): calcd. for $\left[\mathrm{C}_{21} \mathrm{H}_{15} \mathrm{BrClF}_{2} \mathrm{NO}_{2}+\mathrm{H}\right]^{+}: 466.0016$ found: 466.0006

MP: $40.3-44.5^{\circ} \mathrm{C}$ 


\section{Derivatization of $\mathrm{CF}_{2} \mathrm{Br}$ Heterocycles}

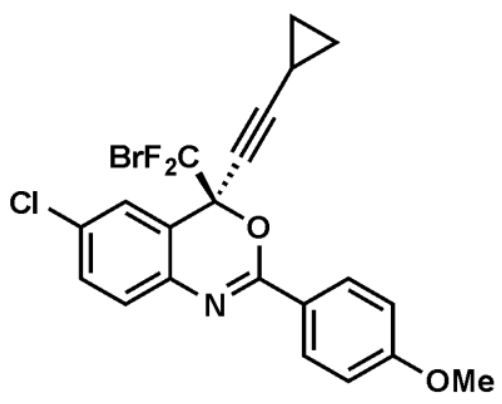

$4 \mathrm{~m}$

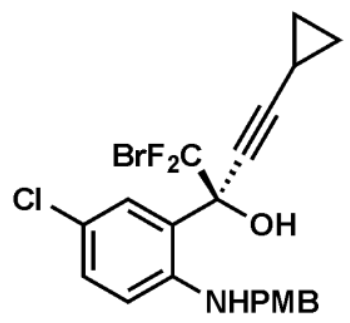

5

5: To a stirred solution of $\mathbf{4 m}(210 \mathrm{mg}, 0.45 \mathrm{mmol}, 1.0$ equiv.) in $4.5 \mathrm{~mL}$ of $\mathrm{AcOH}$, was added $140 \mathrm{mg}$ of $\mathrm{NaBH}_{3} \mathrm{CN}$ (2.3 mmol, 5 equiv.) portionwise. After stirring the reaction overnight, $4.5 \mathrm{~mL}$ of $\mathrm{H}_{2} \mathrm{O}$ was added dropwise. After an additional hour of stirring, the reaction was diluted with DCM (30 mL), and the reaction was neutralized with saturated $\mathrm{NaHCO}_{3}$. The layers were then separated and the aqueous layer was extracted three times with DCM (40 mL each). The

combined organic layers were dried with $\mathrm{Na}_{2} \mathrm{SO}_{4}$, filtered, and solvent removed in vacuo to yield $190 \mathrm{mg}(90 \%)$ of the title compound as a pale-yellow oil.

${ }^{1} \mathbf{H}$ NMR $\left(600 \mathrm{MHz}, \mathrm{CDCl}_{3}\right) \delta 7.61(\mathrm{~d}, J=2.6 \mathrm{~Hz}, 1 \mathrm{H}), 7.24(\mathrm{~d}, J=8.6 \mathrm{~Hz}, 2 \mathrm{H}), 7.14(\mathrm{dd}, J=8.8$, $2.6 \mathrm{~Hz}, 1 \mathrm{H}), 6.87(\mathrm{~d}, J=8.6 \mathrm{~Hz}, 2 \mathrm{H}), 6.59(\mathrm{~d}, J=8.9 \mathrm{~Hz}, 1 \mathrm{H}), 4.24$ (s, 2H), 3.80 (s, 3H), $1.38-$ $1.32(\mathrm{~m}, 1 \mathrm{H}), 0.91-0.85(\mathrm{~m}, 2 \mathrm{H}), 0.81-0.76(\mathrm{~m}, 2 \mathrm{H})$.

${ }^{13} \mathrm{C}$ NMR $\left(151 \mathrm{MHz}, \mathrm{CDCl}_{3}\right) \delta 159.0,145.9,131.2,130.8,130.5,128.6,128.4(\mathrm{t}, J=317.2 \mathrm{~Hz})$, 121.5, 119.0, 114.8, 114.3, 94.7, 80.2 (t, $J=2.4 \mathrm{~Hz}), 72.1$ (d, $J=4.8 \mathrm{~Hz}), 55.5,48.2$, 8.6, 8.6, -0.4.

${ }^{19}$ F NMR $\left(565 \mathrm{MHz}, \mathrm{CDCl}_{3}\right) \delta-56.3(\mathrm{~d}, J=156.1 \mathrm{~Hz}),-59.2(\mathrm{~d}, J=156.9 \mathrm{~Hz})$.

HRMS (ESI+): calcd. for $\left[\mathrm{C}_{21} \mathrm{H}_{19} \mathrm{BrClF}_{2} \mathrm{NO}_{2}+\mathrm{H}\right]^{+}: 470.0329$ found: 470.0335.

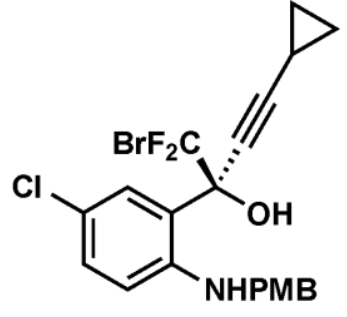

5

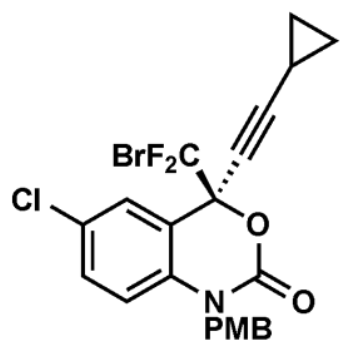

N-PMB BDF-Efavirenz

N-PMB BDF-efavirenz (S10): To a stirred solution of 5 (187 mg, $0.40 \mathrm{mmol}, 1.0$ equiv.) and $0.17 \mathrm{~mL}$ of $\mathrm{NEt}_{3}$ (1.2 mmol, 3 equiv.) in $2.0 \mathrm{~mL}$ of anhydrous toluene under $\mathrm{N}_{2}$ at $0{ }^{\circ} \mathrm{C}$, was added a solution of $48 \mathrm{mg}$ of triphosgene ( $0.16 \mathrm{mmol}, 0.4$ equiv.) in $2 \mathrm{~mL}$ of toluene, dropwise. After stirring the solution for 1 hour, the reaction was allowed to warm to room temperature and 
stirred for an additional hour. The reaction was then quenched with $0.5 \mathrm{~mL}$ of methanol and allowed to stir for an additional 30 minutes. The precipitate was then filtered off, and the solid washed with $20 \mathrm{~mL}$ of $\mathrm{Et}_{2} \mathrm{O}$. The solvent was removed under reduced pressure and the compound was purified by column chromatography on $\mathrm{SiO}_{2}$ (pentane to $1: 4 \mathrm{Et}_{2} \mathrm{O} /$ pentane gradient) to yield $163 \mathrm{mg}(82 \%)$ of the title compound as a pale-yellow solid.

${ }^{1} \mathbf{H}$ NMR $\left(600 \mathrm{MHz}, \mathrm{CDCl}_{3}\right) \delta 7.57(\mathrm{~d}, J=1.4 \mathrm{~Hz}, 1 \mathrm{H}), 7.29-7.24(\mathrm{~m}, 1 \mathrm{H}), 7.20-7.17(\mathrm{~m}, 2 \mathrm{H})$, $6.86-6.84(\mathrm{~m}, 2 \mathrm{H}), 6.82(\mathrm{~d}, J=8.9 \mathrm{~Hz}, 1 \mathrm{H}), 5.10(\mathrm{~d}, J=16.3 \mathrm{~Hz}, 1 \mathrm{H}), 5.04(\mathrm{~d}, J=16.3 \mathrm{~Hz}, 1 \mathrm{H})$, $3.78(\mathrm{~s}, 3 \mathrm{H}), 1.45-1.36(\mathrm{~m}, 1 \mathrm{H}), 0.95-0.90(\mathrm{~m}, 2 \mathrm{H}), 0.89-0.83(\mathrm{~m}, 2 \mathrm{H})$.

${ }^{13}$ C NMR (151 MHz, $\left.\mathrm{CDCl}_{3}\right) \delta 159.3,148.6,135.6,131.4,128.9,128.8,128.1,127.0,122.4(\mathrm{t}$, $J=316.7 \mathrm{~Hz}), 117.9,116.0,114.5,96.0,82.4(\mathrm{t}, J=27.9 \mathrm{~Hz}), 68.0(\mathrm{~d}, J=3.9 \mathrm{~Hz}), 55.4,48.1,8.9$, $8.9,-0.3$.

${ }^{19} \mathbf{F}$ NMR $\left(565 \mathrm{MHz}, \mathrm{CDCl}_{3}\right) \delta-59.8(\mathrm{~d}, J=165.5 \mathrm{~Hz}),-61.4(\mathrm{~d}, J=165.3 \mathrm{~Hz})$.

HRMS (ESI+): calcd. for $\left[\mathrm{C}_{22} \mathrm{H}_{17} \mathrm{BrClF}_{2} \mathrm{NO}_{3}+\mathrm{MeCN}+\mathrm{Na}\right]^{+}: 559.0206$ found: 559.0179 .

MP: $73.2-78.2^{\circ} \mathrm{C}$

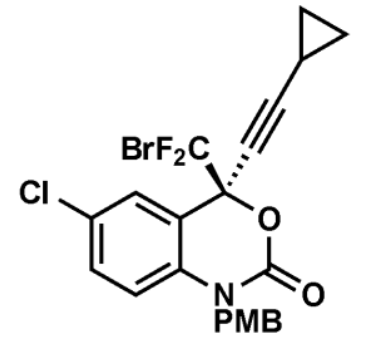

N-PMB BDF-Efavirenz

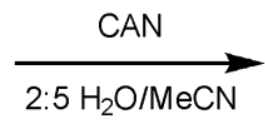

BDF-efavirenz: To a stirred solution of $150 \mathrm{mg}$ of $\mathbf{S 1 0}(0.30 \mathrm{mmol}, 1.0$ equiv.) in $1.8 \mathrm{~mL}$ of a 2:5 mixture of $\mathrm{H}_{2} \mathrm{O} / \mathrm{MeCN}$ was added $490 \mathrm{mg}$ of ceric ammonium nitrate ( 0.9 mmol, 3 equiv.) portionwise. The solution was stirred for 1 hour, and diluted with $3 \mathrm{~mL}$ of DCM. The solvent was dried with $\mathrm{Na}_{2} \mathrm{SO}_{4}$, the solid filtered, and the solvent removed under reduced pressure. The residue was purified by column chromatography on $\mathrm{SiO}_{2}\left(1: 9 \mathrm{Et}_{2} \mathrm{O}\right.$ /pentane to 1:2 $\mathrm{Et}_{2} \mathrm{O}$ /pentane gradient) to yield $90 \mathrm{mg}(80 \%)$ of the title compound as a pale-yellow solid. Crystals suitable for X-ray diffraction were obtained by recrystallization from cyclohexane.

${ }^{1} \mathbf{H}$ NMR $\left(600 \mathrm{MHz}, \mathrm{CDCl}_{3}\right) \delta 8.64(\mathrm{~s}, 1 \mathrm{H}), 7.54(\mathrm{~s}, 1 \mathrm{H}), 7.36(\mathrm{dd}, J=8.5,2.3 \mathrm{~Hz}, 1 \mathrm{H}), 6.80(\mathrm{~d}$, $J=8.5 \mathrm{~Hz}, 1 \mathrm{H}), 1.43-1.37(\mathrm{~m}, 1 \mathrm{H}), 0.95-0.91(\mathrm{~m}, 2 \mathrm{H}), 0.88-0.84(\mathrm{~m}, 2 \mathrm{H})$.

${ }^{13} \mathrm{C}$ NMR $\left(151 \mathrm{MHz}, \mathrm{CDCl}_{3}\right) \delta 148.5,133.6,131.8,129.1,128.8,122.2(\mathrm{t}, J=316.6 \mathrm{~Hz}), 116.1$, $115.8,96.4,83.8(\mathrm{t}, J=28.4 \mathrm{~Hz}), 67.7(\mathrm{~d}, J=3.4 \mathrm{~Hz}), 8.9,8.9,-0.4$.

${ }^{19}$ F NMR $\left(565 \mathrm{MHz}, \mathrm{CDCl}_{3}\right) \delta-60.0(\mathrm{~d}, J=165.0 \mathrm{~Hz}),-61.5(\mathrm{~d}, J=165.2 \mathrm{~Hz})$. 
HRMS (ESI+): calcd. for $\left[\mathrm{C}_{14} \mathrm{H}_{9} \mathrm{BrClF}_{2} \mathrm{NO}_{2}+\mathrm{MeCN}+\mathrm{Na}\right]^{+}$: 438.9631 found: 438.9606.

MP: $112.7-118.1^{\circ} \mathrm{C}$

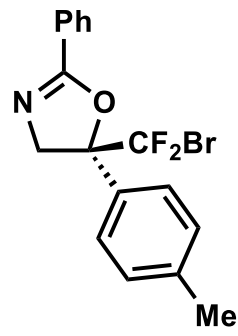

$2 a$

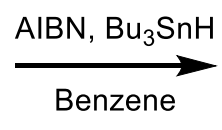

6a: To a flame dried 1-dram vial, was added $18 \mathrm{mg}$ of $\mathbf{2 a}(0.050 \mathrm{mmol}, 1.0$ equiv.) and 0.8 $\mathrm{mg}$ of AIBN (0.005 mmol, 0.1 equiv.). After evacuation and backfilling with $\mathrm{N}_{2}(3 \mathrm{x}), 0.5 \mathrm{~mL}$ of anhydrous, degassed benzene was added, followed by $26 \mu \mathrm{L}$ of $\mathrm{Bu}_{3} \mathrm{SnH}$ ( $0.10 \mathrm{mmol}, 2.0$ equiv.). The resulting solution was heated and stirred at $90{ }^{\circ} \mathrm{C}$ overnight. After cooling back to room temperature, the product was purified by column chromatography on $\mathrm{SiO}_{2}$ (pentane to 1:9 $\mathrm{Et}_{2} \mathrm{O} /$ pentane gradient). The product was then dissolved in $\mathrm{Et}_{2} \mathrm{O}$, and passed through a $10 \%$ $\mathrm{KF} / \mathrm{SiO}_{2}$ plug to remove trace tin species. Removal of the solvent resulted under reduced pressure yielded $13 \mathrm{mg}(90 \%)$ of $\mathbf{6 a}$ as a colorless oil.

${ }^{1} \mathbf{H}$ NMR $\left(600 \mathrm{MHz}, \mathrm{CDCl}_{3}\right) \delta 8.04(\mathrm{~d}, J=7.0 \mathrm{~Hz}, 2 \mathrm{H}), 7.54-7.49(\mathrm{~m}, 1 \mathrm{H}), 7.46(\mathrm{t}, J=7.6 \mathrm{~Hz}$, 2H), $7.38(\mathrm{~d}, J=8.0 \mathrm{~Hz}, 2 \mathrm{H}), 7.23(\mathrm{~d}, J=7.8 \mathrm{~Hz}, 2 \mathrm{H}), 5.92(\mathrm{t}, J=55.8 \mathrm{~Hz}, 1 \mathrm{H}), 4.66(\mathrm{~d}, J=15.1$ $\mathrm{Hz}, 1 \mathrm{H}), 4.24$ (d, $J=15.1 \mathrm{~Hz}, 1 \mathrm{H}), 2.37(\mathrm{~s}, 3 \mathrm{H})$.

${ }^{13}$ C NMR $\left(151 \mathrm{MHz}, \mathrm{CDCl}_{3}\right) \delta 162.9,139.0,134.5(\mathrm{~d}, J=2.1 \mathrm{~Hz}), 131.9,129.5,128.6,128.5$, 127.2, 126.0, $114.8(\mathrm{t}, J=249.6 \mathrm{~Hz}), 86.5(\mathrm{t}, J=23.6 \mathrm{~Hz}), 62.4,21.3$.

${ }^{19} \mathbf{F}$ NMR $\left(565 \mathrm{MHz}, \mathrm{CDCl}_{3}\right) \delta-130.2(\mathrm{dd}, J=283.7,56.2 \mathrm{~Hz}, 1 \mathrm{~F}),-130.9(\mathrm{dd}, J=283.9,55.5 \mathrm{~Hz}$, $1 \mathrm{~F})$.

HRMS (ESI+): calcd. for $\left[\mathrm{C}_{16} \mathrm{H}_{13} \mathrm{~F}_{2} \mathrm{NO}+\mathrm{H}\right]^{+}: 274.1038$ found: 274.1022.
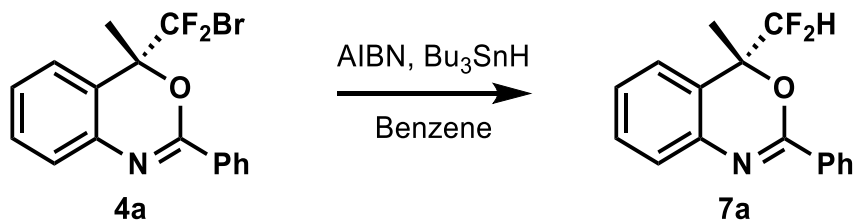

7a: Prepared analogously to above by using $18 \mathrm{mg}$ of $\mathbf{4 a}$ ( $0.050 \mathrm{mmol}, 1.0$ equiv.), yielding $14 \mathrm{mg}(99 \%)$ of $\mathbf{7 a}$ as a colorless oil. 
${ }^{1} \mathbf{H}$ NMR $\left(600 \mathrm{MHz}, \mathrm{CDCl}_{3}\right) \delta 8.15(\mathrm{~d}, J=7.2 \mathrm{~Hz}, 1 \mathrm{H}), 7.52(\mathrm{t}, J=7.3 \mathrm{~Hz}, 1 \mathrm{H}), 7.46(\mathrm{t}, J=7.6 \mathrm{~Hz}$, $2 \mathrm{H}), 7.40(\mathrm{td}, J=7.4,6.6,2.1 \mathrm{~Hz}, 1 \mathrm{H}), 7.35(\mathrm{~d}, J=7.3 \mathrm{~Hz}, 1 \mathrm{H}), 7.28-7.23(\mathrm{~m}, 2 \mathrm{H}), 5.83(\mathrm{t}, J=$ $55.6 \mathrm{~Hz}, 1 \mathrm{H}), 1.87(\mathrm{t}, J=1.6 \mathrm{~Hz}, 3 \mathrm{H})$.

${ }^{13} \mathbf{C}$ NMR $\left(151 \mathrm{MHz}, \mathrm{CDCl}_{3}\right) \delta 155.6,139.3,132.1,131.8,130.3,128.5,128.2,127.2,125.8$, 124.9, 122.8, 114.6 (dd, $J=252.6,250.4 \mathrm{~Hz}), 78.6(\mathrm{t}, J=23.2 \mathrm{~Hz}), 20.4(\mathrm{t}, J=2.5 \mathrm{~Hz})$.

${ }^{19} \mathbf{F}$ NMR $\left(565 \mathrm{MHz}, \mathrm{CDCl}_{3}\right) \delta-130.8(\mathrm{dd}, J=282.2,55.3 \mathrm{~Hz}, 1 \mathrm{~F}),-132.2(\mathrm{dd}, J=282.3,56.1 \mathrm{~Hz}$, $1 \mathrm{~F})$.

HRMS (ESI+): calcd. for $\left[\mathrm{C}_{17} \mathrm{H}_{15} \mathrm{~F}_{2} \mathrm{NO}+\mathrm{H}\right]^{+}: 288.1195$ found: 288.1171.

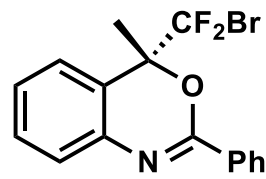

$4 a$

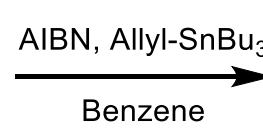

Benzene

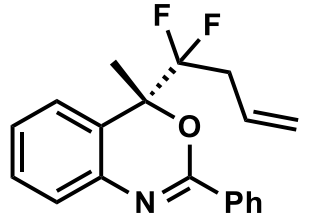

$7 b$

7b: To a flame dried 1-dram vial, was added $18 \mathrm{mg}$ of $4 \mathbf{a}(0.050 \mathrm{mmol}, 1.0$ equiv.) and 2 $\mathrm{mg}$ of AIBN (0.010 mmol, 0.2 equiv.). After evacuation and backfilling with $\mathrm{N}_{2}(3 \mathrm{x}), 0.5 \mathrm{~mL}$ of anhydrous, degassed benzene was added, followed by $30 \mu \mathrm{L}$ of $\mathrm{Bu}_{3} \mathrm{Sn}$-allyl $(0.10 \mathrm{mmol}, 2.0$ equiv.). The resulting solution was heated and stirred at $90{ }^{\circ} \mathrm{C}$ overnight. After cooling back to room temperature, the product was purified by column chromatography on $\mathrm{SiO}_{2}$ (pentane to 1:9 $\mathrm{Et}_{2} \mathrm{O} /$ pentane gradient). The product was then dissolved in $\mathrm{Et}_{2} \mathrm{O}$, and passed through a $10 \%$ $\mathrm{KF} / \mathrm{SiO}_{2}$ plug to remove trace tin species. Removal of the solvent resulted under reduced pressure yielded $14 \mathrm{mg}(91 \%)$ of $\mathbf{7 b}$ as a colorless oil.

${ }^{1} \mathbf{H}$ NMR $\left(600 \mathrm{MHz}, \mathrm{CDCl}_{3}\right) \delta 8.17-8.13(\mathrm{~m}, 2 \mathrm{H}), 7.54-7.50(\mathrm{~m}, 1 \mathrm{H}), 7.49-7.44(\mathrm{~m}, 2 \mathrm{H}), 7.37$ $(\operatorname{td}, J=7.5,1.5 \mathrm{~Hz}, 1 \mathrm{H}), 7.32(\mathrm{dd}, J=7.9,1.4 \mathrm{~Hz}, 1 \mathrm{H}), 7.28(\mathrm{~d}, J=7.8 \mathrm{~Hz}, 1 \mathrm{H}), 7.23(\mathrm{td}, J=7.5$, $1.4 \mathrm{~Hz}, 1 \mathrm{H}), 5.76$ (ddt, $J=17.2,10.2,6.9 \mathrm{~Hz}, 1 \mathrm{H}), 5.12(\mathrm{dd}, J=10.3,1.5 \mathrm{~Hz}, 1 \mathrm{H}), 5.08$ (dd, $J=$ 17.1, $1.6 \mathrm{~Hz}, 1 \mathrm{H}), 2.80-2.52(\mathrm{~m}, 2 \mathrm{H}), 1.89$ (d, $J=1.4 \mathrm{~Hz}, 3 \mathrm{H})$.

${ }^{13}$ C NMR $\left(151 \mathrm{MHz}, \mathrm{CDCl}_{3}\right) \delta 155.3,138.8,132.2,131.8,130.0,128.5,128.2(\mathrm{t}, J=4.7 \mathrm{~Hz})$, 128.0, 127.1, 125.9, 125.4 (d, $J=4.2 \mathrm{~Hz}), 123.6(\mathrm{~d}, J=2.7 \mathrm{~Hz}), 122.4(\mathrm{dd}, J=254.6,250.8 \mathrm{~Hz})$, 120.5, 81.1 (dd, $J=29.7,26.8 \mathrm{~Hz}), 36.4(\mathrm{t}, J=24.5 \mathrm{~Hz}), 22.9(\mathrm{t}, J=2.6 \mathrm{~Hz})$.

${ }^{19} \mathbf{F}$ NMR $\left(565 \mathrm{MHz}, \mathrm{CDCl}_{3}\right) \delta-110.2(\mathrm{dd}, J=253.8,26.9 \mathrm{~Hz}, 1 \mathrm{~F}),-114.4(\mathrm{dd}, J=252.8,27.2 \mathrm{~Hz}$, $1 \mathrm{~F})$.

HRMS (ESI+): calcd. for $\left[\mathrm{C}_{19} \mathrm{H}_{17} \mathrm{~F}_{2} \mathrm{NO}+\mathrm{H}\right]^{+}: 314.1351$ found: 314.1331 . 


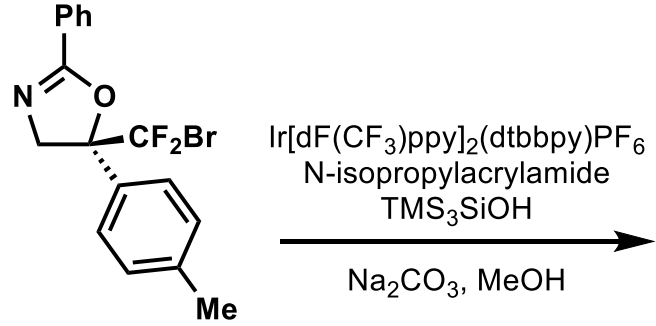

2a

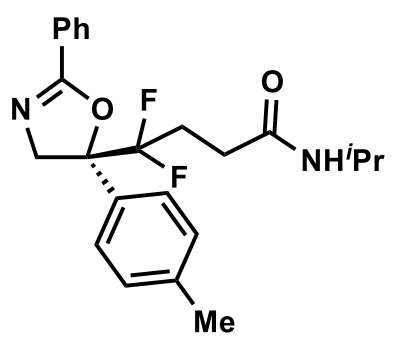

6b

6b: A flame dried 1-dram vial was charged with $18 \mathrm{mg}$ of $\mathbf{2 a}(0.050 \mathrm{mmol}, 1.0$ equiv. $), 11$ mg of $N$-isopropylacrylamide ( $0.10 \mathrm{mmol}, 2.0$ equiv.), $1 \mathrm{mg}$ of $\operatorname{Ir}\left[\mathrm{dF}\left(\mathrm{CF}_{3}\right) \text { ppy }\right]_{2}(\mathrm{dtbbpy}) \mathrm{PF}_{6}(0.001$ mmol, $2 \mathrm{~mol} \%$ ), $12 \mathrm{mg}$ of $\mathrm{TMS}_{3} \mathrm{SiOH}$ (0.050 mmol, 1.0 equiv.), and $11 \mathrm{mg}$ of $\mathrm{Na}_{2} \mathrm{CO}_{3}(0.1 \mathrm{mmol}$, 2.0 equiv.). After the vial was evacuated and backfilled with $\mathrm{N}_{2}$ three times, $0.5 \mathrm{~mL}$ of degassed $\mathrm{MeOH}$ was added. The reaction was stirred at room temperature under irradiation by blue LED's for 1 hour. After this time, the solvent was removed under reduced pressure, and the resulting residue was purified by column chromatography on $\mathrm{SiO}_{2}\left(1: 9 \mathrm{Et}_{2} \mathrm{O} /\right.$ pentane to $1: 3 \mathrm{Et} 2 \mathrm{O} /$ pentane gradient) to yield $14 \mathrm{mg}(70 \%)$ of title compound as a white solid.

${ }^{1} \mathbf{H}$ NMR $\left(600 \mathrm{MHz}, \mathrm{CDCl}_{3}\right) \delta 8.03(\mathrm{~d}, J=6.9 \mathrm{~Hz}, 2 \mathrm{H}), 7.52(\mathrm{t}, J=7.4 \mathrm{~Hz}, 1 \mathrm{H}), 7.45(\mathrm{t}, J=7.5 \mathrm{~Hz}$, $2 \mathrm{H}), 7.38(\mathrm{~d}, J=6.8 \mathrm{~Hz}, 2 \mathrm{H}), 7.19(\mathrm{~d}, J=8.0 \mathrm{~Hz}, 2 \mathrm{H}), 5.18(\mathrm{~s}, 1 \mathrm{H}), 4.81(\mathrm{~d}, J=15.2 \mathrm{~Hz}, 1 \mathrm{H}), 4.19$ $(\mathrm{dd}, J=15.2,1.9 \mathrm{~Hz}, 1 \mathrm{H}), 4.07-3.96(\mathrm{~m}, 1 \mathrm{H}), 2.49-2.21(\mathrm{~m}, 6 \mathrm{H}), 2.02$ (dddt, $J=30.0,14.7,9.7$, $5.2 \mathrm{~Hz}, 1 \mathrm{H}), 1.10(\mathrm{t}, J=6.9 \mathrm{~Hz}, 6 \mathrm{H})$.

${ }^{13} \mathrm{C}$ NMR $\left(151 \mathrm{MHz}, \mathrm{CDCl}_{3}\right) \delta 170.3,162.5,138.6,136.0(\mathrm{~d}, J=4.1 \mathrm{~Hz}), 131.8,129.4,128.6$, 128.4, 127.3, 125.9 (d, $J=1.9 \mathrm{~Hz}), 123.2$ (dd, $J=252.7,247.3 \mathrm{~Hz}), 88.4$ (dd, $J=31.5,26.4 \mathrm{~Hz})$, 63.9, 41.6, 29.9, 28.5, 28.1 (t, $J=23.5 \mathrm{~Hz}), 22.9$ (d, $J=2.2 \mathrm{~Hz}), 21.2$.

${ }^{19}$ F NMR $\left(565 \mathrm{MHz}, \mathrm{CDCl}_{3}\right) \delta-112.4(\mathrm{dd}, J=251.6,32.3 \mathrm{~Hz}, 1 \mathrm{~F}),-115.2(\mathrm{dd}, J=251.3,31.4 \mathrm{~Hz}$, $1 \mathrm{~F})$.

HRMS (ESI+): calcd. for $\left[\mathrm{C}_{23} \mathrm{H}_{26} \mathrm{~F}_{2} \mathrm{~N}_{2} \mathrm{O}_{2}+\mathrm{H}\right]^{+}: 401.2035$ found: 401.1998.

MP: $82.9-87.5^{\circ} \mathrm{C}$

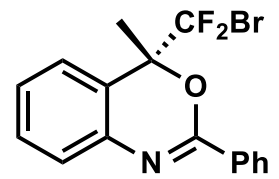

$4 a$
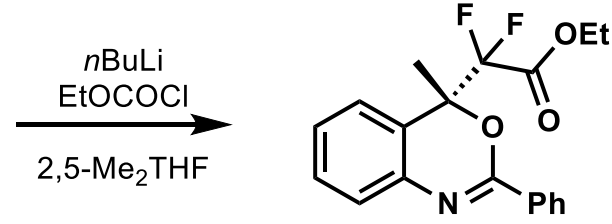

7c

7c: To a solution of $18 \mathrm{mg}$ of $\mathbf{4 a}(0.050 \mathrm{mmol}, 1.0$ equiv.) in $0.5 \mathrm{~mL}$ of anhydrous $2,5-$ $\mathrm{Me}_{2}$ THF was added $40 \mu \mathrm{L}$ of ethyl chloroformate $(0.20 \mathrm{mmol}, 4.0$ equiv.). After the solution was cooled to $-130{ }^{\circ} \mathrm{C}$ by use of a pentane/liquid $\mathrm{N}_{2}$ cooling bath, $40 \mu \mathrm{L}$ of an $n$ BuLi solution $(2.5 \mathrm{M}$ in hexane, $0.10 \mathrm{mmol}, 2.0$ equiv.) was added dropwise. After complete addition, the reaction was stirred for $20 \mathrm{~min}$ at $-130{ }^{\circ} \mathrm{C}$, and then allowed to warm to room temperature. The solvent was 
then removed under reduced pressure, and the residue was purified by column chromatography on $\mathrm{SiO}_{2}$ (pentane to 1:4 $\mathrm{Et}_{2} \mathrm{O}$ /pentane gradient) to yield $10 \mathrm{mg}(56 \%)$ of $7 \mathrm{c}$ as colorless oil.

${ }^{1} \mathbf{H}$ NMR $\left(600 \mathrm{MHz}, \mathrm{CDCl}_{3}\right) \delta 8.15-8.09(\mathrm{~m}, 2 \mathrm{H}), 7.53-7.48(\mathrm{~m}, 1 \mathrm{H}), 7.47-7.42(\mathrm{~m}, 2 \mathrm{H}), 7.39$ $(\mathrm{ddd}, J=7.8,7.0,1.8 \mathrm{~Hz}, 1 \mathrm{H}), 7.31(\mathrm{dd}, J=7.8,0.9 \mathrm{~Hz}, 1 \mathrm{H}), 7.29-7.23(\mathrm{~m}, 2 \mathrm{H}), 4.13(\mathrm{qq}, J=$ 10.7, 7.2 Hz, 2H), 2.00 (s, 3H), 1.03 (t, $J=7.2 \mathrm{~Hz}, 3 \mathrm{H})$.

${ }^{13}$ C NMR $\left(151 \mathrm{MHz}, \mathrm{CDCl}_{3}\right) \delta 162.7(\mathrm{t}, J=31.7 \mathrm{~Hz}), 155.1,139.0,132.0,131.7,130.5,128.4$, 128.2, 127.2, 125.9, 125.5, 121.6, $113.9(\mathrm{dd}, J=263.8,261.9 \mathrm{~Hz}), 80.0$ (t, $J=26.8 \mathrm{~Hz}), 63.4,22.6$, 13.5 .

${ }^{19}$ F NMR $\left(565 \mathrm{MHz}, \mathrm{CDCl}_{3}\right) \delta-115.7(\mathrm{~d}, J=254.9 \mathrm{~Hz}, 1 \mathrm{~F}),-117.7(\mathrm{~d}, J=254.9 \mathrm{~Hz}, 1 \mathrm{~F})$.

HRMS (ESI+): calcd. for $\left[\mathrm{C}_{19} \mathrm{H}_{17} \mathrm{~F}_{2} \mathrm{NO}_{3}+\mathrm{H}\right]^{+}: 346.1249$ found: 346.1225 .

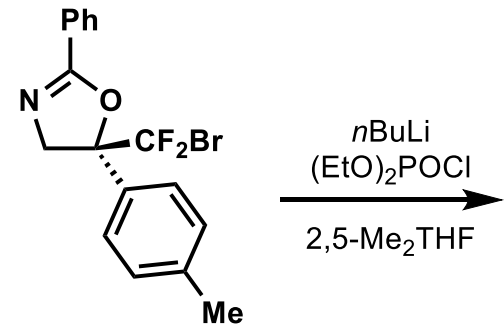

$2 a$

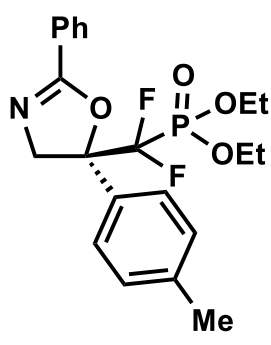

6c

6c: Prepared analogously to $7 \mathbf{b}$ by using $30 \mu \mathrm{L}$ of diethyl chlorophosphate $(0.20 \mathrm{mmol}, 4.0$ equiv.) and $18 \mathrm{mg}$ of $\mathbf{2 a}(0.050 \mathrm{mmol}, 1.0$ equiv.) to yield $9.0 \mathrm{mg}(42 \%)$ of $\mathbf{6 c}$ as a colorless oil.

${ }^{1} \mathbf{H}$ NMR $\left(600 \mathrm{MHz}, \mathrm{CDCl}_{3}\right) \delta 8.06(\mathrm{~d}, J=7.6 \mathrm{~Hz}, 2 \mathrm{H}), 7.53-7.41(\mathrm{~m}, 5 \mathrm{H}), 7.20(\mathrm{~d}, J=7.9 \mathrm{~Hz}$, $2 \mathrm{H}), 4.97(\mathrm{~d}, J=15.3 \mathrm{~Hz}, 1 \mathrm{H}), 4.31(\mathrm{~d}, J=15.3 \mathrm{~Hz}, 1 \mathrm{H}), 4.17-4.07(\mathrm{~m}, 2 \mathrm{H}), 4.06-3.95(\mathrm{~m}, 2 \mathrm{H})$, $2.35(\mathrm{~s}, 3 \mathrm{H}), 1.19(\mathrm{dt}, J=10.1,7.1 \mathrm{~Hz}, 6 \mathrm{H})$.

${ }^{13} \mathrm{C}$ NMR $\left(151 \mathrm{MHz}, \mathrm{CDCl}_{3}\right) \delta 162.5,138.8,134.6,131.8,129.1,128.6,128.5,127.3,126.8$, $121.5-115.5(\mathrm{~m}), 88.3-87.4(\mathrm{~m}), 64.7$ (d, $J=8.0 \mathrm{~Hz}), 64.6(\mathrm{~d}, J=5.5 \mathrm{~Hz}), 29.9,21.3,16.3(\mathrm{~d}$, $J=5.9 \mathrm{~Hz}), 16.3(\mathrm{~d}, J=5.9 \mathrm{~Hz})$.

${ }^{19}$ F NMR $\left(565 \mathrm{MHz}, \mathrm{CDCl}_{3}\right) \delta-116.2(\mathrm{dd}, J=312.2,102.9 \mathrm{~Hz}),-117.8(\mathrm{dd}, J=312.5,103.5 \mathrm{~Hz})$.

${ }^{31} \mathbf{P}$ NMR $\left(243 \mathrm{MHz}, \mathrm{CDCl}_{3}\right) \delta 4.3(\mathrm{t}, J=102.8 \mathrm{~Hz})$.

HRMS (ESI+): calcd. for $\left[\mathrm{C}_{21} \mathrm{H}_{24} \mathrm{~F}_{2} \mathrm{NO}_{4} \mathrm{P}+\mathrm{H}\right]^{+}$: 424.1484 found: 424.1502. 

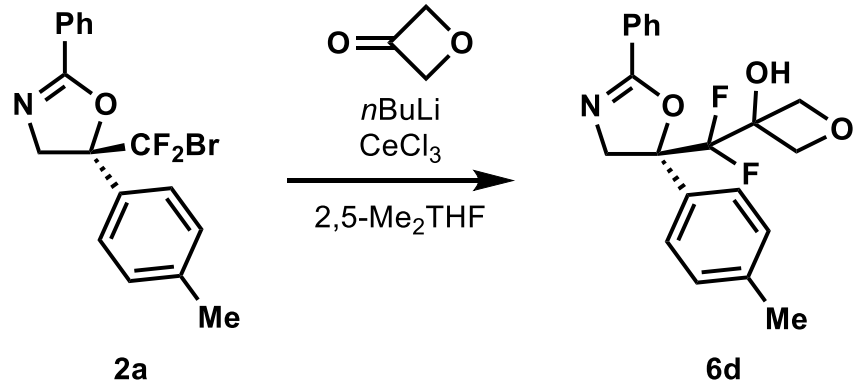

6d: To a solution of $18 \mathrm{mg}$ of $\mathbf{2 a}(0.050 \mathrm{mmol}, 1.0$ equiv. $)$ in $0.5 \mathrm{~mL}$ of anhydrous $2,5-$ $\mathrm{Me}_{2} \mathrm{THF}$ was added $14 \mathrm{mg}$ of 3 -oxetanone ( $0.20 \mathrm{mmol}, 4.0$ equiv.) and $25 \mathrm{mg}$ of $\mathrm{CeCl}_{3}(0.1 \mathrm{mmol}$, 2.0 equiv.). After the solution was cooled to $-130{ }^{\circ} \mathrm{C}$ by use of a pentane/liquid $\mathrm{N}_{2}$ cooling bath, $40 \mu \mathrm{L}$ of an $n \mathrm{BuLi}$ solution (2.5 M in hexane, $0.10 \mathrm{mmol}, 2.0$ equiv.) was added dropwise. After complete addition, the reaction was stirred for $20 \mathrm{~min}$ at $-130{ }^{\circ} \mathrm{C}$, and then allowed to warm to room temperature. The solvent was then removed under reduced pressure, and the residue was purified by column chromatography on $\mathrm{SiO}_{2}$ (pentane to $1: 4 \mathrm{Et}_{2} \mathrm{O} /$ pentane gradient) to yield 10 $\mathrm{mg}(54 \%)$ of $\mathbf{6 d}$ as colorless oil.

${ }^{1} \mathbf{H}$ NMR $\left(600 \mathrm{MHz}, \mathrm{CDCl}_{3}\right) \delta 7.99(\mathrm{~d}, J=7.3 \mathrm{~Hz}, 2 \mathrm{H}), 7.53(\mathrm{t}, J=7.4 \mathrm{~Hz}, 1 \mathrm{H}), 7.48-7.41(\mathrm{~m}$, $4 \mathrm{H}), 7.21(\mathrm{~d}, J=8.0 \mathrm{~Hz}, 2 \mathrm{H}), 4.95(\mathrm{~d}, J=15.3 \mathrm{~Hz}, 1 \mathrm{H}), 4.70(\mathrm{~d}, J=7.7 \mathrm{~Hz}, 1 \mathrm{H}), 4.37$ (dd, $J=7.7$, $3.0 \mathrm{~Hz}, 1 \mathrm{H}), 4.33(\mathrm{~d}, J=15.3 \mathrm{~Hz}, 1 \mathrm{H}), 4.19(\mathrm{~d}, J=7.9 \mathrm{~Hz}, 1 \mathrm{H}), 4.07$ (d, J=8.2 Hz, 1H), 2.36 (s, $3 \mathrm{H})$.

${ }^{13} \mathrm{C}$ NMR $\left(151 \mathrm{MHz}, \mathrm{CDCl}_{3}\right) \delta$ 162.0, 139.5, 134.7 (d, J= 3.3 Hz), 132.0, 129.6, 128.8, 128.3, 126.9, 126.0, $119.3(\mathrm{dd}, J=256.7,252.5 \mathrm{~Hz}), 88.2(\mathrm{dd}, J=30.7,25.1 \mathrm{~Hz}), 79.4(\mathrm{t}, J=4.4 \mathrm{~Hz}), 79.0$ $(\mathrm{dd}, J=6.7,2.8 \mathrm{~Hz}), 65.0(\mathrm{~d}, J=2.6 \mathrm{~Hz}), 21.3$. (One resonance missing)

${ }^{19}$ F NMR $\left(565 \mathrm{MHz}, \mathrm{CDCl}_{3}\right) \delta-120.1(\mathrm{~d}, J=272.1 \mathrm{~Hz}),-124.5(\mathrm{~d}, J=271.9 \mathrm{~Hz})$.

HRMS (ESI+): calcd. for $\left[\mathrm{C}_{20} \mathrm{H}_{19} \mathrm{~F}_{2} \mathrm{NO}_{3}+\mathrm{H}\right]^{+}: 360.1406$ found: 360.1421 .

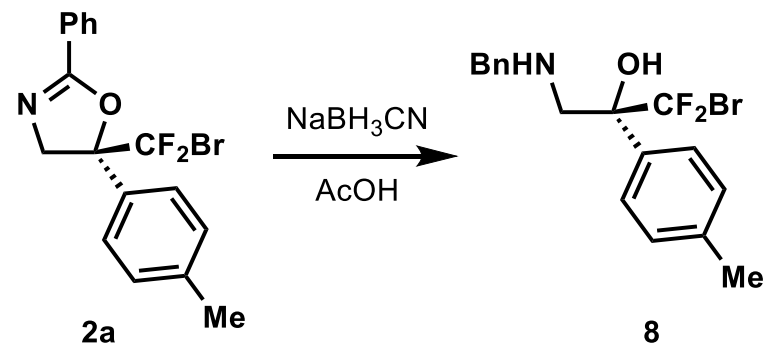

8: To a stirred solution of $\mathbf{2 a}(18 \mathrm{mg}, 0.05 \mathrm{mmol}, 1.0$ equiv.) in $0.5 \mathrm{~mL}$ of $\mathrm{AcOH}$, was added $16 \mathrm{mg}$ of $\mathrm{NaBH}_{3} \mathrm{CN}$ ( $0.25 \mathrm{mmol}, 5$ equiv.). After stirring the reaction overnight, $0.5 \mathrm{~mL}$ of $\mathrm{H}_{2} \mathrm{O}$ was added dropwise. After an additional hour of stirring, the reaction was diluted with DCM $(10 \mathrm{~mL})$, and the reaction was neutralized with saturated $\mathrm{NaHCO}_{3}$. The layers were then separated and the aqueous layer was extracted three times with DCM (10 mL each). The combined organic 
layers were dried with $\mathrm{Na}_{2} \mathrm{SO}_{4}$, filtered, and solvent removed in vacuo. The product was purified by column chromatography on $\mathrm{SiO}_{2}\left(1: 9 \mathrm{Et}_{2} \mathrm{O} /\right.$ pentane to $1: 2 \mathrm{Et}_{2} \mathrm{O} /$ pentane gradient $)$ to yield 17 $\mathrm{mg}(98 \%)$ of pure 8 as a colorless oil. Title compound was then dissolved in $\mathrm{Et}_{2} \mathrm{O}, 1.0$ equiv. of $\mathrm{HCl}\left(2 \mathrm{M}\right.$ in $\left.\mathrm{Et}_{2} \mathrm{O}\right)$ was added, and the solvent was removed under reduced pressure. Crystals suitable for X-ray diffraction were obtained by slow diffusion of $\mathrm{Et}_{2} \mathrm{O}$ into a solution of $\mathbf{8} \cdot \mathbf{H C l}$ dissolved in $\mathrm{MeOH}$.

${ }^{1} \mathbf{H}$ NMR $\left(600 \mathrm{MHz}, \mathrm{CDCl}_{3}\right) \delta 7.47(\mathrm{~d}, J=8.0 \mathrm{~Hz}, 2 \mathrm{H}), 7.33(\mathrm{t}, J=7.4 \mathrm{~Hz}, 2 \mathrm{H}), 7.28(\mathrm{~d}, J=7.1$ $\mathrm{Hz}, 1 \mathrm{H}), 7.24(\mathrm{~d}, J=6.9 \mathrm{~Hz}, 2 \mathrm{H}), 7.19(\mathrm{~d}, J=8.0 \mathrm{~Hz}, 2 \mathrm{H}), 3.78(\mathrm{~s}, 2 \mathrm{H}), 3.51(\mathrm{~d}, J=12.8 \mathrm{~Hz}, 1 \mathrm{H})$, $3.10(\mathrm{~d}, J=12.8 \mathrm{~Hz}, 1 \mathrm{H}), 2.36(\mathrm{~s}, 3 \mathrm{H})$.

${ }^{13} \mathbf{C}$ NMR $\left(151 \mathrm{MHz}, \mathrm{CDCl}_{3}\right) \delta 139.2,138.5,135.9,129.1,128.8,128.1,127.6,127.4(\mathrm{t}, J=314.0$ $\mathrm{Hz}), 126.8,78.2(\mathrm{t}, J=22.1 \mathrm{~Hz}), 54.2,52.9,21.3$.

${ }^{19}$ F NMR $\left(565 \mathrm{MHz}, \mathrm{CDCl}_{3}\right) \delta-57.0(\mathrm{~s})$.

HRMS (ESI+): calcd. for $\left[\mathrm{C}_{17} \mathrm{H}_{18} \mathrm{BrF}_{2} \mathrm{NO}+\mathrm{H}\right]^{+}: 370.0613$ found: 370.0592 . 


\section{X-ray Crystallographic Data:}

X-ray Crystallography. Crystals were coated with Paratone-N oil, attached to a Kapton loop, and mounted onto a Rigaku XtaLAB P200 equipped with a MicroMax 007HF rotating anode and Pilatus3 R 200K-A hybrid pixel array detector. Data were collected using $\mathrm{Cu} \mathrm{K} \alpha$ radiation $(\lambda=$ $1.5418 \AA$ ). Crystals were kept at $100(2) \mathrm{K}$ throughout the collection. Data collection was performed with CrysAlisPro ${ }^{11}$. Data processing was done with CrysAlisPro and included a multiscan absorption correction applied using the SCALE3 ABSPACK scaling algorithm within CrysAlisPro.

\section{BDF-efavirenz:}

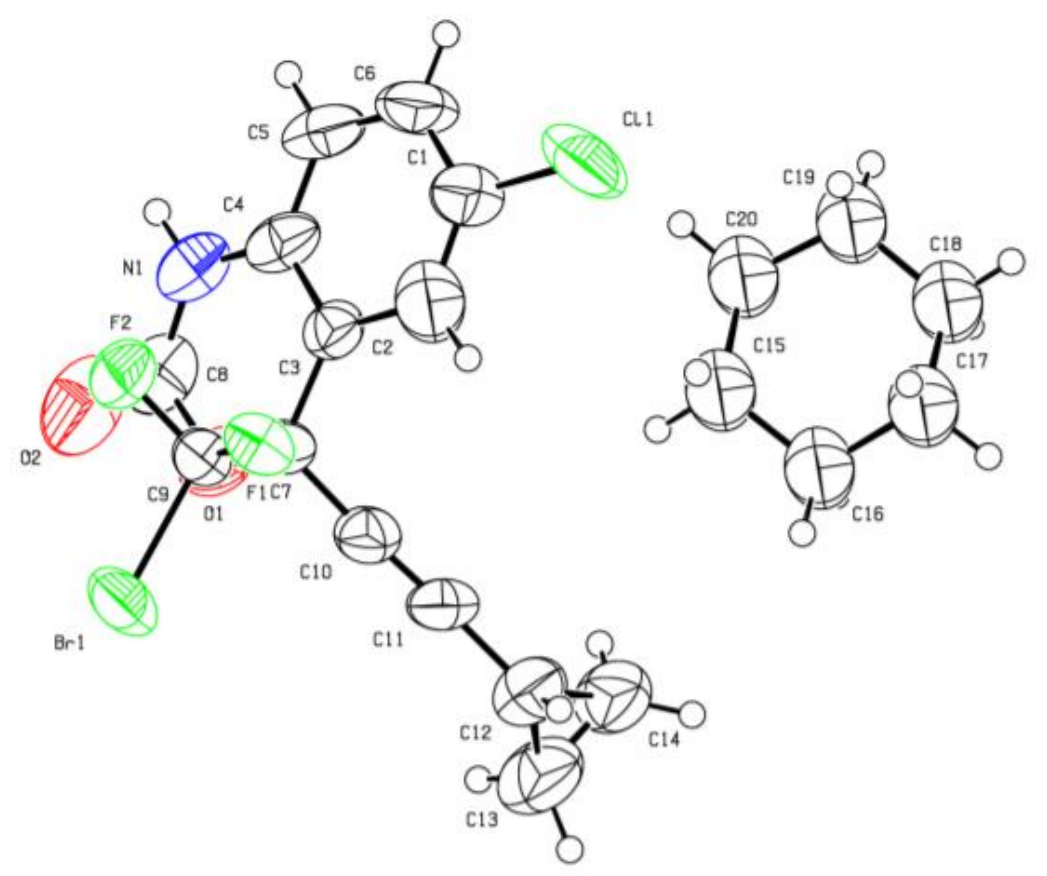

Figure S1. Full ORTEP of BDF-efavirenz (cyclohexane removed for clarity)

Table 1. Crystallographic data ${ }^{a}$ for BDF-efavirenz.

BDF-efavirenz

\begin{tabular}{ll}
\hline Chemical formula & $\mathrm{C}_{14} \mathrm{H}_{9} \mathrm{BrClF}_{2} \mathrm{NO}_{2}$ \\
FW & 460.73 \\
Crystal system, space group & Hexagonal, $P 6_{2}$ \\
Temperature (K) & $100(2)$ \\
$a(\AA)$ & $18.0955(2)$ \\
$b(\AA)$ & $18.0955(2)$ \\
$c(\AA)$ & $10.6307(1)$ \\
$V\left(\AA^{3}\right)$ & $3014.63(7)$ \\
$Z$ & 6
\end{tabular}




\begin{tabular}{ll}
$\mu\left(\mathrm{mm}^{-1}\right)$ & 4.36 \\
$F(000)$ & 1308 \\
Crystal size $(\mathrm{mm})$ & $0.13 \times 0.16 \times 0.5$ \\
$\theta$ range $\left(^{\circ}\right)$ for cell measurement & $2.8-79.4$ \\
Radiation type & $\mathrm{Cu} K \alpha$ \\
No. of reflections for cell & 129069 \\
measurement & $-22 \leq \mathrm{h} \leq 22$ \\
Index ranges & $-22 \leq \mathrm{k} \leq 23$ \\
& $-13 \leq 1 \leq 12$ \\
& $129069 / 4219$ \\
Reflections collected / unique & 0.109 \\
$R_{\text {int }}$ & $4219 / 1 / 203$ \\
Data / restraints / parameters & 1.850 \\
Goodness-of-fit on $\mathrm{F}^{2}$ & $\mathrm{R}_{1}=0.109, w \mathrm{R}_{2}=0.366$ \\
Final R indices & \\
Largest diff. peak and hole, $\AA^{-3}$ & 1.04 and -1.26 \\
\hline
\end{tabular}

${ }^{a}$ Obtained with a rotating-anode X-ray tube, Rigaku $\mathrm{Cu} K_{\alpha}(\lambda=1.54184 \AA$ A $)$ X-ray source.

${ }^{b} \mathrm{R}_{1}=\sum|| F_{0}\left|-F_{\mathrm{C}} \| / \sum\right| F_{0} \mid, \mathrm{R}_{2}=\left\{\sum\left[w\left(F_{0}^{2}-F_{0}^{2}\right)^{2}\right] / \sum\left[w\left(F_{0}^{2}\right)^{2}\right]\right\}^{1 / 2}$. 


\section{8•HCl:}

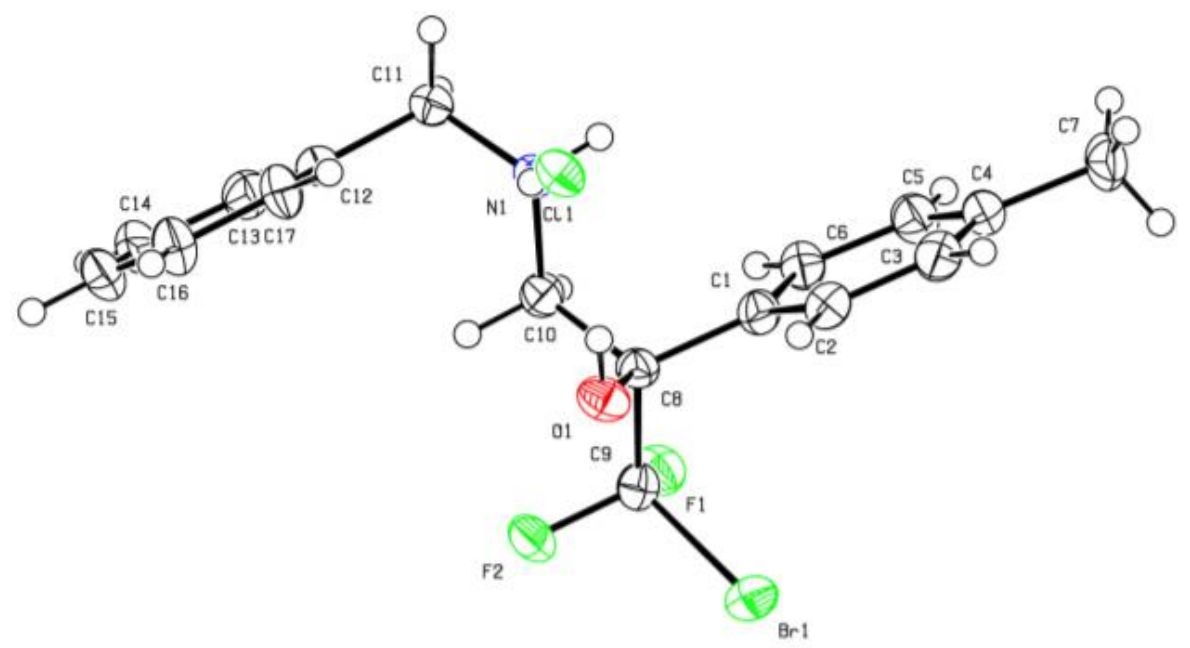

Figure S2. Full ORTEP of $\mathbf{8} \cdot \mathbf{H C l}$

Table 2. Crystallographic data ${ }^{a}$ for $\mathrm{CF} 2 \mathrm{Br} \_a m i n o a l c o h o l(\mathbf{8} \cdot \mathbf{H C l})$

\begin{tabular}{ll}
\hline & \multicolumn{1}{c}{ CF2Br_aminoalcohol } \\
\hline Chemical formula & $\mathrm{C}_{17} \mathrm{H}_{19} \mathrm{BrClF}_{2} \mathrm{NO}$ \\
FW & 406.68 \\
Crystal system, space group & Monoclinic, $P_{1}{ }_{1}$ \\
Temperature (K) & $100(2)$ \\
$a(\AA)$ & $11.3777(2)$ \\
$b(\AA)$ & $6.86536(10)$ \\
$c(\AA)$ & $11.42344(18)$ \\
$V\left(\AA^{3}\right)$ & $862.58(2)$ \\
$Z$ & 2 \\
$\mu\left(\mathrm{mm}{ }^{-1}\right)$ & 4.88 \\
$F(000)$ & 412 \\
Crystal size (mm) & $0.05 \times 0.08 \times 0.52$ \\
$\theta$ range $\left(^{\circ}\right)$ for cell measurement & $4.0-79.0$ \\
Radiation type & $\mathrm{Cu} K \alpha$ \\
No. of reflections for cell & 11764 \\
measurement & \\
Index ranges & $-14 \leq \mathrm{h} \leq 14$ \\
& $-8 \leq \mathrm{k} \leq 7$
\end{tabular}


$-14 \leq 1 \leq 14$

Reflections collected / unique

11764 / 3333

$R_{\text {int }}$

0.053

Data / restraints / parameters

$3333 / 1 / 210$

Goodness-of-fit on $\mathrm{F}^{2}$

1.168

Final R indices $^{b}$

$\mathrm{R}_{1}=0.053, w \mathrm{R}_{2}=0.155$

Largest diff. peak and hole, $\mathrm{e} \AA^{-3}$

1.11 and -0.6

${ }^{a}$ Obtained with a rotating-anode X-ray tube, Rigaku $\mathrm{Cu} K_{\alpha}(\lambda=1.54184 \AA$ A $)$ X-ray source.

${ }^{b} \mathrm{R}_{1}=\sum|| F_{0}\left|-F_{\mathrm{C}} \| / \sum\right| F_{0} \mid, \mathrm{R}_{2}=\left\{\sum\left[w\left(F_{0}^{2}-F_{0}^{2}\right)^{2}\right] / \Sigma\left[w\left(F_{0}^{2}\right)^{2}\right]\right\}^{1 / 2}$. 


\section{References}

1. Wang, Y.-M.; Wu, J.; Hoong, C.; Rauniyar, V.; Toste, F. D. Enantioselective Halocyclization Using Reagents Tailored for Chiral Anion Phase-Transfer Catalysis J. Am. Chem. Soc. 2012, $134,12928-12931$.

2. Paudler, W. W. and Jovanovic, M. V. Bromination of Some Pyridine and Diazine N-Oxides $J$. Org. Chem. 1983, 48, 1064-1069.

3. Yao, S.-J.; Ren, Z.-H.; Wang, Y.-Y.; Guan, Z.-H. Friedel-Crafts Fluoroacetylation of Indoles with Fluorinated Acetic Acids for the Synthesis of Fluoromethyl Indol-3-yl Ketones under Catalyst- and Additive-Free Conditions J. Org. Chem. 2016, 81, 4226-4234.

4. Góme, A. B.; González, M. A. C.; Lübcke, M.; Johansson, M. J.; Schou, M.; Szabó, K. J. Synthesis of trifluoromethyl moieties by late-stage copper (I) mediated nucleophilic fluorination Journal of Fluorine Chemistry 2017, 194, 51-57.

5. Miura, T.; Ito, Y. Murakami, M. Synthesis of gem-Difluoroalkenes via $\beta$-Fluoride Elimination of Organorhodium(I) Chem. Lett. 2008, 37, 1006-1007.

6. Fort, D. A.; Woltering, T. J.; Nettekoven, M.; Knust, H.; Bach, T. Conformationally restricted pyrrolidines by intramolecular [2+2] photocycloaddition reactions Chem. Commun. 2013, 49, 2989-2991.

7. Albert, K.; Gardinier, M. K; Hembre, E. J.; Hong, J. E; Jungheim, L. N.; Muehl, B. S.; Remich, D. M.; Robertson, M. A.; Savin, K. A. Triazole Derivatives as Tachykinin Receptor Antagonists U.S. Patent WO03091226A1 April 22 2003.

8. Kim, J. H.; Choi, S. J.; Jeong, I. H. Consecutive cross-coupling reactions of 2,2-difluoro-1iodoethenyl tosylate with boronic acids: efficient synthesis of 1,1-diaryl-2,2-difluoroethenes Beilstein J. Org. Chem. 2013, 9, 2470-2475.

9. Ichikawa, J.; Wada, Y.; Kuroki, H.; Mihara, J; Nadano, R. Intramolecular cyclization of $\beta, \beta$ difluorostyrenes bearing an iminomethyl or a diazenyl group at the ortho position: synthesis of 3fluorinated isoquinoline and cinnoline derivatives Org. Biomol. Chem., 2007, 5, 3956-3962.

10. Klussmann, M.; Ratjen, L.; Hoffmann, S.; Wakchaure, V.; Goddard, R.; List, B. Synthesis of TRIP and Analysis of Phosphate Salt Impurities Synlett 2010, 14, 2189-2192. 
11. Rigaku Oxford Diffraction, CrysAlisPro Software system (Rigaku Corporation, Oxford, UK, 2015). 
Chiral HPLC separation conditions and retention times for products: Qa': OD 99.5:0.5 hexanes/isopropanol, $1 \mathrm{ml} / \mathrm{min}, \mathrm{t}_{\mathrm{m}}=9.3 \mathrm{~min}, \mathrm{t}_{\mathrm{M}}=6.1 \mathrm{~min}$.
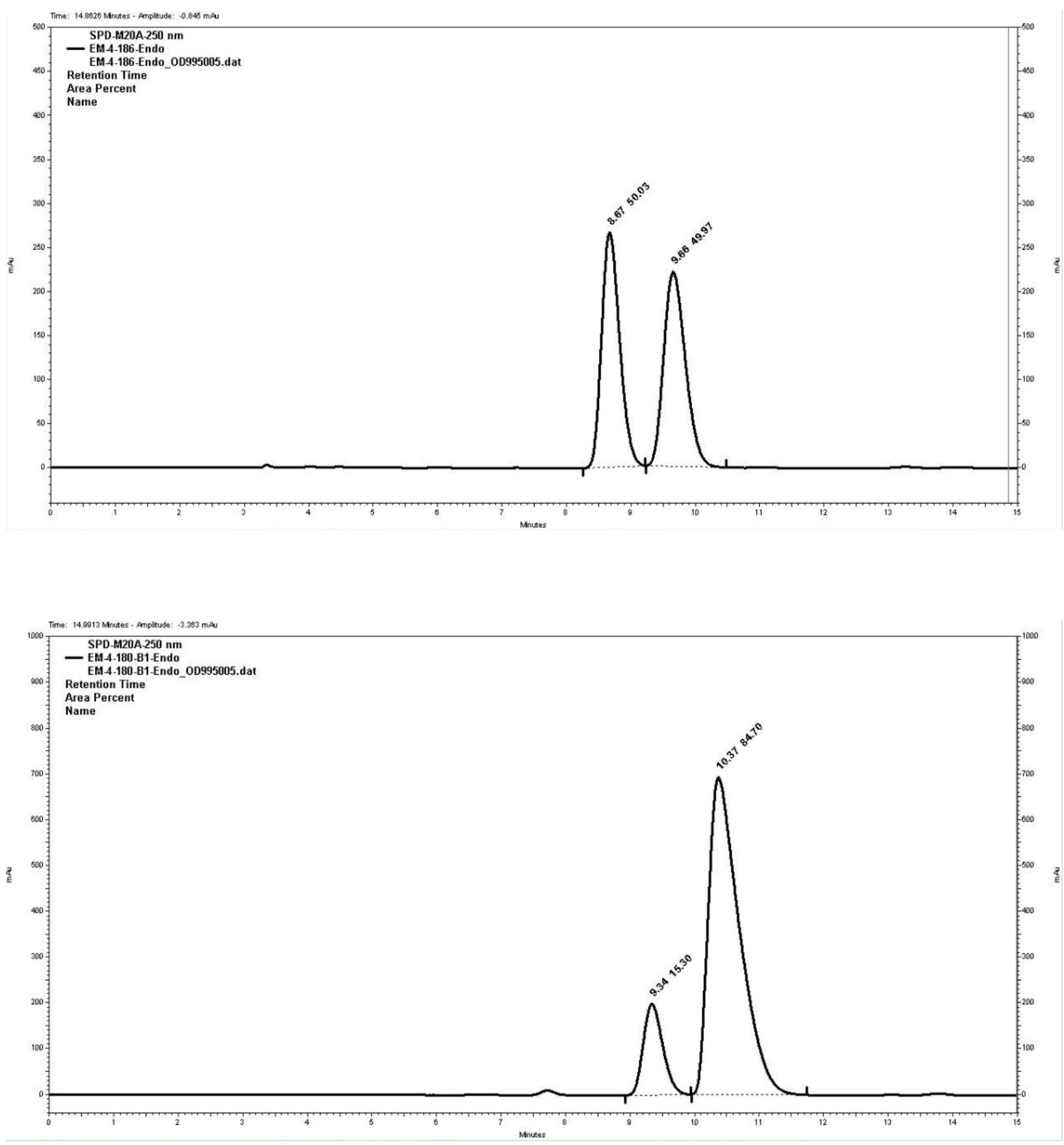

S53 
2a: IB 99:01 hexanes/isopropanol, $1 \mathrm{ml} / \mathrm{min}, \mathrm{t}_{\mathrm{m}}=5.8 \mathrm{~min}, \mathrm{t}_{\mathrm{M}}=6.1 \mathrm{~min}$.
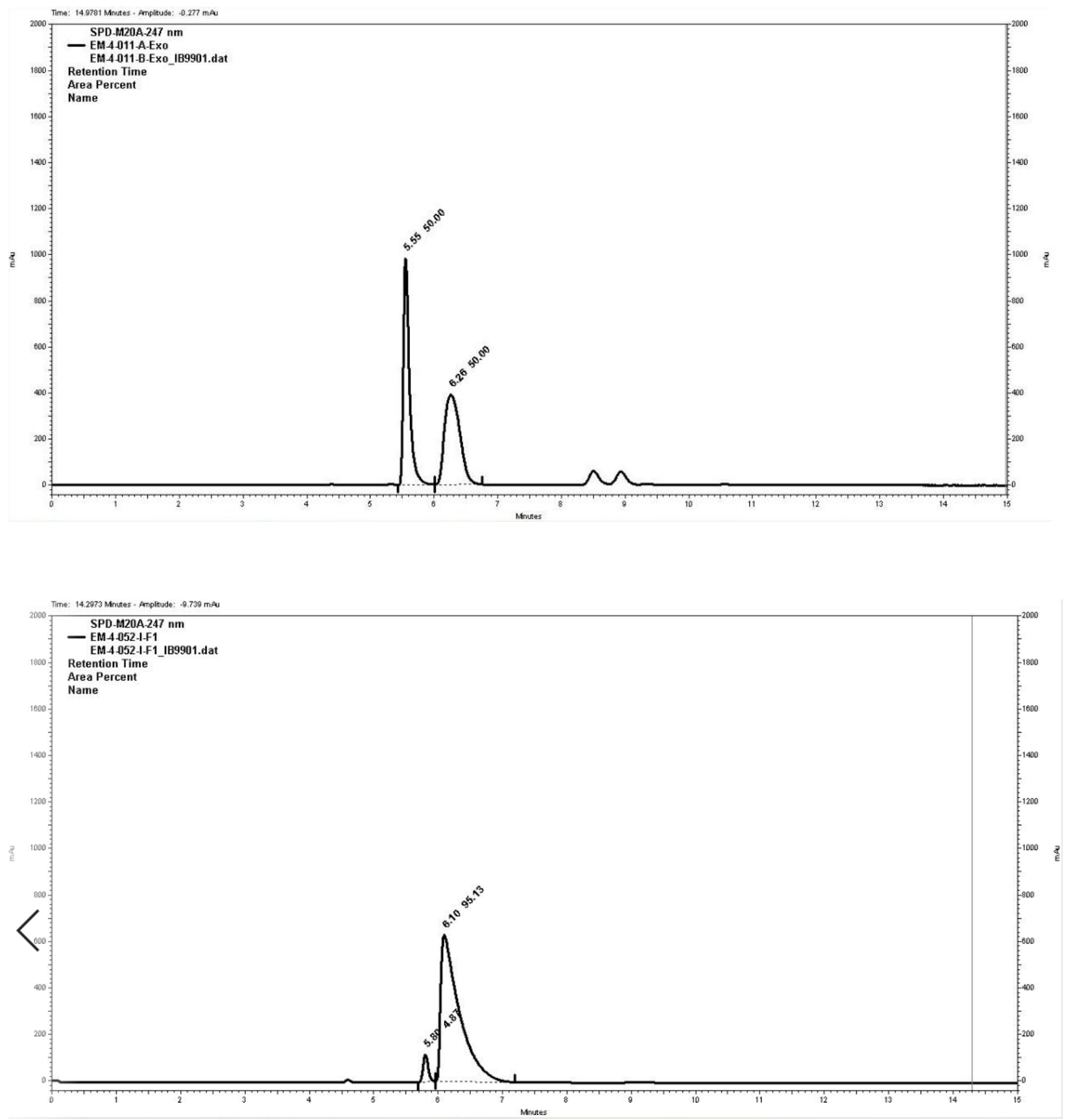
2b: IB 99:01 hexanes/isopropanol, $1 \mathrm{ml} / \mathrm{min}, \mathrm{t}_{\mathrm{m}}=11.3 \mathrm{~min}, \mathrm{t}_{\mathrm{M}}=11.8 \mathrm{~min}$.
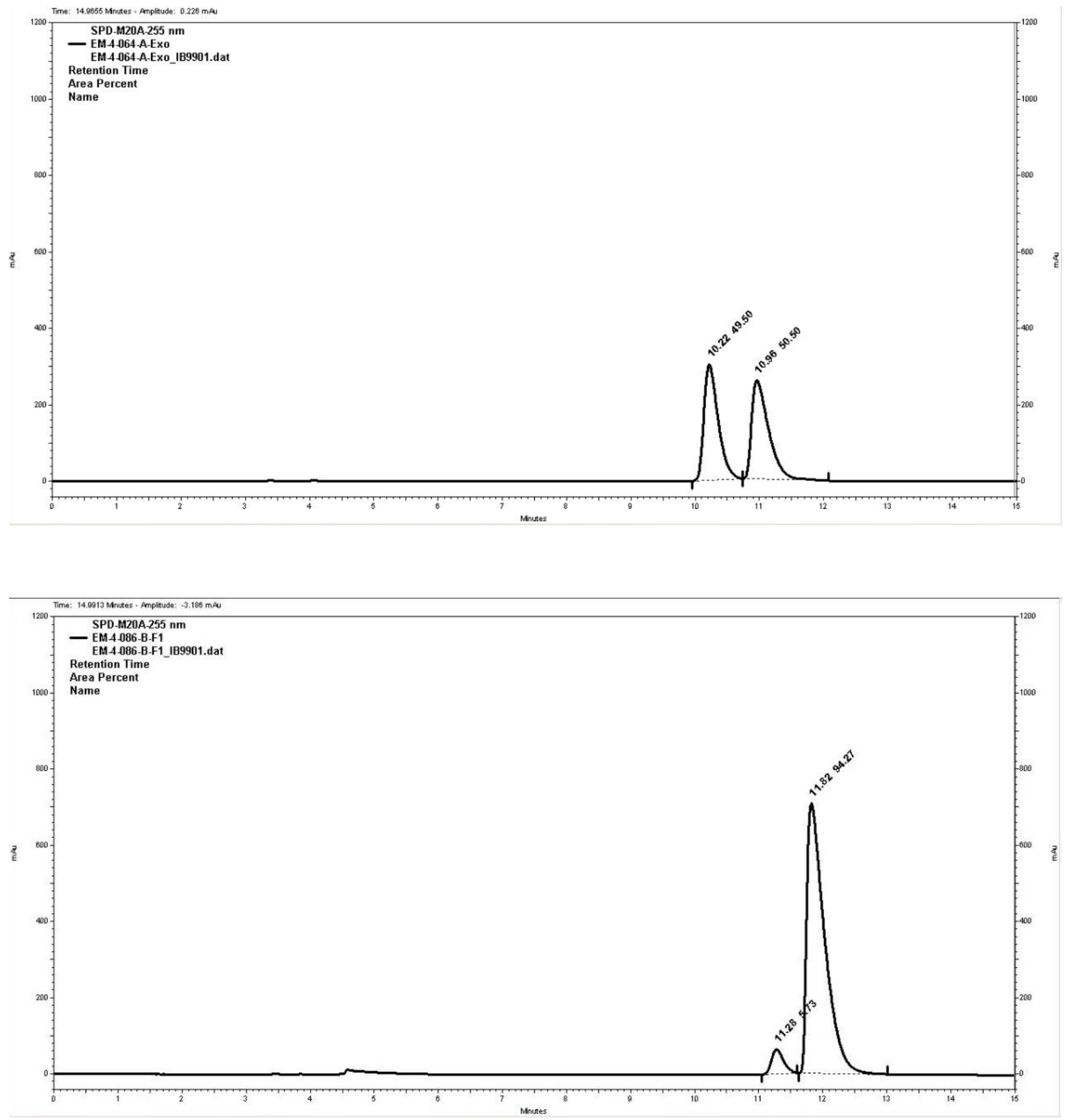
2c: IB 99:01 hexanes/isopropanol, $1 \mathrm{ml} / \mathrm{min}, \mathrm{t}_{\mathrm{m}}=5.4 \mathrm{~min}, \mathrm{t}_{\mathrm{M}}=5.6 \mathrm{~min}$.
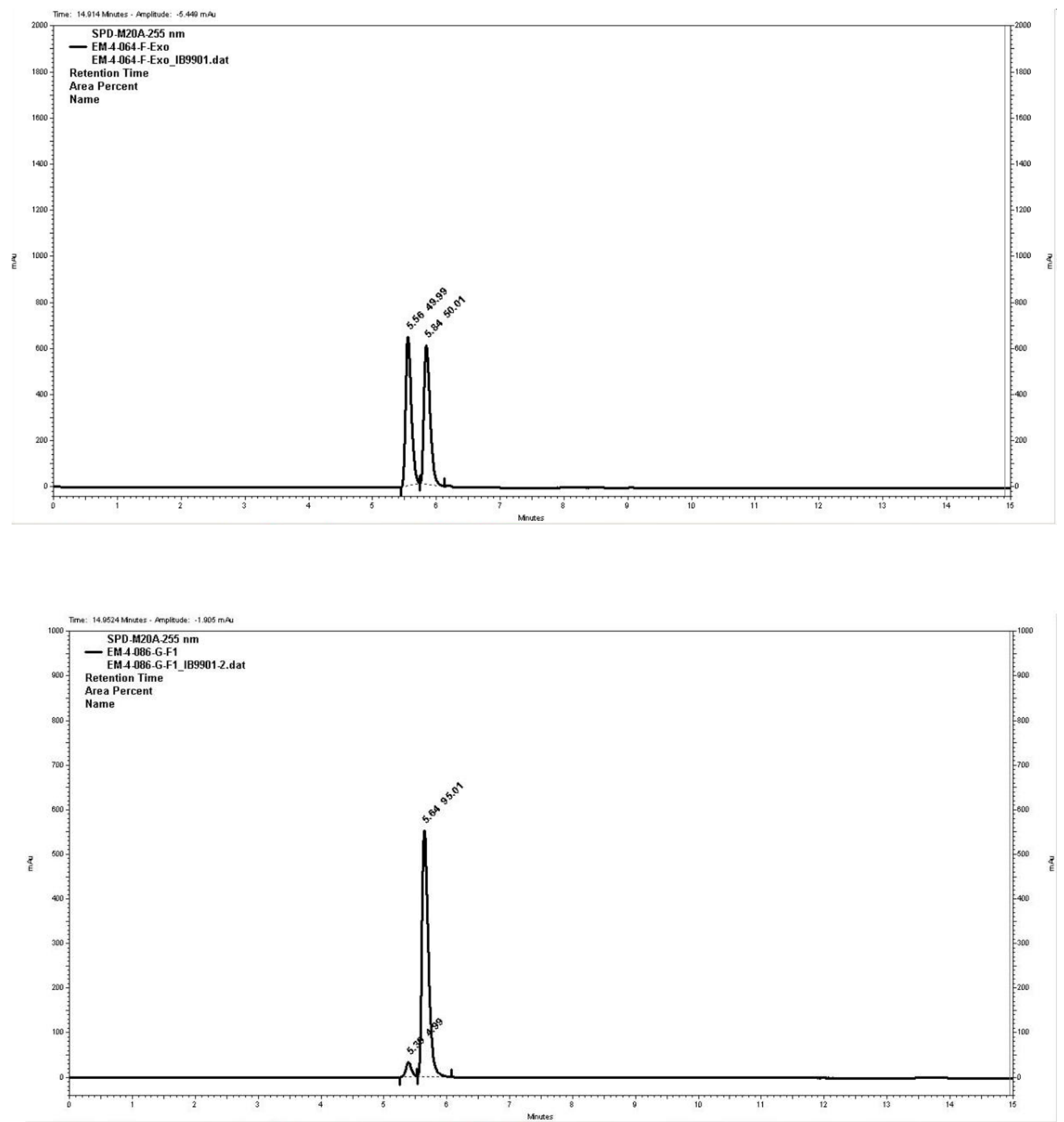
2d: IB 99.9:0.1 hexanes/isopropanol, $1 \mathrm{ml} / \mathrm{min}, \mathrm{t}_{\mathrm{m}}=11.3 \mathrm{~min}, \mathrm{t}_{\mathrm{M}}=12.2 \mathrm{~min}$.
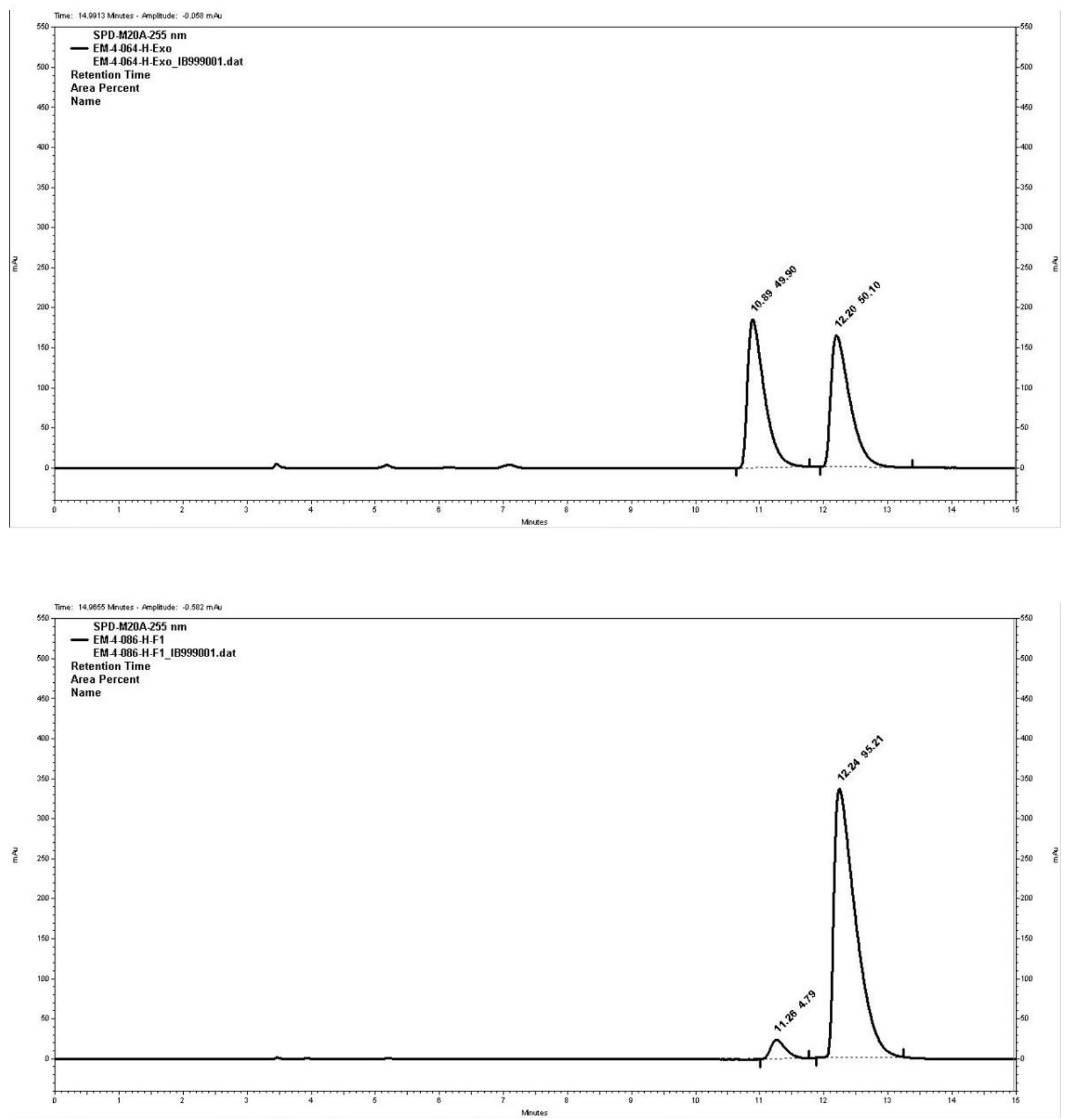
2e: IA 99:01 hexanes/isopropanol, $1 \mathrm{ml} / \mathrm{min}, \mathrm{t}_{\mathrm{m}}=10.6 \mathrm{~min}, \mathrm{t}_{\mathrm{M}}=8.8 \mathrm{~min}$.
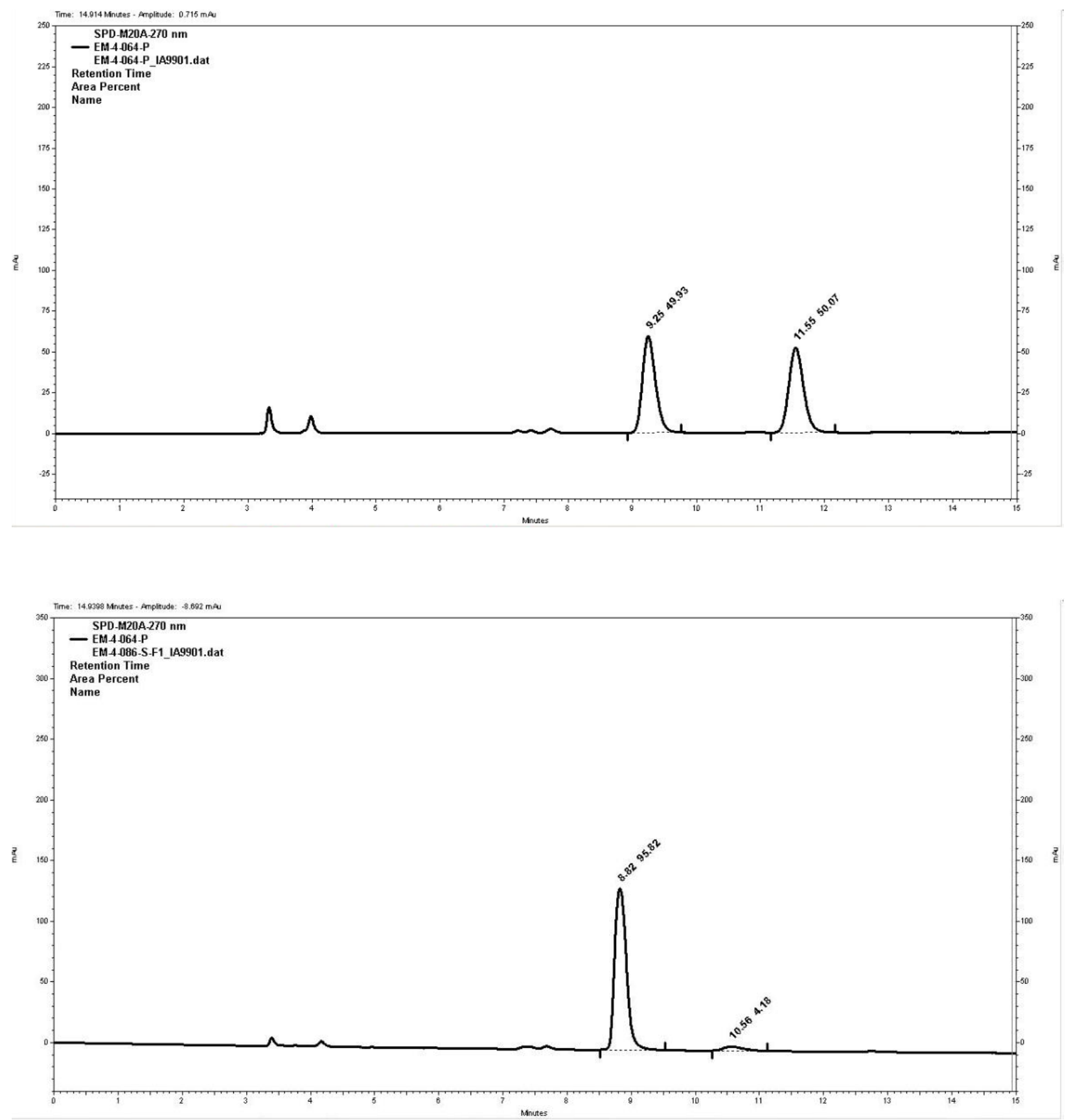
2f: IB 99:01 hexanes/isopropanol, $1 \mathrm{ml} / \mathrm{min}, \mathrm{t}_{\mathrm{m}}=7.5 \mathrm{~min}, \mathrm{t}_{\mathrm{M}}=8.3 \mathrm{~min}$.
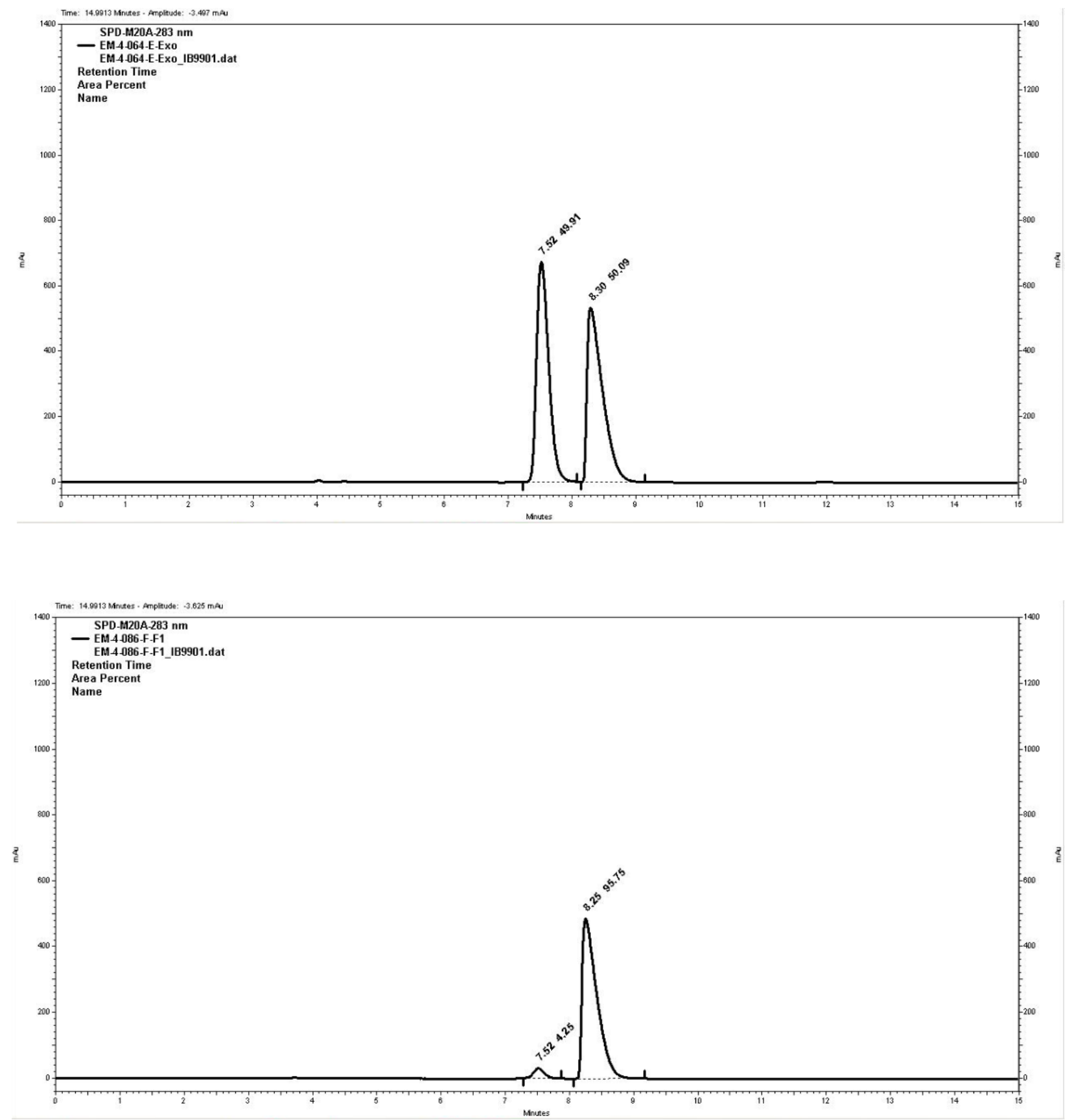
2g: IB 99:01 hexanes/isopropanol, $1 \mathrm{ml} / \mathrm{min}, \mathrm{t}_{\mathrm{m}}=7.5 \mathrm{~min}, \mathrm{t}_{\mathrm{M}}=9.1 \mathrm{~min}$.
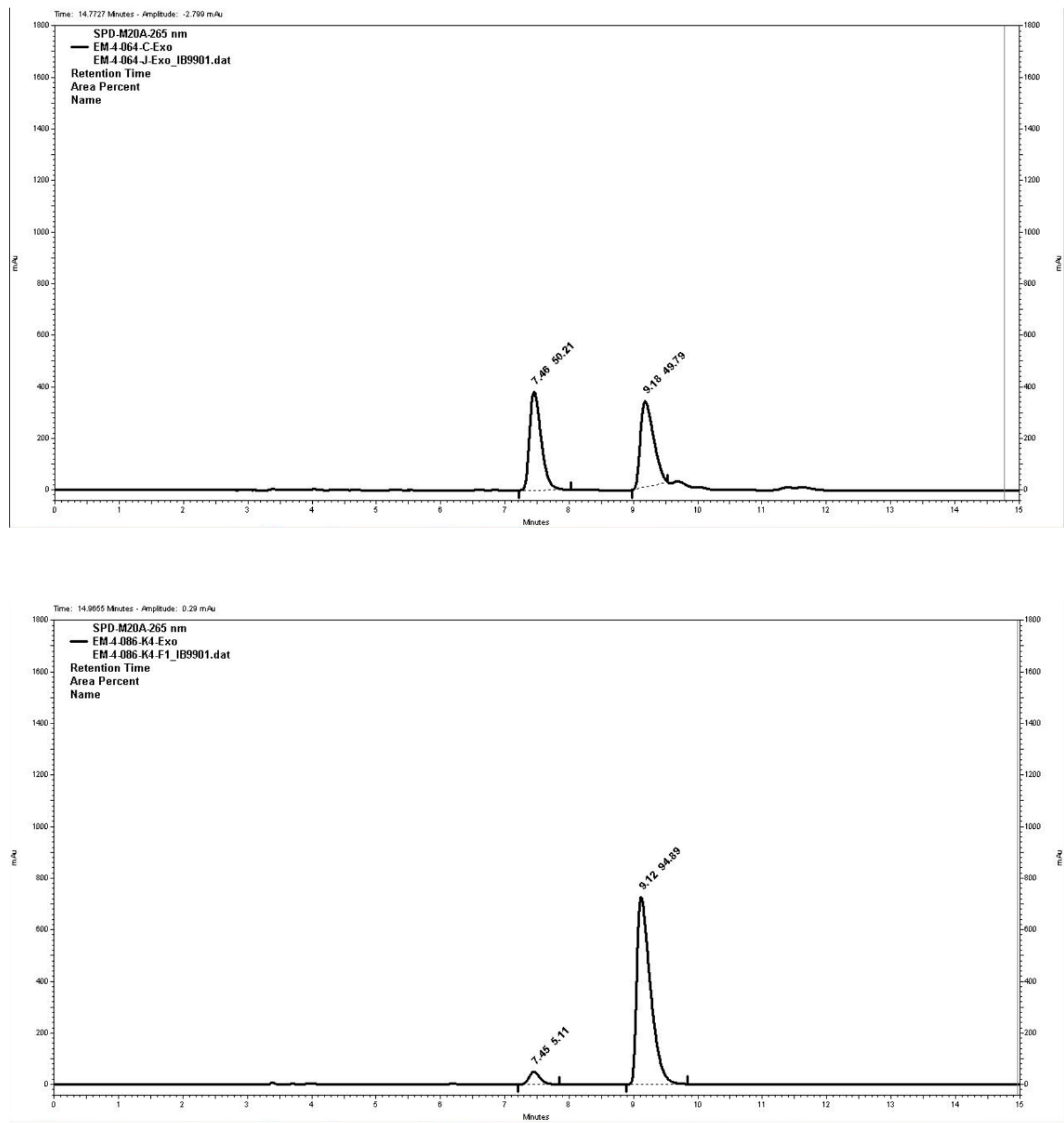
2h: IB 99:01 hexanes/isopropanol, $1 \mathrm{ml} / \mathrm{min}, \mathrm{t}_{\mathrm{m}}=8.7 \mathrm{~min}, \mathrm{t}_{\mathrm{M}}=9.4 \mathrm{~min}$.
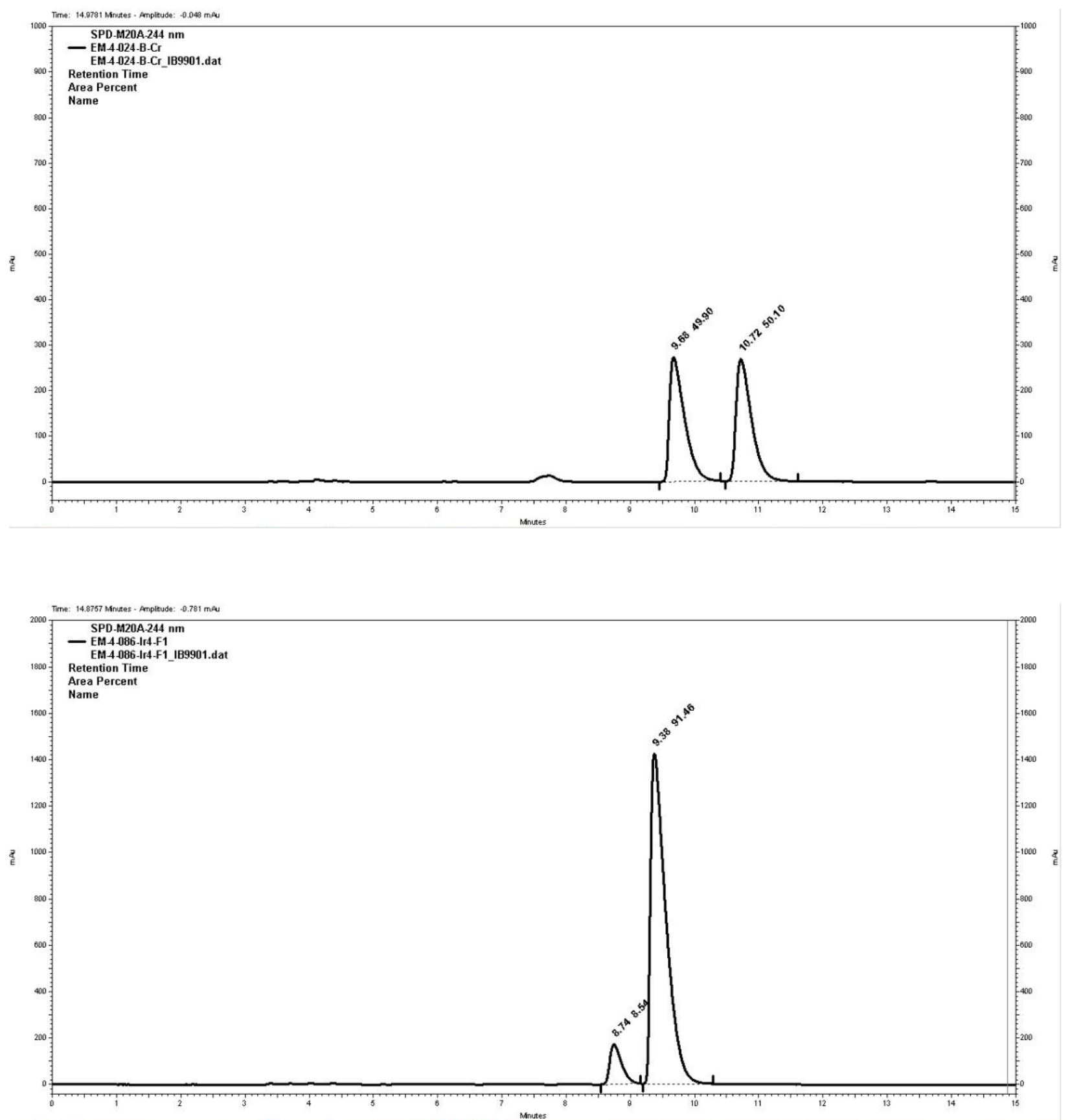
2i: IA 90:10 hexanes/isopropanol, $1 \mathrm{ml} / \mathrm{min}, \mathrm{t}_{\mathrm{m}}=16.3 \mathrm{~min}, \mathrm{t}_{\mathrm{M}}=20.3 \mathrm{~min}$.
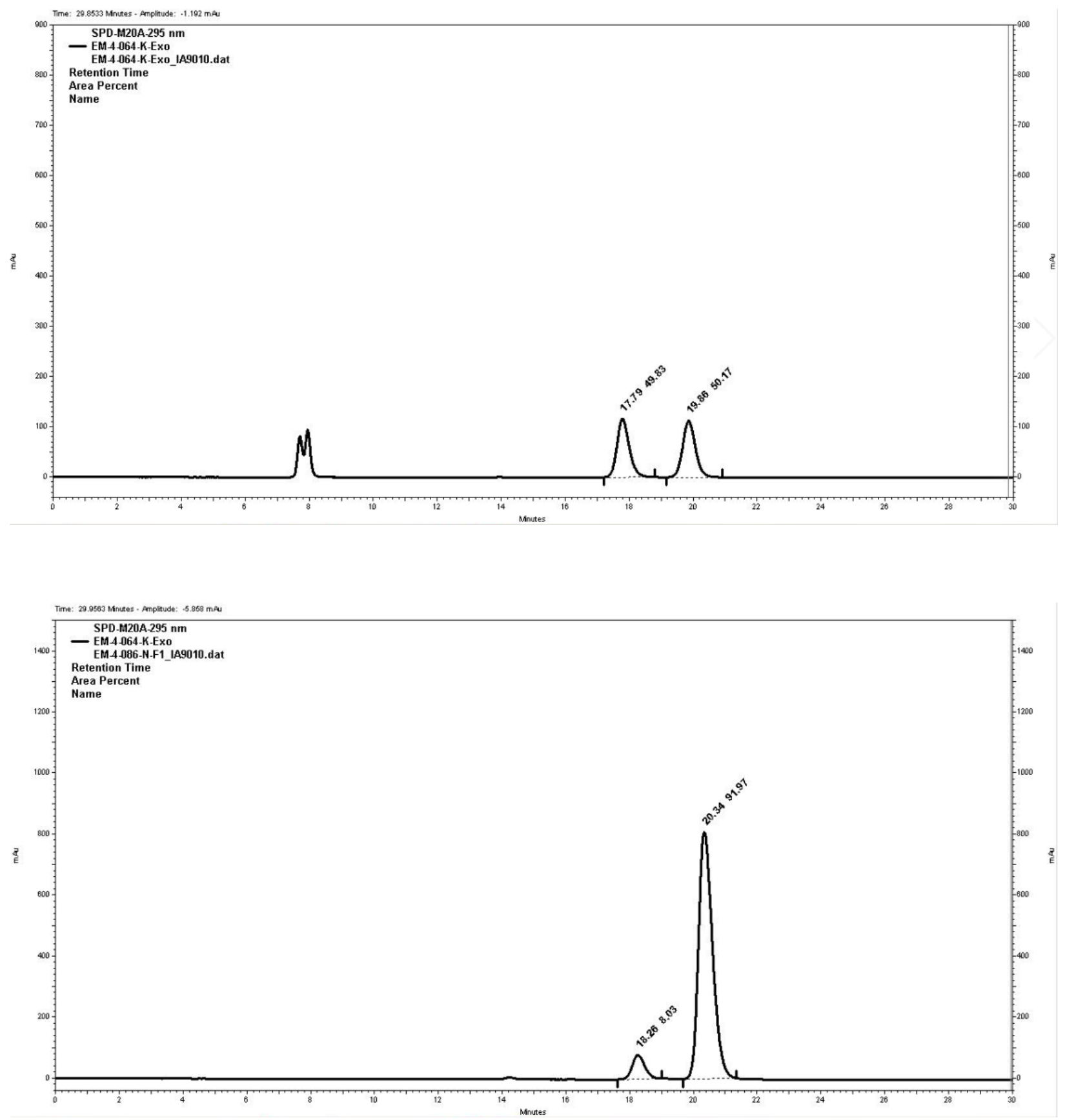
2j: IB 99:01 hexanes/isopropanol, $1 \mathrm{ml} / \mathrm{min}, \mathrm{t}_{\mathrm{m}}=7.8 \mathrm{~min}, \mathrm{t}_{\mathrm{M}}=6.0 \mathrm{~min}$.
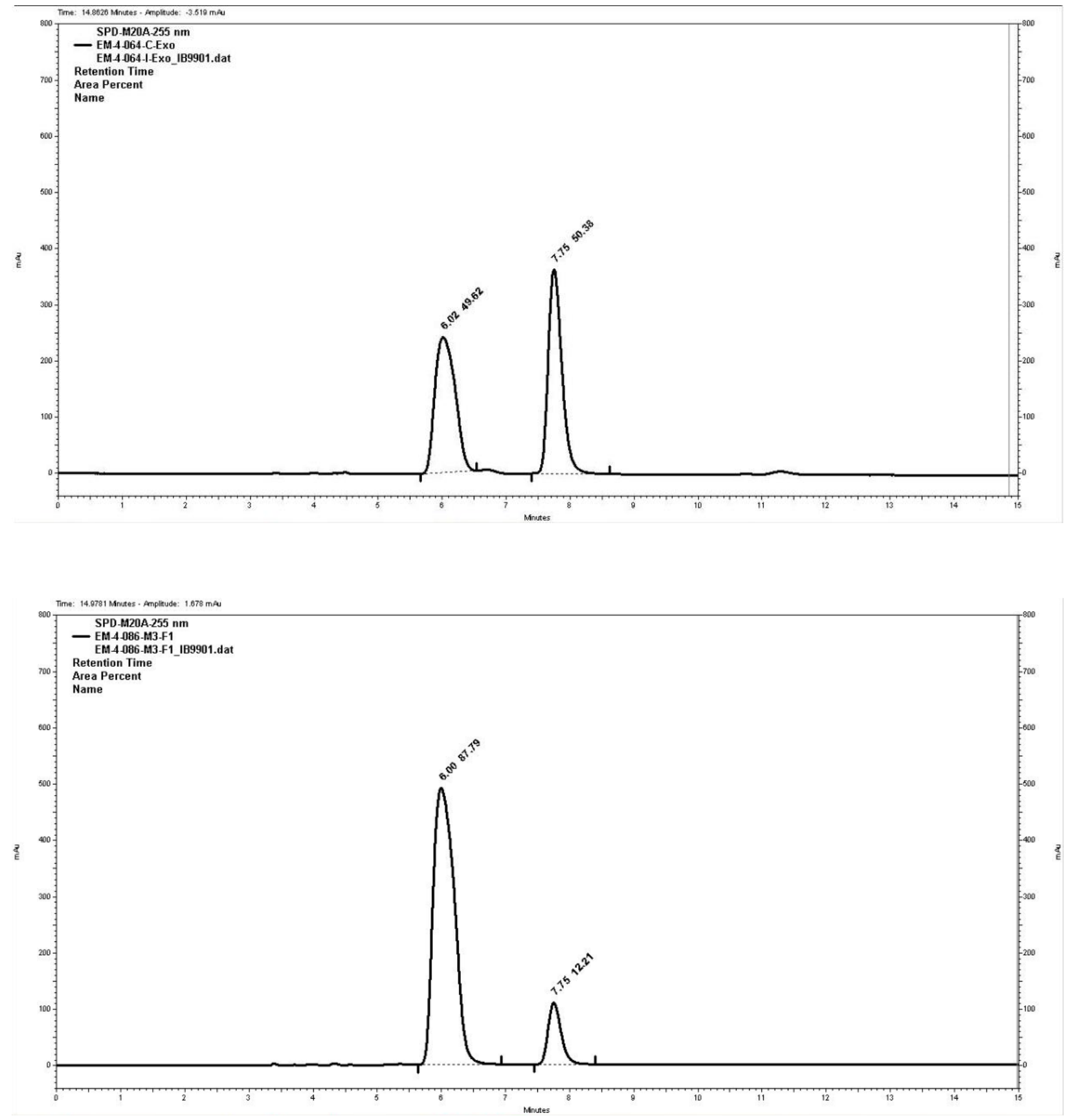
2k: IC 95:05 hexanes/isopropanol, $1 \mathrm{ml} / \mathrm{min}, \mathrm{t}_{\mathrm{m}}=12.2 \mathrm{~min}, \mathrm{t}_{\mathrm{M}}=15.1 \mathrm{~min}$.
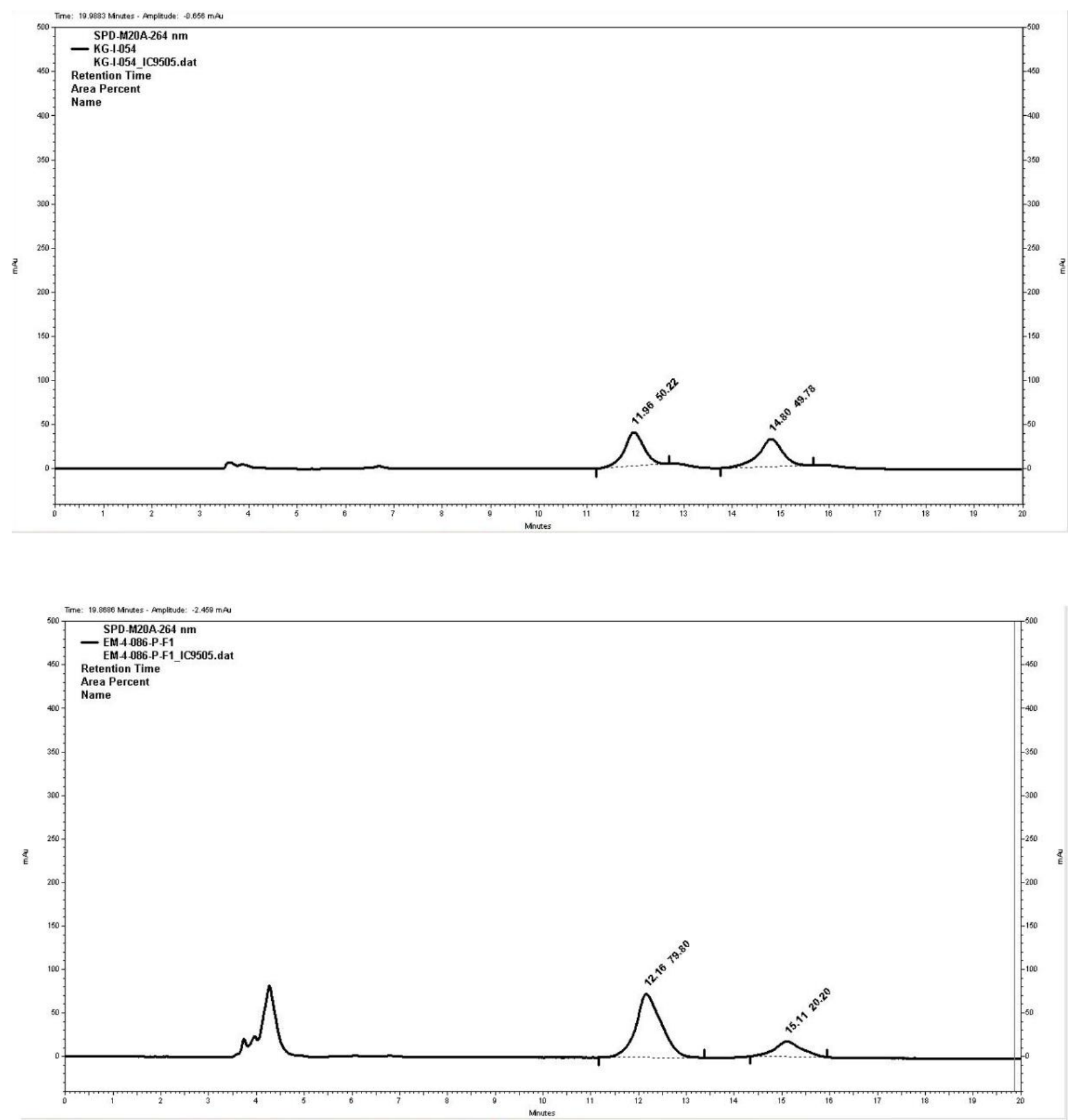
2l·H $\mathbf{H}_{2}$ O: IA 95:05 hexanes/isopropanol, $1 \mathrm{ml} / \mathrm{min}, \mathrm{t}_{\mathrm{m}}=19.6 \mathrm{~min}, \mathrm{t}_{\mathrm{M}}=27.0 \mathrm{~min}$.
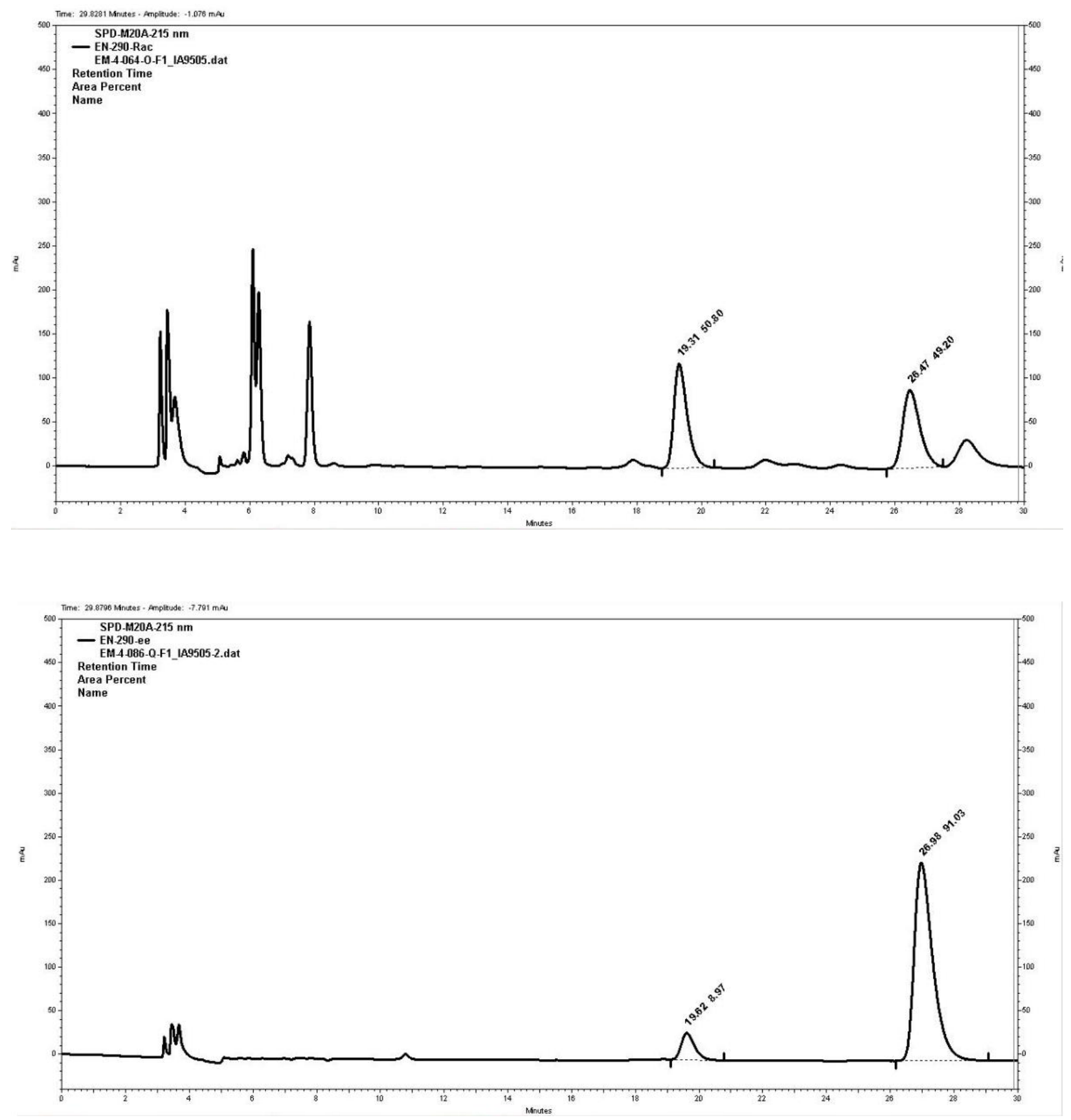
4a: IB 99.5:0.5 hexanes/isopropanol, $1 \mathrm{ml} / \mathrm{min}, \mathrm{t}_{\mathrm{m}}=6.2 \mathrm{~min}, \mathrm{t}_{\mathrm{M}}=6.5 \mathrm{~min}$.
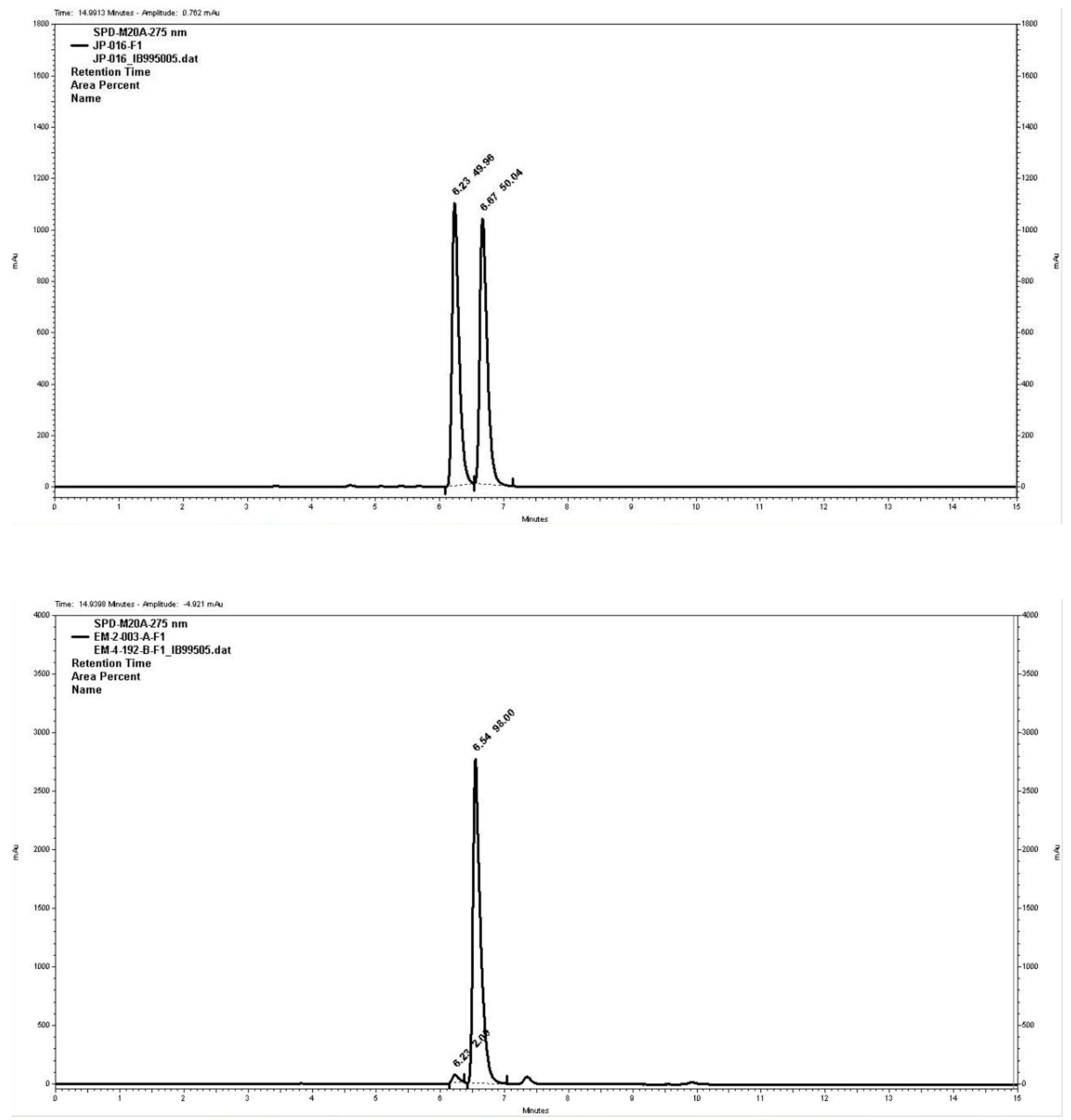
4b: IB 99.9:0.1 hexanes/isopropanol, $1 \mathrm{ml} / \mathrm{min}, \mathrm{t}_{\mathrm{m}}=7.0 \mathrm{~min}, \mathrm{t}_{\mathrm{M}}=8.1 \mathrm{~min}$.
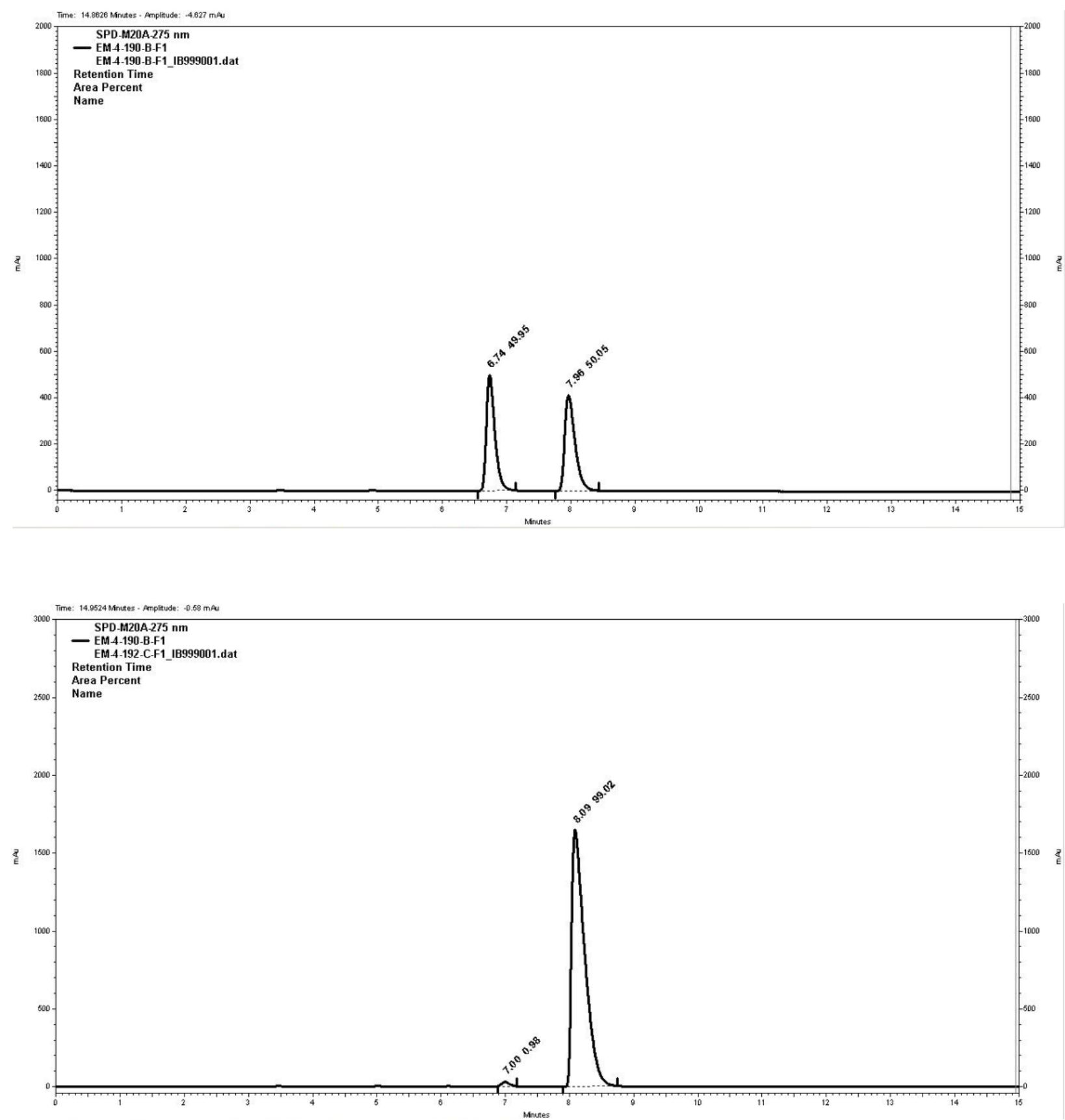
4c: $\mathrm{OJ} 100 \%$ hexanes, $1 \mathrm{ml} / \mathrm{min}, \mathrm{t}_{\mathrm{m}}=9.0 \mathrm{~min}, \mathrm{t}_{\mathrm{M}}=7.4 \mathrm{~min}$.
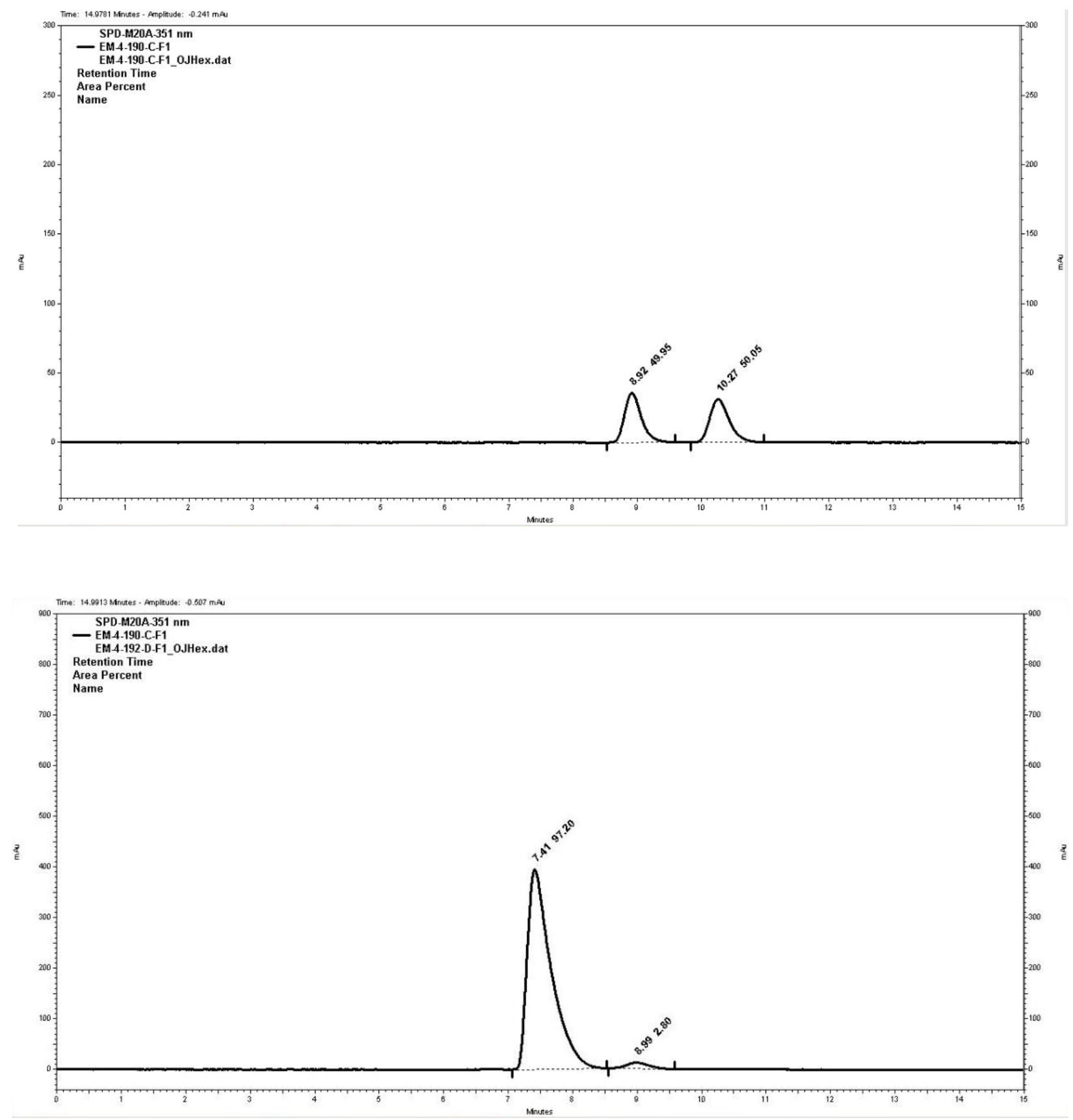
4d: IB 99.5:0.5 hexanes $/ \mathrm{THF}, 1 \mathrm{ml} / \mathrm{min}, \mathrm{t}_{\mathrm{m}}=17.9 \mathrm{~min}, \mathrm{t}_{\mathrm{M}}=16.5 \mathrm{~min}$.
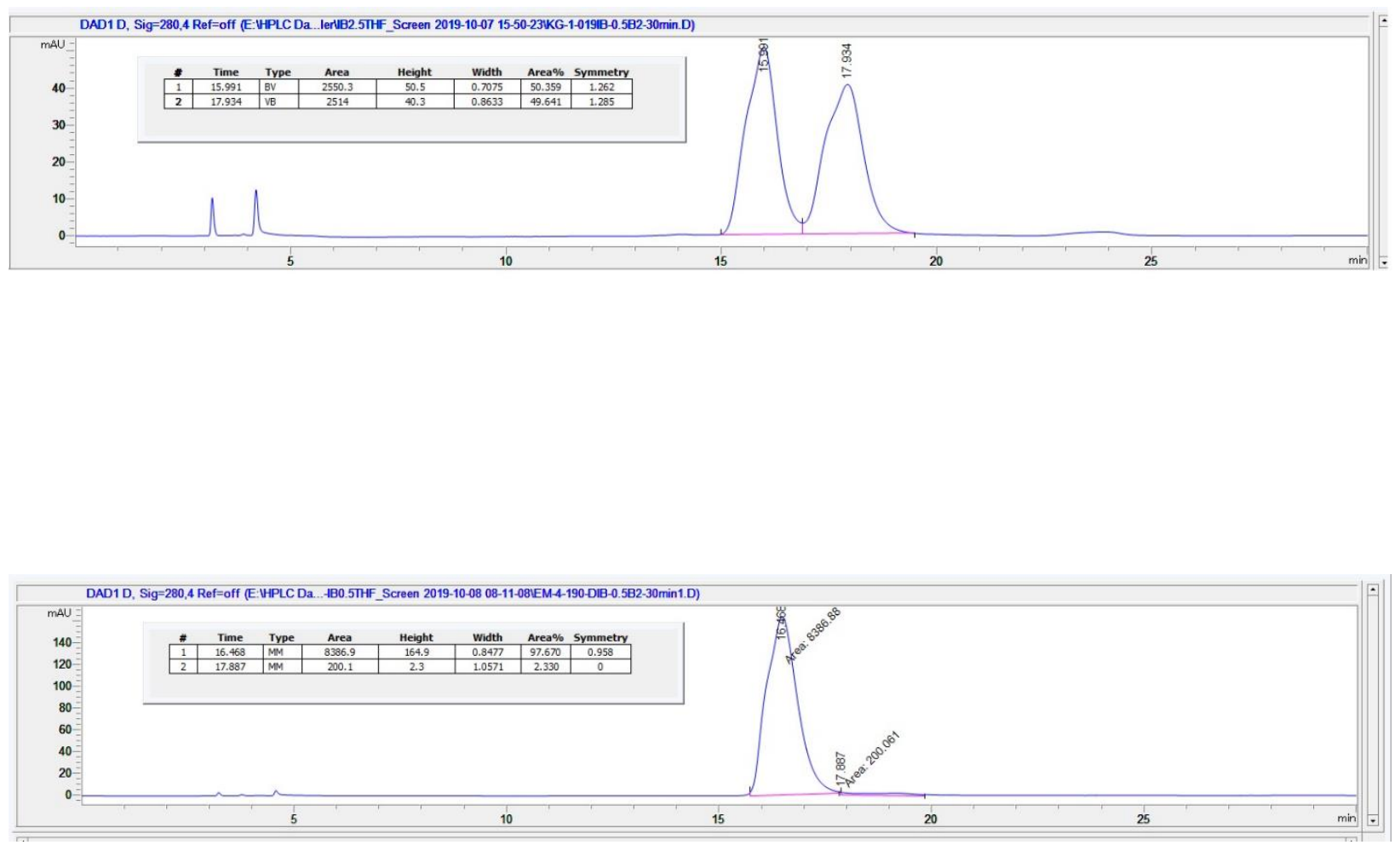
4e: IC 99.9:0.1 hexanes/isopropanol, $1 \mathrm{ml} / \mathrm{min}, \mathrm{t}_{\mathrm{m}}=8.9 \mathrm{~min}, \mathrm{t}_{\mathrm{M}}=5.4 \mathrm{~min}$.
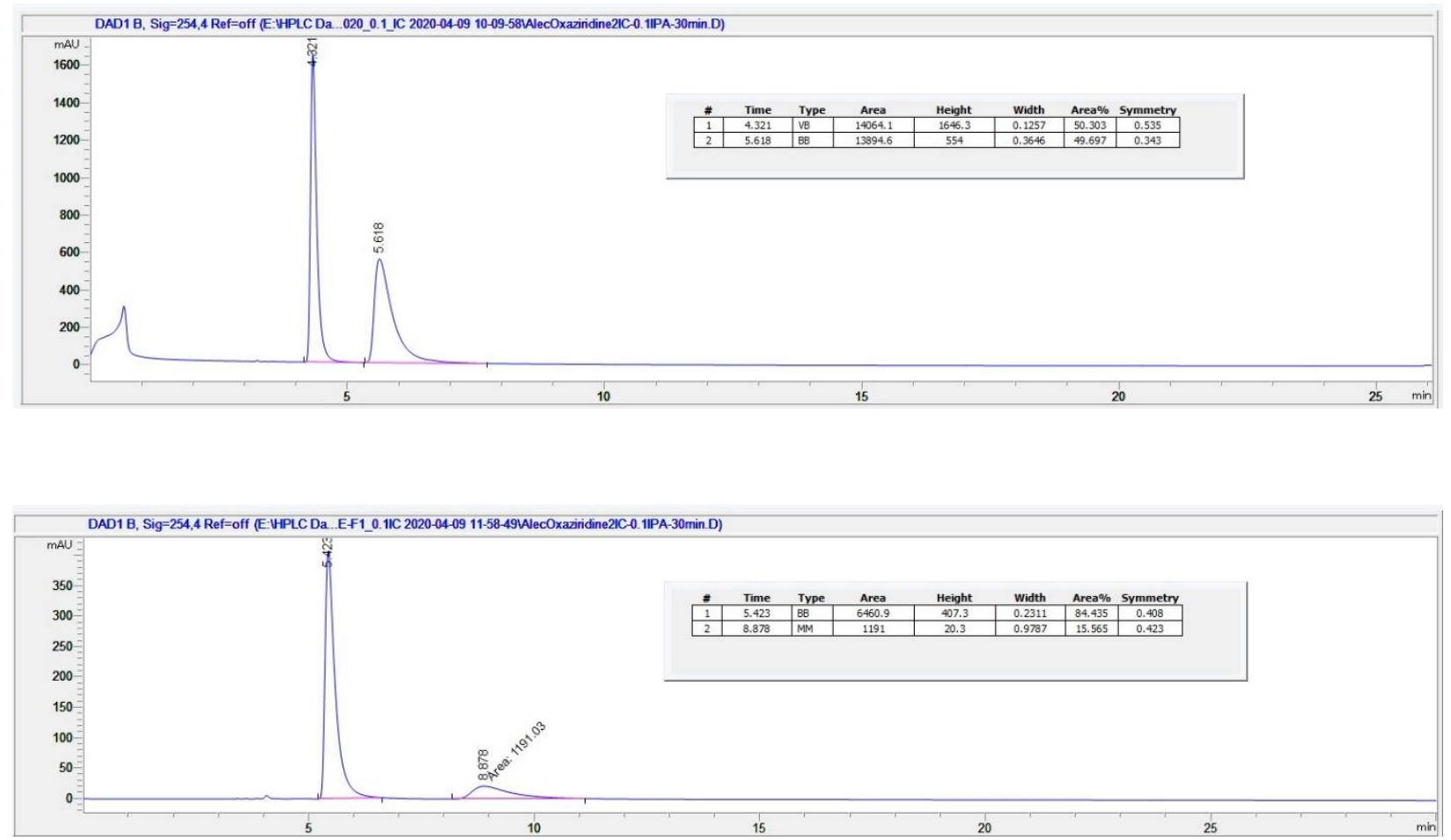
4f: IB 99.9:0.1 hexanes/isopropanol, $0.5 \mathrm{ml} / \mathrm{min}, \mathrm{t}_{\mathrm{m}}=14.6 \mathrm{~min}, \mathrm{t}_{\mathrm{M}}=13.6 \mathrm{~min}$.
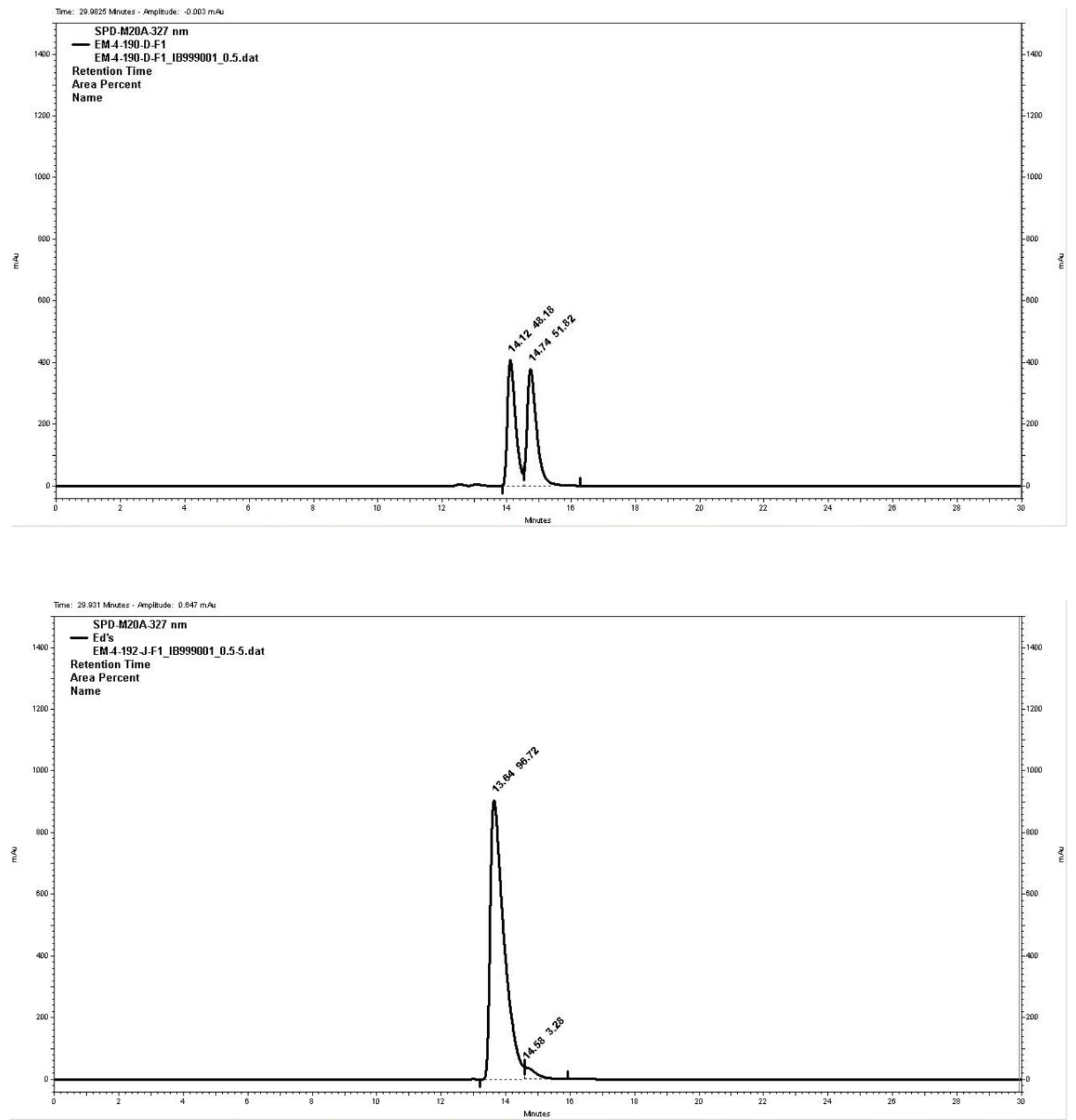
4g: IC $100 \%$ hexanes, $1 \mathrm{ml} / \mathrm{min}, \mathrm{t}_{\mathrm{m}}=18.5 \mathrm{~min}, \mathrm{t}_{\mathrm{M}}=14.2 \mathrm{~min}$.
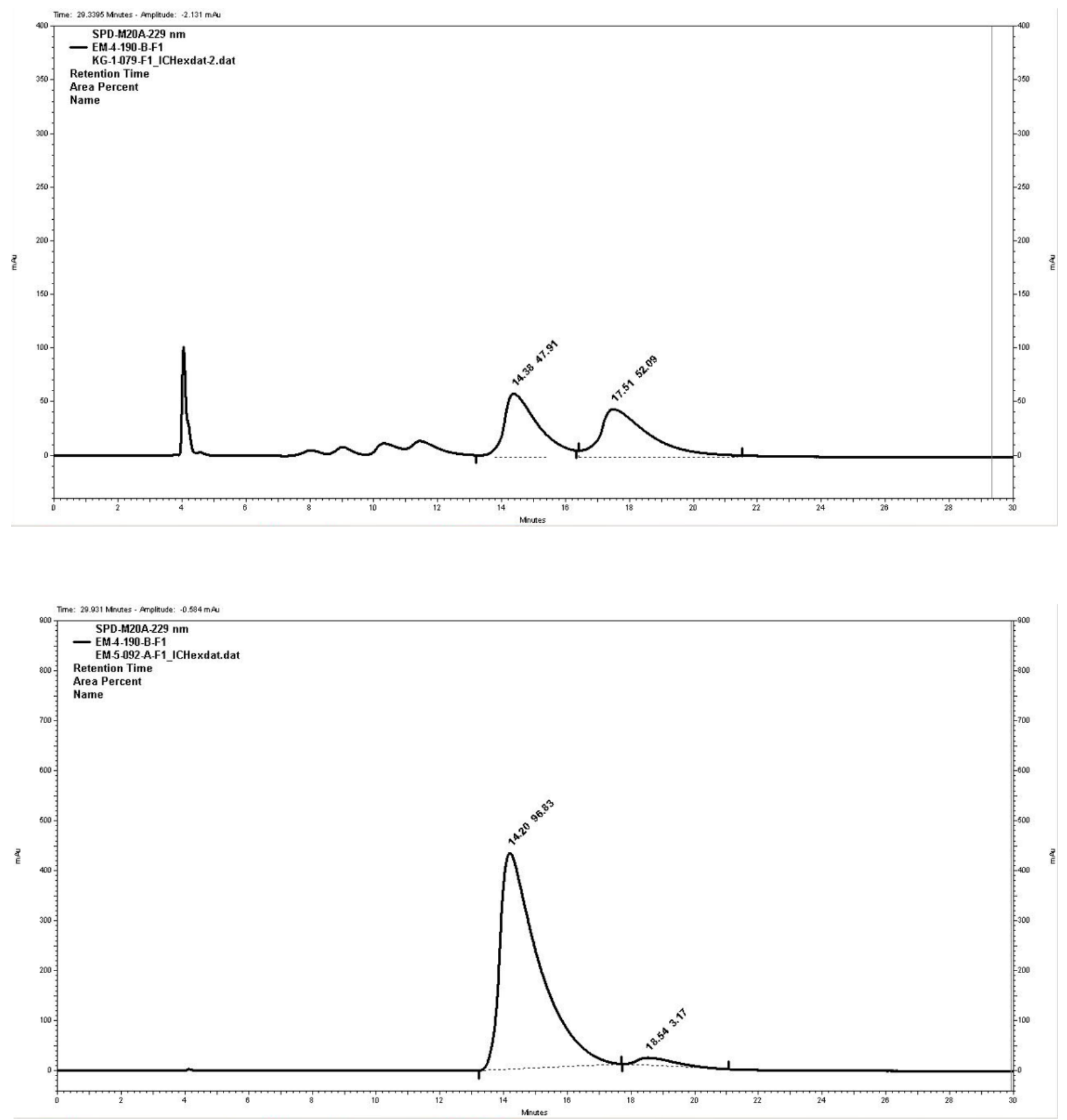
4h: IB 99.9:0.1 hexanes/isopropanol, $1 \mathrm{ml} / \mathrm{min}, \mathrm{t}_{\mathrm{m}}=6.0 \mathrm{~min}, \mathrm{t}_{\mathrm{M}}=5.9 \mathrm{~min}$.
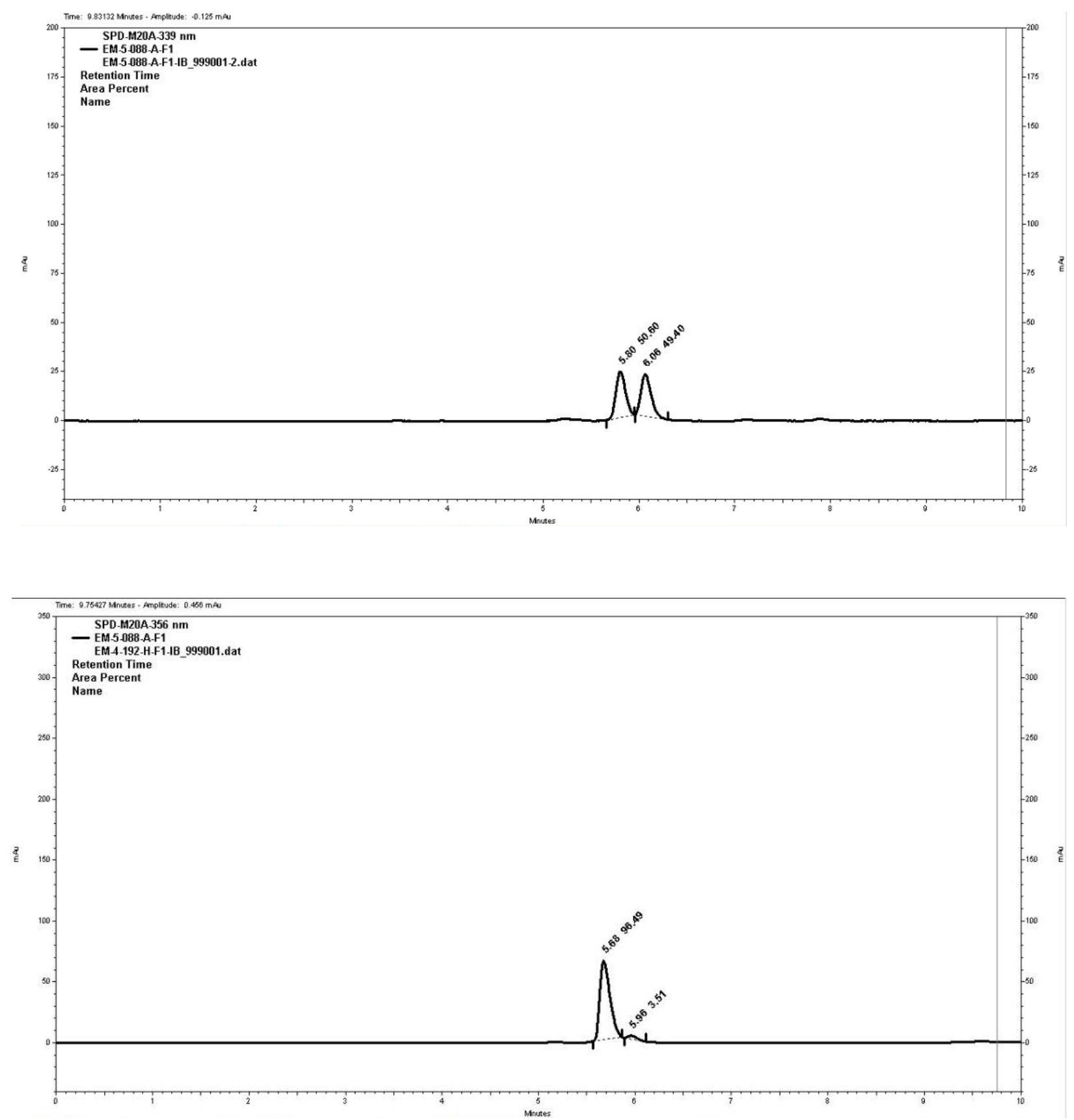
4i: IB $100 \%$ hexanes, $1 \mathrm{ml} / \mathrm{min}, \mathrm{t}_{\mathrm{m}}=7.3 \mathrm{~min}, \mathrm{t}_{\mathrm{M}}=6.5 \mathrm{~min}$.
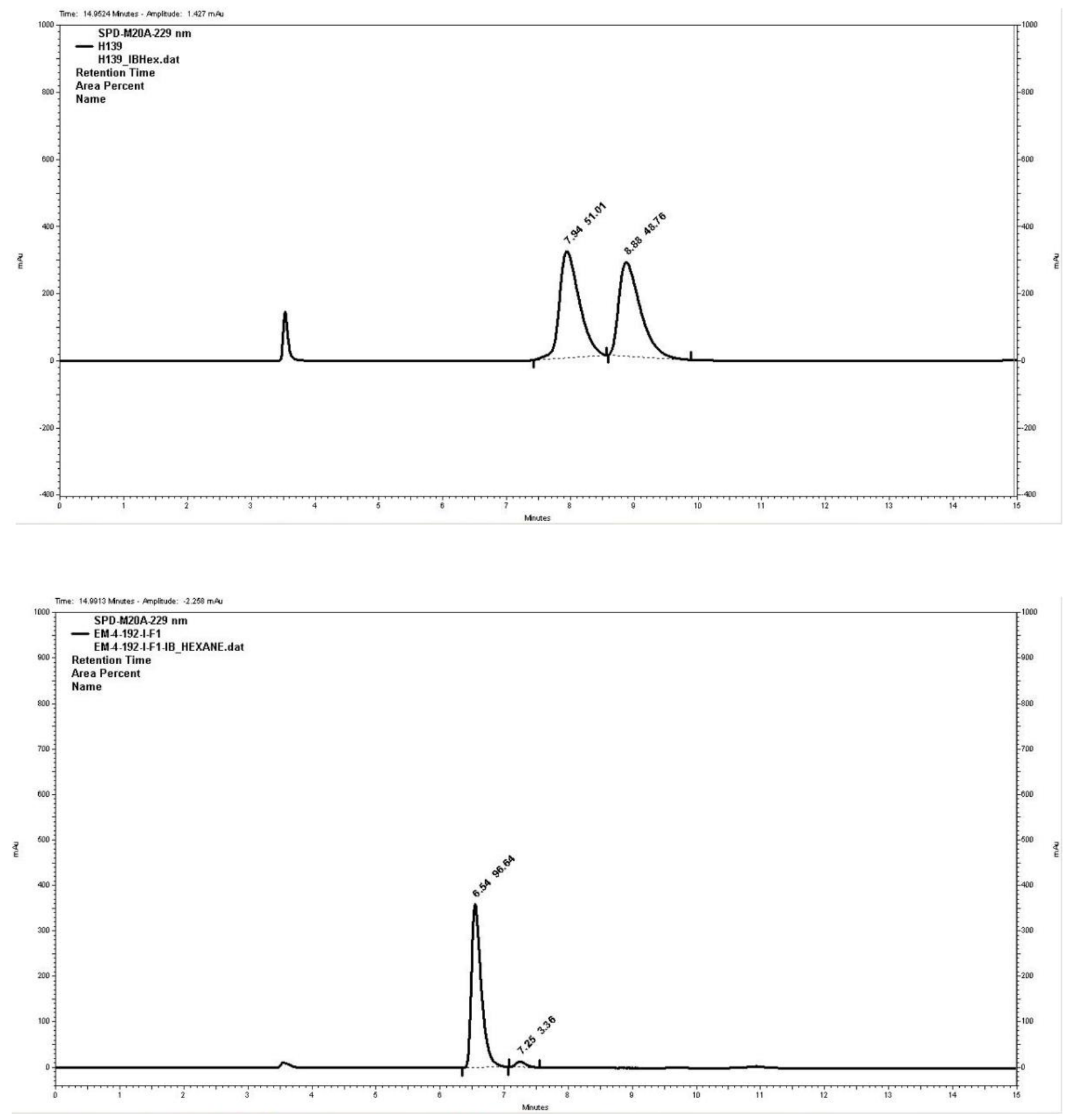
4j: IB 99:01 hexanes/isopropanol, $1 \mathrm{ml} / \mathrm{min}, \mathrm{t}_{\mathrm{m}}=10.3 \mathrm{~min}, \mathrm{t}_{\mathrm{M}}=11.4 \mathrm{~min}$.
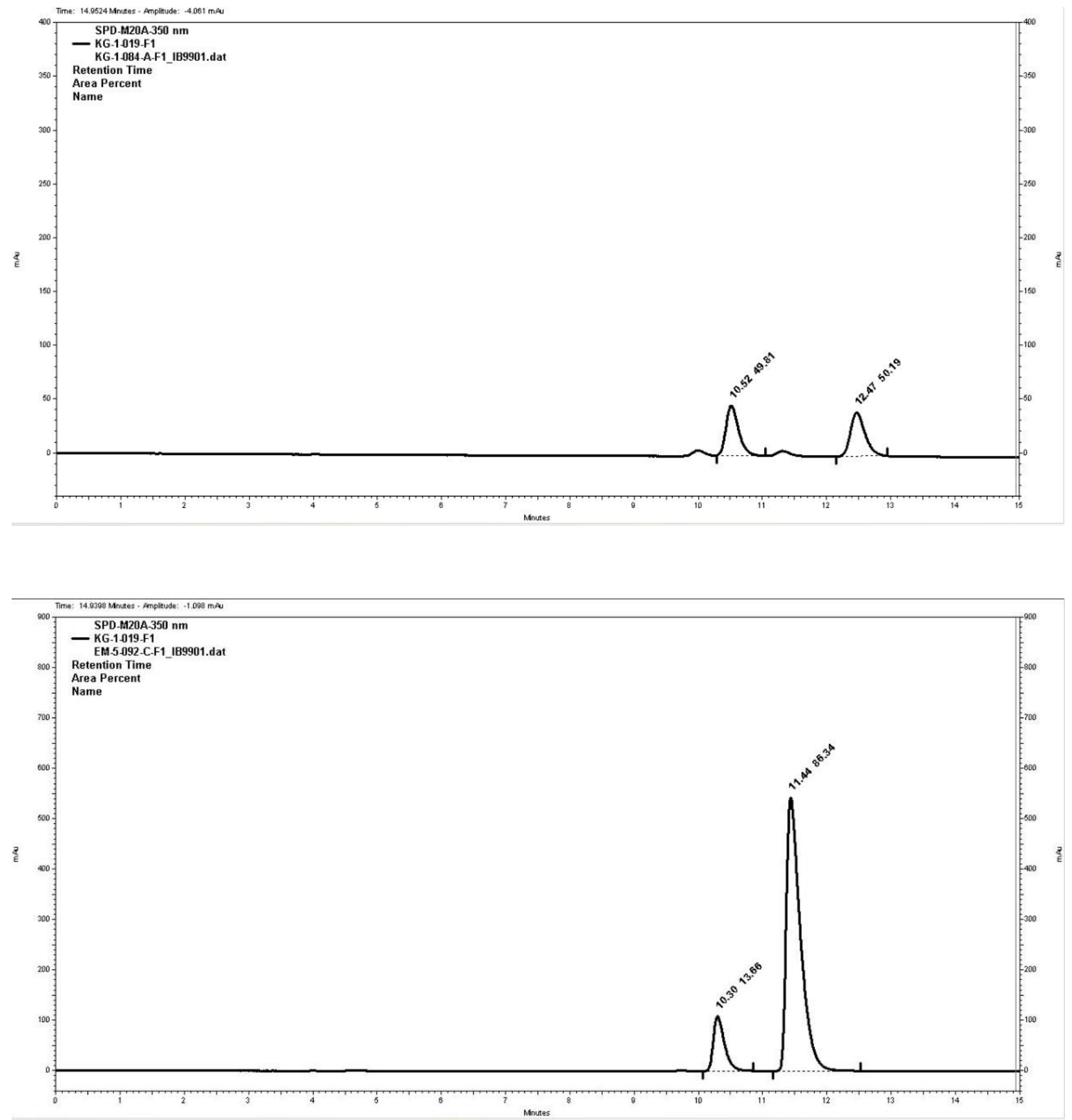
4k: IC 95:05 hexanes/isopropanol, $1 \mathrm{ml} / \mathrm{min}, \mathrm{t}_{\mathrm{m}}=28.8 \mathrm{~min}, \mathrm{t}_{\mathrm{M}}=33.0 \mathrm{~min}$.
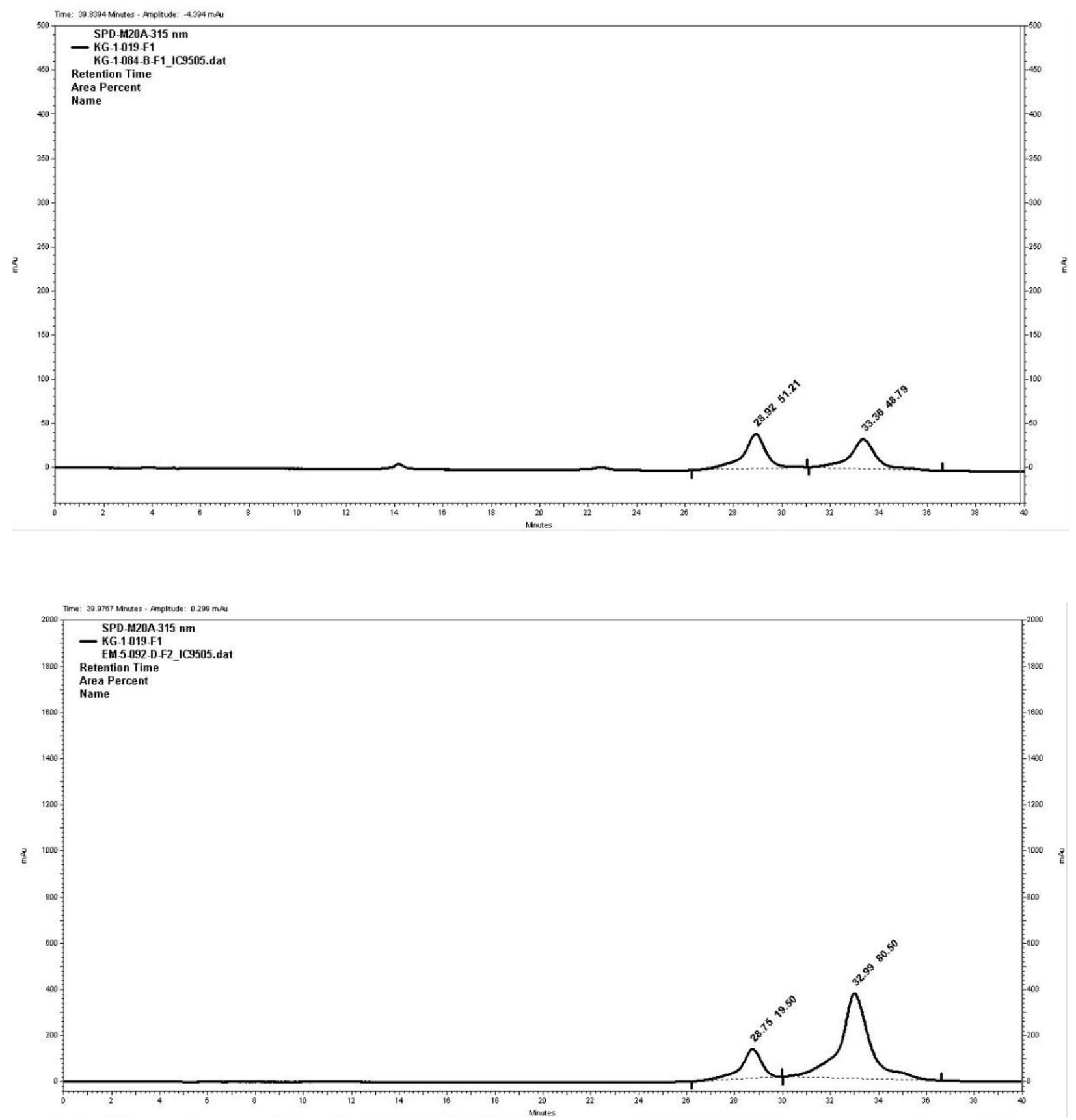
41: IA 95:05 hexanes/THF, $1 \mathrm{ml} / \mathrm{min}, \mathrm{t}_{\mathrm{m}}=6.6 \mathrm{~min}, \mathrm{t}_{\mathrm{M}}=6.1 \mathrm{~min}$.
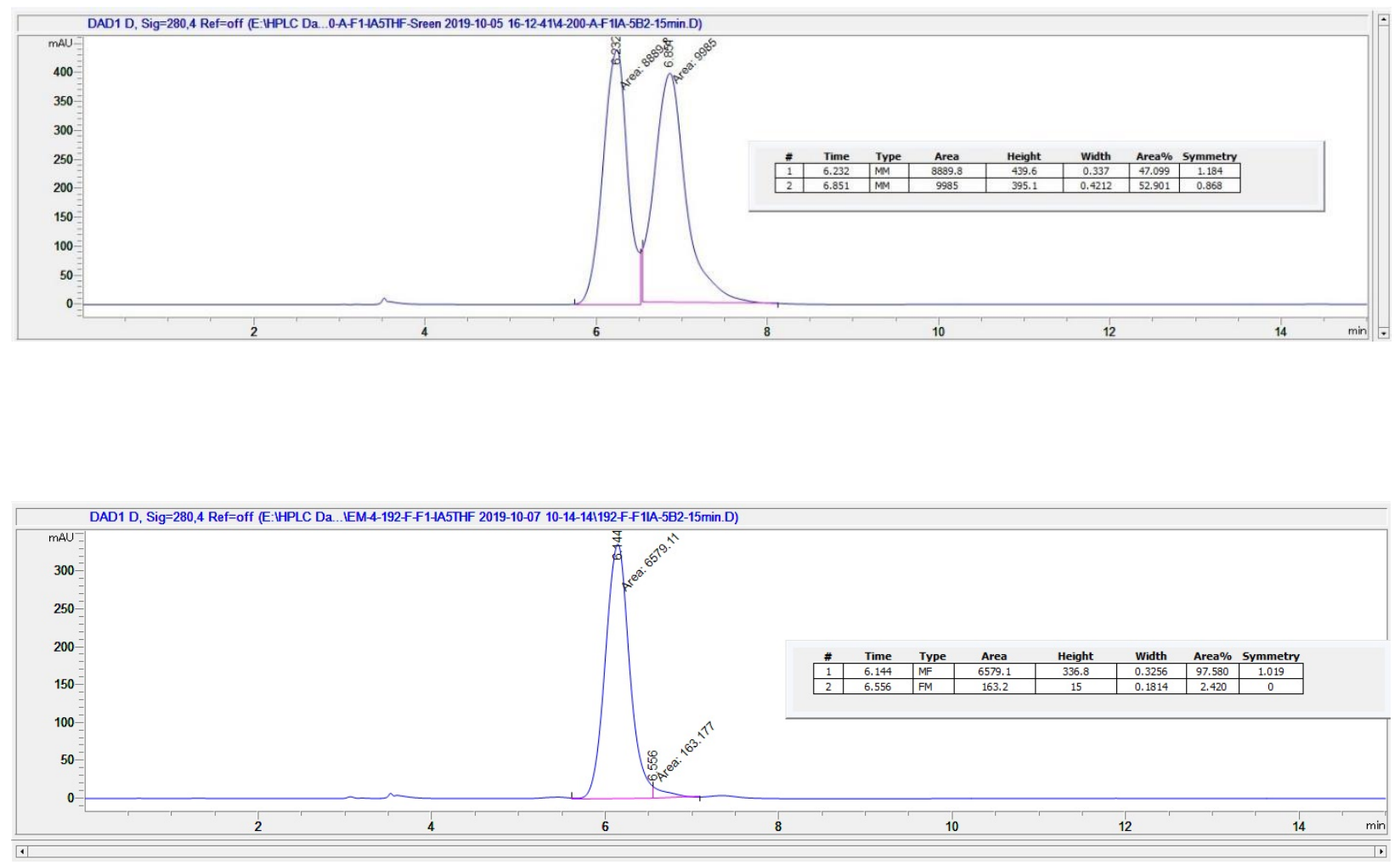
4m: IA 99:01 hexanes/isopropanol, $1 \mathrm{ml} / \mathrm{min}, \mathrm{t}_{\mathrm{m}}=6.9 \mathrm{~min}, \mathrm{t}_{\mathrm{M}}=7.8 \mathrm{~min}$.
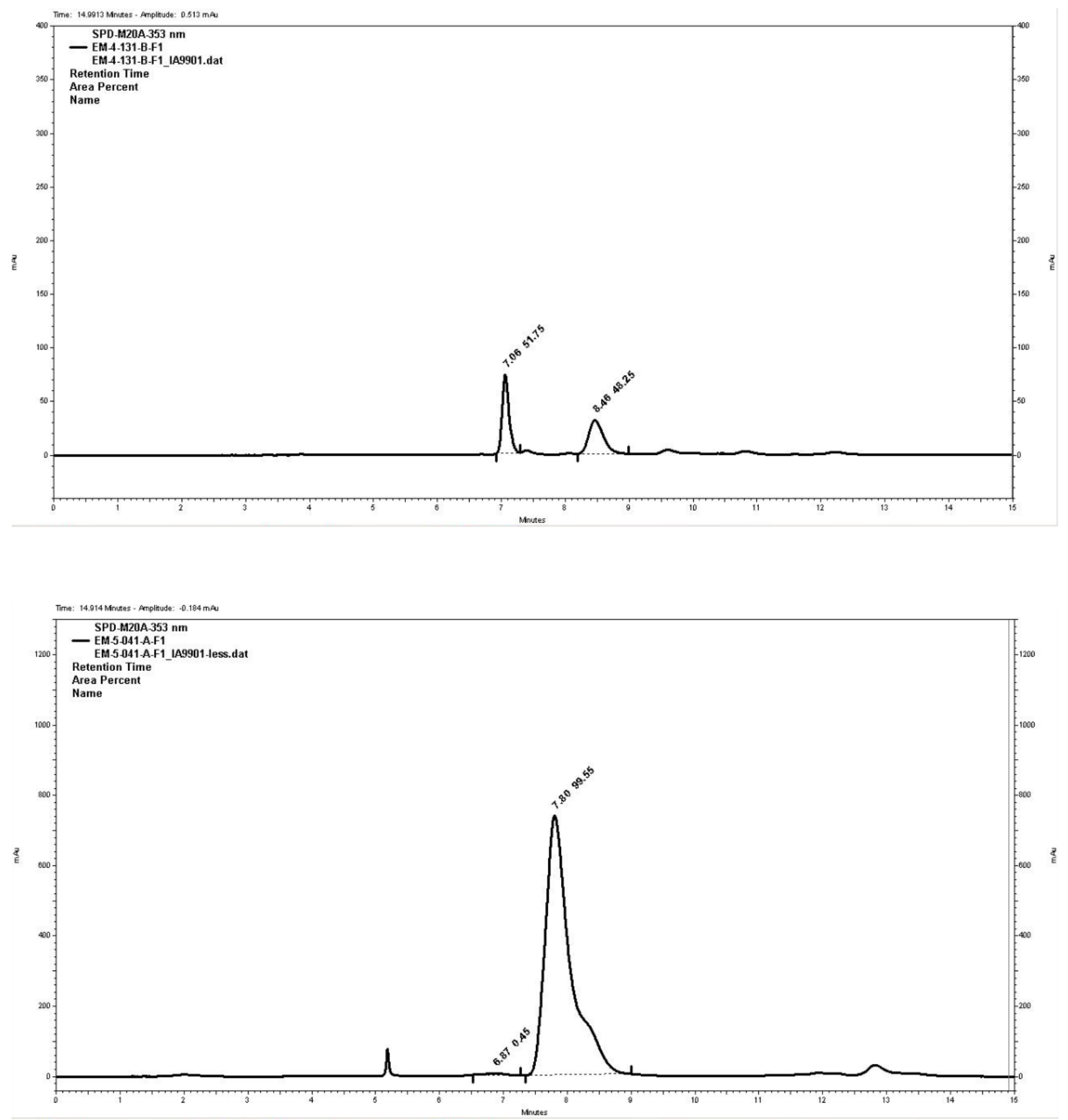
BDF-efavirenz: IB 90:10 hexanes/isopropanol, $1 \mathrm{ml} / \mathrm{min}, \mathrm{t}_{\mathrm{m}}=7.7 \mathrm{~min}, \mathrm{t}_{\mathrm{M}}=8.2 \mathrm{~min}$.
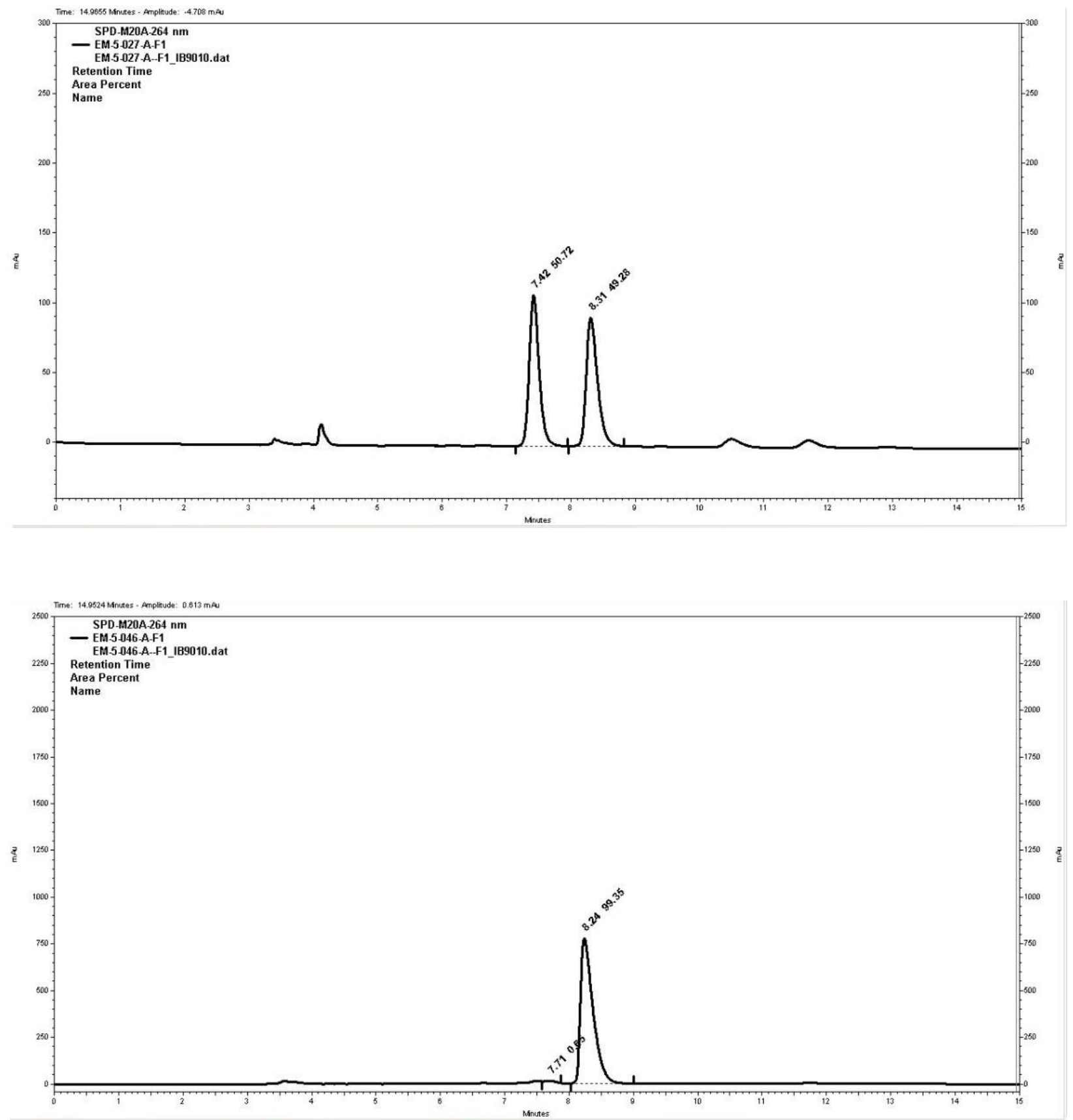
6a: IA 99:01 hexanes/isopropanol, $1 \mathrm{ml} / \mathrm{min}, \mathrm{t}_{\mathrm{m}}=23.6 \mathrm{~min}, \mathrm{t}_{\mathrm{M}}=26.4 \mathrm{~min}$.
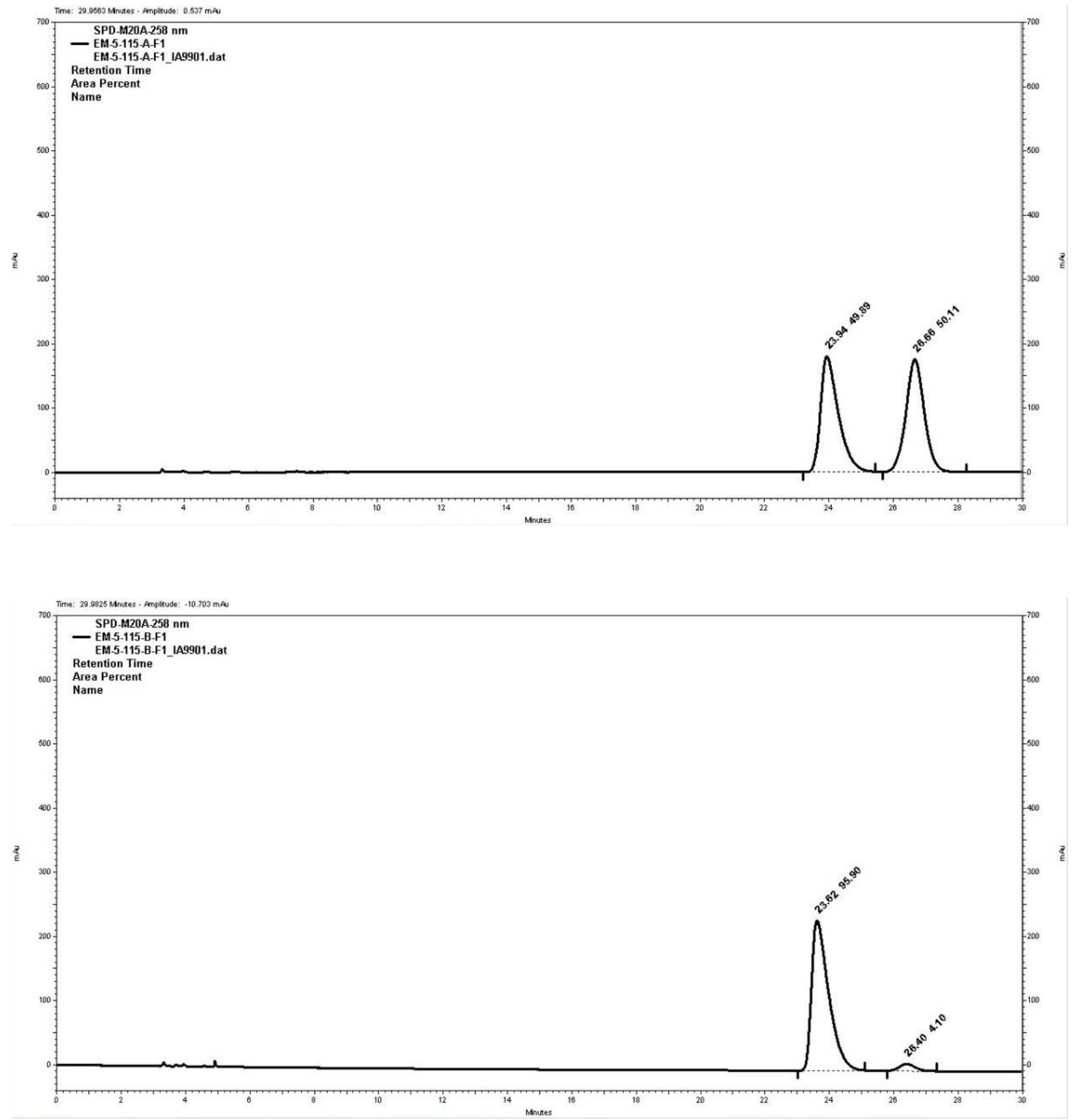
6b: IC 85:15 hexanes/isopropanol, $1 \mathrm{ml} / \mathrm{min}, \mathrm{t}_{\mathrm{m}}=7.9 \mathrm{~min}, \mathrm{t}_{\mathrm{M}}=9.7 \mathrm{~min}$.
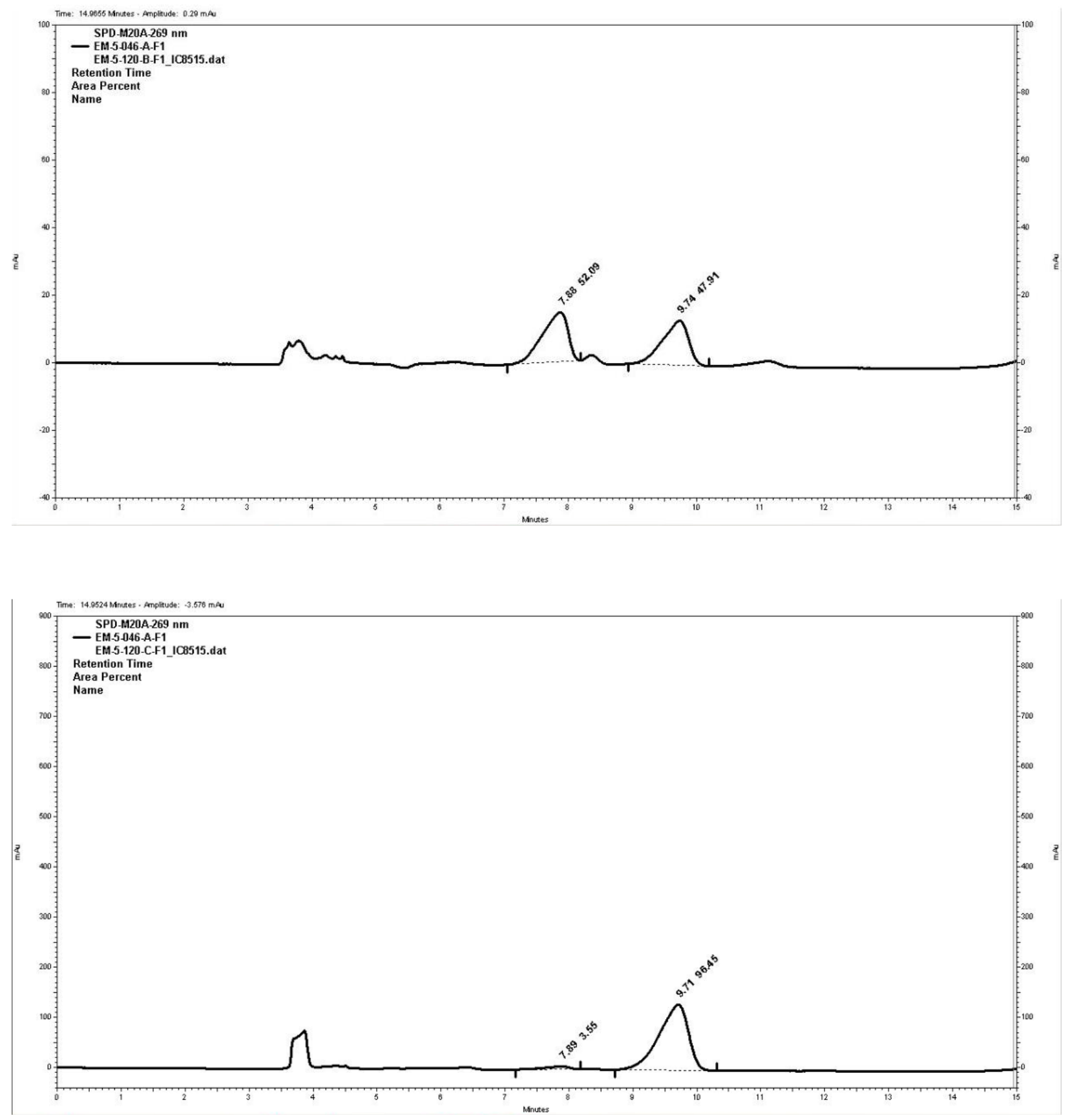
6c: IC 85:15 hexanes/isopropanol, $1 \mathrm{ml} / \mathrm{min}, \mathrm{t}_{\mathrm{m}}=12.3 \mathrm{~min}, \mathrm{t}_{\mathrm{M}}=17.0 \mathrm{~min}$.
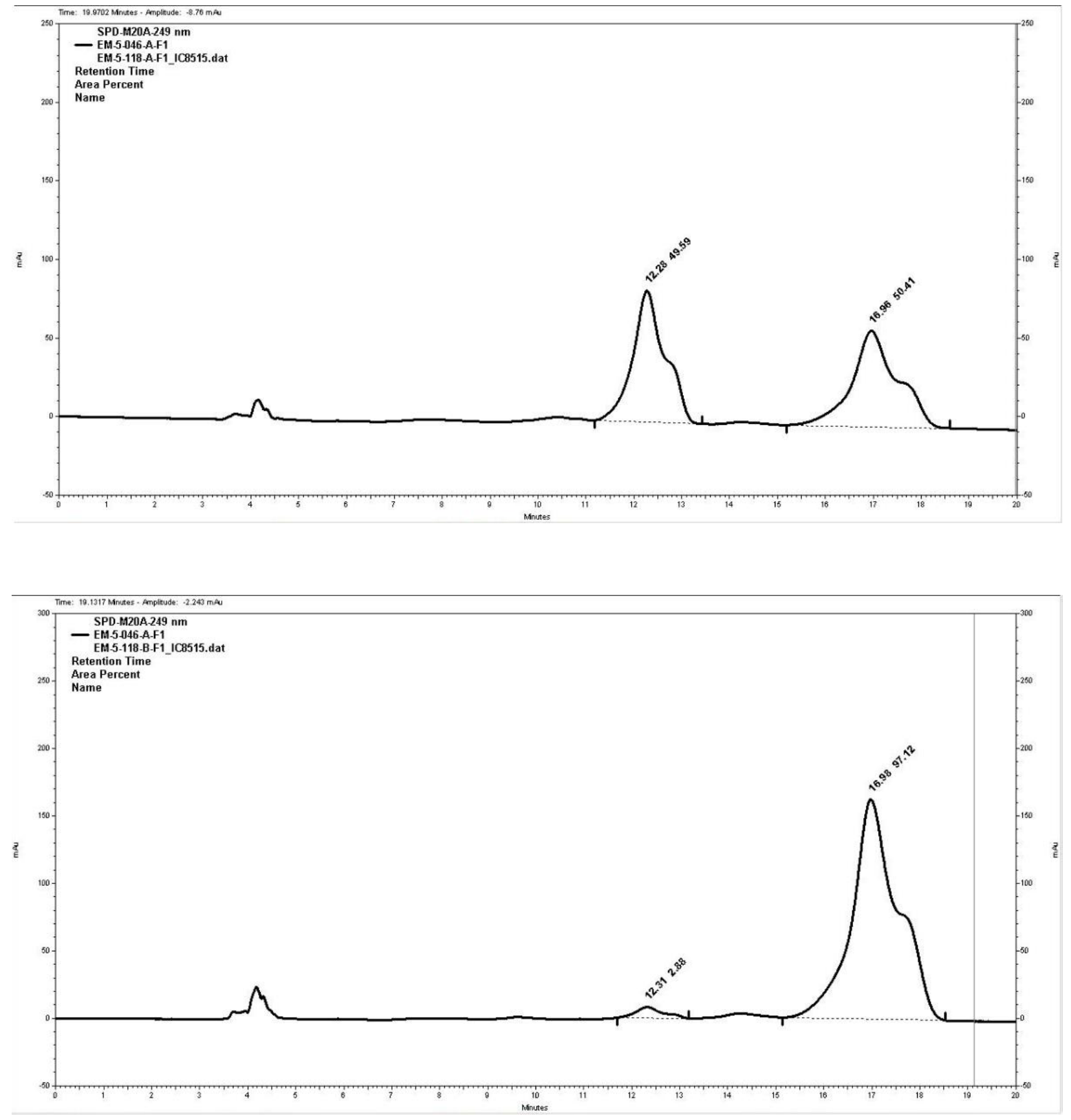
6d: IA 95:05 hexanes/isopropanol, $1 \mathrm{ml} / \mathrm{min}, \mathrm{t}_{\mathrm{m}}=26.9 \mathrm{~min}, \mathrm{t}_{\mathrm{M}}=20.4 \mathrm{~min}$.
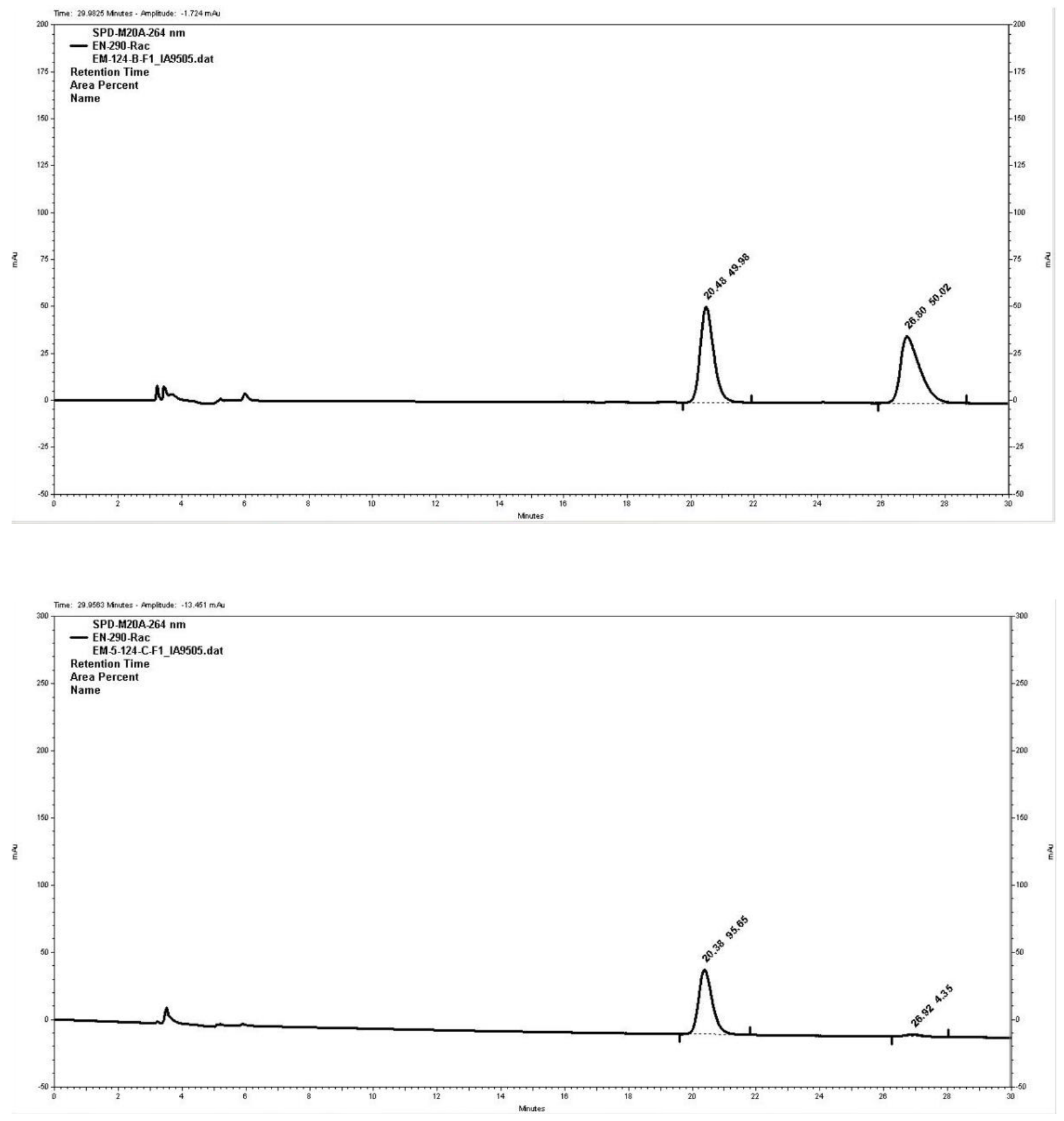
7a: IA 99.9:0.1 hexanes/isopropanol, $1 \mathrm{ml} / \mathrm{min}, \mathrm{t}_{\mathrm{m}}=8.5 \mathrm{~min}, \mathrm{t}_{\mathrm{M}}=9.1 \mathrm{~min}$.
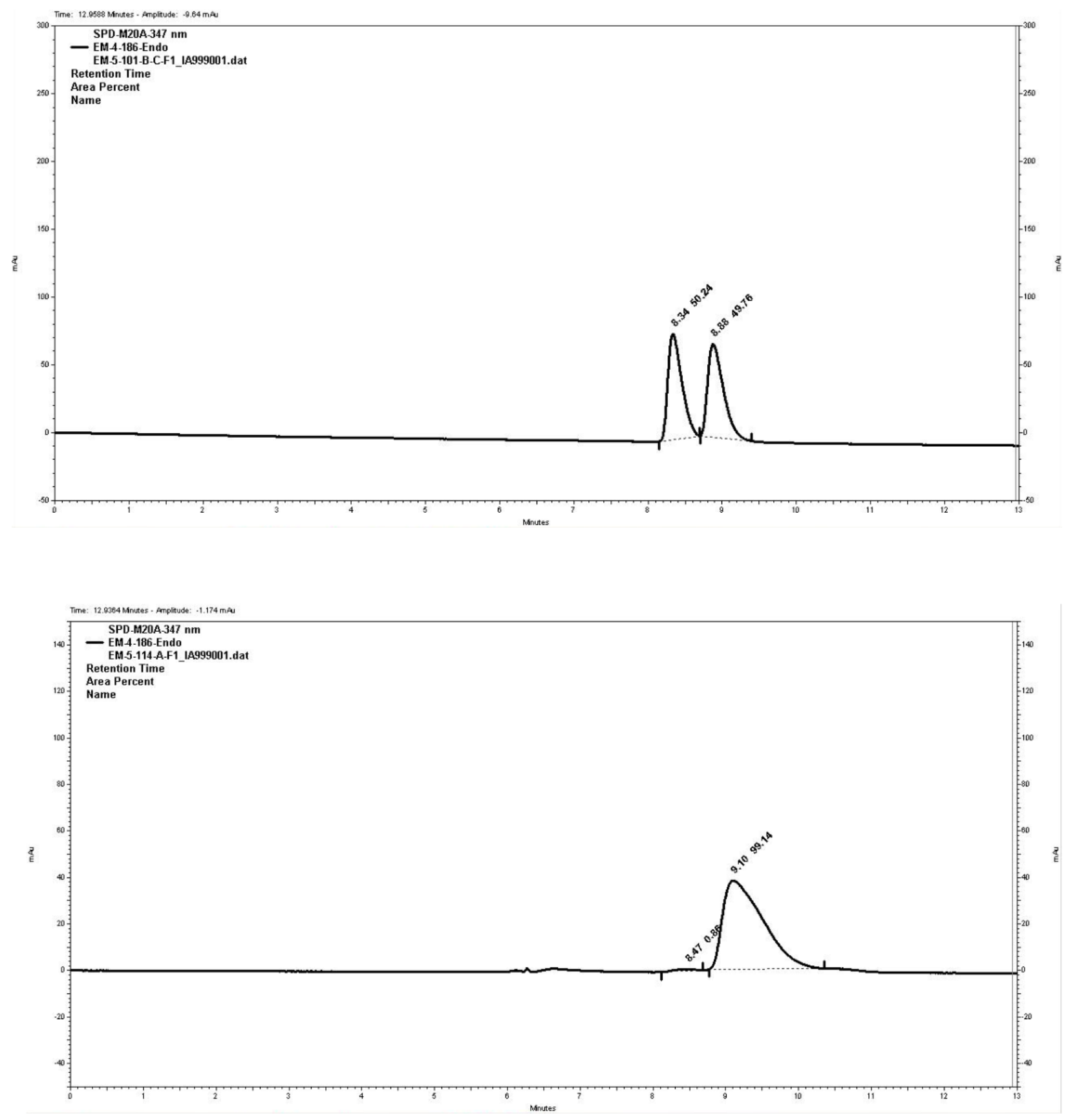
7b: IC 99.5:0.5 hexanes/isopropanol, $1 \mathrm{ml} / \mathrm{min}, \mathrm{t}_{\mathrm{m}}=7.1 \mathrm{~min}, \mathrm{t}_{\mathrm{M}}=5.3 \mathrm{~min}$.
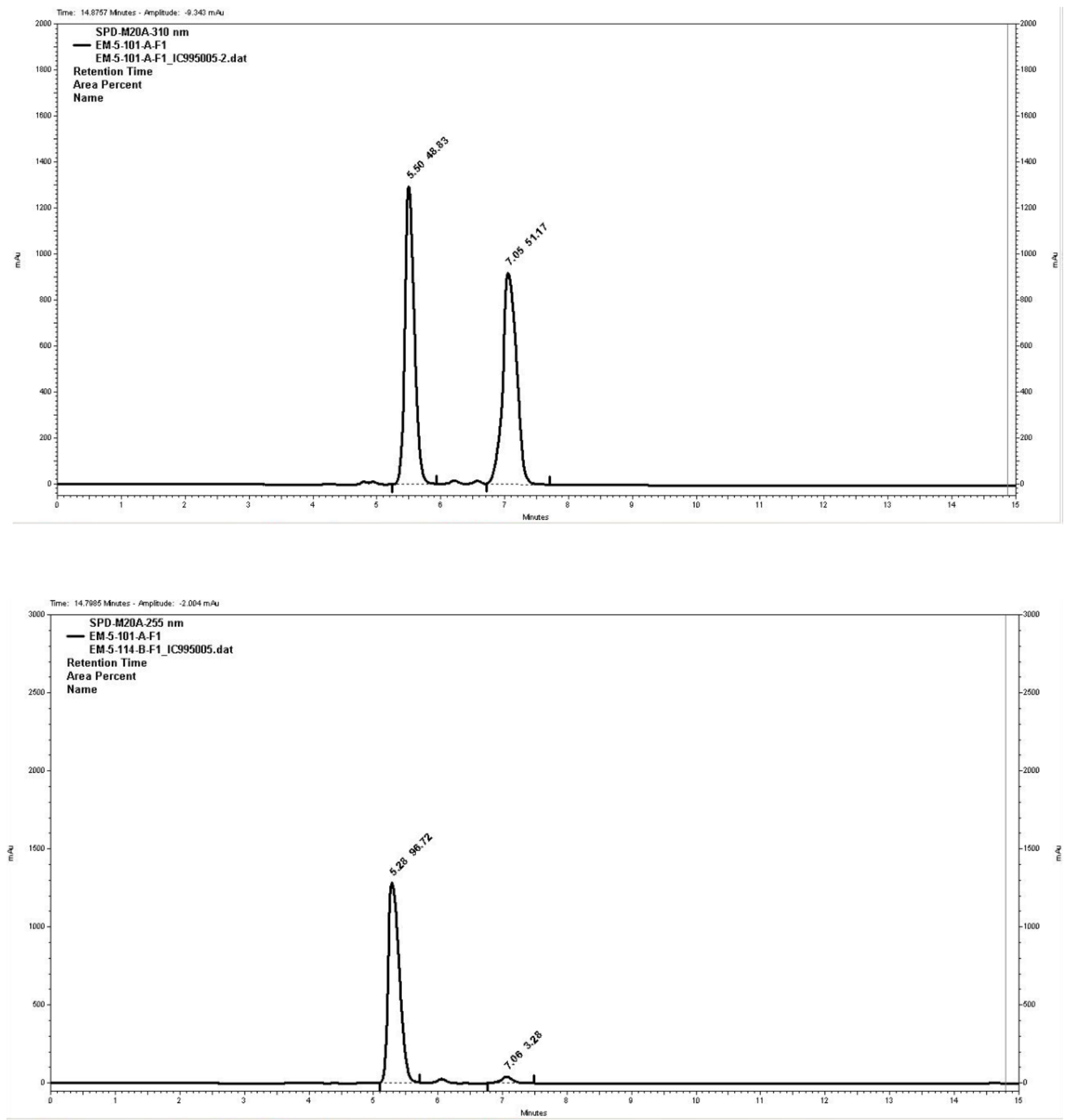
7c: ODH 98:02 hexanes/isopropanol, $1 \mathrm{ml} / \mathrm{min}, \mathrm{t}_{\mathrm{m}}=6.5 \mathrm{~min}, \mathrm{t}_{\mathrm{M}}=6.8 \mathrm{~min}$.
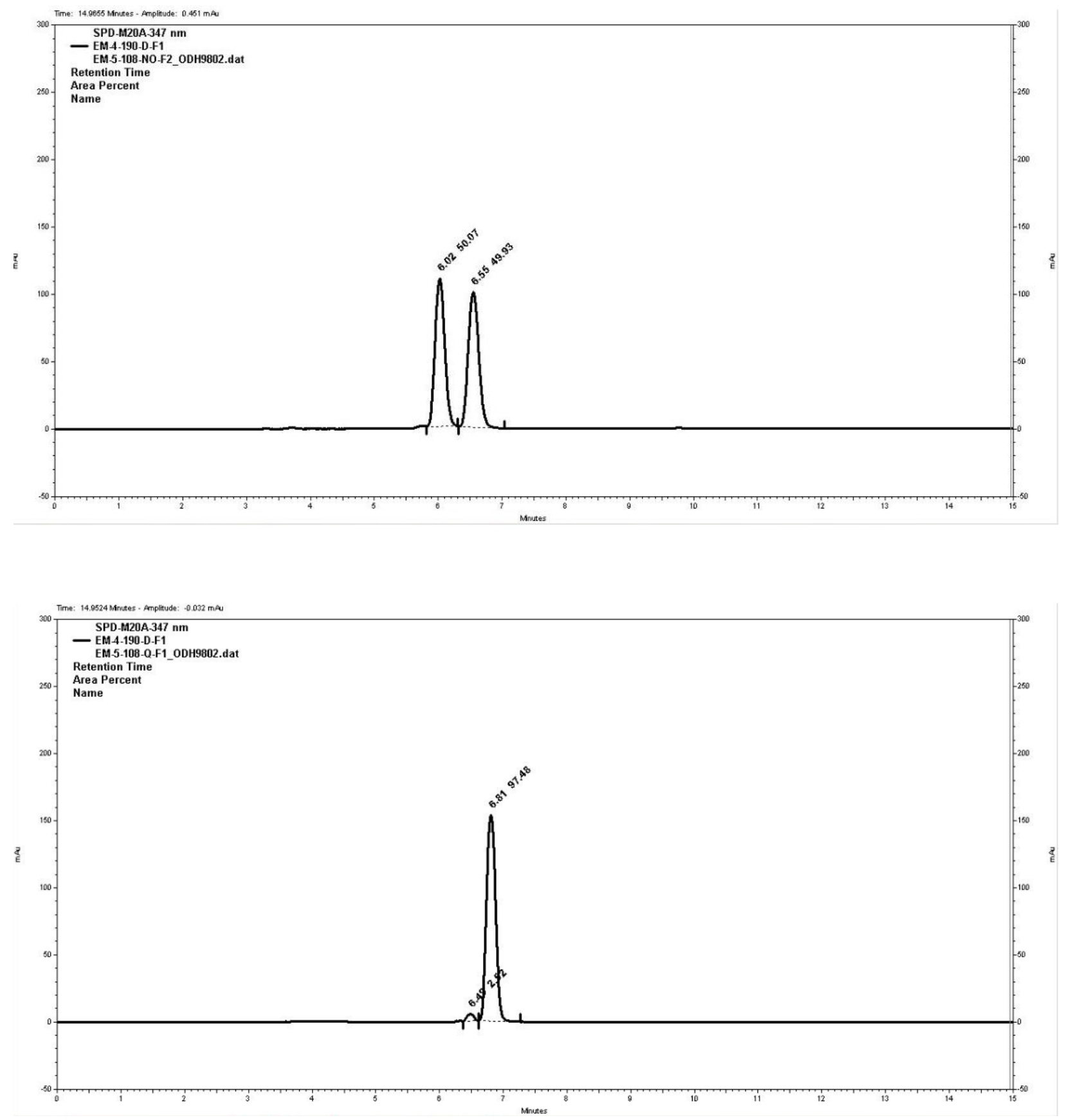
8: IA 99:01 hexanes/isopropanol, $1 \mathrm{ml} / \mathrm{min}, \mathrm{t}_{\mathrm{m}}=12.9 \mathrm{~min}, \mathrm{t}_{\mathrm{M}}=14.9 \mathrm{~min}$.
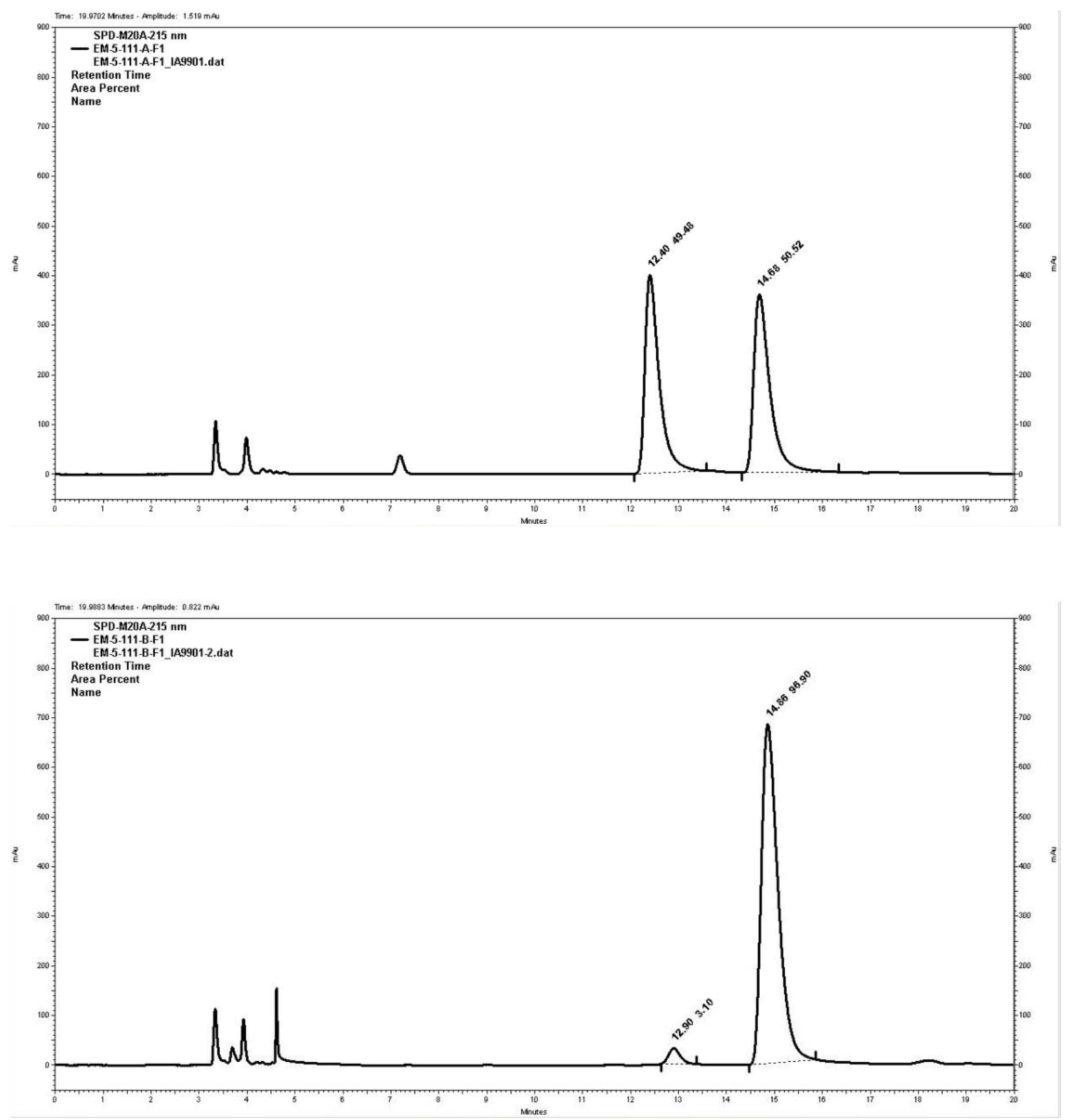


\section{NMR Spectra}

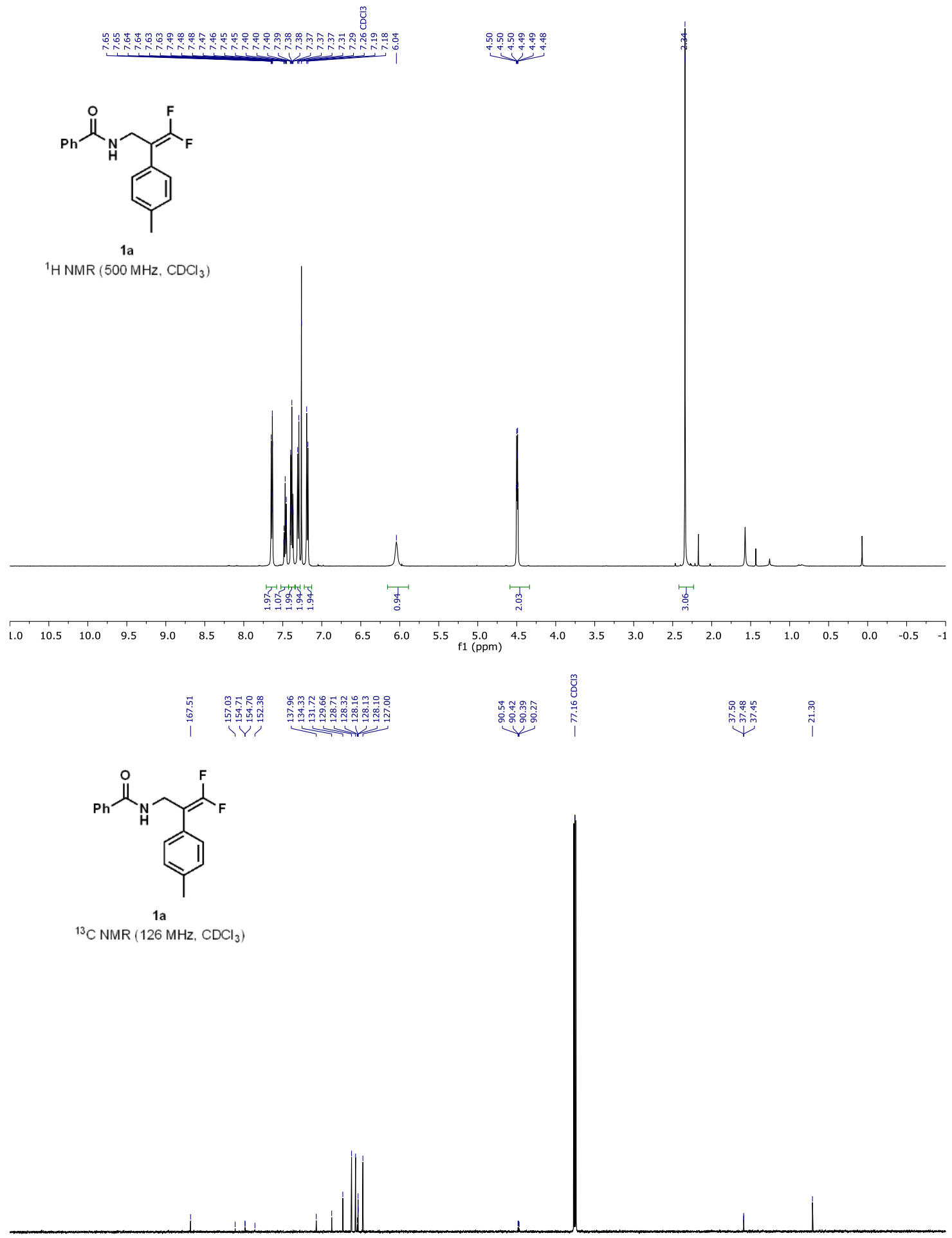

$\begin{array}{lllllllllllllllllllllllll} & 190 & 190 & 180 & 170 & 160 & 150 & 140 & 130 & 120 & 110 & \begin{array}{l}100 \\ \mathrm{f} 1(\mathrm{ppm})\end{array} & 90 & 80 & 70 & 60 & 50 & 40 & 30 & 20 & 10 & 0 & -\end{array}$ 


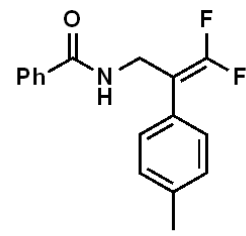

${ }^{19} \mathrm{~F} \mathrm{NMR}\left(470 \mathrm{MHz}, \mathrm{CDCl}_{3}\right)$

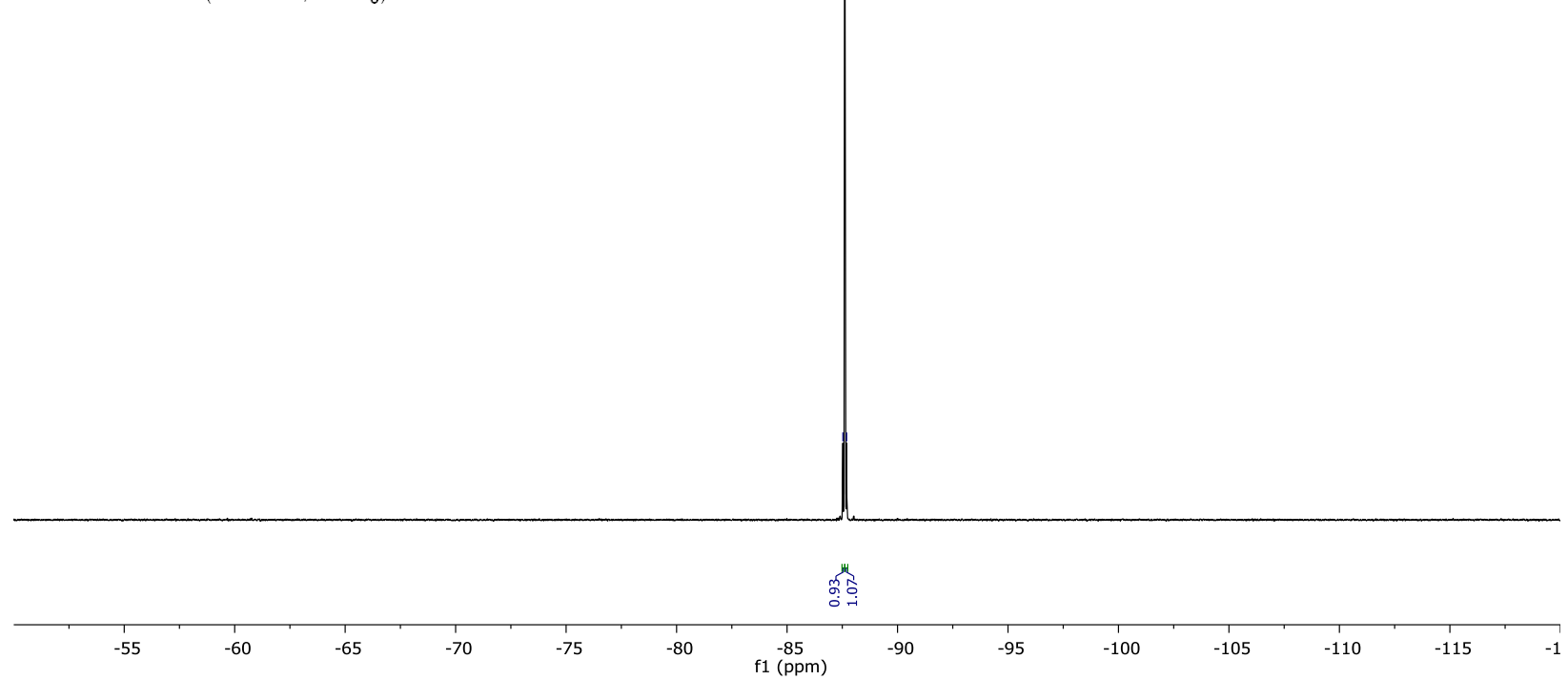




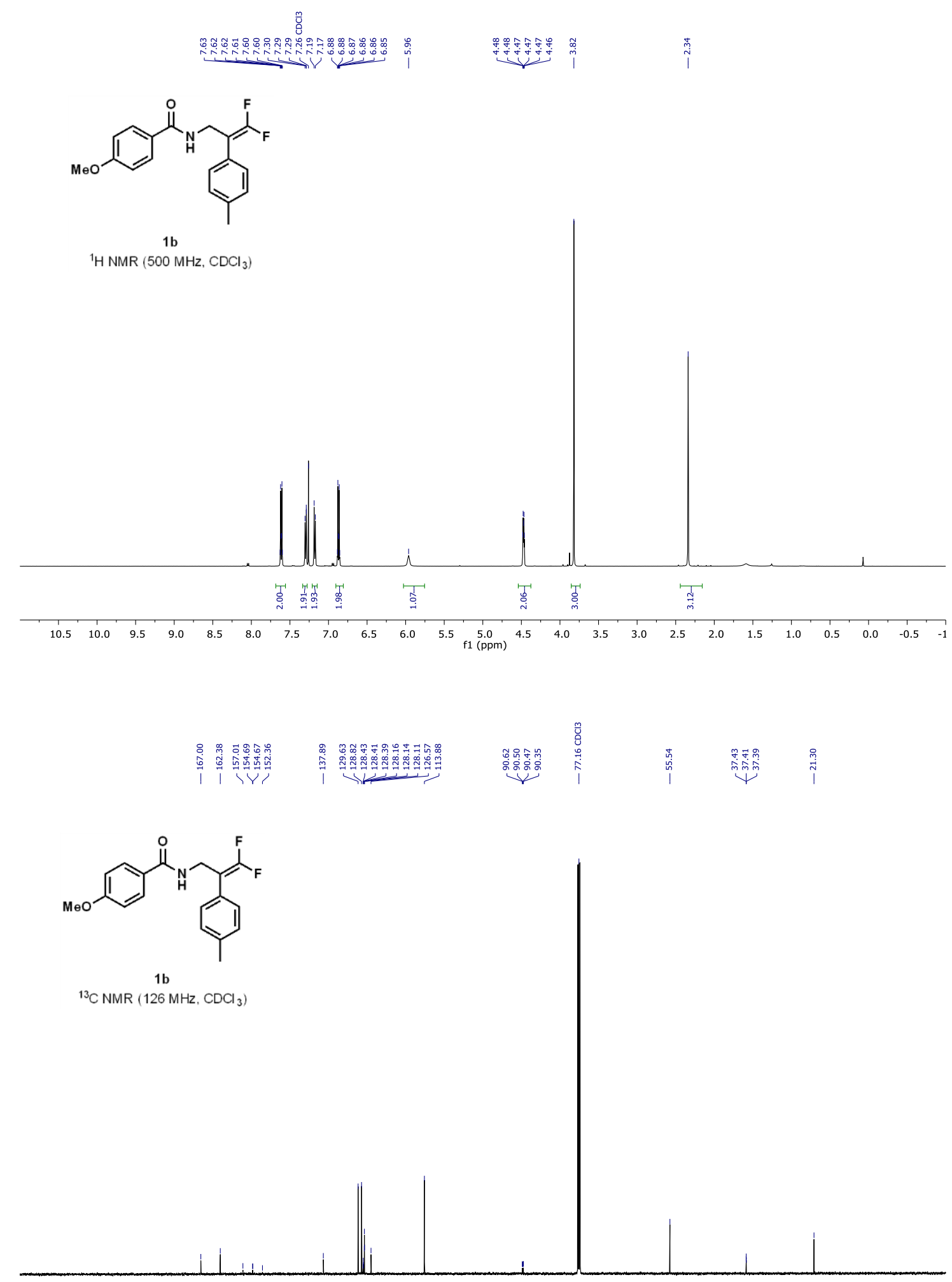

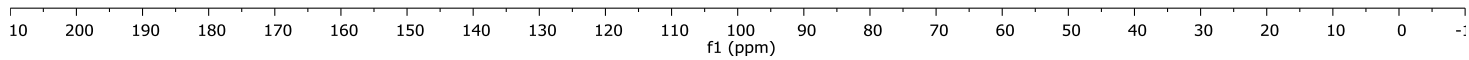




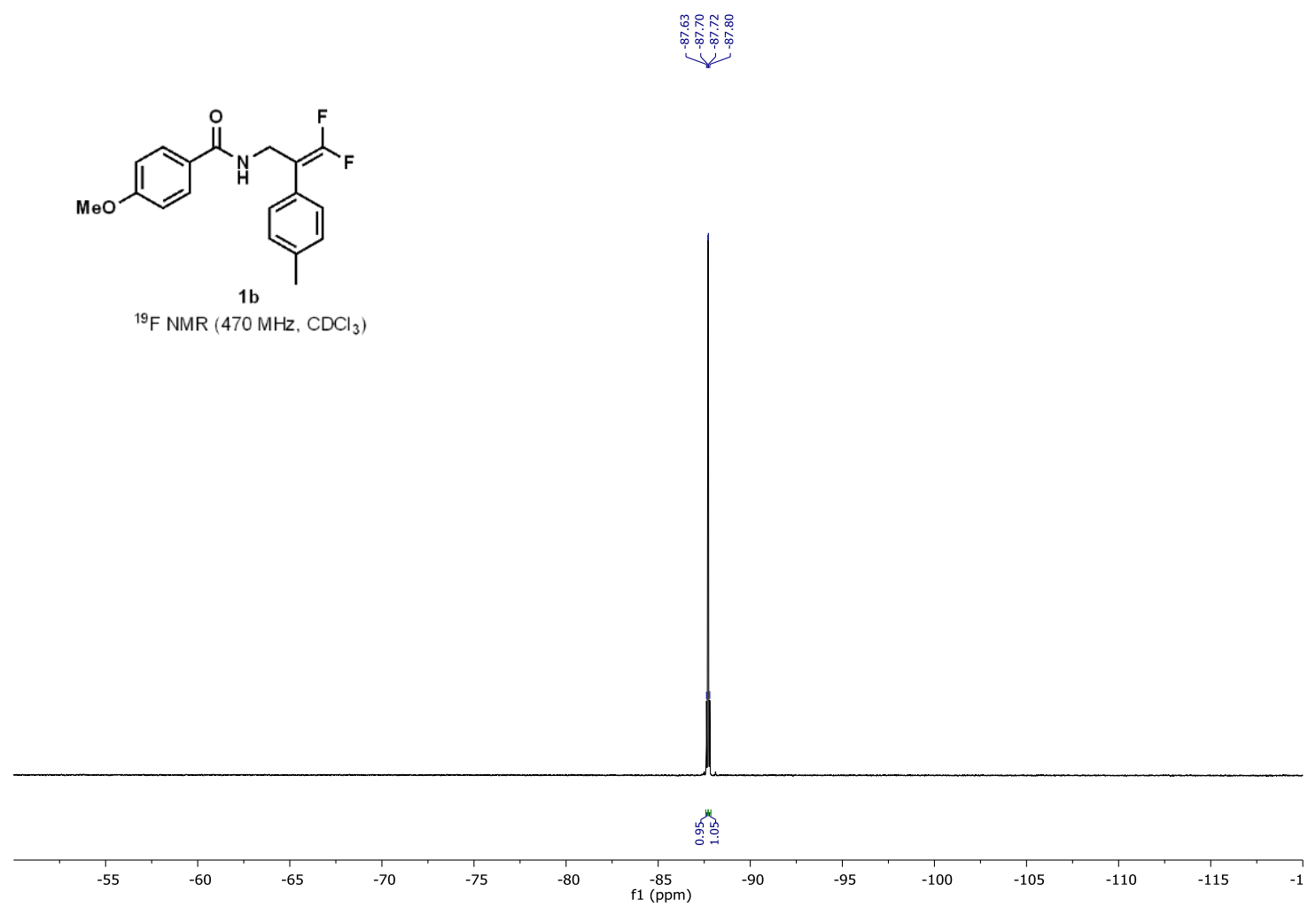



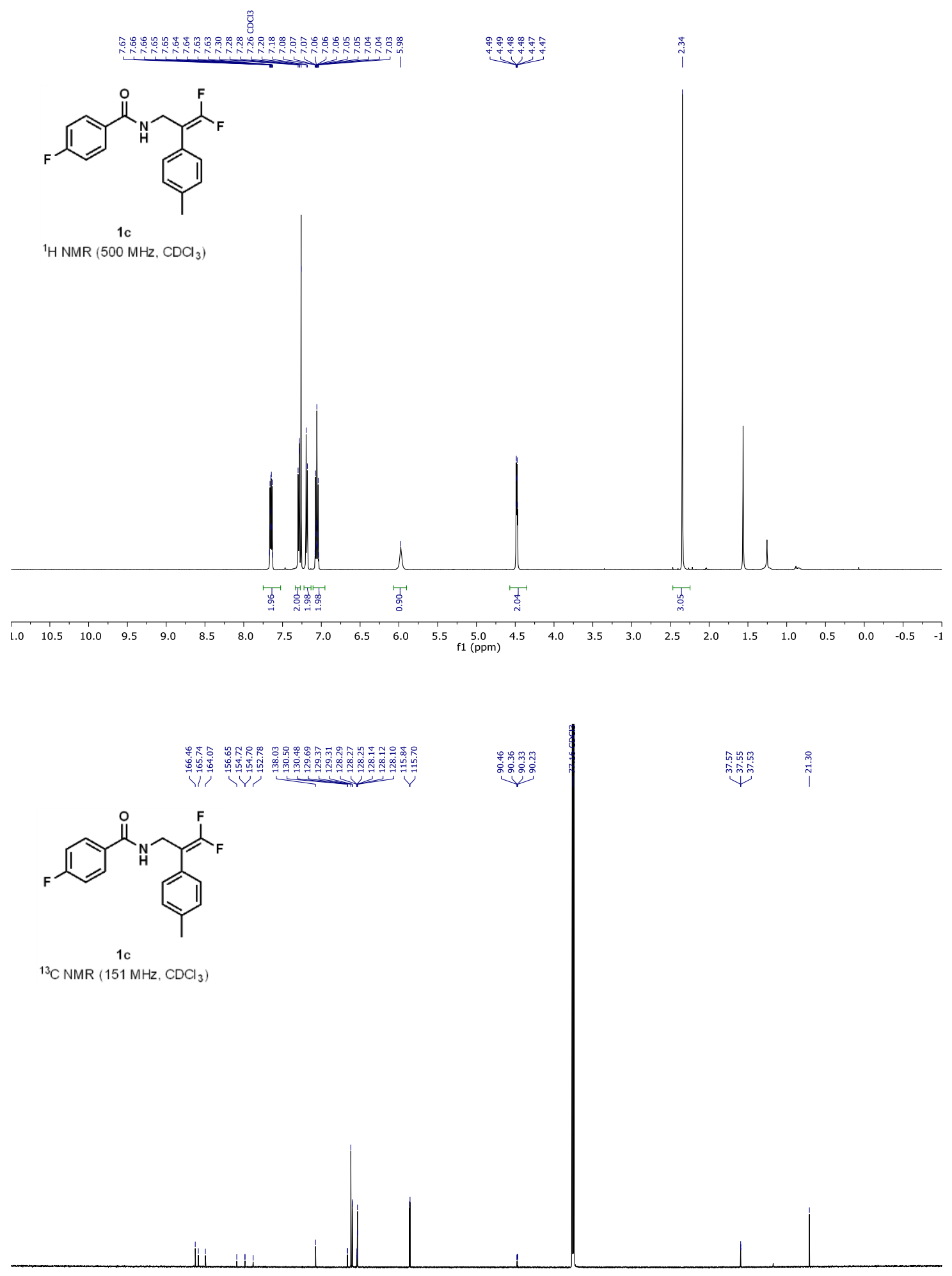


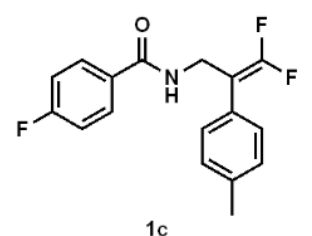

议

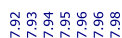

50ㅎำ

${ }^{19} \mathrm{~F} \mathrm{NMR}\left(470 \mathrm{MHz}, \mathrm{CDCl}_{3}\right)$

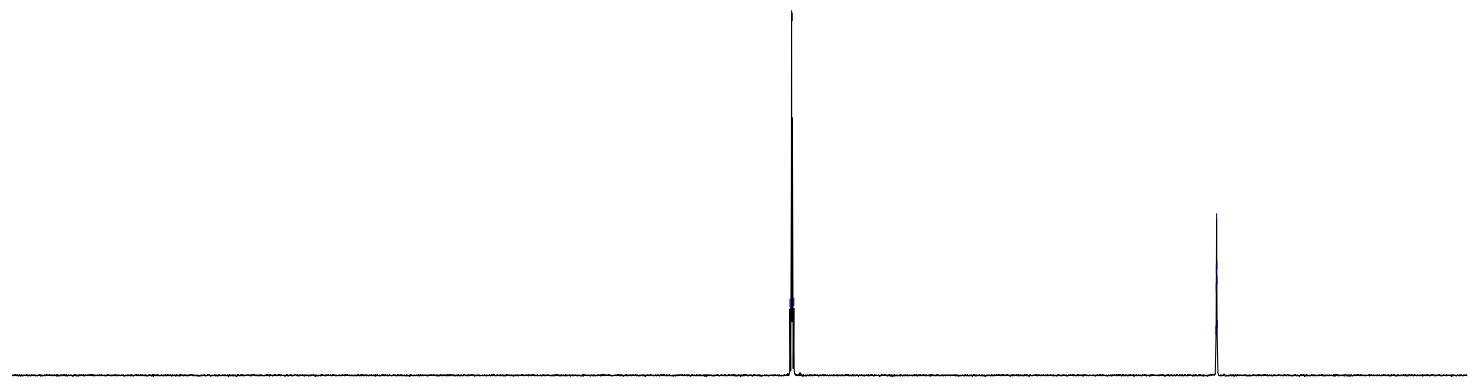

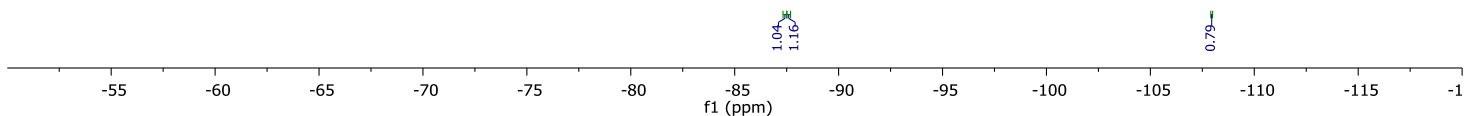




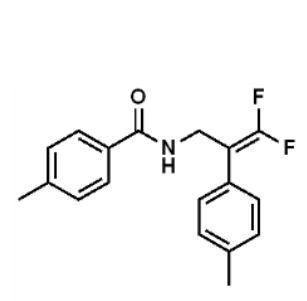

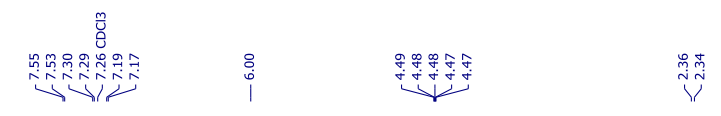

${ }^{1} \mathrm{H} \mathrm{NMR}\left(600 \mathrm{MHz}, \mathrm{CDCl}_{3}\right.$ )
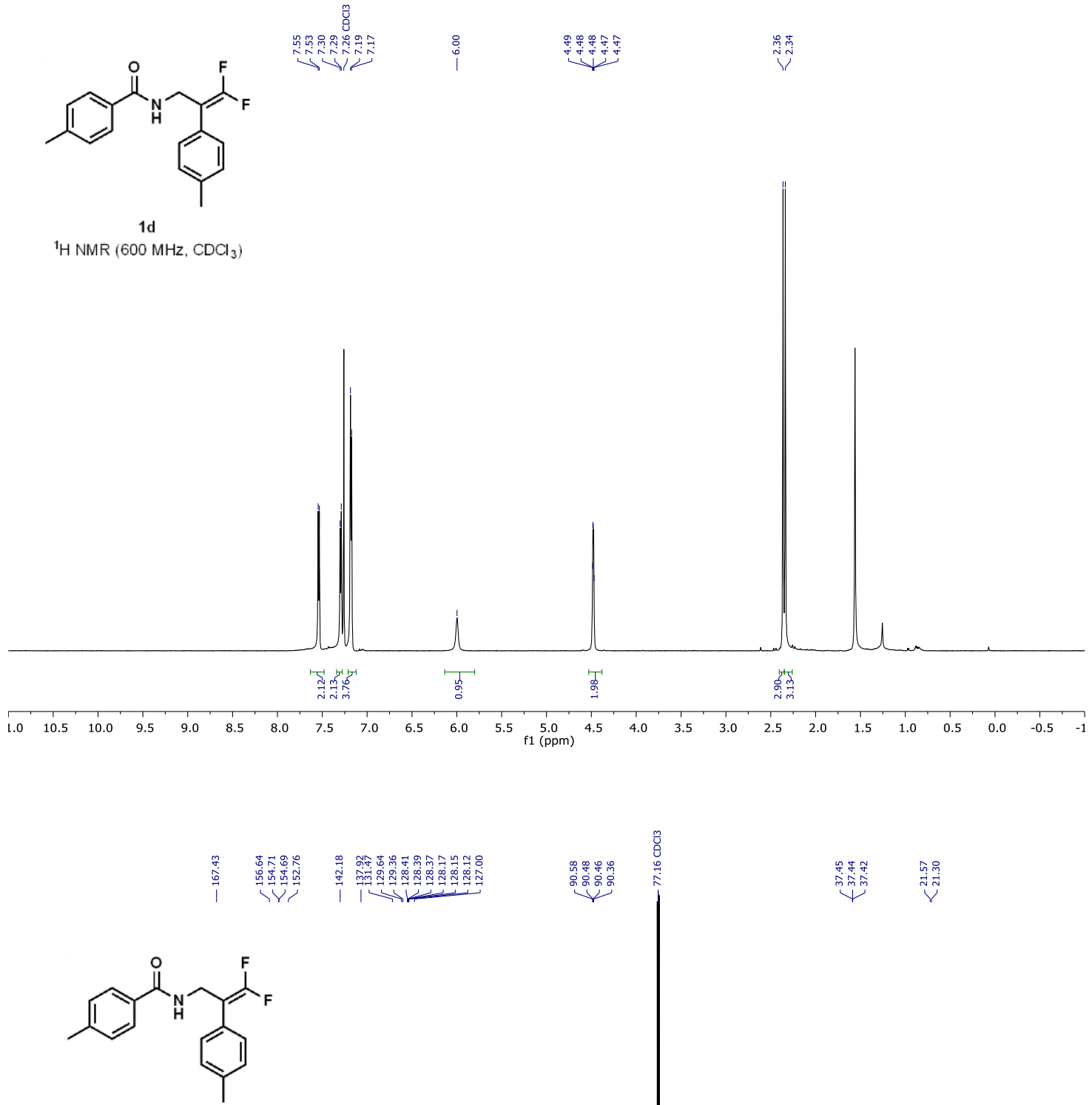

1d

${ }^{13} \mathrm{CNMR}\left(151 \mathrm{MHz}, \mathrm{CDCl}_{3}\right)$

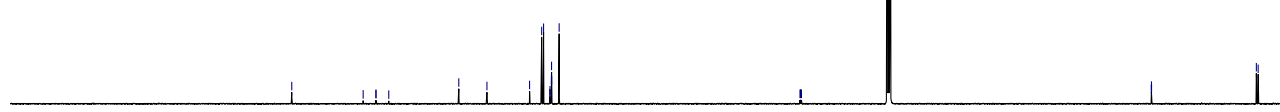

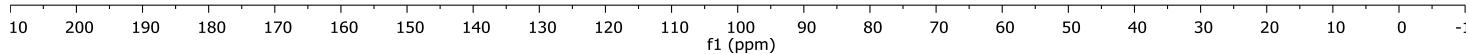




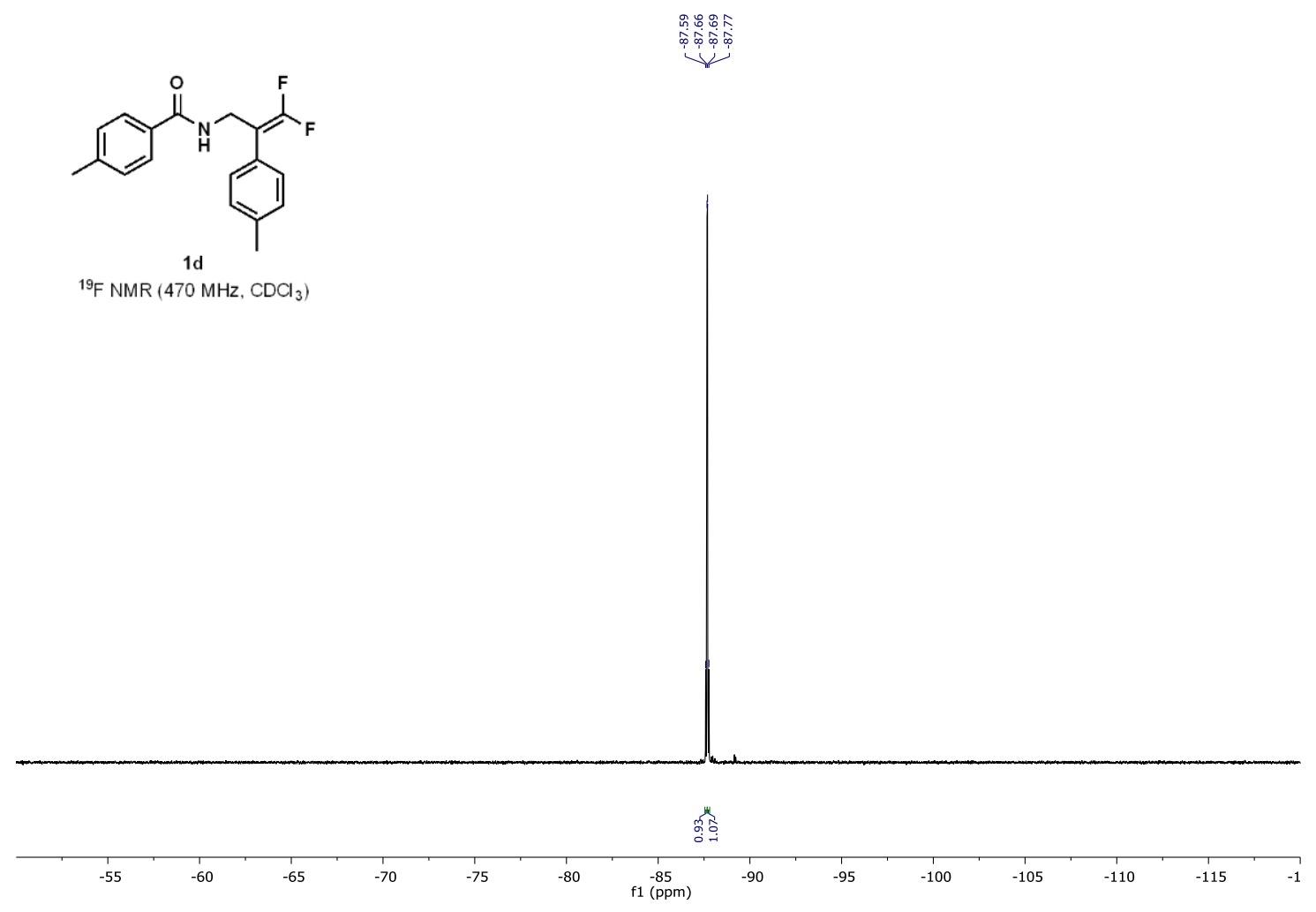




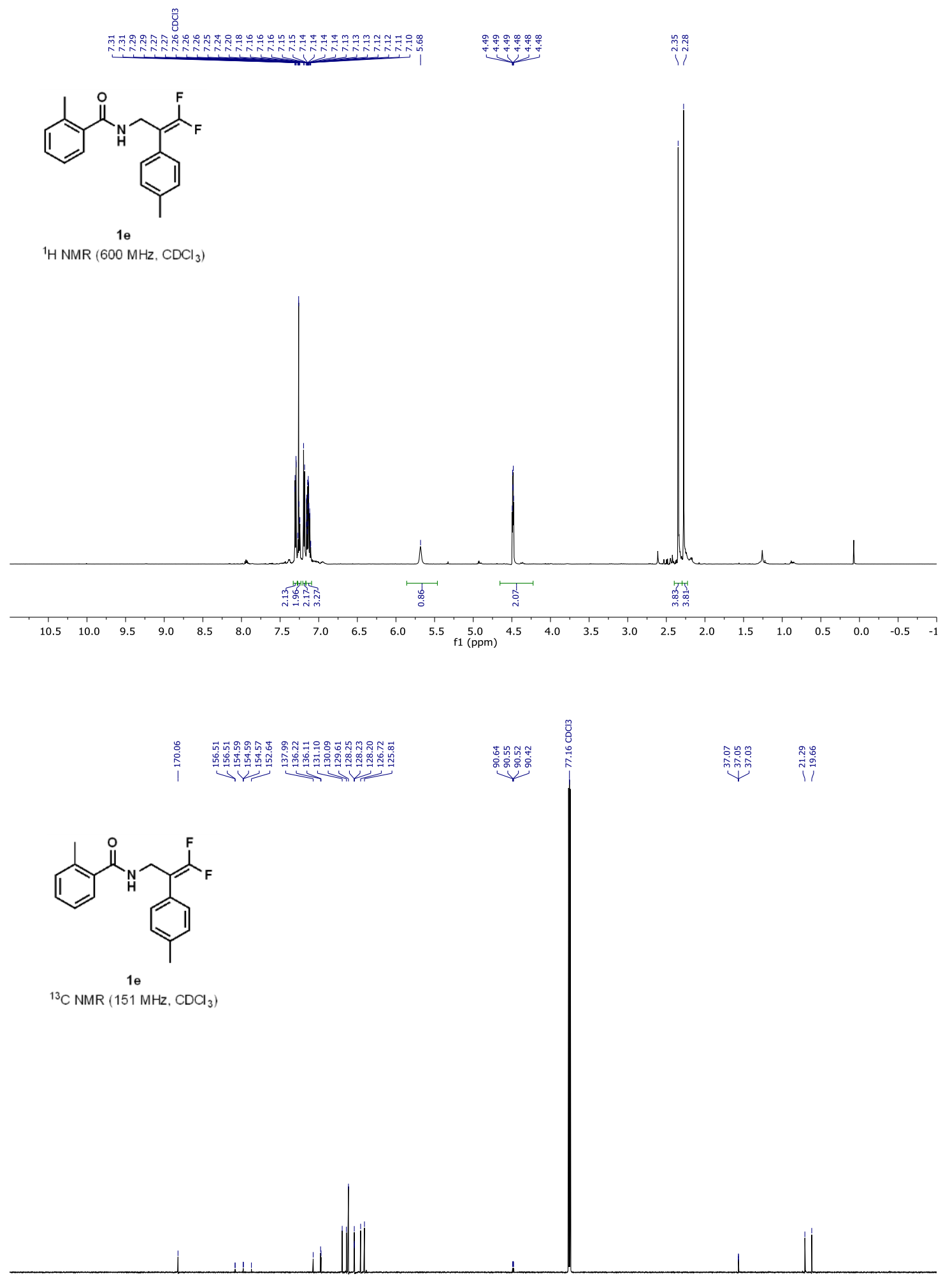

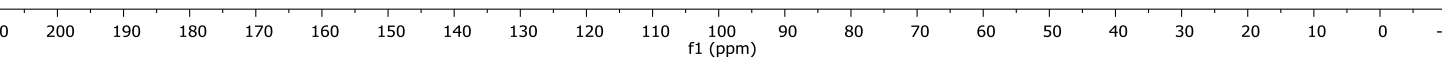




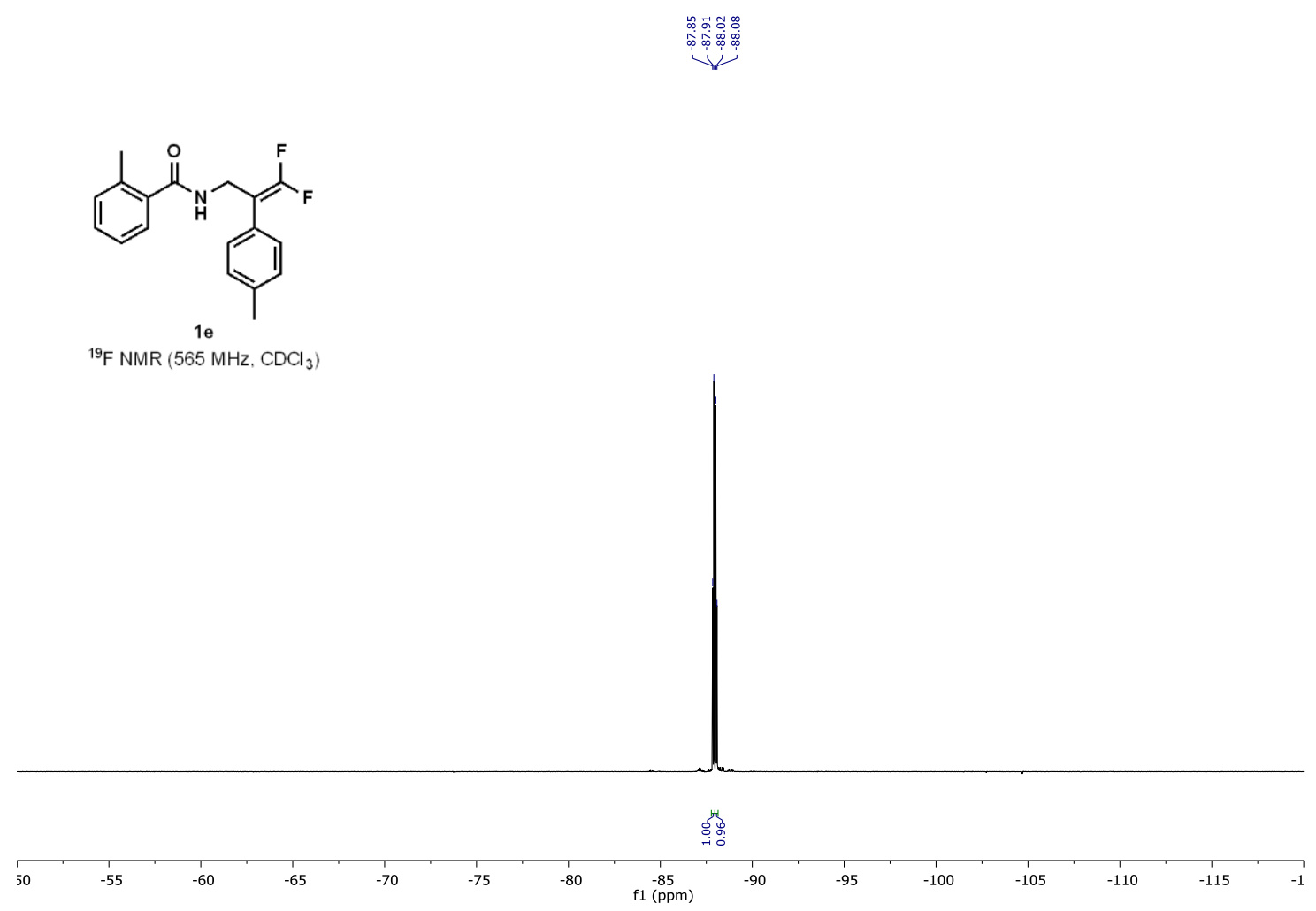




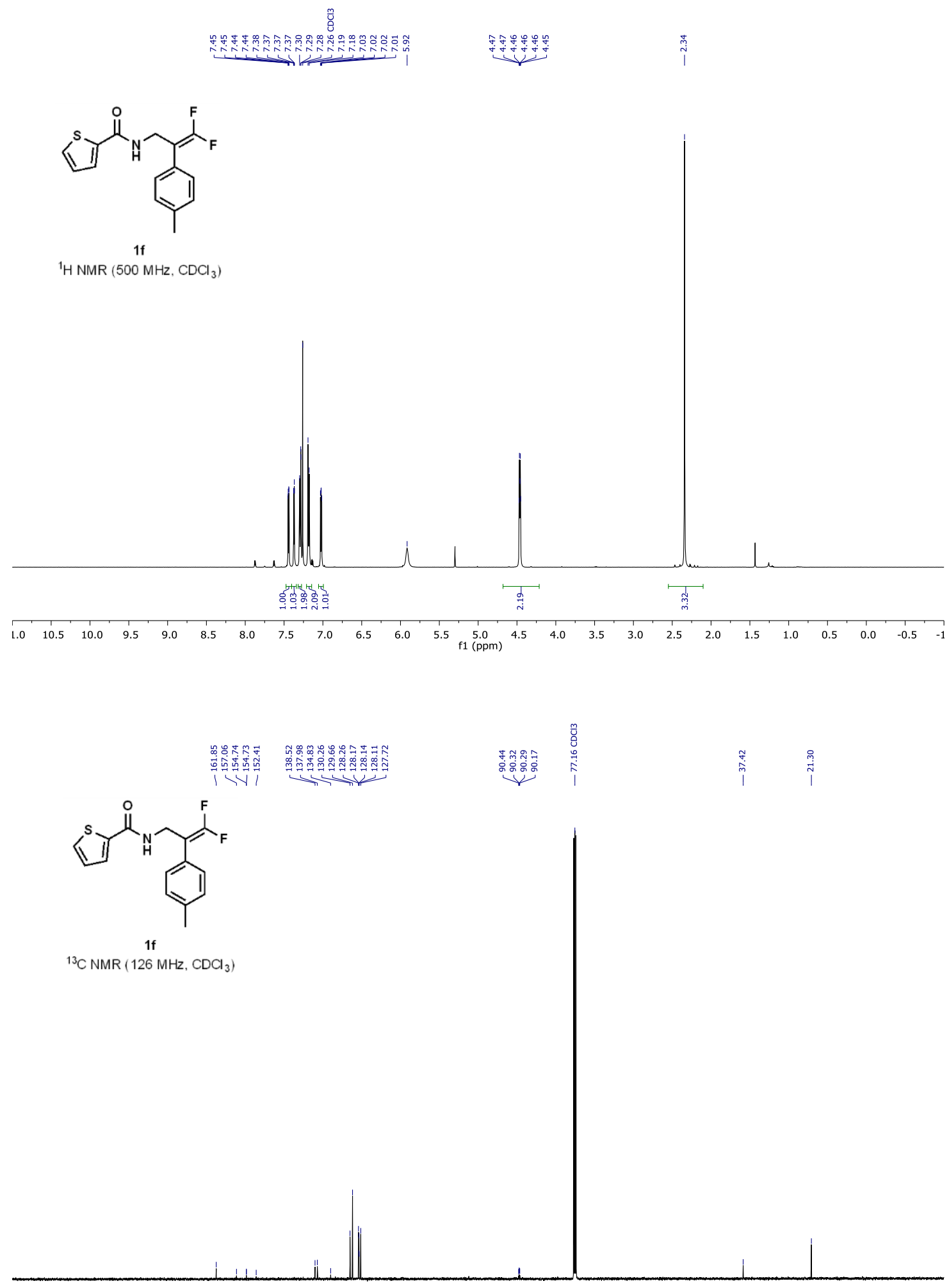

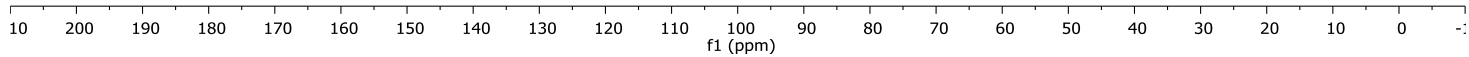




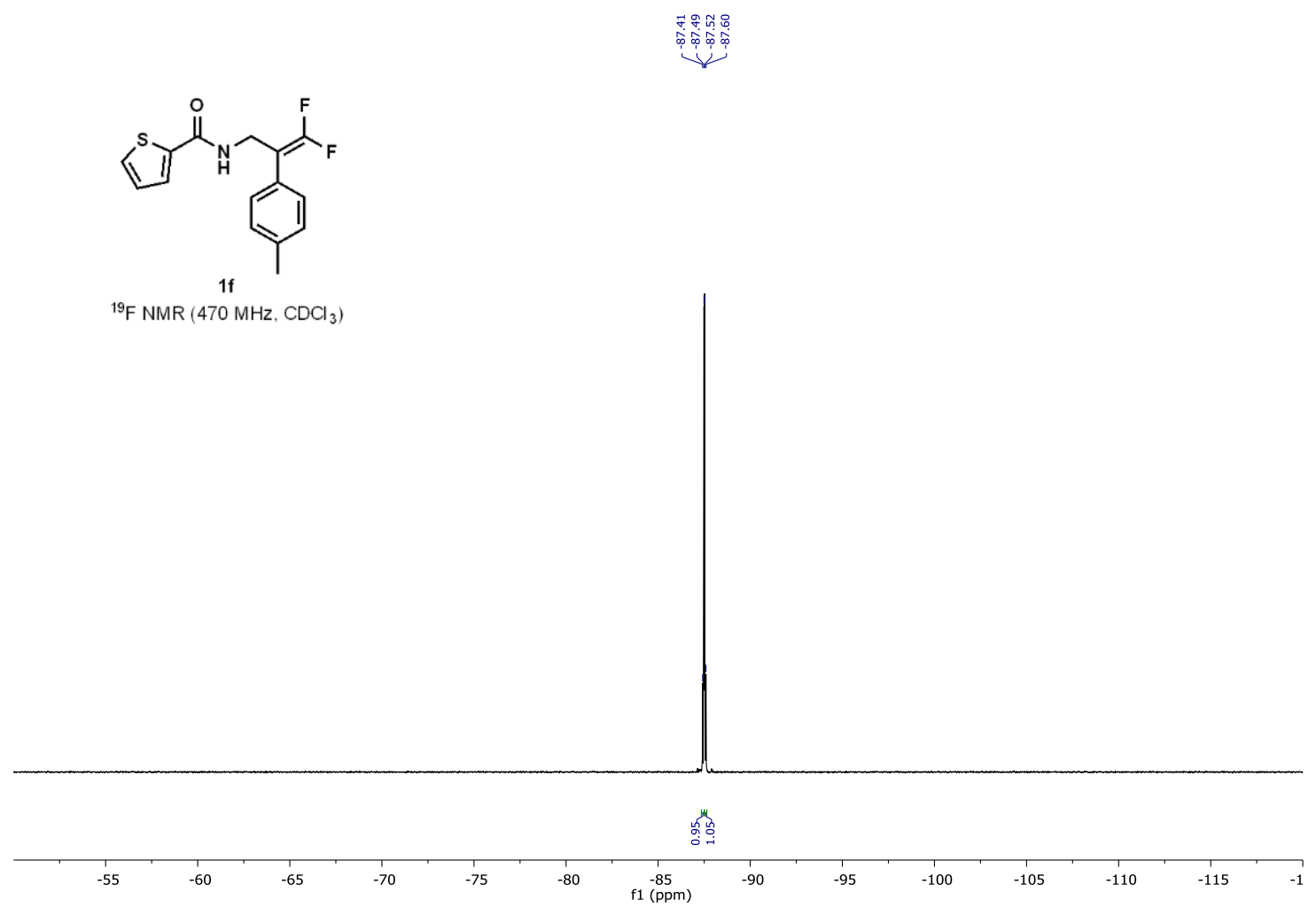



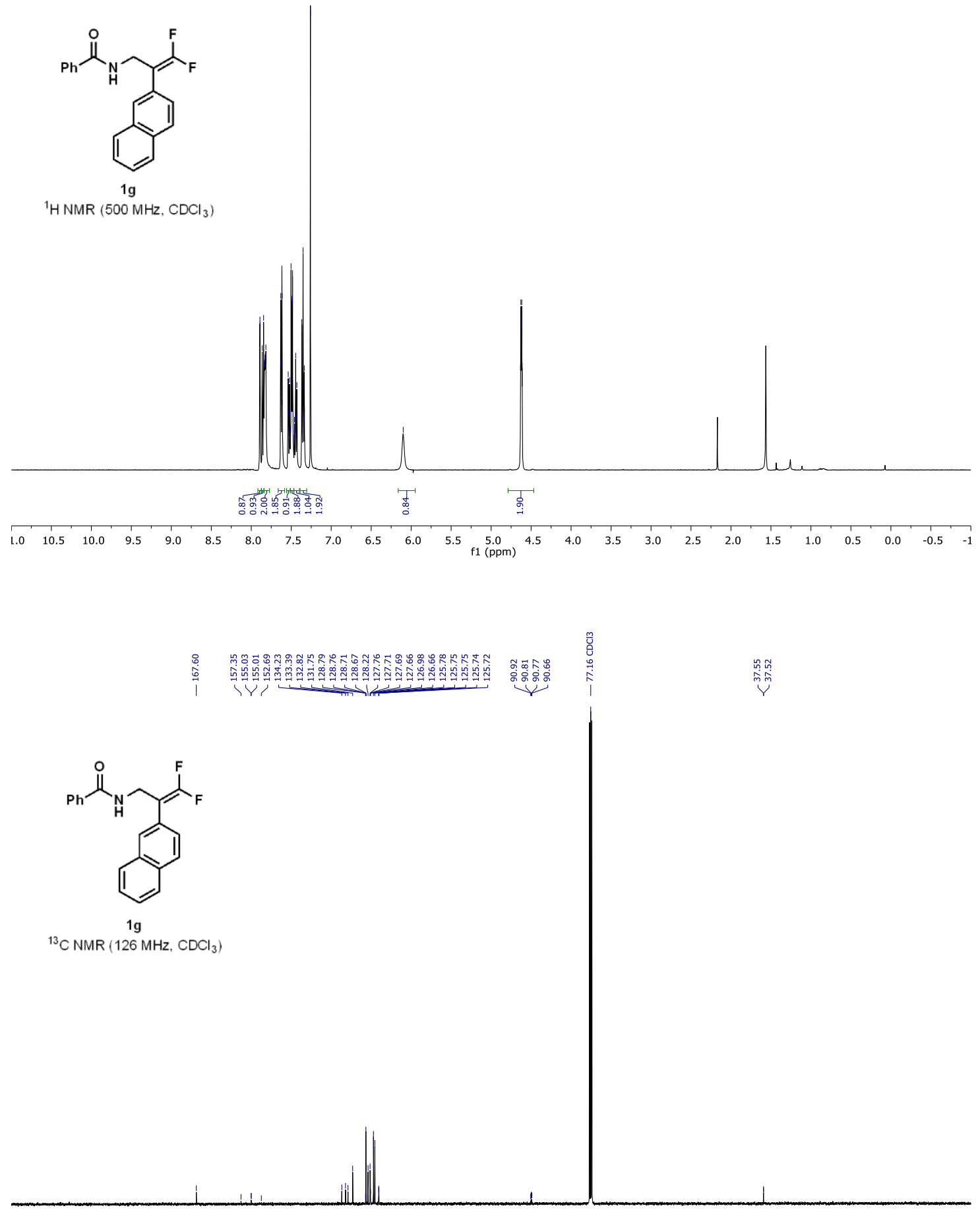


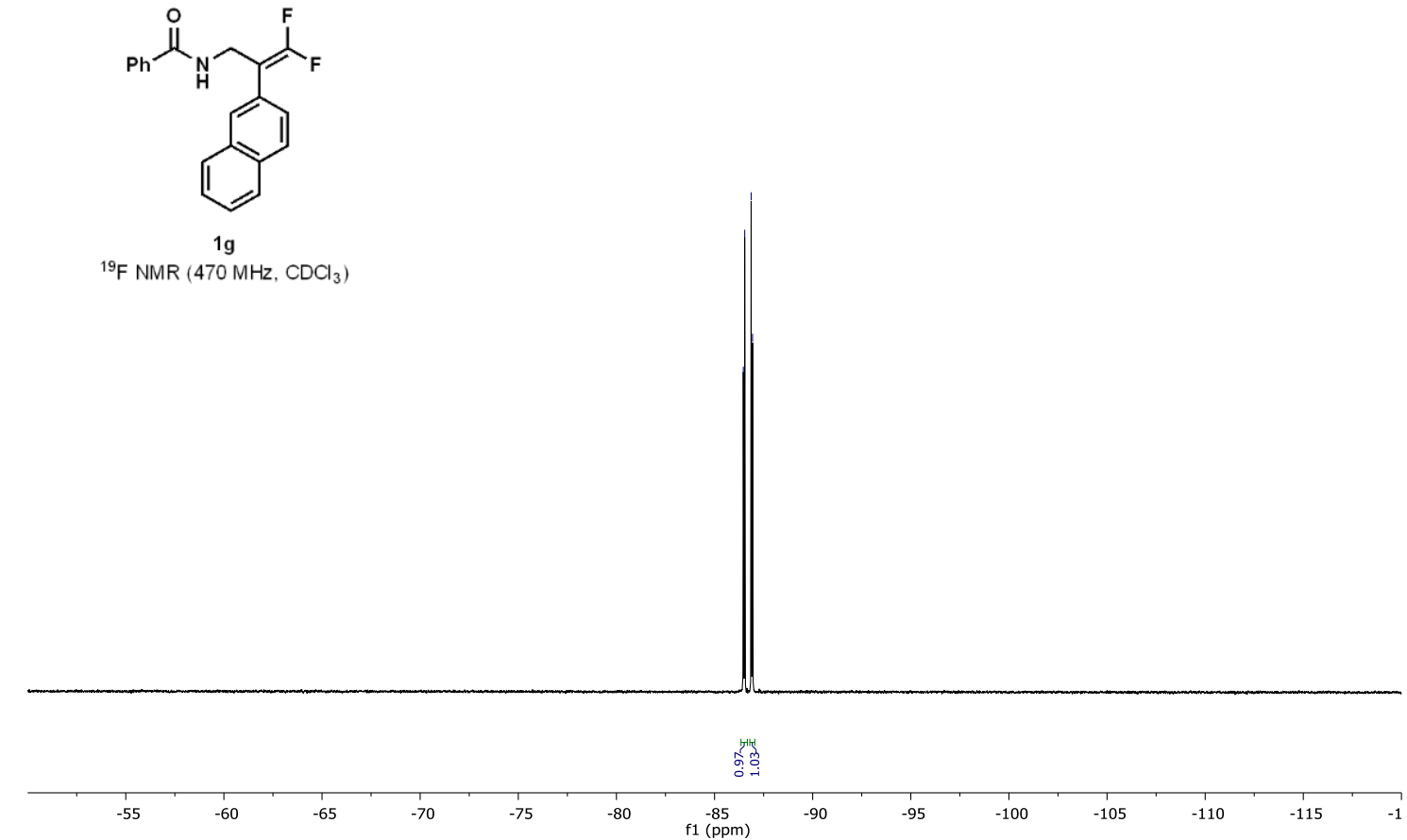

${ }^{19} \mathrm{~F} \mathrm{NMR}\left(470 \mathrm{MHz}, \mathrm{CDCl}_{3}\right)$ 

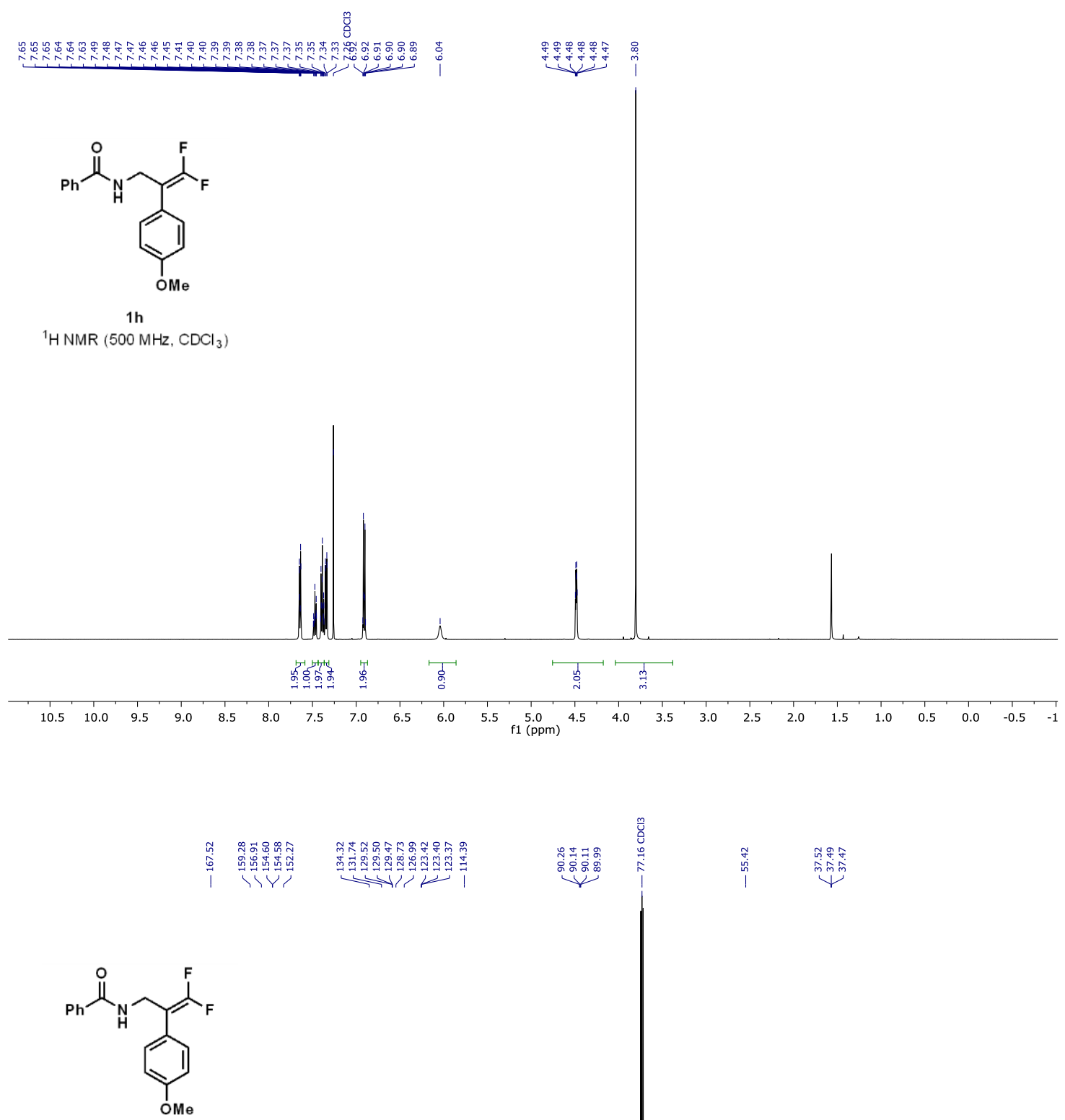

$1 \mathrm{~h}$

${ }^{13} \mathrm{CNMR}\left(126 \mathrm{MHz}, \mathrm{CDCl}_{3}\right)$

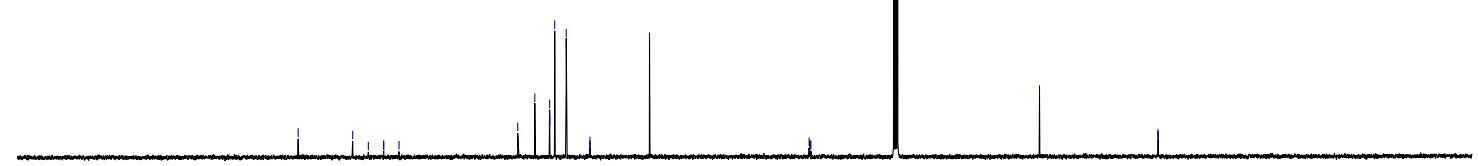

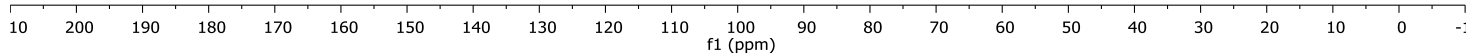




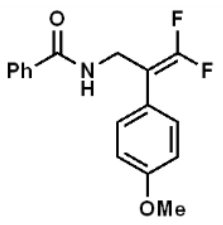

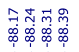

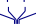

${ }^{9} \mathrm{~F}$ NMR $\left(470 \mathrm{MHz}, \mathrm{CDCl}_{3}\right)$

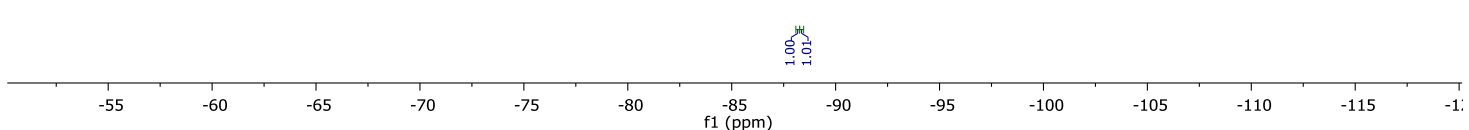



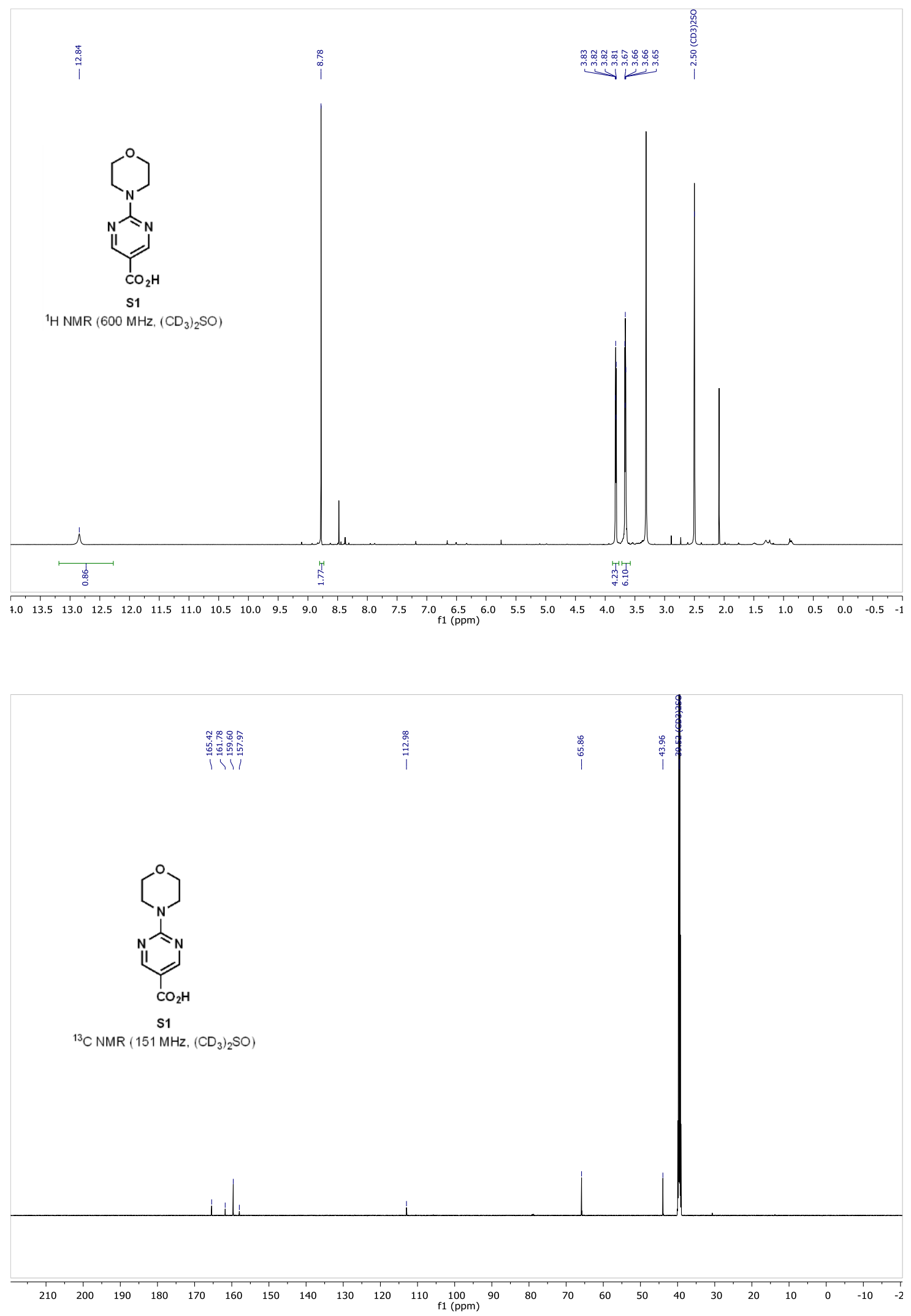

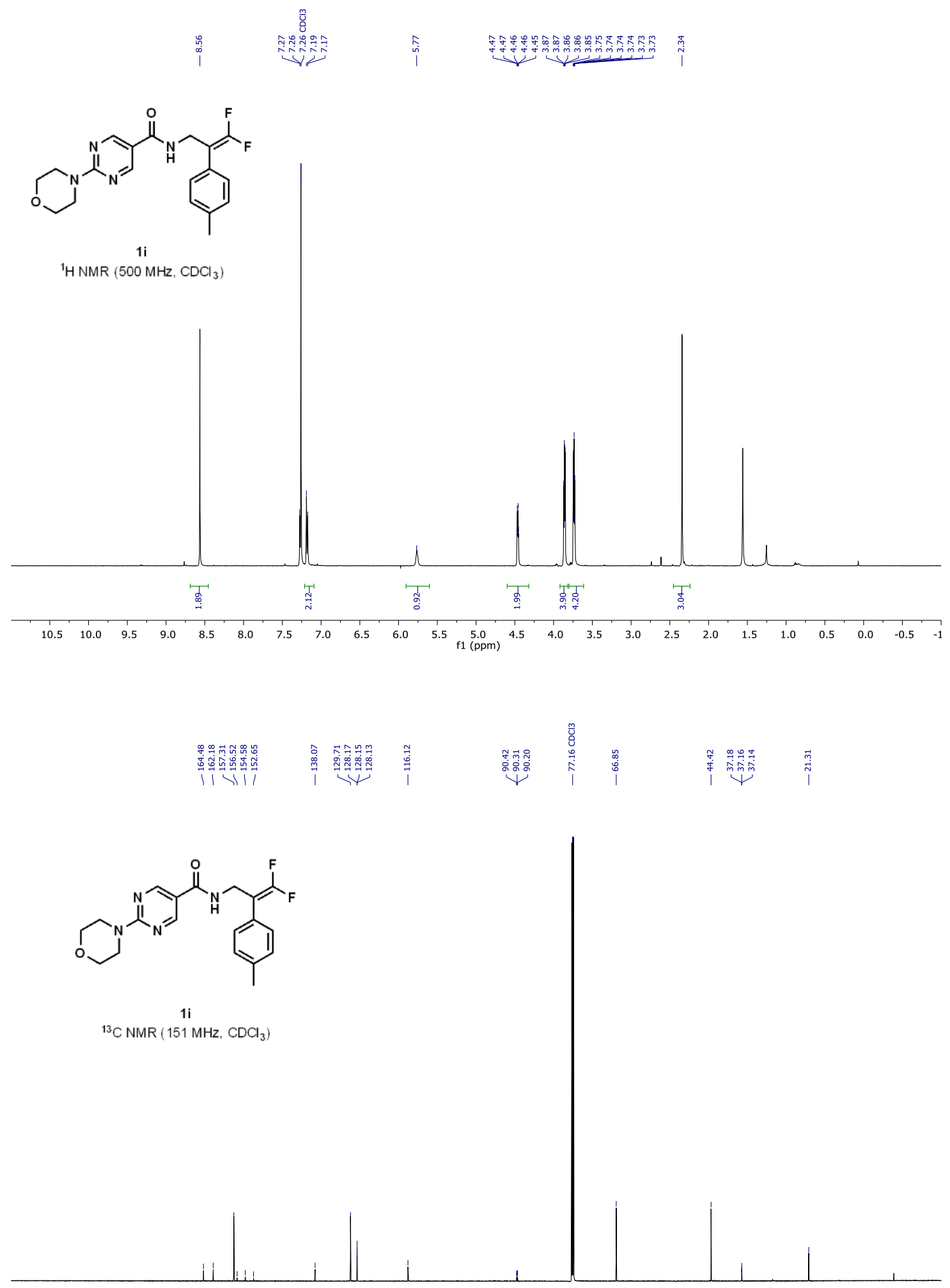

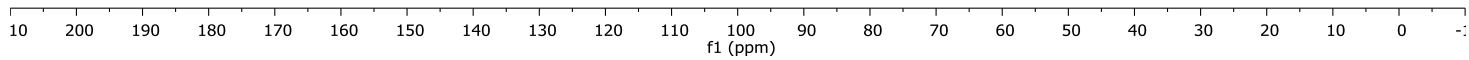




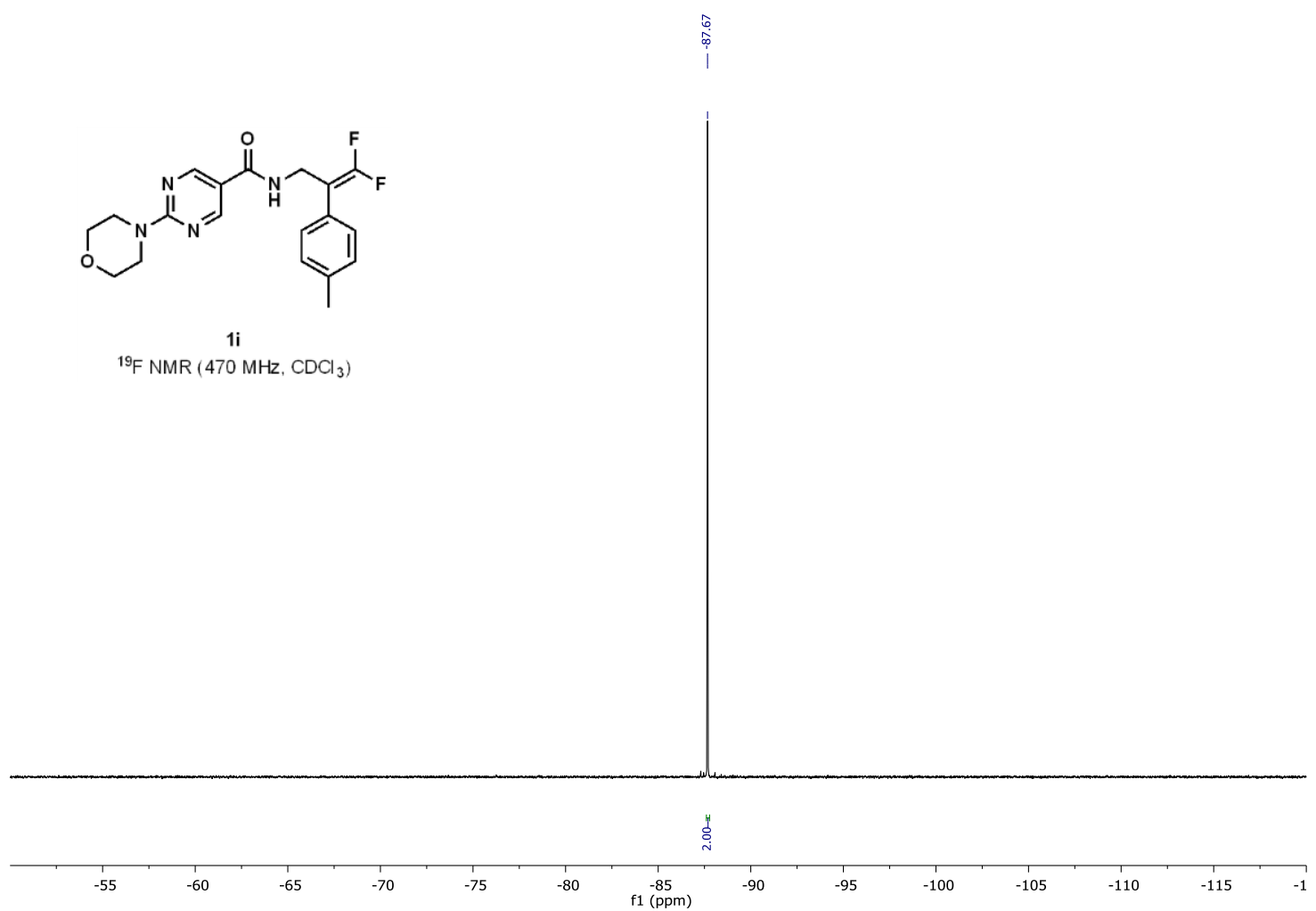



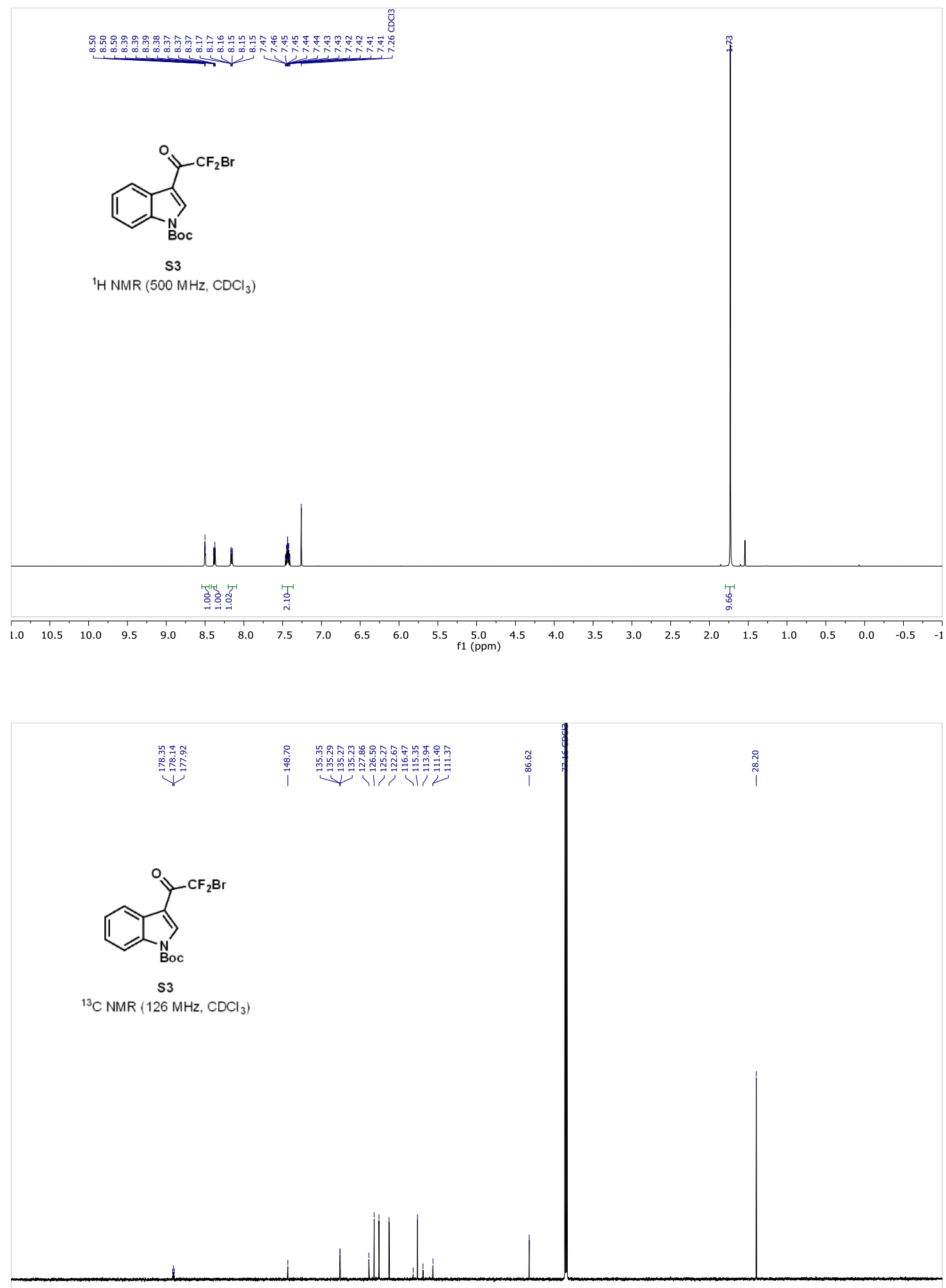

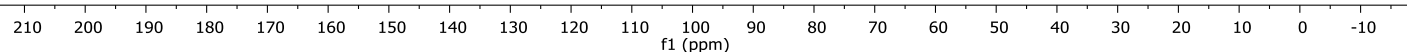




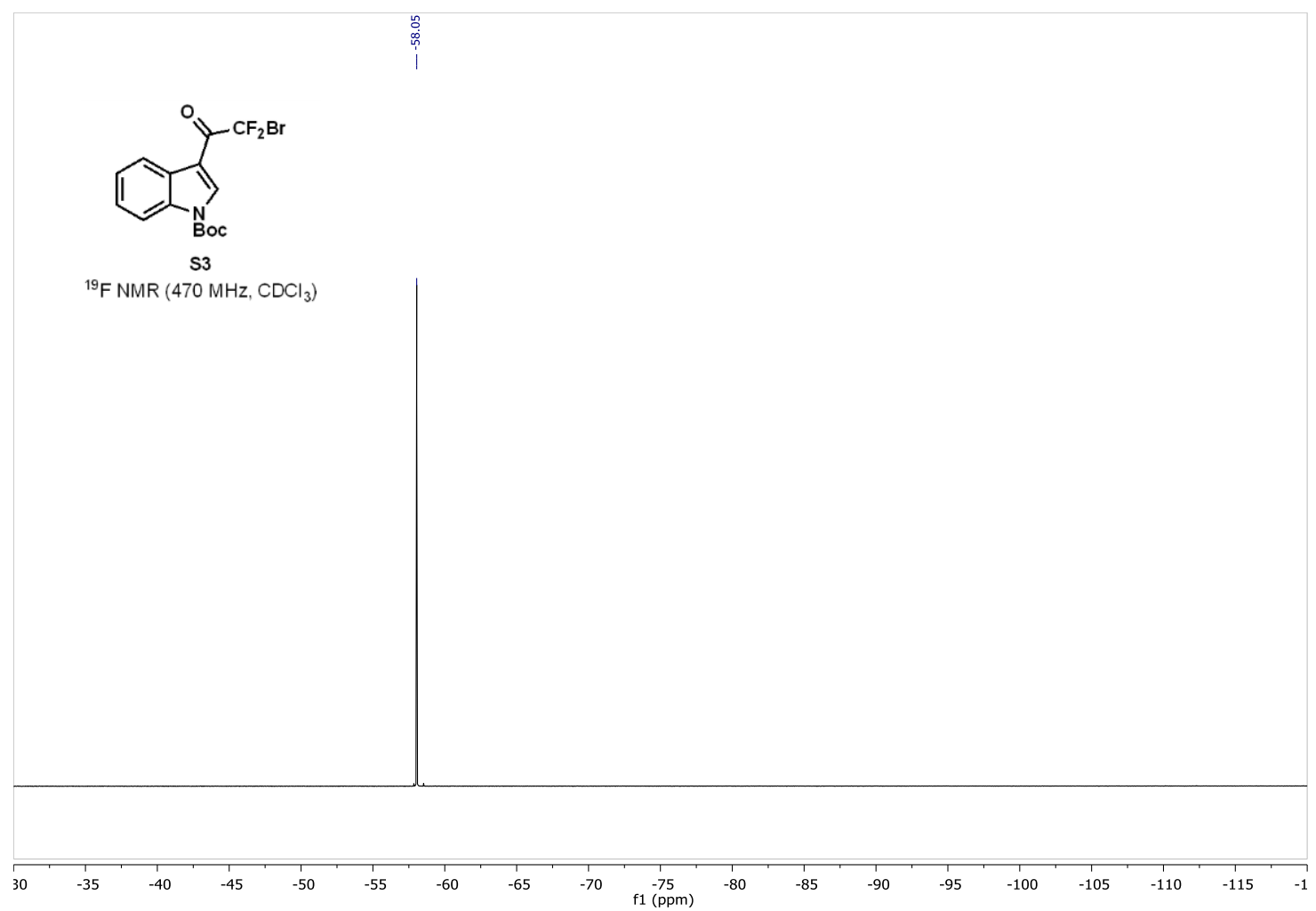



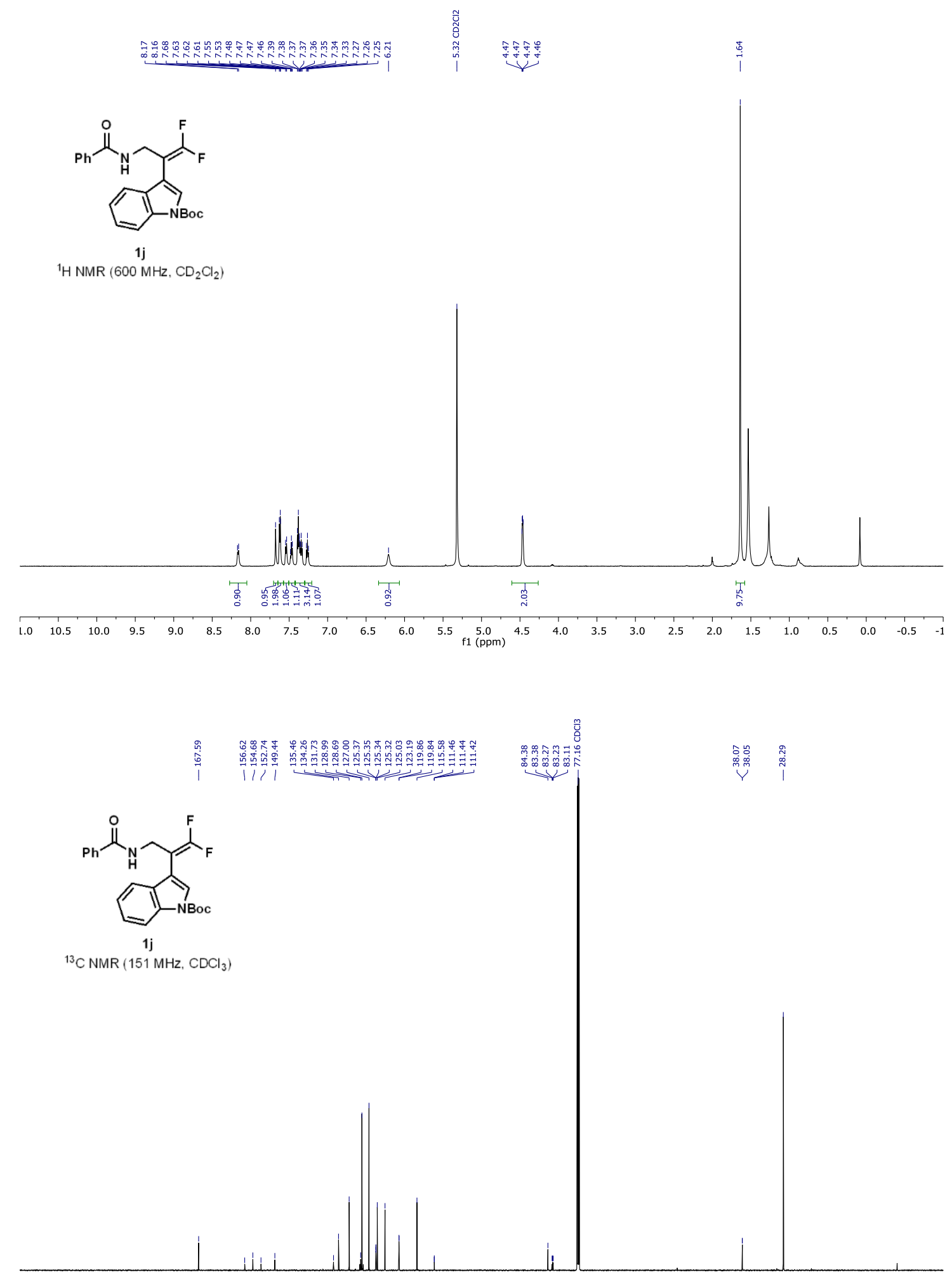

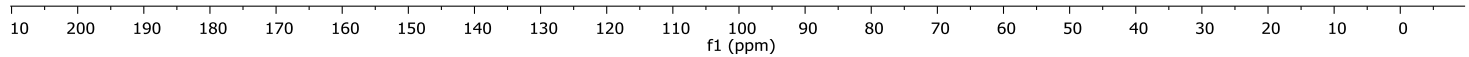




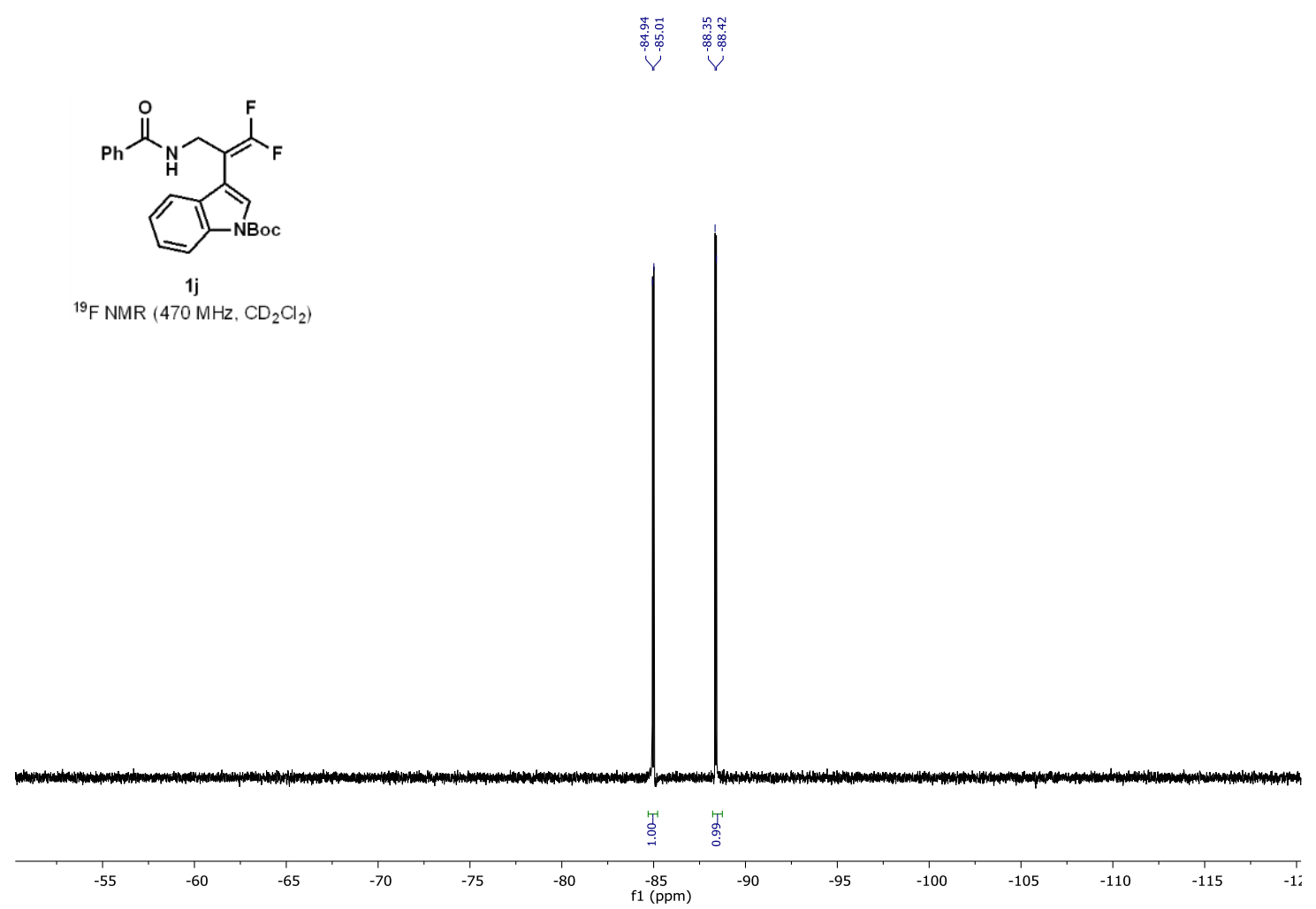



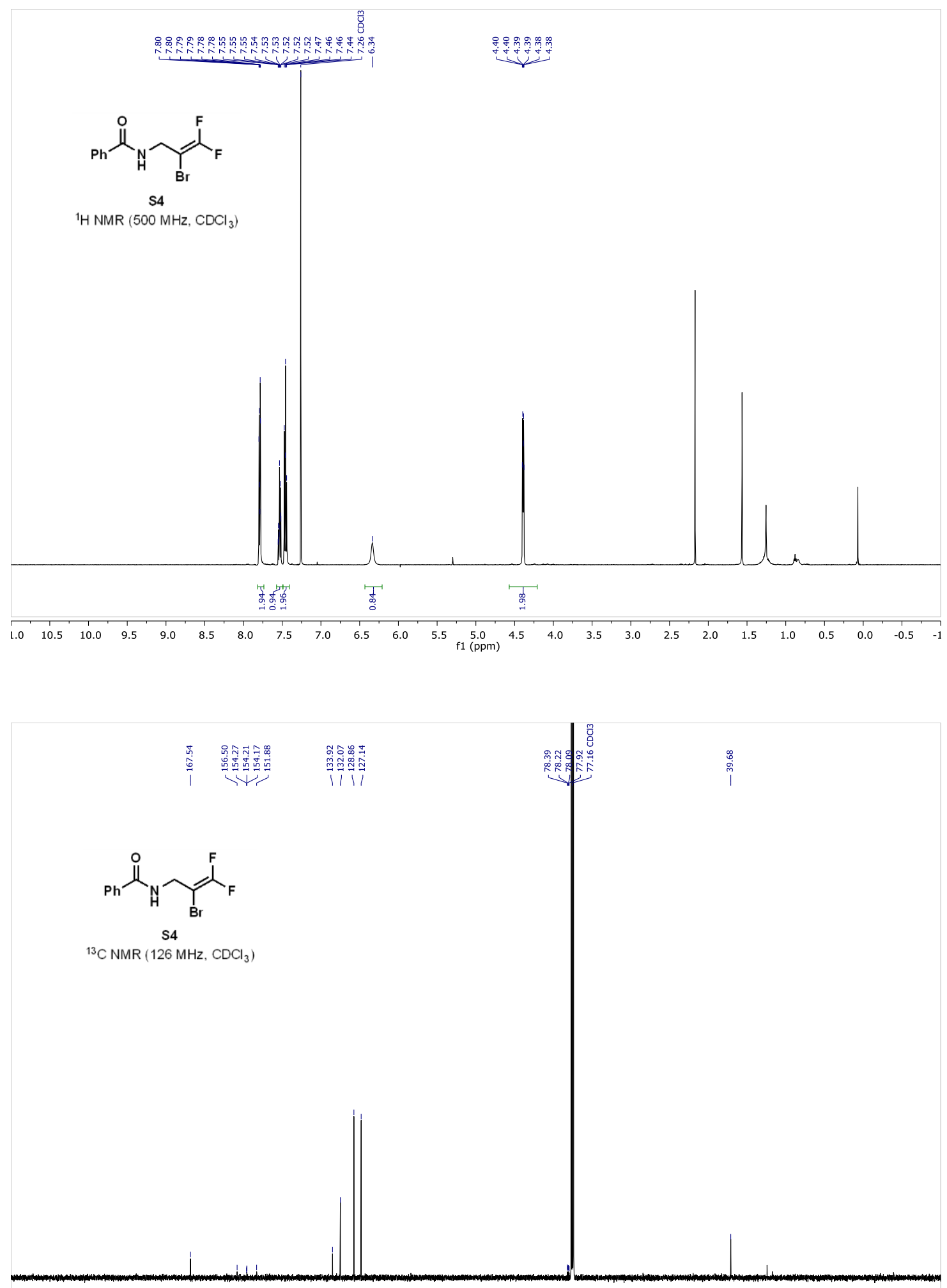


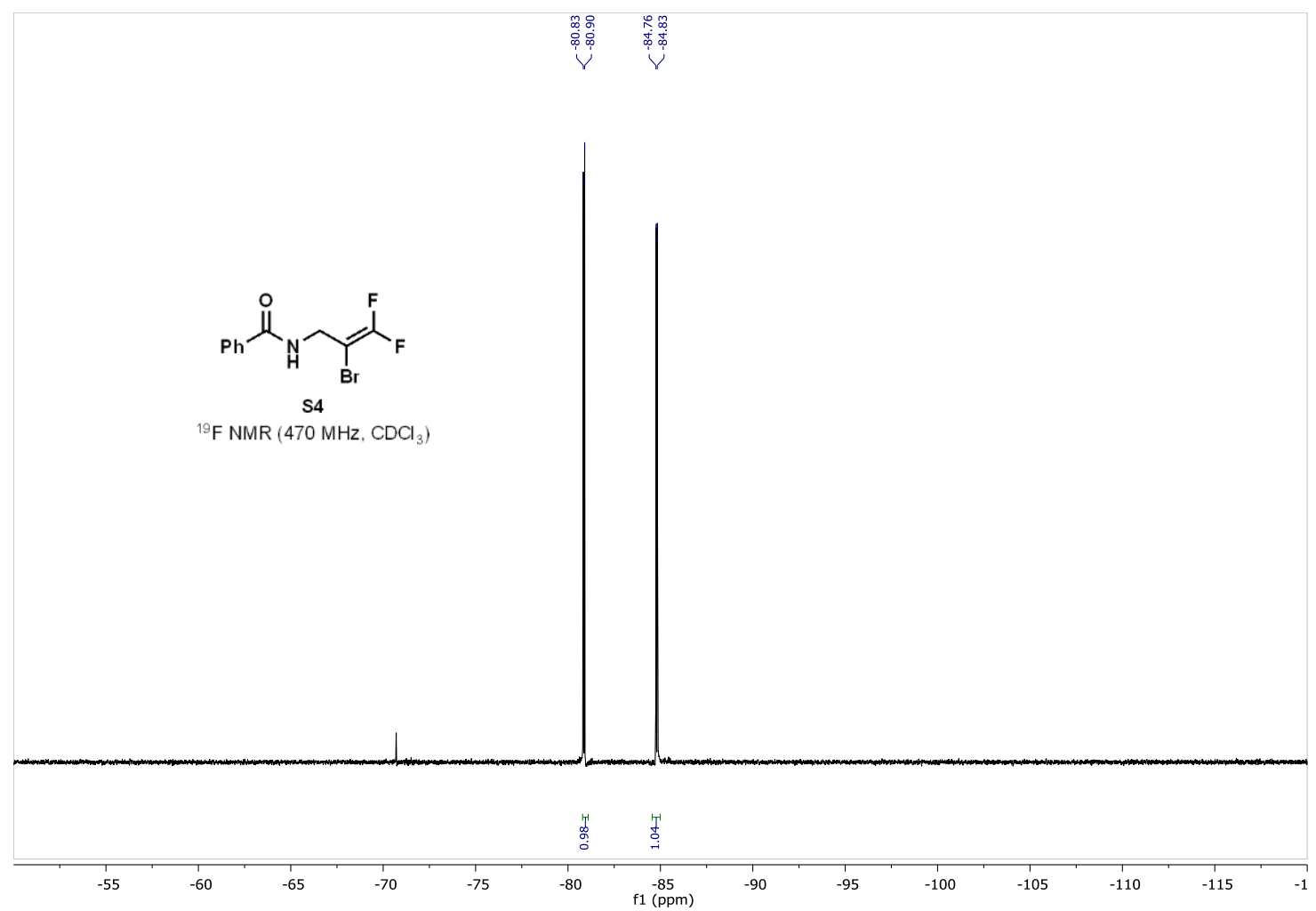



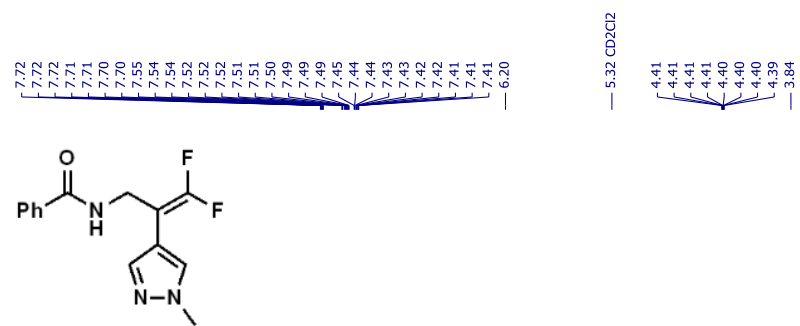

${ }^{1} \mathrm{H} \operatorname{NMR}\left(500 \mathrm{MHz}, \mathrm{CD}_{2} \mathrm{Cl}_{2}\right)$
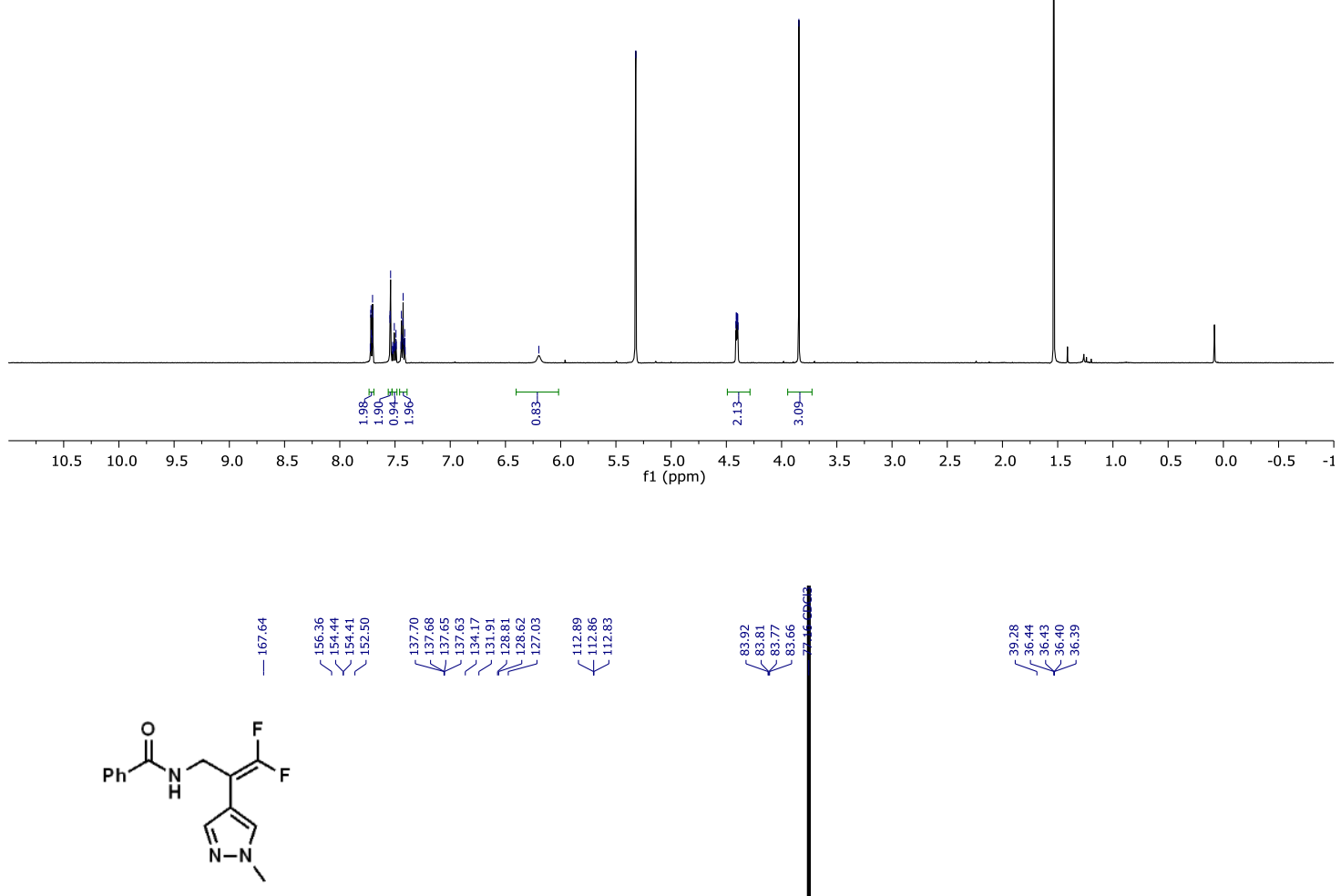

$1 \mathrm{k}$

${ }^{13} \mathrm{C} \mathrm{NMR}\left(151 \mathrm{MHz}, \mathrm{CDCl}_{3}\right)$

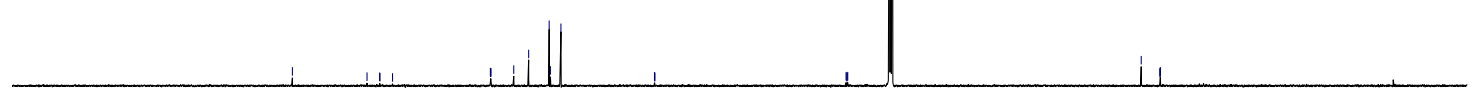

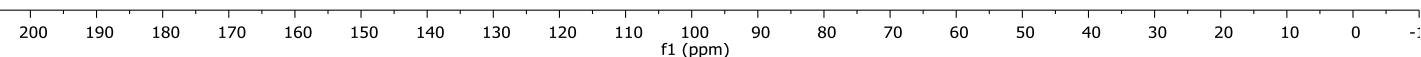




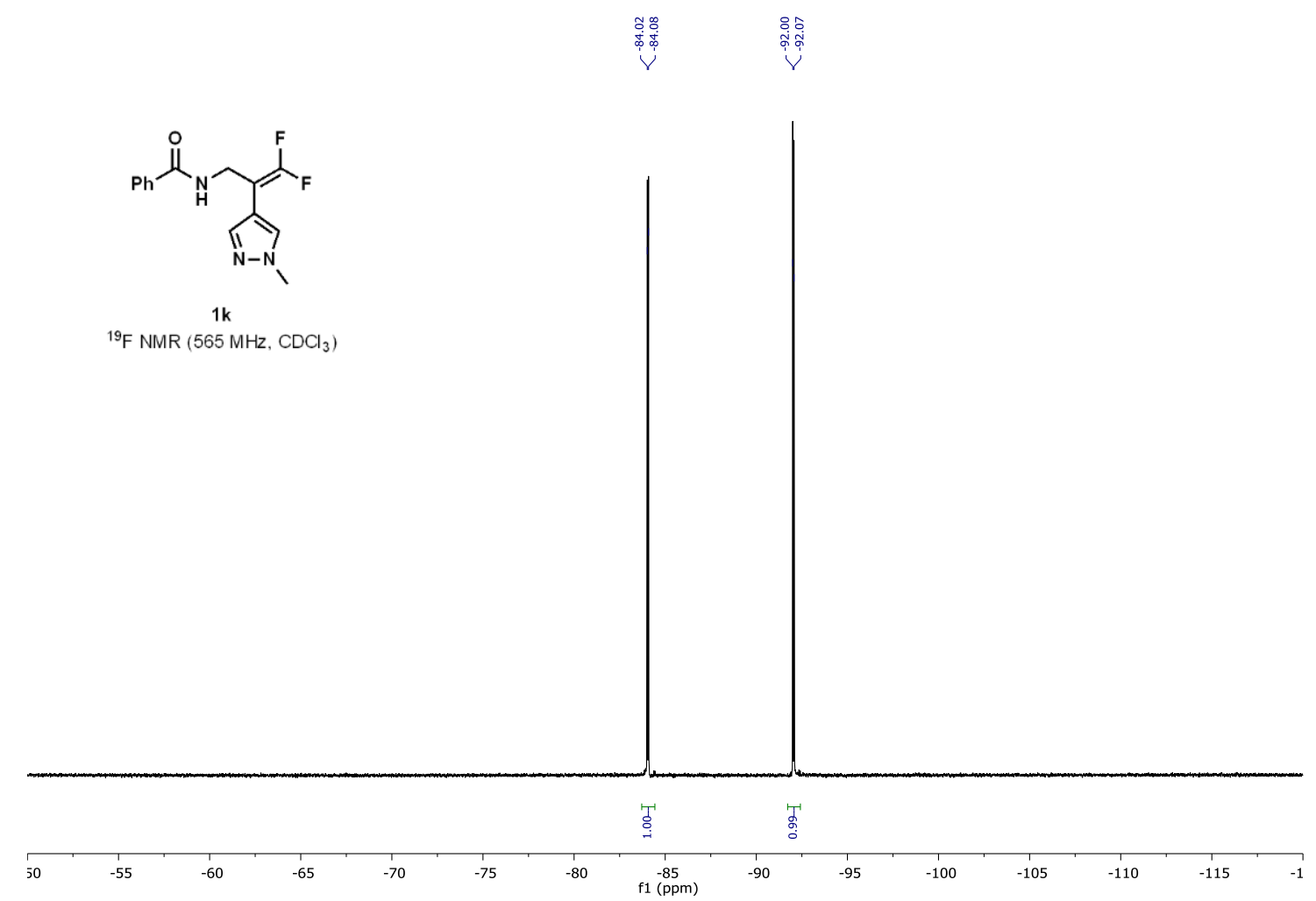



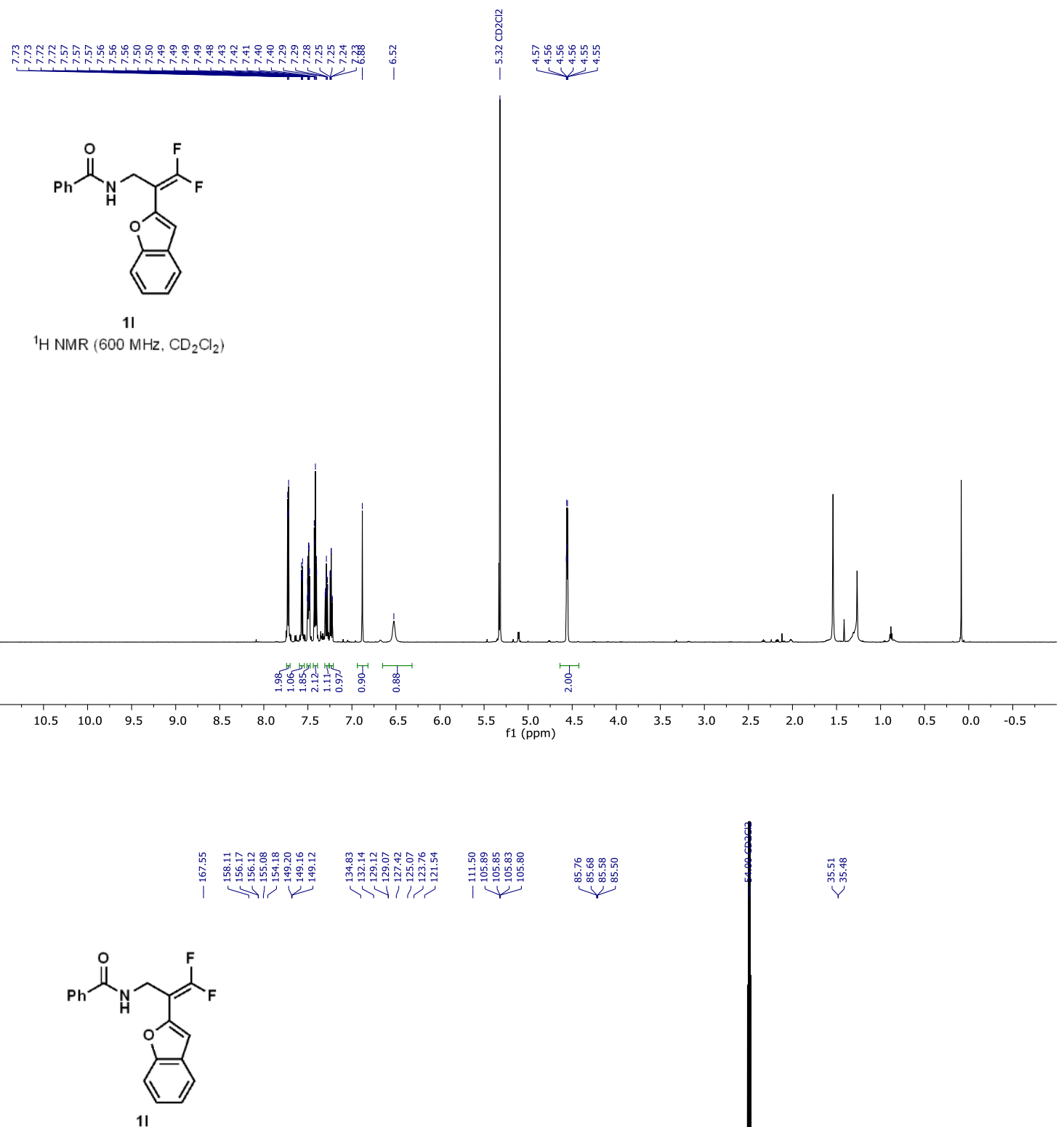

${ }^{13} \mathrm{CNMR}\left(151 \mathrm{MHz}, \mathrm{CD}_{2} \mathrm{Cl}_{2}\right)$

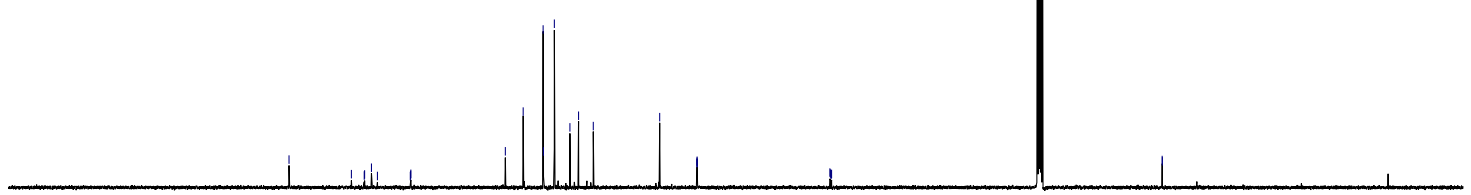

10
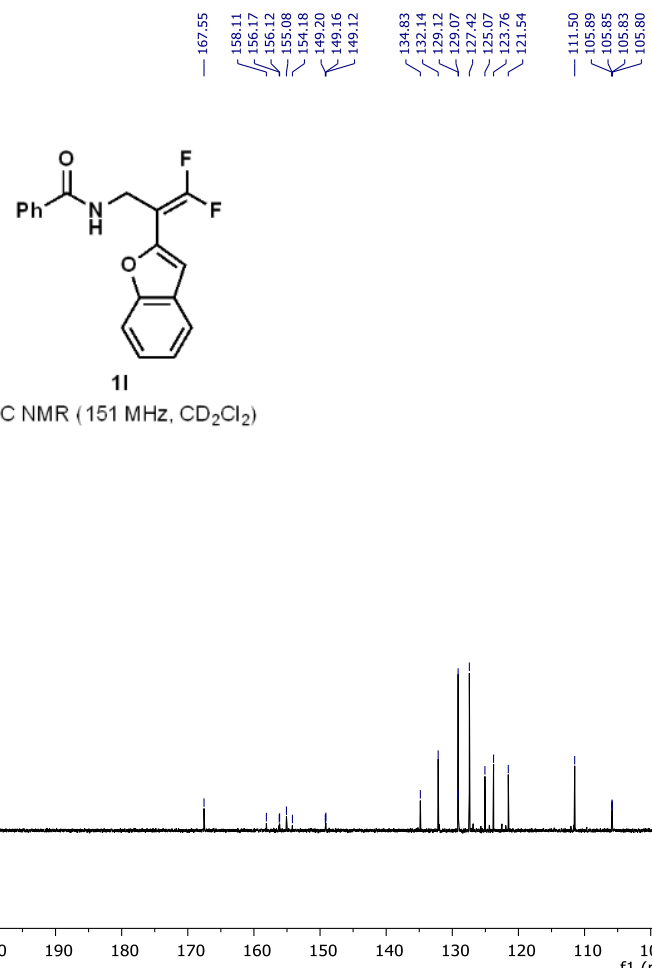

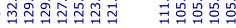

रप्रा1?

14

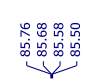

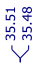

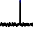




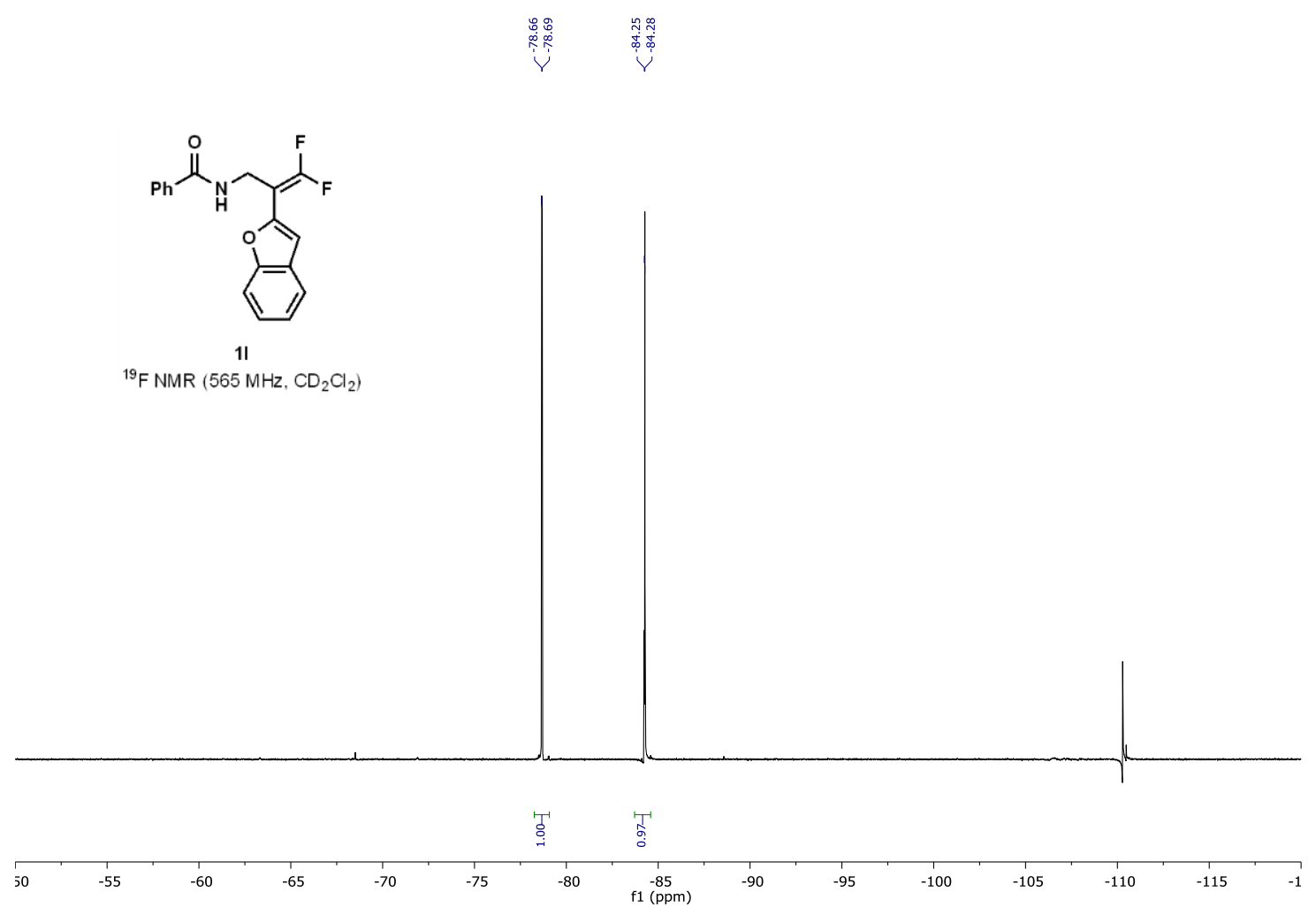



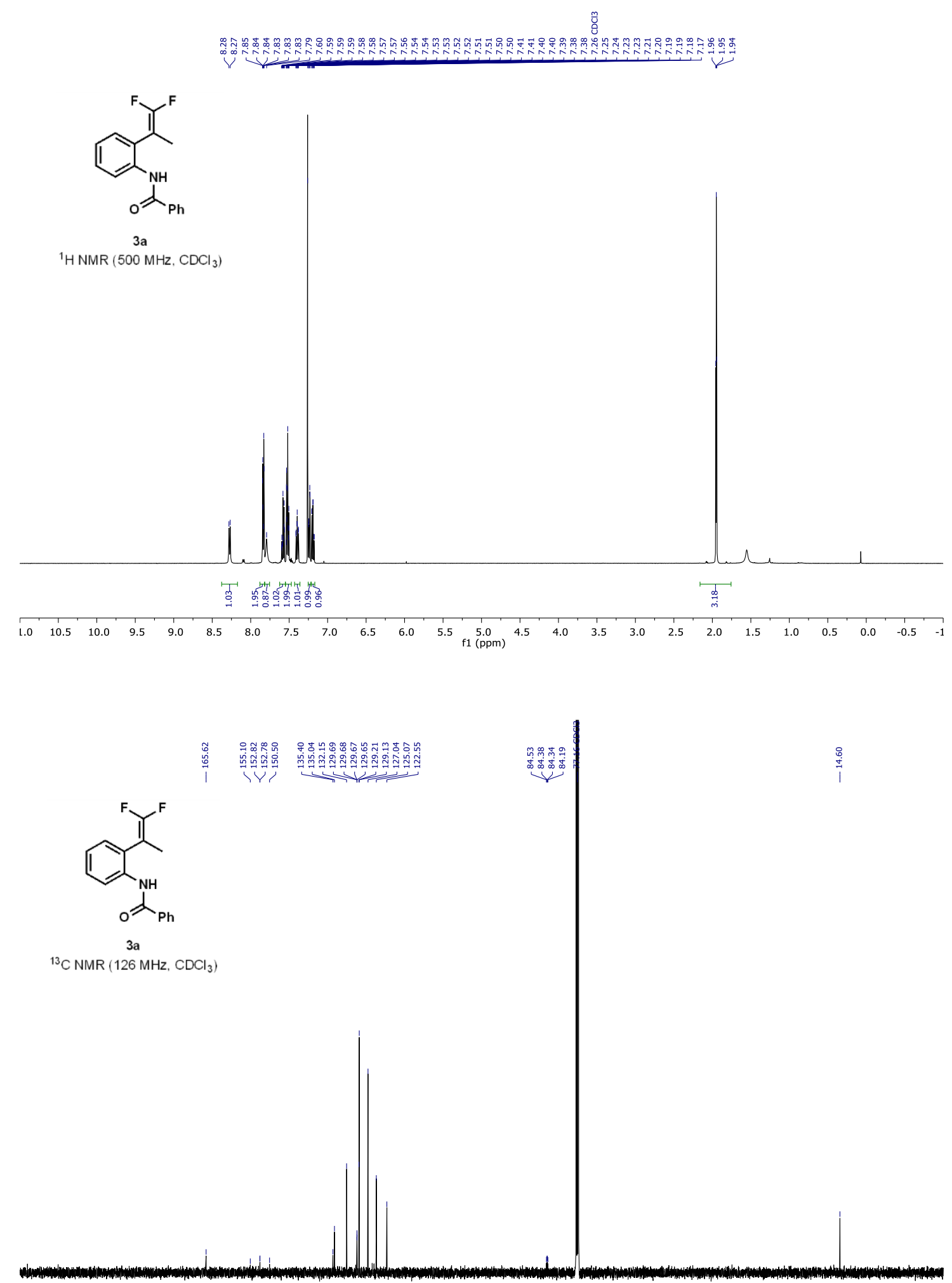

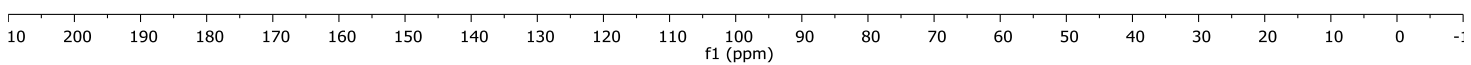




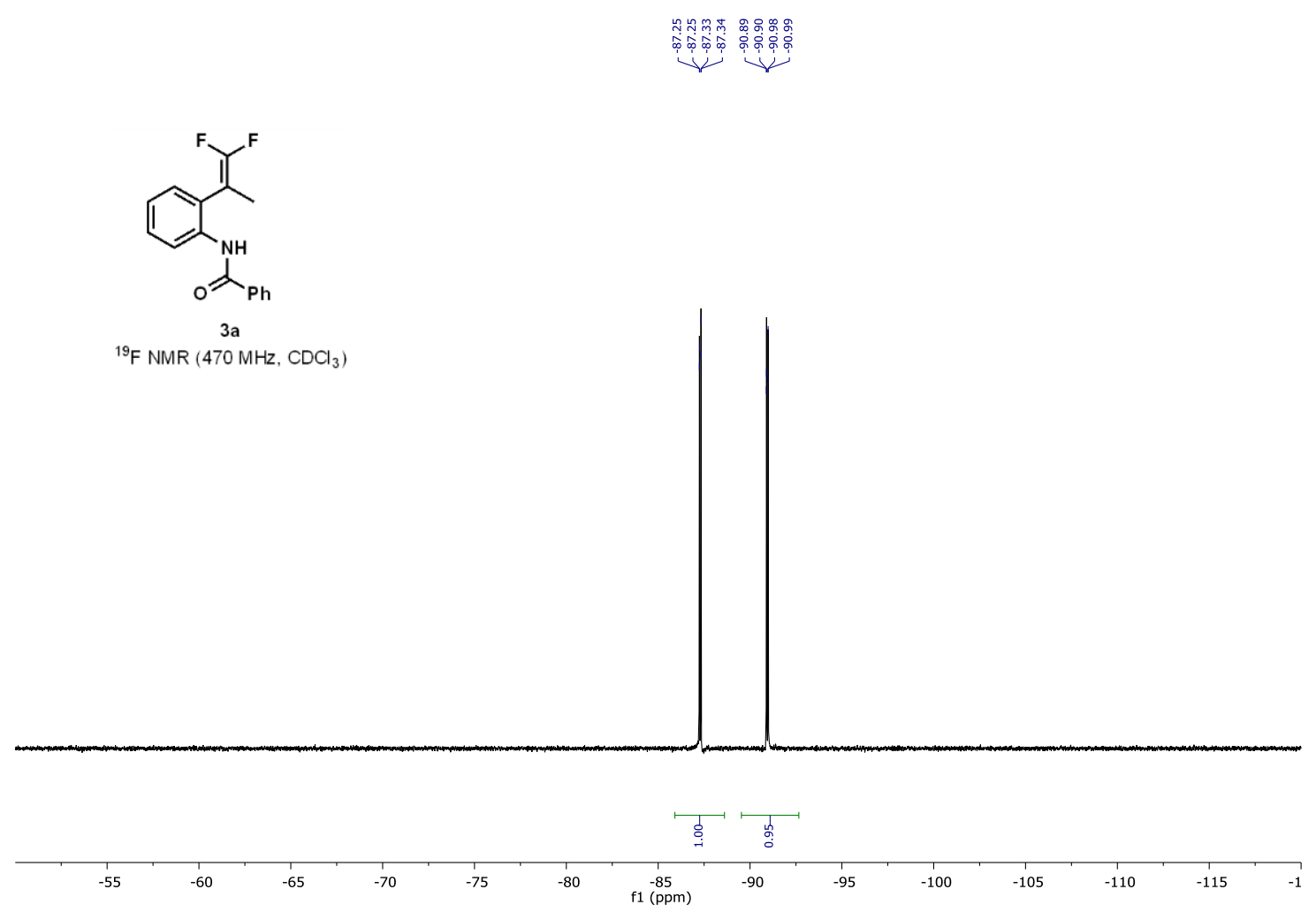



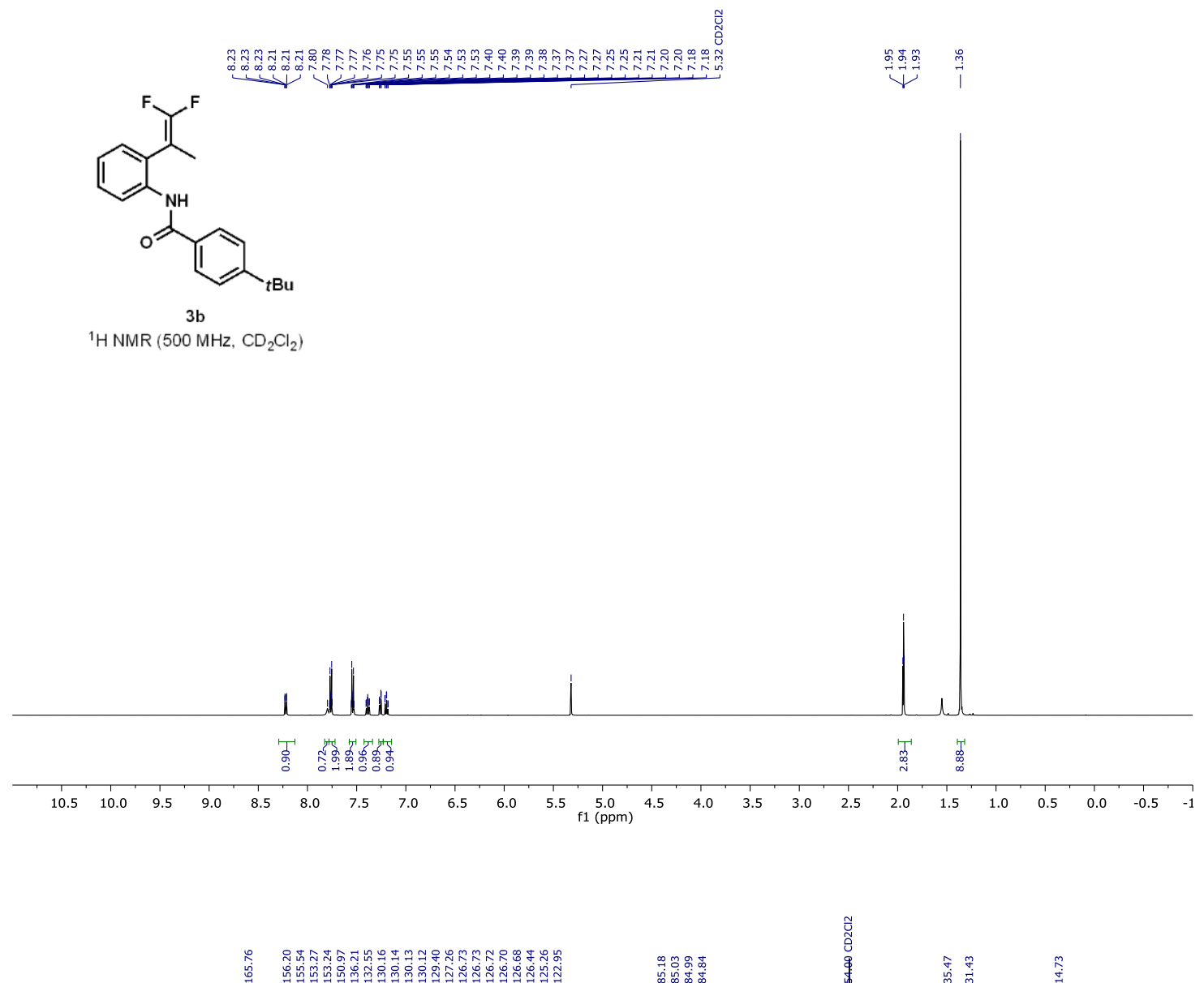

$\underbrace{3}$
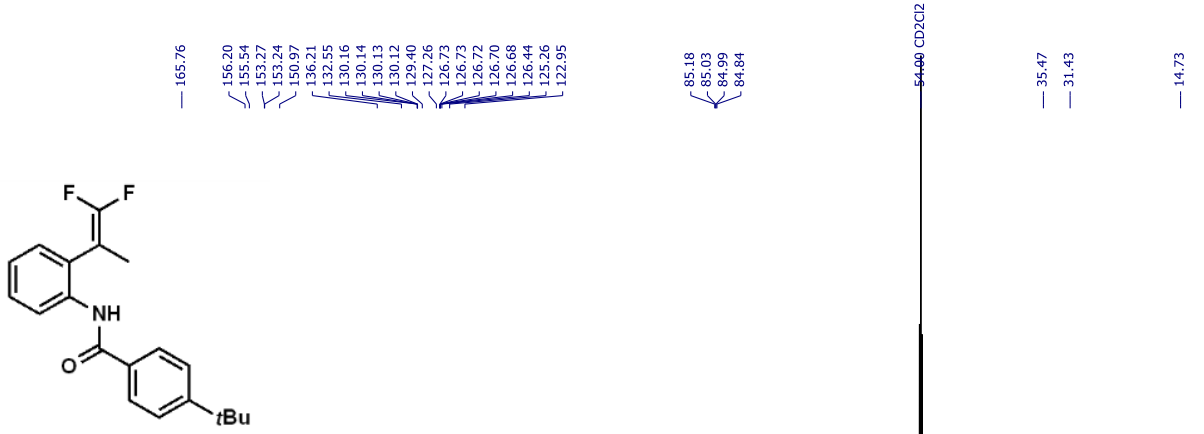

$3 b$

${ }^{13} \mathrm{CNMR}\left(126 \mathrm{MHz}, \mathrm{CD}_{2} \mathrm{Cl}_{2}\right.$ )

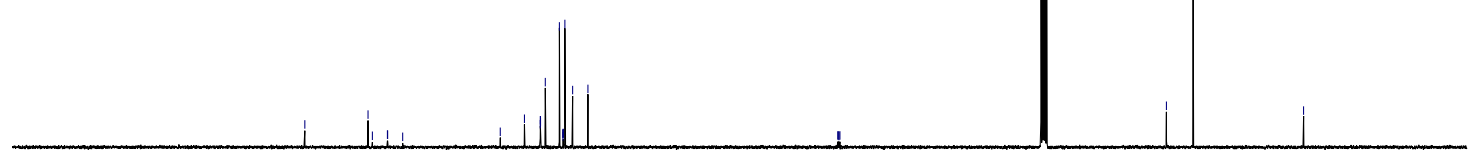

f1 (ppm) 


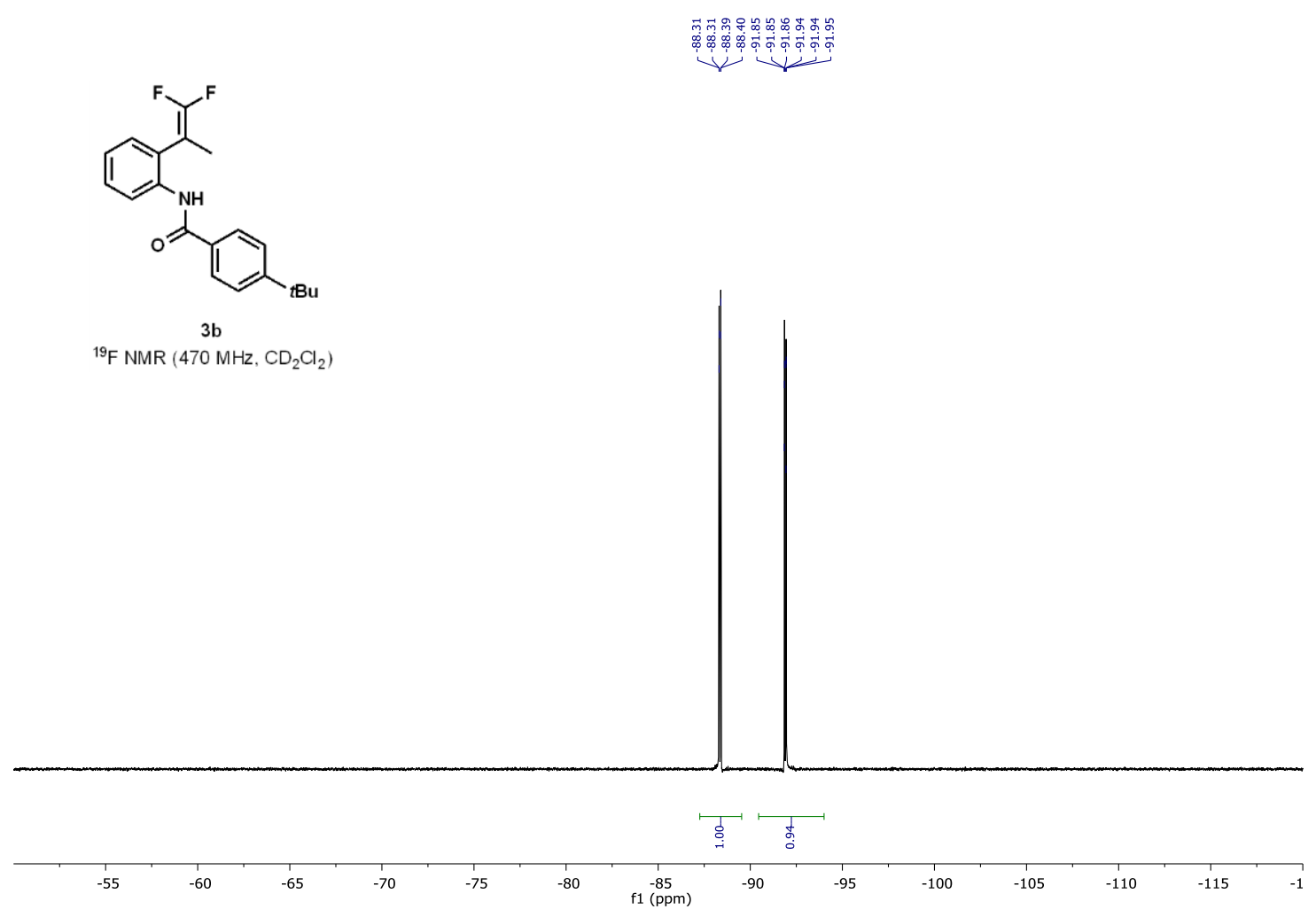




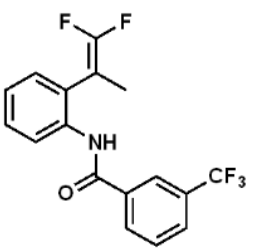

$3 c$

${ }^{1} \mathrm{H} \operatorname{NMR}\left(500 \mathrm{MHz}, \mathrm{CDCl}_{3}\right)$
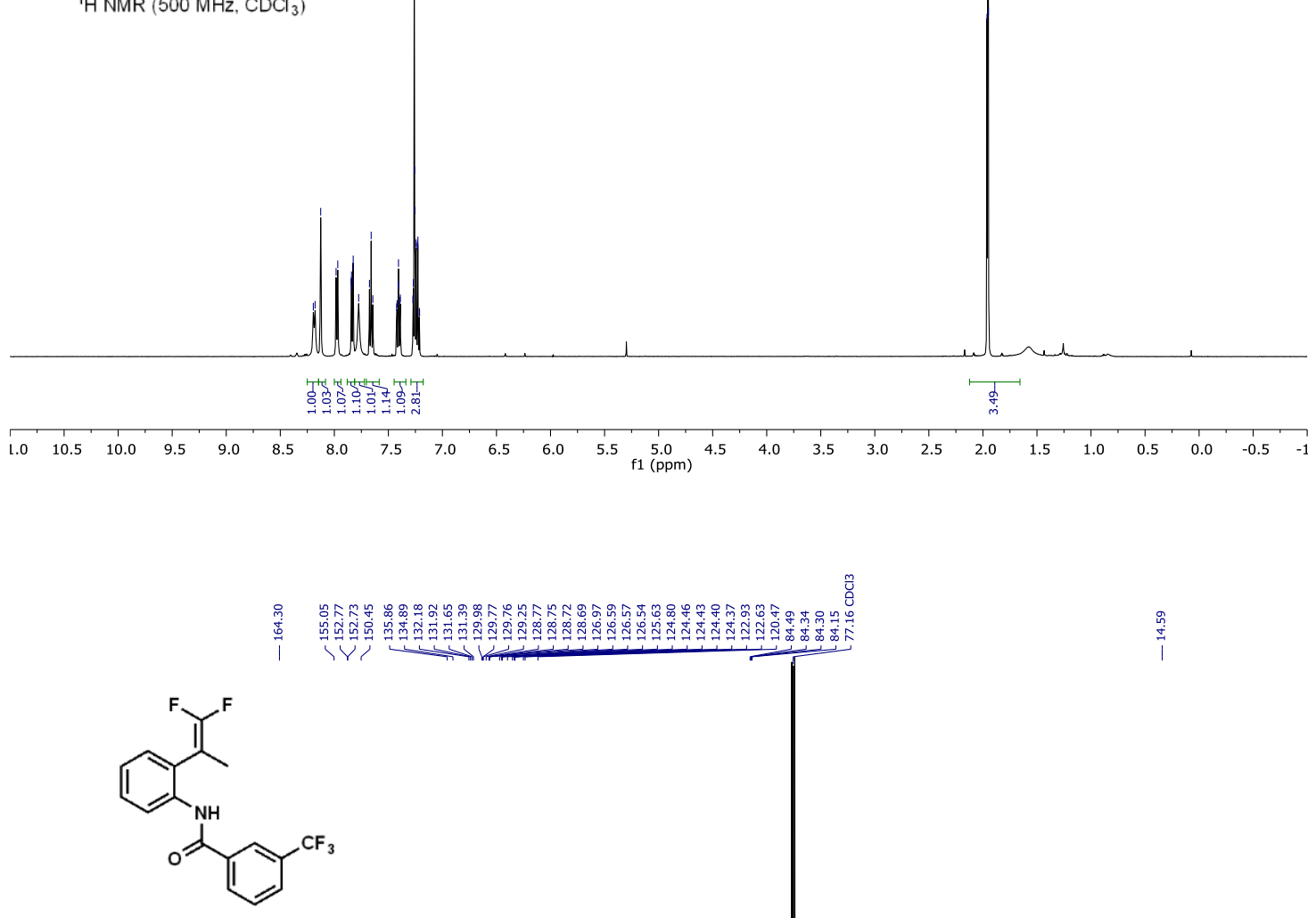

$3 \mathrm{c}$

${ }^{13} \mathrm{C}$ NMR ( $\left.126 \mathrm{MHz}, \mathrm{CDCl}_{3}\right)$

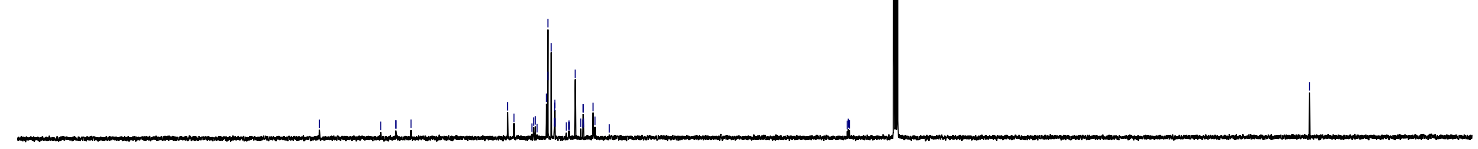

10

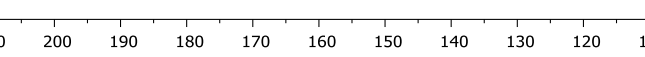

f1 $(\mathrm{ppm})$ 


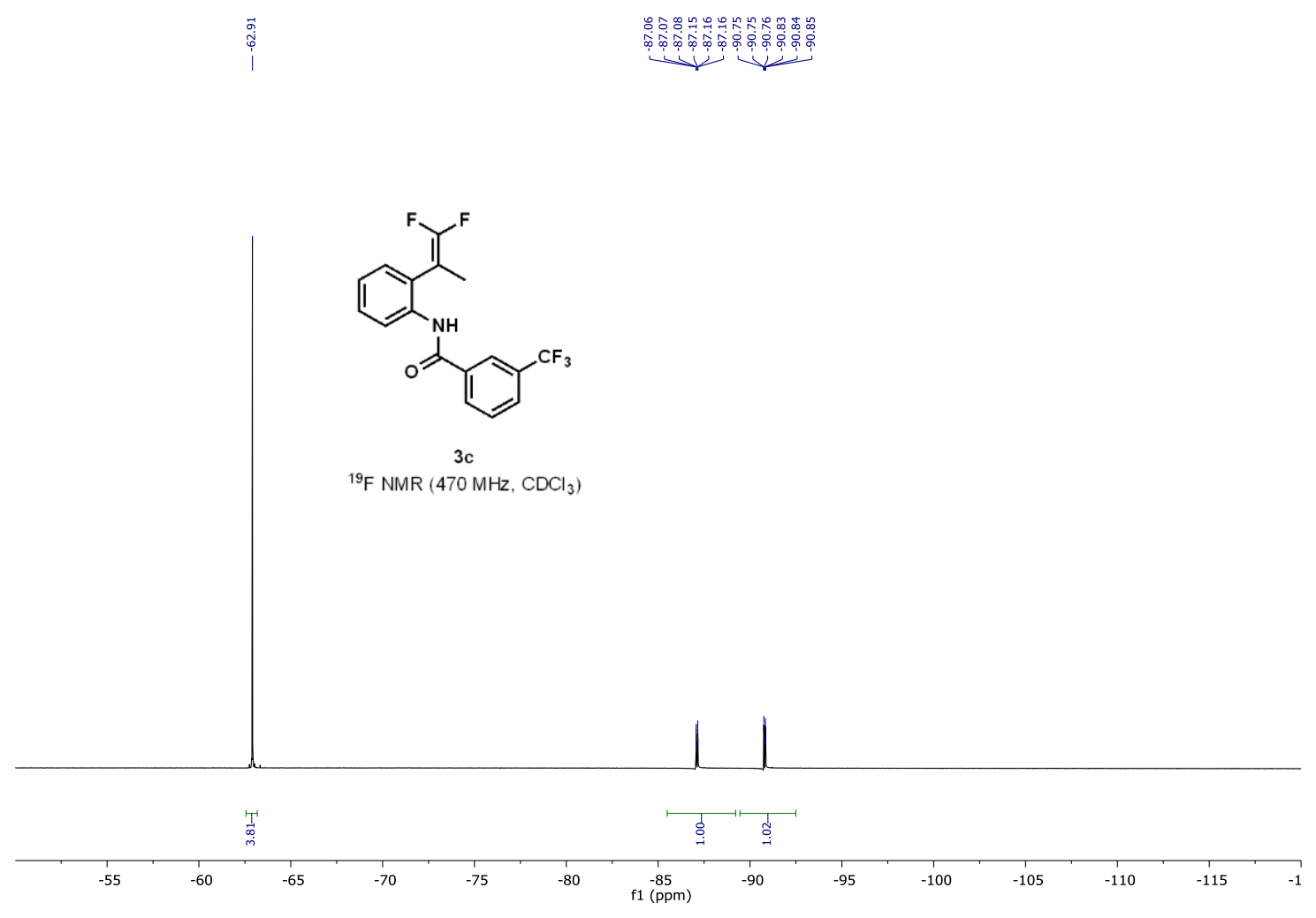



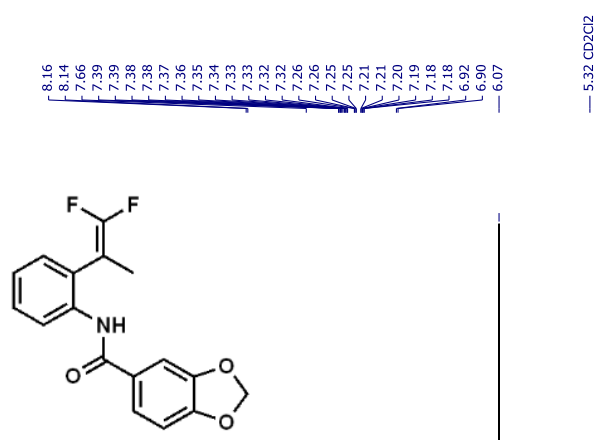

3d

${ }^{1} \mathrm{H} \mathrm{NMR}\left(600 \mathrm{MHz}, \mathrm{CD}_{2} \mathrm{Cl}_{2}\right)$

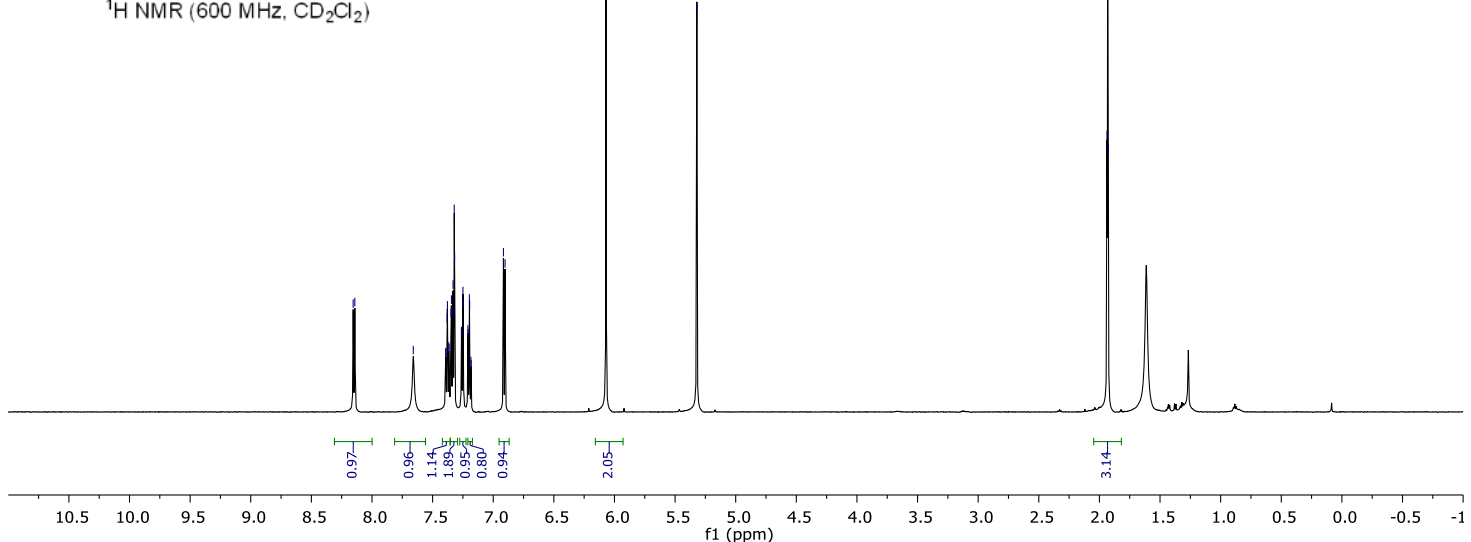

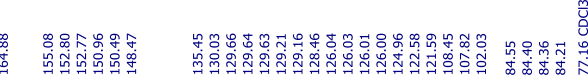

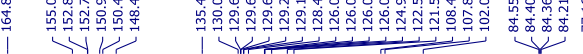

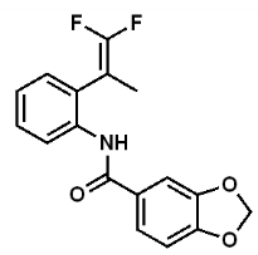

$3 d$

${ }^{13} \mathrm{C} \mathrm{NMR}$ ( $126 \mathrm{MHz}, \mathrm{CDCl}_{3}$ )
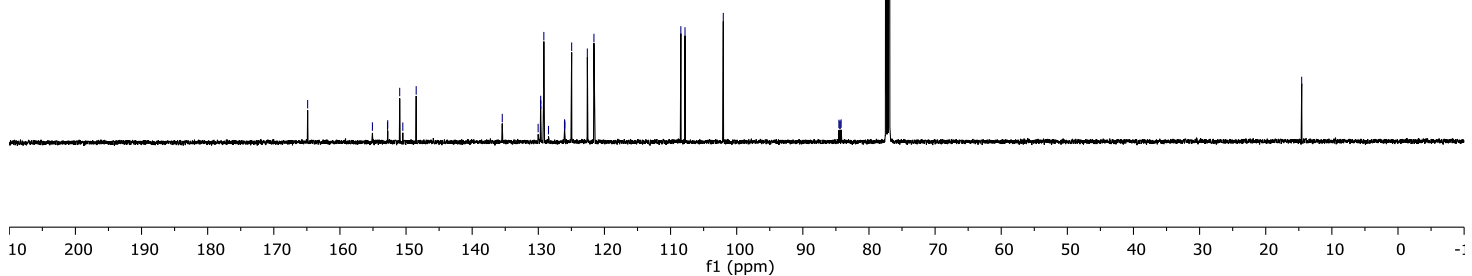


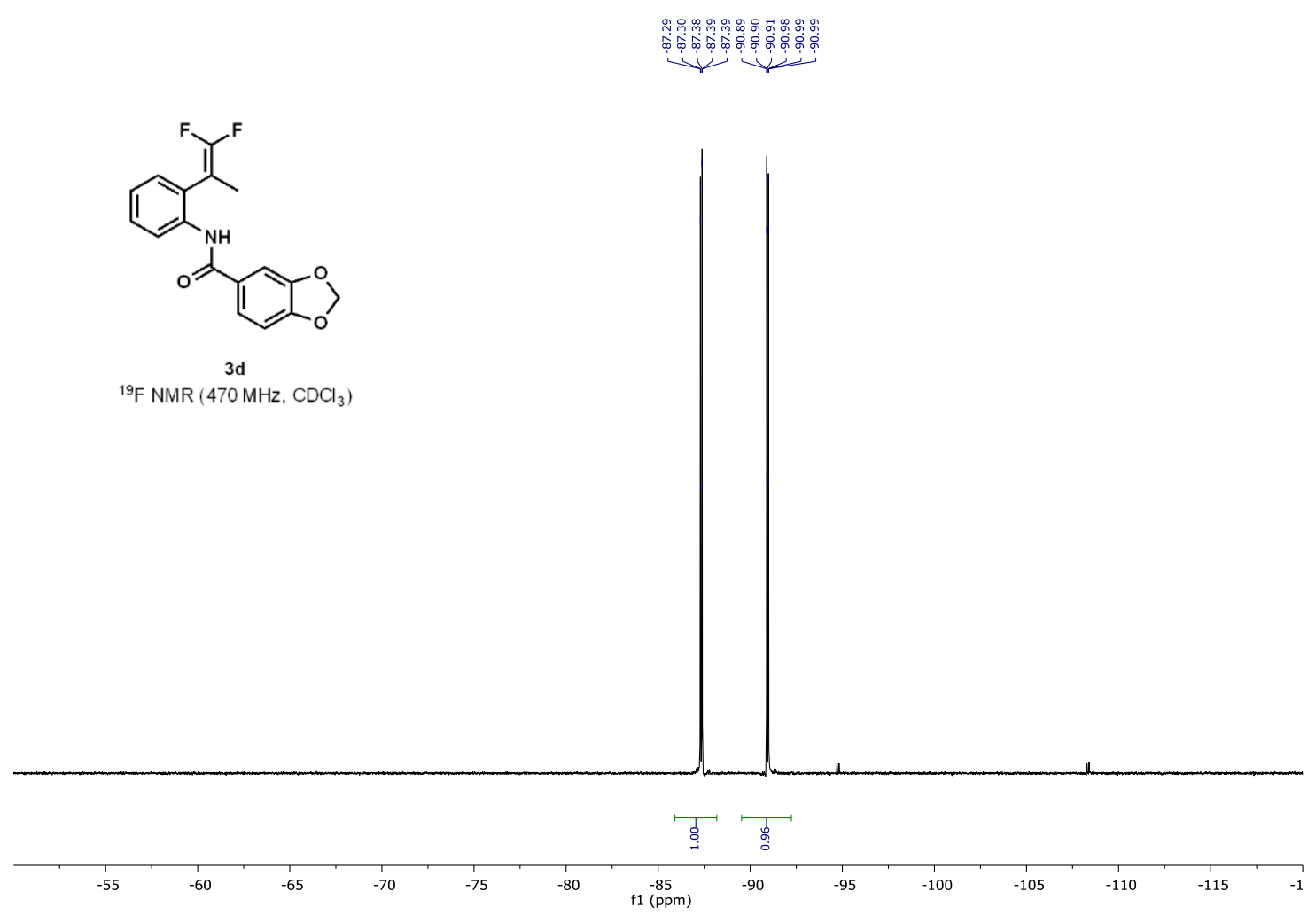



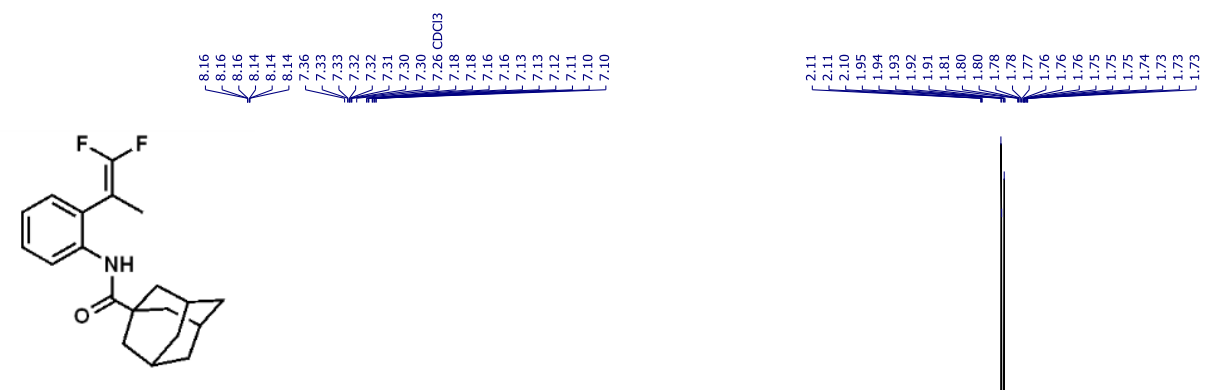

${ }^{1} \mathrm{H} \mathrm{NMR}\left(500 \mathrm{MHz}, \mathrm{CDCl}_{3}\right)$
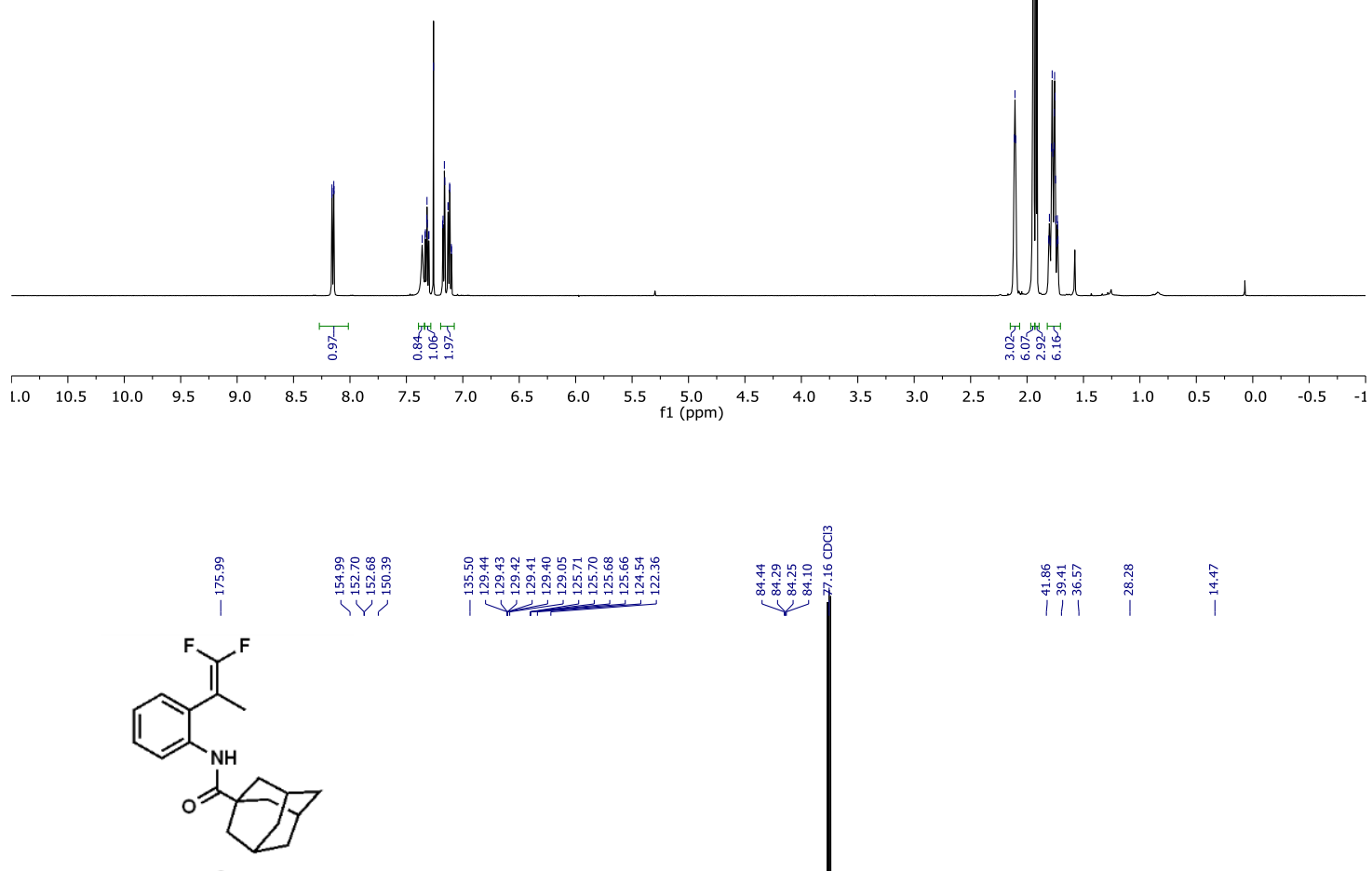

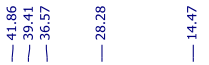

${ }^{13} \mathrm{C} \mathrm{NMR}\left(126 \mathrm{MHz}, \mathrm{CDCl}_{3}\right.$

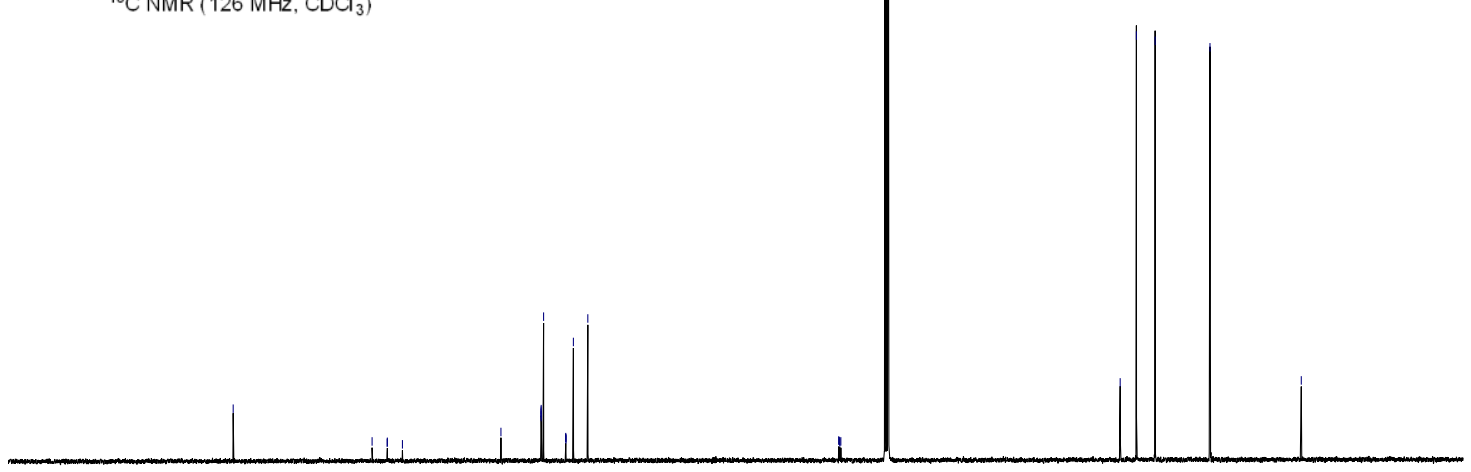

10

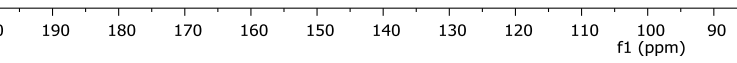




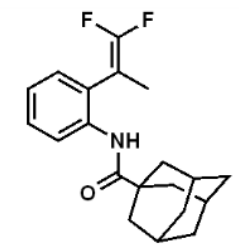

$3 e$

${ }^{19} \mathrm{~F} \mathrm{NMR}\left(376 \mathrm{MHz}, \mathrm{CDCl}_{3}\right)$

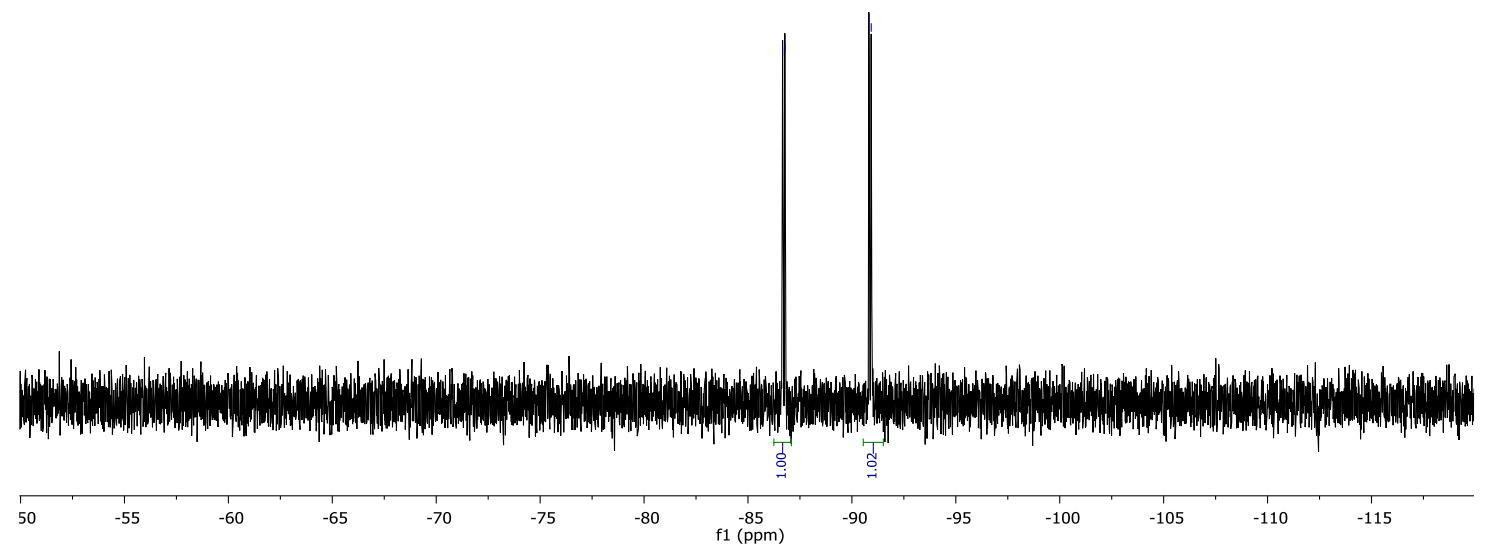

6 

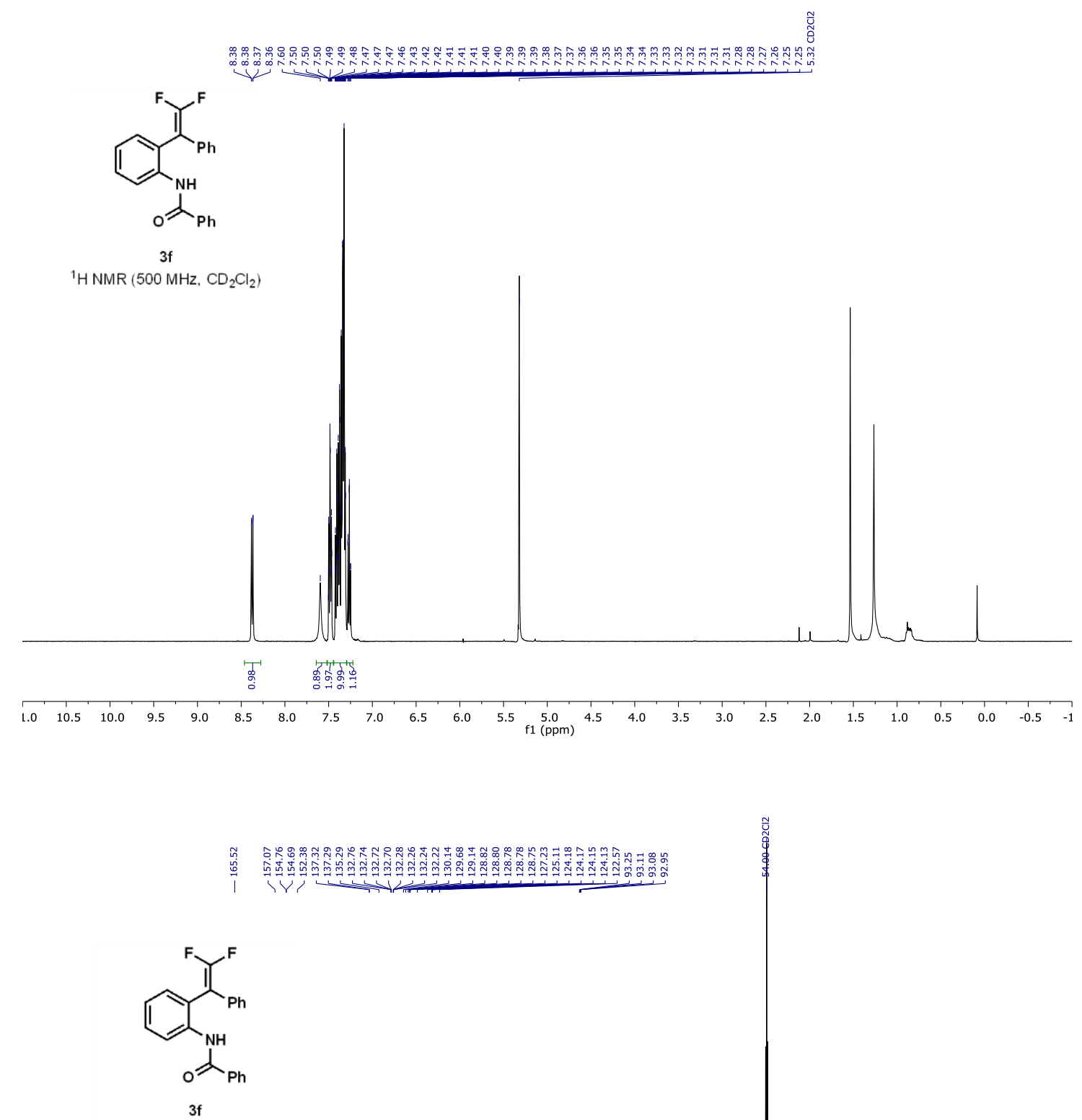

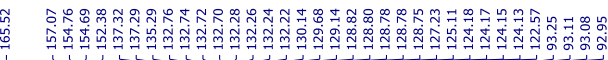

${ }^{13} \mathrm{CNMR}\left(126 \mathrm{MHz}, \mathrm{CD}_{2} \mathrm{Cl}_{2}\right)$

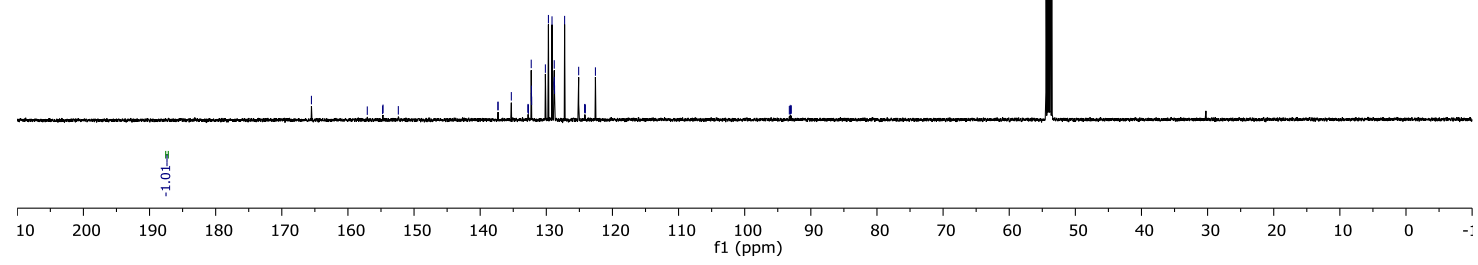




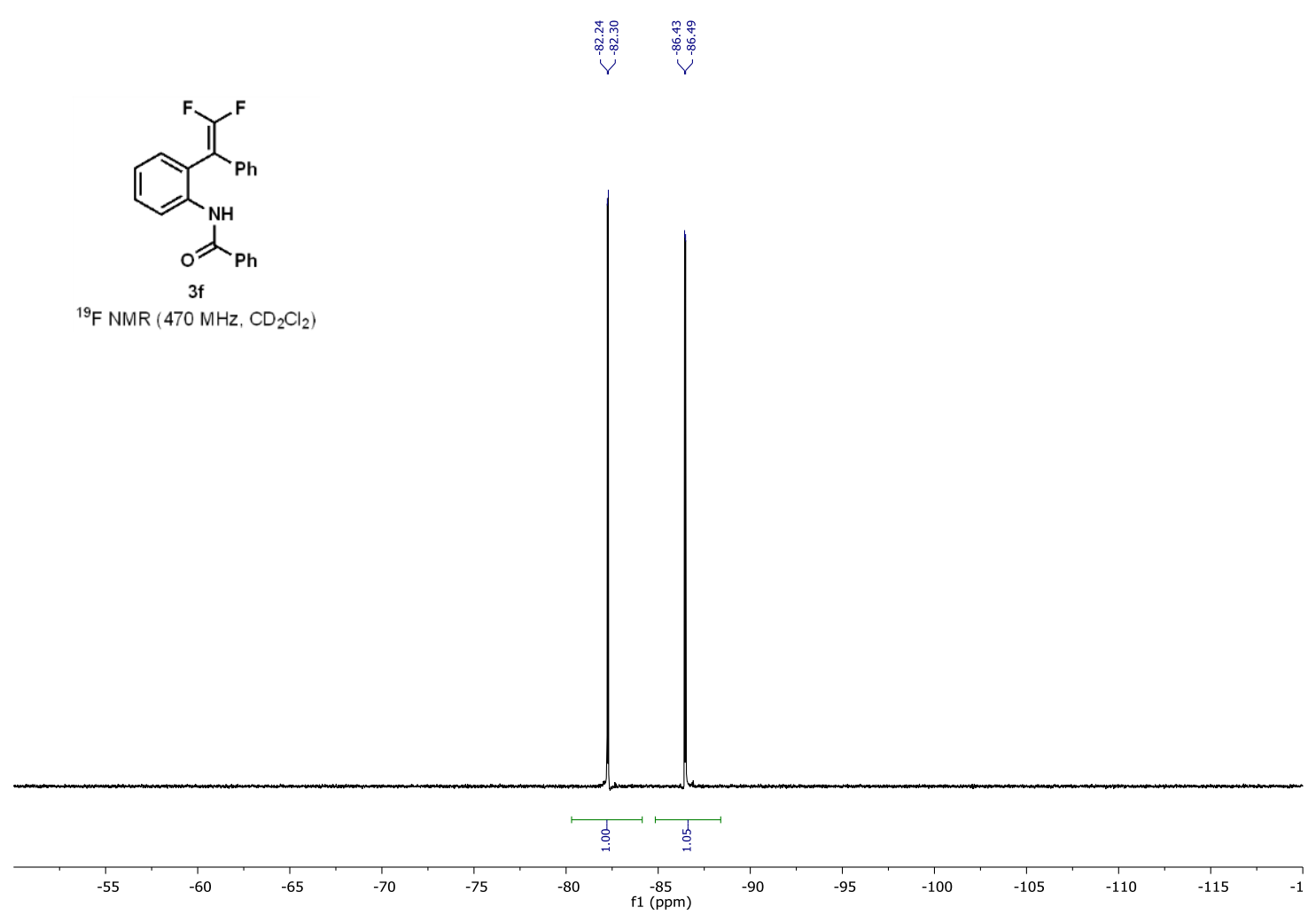



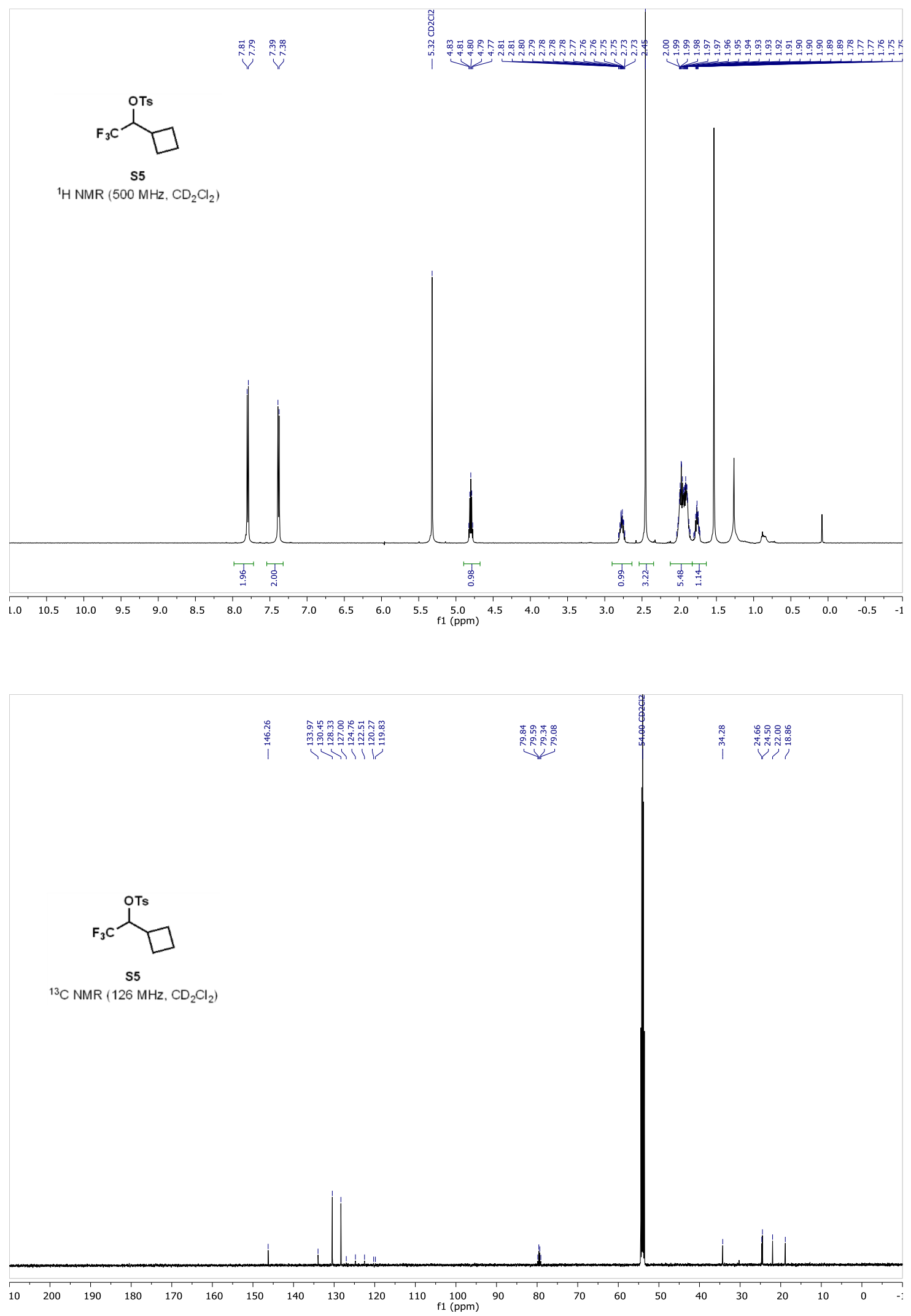


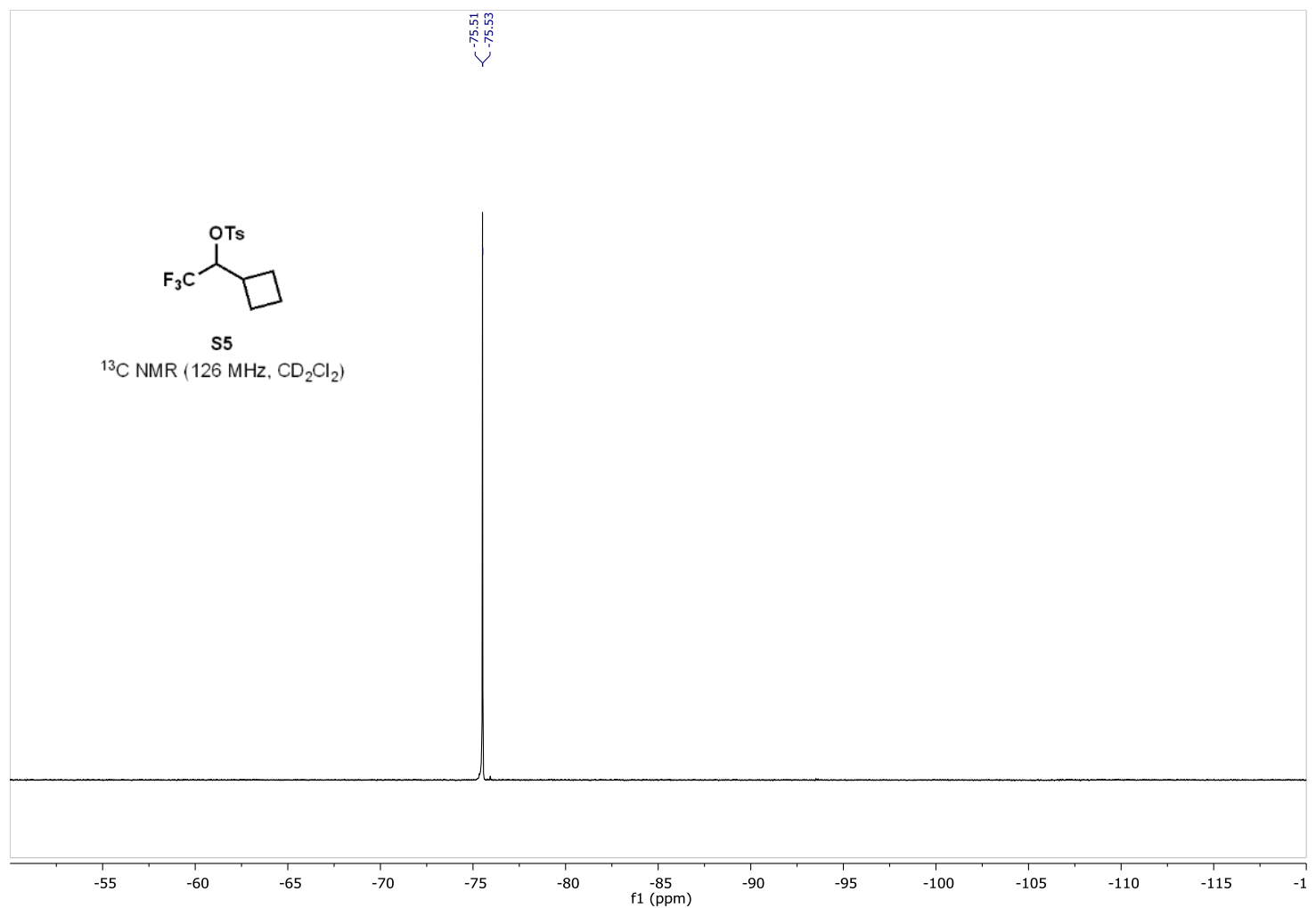



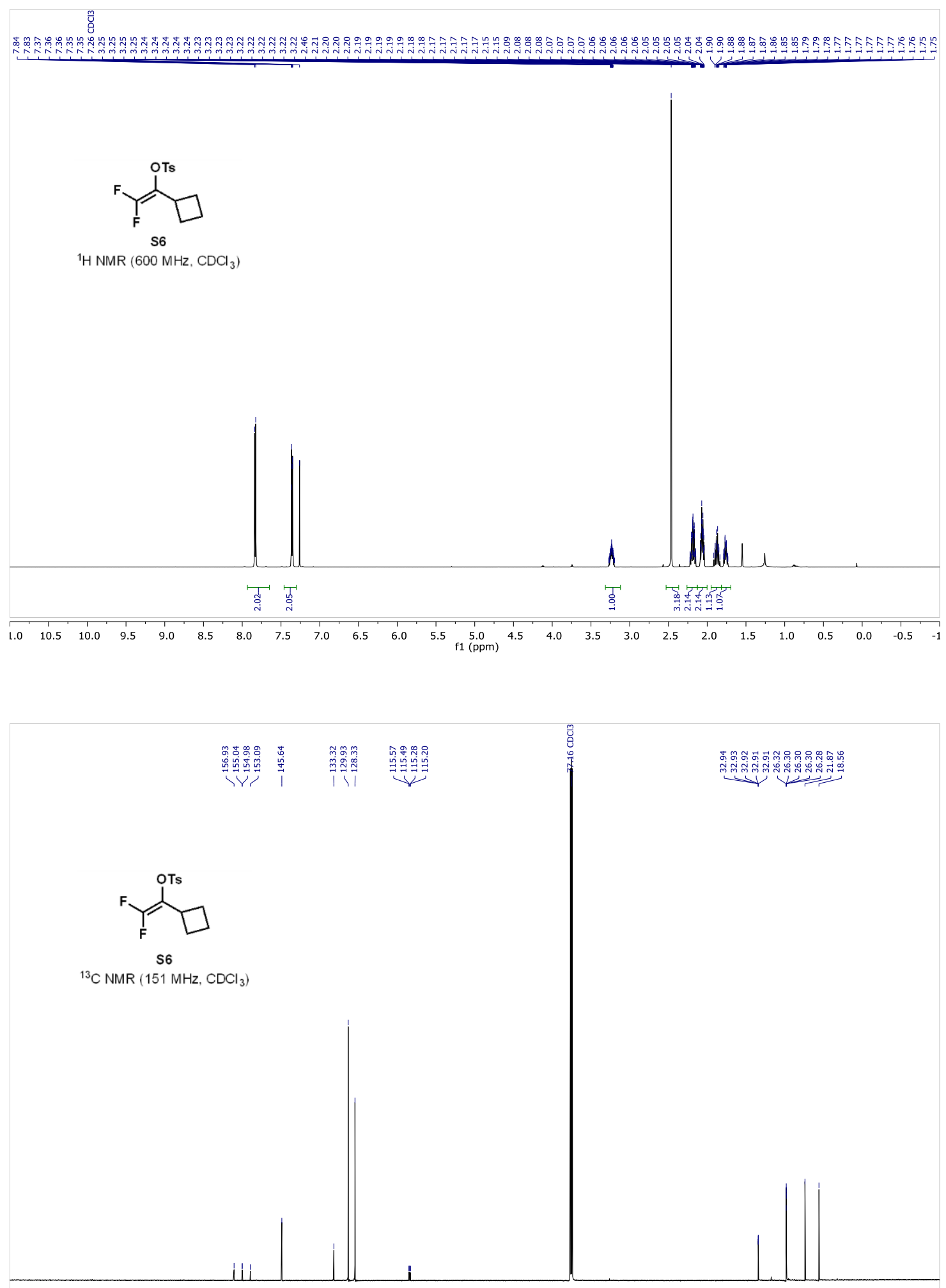

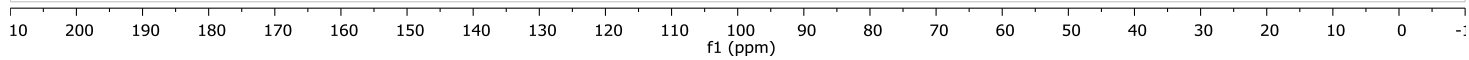




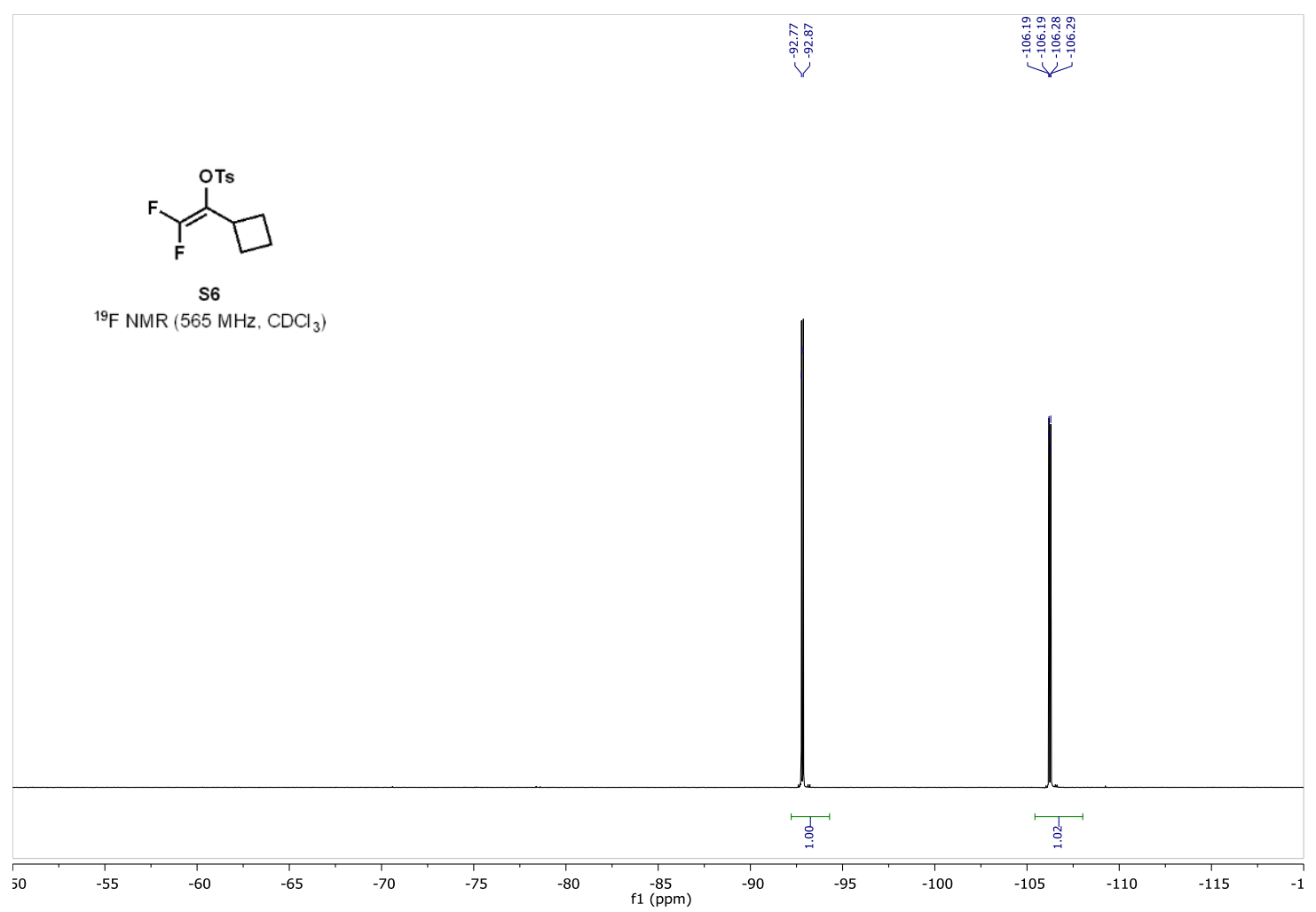



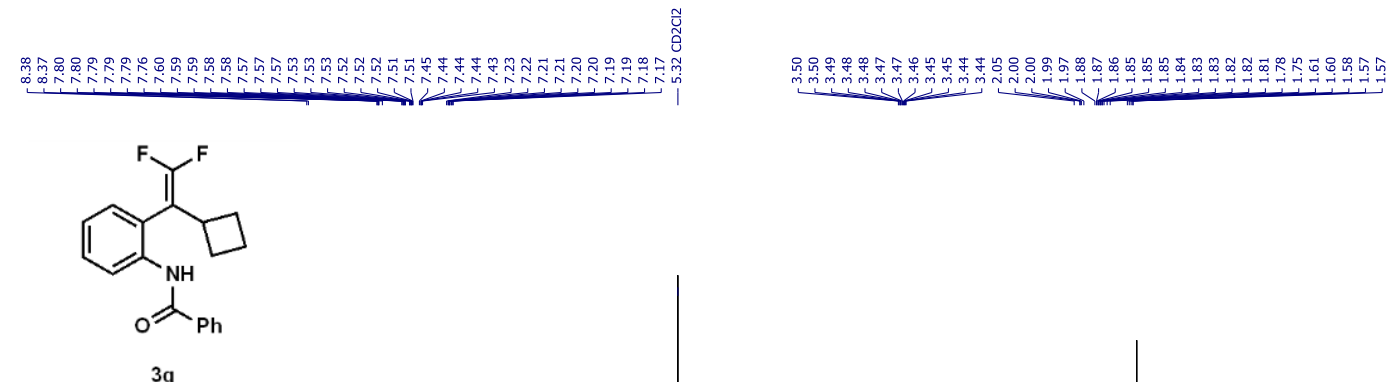

${ }^{1} \mathrm{HNMR}\left(600 \mathrm{MHz}, \mathrm{CD}_{2} \mathrm{Cl}_{2}\right)$
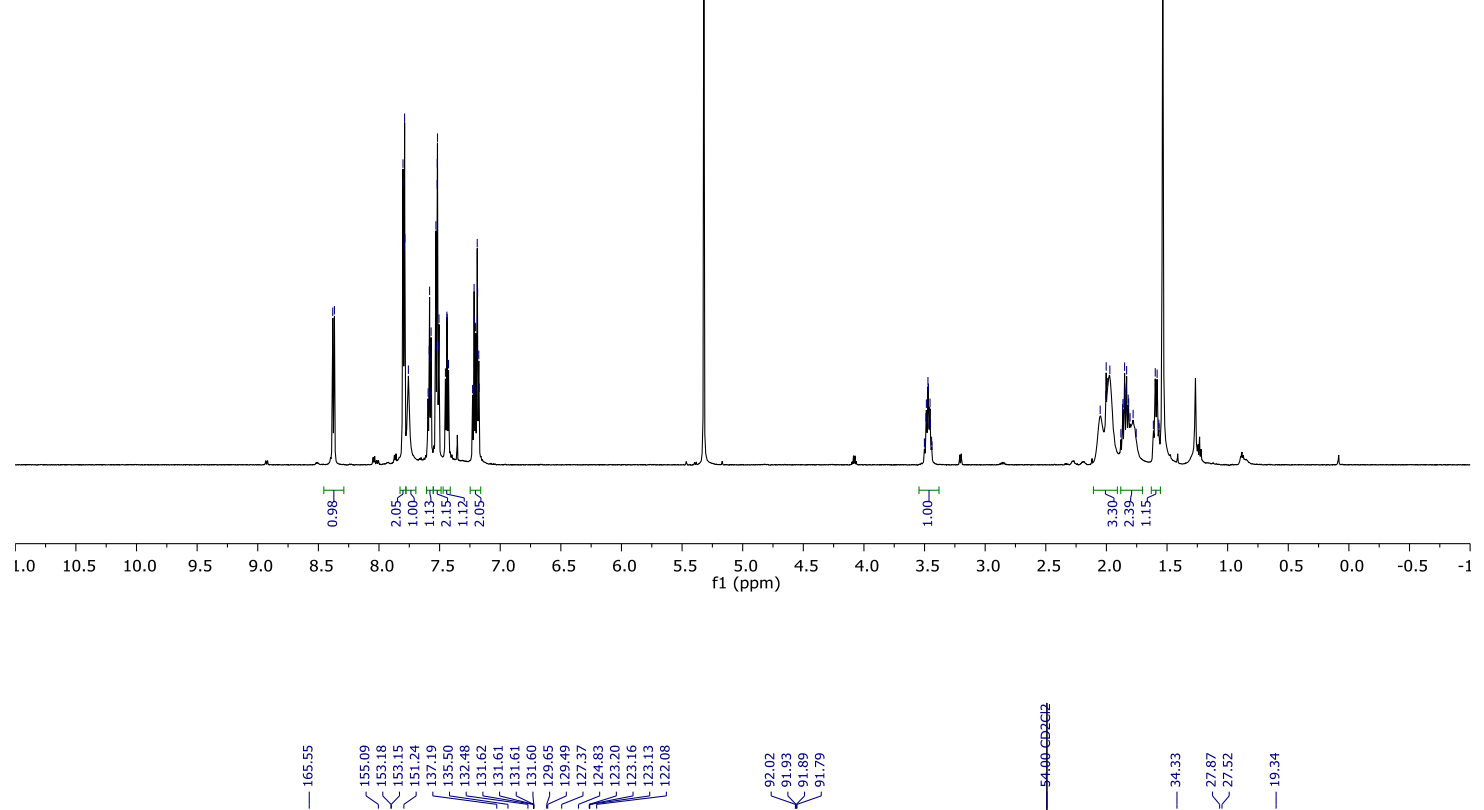

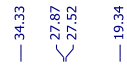

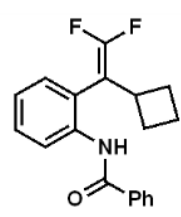

$3 \mathrm{~g}$

${ }^{13} \mathrm{C}$ NMR $\left(151 \mathrm{MHz}, \mathrm{CD}_{2} \mathrm{Cl}_{2}\right)$

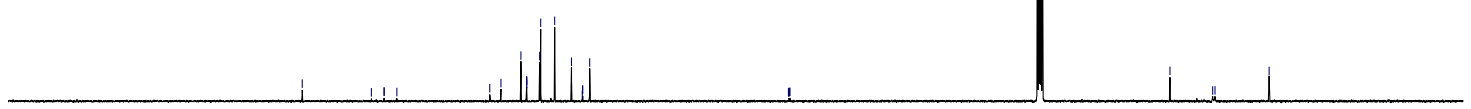

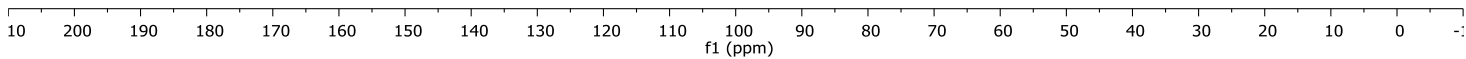




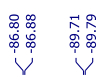

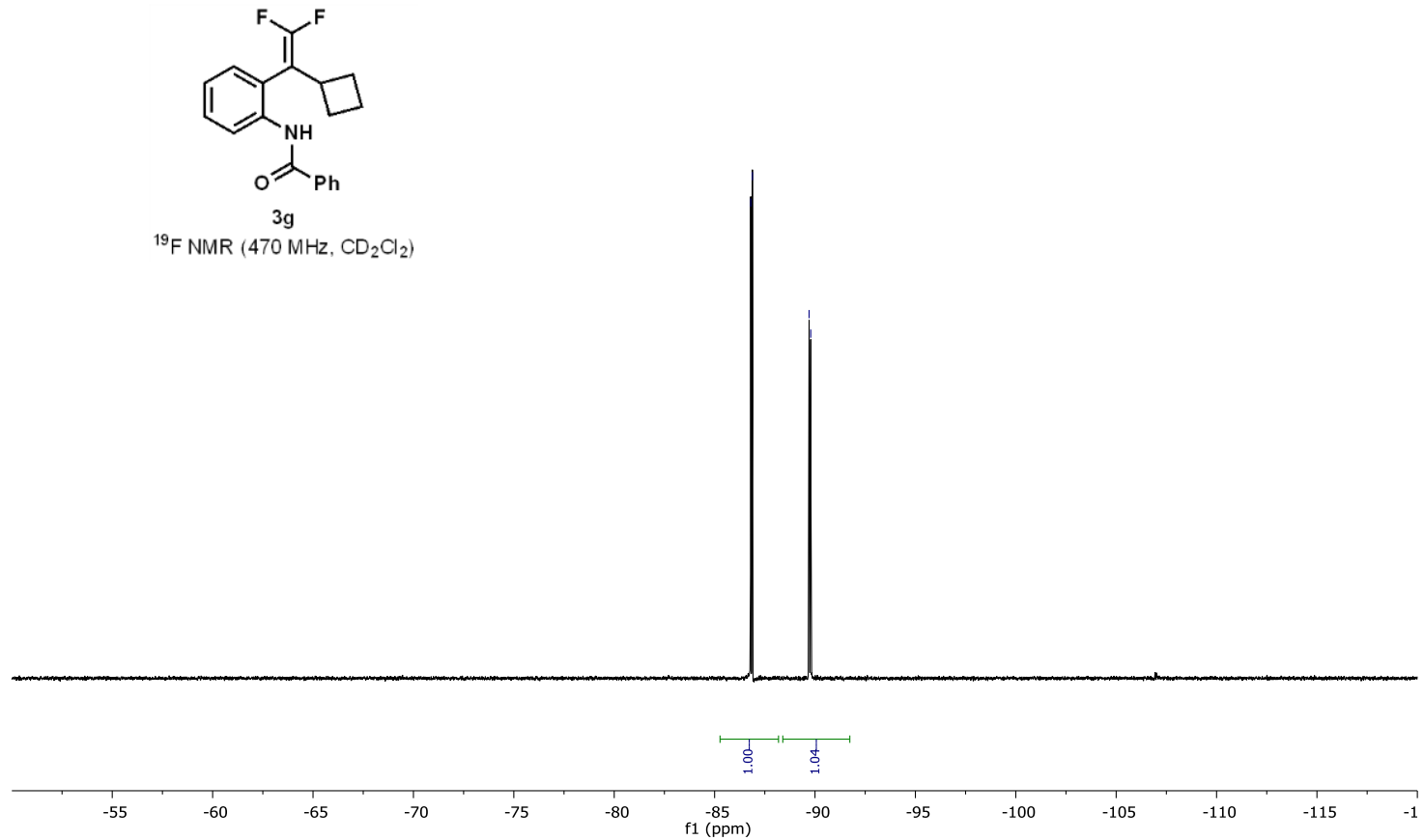



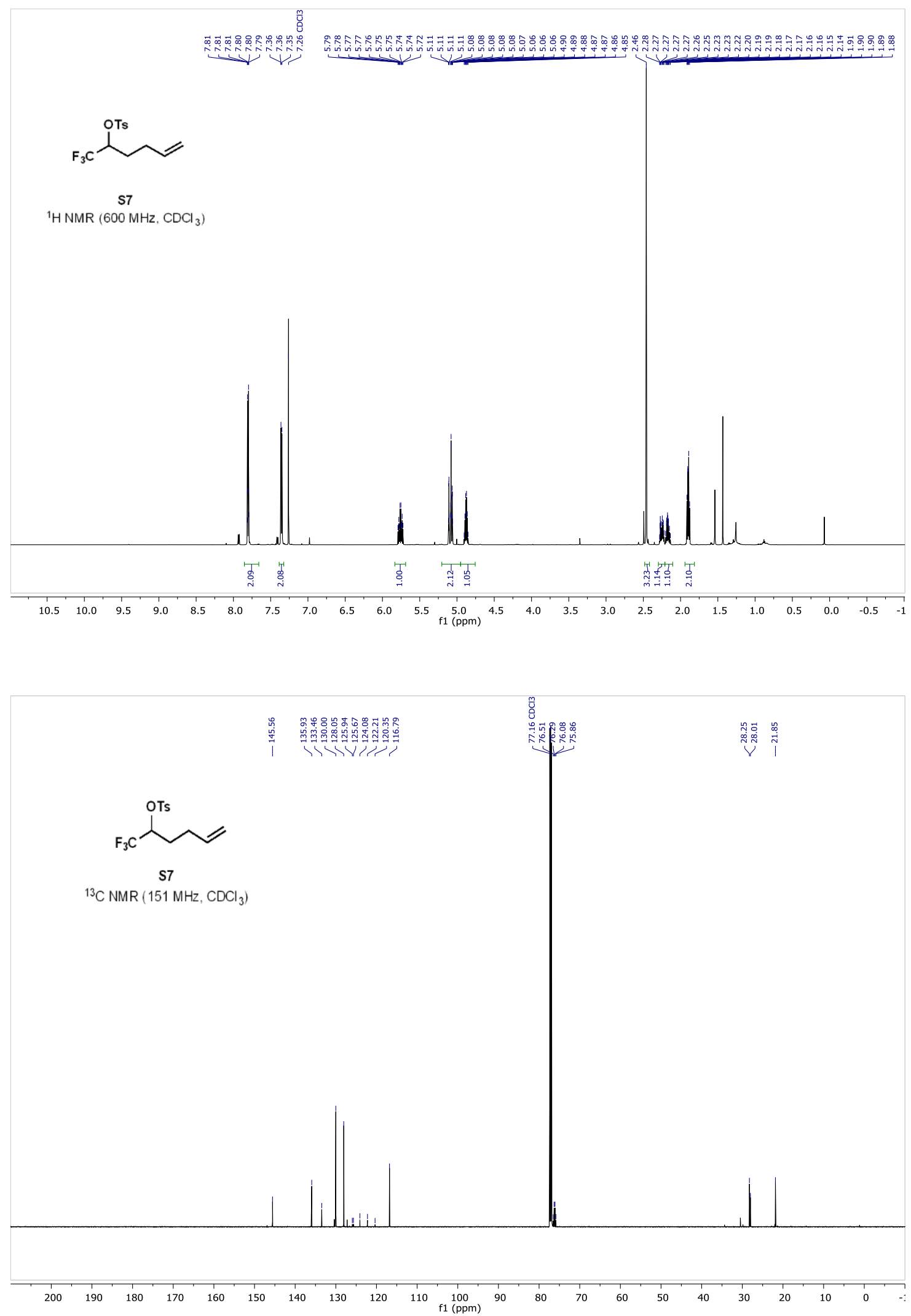


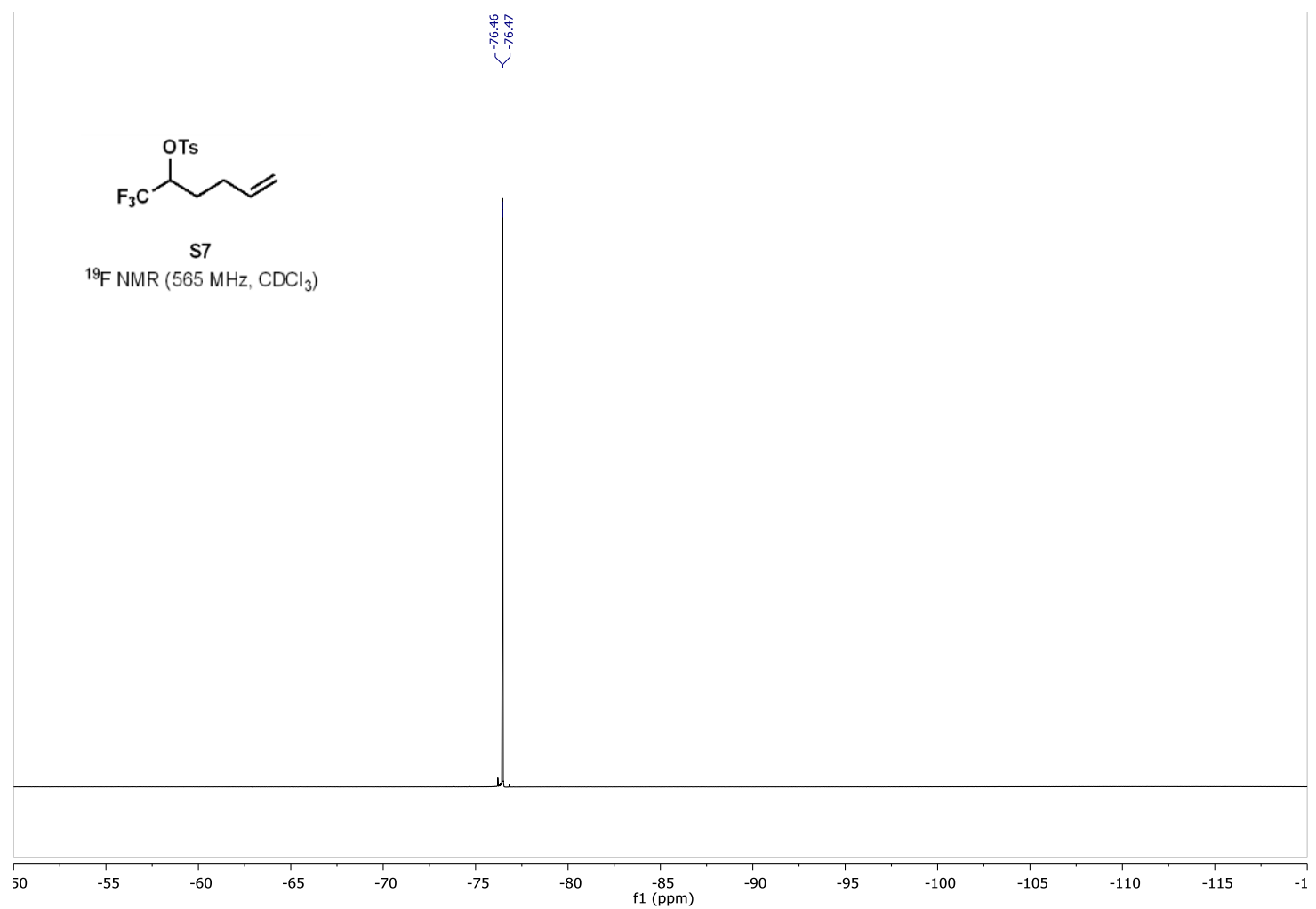




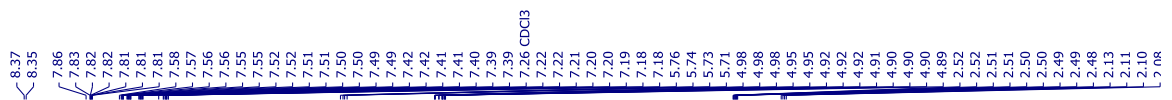
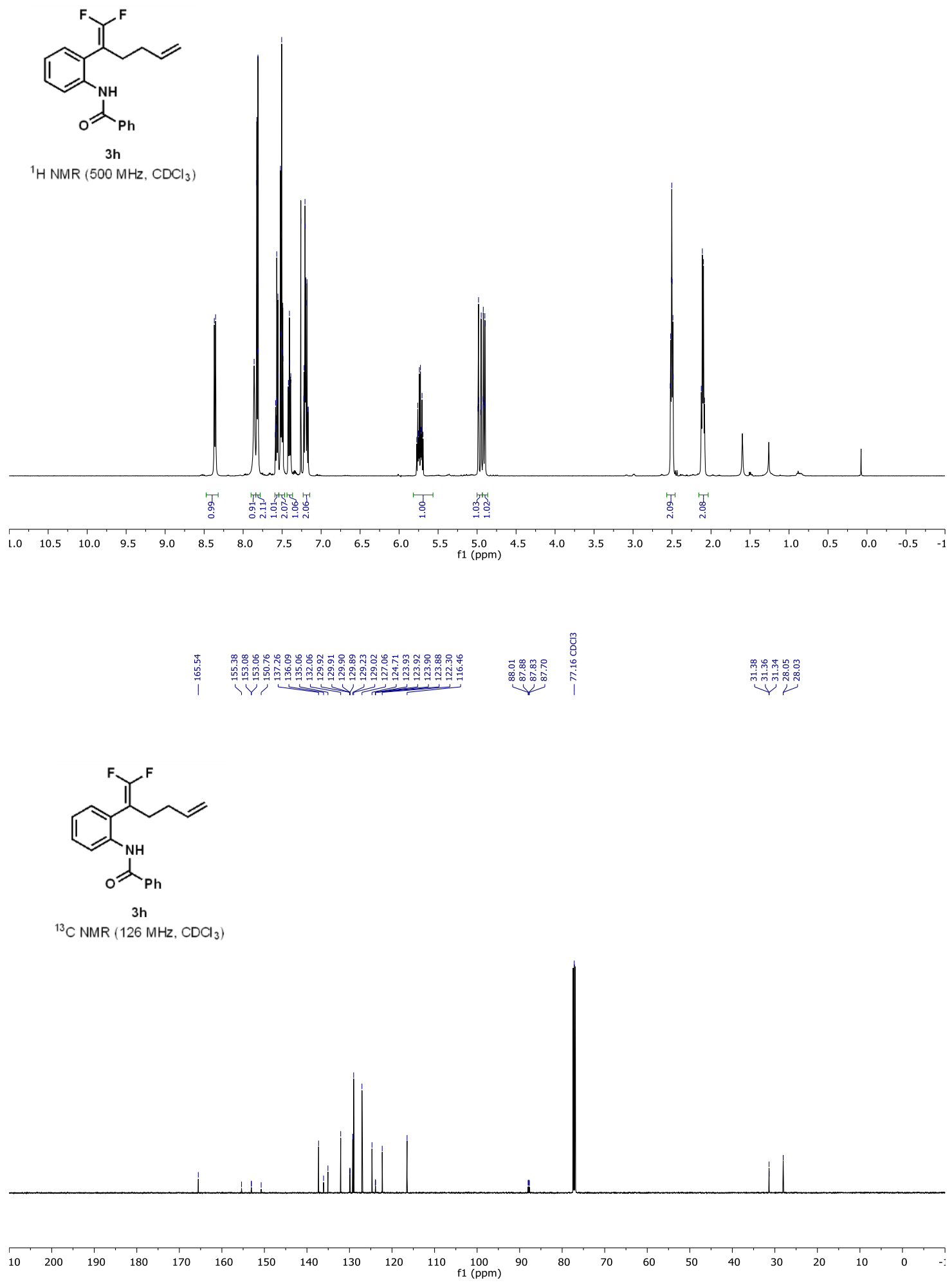

S137 


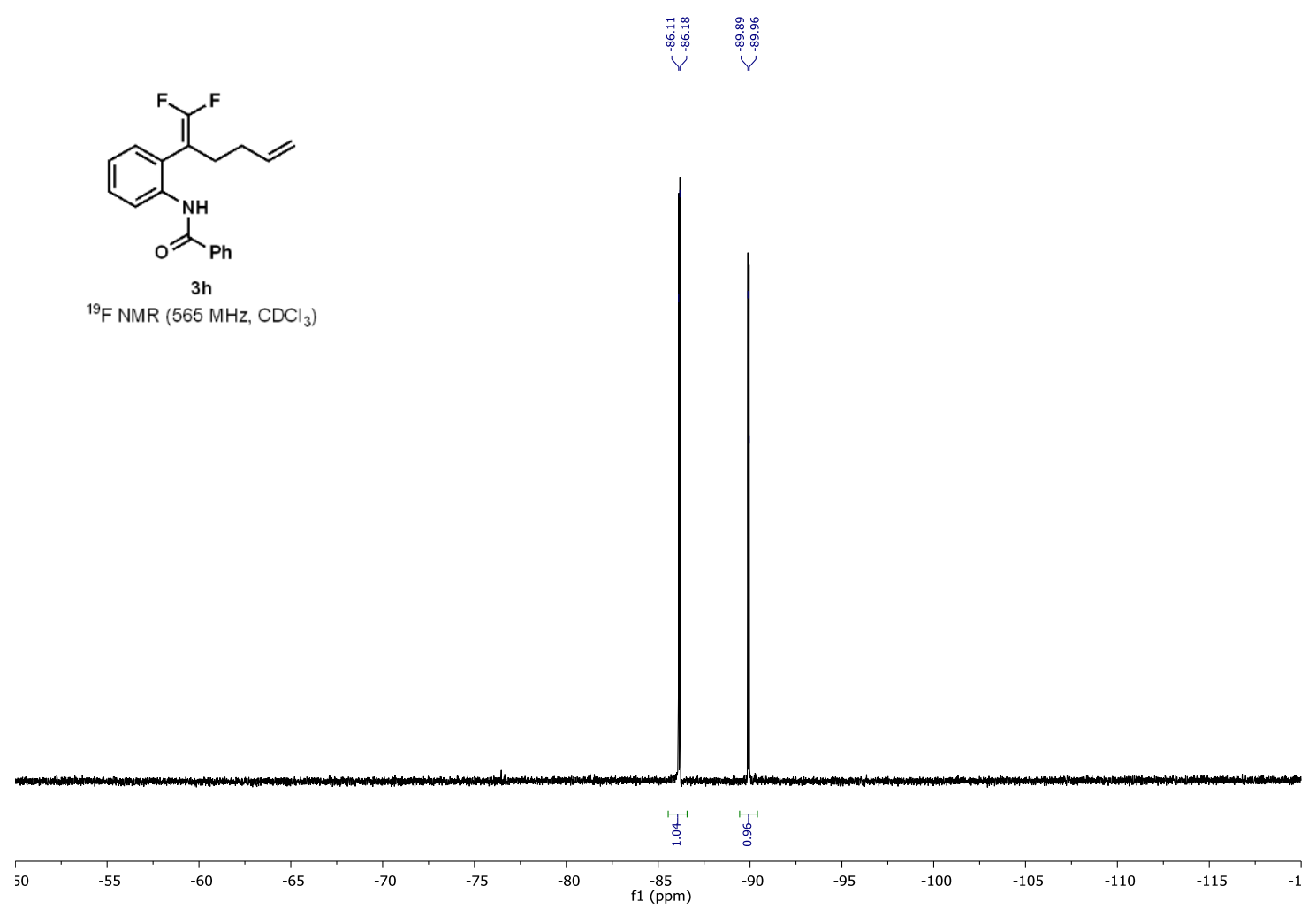




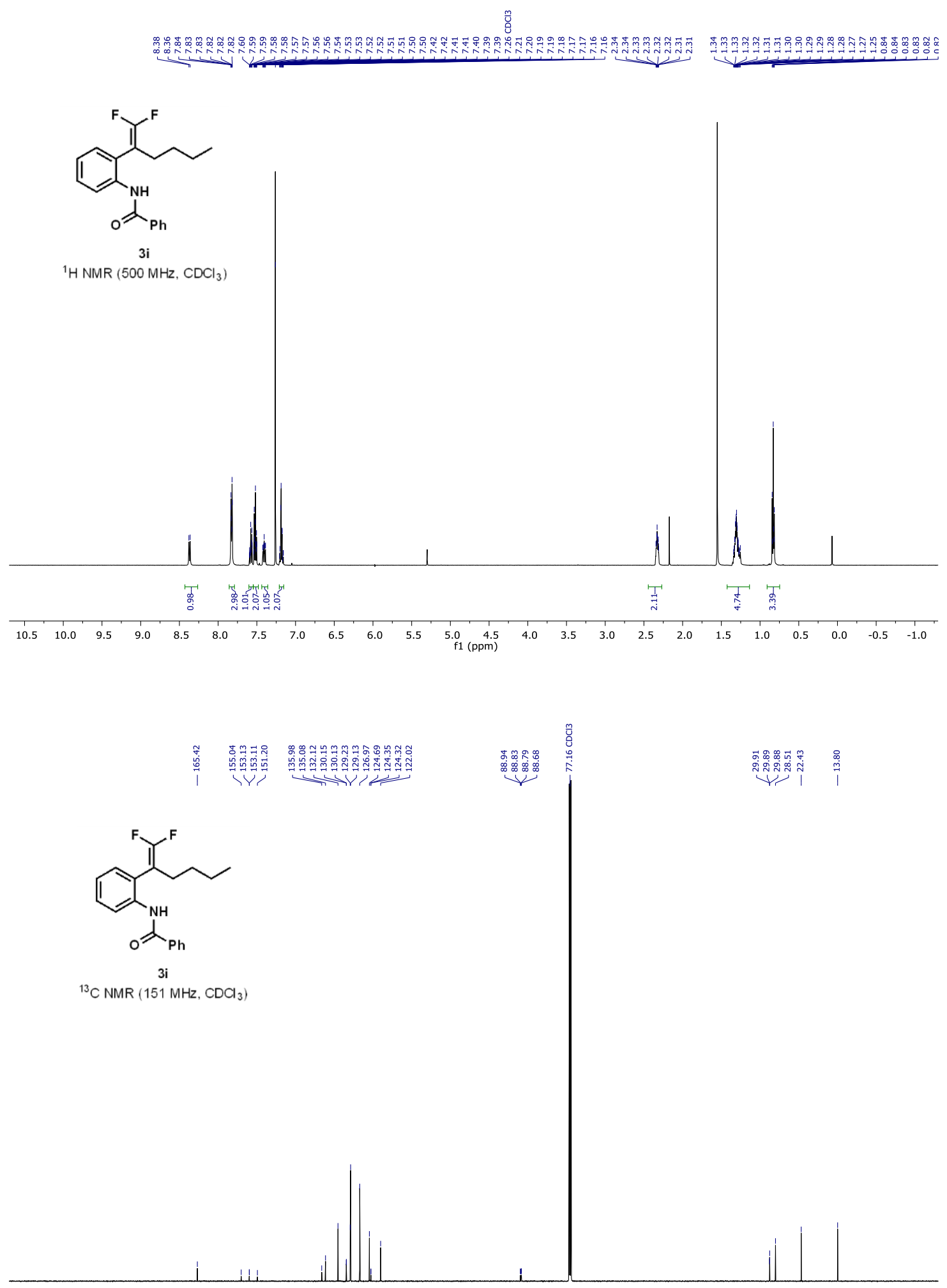

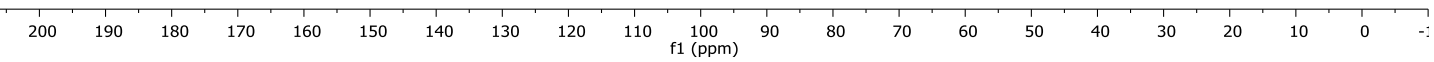




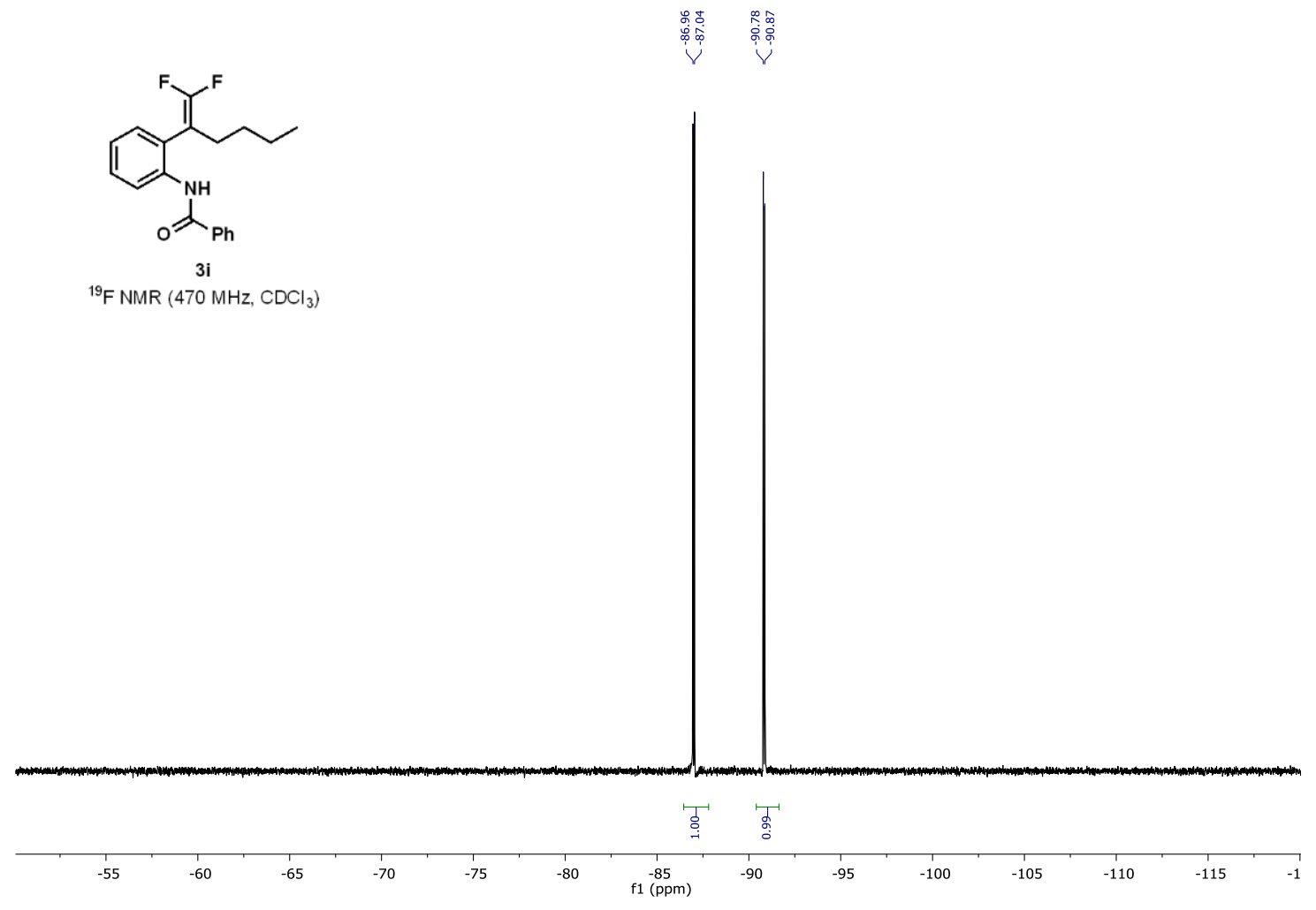



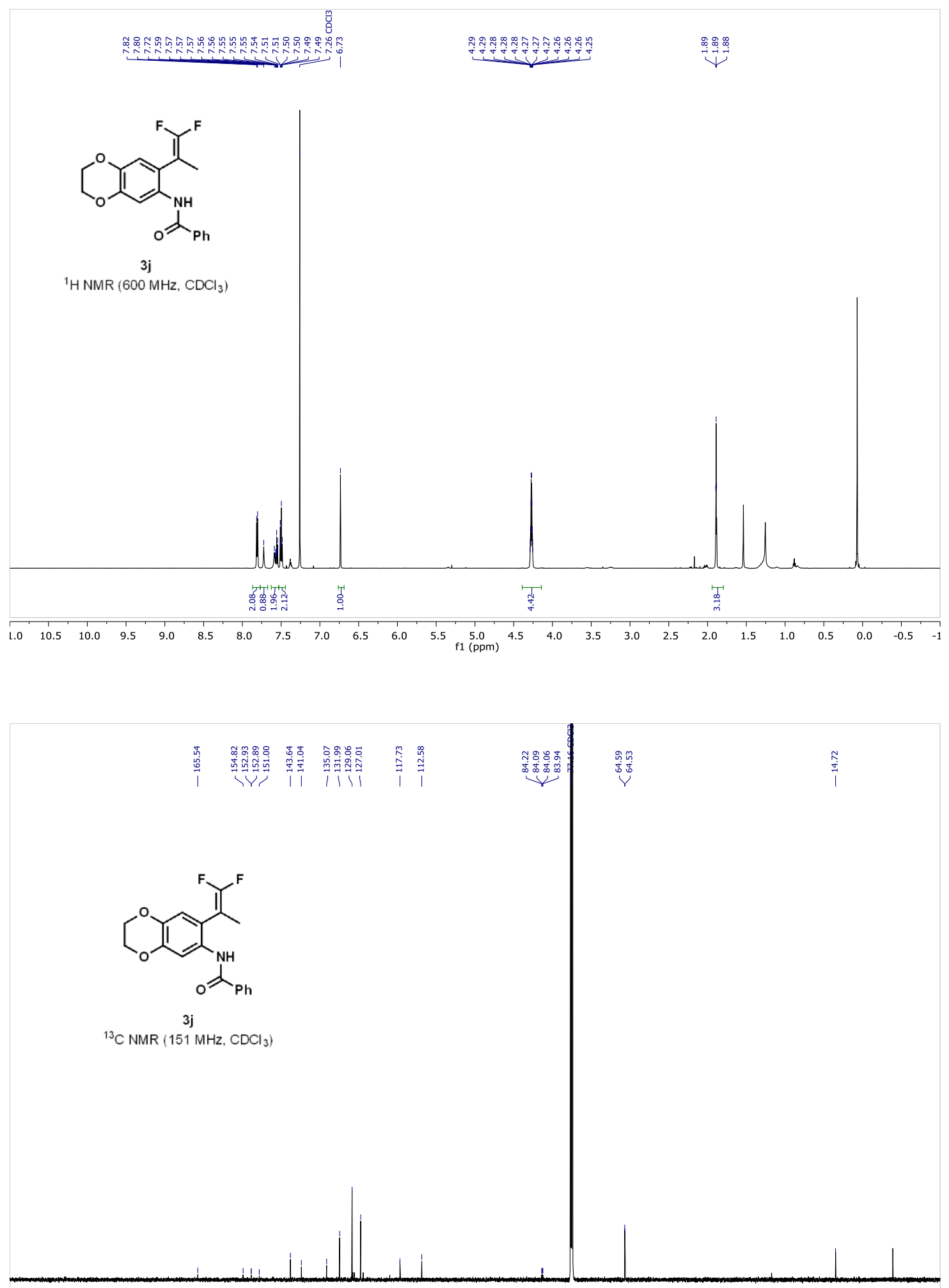


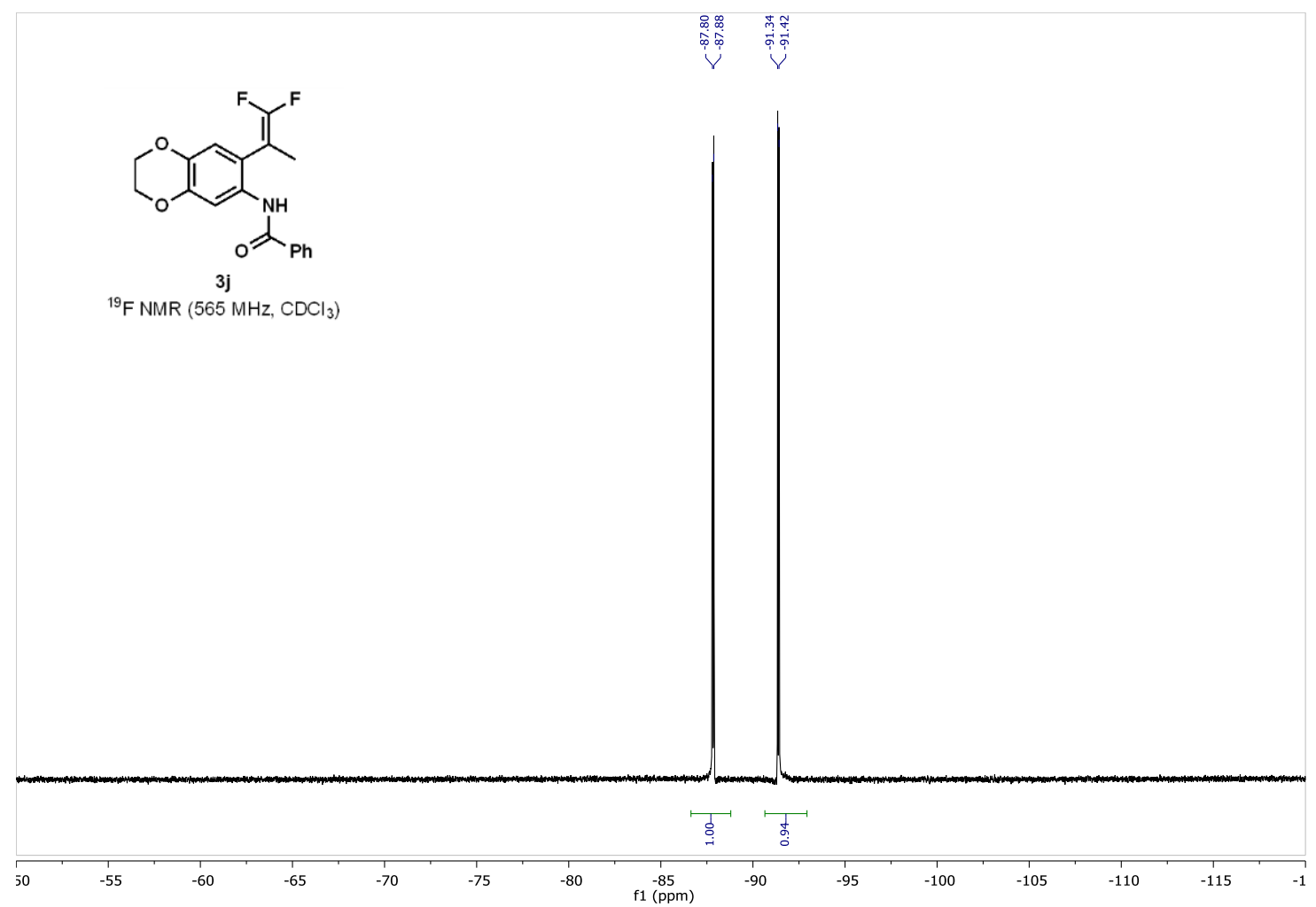



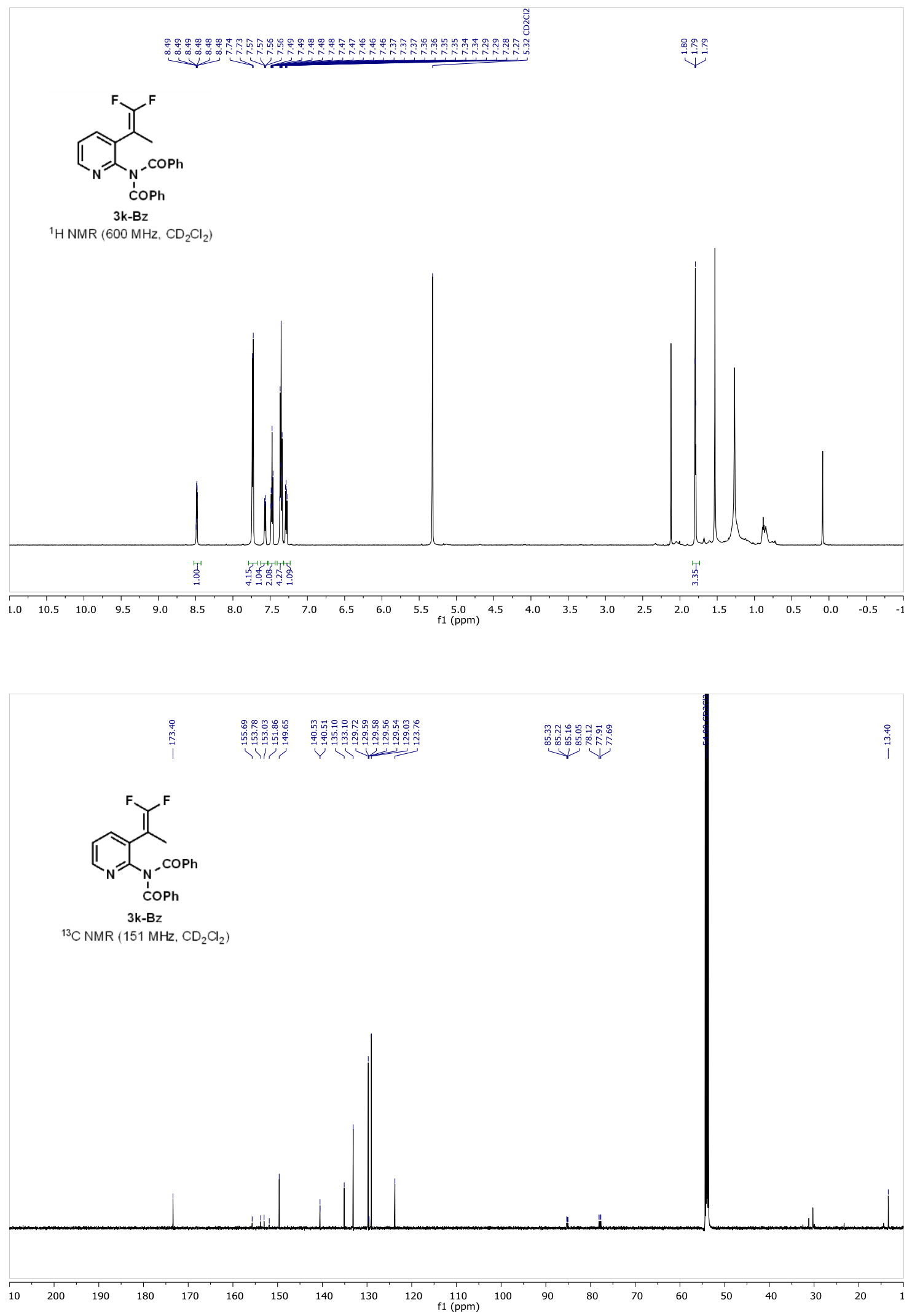


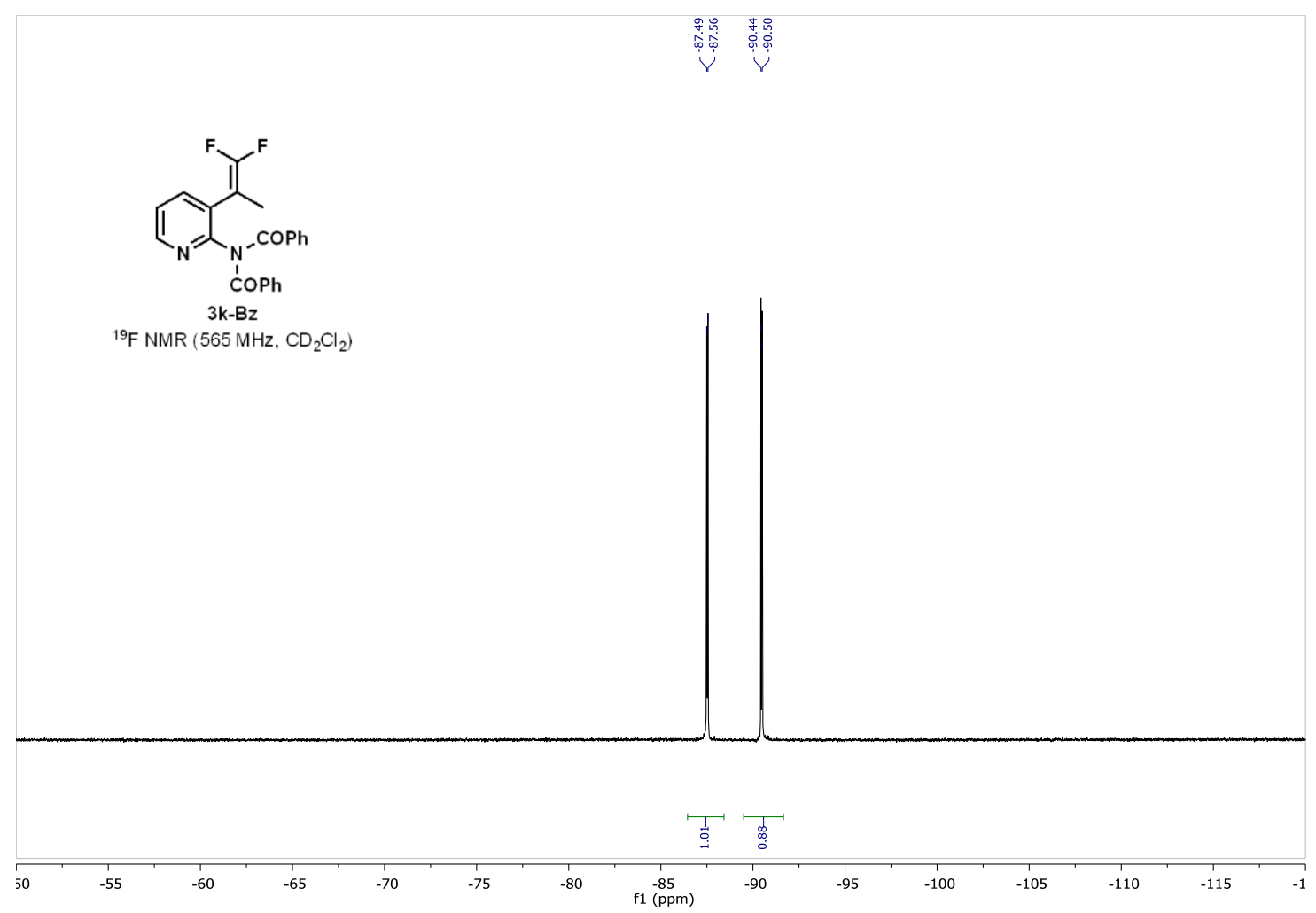




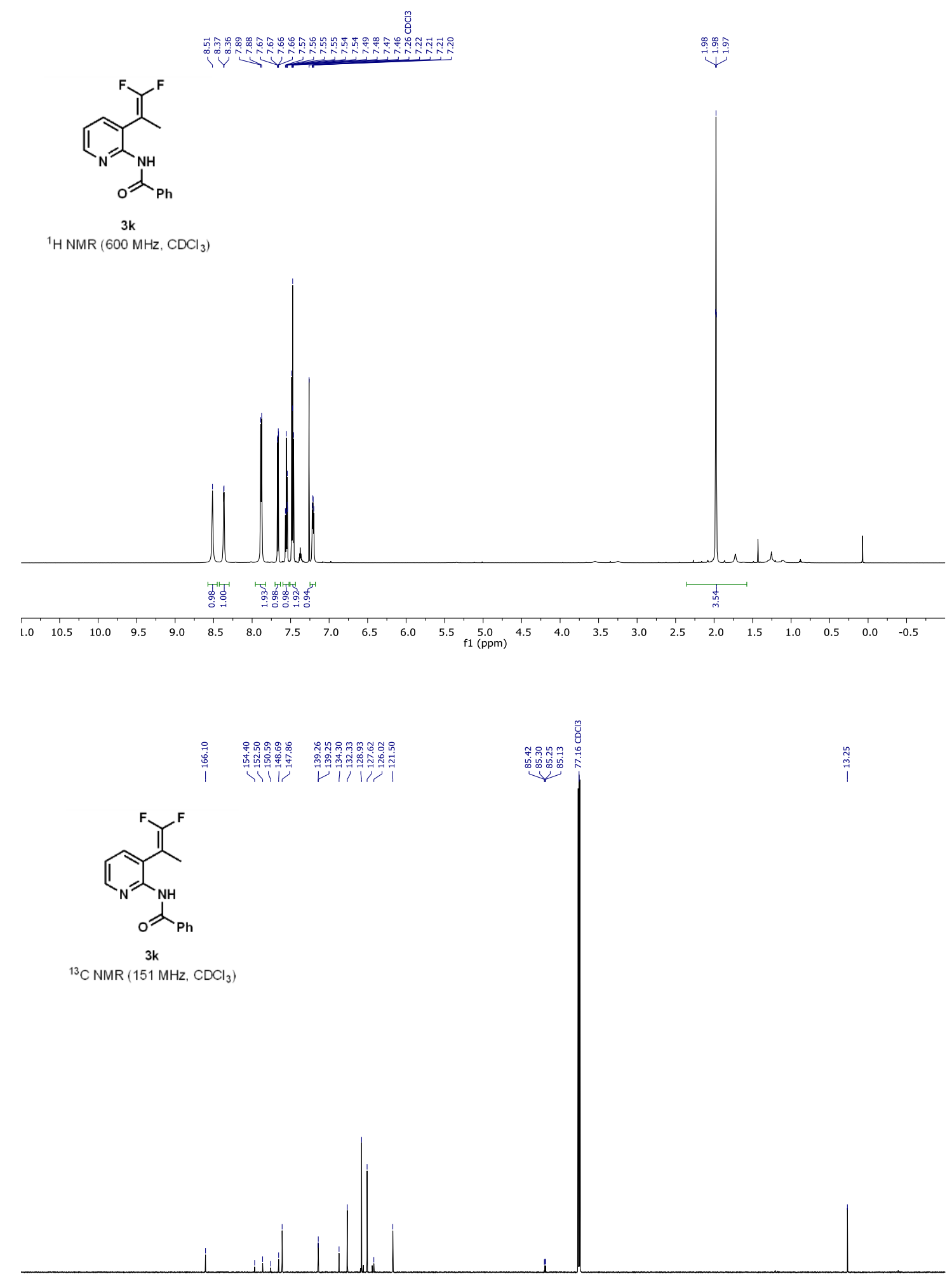

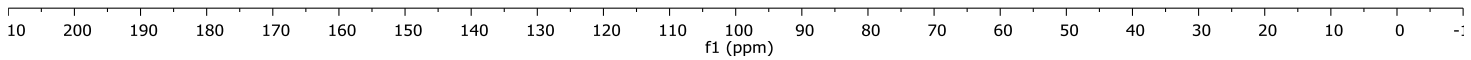




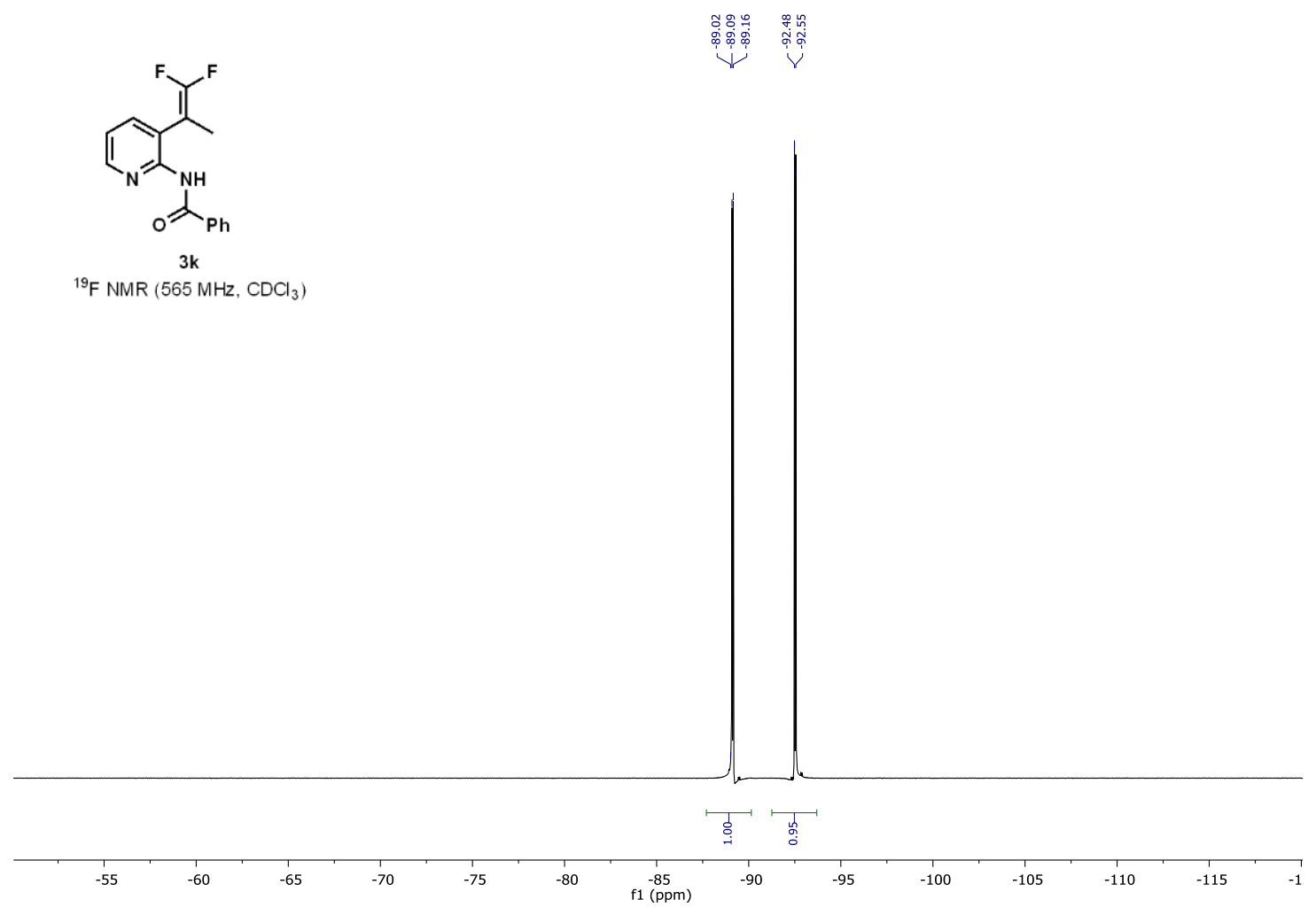


$\underbrace{\underbrace{2}}$
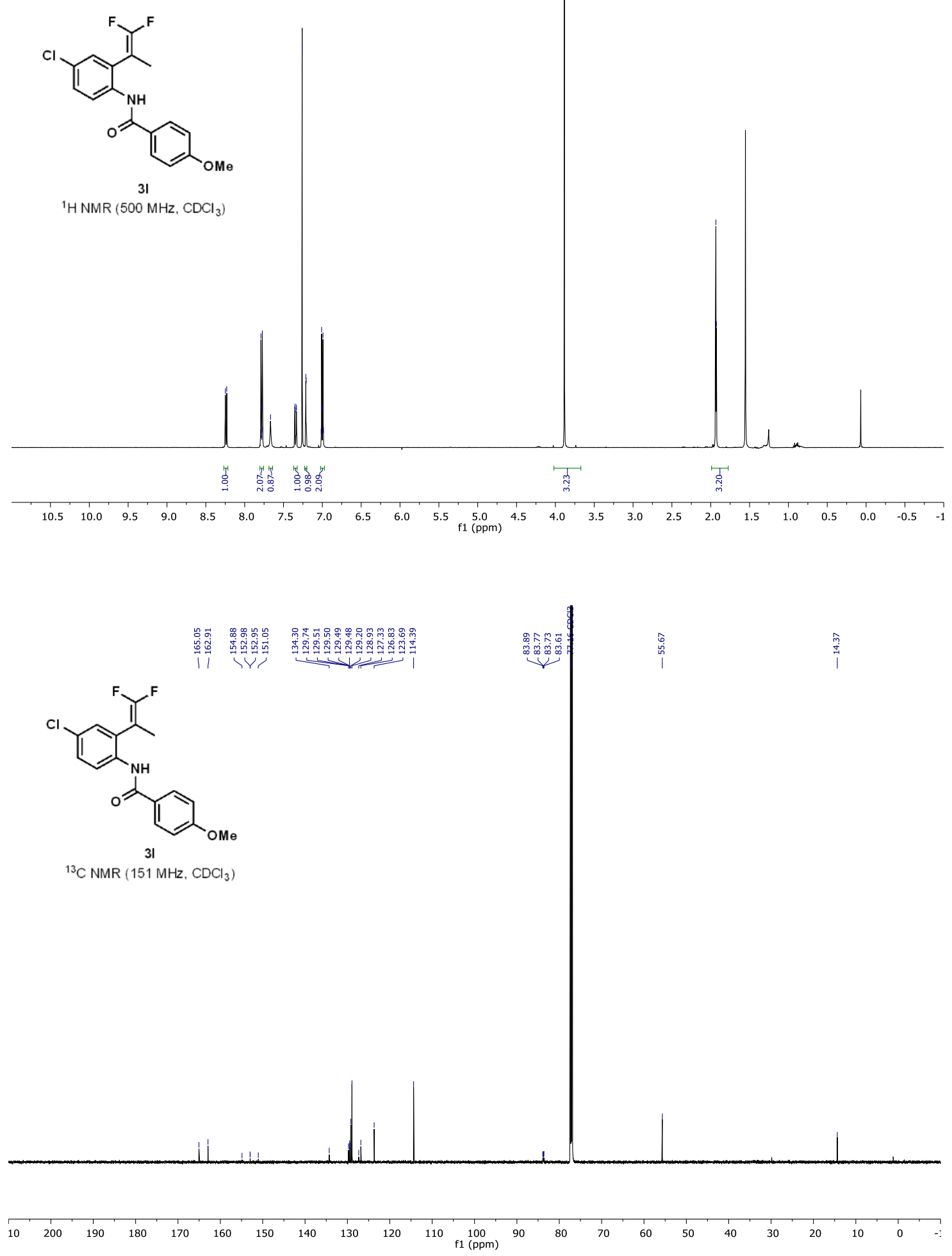

S147 

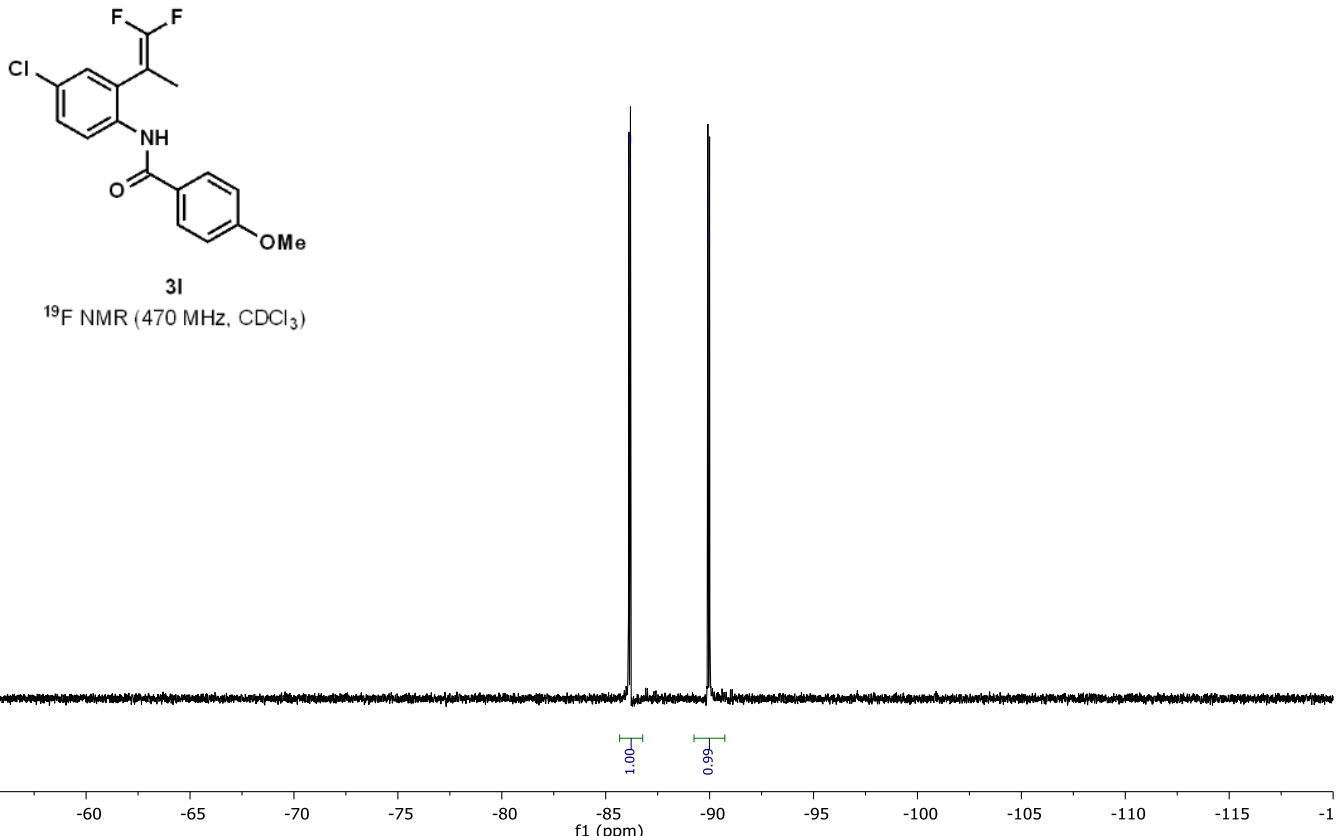

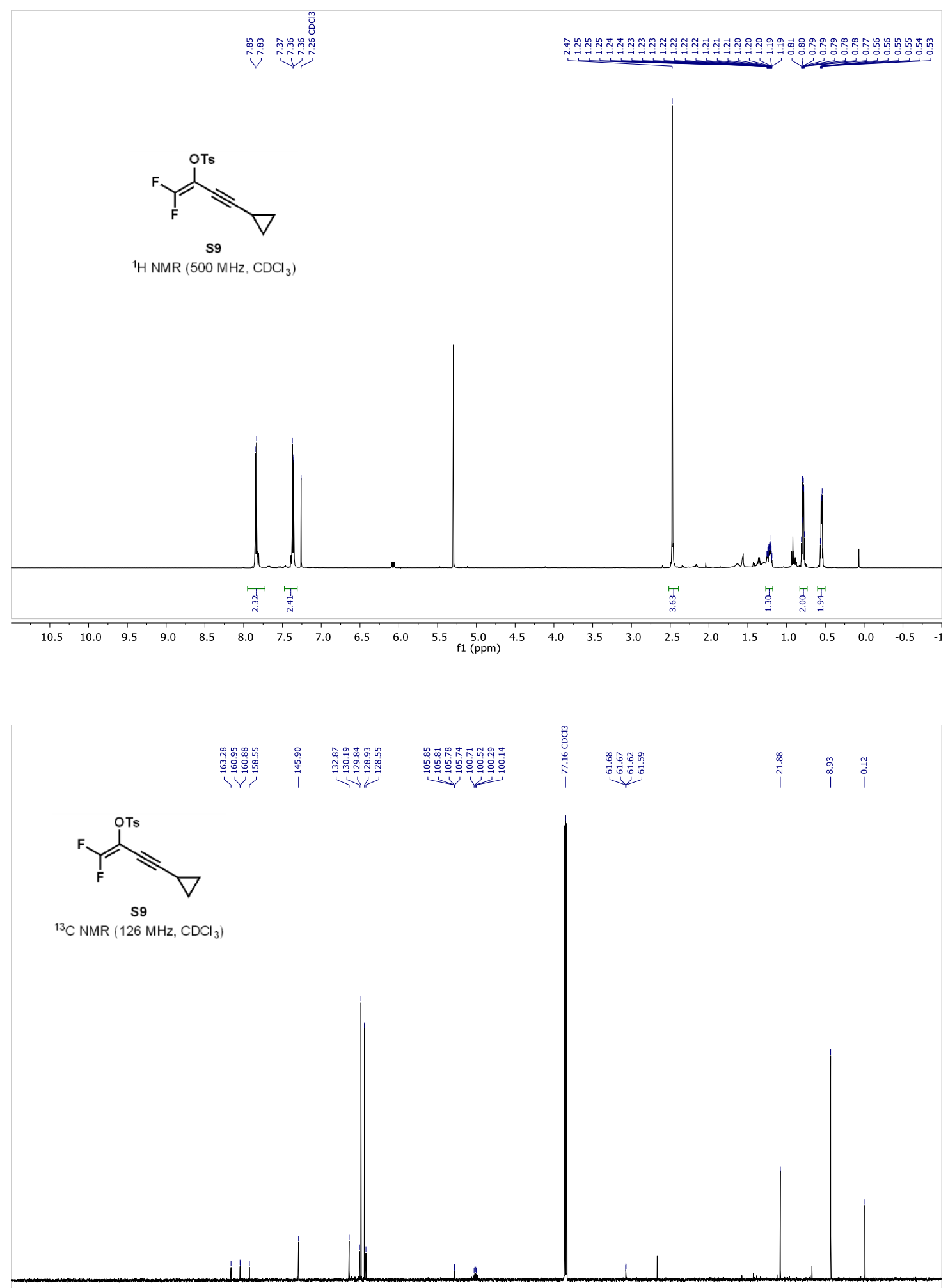

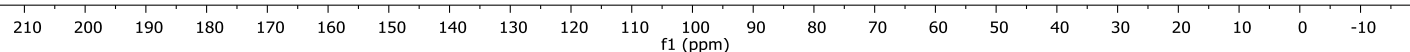




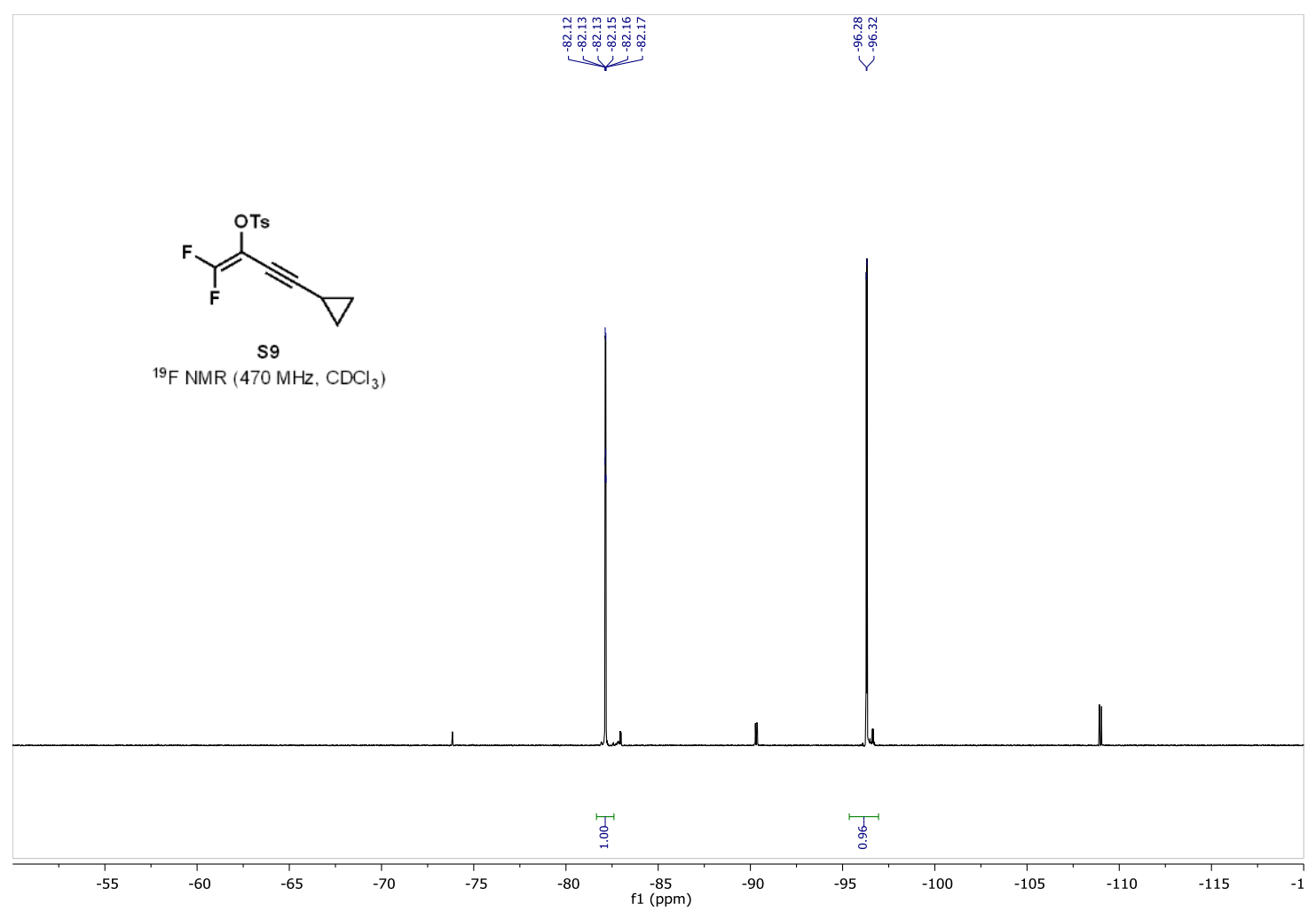




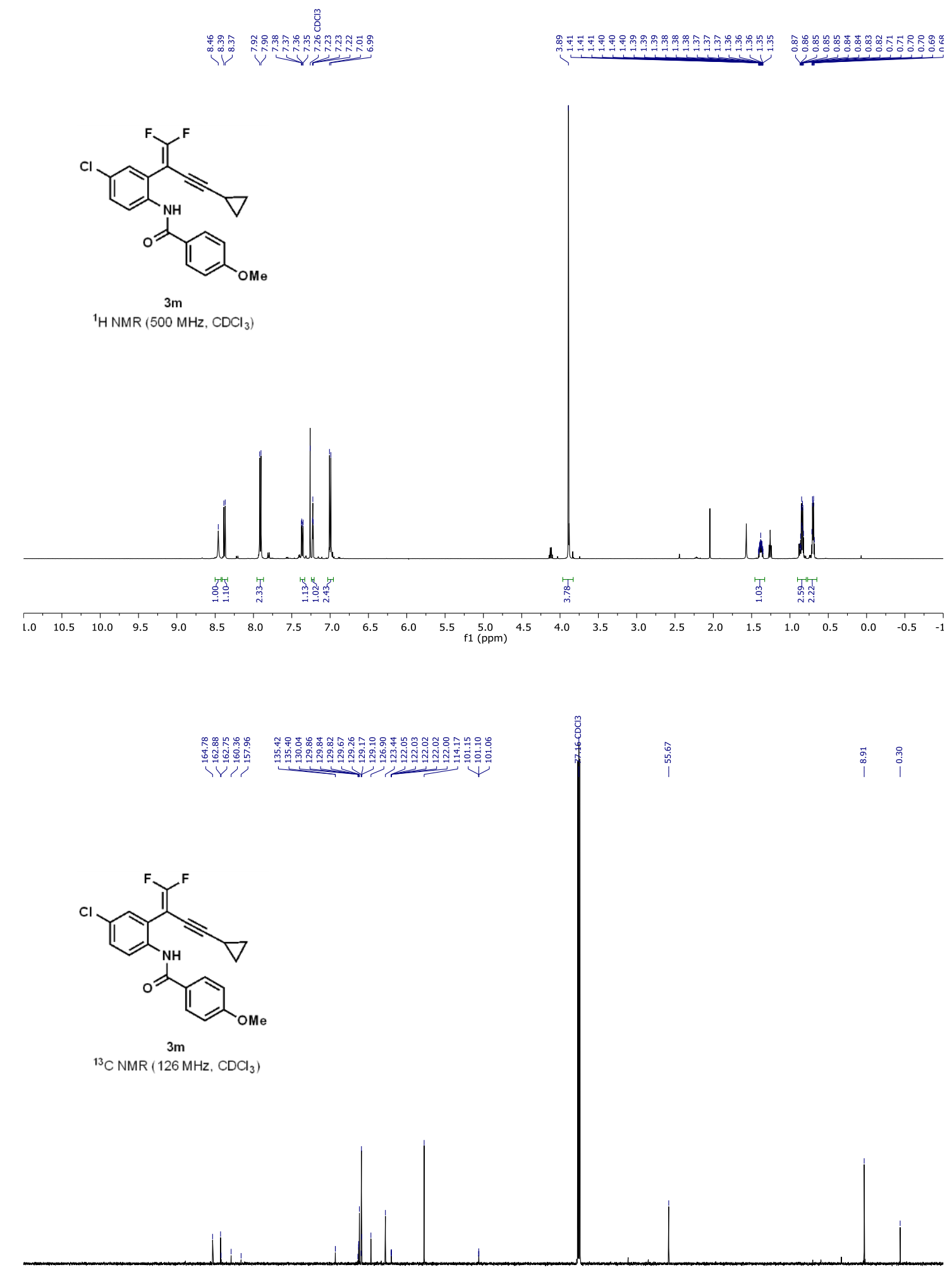

(10.5

$\begin{array}{ll}\overrightarrow{0} & 0 \\ 0 & 0 \\ 0 & 0\end{array}$ 


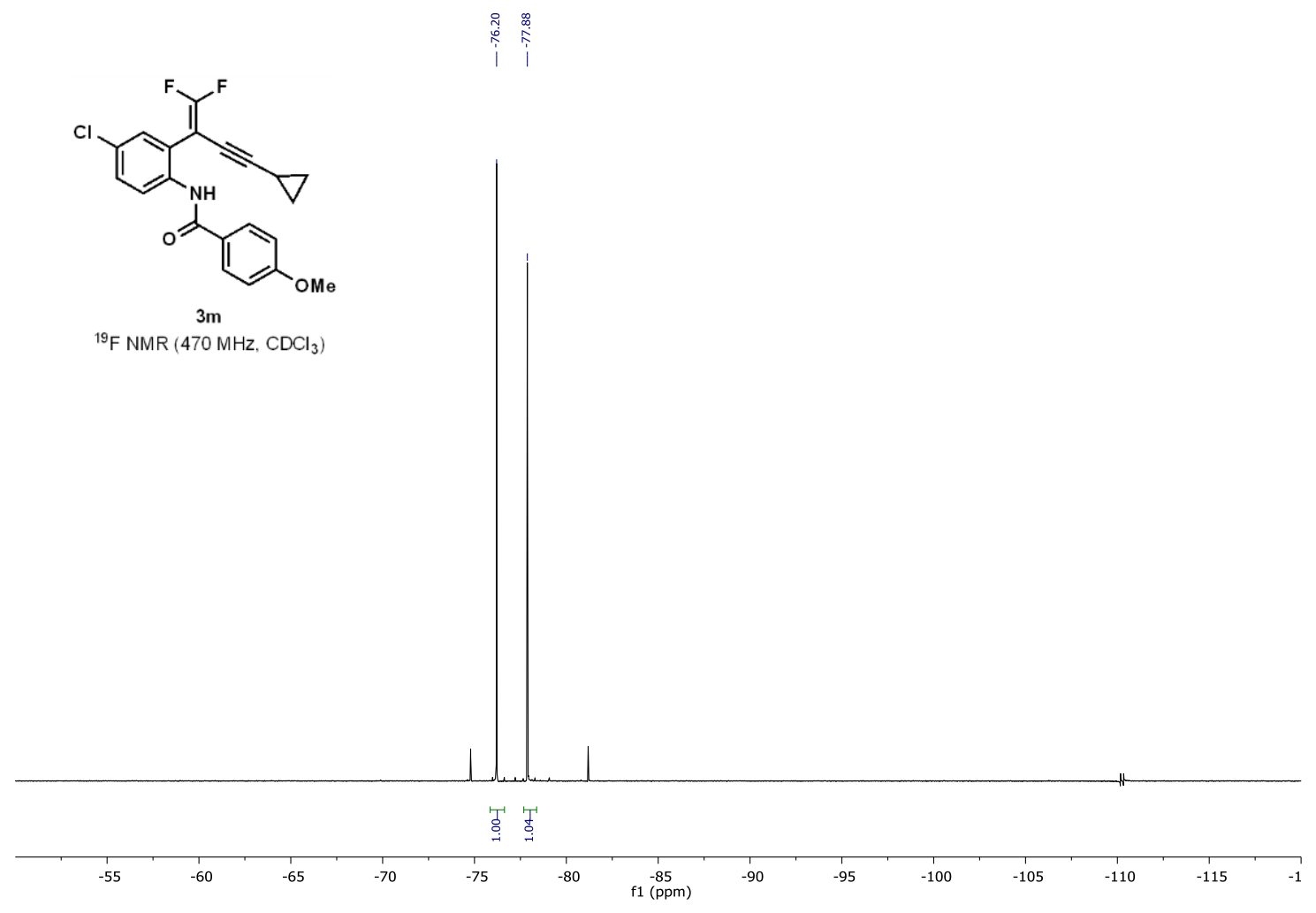



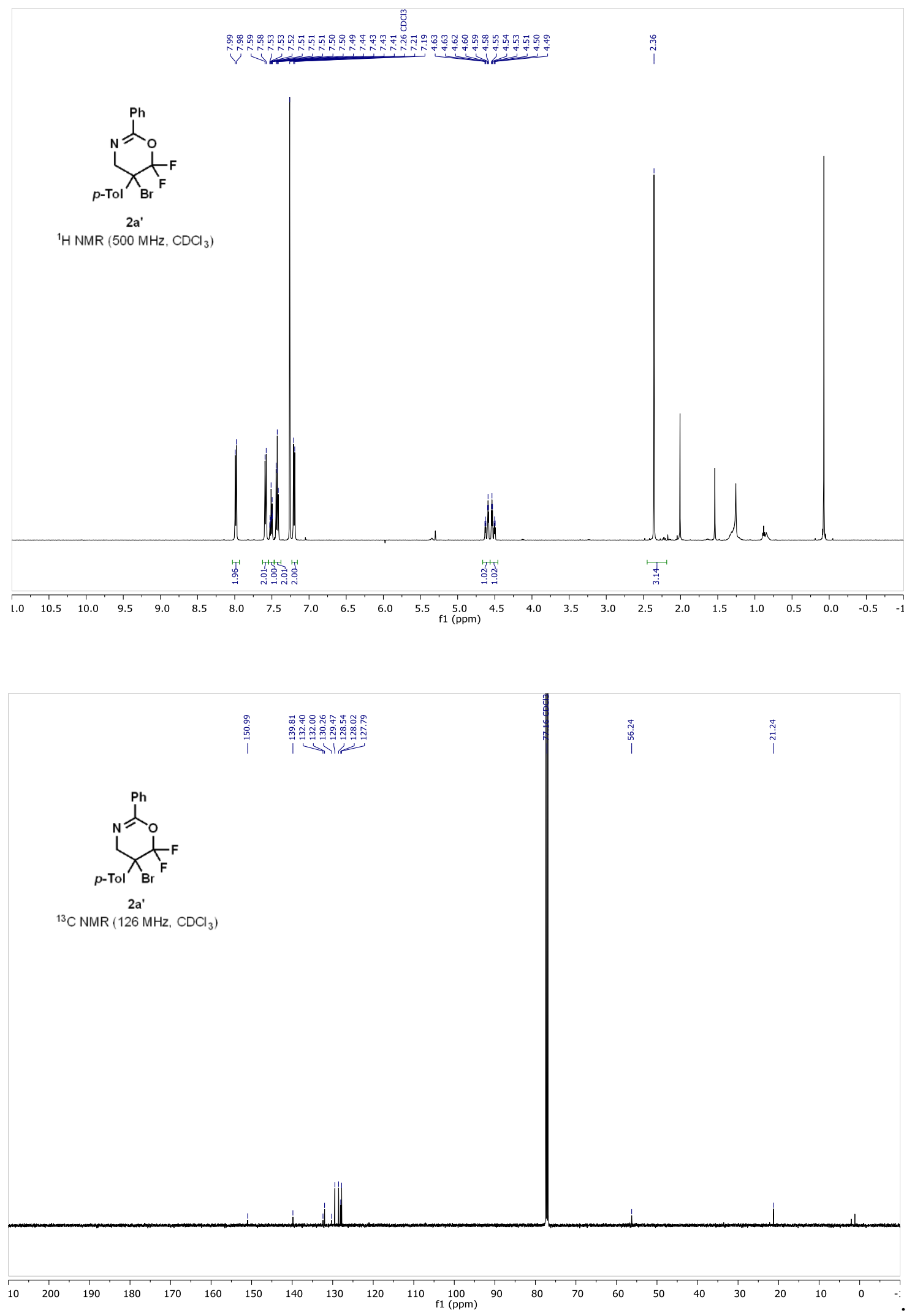


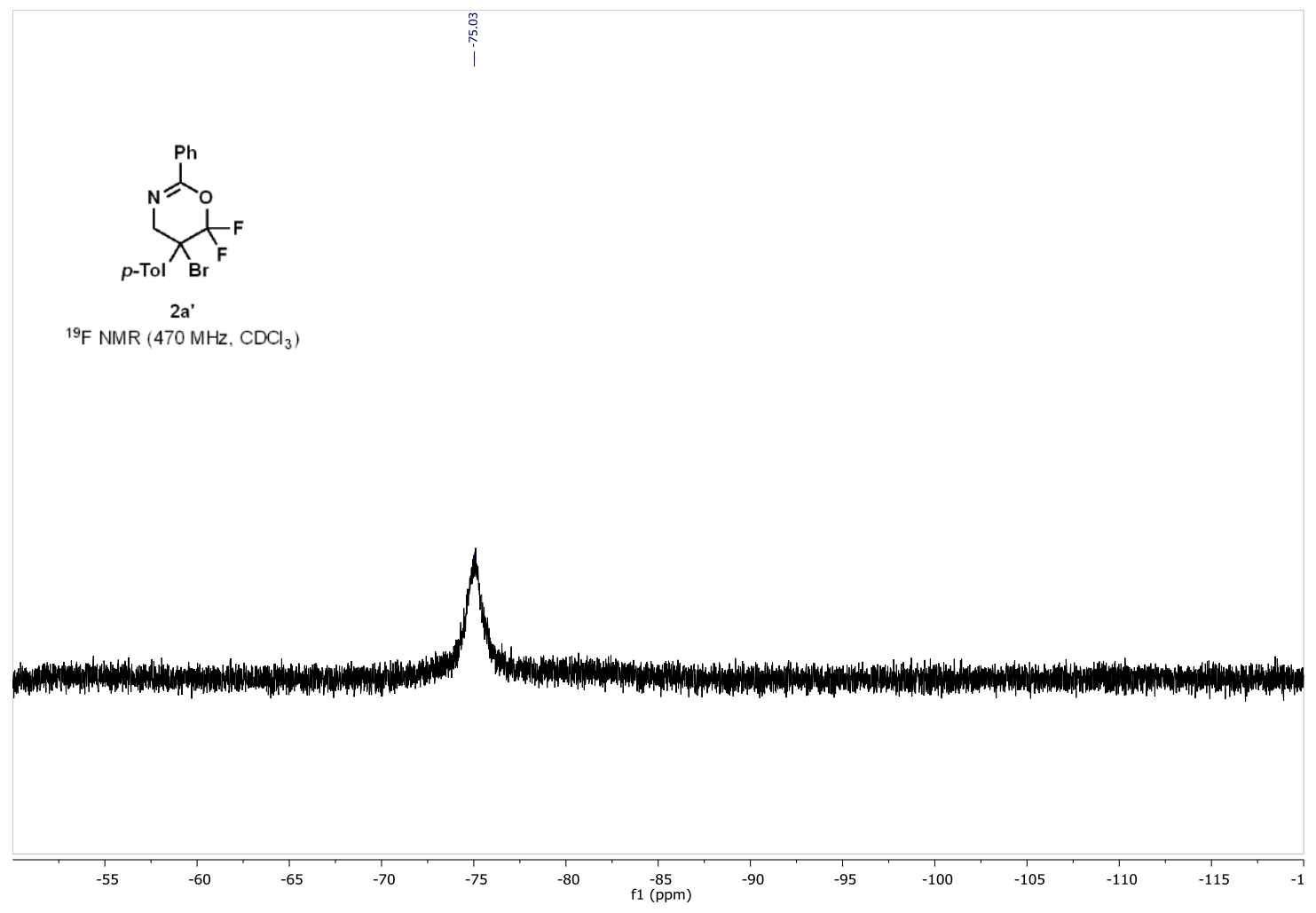



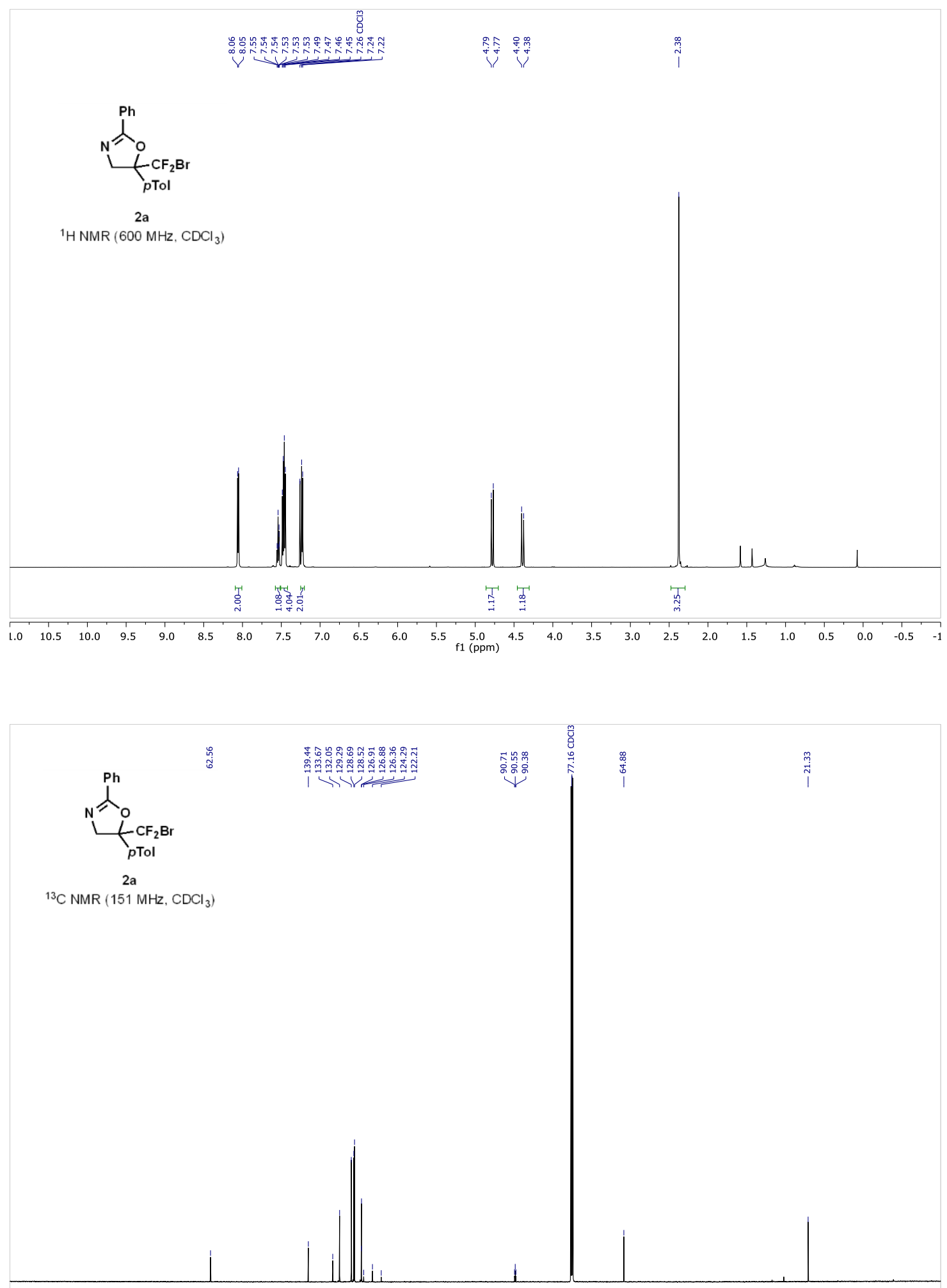

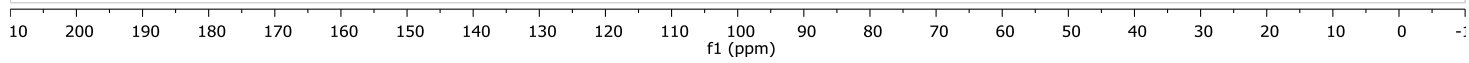




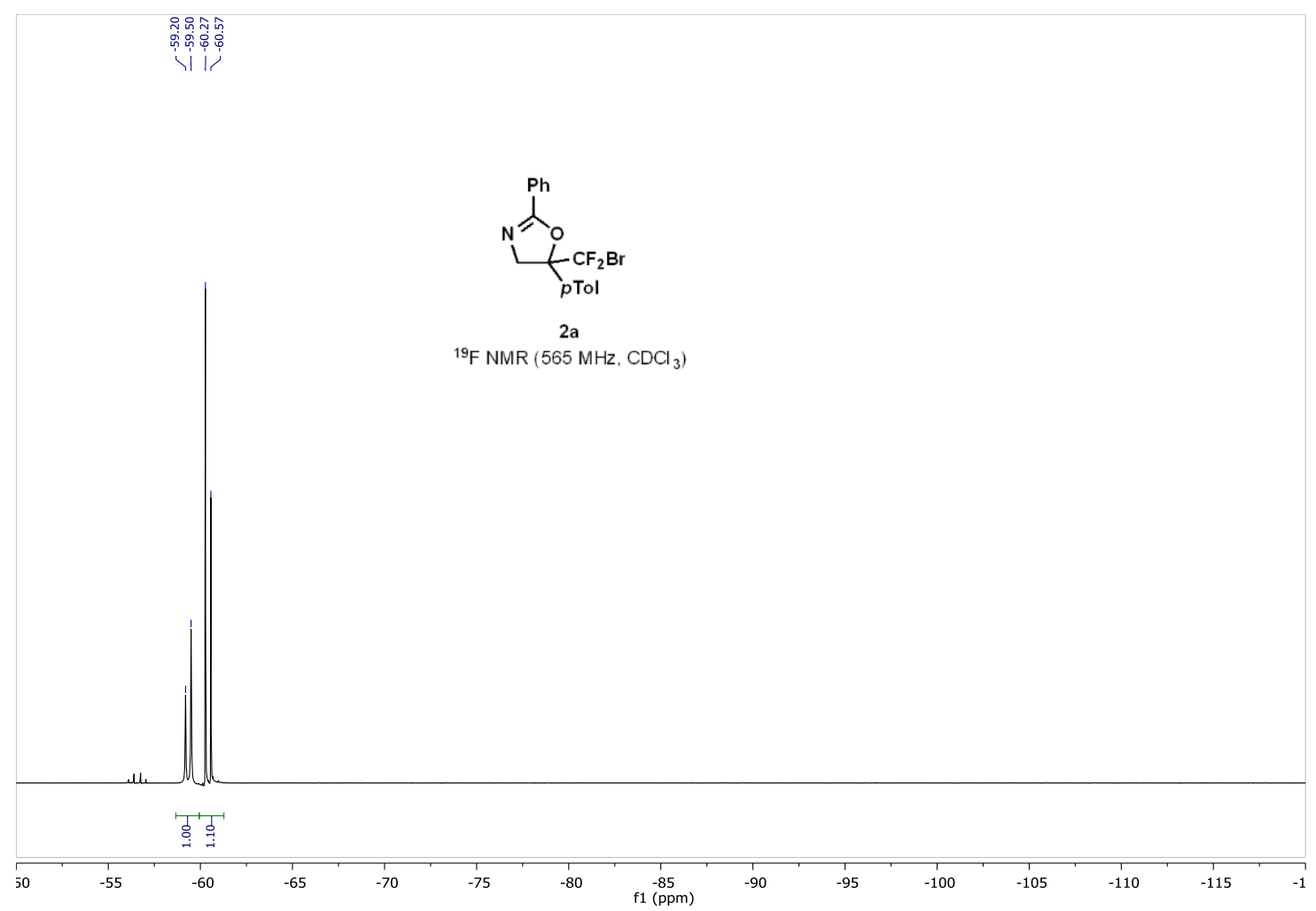



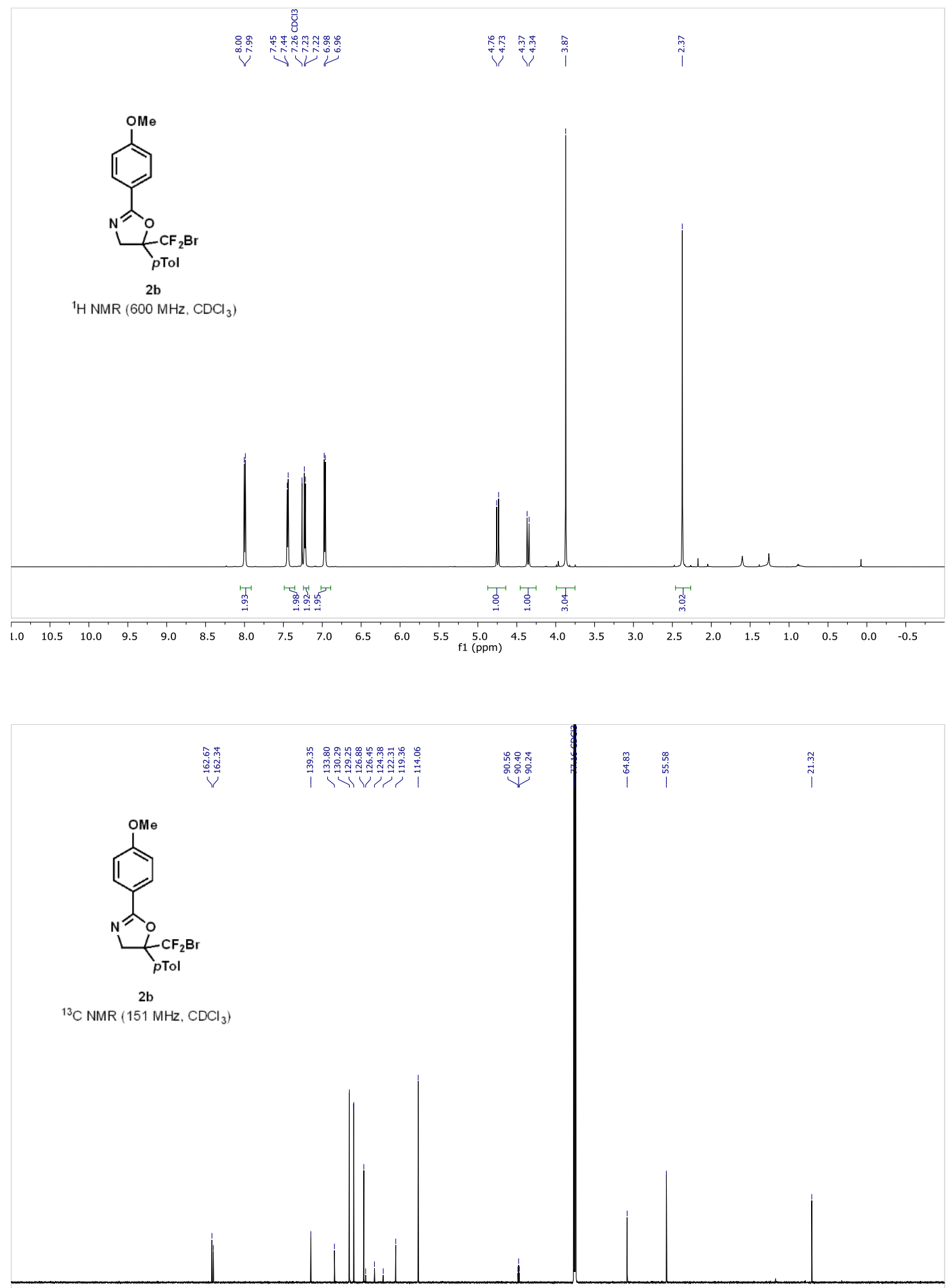

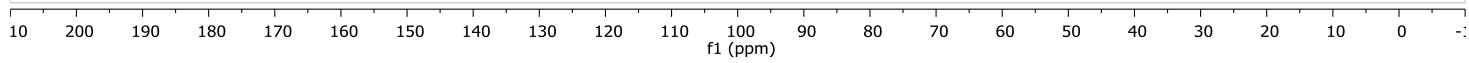




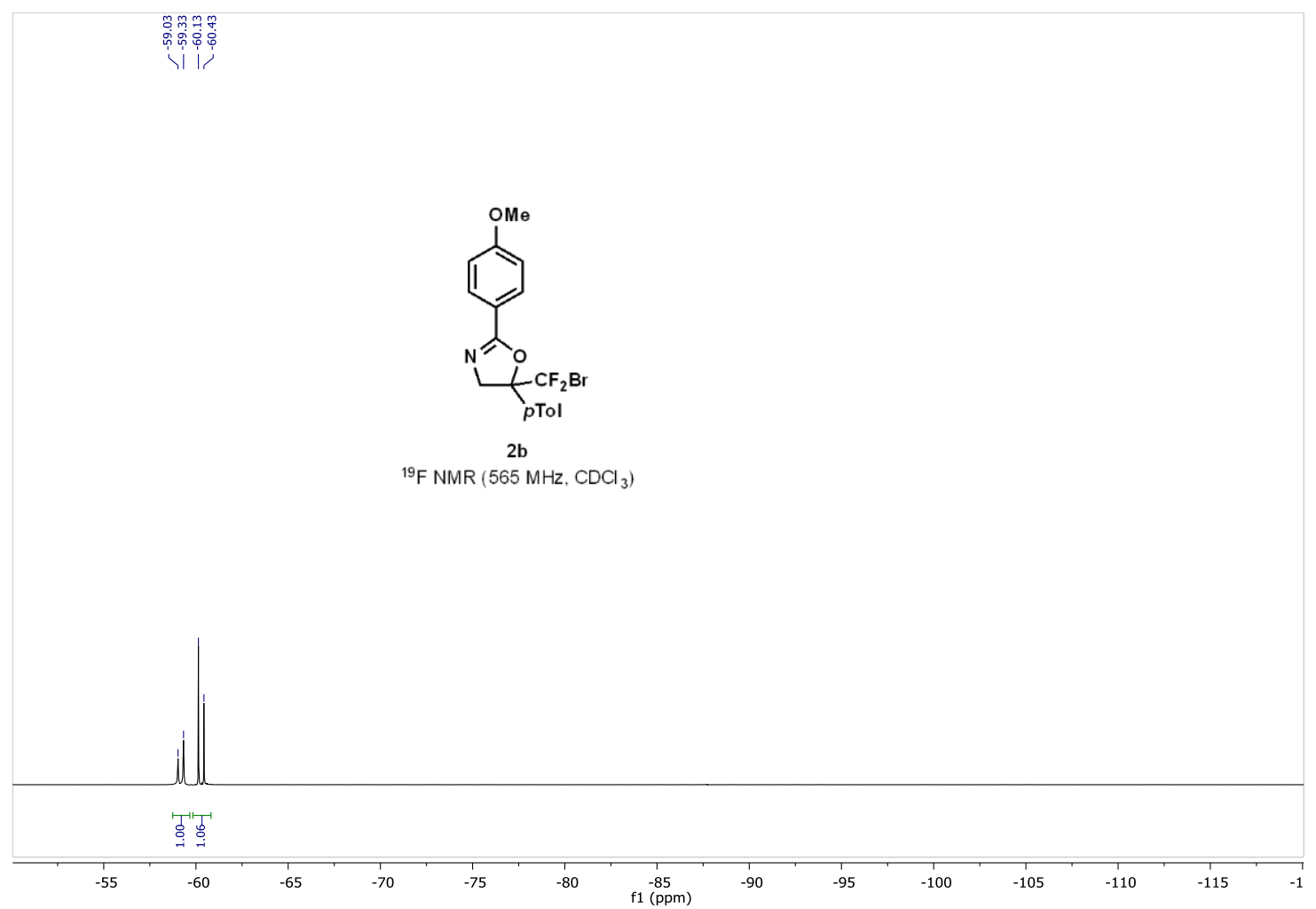



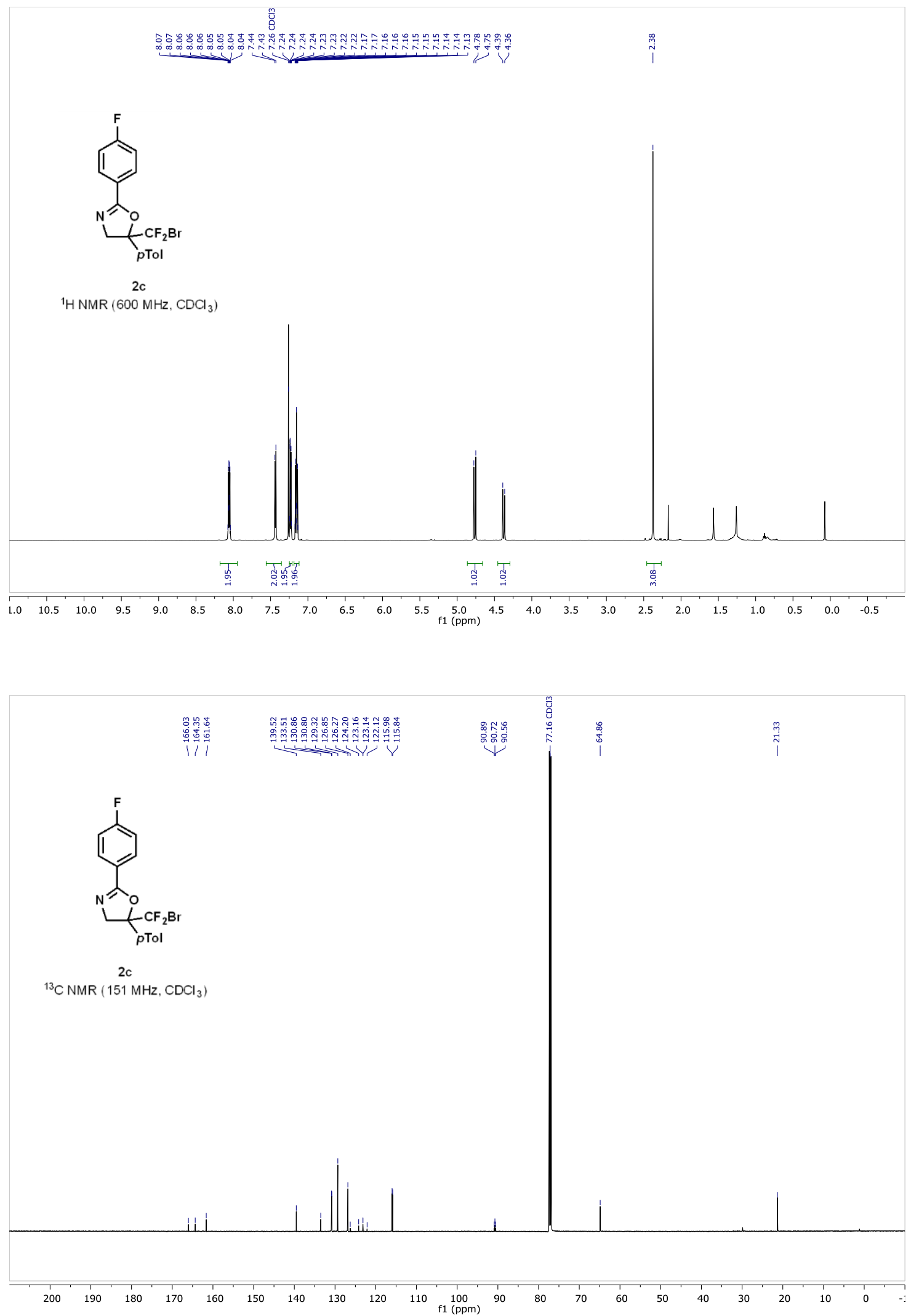


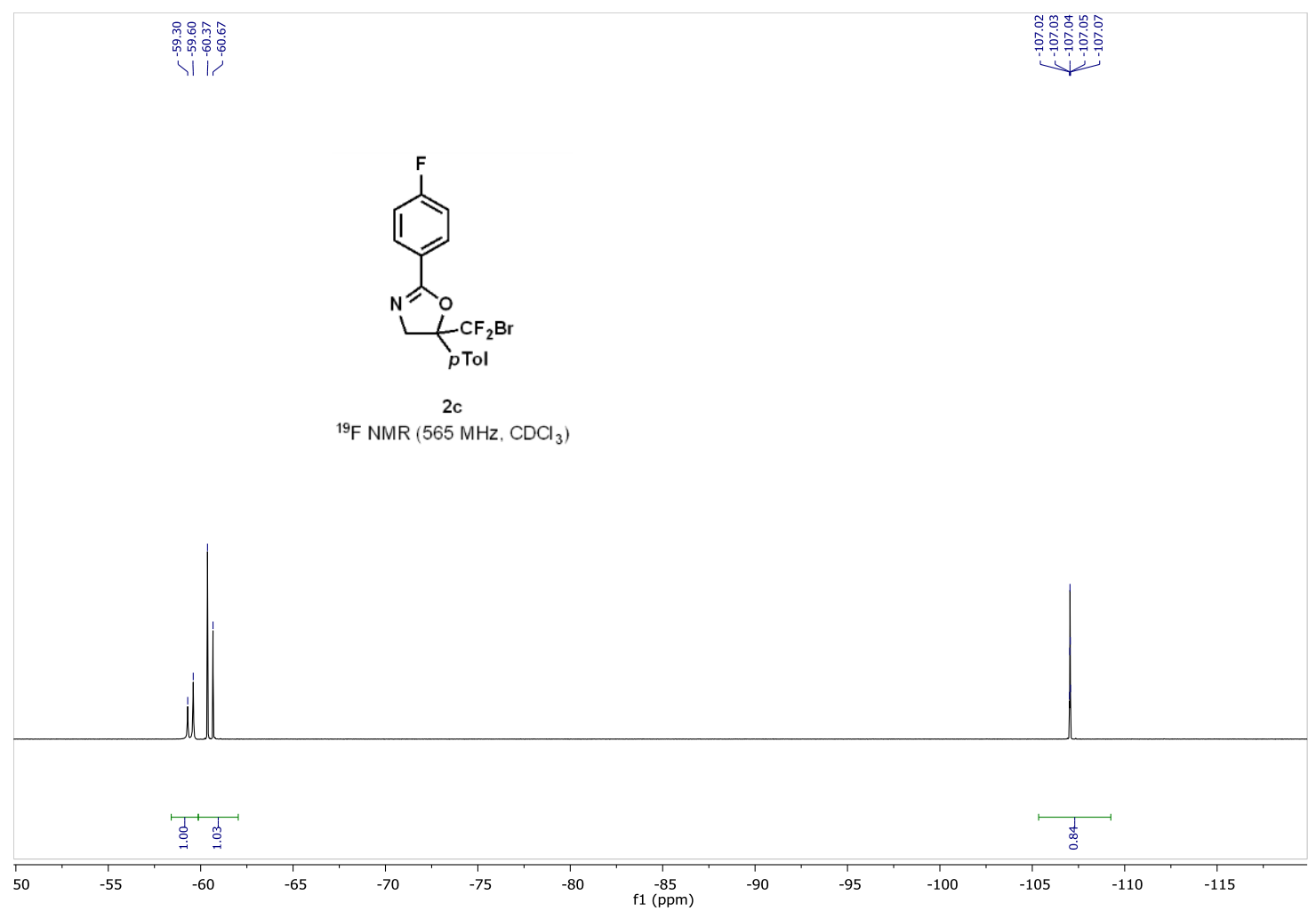



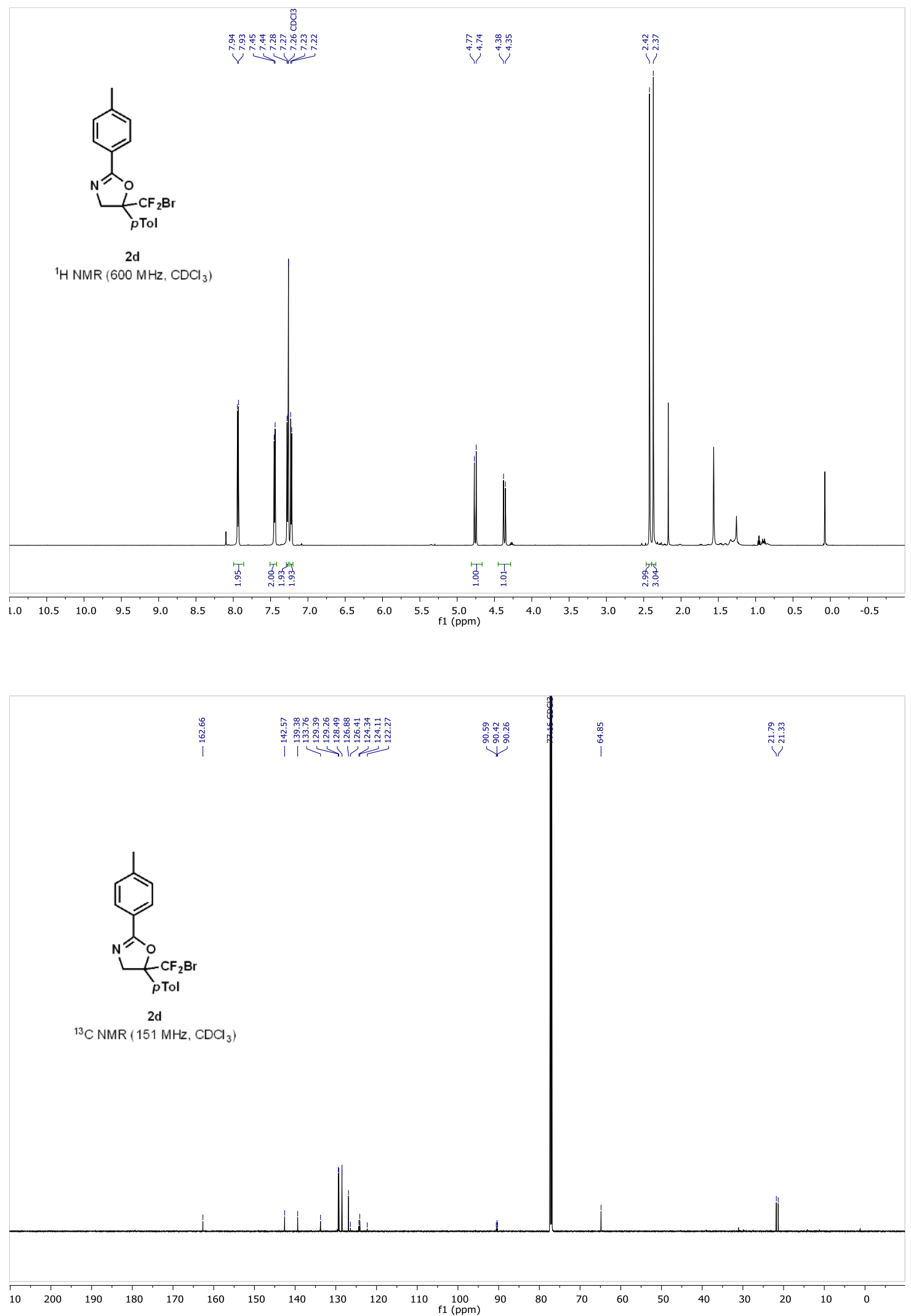


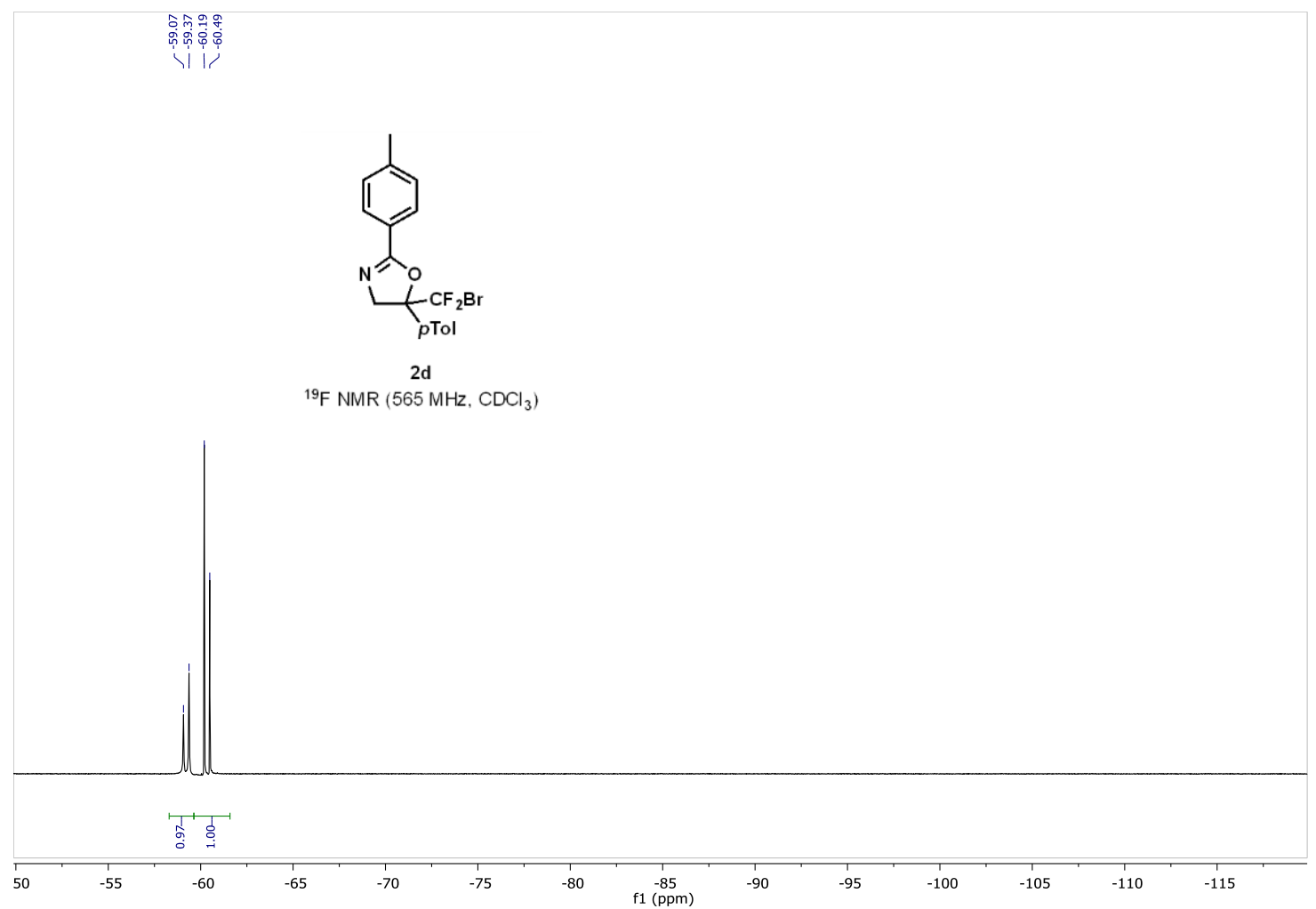



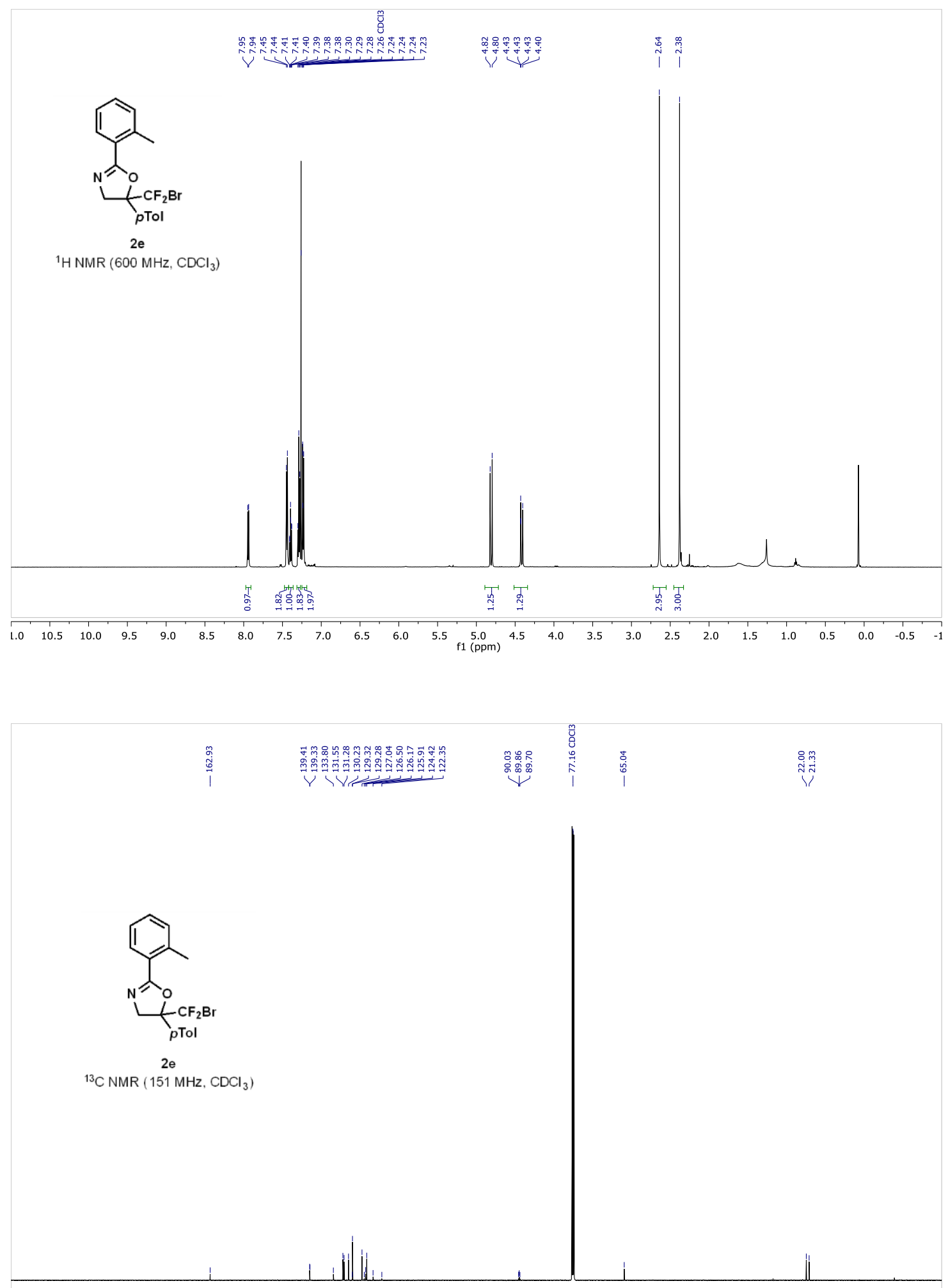

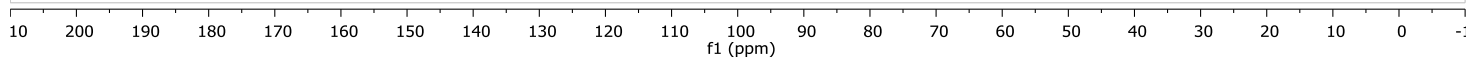




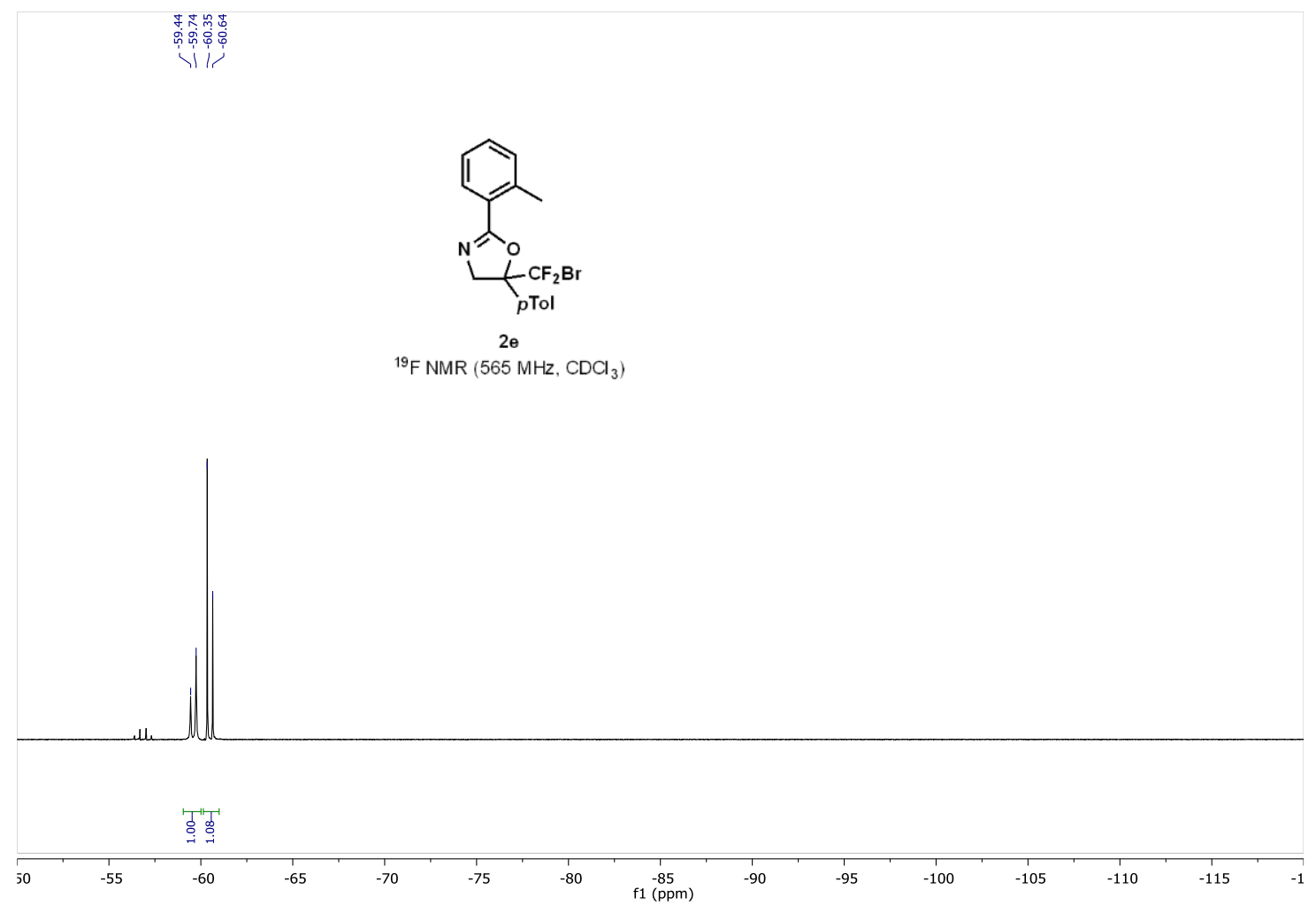



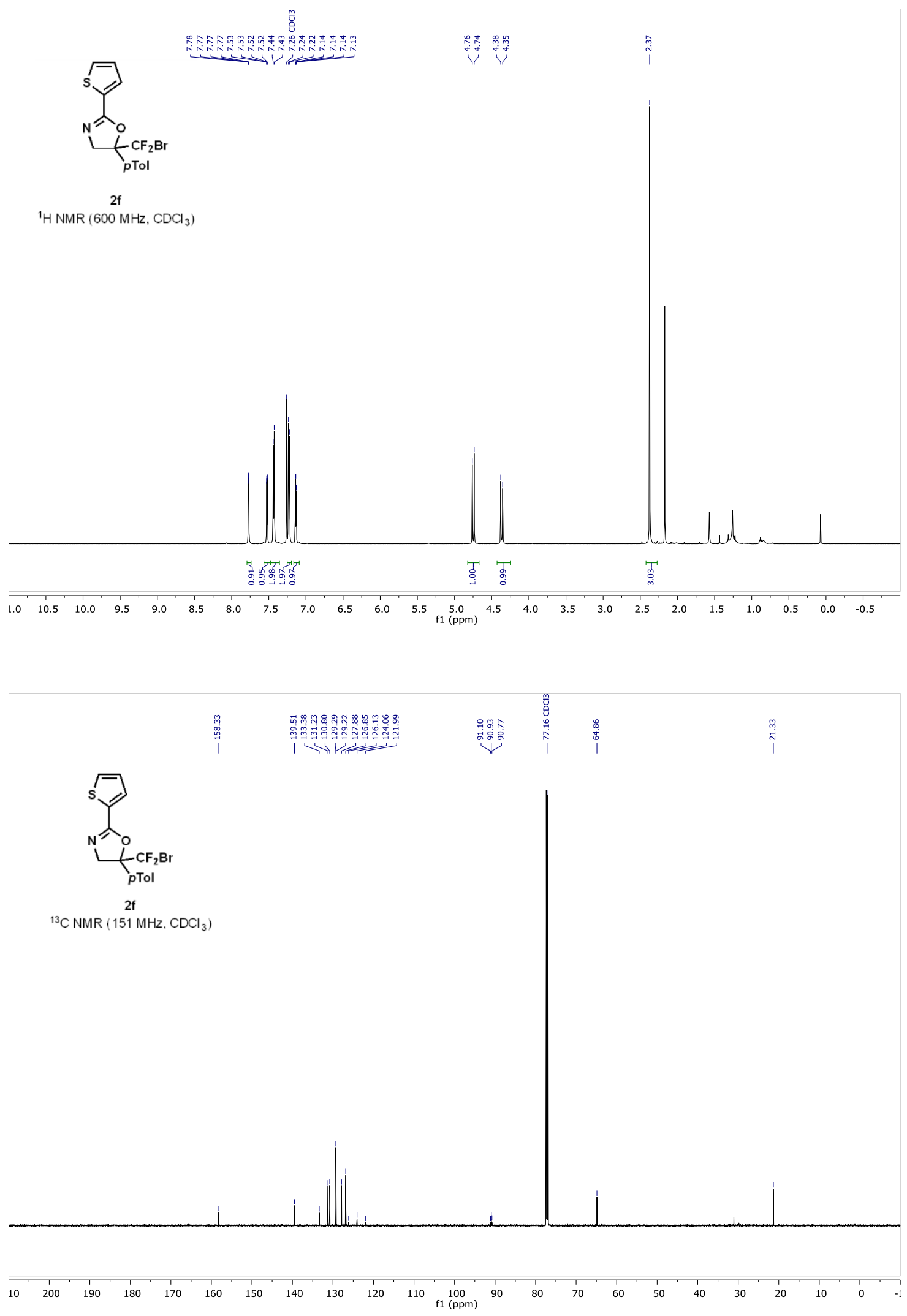

S165 


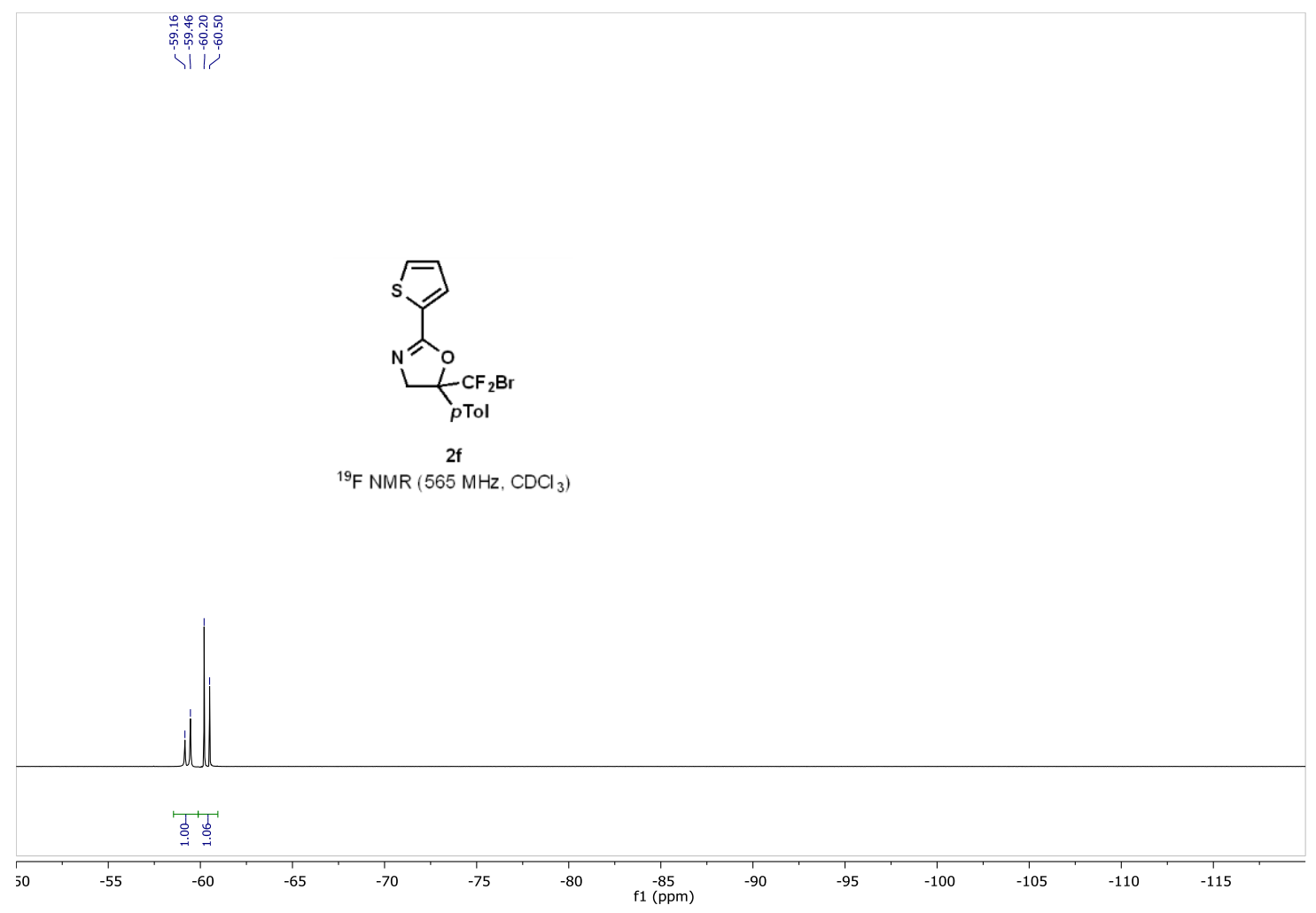



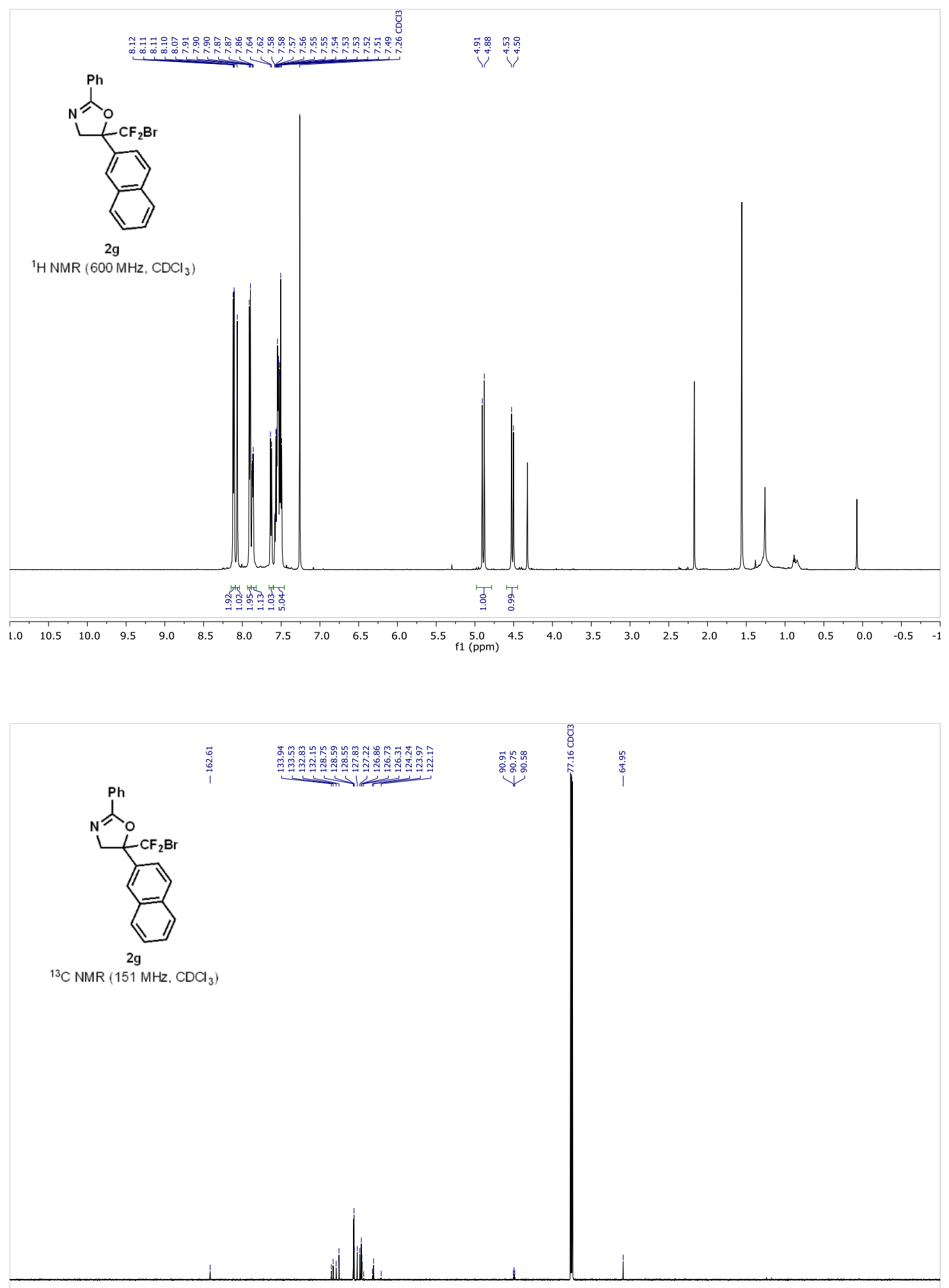

\begin{tabular}{llllllllllllllllllllllll}
\hline 10 & 200 & 190 & 180 & 170 & 160 & 150 & 140 & 130 & 120 & 110 & 100 & 10 & 80 & 70 & 60 & 50 & 40 & 30 & 20 & 10 & 0 & -
\end{tabular} 


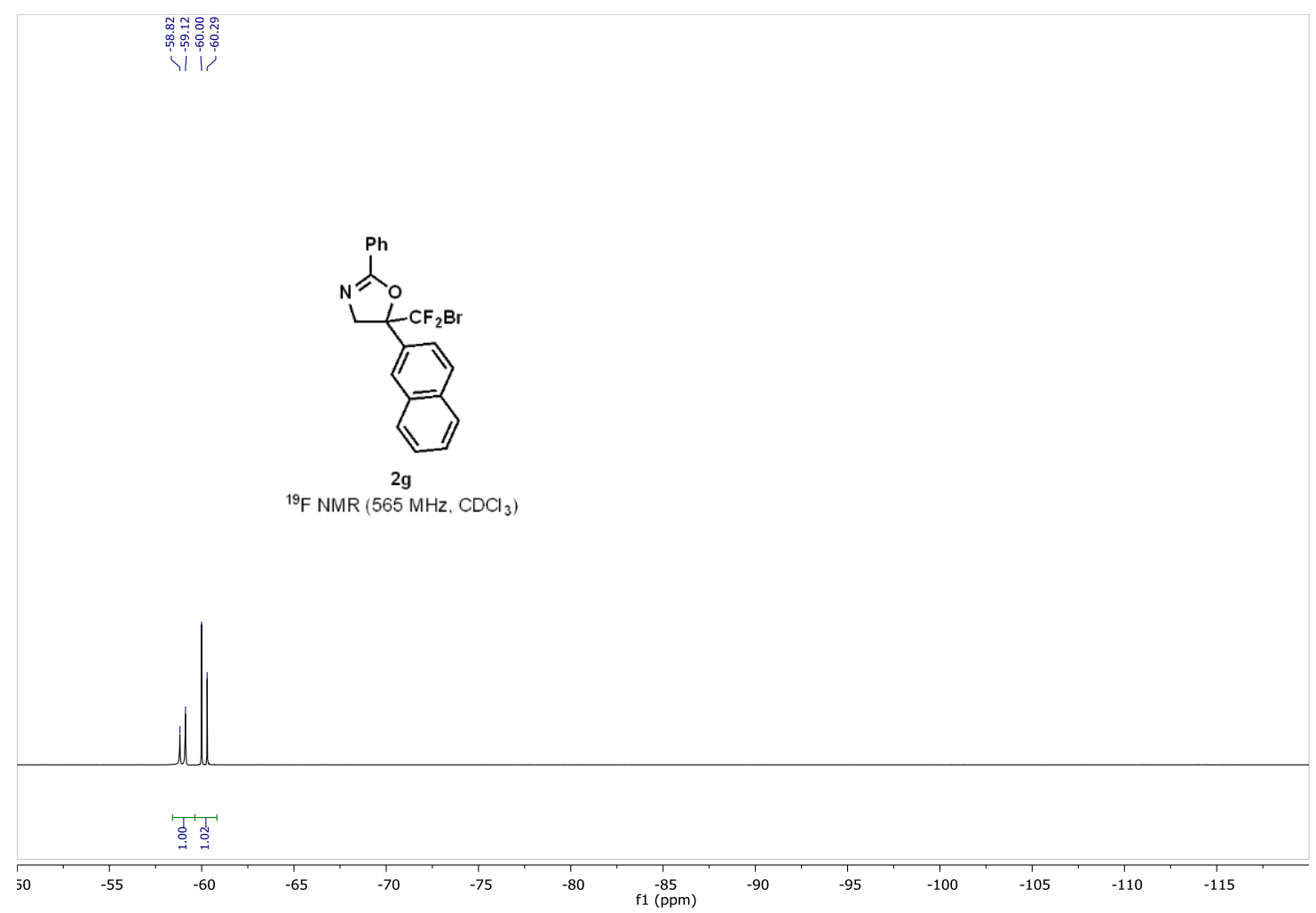



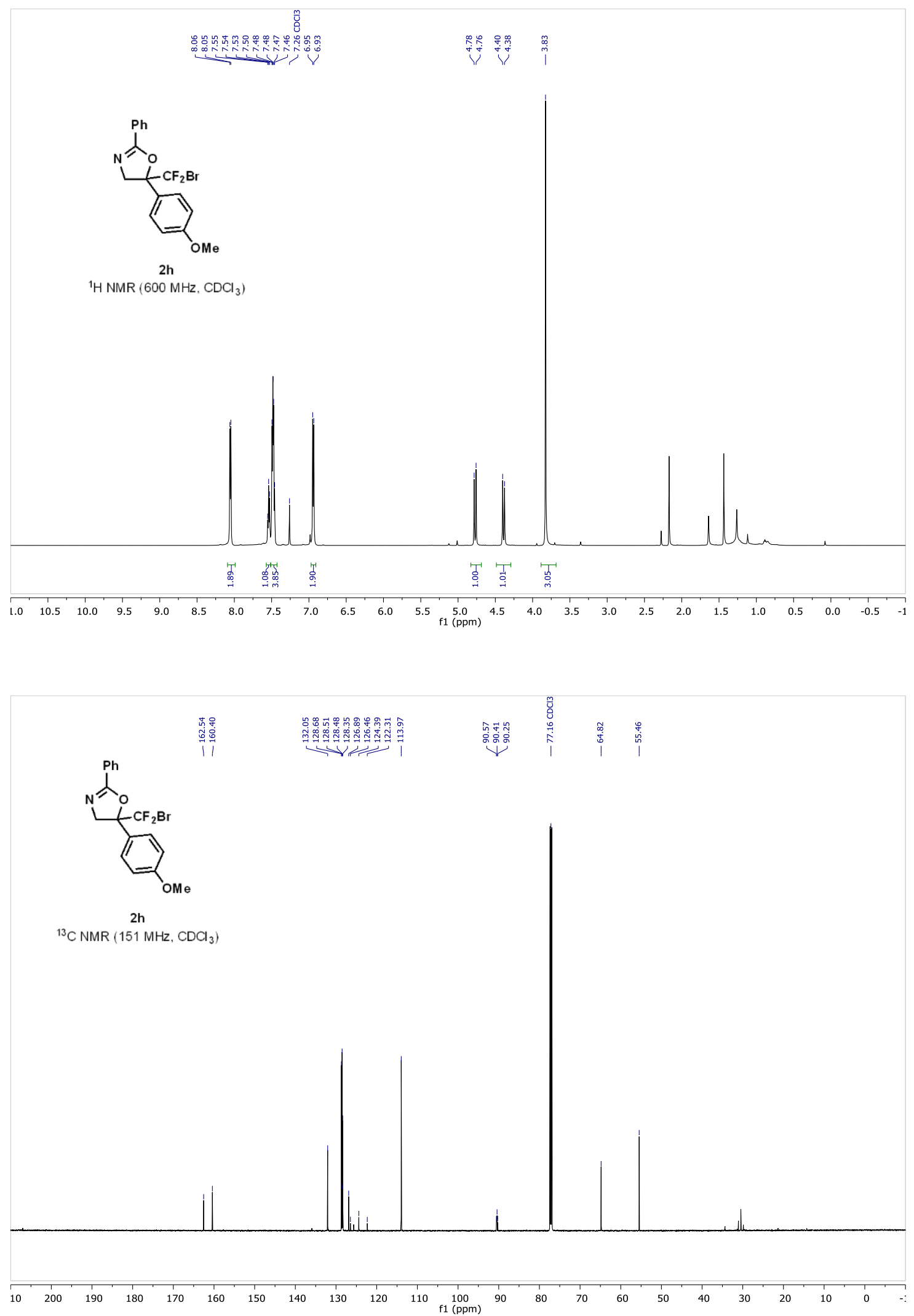


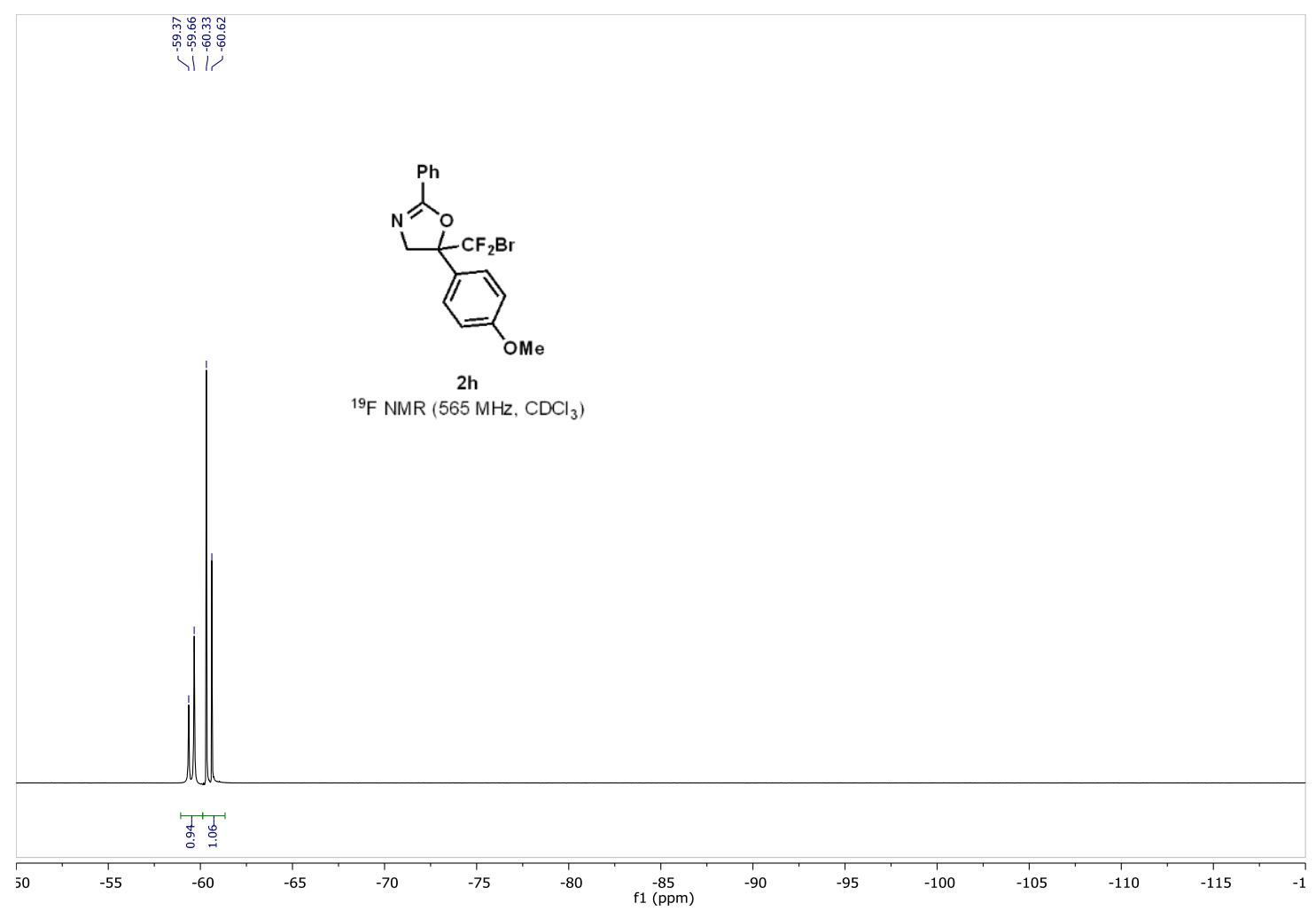



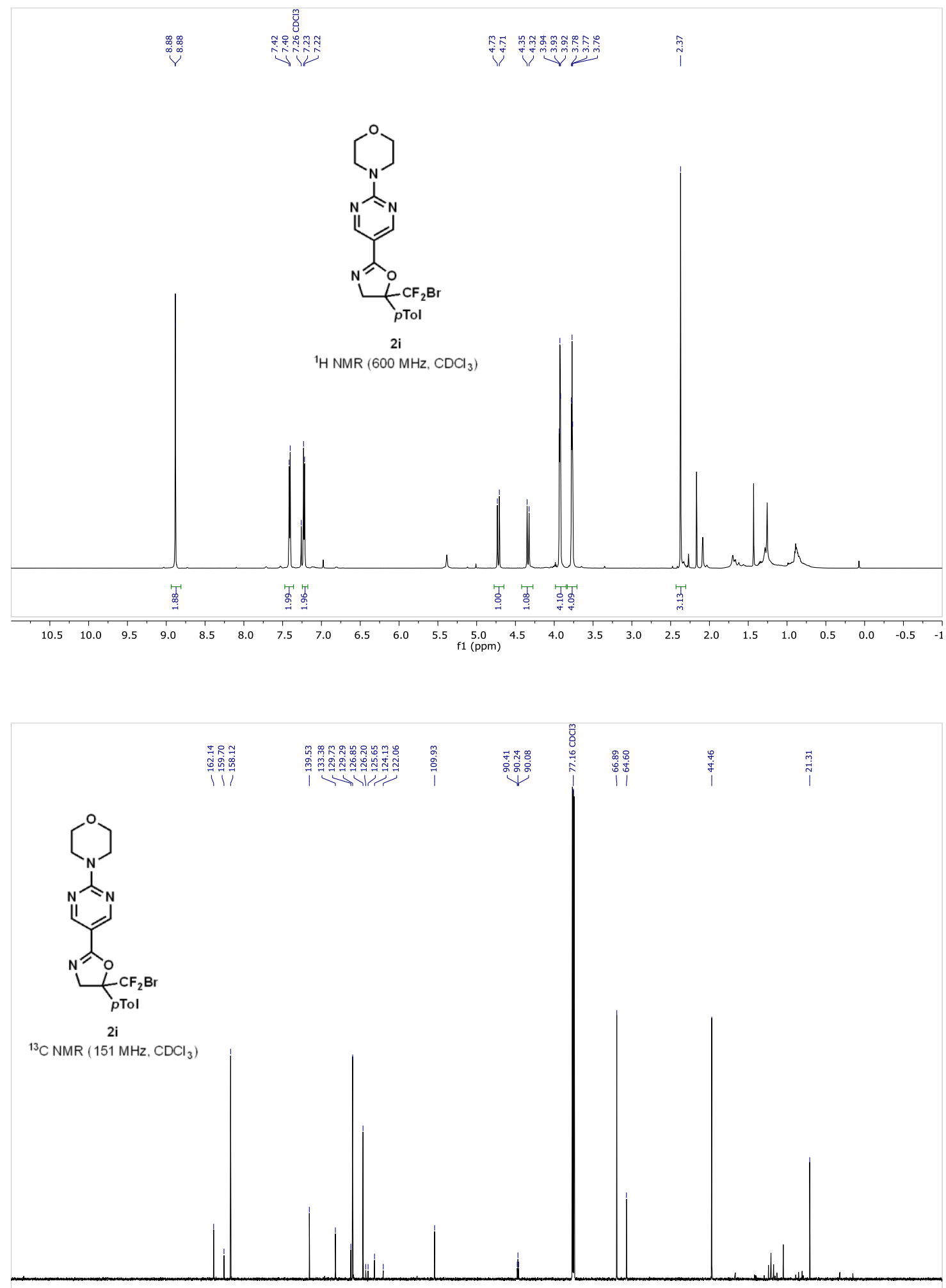

\begin{tabular}{llllllllllllllllllllllll}
\hline 10 & 200 & 190 & 180 & 170 & 160 & 150 & 140 & 130 & 120 & 110 & 100 & 10 & 80 & 70 & 60 & 50 & 40 & 30 & 20 & 10 & 0 & -
\end{tabular} 


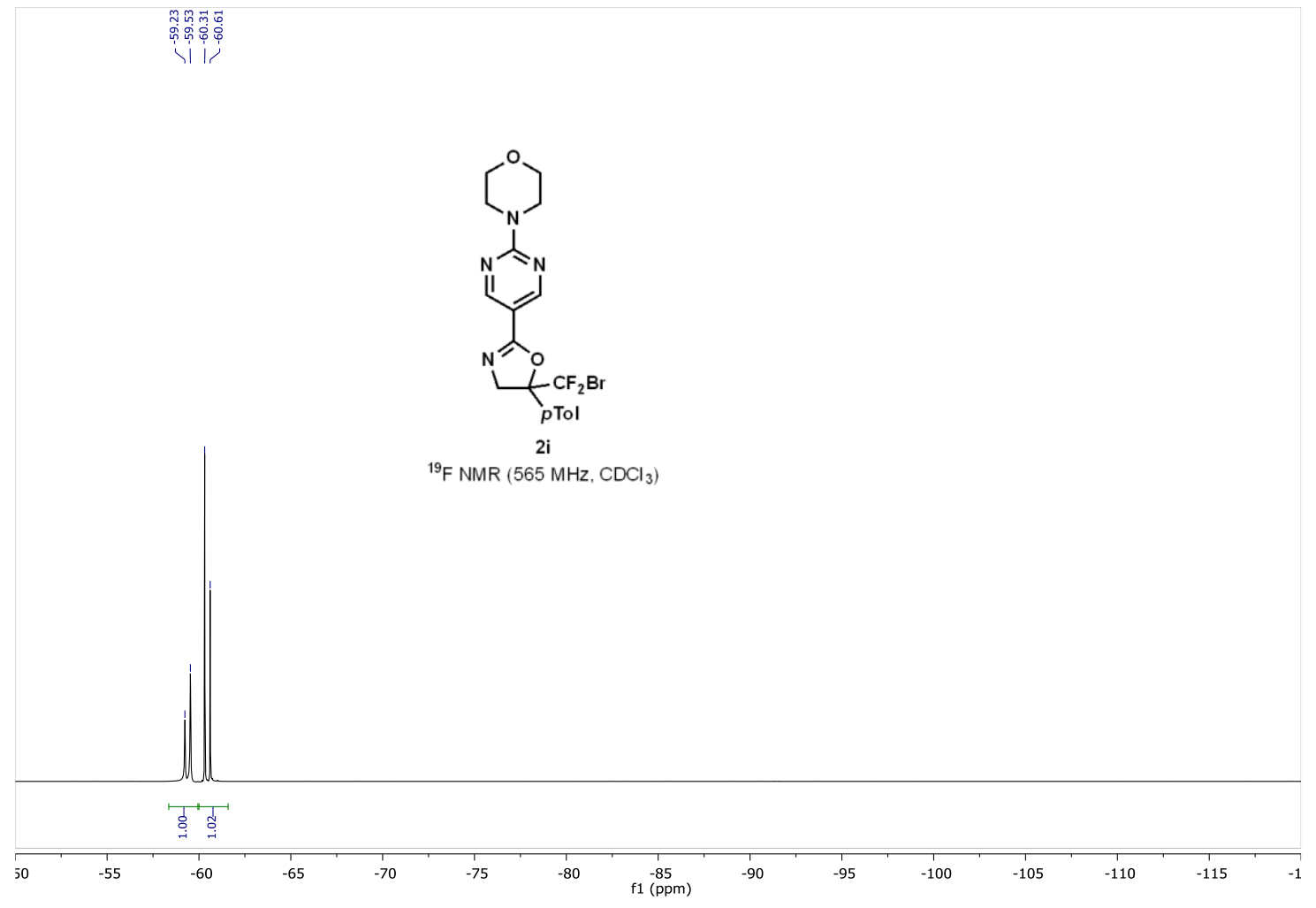



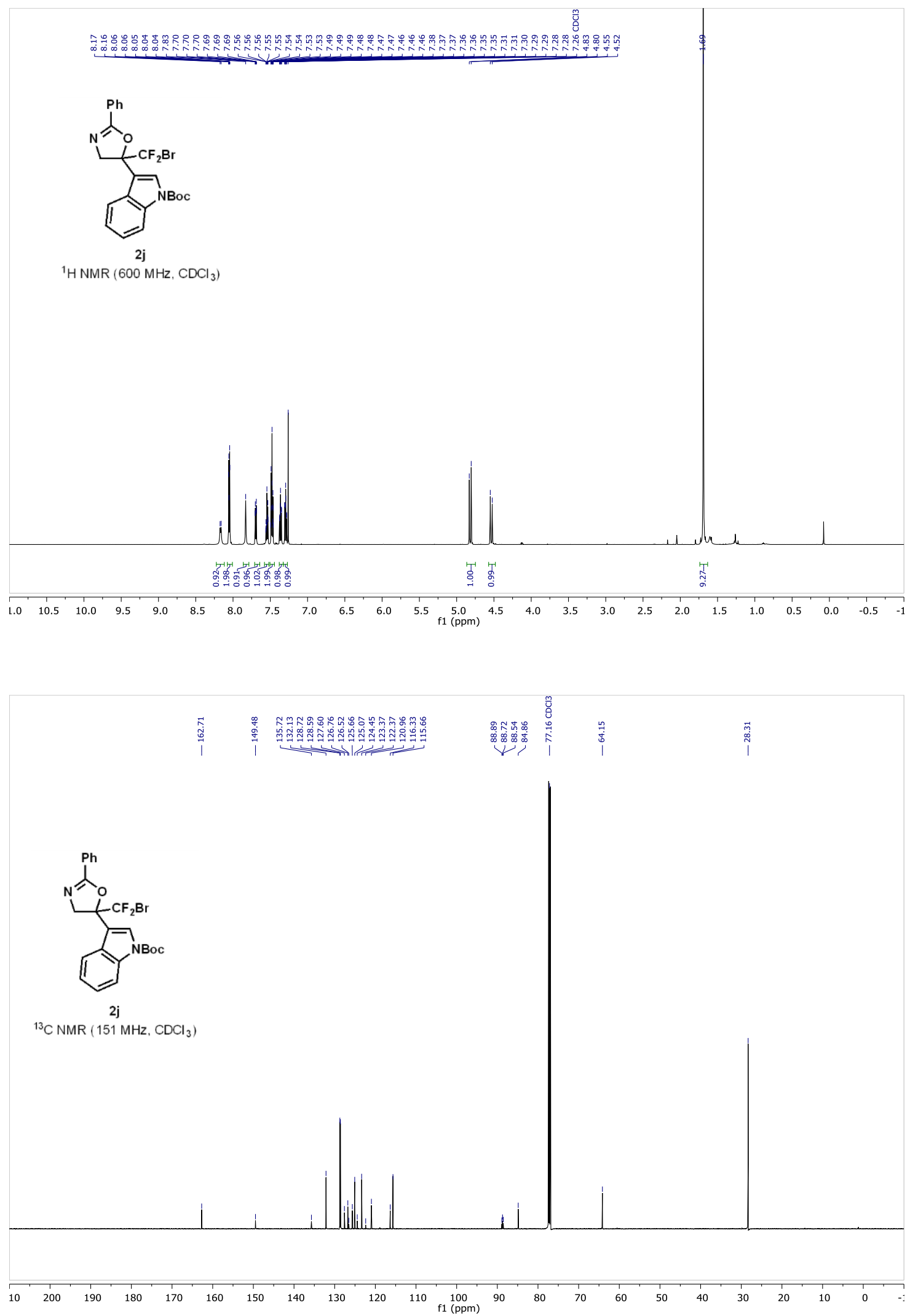


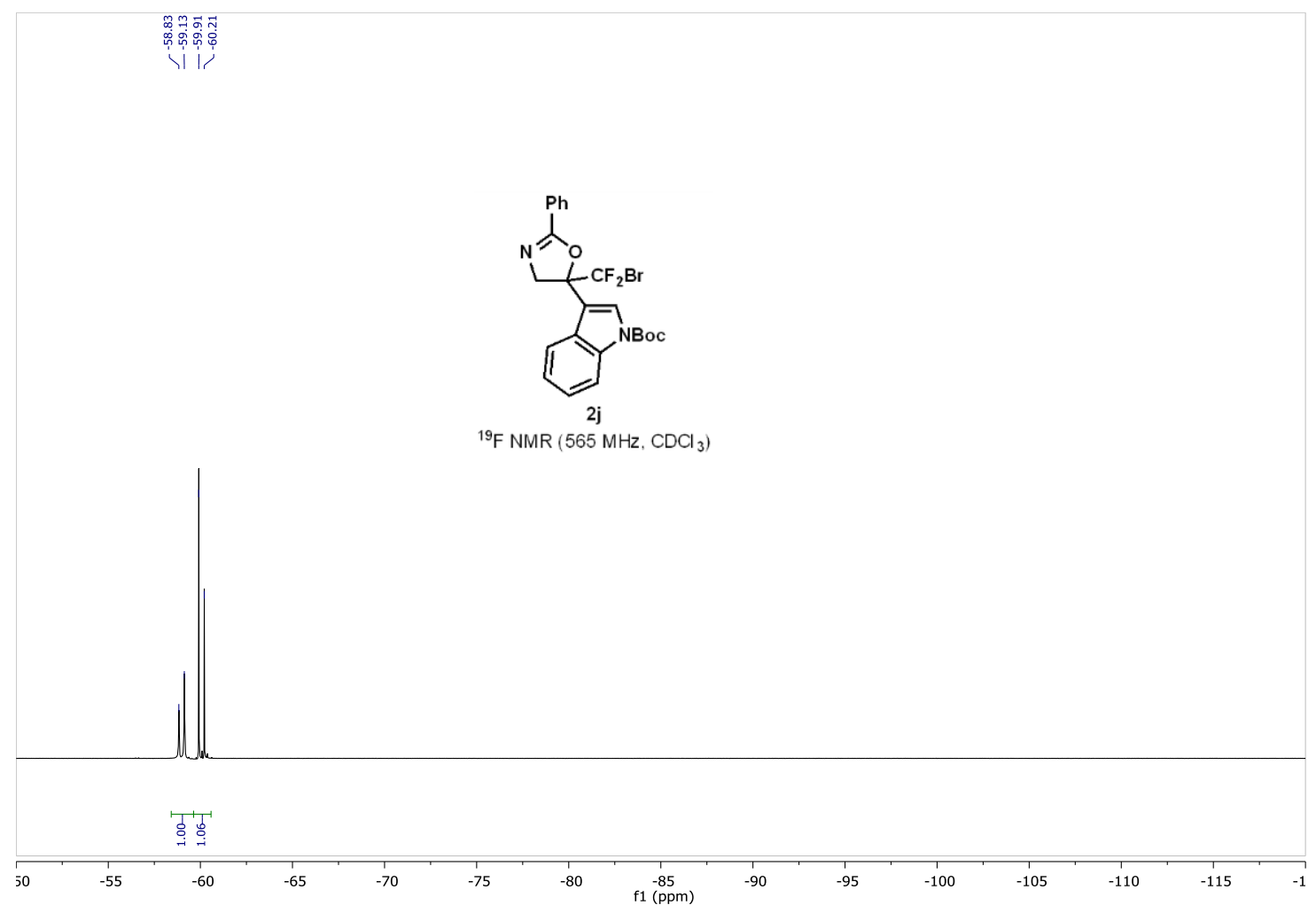



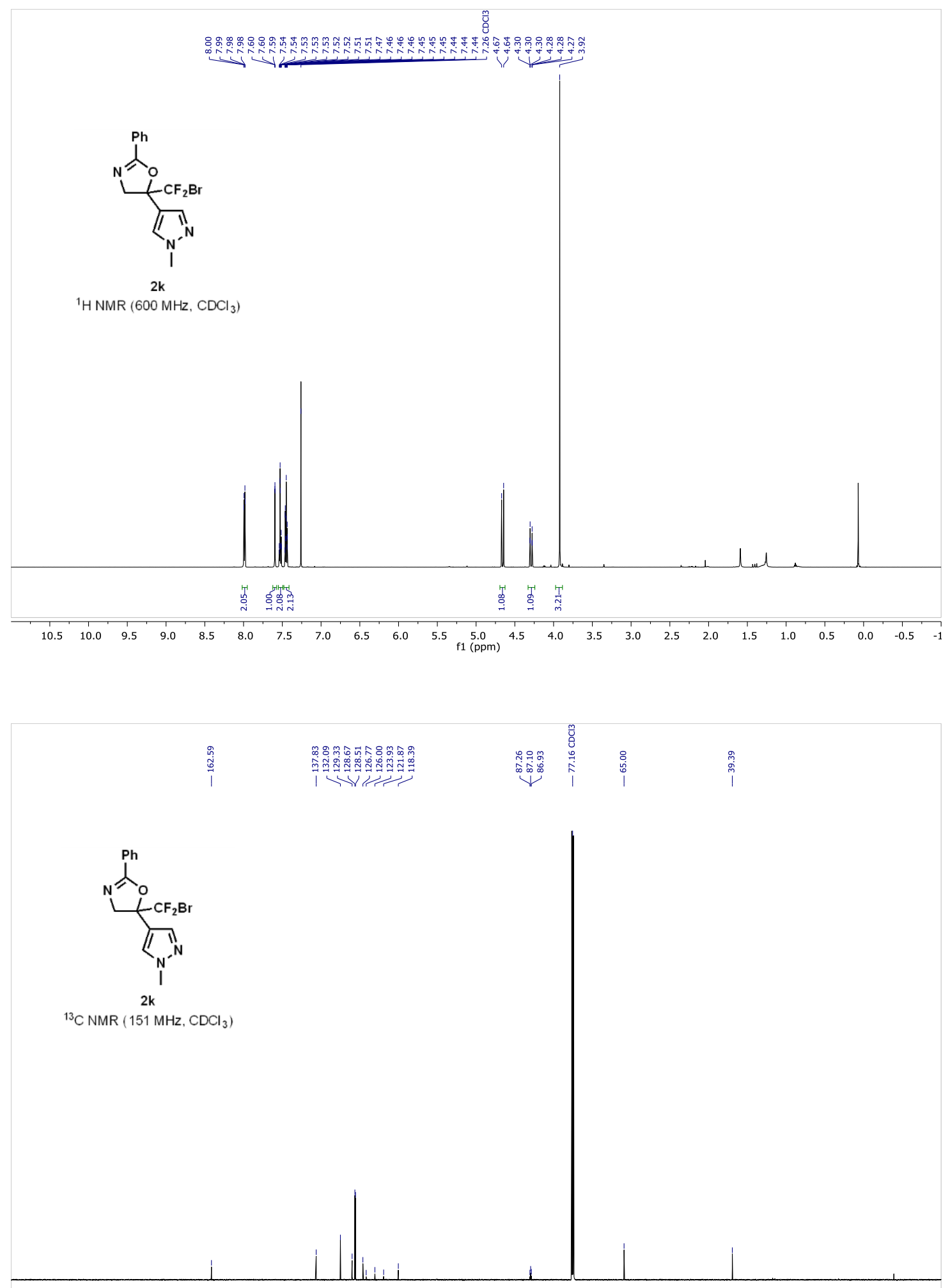

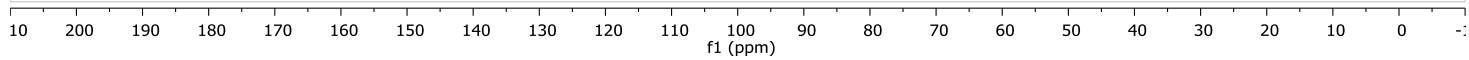




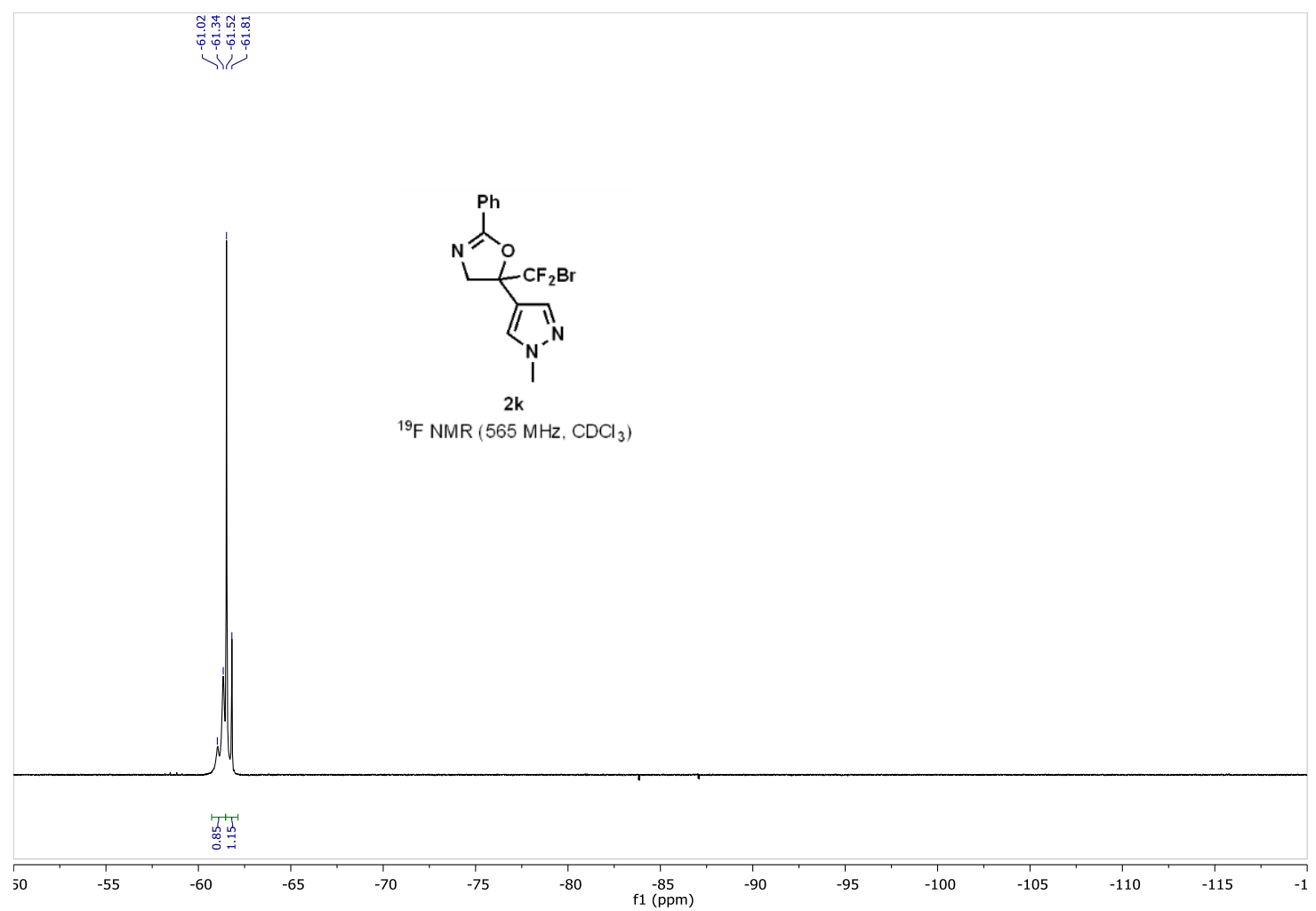



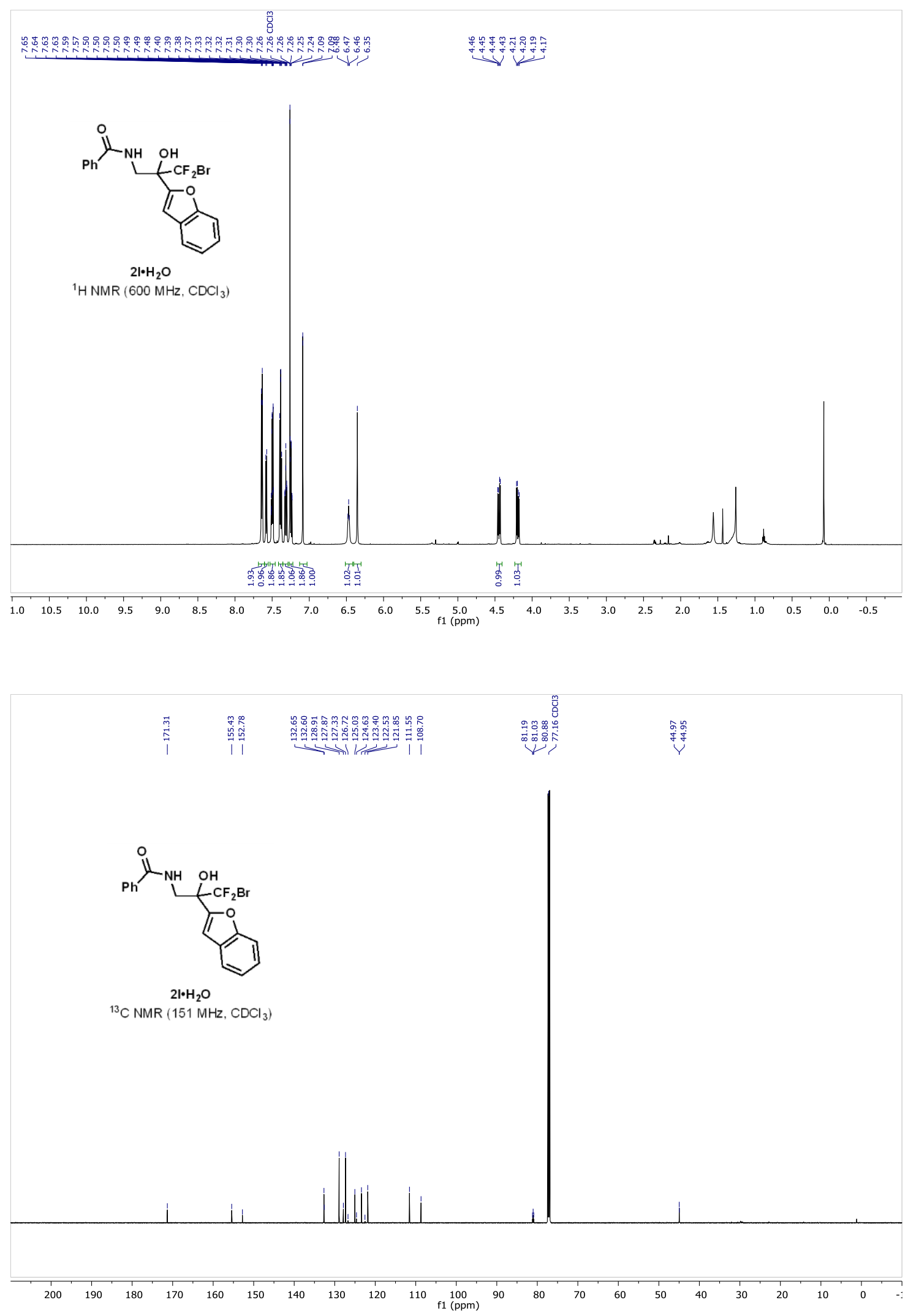


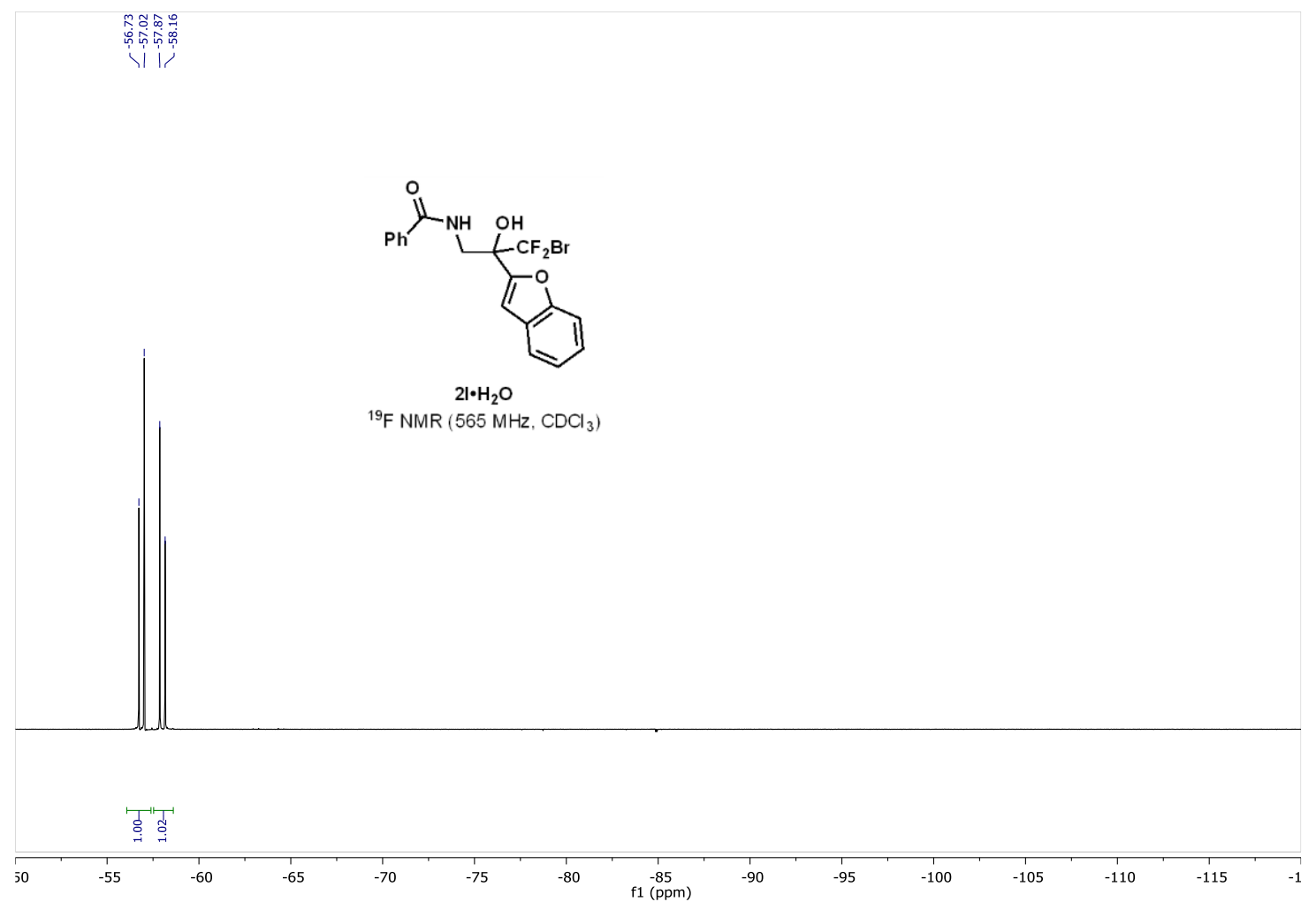



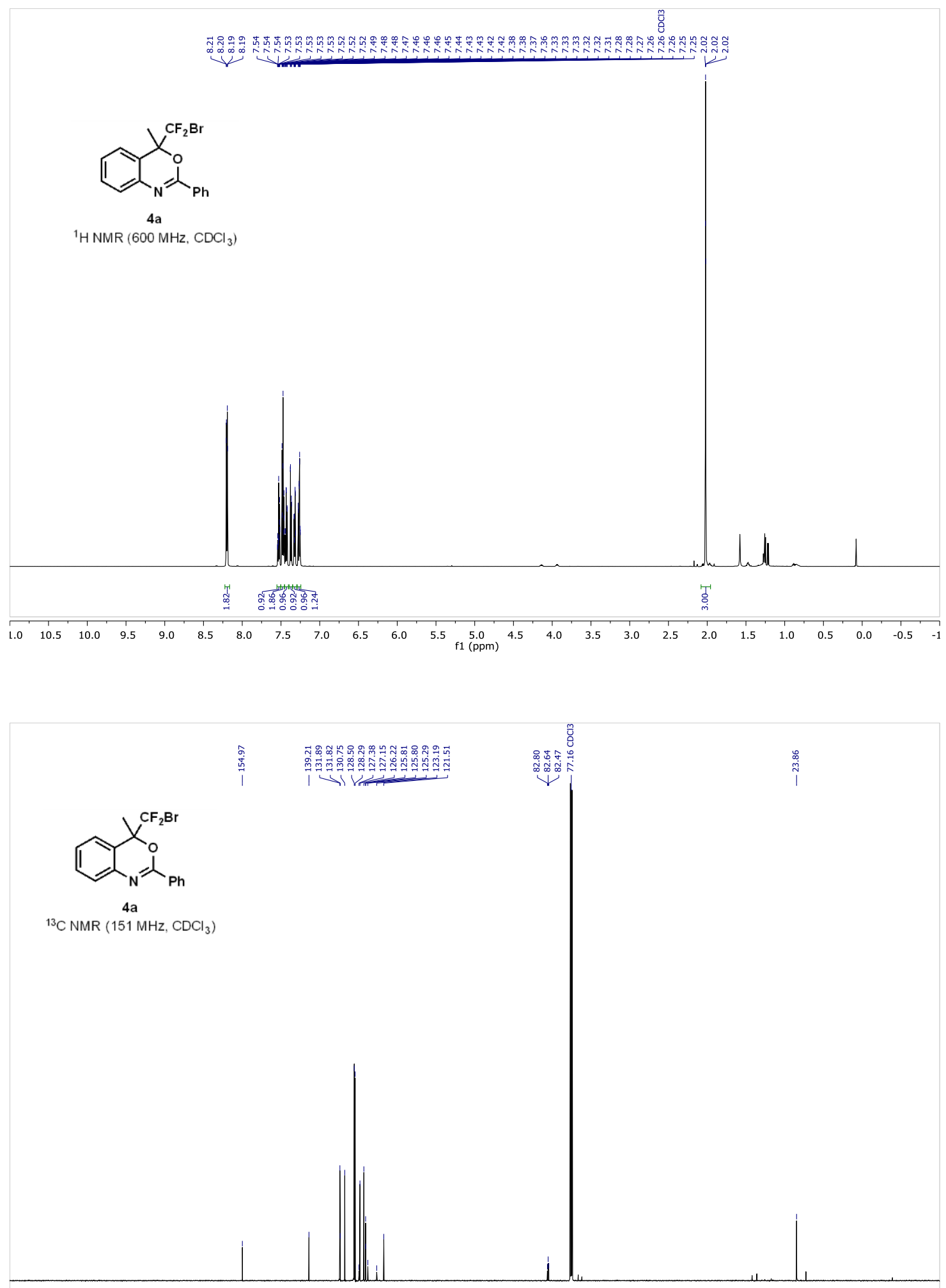

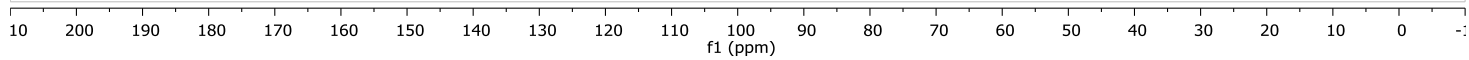




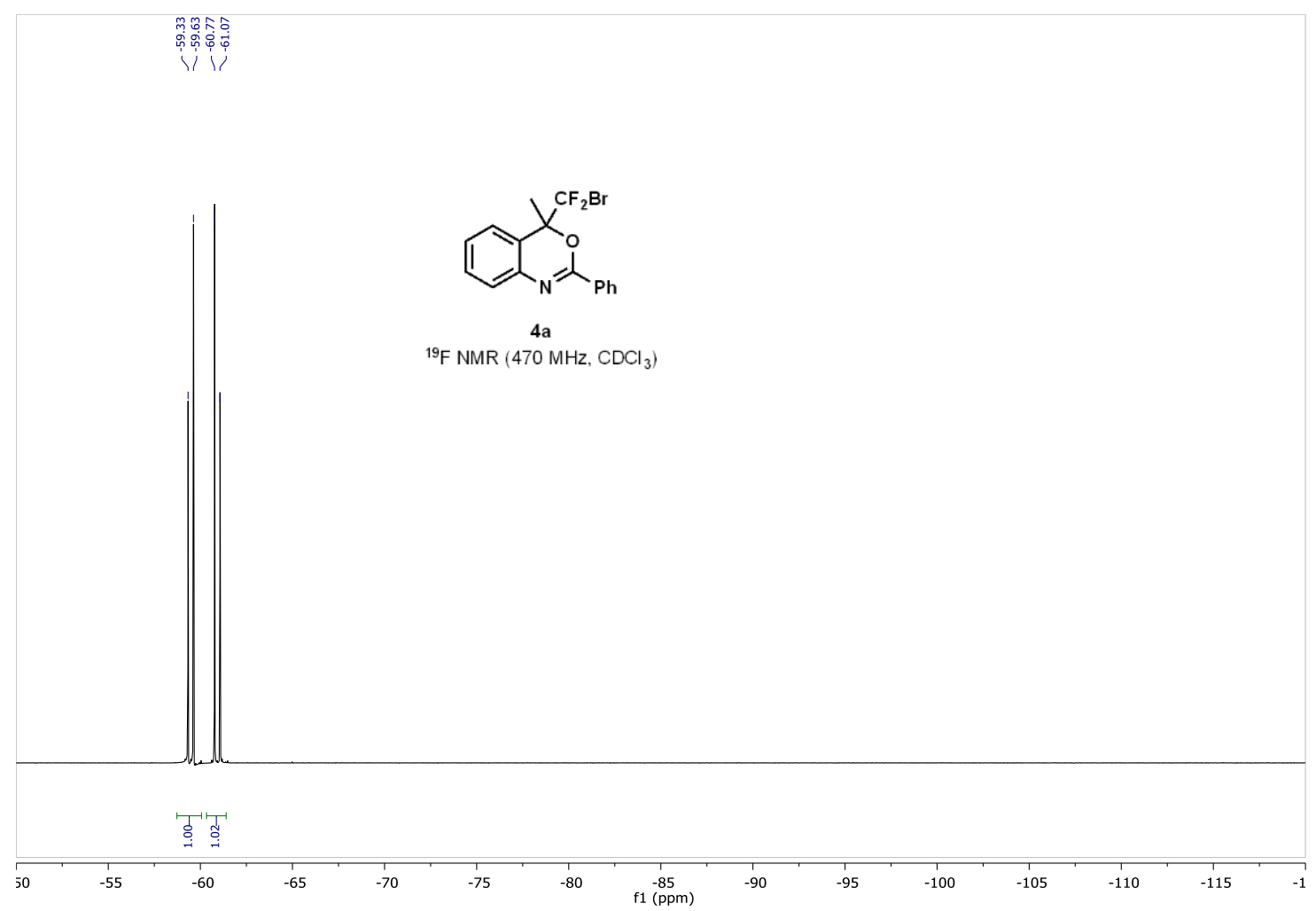



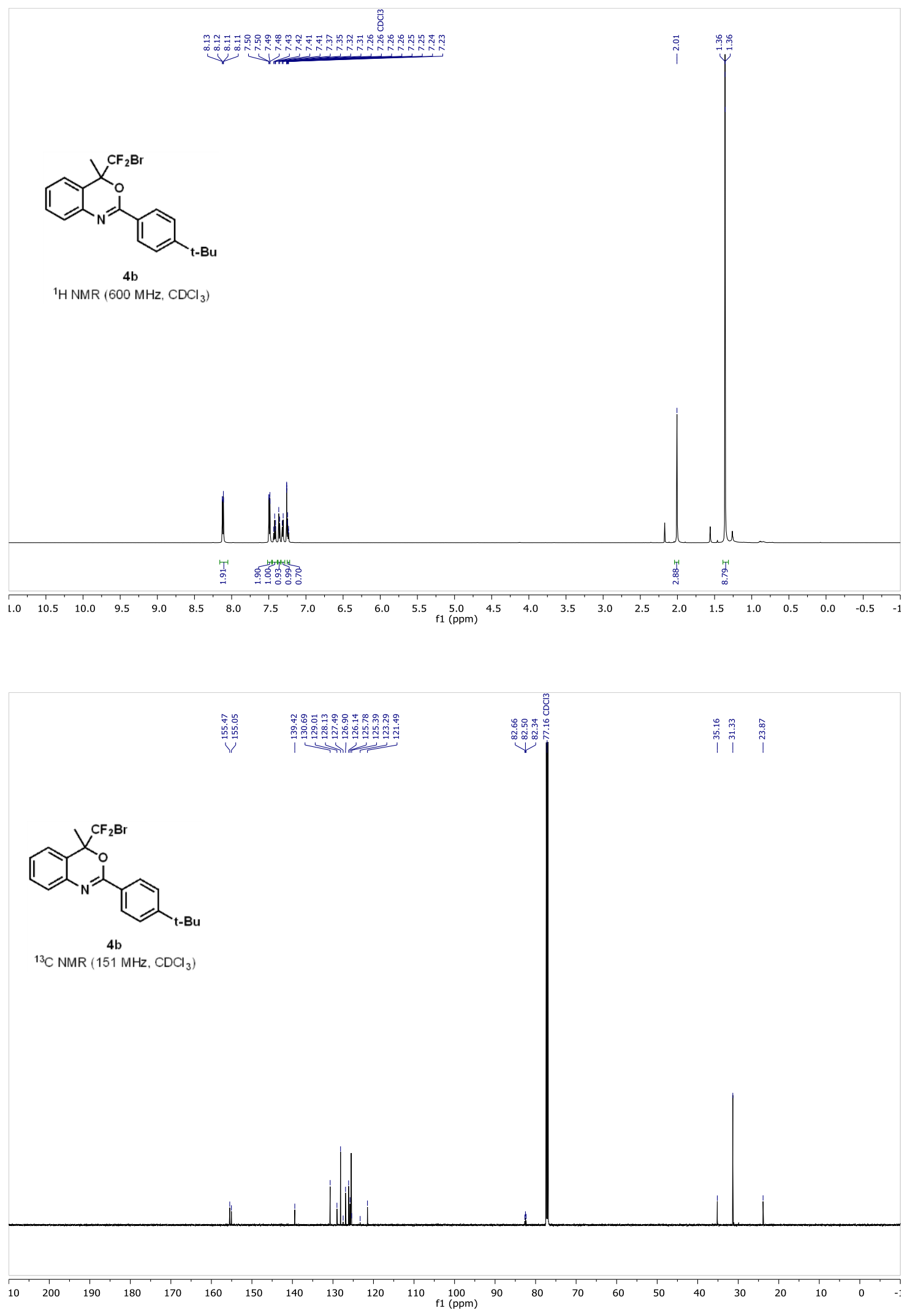

S181 


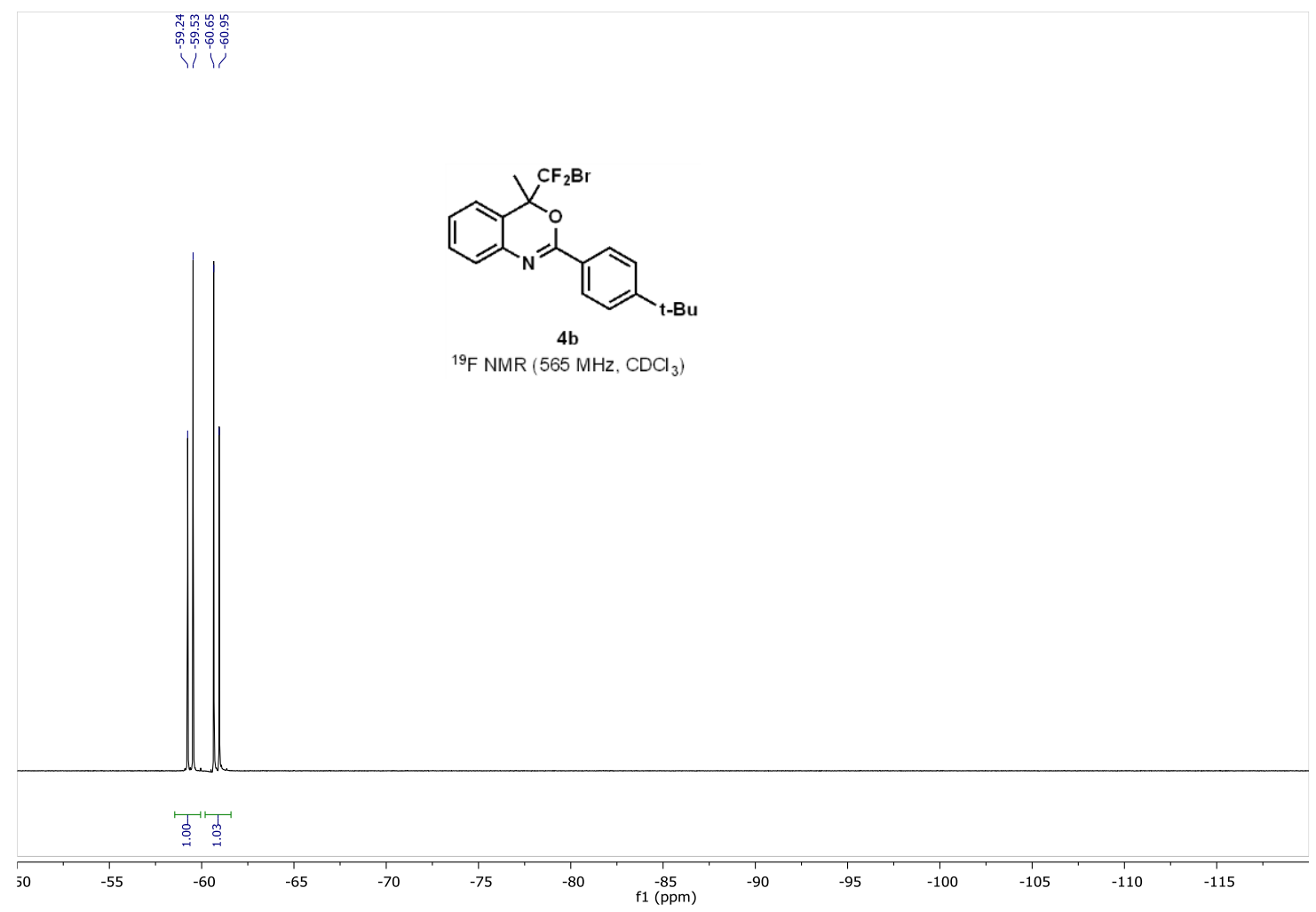



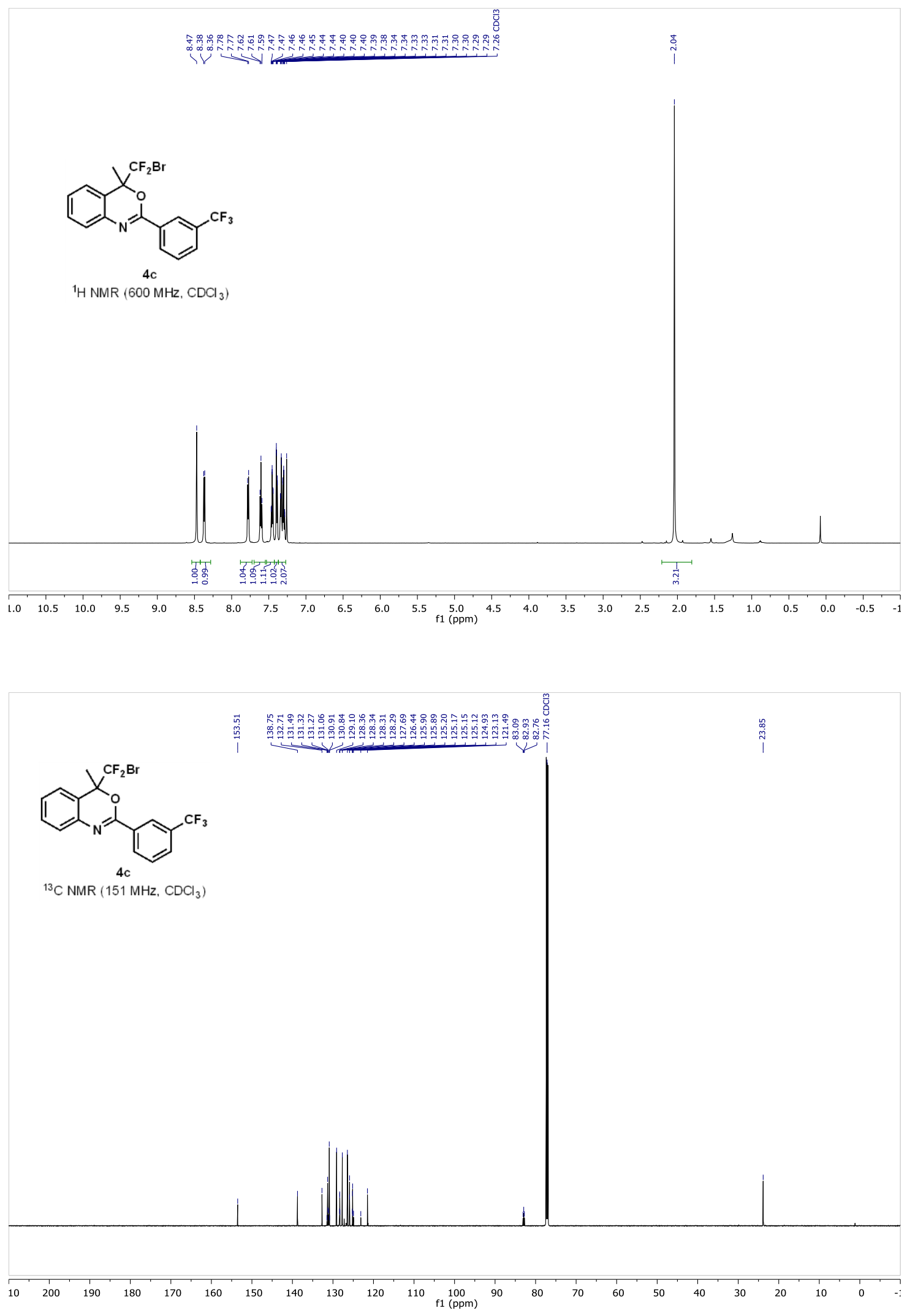

S183 


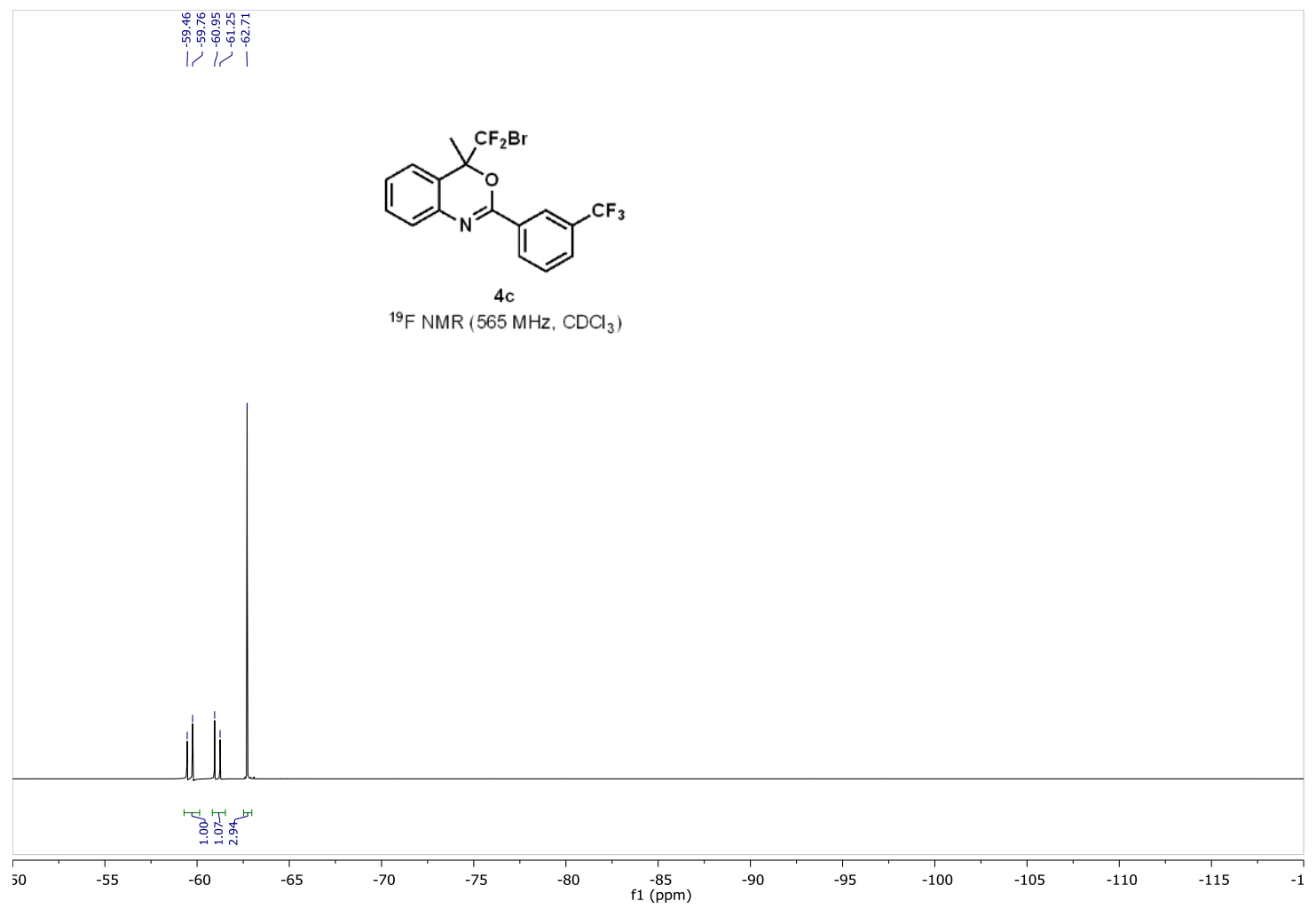



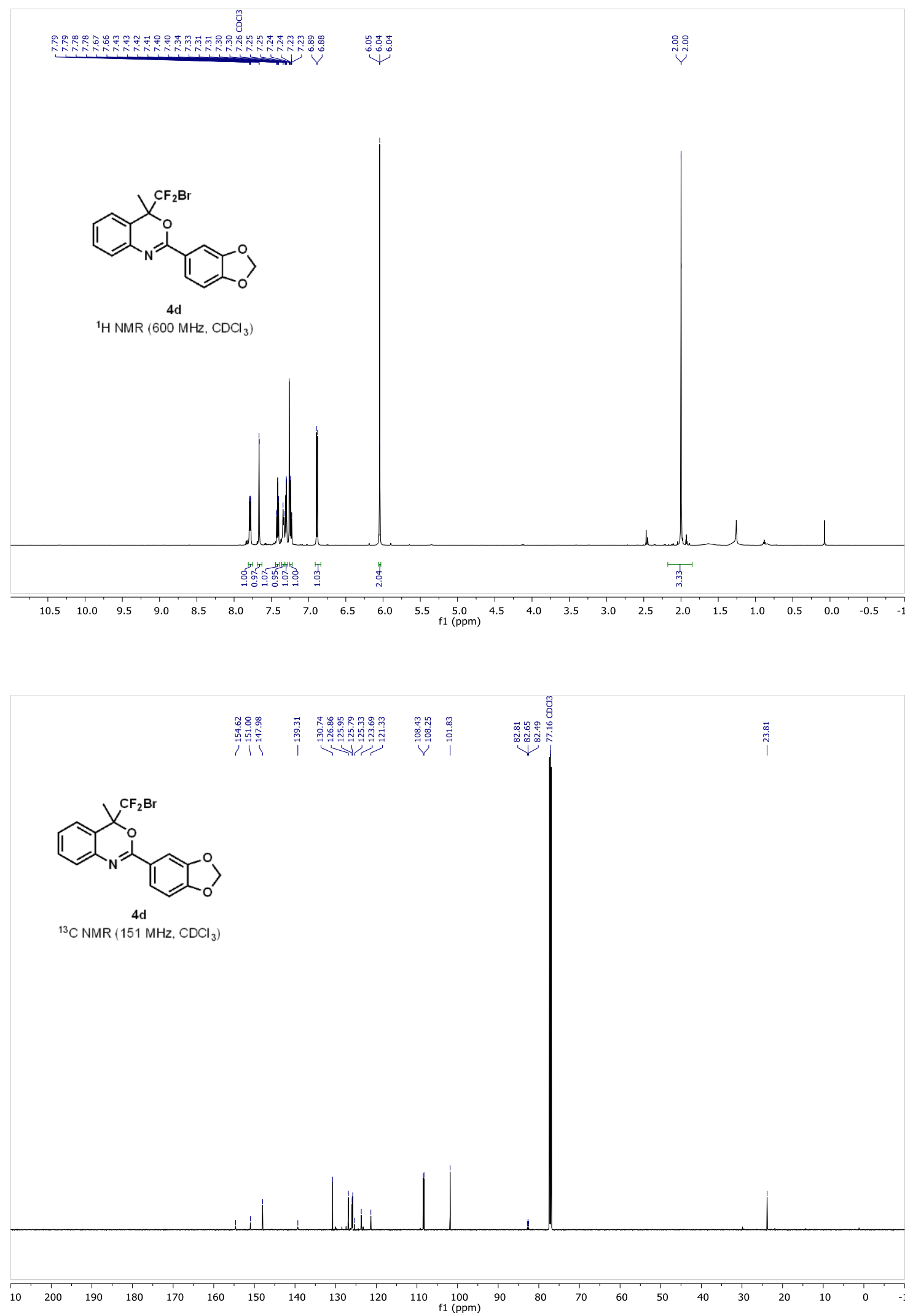


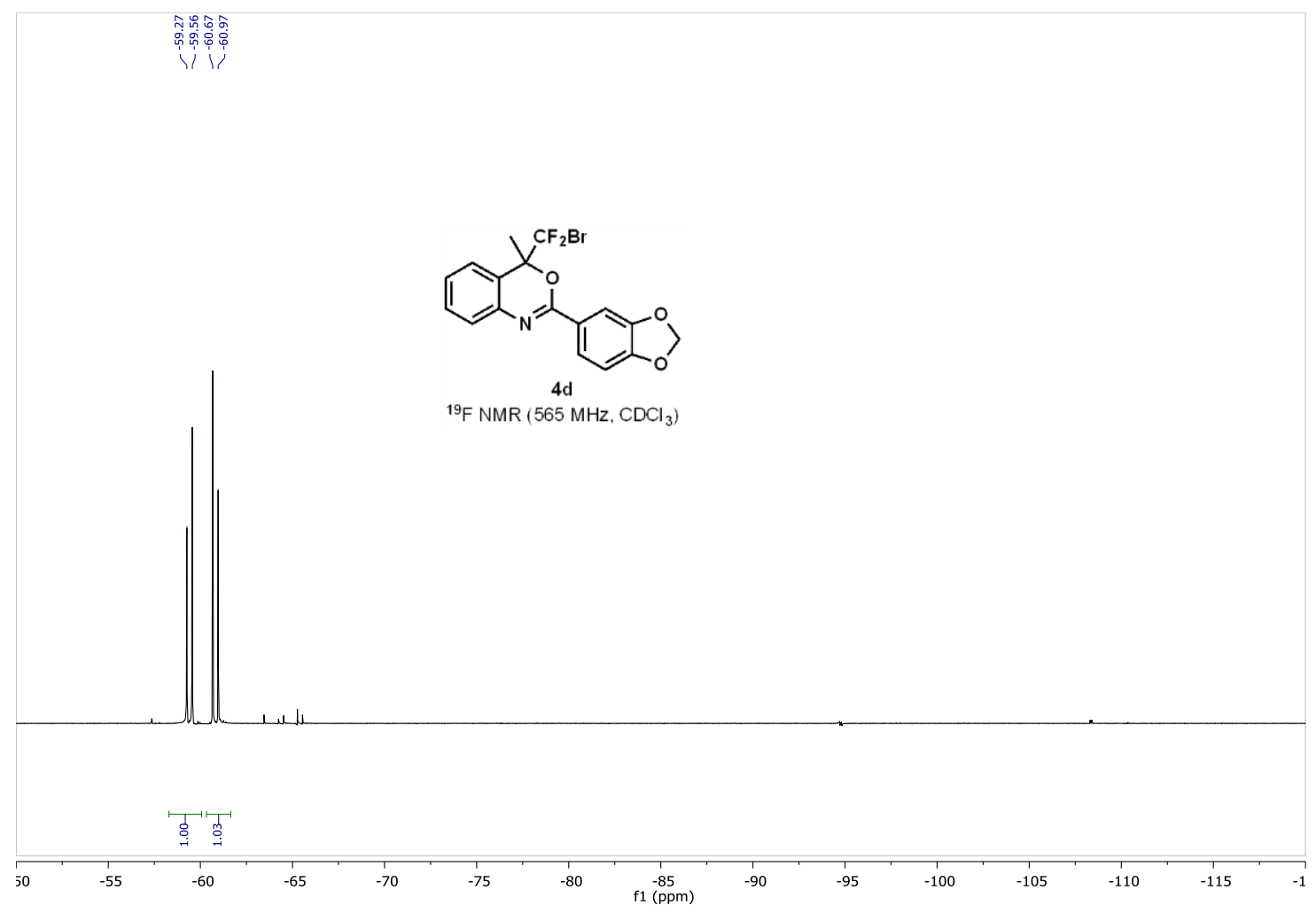



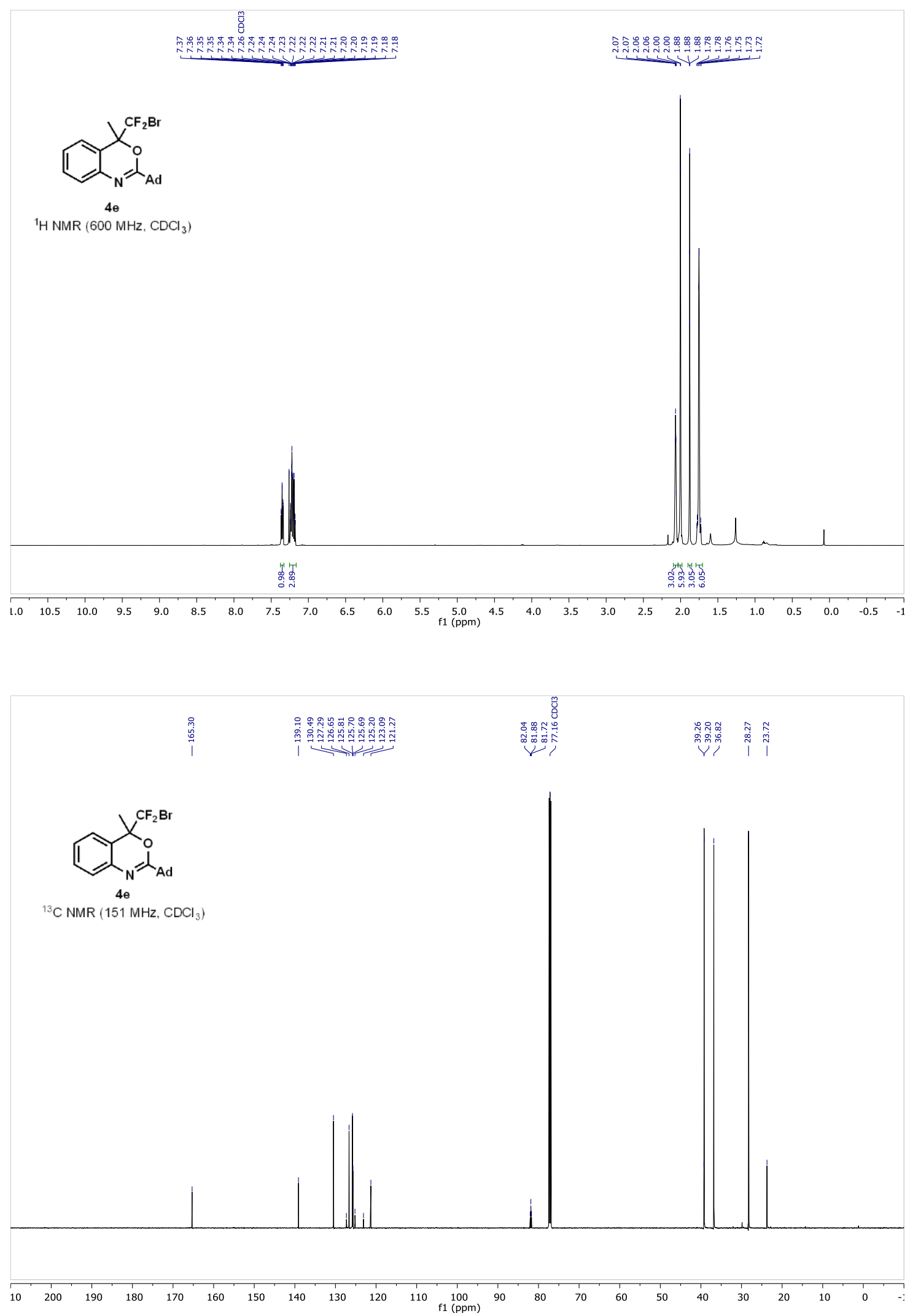


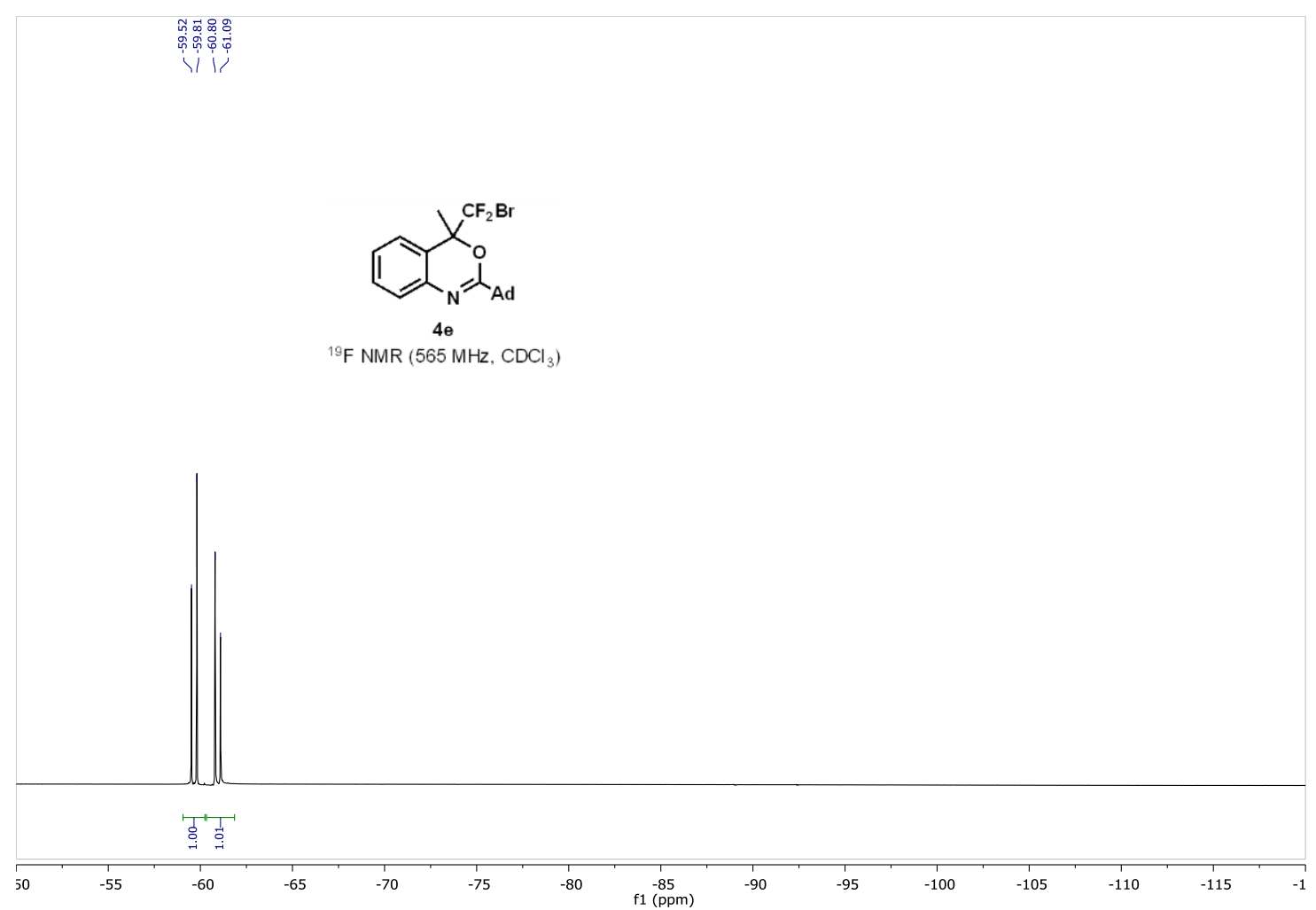



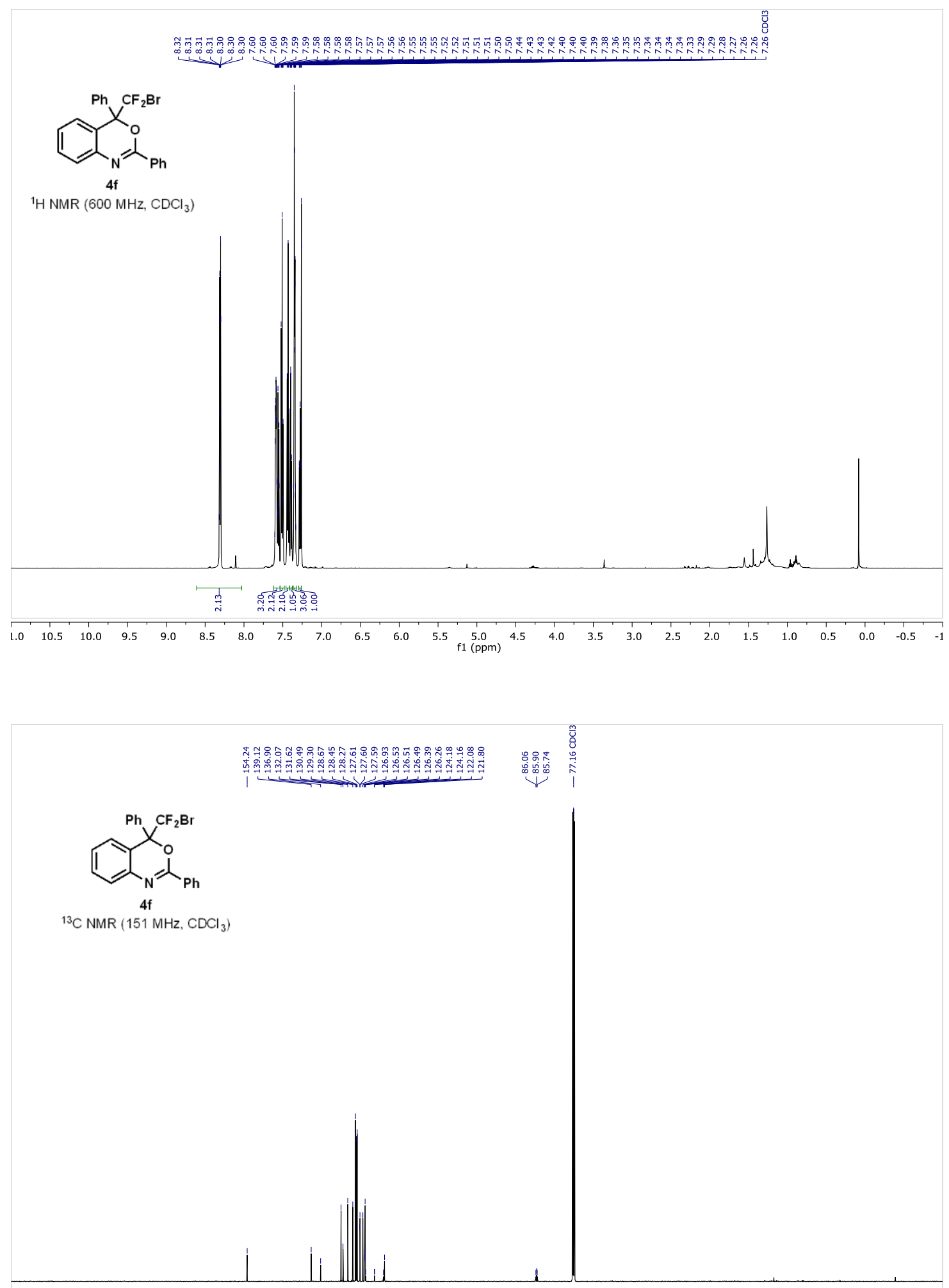

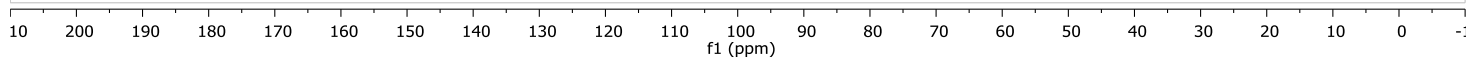




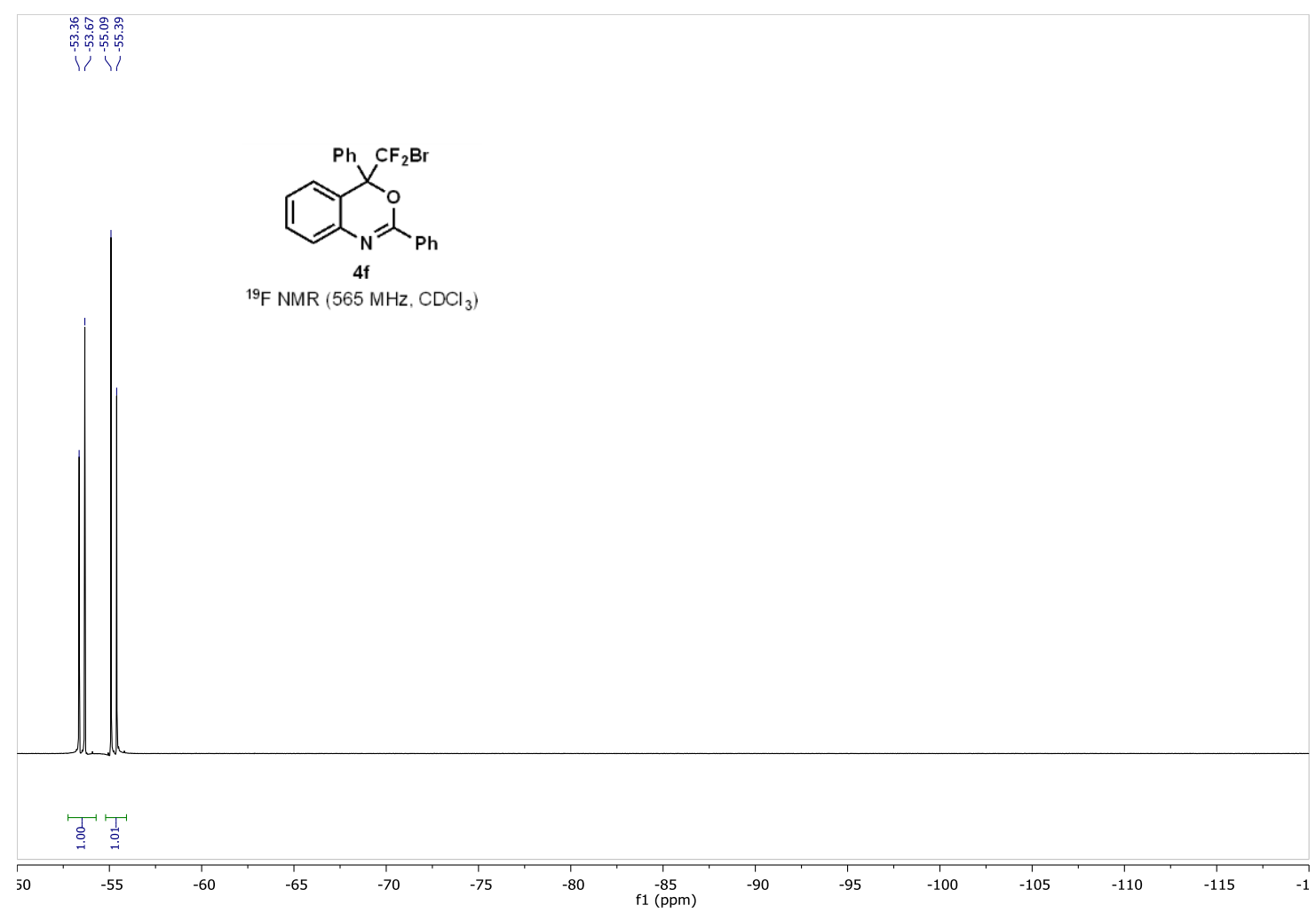



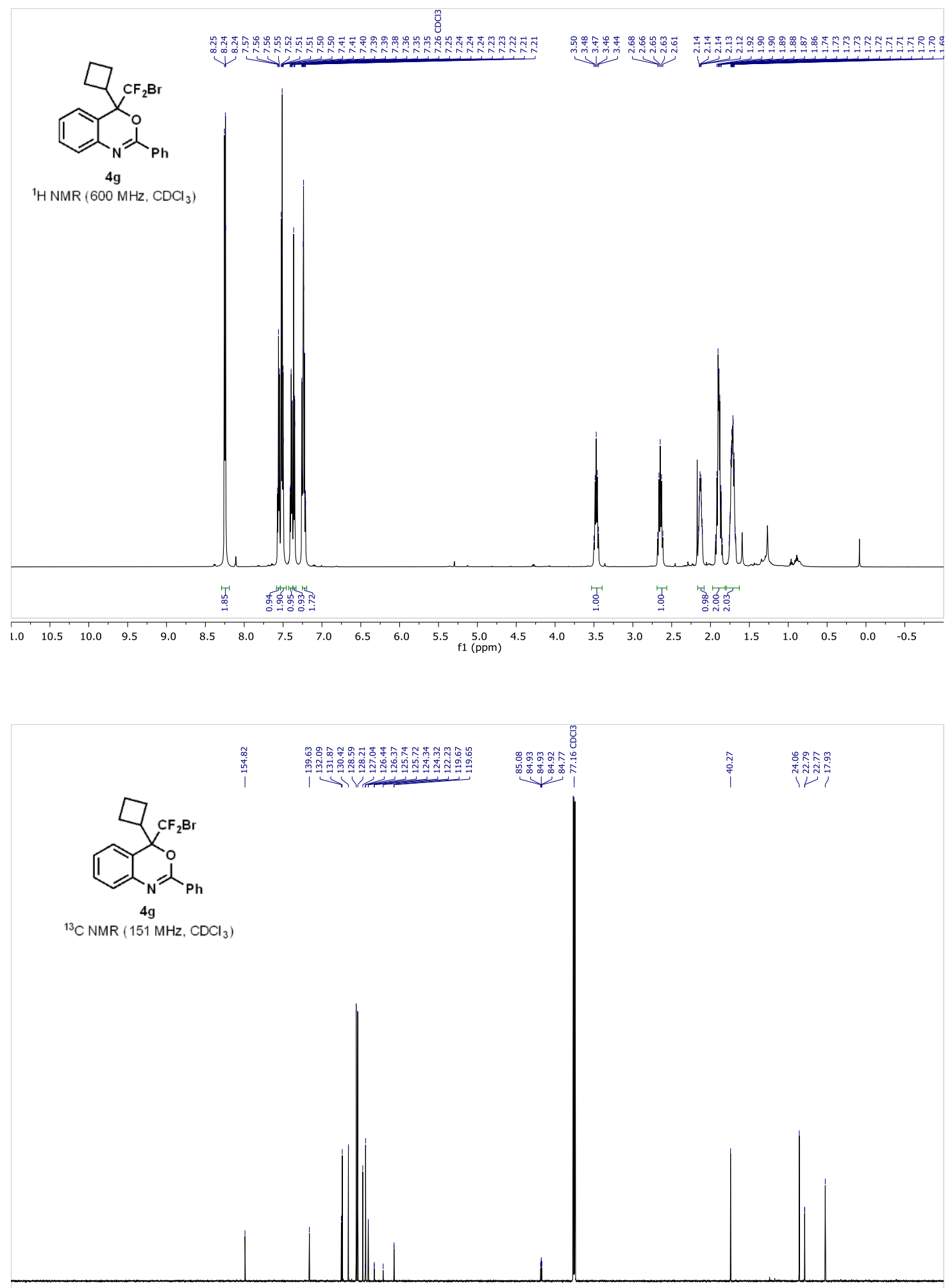

\begin{tabular}{llllllllllllllllllllllll}
\hline 10 & 200 & 190 & 180 & 170 & 160 & 150 & 140 & 130 & 120 & 110 & 100 & 10 & 80 & 70 & 60 & 50 & 40 & 30 & 20 & 10 & 0 & -
\end{tabular} 


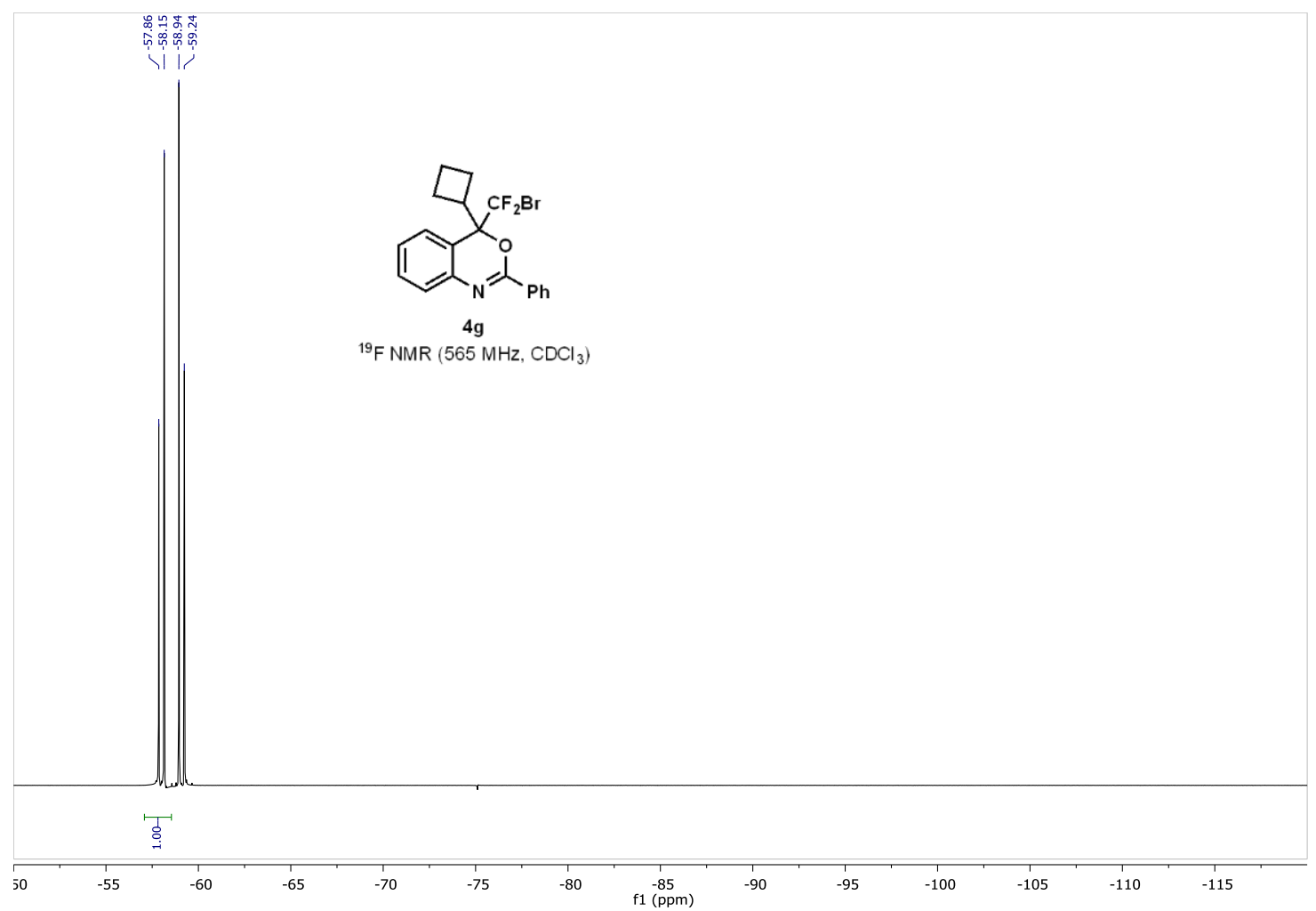



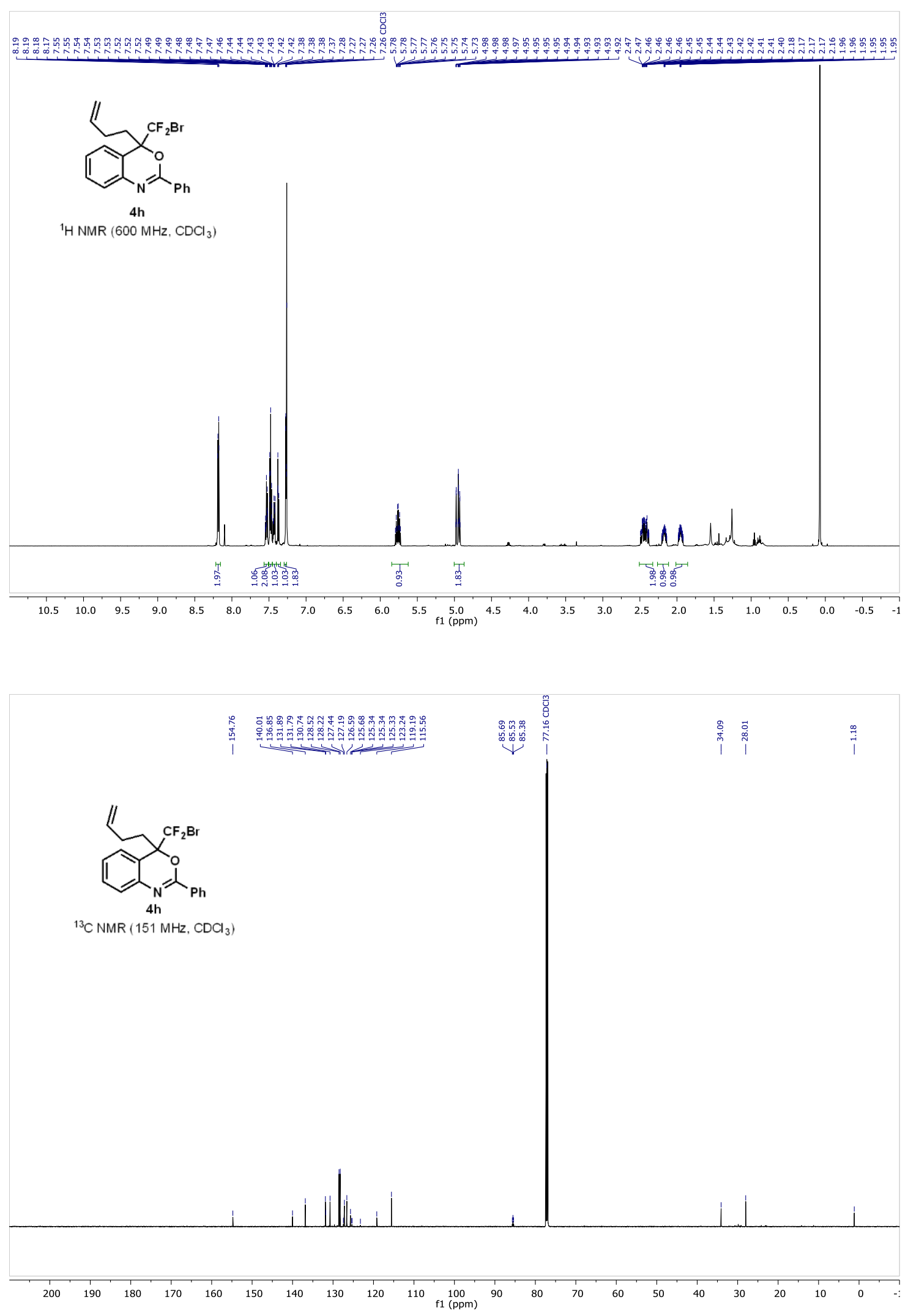


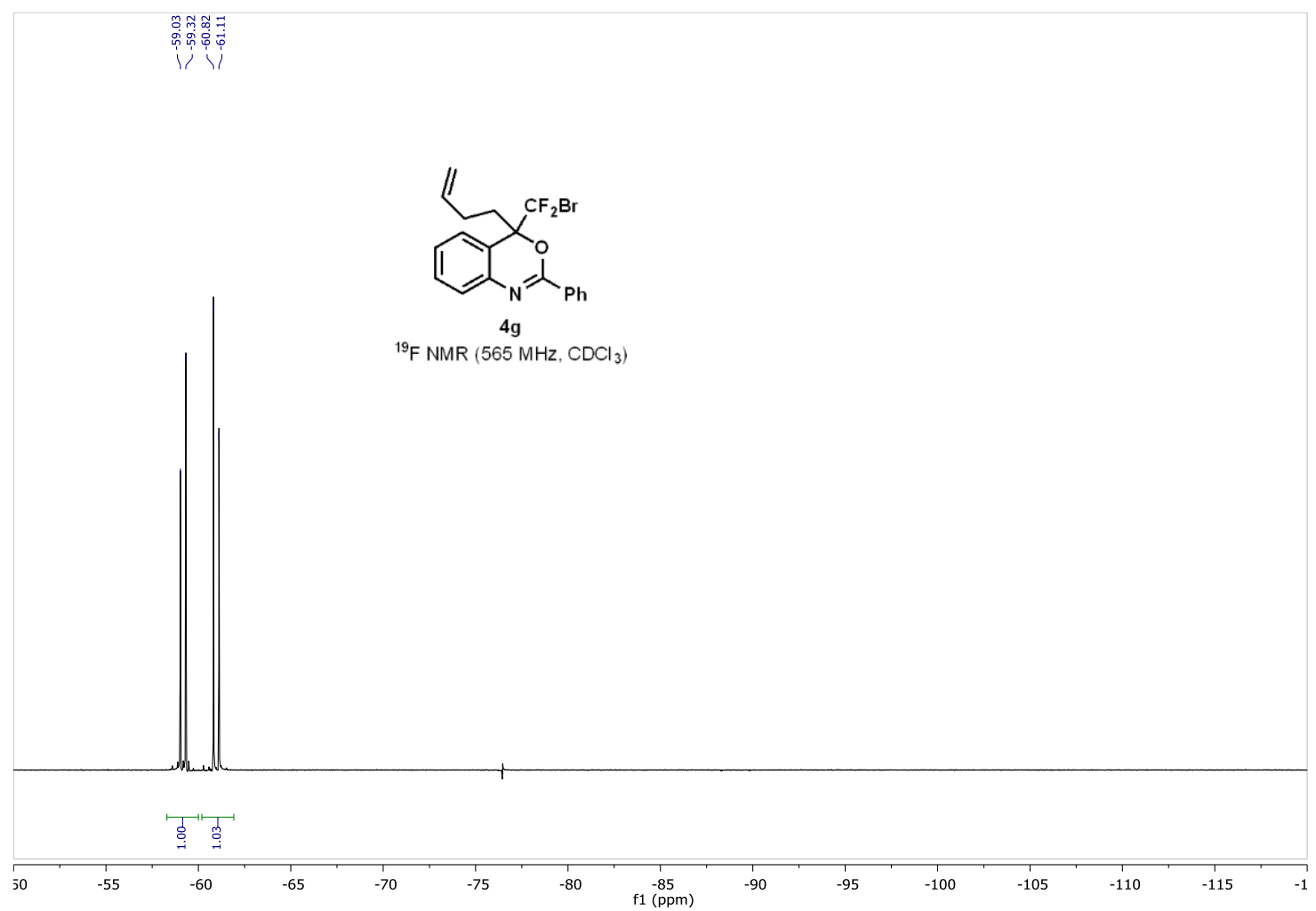



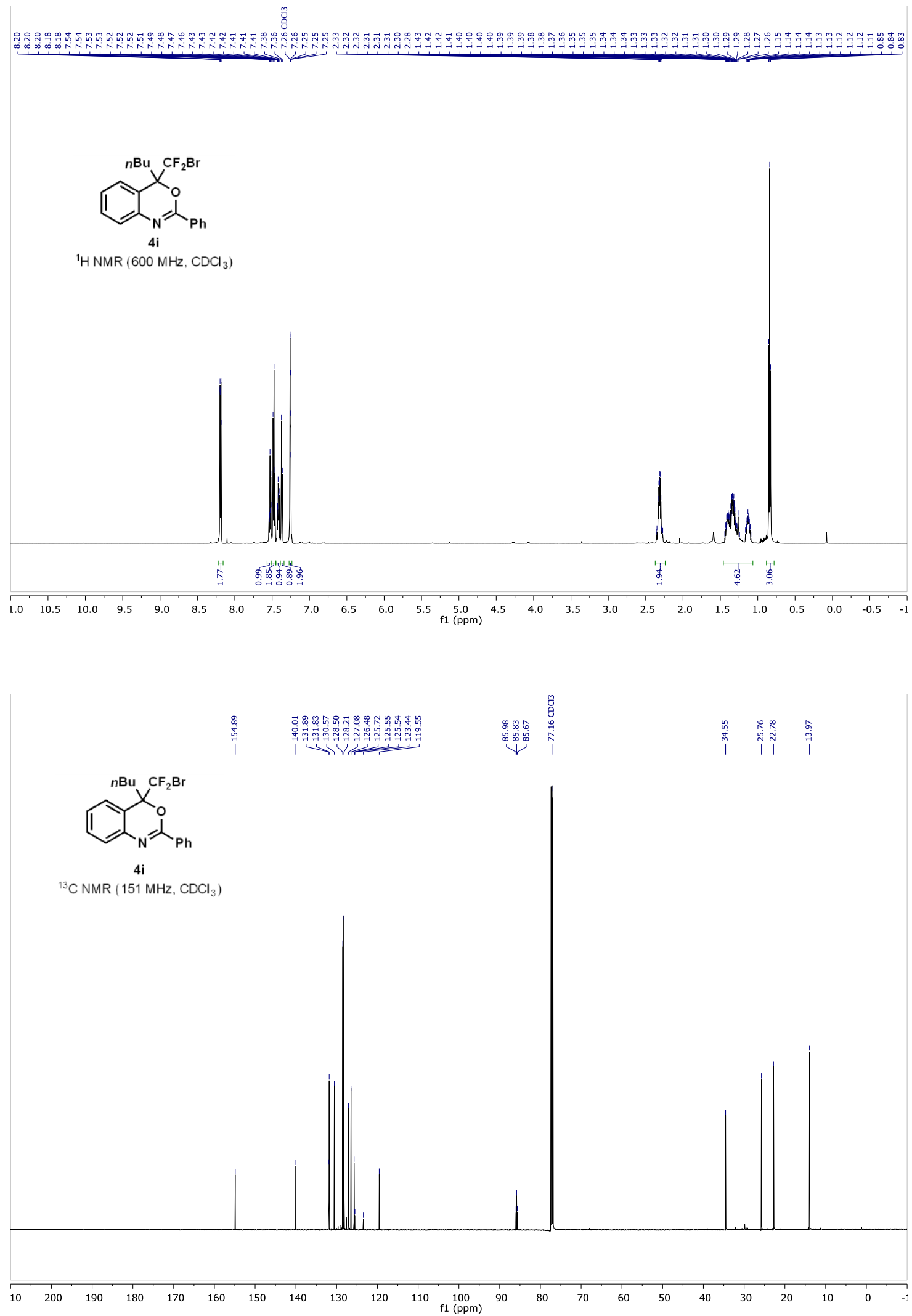


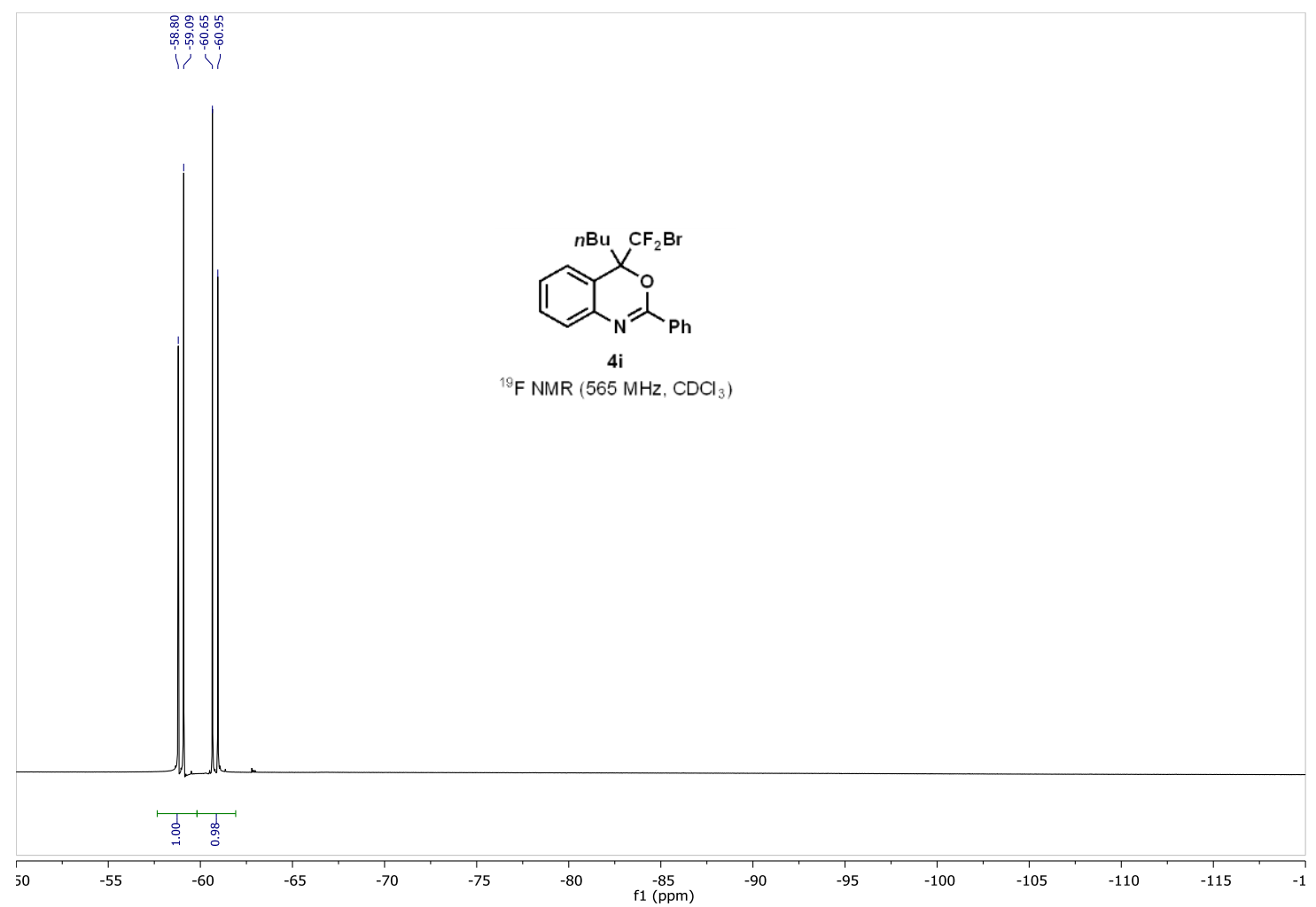



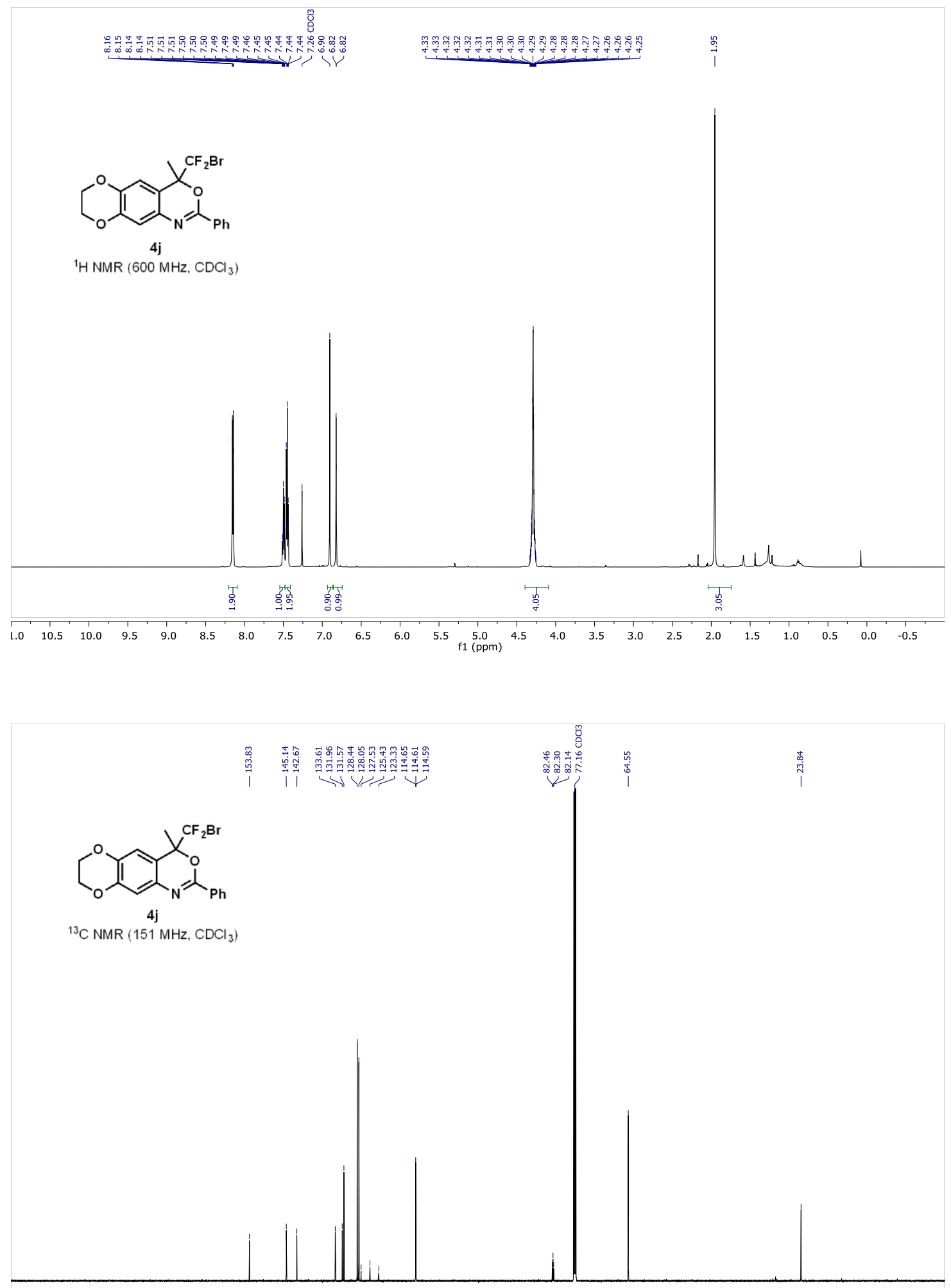

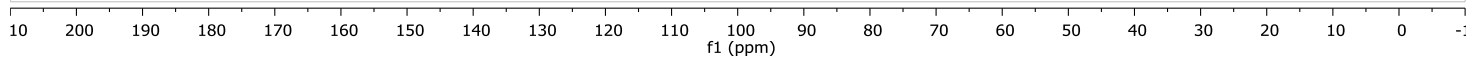




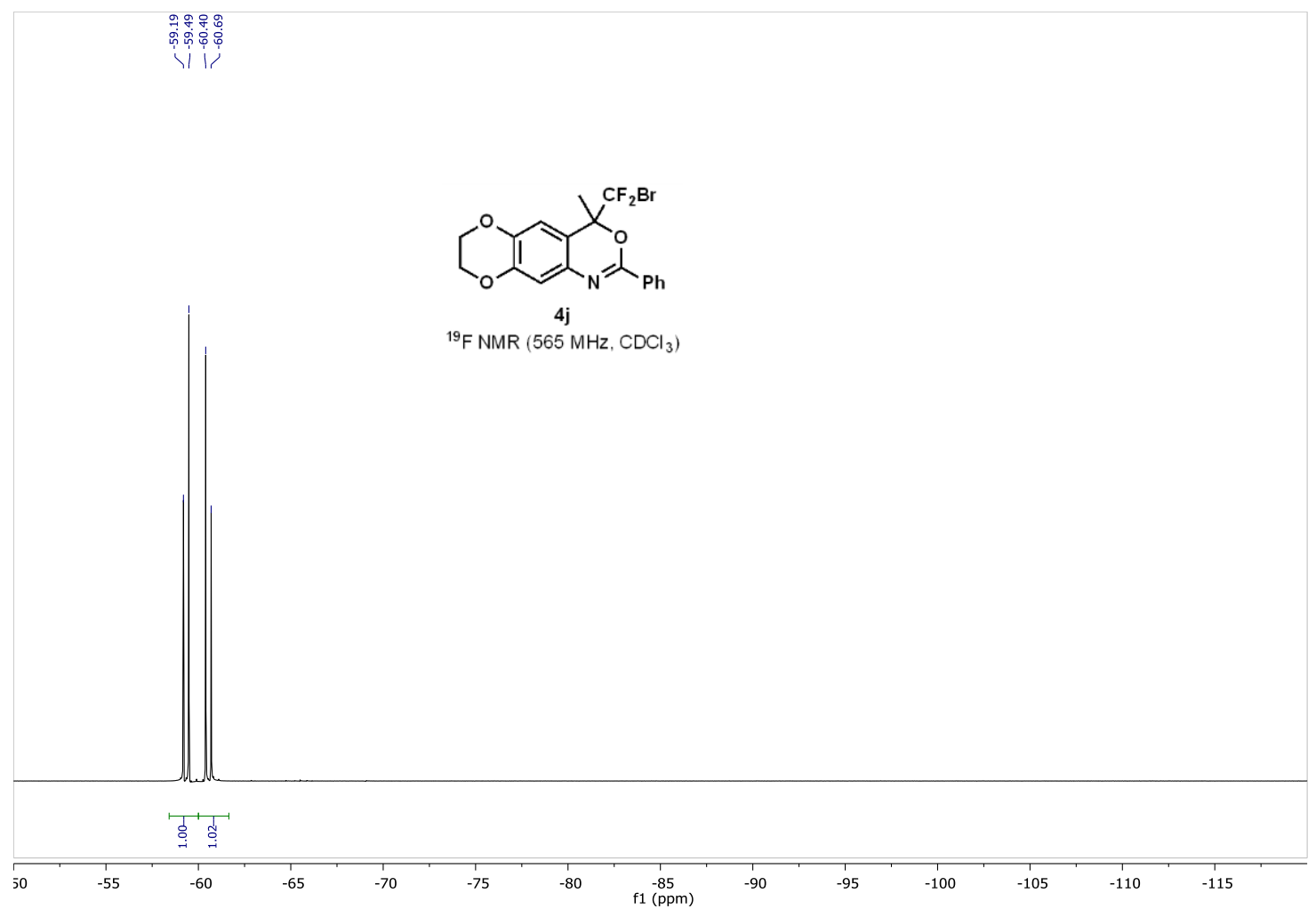



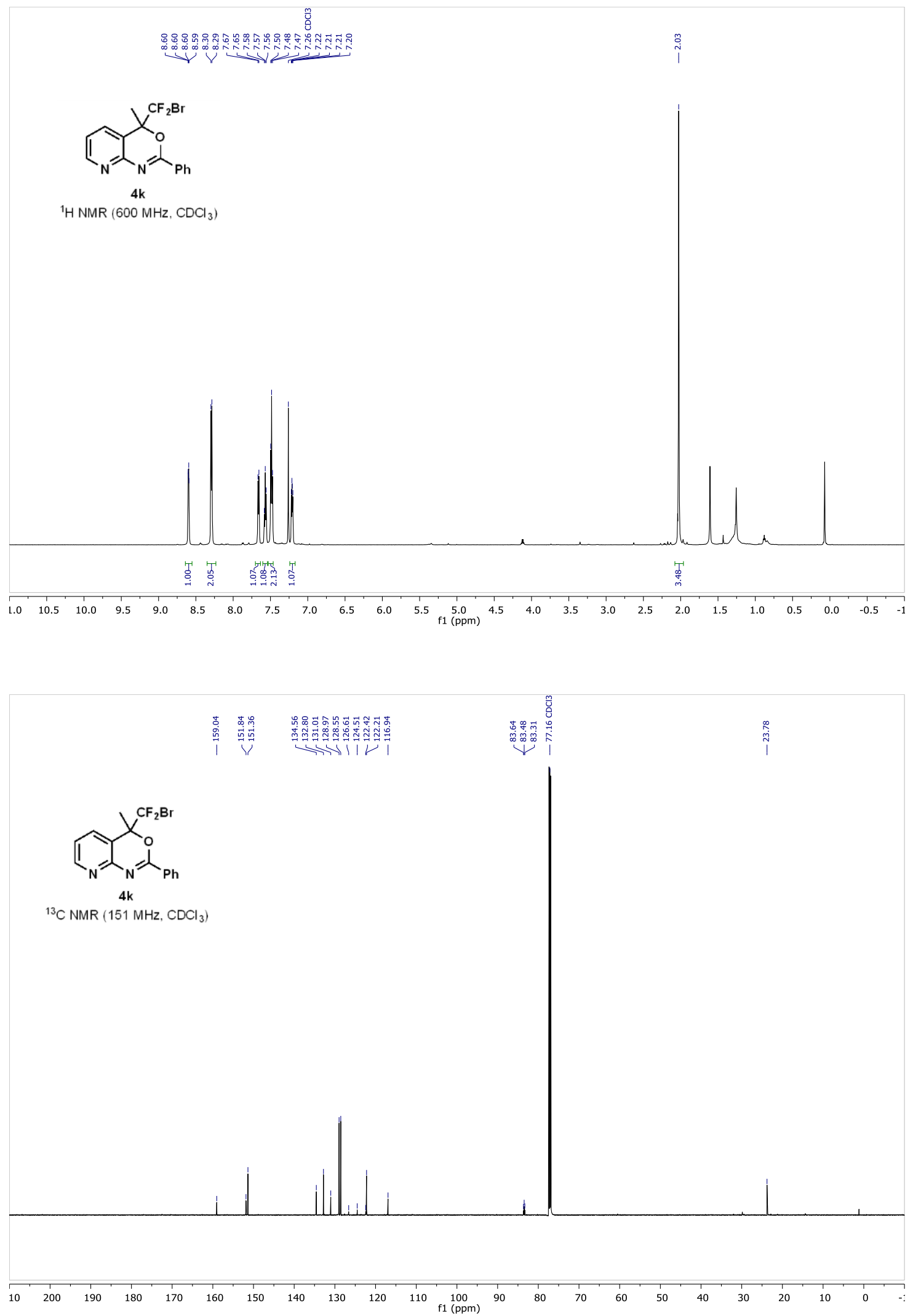


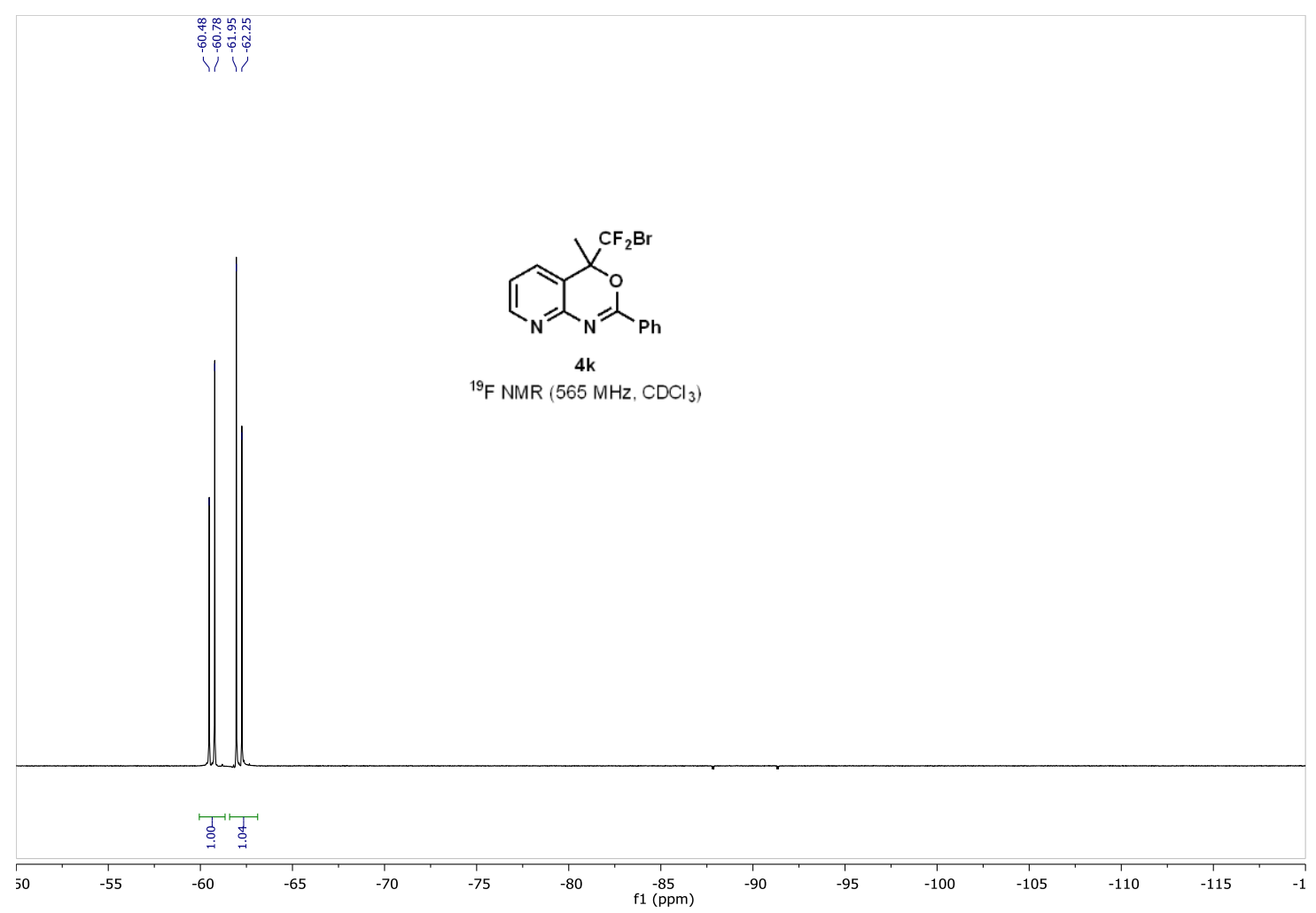



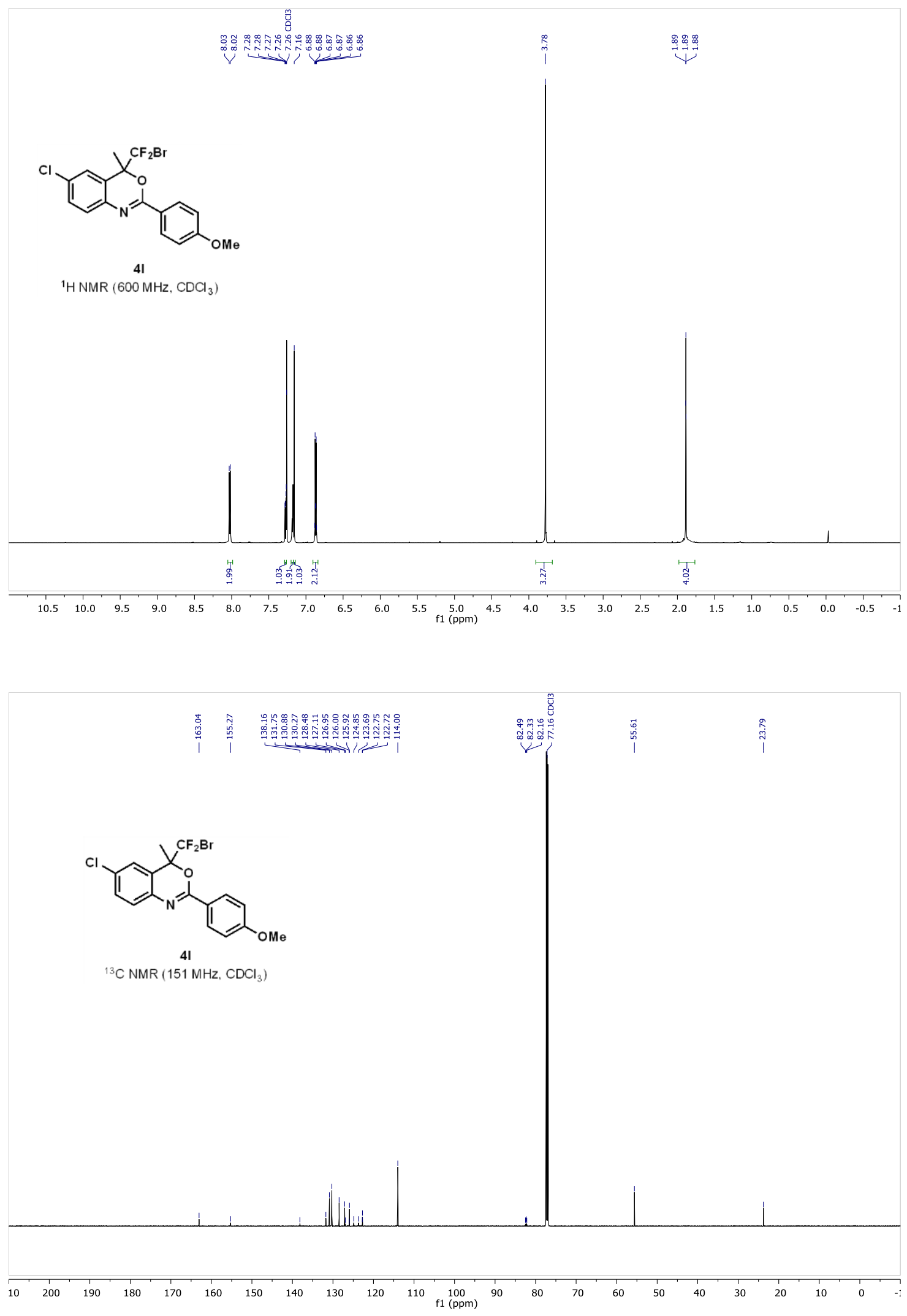

S201 


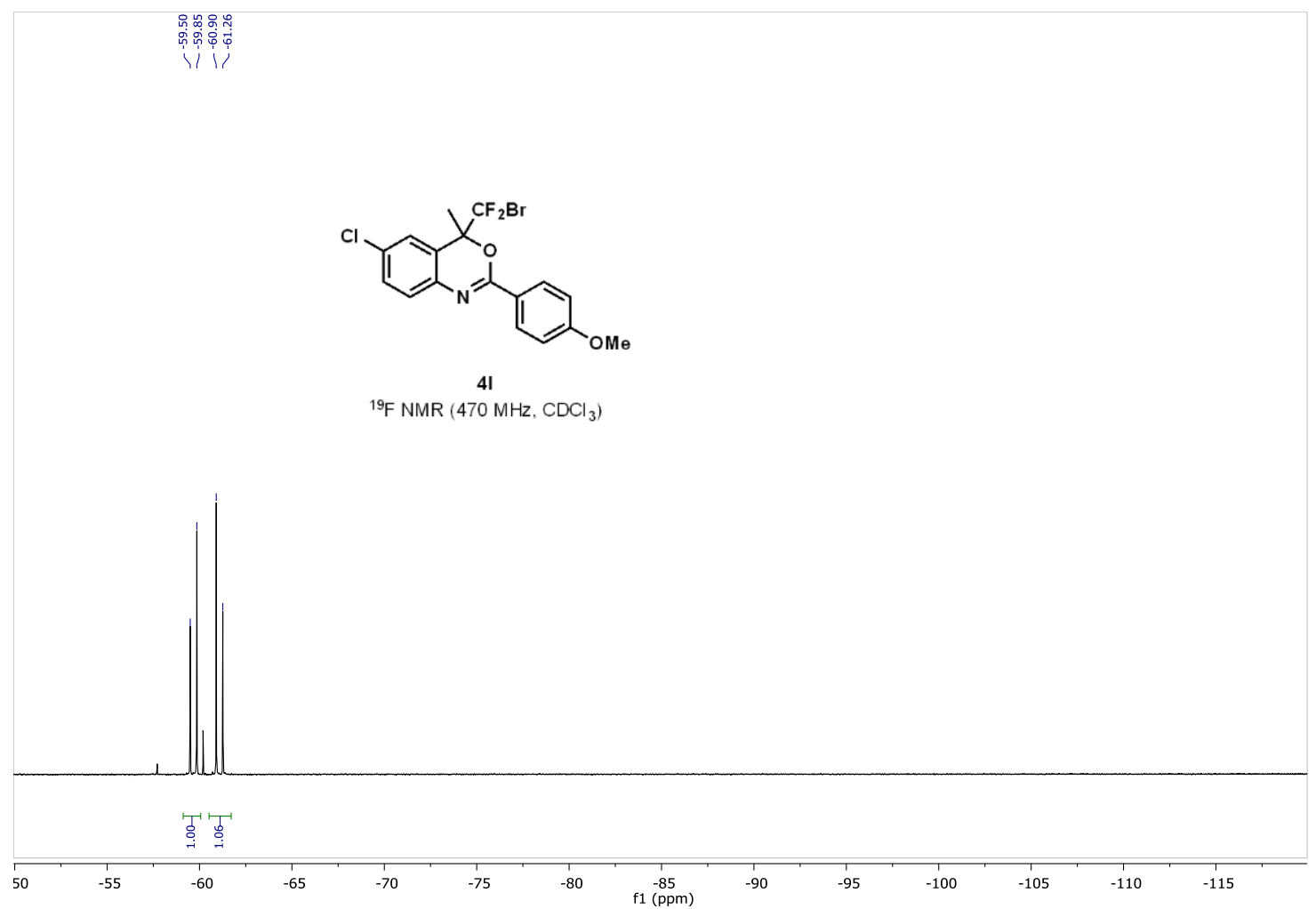



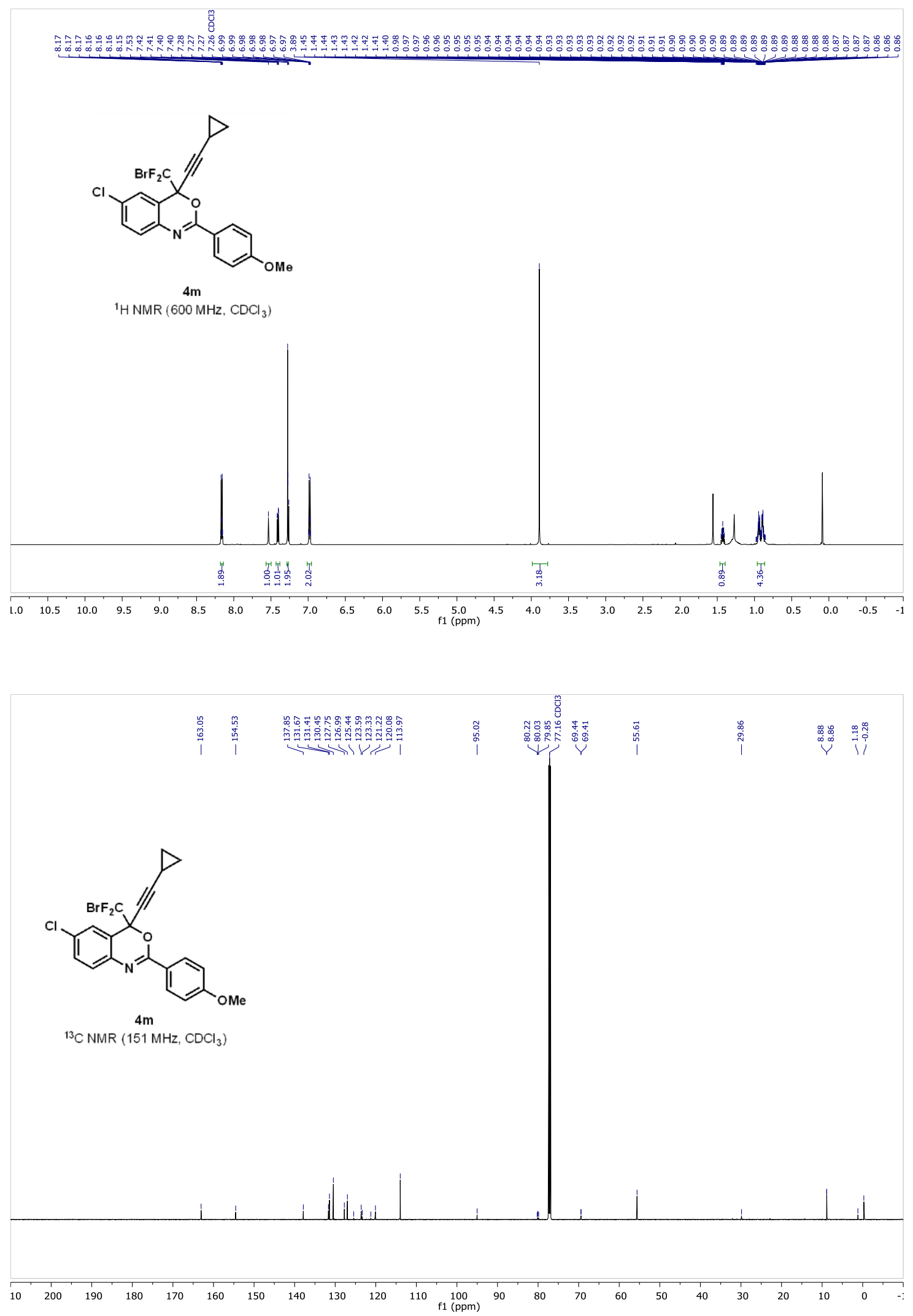


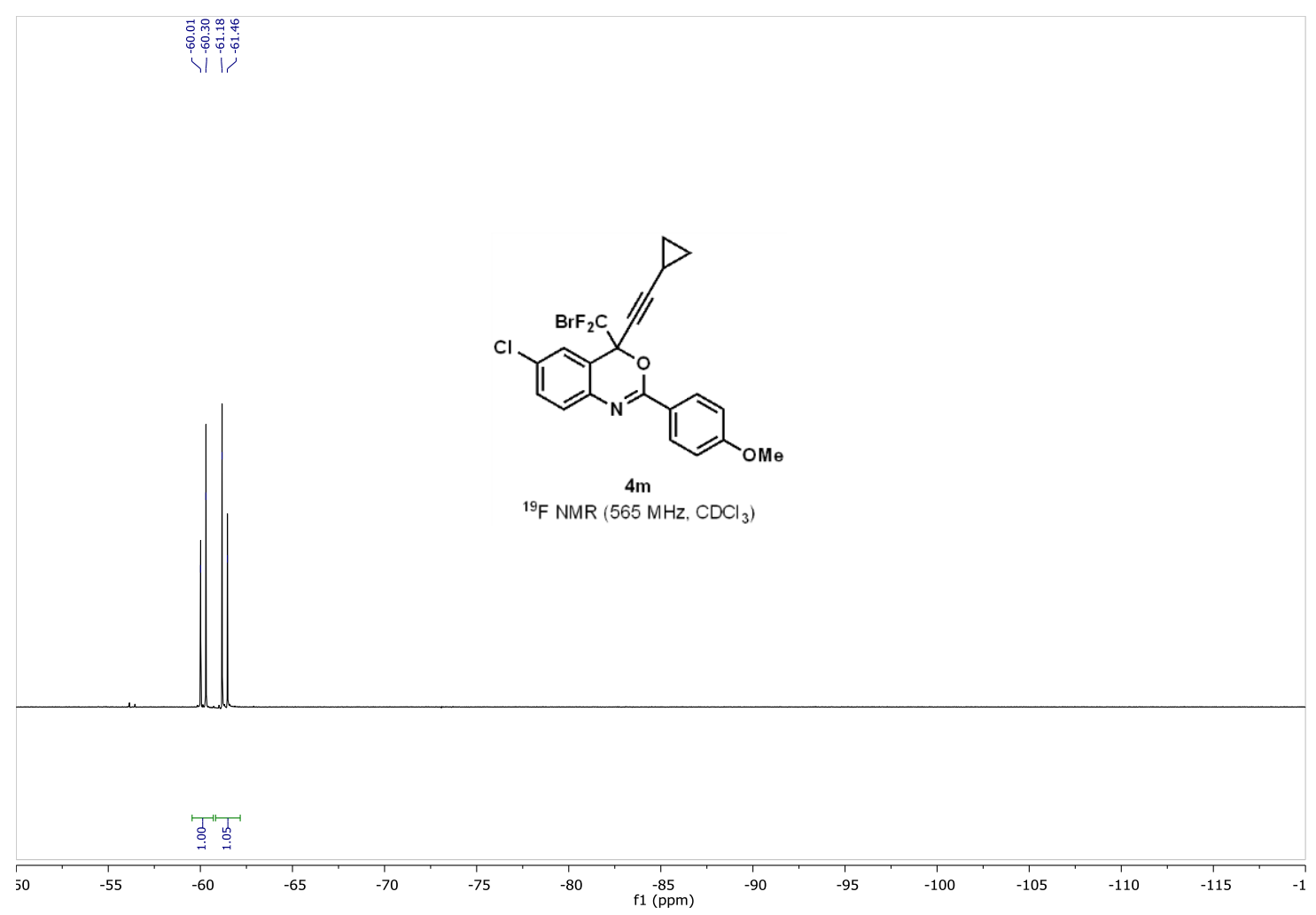



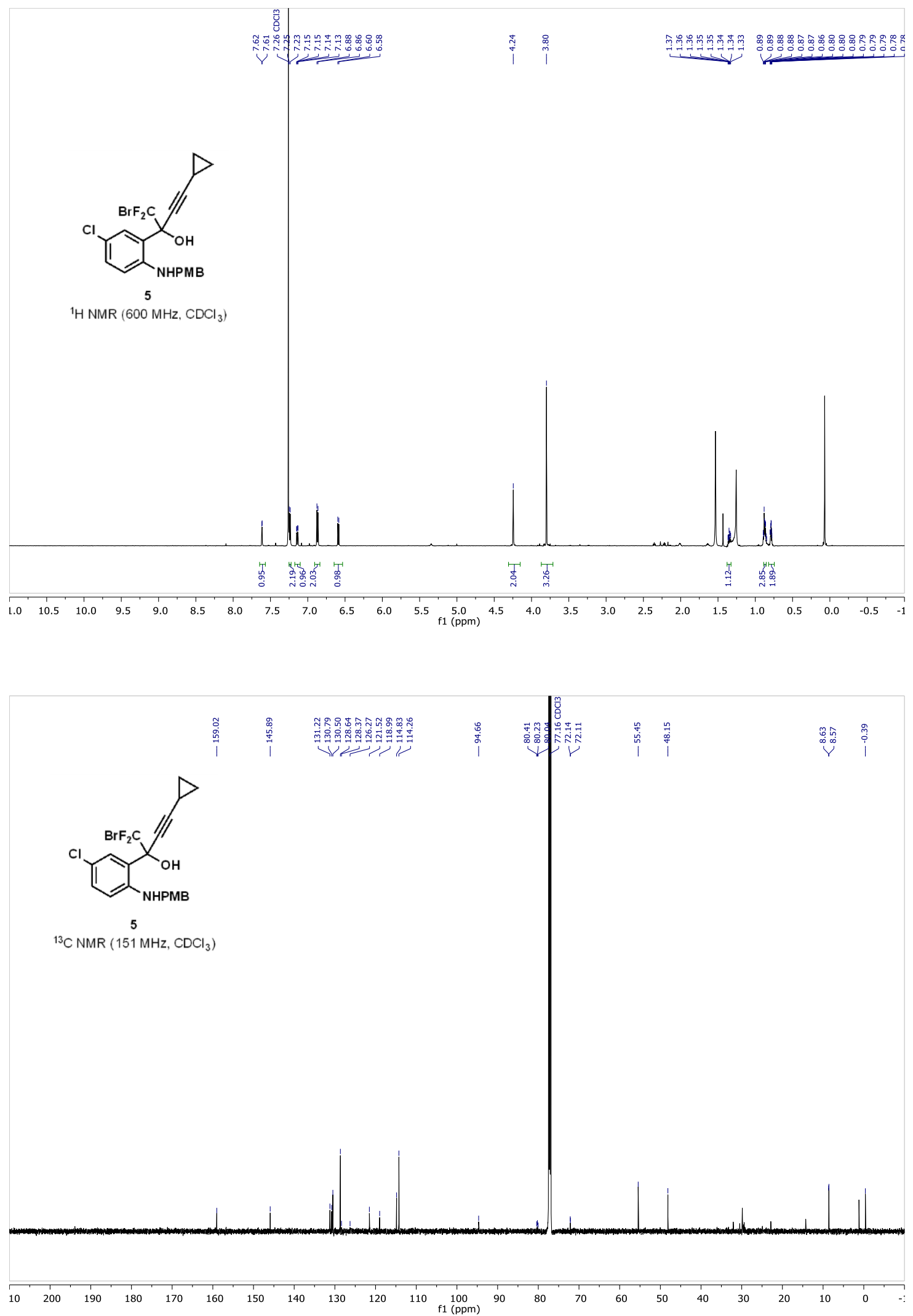


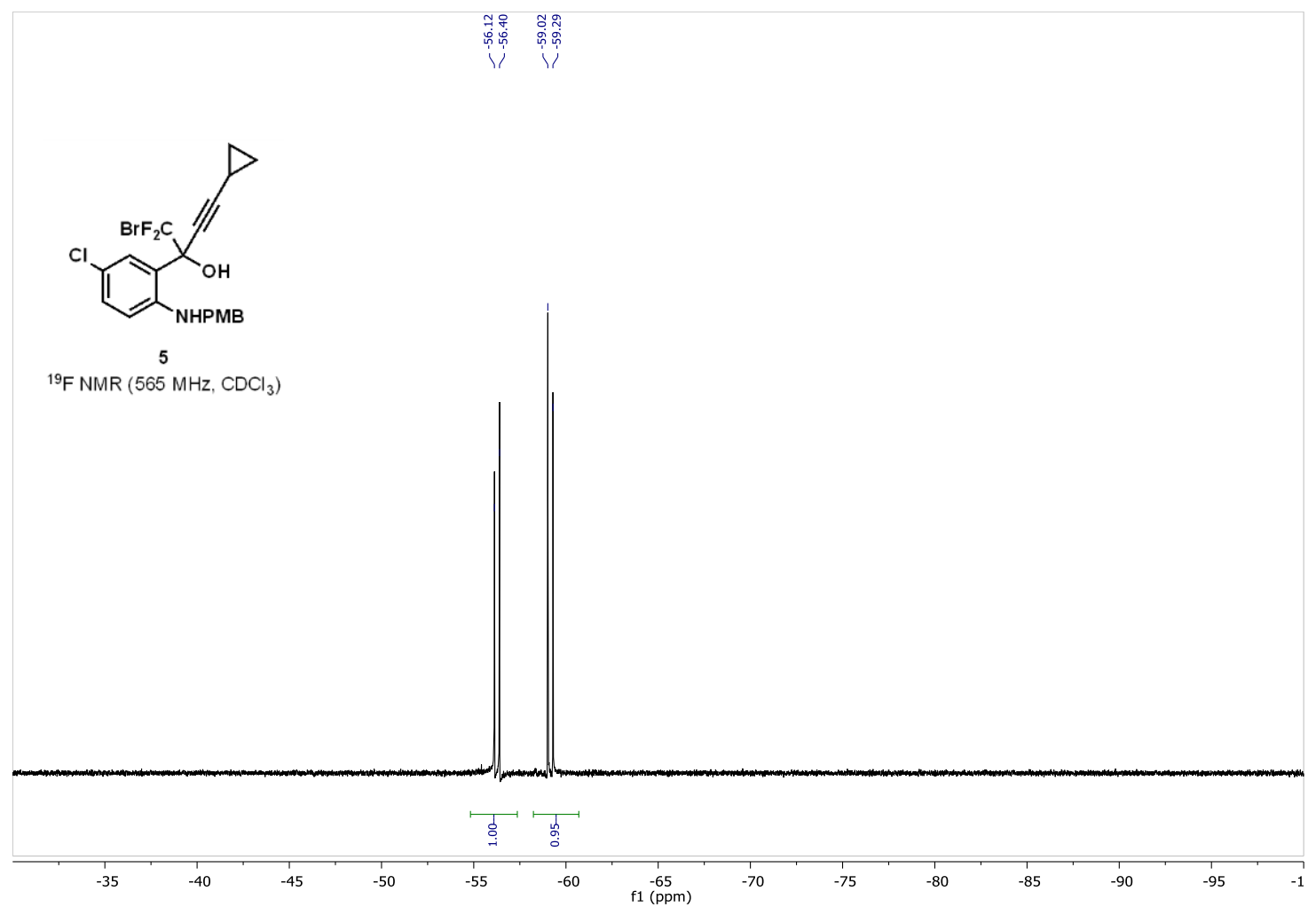



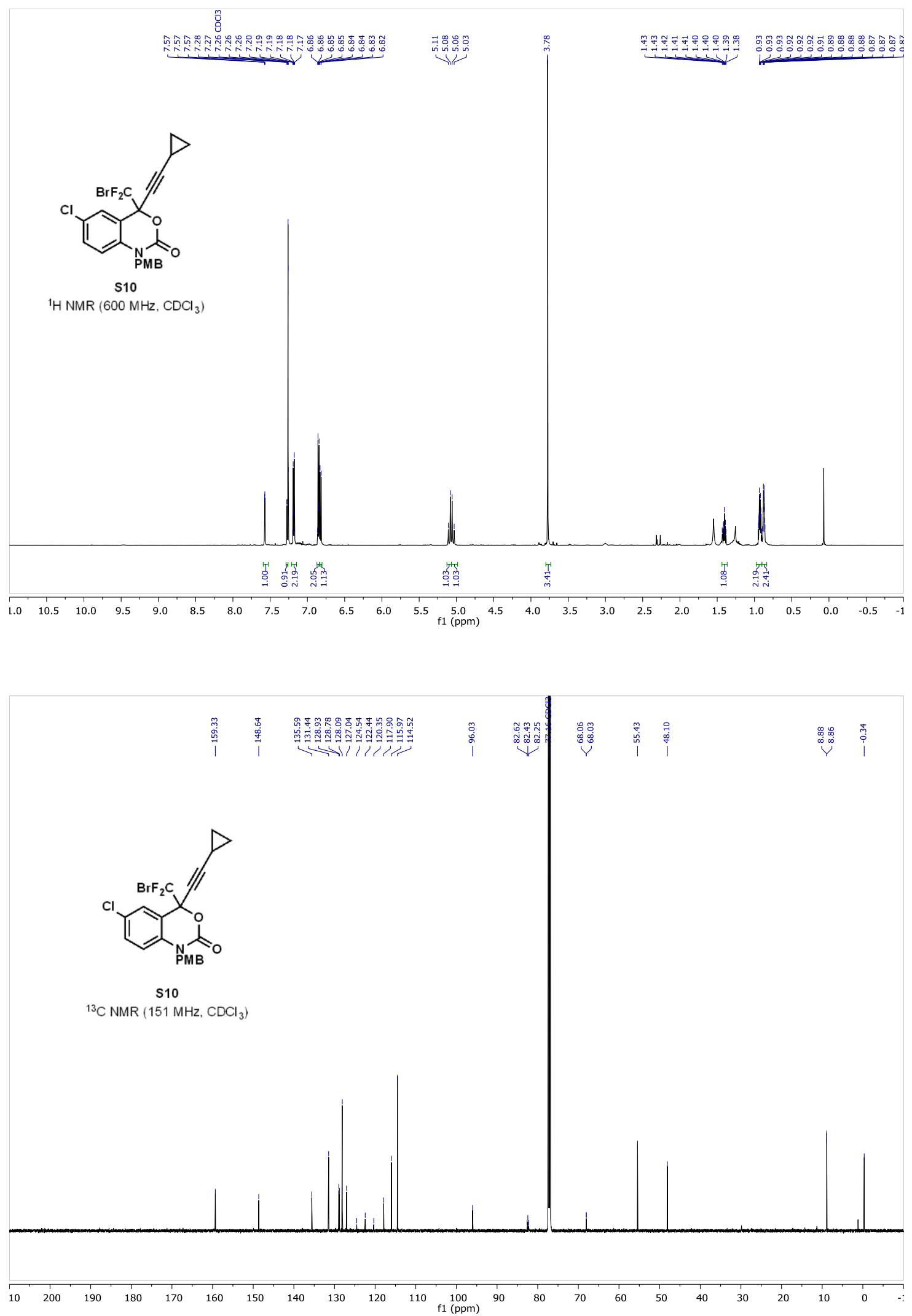


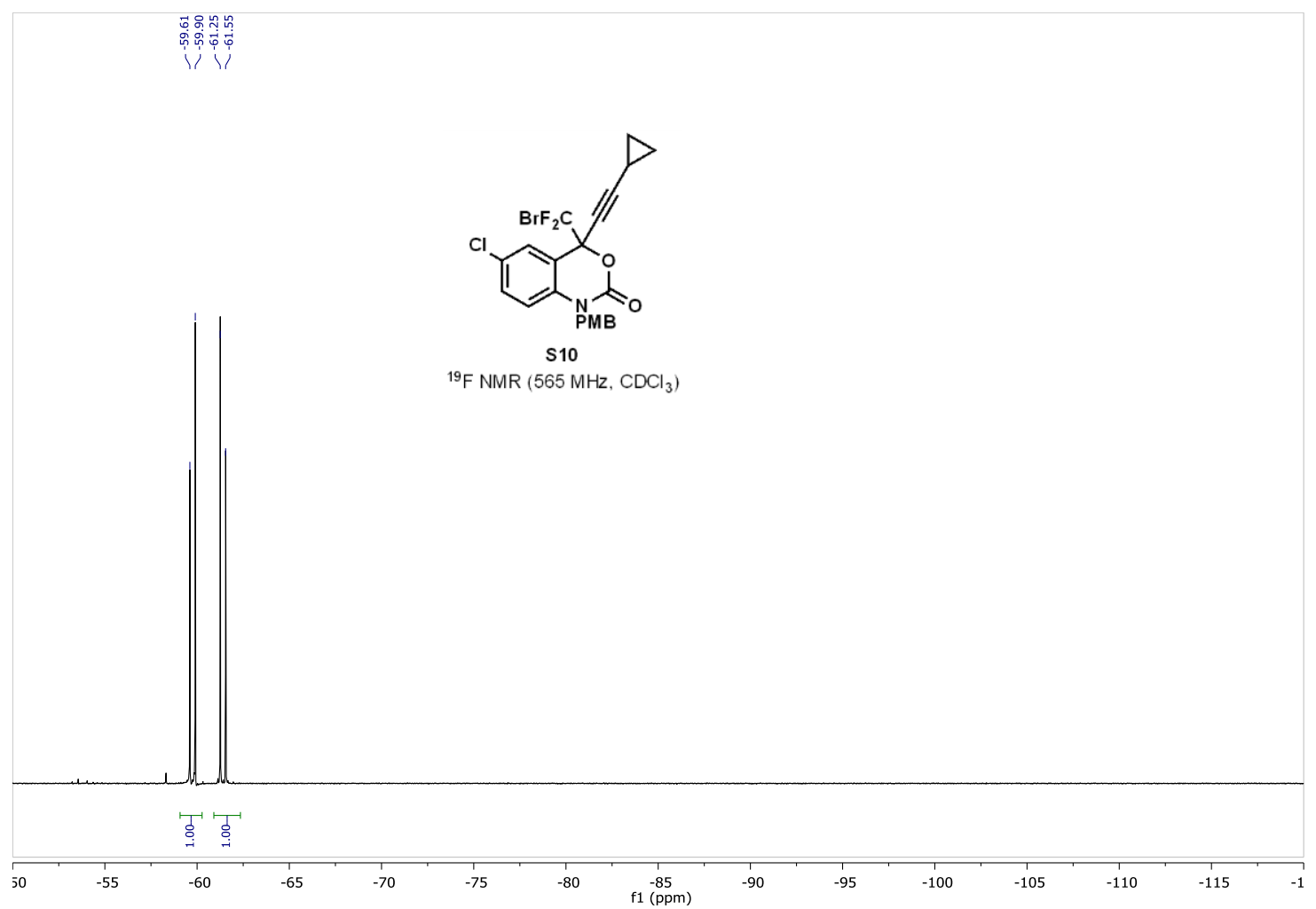



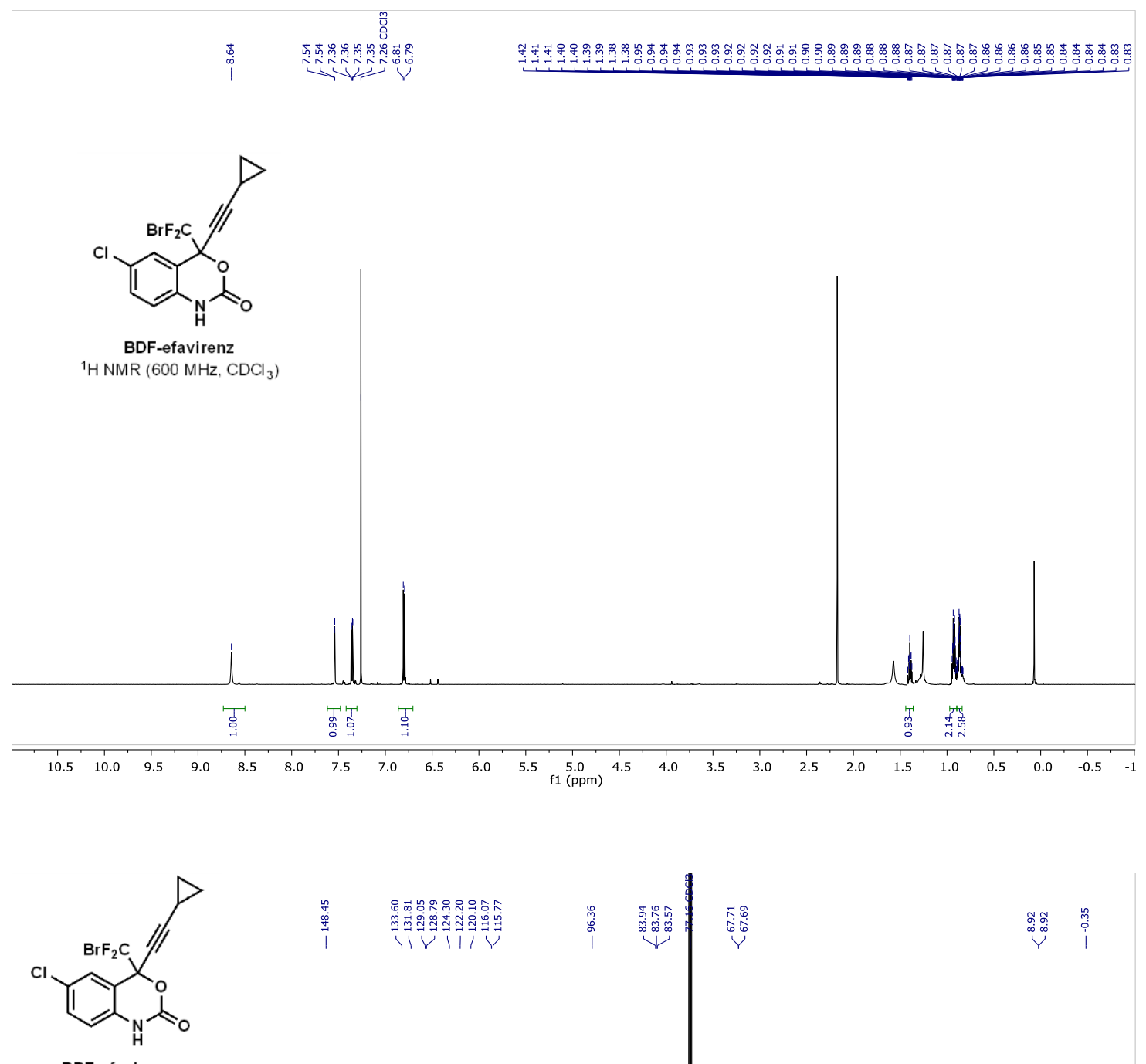

${ }^{13} \mathrm{CNMR}\left(151 \mathrm{MHz}, \mathrm{CDCl}_{3}\right)$
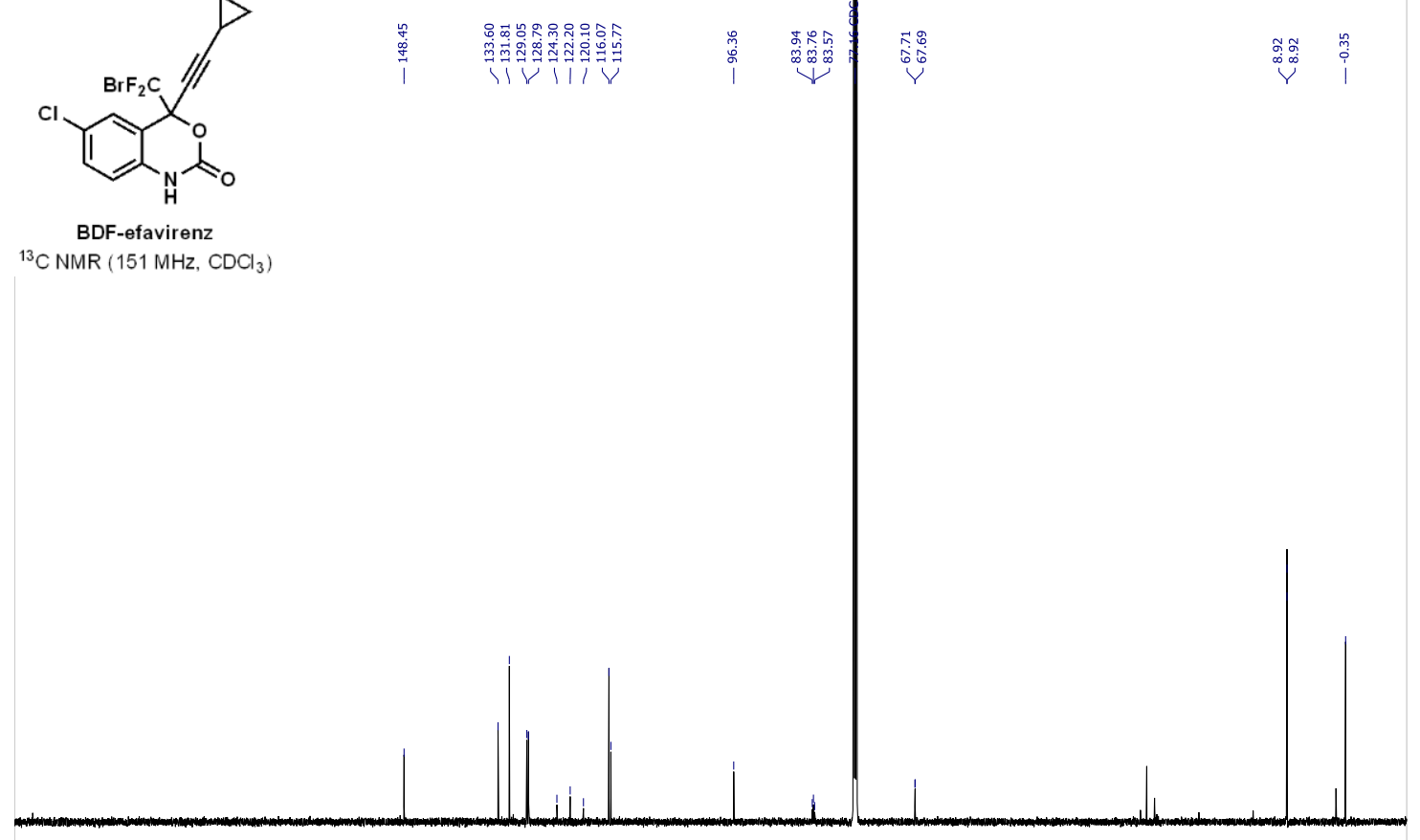

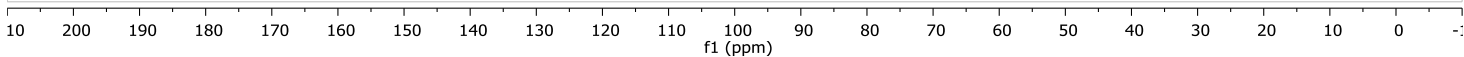




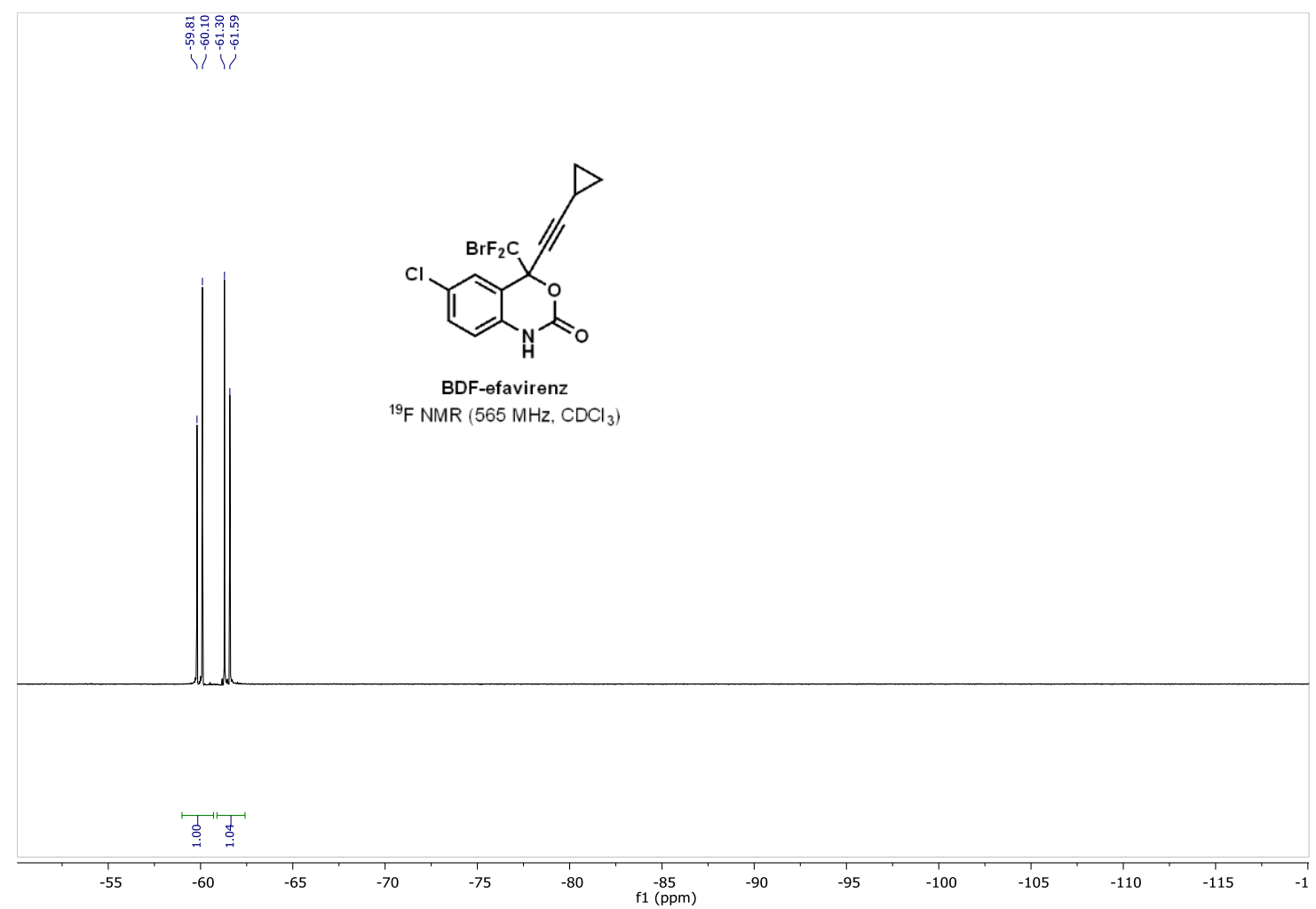



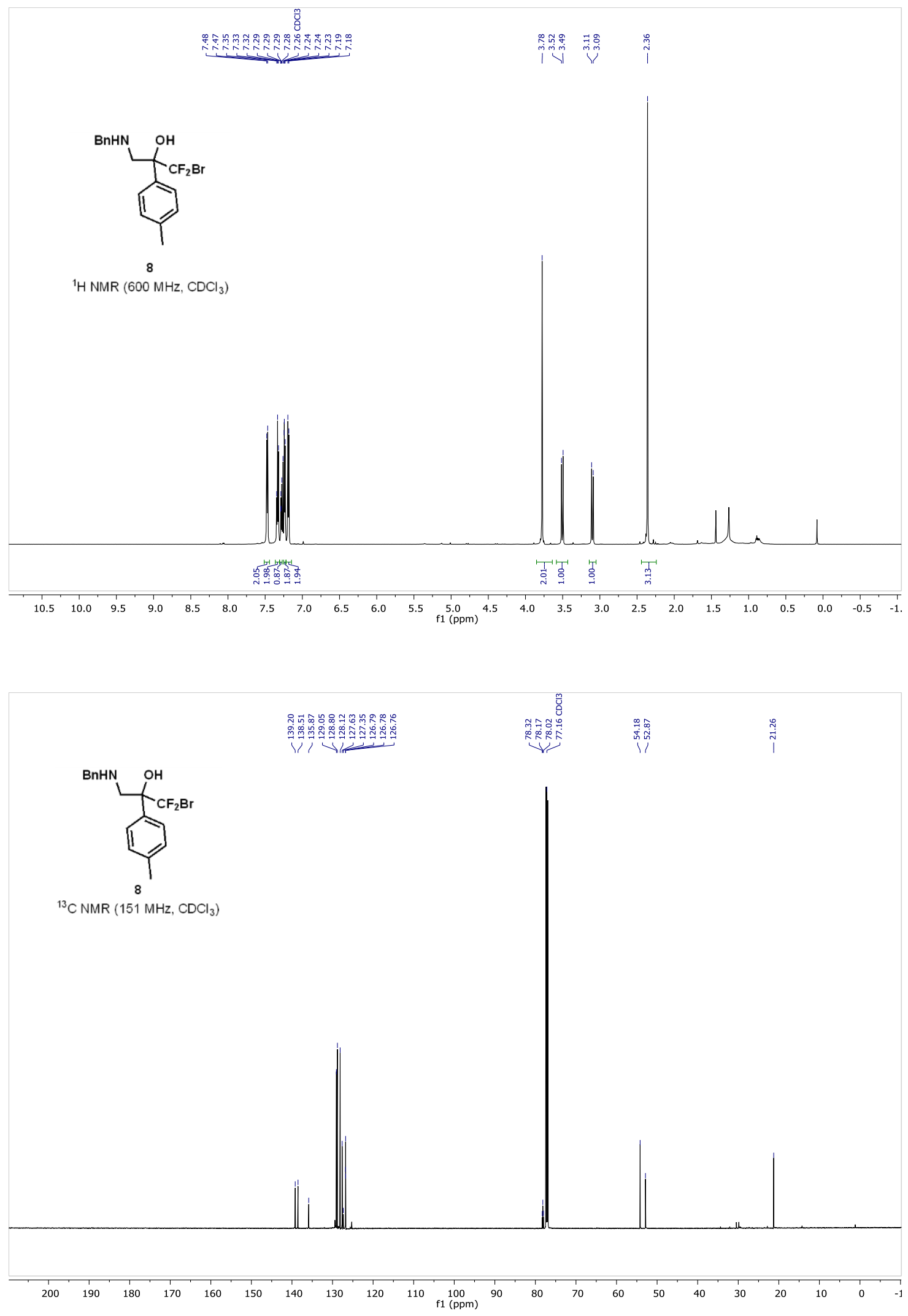

S211 


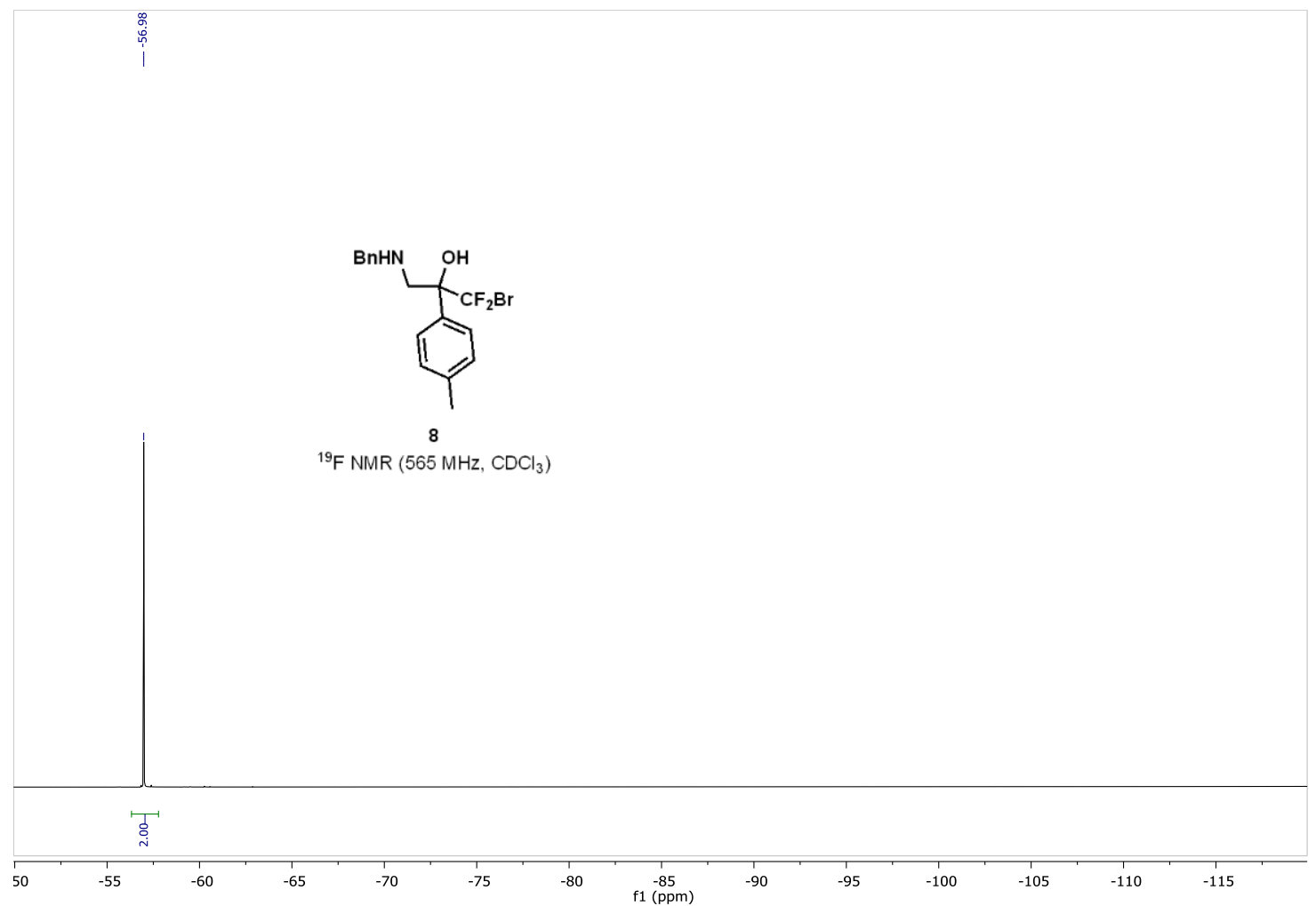



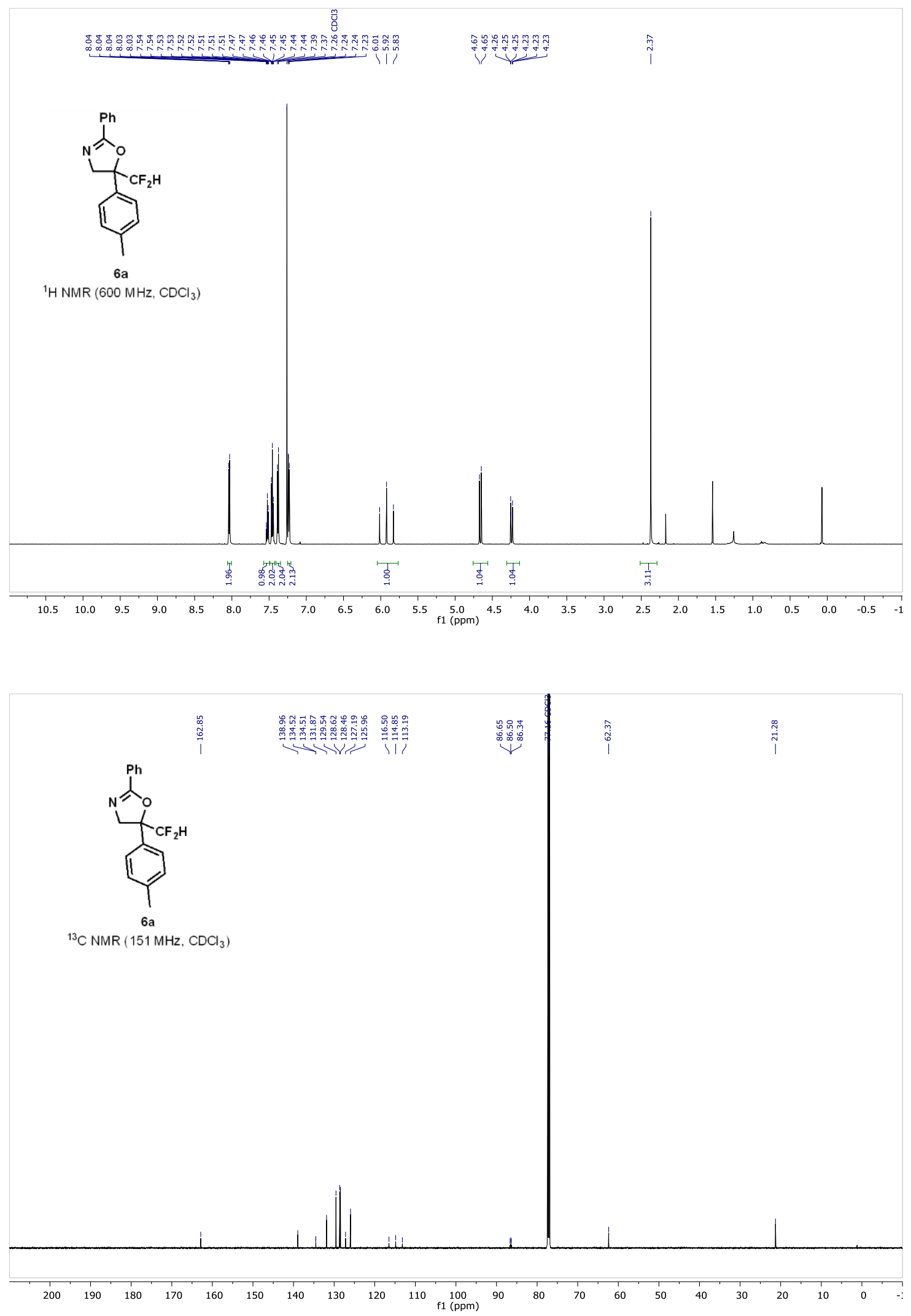


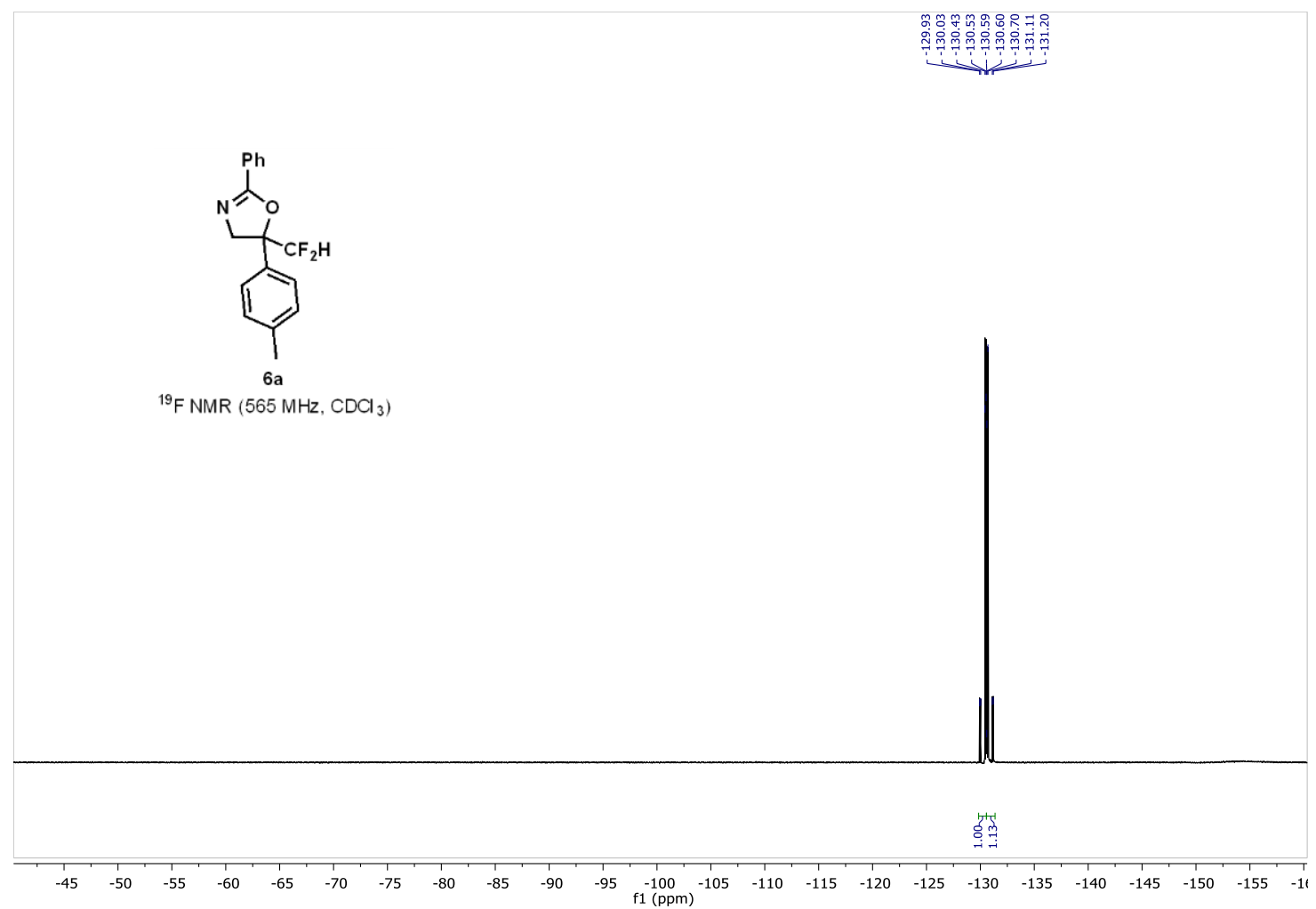



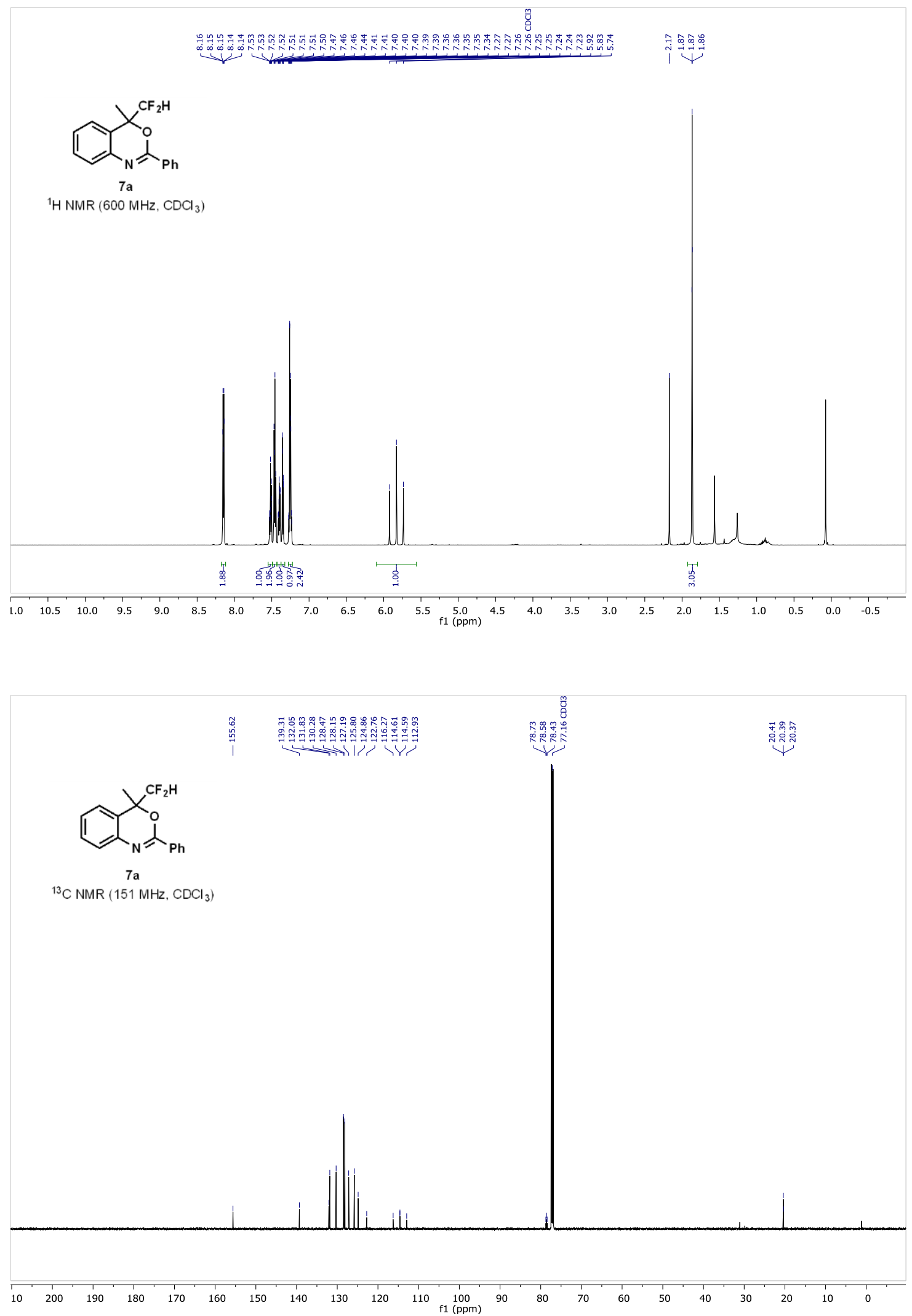


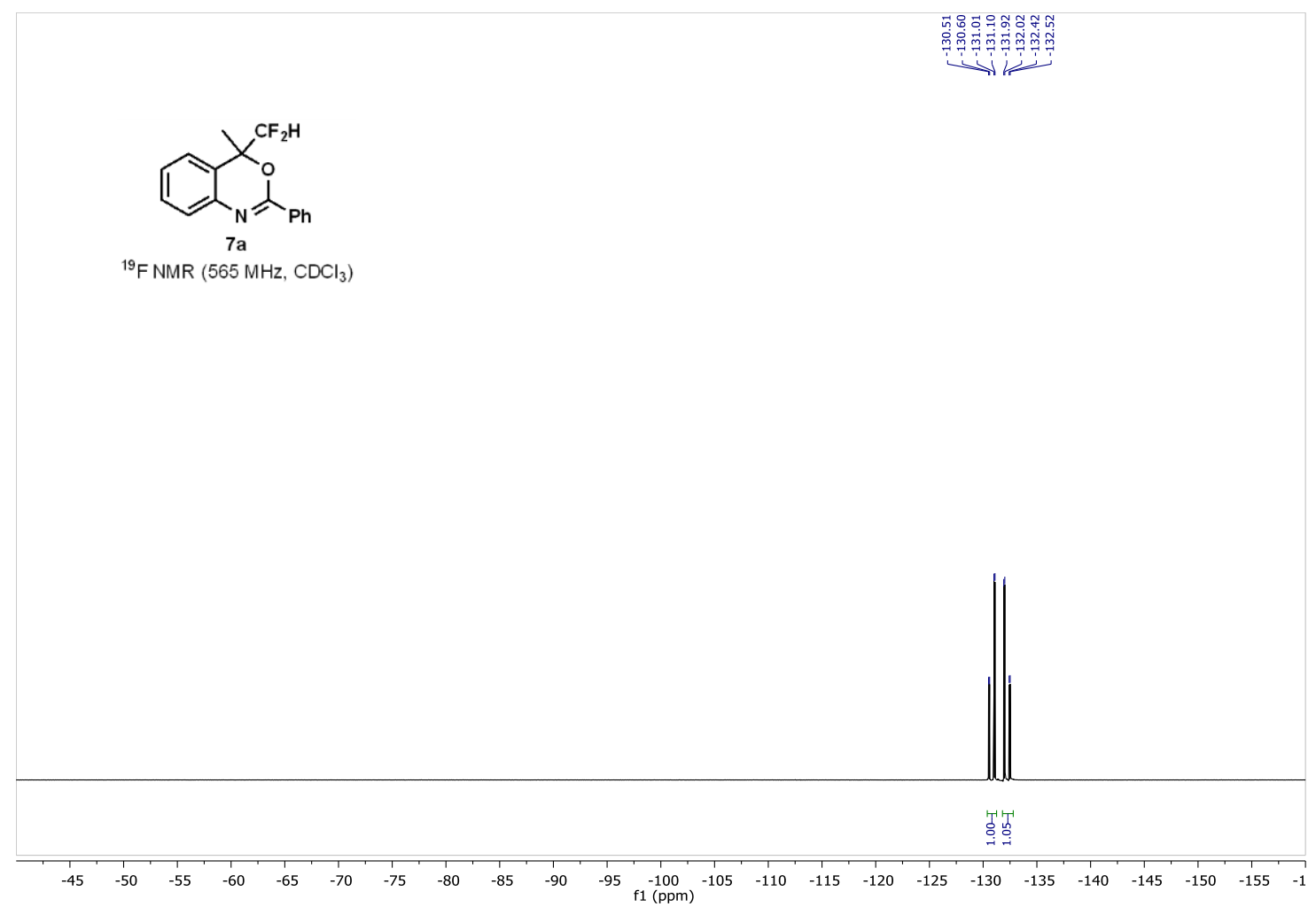



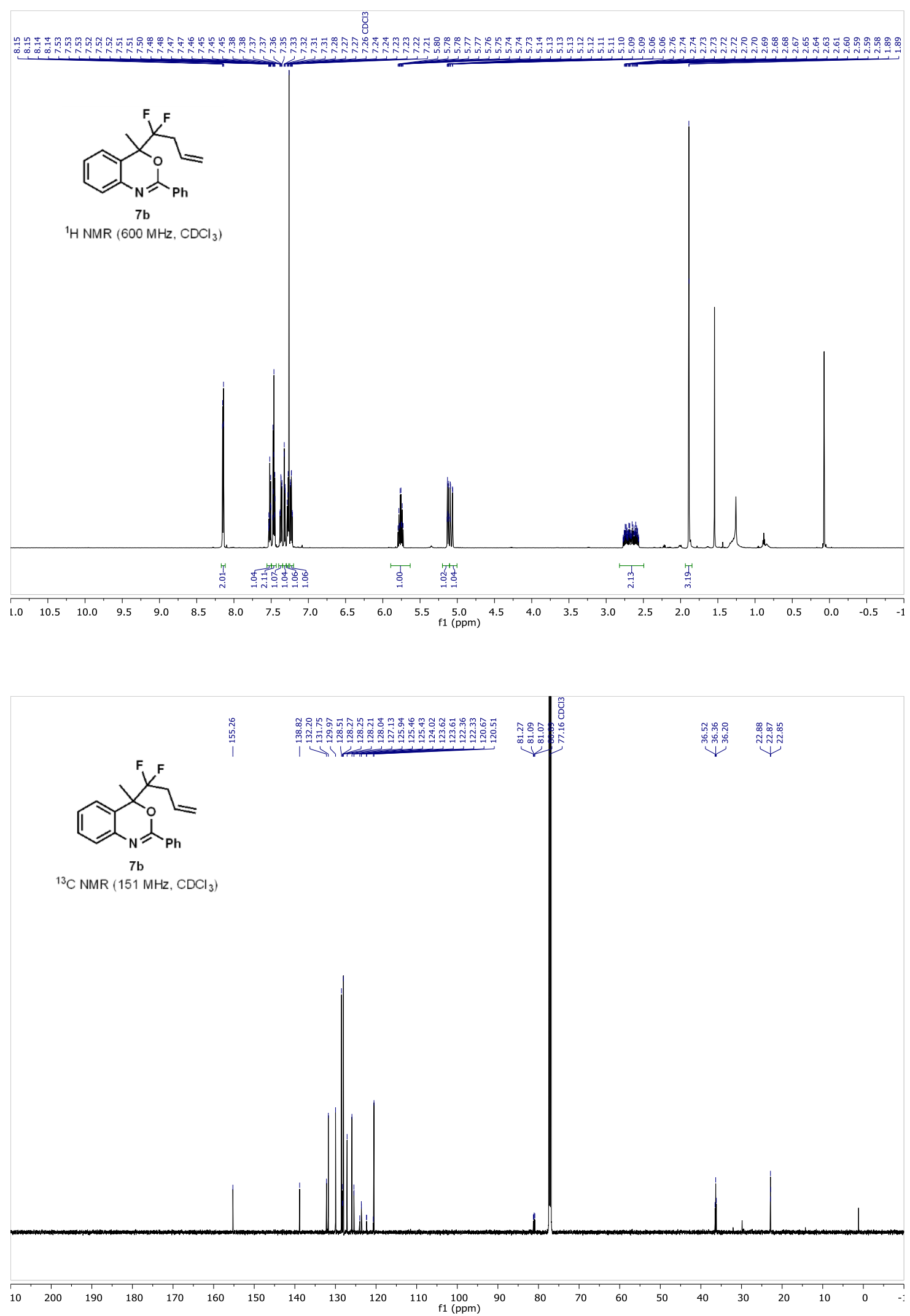


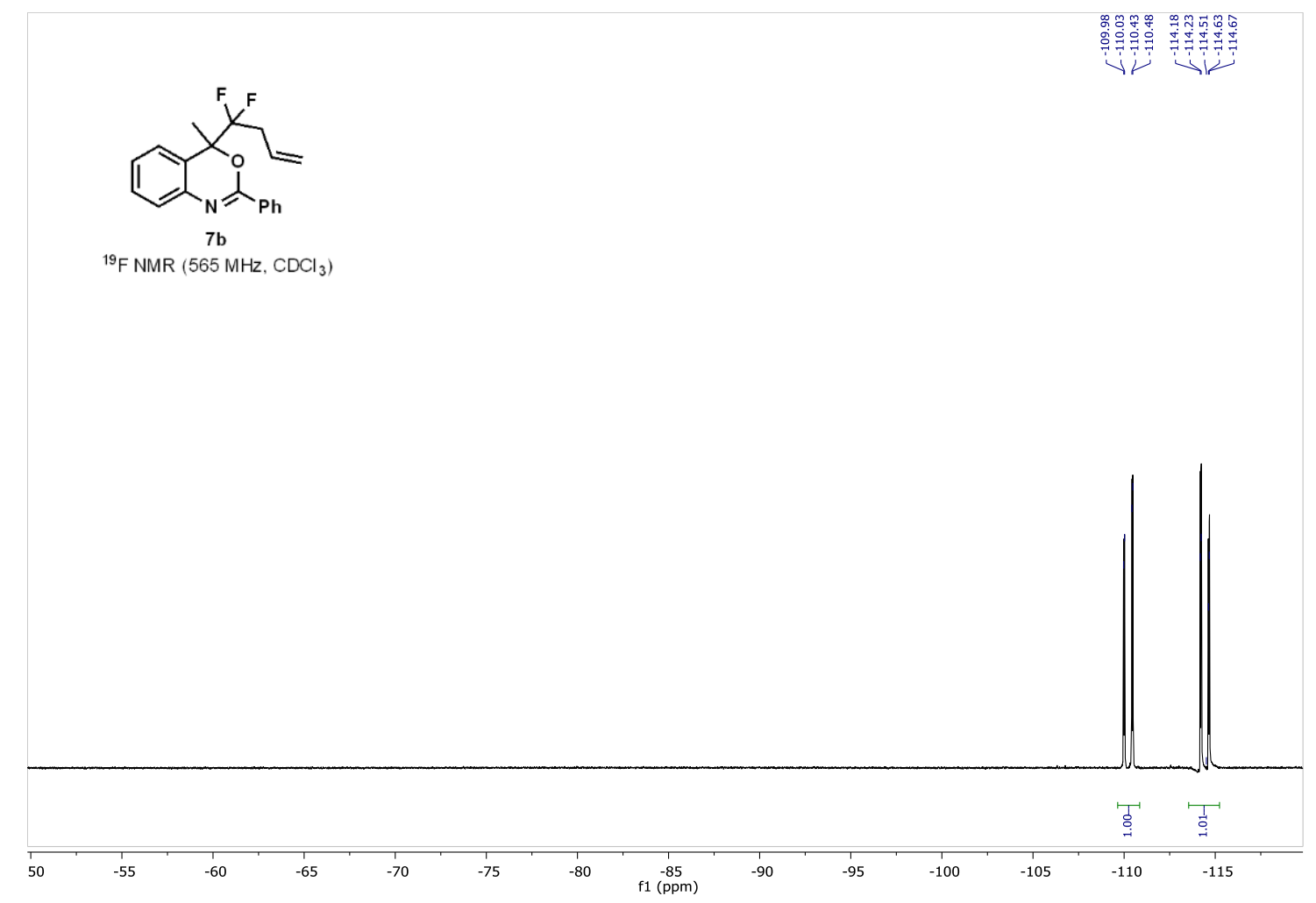



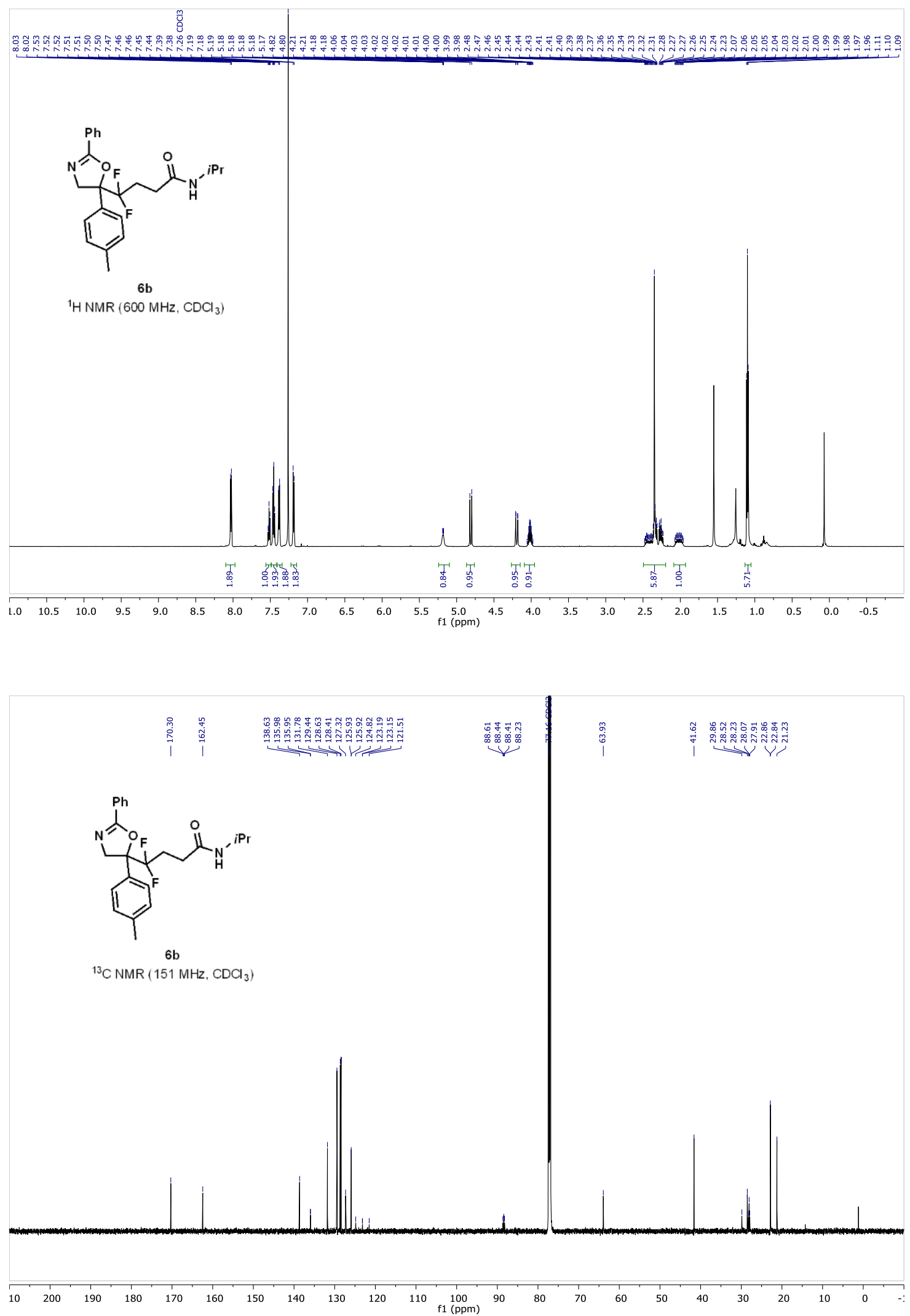


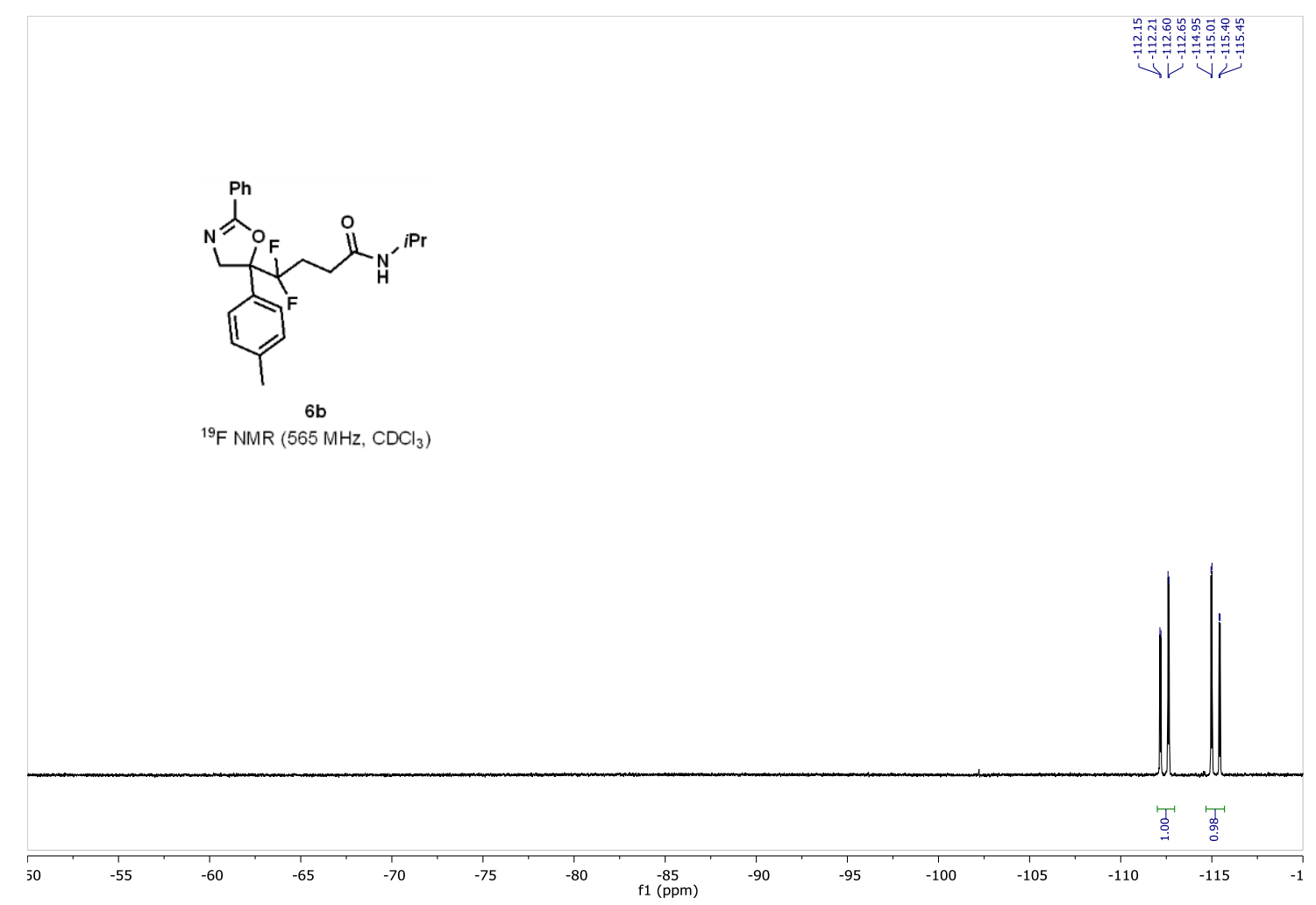



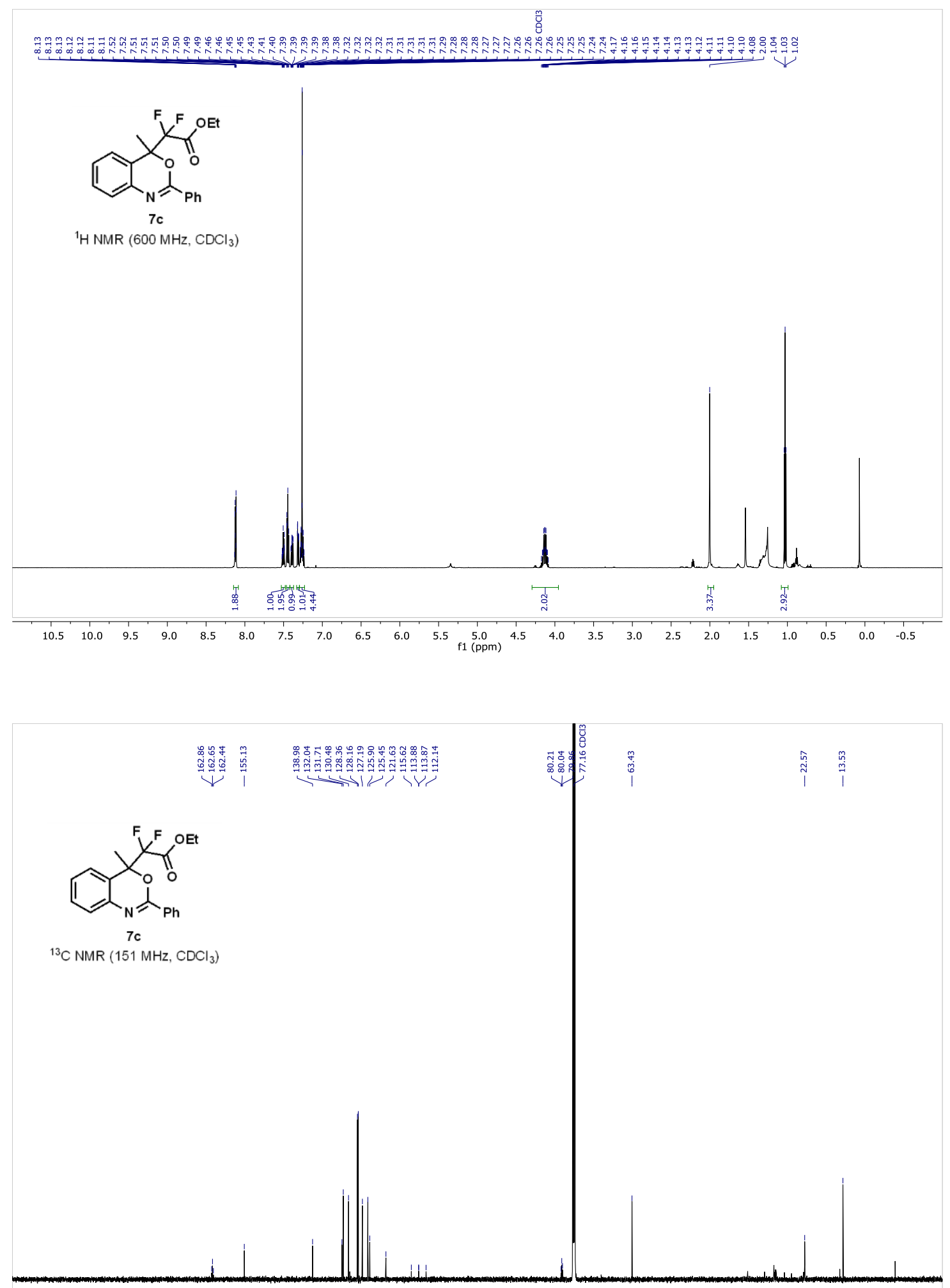


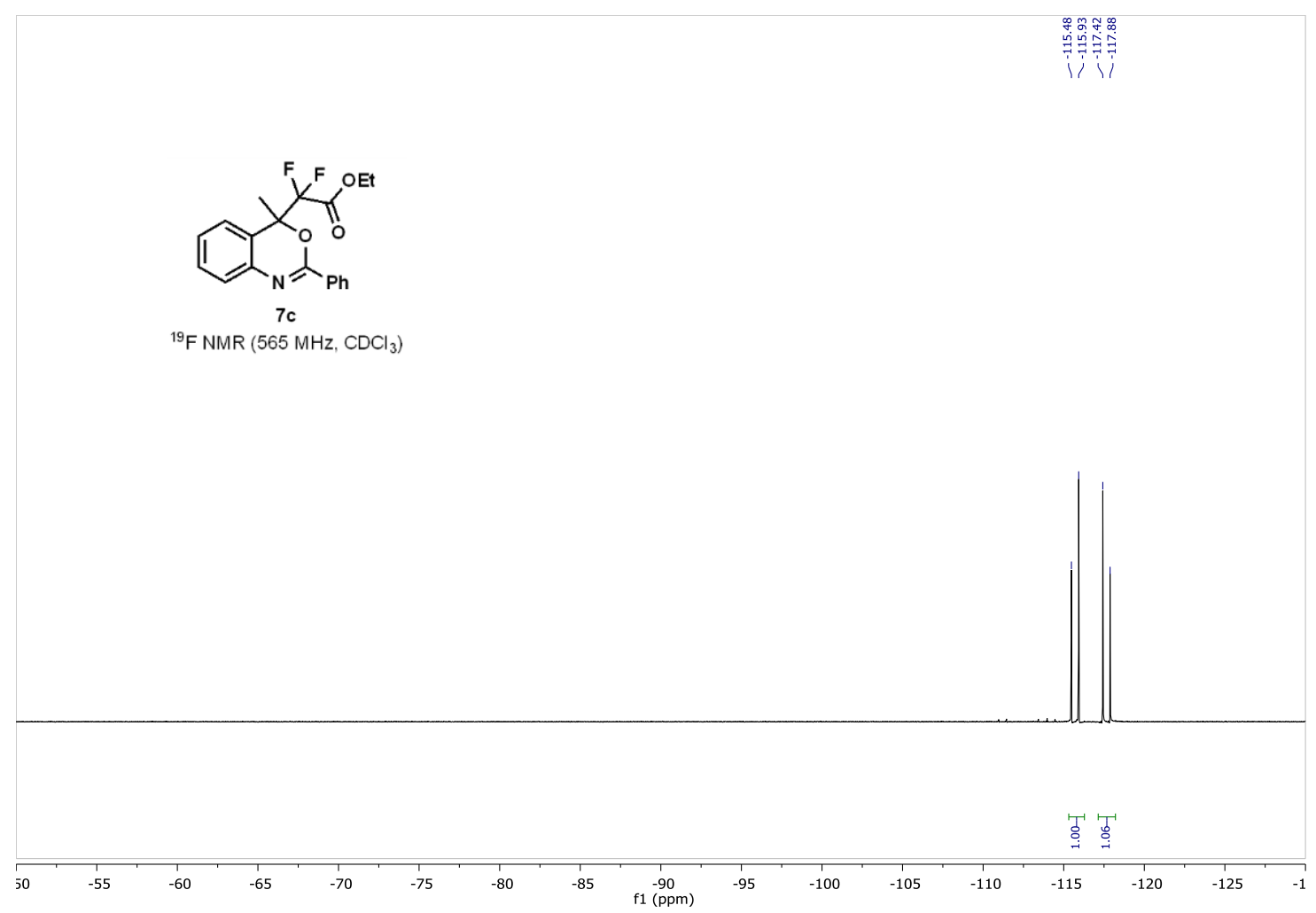



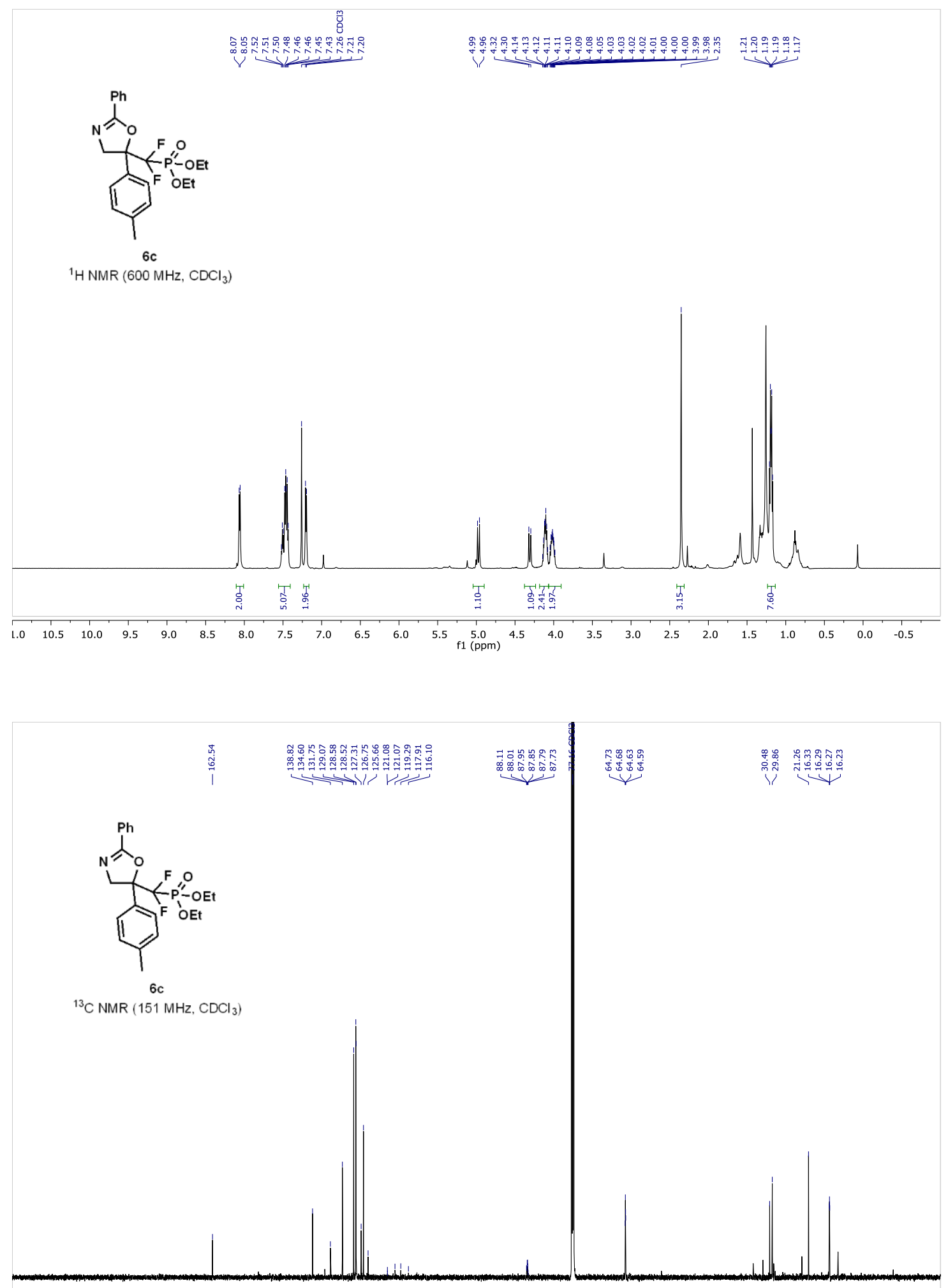


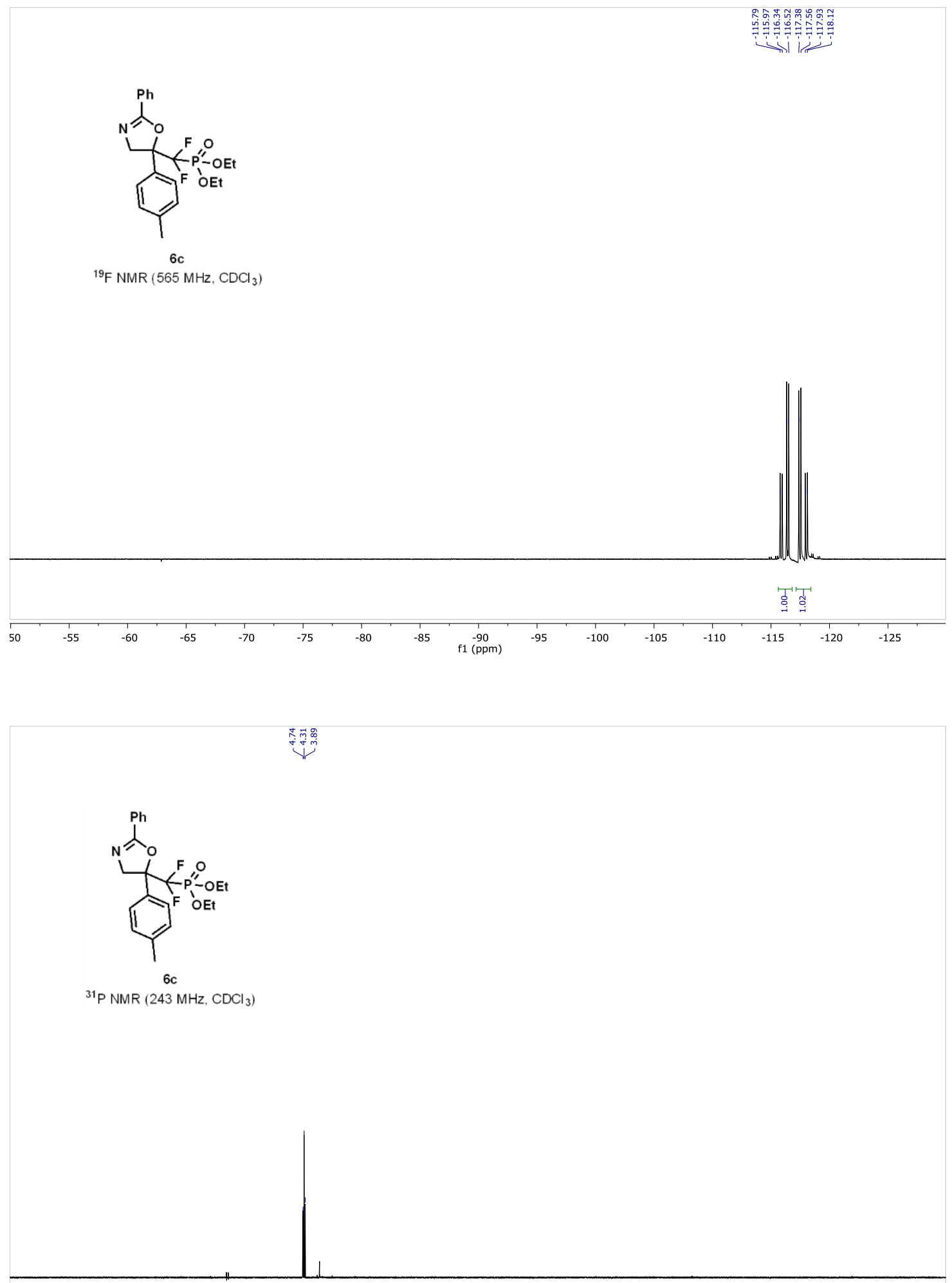

\begin{tabular}{lllllllllllllllllllllll}
\hline 20 & 100 & 80 & 60 & 40 & 20 & 0 & -20 & -40 & -60 & -80 & -100 & -120 & -140 & -160 & -180 & -200 & -220 & -240
\end{tabular} 

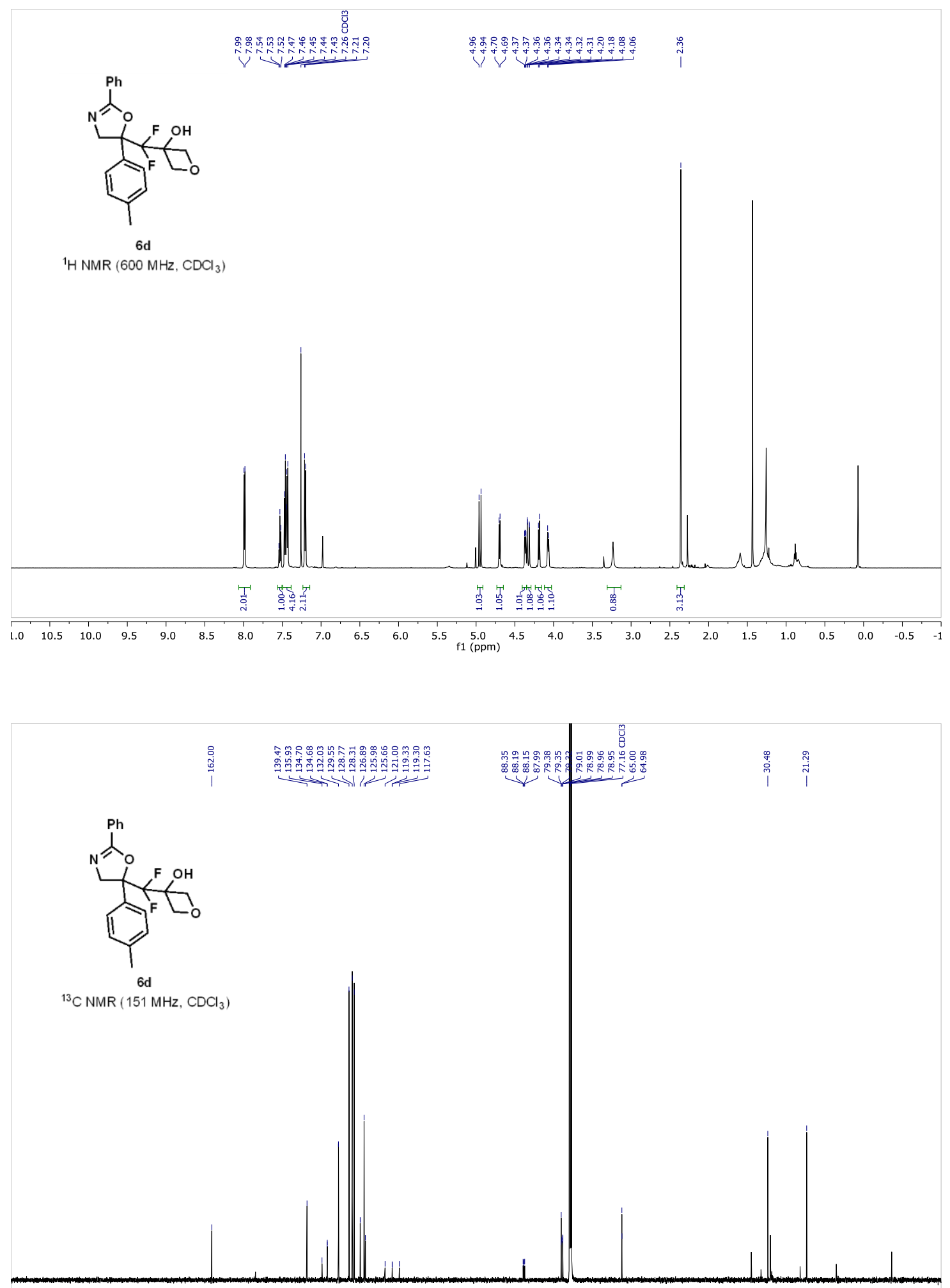


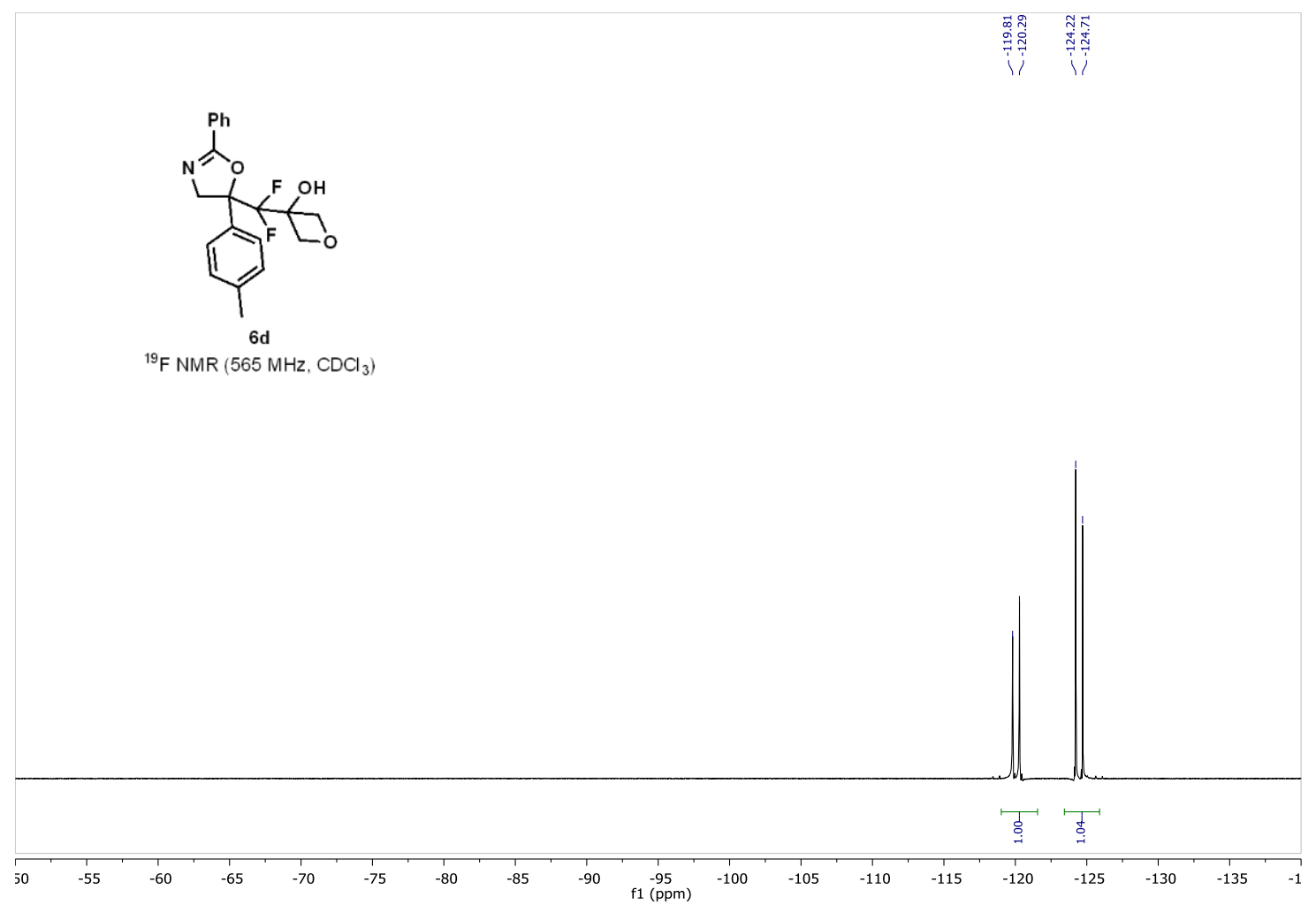

